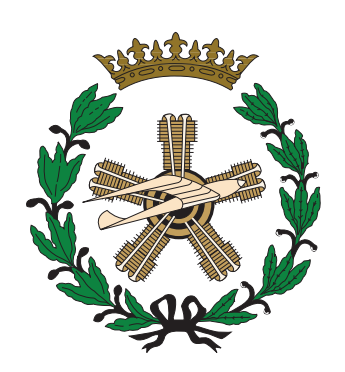

\title{
Optimización paramétrica de las características aerodinámicas de una aeronave de configuración geométrica no convencional definida con alas convergentes
}

Tesis Doctoral

Javier Pérez Álvarez

Ingeniero Aeronáutico

Marzo, 2017 

IDR/UPM

ESCUELA TÉCNICA SUPERIOR DE INGENIEROS AERONÁUTICOS

\title{
Optimización paramétrica de las características aerodinámicas de una aeronave de configuración geométrica no convencional definida con alas convergentes
}

TESIS DOCTORAL

\author{
Autor: \\ JAVIER PÉREZ ÁLVAREZ \\ Ingeniero Aeronáutico
}

Dirigida por:

CRISTINA CUERNO REJADO

Doctor Ingeniero Aeronáutico

Marzo, 2017 



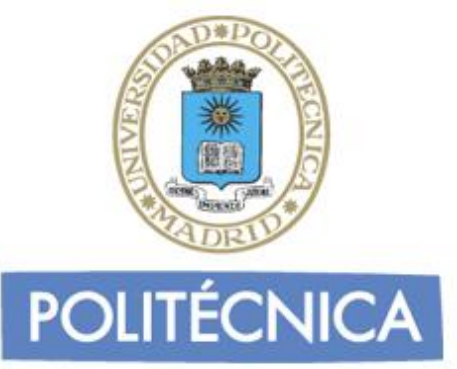

Tribunal nombrado por el Sr. Rector Magfco. de la Universidad Politécnica de Madrid, el día. de. .de 2017.

Presidente:

Vocal:

Vocal:

Vocal:

Secretario:

Suplente:

Suplente:

Realizado el acto de defensa y lectura de la Tesis el día. de. de 2017 en la E.T.S.I. Aeronáuticos.

Calificación

EL PRESIDENTE LOS VOCALES 

A mi mujer Toñi por su paciencia más que infinita en estos 23 años. 



\section{Agradecimientos}

Son muchas las personas a quienes debo dar gracias por contribuir, de alguna forma, en la realización de este trabajo, por lo que estoy seguro, y por eso pido disculpas de ante mano, que me olvidaré de alguna.

En primer lugar me gustaría dar las gracias a mis codirectores de tesis, el profesor José Meseguer y la profesora Cristina Cuerno, quienes me han dado la oportunidad de poder completar esta etapa proporcionando la idea central de la Tesis y siendo comprensivos con las dificultades que han ido apareciendo en el camino. A pesar de sus múltiples ocupaciones, la profesora Cristina Cuerno siempre ha tenido tiempo, incluso en vacaciones, para mis consultas y dudas, manteniendo siempre una actitud positiva, su inestimable ayuda, su paciencia y su generosidad siempre han estado ahí sin reservas. Son innumerables las veces que el Profesor José Meseguer ha puesto su gran conocimiento a mi servicio y me ha brindado su ayuda, su consejo y su guía académica, por eso y por mucho más, su fallecimiento ha significado para todos nosotros una pérdida irrecuperable tanto humana como académica, y a pesar de que cuestiones administrativas (que a día de hoy todavía no puedo ni quiero entender) no han permitido que su nombre aparezca como codirector de la Tesis es mi deber y mi obligación hacerlo constar.

En segundo lugar es muy importante, al menos para mí, agradecer a Álvaro Cuerva y a Oscar López los años de ayuda, consejos y de amistad, con ellos las tentaciones de perder el rumbo de la honradez académica son mucho menores, siempre han sido y serán un ejemplo a seguir.

A todos mis compañeros del área de Expresión Gráfica en la Ingeniería quienes, incluso después de 22 años de docencia, siguen estando ahí y eso que, con mi personalidad, a veces se lo pongo muy difícil.

A todo el personal laboral, personal de administración y servicios y compañeros, sin excepción, del Instituto Universitario de Microgravedad "Ignacio Da Riva" (especialmente a los tres de las ocho) quienes sin su ayuda muchas de las tareas necesarias para la realización de la Tesis hubieran sido imposibles de llevar a cabo. No quisiera personalizar en nadie pero es necesario reconocer que la desinteresada ayuda y predisposición de Carlos, Javi, Manolo, Luis, Patricia y Donato es incuestionable, me pregunto a cuantos compañeros han ayudado y ayudarán

Y por último, no me puedo olvidar de mi familia, especialmente a mis padres quienes han fallecido recientemente y no podrán ver este final y por supuesto a Toñi, quien al fin y al cabo es siempre ella la que más celebra y se alegra con mis triunfos y más sufre con mis fracasos, lo intentaría pero sé que es imposible encontrar palabras de agradecimiento para ella. 



\section{Resumen}

En los últimos 50 años una de las principales áreas de innovación en el campo del diseño aeronáutico es la búsqueda de mejoras que optimicen la eficiencia aerodinámica, la eficiencia estructural y el peso de la aeronave.

Hasta la fecha, muchos de los avances destinados a la mejora de estos parámetros, en la aviación comercial, se han realizado conservando la configuración convencional, es decir, aeronaves de fuselaje esbelto, alas en situación media o baja, estabilizadores vertical y horizontal unidos al cono de cola y motores montados bajo ala o unidos en la parte trasera del fuselaje.

Por otro lado existe una alternativa que pasa por apostar por el desarrollo de configuraciones geométricas no convencionales, las cuales ya son consideradas como opciones reales por las grandes empresas aeronáuticas. Es este el caso de un modelo de configuración no convencional, denominado avión de alas convergentes, que consiste en un diseño que utiliza dos alas, una delantera y otra trasera ambas unidas en tándem de tal manera que tienen forma de diamante tanto vistas en planta como en alzado.

A juicio del autor, es un reto conseguir que las compañías aéreas sean partícipes en favorecer el entorno social para hacer atractivos al pasajero cambios tan significativos en la morfología de las aeronaves. Sin embargo existe una alternativa con mayor predisposición a la implantación de estos cambios como es el caso de los sistemas de aeronaves no tripuladas (UAS). Este sector es uno de los más dinámicos dentro de la industria aeroespacial, y se prevé que el mercado sea el doble en una década, por lo que esta situación hace de estos sistemas un marco excelente para la investigación, desarrollo y ejecución de nuevas configuraciones, no convencionales, más fáciles de implementar que en la aviación comercial.

Con esta idea, se ha realizado la optimización aerodinámica de una configuración geométrica no convencional del tipo de alas convergentes, realizada por medio del estudio paramétrico de diferentes configuraciones de modelos físicos de ensayos. Cada modelo de ensayo está caracterizado por parámetros geométricos que definen la posición relativa del ala trasera con respecto a la delantera, estos parametros pueden sustituirse por variación en los valores del ángulo diedro y ángulo de flecha de dicha ala, permitiendo, así, la definición de 25 configuraciones de ensayo diferentes.

Para la medida de los coeficientes aerodinámicos del sistema se ha realizado ensayos en túnel aerodinámico, donde los datos presentados corresponden a los coeficientes de sustentación, $C_{L}$, resistencia, $C_{D}$, resistencia

inducida, $C_{D i}$, parámetro de potencia mínima requerida, $C_{L}^{3 / 2} / C_{D}$, y la eficiencia aerodinámica, $E$. Los resultados muestran la influencia sobre los coeficientes aerodinámicos de los parámetros de ángulo de flecha y de ángulo diedro del ala trasera.

Además de lo anterior, se ha elaborado un modelo numérico simple basado en aproximar las características aerodinámicas de cada ala por medio de una línea o herradura de torbellinos, con el propósito de obtener resultados rápidos, sea cual sea el valor de las variables de estudio, y que facilite el análisis de sensibilidad de parámetros, propio de fases iniciales de diseño conceptual en un proyecto de aeronave. 



\begin{abstract}
In the last 50 years one of the main areas of innovation in the field of aeronautical design is the search for improvements to optimize the aerodynamic efficiency, the structural efficiency and the weight of the aircraft.

To date, many of the advances made to improve these parameters in commercial aviation have been carried out in a conventional configuration, in other words, slender fuselage aircraft, wings in medium or low position, vertical and horizontal stabilizers attached to Tail cone and engines mounted under wing or attached to the rear of the fuselage.

On the other hand there is an alternative which goes through to opt for the development of non-conventional geometric configurations, which are already considered as real options by large aeronautical companies. This is the case of an unconventional configuration model, called joined-wing aircraft, which consists of a design that uses two wings, one front and one rear, both tandem joined in such way that they have diamond shape view in front and top views.
\end{abstract}

In the opinion of the author, it is a challenge to get the airlines to participate to favour the social environment to make attractive, to the passenger, changes so significant in the morphology of the aircraft. However, there is an alternative with a greater predisposition to the implementation of these changes as it is the case of Unmanned Aircraft Systems (UAS).

This sector is one of the most dynamic in the aerospace industry, and it is expected that the market will be double in a decade, so this situation makes these systems an excellent framework for research, development and implementation of these new non-conventional configurations, easier to implement than commercial aviation.

With this idea, the aerodynamic optimization of an unconventional geometric configuration of the joined-wing has been conducted by the parametric study of different configurations of physical models of tests. Each test model is characterized by geometric parameters that define the relative position of the rear wing with respect to the front wing.

These parameters can be substituted by variation in the values of the dihedral angle and swept angle of the rear wing, thus allowing the definition of 25 different test configurations.

In order to measure the aerodynamic coefficients of the system, aerodynamic tunnel tests were performed. The data presented correspond to the coefficients of lift, $C_{L}$, drag, $C_{D}$, induced drag, $C_{D i}$, required minimum power parameter, $C_{L}^{3 / 2} / C_{D}$, and the aerodynamic efficiency, $E$. The results show the influence on the aerodynamic coefficients of the angle parameters of the swept and dihedral angle of the rear wing.

In addition to the above, a simple numerical model has been developed based on the approximation of the aerodynamic characteristics of each wing by means of replacing the wing vorticity with a horseshoe of vortices, in order to obtain fast results, whatever the value of the study variables and, in the other way, to facilitate the sensitivity analysis of parameters, typical of initial phases of conceptual design in an aircraft project. 



\section{Índice general}

Índice general I

Índice de figuras

$\begin{array}{ll}\text { Índice de tablas } & \text { XVII }\end{array}$

$\begin{array}{lll}\text { Nomenclatura } & \text { XVIII }\end{array}$

1 Introducción $\quad 1$

1.1. Objetivos de la Tesis . . . . . . . . . . . . . . . . . . 9

1.2. Organización de la Tesis . . . . . . . . . . . . . . . . . . . 10

1.3. Publicaciones realizadas . . . . . . . . . . . . . . . . 12

2 Antecedentes $\quad 15$

2.1. Introducción. . . . . . . . . . . . . . . . . . 15

2.2. El concepto de aeronave de alas convergentes. . . . . . . . . . . 15

2.3. Influencia en la estructura de las alas . . . . . . . . . . . . . . . . . . 20

2.4. Aerodinámica de la aeronave de alas convergentes. . . . . . . . . . . . 26

3 Influencia de la configuración sobre los coeficientes aerodinámicos. 29

3.1. Introducción. . . . . . . . . . . . . . . . . . . . . . . 29

3.2. Influencia en el coeficiente de sustentación. . . . . . . . . . . . . 29

3.3. Influencia en el coeficiente de resistencia inducida. . . . . . . . . . . . 35

4 Ensayos para la determinación de los coeficientes aerodinámicos $\quad 45$

4.1. Introducción. . . . . . . . . . . . . . . . . . 45

4.2. Modelo físico de ensayo . . . . . . . . . . . . . . . . . . 46

4.3. Equipos de medida . . . . . . . . . . . . . . . . . 53

4.3.1. Túnel aerodinámico A9 . . . . . . . . . . . . . . . 53

4.3.2. Sensor célula de carga Fuerza/Par Delta SI-330-30 . . . . . . . 55

4.3.3. Equipo de anemometría de hilo caliente . . . . . . . . . 56

4.4. Disposición del modelo en la cámara de ensayo del túnel A9 y parámetros de ensayo . . . . . . . . . . . . . . . . . . 57

4.4.1. Montaje del modelo de ensayo en la cámara del túnel A9 . . . 57

4.4.2. Parámetros de ensayo . . . . . . . . . . . . . . 57 
4.5. Resultados de ensayo . . . . . . . . . . . . . . . . . . 62

$\begin{array}{lll}5 & \text { Modelo numérico simplificado } & 77\end{array}$

5.1. Introducción . . . . . . . . . . . . . . . . . . 77

5.2. Modelo de líneas sustentadoras . . . . . . . . . . . . . . . . 78

5.2.1. Definición del modelo de linea sustentadora . . . . . . . . . . 78

5.2.2. Parámetros geométricos y sistemas de referencia . . . . . . . . 80

5.2.3. Cálculo de velocidades inducidas . . . . . . . . . . . . 83

5.2.4. Cálculo de las fuerzas aerodinámicas . . . . . . . . . . . . . 95

5.2.5. Comparación de resultados numéricos y experimentales . . . . 103

6 Conclusiones y líneas de investigación futuras $\quad 107$

$\begin{array}{ll}\text { Bibliografía } & 117\end{array}$

Apéndices

A Modelo analítico y geométrico. Aplicación al modelo de alas convergentes

A.1. Introducción . . . . . . . . . . . . . . . . . . . 125

A.2. El modelo analítico de Letcher . . . . . . . . . . . . . . . . . . . . 125

A.3. El modelo geométrico de DeYoung. . . . . . . . . . . . . . . . . . . 130

A.4. Aplicación del método para el modelo de alas convergentes. . . . . . . . 131

B Calibración túnel A9 $\quad 139$

B.1. Introducción. . . . . . . . . . . . . . . . . . . . . . 139

B.2. Procedimiento de calibración. . . . . . . . . . . . . . . . . 139

C Resultados de ensayo $1 \quad 143$

C.1. Introducción. . . . . . . . . . . . . . . . . . . . . . 143

C.2. Coeficientes aerodinámicos en función del ángulo ángulo de ataque, $\alpha .145$

C.3. Coeficientes aerodinámicos en función del coeficiente de resistencia,

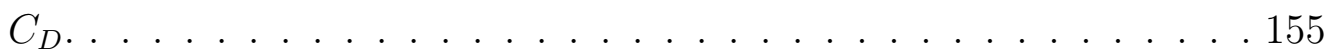

C.4. Coeficientes aerodinámicos en función del coeficiente de sustentación,

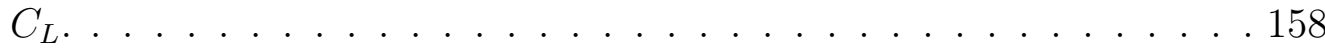

$\begin{array}{lr}\text { D Resultados de ensayo } 2 & 161\end{array}$

D.1. Introducción. . . . . . . . . . . . . . . . . . . 161

D.2. Coeficientes aerodinámicos en función del ángulo diedro, $\delta_{R}$, y del ángulo de flecha, $\varphi_{R}$, del ala trasera para ángulos de ataque, $\alpha=$ $\left[-14^{\circ}, 15^{\circ}\right]$. . . . . . . . . . . . . . . . . . 162

$\begin{array}{lr}\text { E Resultados modelo numérico } & \mathbf{1 8 5}\end{array}$

E.1. Introducción. . . . . . . . . . . . . . . . . . . 185

E.2. Coeficientes aerodinámicos en función del ángulo ángulo de ataque, $\alpha .185$ 


\section{Índice de figuras}

1.1. Variación del precio del combustible de aviación [Jupp 2016] . . . . . . . . 2

1.2. Previsión temporal de la reducción de emisiones de $\mathrm{CO}_{2}$ en función de acciones ejecutadas [Tyler 2015]. . . . . . . . . . . . . . . 3

1.3. Representación artística del concepto de aeronave con configuración box wing de Lockheed Martin (CNASA/Lockheed Martin). . . . . . . . . . 6

1.4. Representación esquemática de aeronave con configuración de alas convergentes. . . . . . . . . . . . . . . . . 7

1.5. Representación esquemática de diferentes formas en planta de UAS. . . . 7

2.1. Foto de reconstrucción del planeador diseñado por Reinhold Platz en 1920, donde se pueden observar los dos largueros en cruz y las alas delantera y trasera [Platz 1924] . . . . . . . . . . . . . . . . 16

2.2. Aeronave de alas múltiples diseñada y construida por Ben Brown en 1932 [Wolkovitch 1982]. . . . . . . . . . . . . . . . . 16

2.3. Modelo de ensayo de aplicaciones agrícolas de Elbert L. Rutan medido en Langley [Chambers 2005]. . . . . . . . . . . . . . . . . . . . . 17

2.4. El investigador Mike Durham con el modelo de configuración de alas convergentes para el estudio de flameo en vehículos con alas de gran alargamiento [Chambers 2005].

2.5. NASA-AD1 modificado en dos modelos de alas convergentes de ensayo en túnel [Smith and Stonum 1989]. . . . . . . . . . . . . . . . 18

2.6. Imagen artística del modelo de aeronave de alas convergentes Boeing EX [Chambers 2005]. . . . . . . . . . . . . . . . . . . . . . . . 18

2.7. Vista superior de la aeronave de alas convergentes Boeing SensorCraft [Nangia and Palmer 2006]. . . . . . . . . . . . . . . . . . . 19

2.8. Modelo de alas convergentes "Guizhou Soar Dragon" (C)www.MilitaryFactory.com). . . . . . . . . . . . . . .

2.9. Distribución de espesores del cajón de torsión según Samuels [Samuels 1982]: (a) modelo de referencia; (b) modelo de alas convergentes. Las zonas sombreadas representan la distribución de esfuerzos sobre el cajón de torsión del ala . . . . . . . . . . . . . . . . . . 21

2.10. Esquema de descomposición de la fuerza de sustentación, $L$, en fuerzas paralelas y normales al plano del eje de flexión definido por la unión de los puntos 1/4 de las secciones en el encastre del ala delantera y del ala trasera. 
2.11. Peso de la estructura de las superficies sustentadoras, $W_{S}$, en función del alargamiento, $\Lambda$, y de la envergadura efectiva, $b$, para el modelo de ala convergentes y para el modelo convencional [Wolkovitch 1986]. . . . . . . 22

2.12. Relación entre el peso de la estructura de las superficies sustentadoras, $W_{s j w} / W_{\text {sref }}$ en función de la relación de envergaduras, $B$, para el modelo de alas convergentes y para el modelo convencional [Wolkovitch 1986]. . . 23

2.13. Esquema general de la definición geométrica del parámetro cuerda promedio d, [Wolkovitch 1986]. . . . . . . . . . . . . . . . . . 23

2.14. Esquema de cajón de torsión simétrico y no simétrico [Kroo et al. 1991]. 24

2.15. Relación entre el peso del modelo de alas convergentes y el modelo de referencia (aeronave convencional equivalente) con la posición de unión de las dos alas, para cada caso de cajón de torsión (simétrico y no simétrico) y para diferentes posiciones relativas de ala trasera con respecto a delantera, $l_{t},[$ Kroo et al. 1991]. . . . . . . . . . . . . . 25

2.16. Vista en alzado y planta de la representación esquemática del modelo de alas convergentes con los principales parámetros geométricos. . . . . . . .

2.17. Relación entre el coeficiente de resistencia y el parámetro de relación de envergaduras, $B$, para diferentes posiciones relativas de ala trasera con respecto a delantera [Kroo et al. 1991]. Variación del valor del coeficiente de resistencia normalizado con el valor del coeficiente de resistencia para $l_{t}=12 \mathrm{~m} \mathrm{y} B=1 \ldots \ldots \ldots \ldots \ldots \ldots$. . . . . . . . . . . . . . . . .

2.18. Modelo de alas convergentes Houck [Oligney et al. 2008]. . . . . . . . . . 28

3.1. Sección transversal típica del modelo biplano donde se muestra un esquema de los parámetros geométricos característicos: Decalaje $(\delta)$, separación longitudinal (stagger, s) y separación vertical $\left(\right.$ gap,$\left.G_{B}\right) \ldots$. . 30

3.2. Sección transversal típica del modelo de alas convergentes donde se muestra un esquema de los parámetros geométricos de cada ala. . . . . . 31

3.3. Variación del factor de envergadura del monoplano equivalente, $k$, en función de la separación vertical entre el ala delantera y el ala trasera, $G_{B}$, [Stinton 1983; Roedts 2009]. . . . . . . . . . . . . . . 32

3.4. Variación del factor de Munk, $F_{M}$, a lo largo de la envergadura del ala trasera, $b_{R}$, y en función del ángulo diedro de la misma, $\delta_{R} \ldots \ldots$. . . . . 34

3.5. Sección transversal típica del modelo de alas convergentes donde se muestra, esquemáticamente, el efecto de la presencia de las dos alas [Wolkovitch 1985]. . . . . . . . . . . . . . . . .

3.6. Aproximación de Laitone para el valor de interferencia de Prandtl $\sigma$, [Laitone 1990]. . . . . . . . . . . . . . . . . . 36

3.7. Valor teórico del factor de eficiencia de Oswald, e, para la configuración de alas convergentes con álulas (winglet) simétricos e inclinados un ángulo de valor $\pi / 2$ con respecto al ala [Wolkovitch 1986]. . . . . . . . . . . . . 38

3.8. Esquema de la perturbación de la velocidad $U_{\infty}$, cuando la estela se encuentra y no se encuentra alineada con la corriente libre. . . . . . . . .

3.9. Esquema del movimiento del fluido inducido por los torbellinos del ala.

(a) Esquema con enrollamiento de estela y (b) esquema de estela plana [Bos 1987]. . . . . . . . . . . . . . . . . . . 4 41 
3.10. Esquema de transformación de Schwarz-Christoffel para el modelo de alas en forma de diamante. (a) Vista frontal (plano $y-z$ ) del sistema de alas en forma de diamante; (b1) esquema del perfil proyectado, $P$, sobre el plano de Trefftz ; (b2) esquema del perfil transformado sobre el eje real $\xi$.

3.11. Valor teórico del factor de eficiencia de Oswald, e, para la configuración de alas convergentes en forma de diamante calculado con el método analítico de Letcher y el predictivo de DeYoung [DeYoung 1980; Letcher 1972]. . .

4.1. Esquema donde se muestra la unión rígida entre el ala delantera y el ala trasera. El detalle muestra la unión fija del eje al ala delantera y la unión con el ala trasera haciendo uso de un tornillo prisionero para el bloqueo

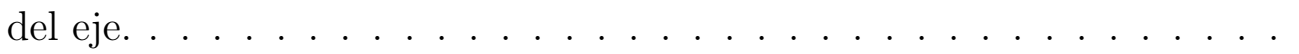

4.2. Variación del coeficiente de sustentación, $c_{l}$, con el ángulo de ataque, $\alpha$, para el perfil NACA0015 y para diferentes valores de número de Reynolds [Cuerva et al. 2006].

4.3. Variación del coeficiente de resistencia, $c_{d}$, con el ángulo de ataque, $\alpha$, para el perfil NACA0015 y para diferentes valores de número de Reynolds [Cuerva et al. 2006]. . . . . . . . . . . . . . . . . . 48

4.4. Máquina de impresión 3D con tecnología FDM. . . . . . . . . . . . 50

4.5. Esquema del modelo de ensayo donde se muestra la unión del ala delantera (1) con el ala trasera $(2,3)$ y fijada en la mesa giratoria (4). La figura muestra dos configuraciones diferentes: $\mathrm{JWC}_{51}$ y $\mathrm{JWC}_{15}$. . . . 50

4.6. Bandeja de impresora 3D con los modelos construidos de la pieza básica para ángulo de flecha del ala trasera, $\varphi_{R}=-10^{\circ}$ y para todo los ángulos diedros del ala trasera, $\delta_{R}=\left[-5^{\circ},-10^{\circ},-15^{\circ},-20^{\circ},-25^{\circ}\right] \ldots \ldots 51$

4.7. Esquema donde se muestra las posiciones del ala trasera con respecto al ala delantera. . . . . . . . . . . . . . . . . 52

4.8. Modelo de ensayo correspondiente a la configuración $\mathrm{JWC}_{15}$, instalado en la cámara de ensayo del túnel A9. . . . . . . . . . . . . . . . . . 53

4.9. Esquema en planta y alzado del Túnel A9: (1) contracción, (2) cámara de ensayos, (3) adaptador y (4) ventiladores. . . . . . . . . . . . 54

4.10. Célula de carga Delta SI-330-30. Transductor (a), cable flexible (b) y sistema de adquisición de datos (c) . . . . . . . . . . . . . .

4.11. Esquema de la sección transversal de la cámara de ensayo donde se muestra la posición del modelo, célula de carga y motor. . . . . . . . . . 56

4.12. Esquema de instalación del equipo de anemometría de hilo caliente DANTEC . . . . . . . . . . . . . . . . . . 57

4.13. Esquema del montaje del modelo en la cámara de ensayo del túnel A9, donde se muestra la distribución de intensidad de turbulencia. (1) Cámara de ensayo, (2) modelo de ensayo, (3) motor giratorio y (4) balanza. 58

4.14. Valores de la convergencia en cada instante en función del tiempo de medida, $t$, para la configuración $\mathrm{JWC}_{15}$ y para un ángulo de ataque $\alpha=0^{\circ}$ : (a) valor medio, $\overline{F_{x}}$, de la fuerza $F_{x}$, (b) valor de la desviación estándar, $\sigma_{F_{x}}$, de la fuerzas $F_{x}$. . . . . . . . . . . . . . . 59

4.15. Valores de la convergencia en cada instante en función del tiempo de medida, $t$, para la configuración $\mathrm{JWC}_{15}$ y para un ángulo de ataque $\alpha=4^{\circ}$ : (a) valor medio, $\overline{F_{x}}$, de la fuerza $F_{x}$, (b) valor de la desviación estándar, $\sigma_{F_{x}}$, de la fuerzas $F_{x}$ 
4.16. Valores de la convergencia en cada instante en función del tiempo de medida, $t$, para la configuración $\mathrm{JWC}_{15}$ y para un ángulo de ataque $\alpha=8^{\circ}$ : (a) valor medio, $\overline{F_{x}}$, de la fuerza $F_{x}$, (b) valor de la desviación estándar, $\sigma_{F_{x}}$, de la fuerzas $F_{x} \ldots \ldots \ldots \ldots \ldots$

4.17. Esquema del proceso de ensayo para la obtención de fuerzas, coeficientes y parámetros aerodinámicos. . . . . . . . . . . . . . .

4.18. Variación de los coeficientes de sustentación, $C_{L}$, y de resistencia, $C_{D}$, en función del ángulo de ataque, $\alpha$, para el ángulo de flecha del ala trasera, $\varphi_{R}=-30^{\circ}$ y para el ángulo diedro del ala trasera, $\delta_{R}=-25^{\circ}$. Número de Reynolds de la corriente libre $R e=250000$. Configuración de referencia $\mathrm{JWC}_{15}$. Las barras verticales representan el valor de la desviación estándar, $\pm \sigma_{C_{L}} \mathrm{y} \pm \sigma_{C_{D}}$, para cada coeficiente respectivamente. 64

4.19. Variación del coeficientes de momento, $C_{M y}$, y de la eficiencia aerodinámica, $E$,en función del ángulo de ataque, $\alpha$, para el ángulo de flecha del ala trasera, $\varphi_{R}=-30^{\circ}$ y para el ángulo diedro del ala trasera, $\delta_{R}=-25^{\circ}$. Número de Reynolds de la corriente libre $\mathrm{Re}=250000$. Configuración de referencia $\mathrm{JWC}_{15}$. Las barras verticales representan el valor de la desviación estándar, $\pm \sigma_{C_{M y}} \mathrm{y} \pm \sigma_{E}$, para cada coeficiente

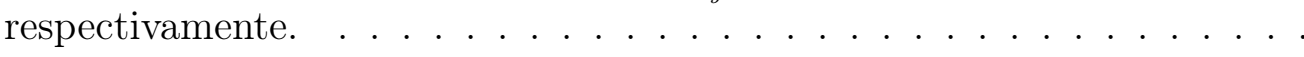

4.20. Variación de los coeficientes de resistencia inducida, $C_{D i}$, y del factor para potencia mínima requerida, $C_{L}^{3 / 2} / C_{D}$, en función del ángulo de ataque, $\alpha$, para el ángulo de flecha del ala trasera, $\varphi_{R}=-30^{\circ}$ y para el ángulo diedro del ala trasera, $\delta_{R}=-25^{\circ}$. Número de Reynolds de la corriente libre $\mathrm{Re}=250000$. Configuración de referencia $\mathrm{JWC}_{15} \ldots \ldots \ldots$

4.21. Variación del coeficiente de sustentación, $C_{L}$, y del coeficiente $C_{L}^{3 / 2}$, en función del coeficiente de resistencia, $C_{D}$, para el ángulo de flecha del ala trasera, $\varphi_{R}=-30^{\circ}$ y para el ángulo diedro del ala trasera, $\delta_{R}=-25^{\circ}$. La pendiente de la línea de trazos es el valor máximo de $C_{L}^{3 / 2} / C_{D}$. Número de Reynolds de la corriente libre $\mathrm{Re}=250000$. Configuración de referencia $\mathrm{JWC}_{15} \ldots \ldots \ldots \ldots \ldots \ldots \ldots \ldots \ldots$

4.22. Variación de la eficiencia aerodinámica, $E$, y del coeficiente de momento, $C_{M y}$, en función del coeficiente de sustentación, $C_{L}$, para el ángulo de flecha del ala trasera, $\varphi_{R}=-30^{\circ}$ y para el ángulo diedro del ala trasera , $\delta_{R}=-25^{\circ}$. Número de Reynolds de la corriente libre Re $=250000$. Configuración de referencia $\mathrm{JWC}_{15}$. . . . . . . . . . . . . .

4.23. Variación de los coeficientes de resistencia inducida, $C_{D i}$, en función del cuadrado del coeficiente de sustentación, $C_{L}$, para el ángulo de flecha del ala trasera, $\varphi_{R}=-30^{\circ}$ y para el ángulo diedro del ala trasera, $\delta_{R}=-25^{\circ}$. Número de Reynolds de la corriente libre $R e=250000$. Configuración de referencia $\mathrm{JWC}_{15} \ldots \ldots \ldots \ldots \ldots$

4.24. Comparación del coeficiente de sustentación, $C_{L}$, del modelo de alas convergentes $(\mathrm{JW})$, de el ala delantera $(\mathrm{FW})$, del ala trasera $(\mathrm{RW})$ y de las suma entre ambas alas $(\mathrm{FW}+\mathrm{RW})$, en función del ángulo de ataque, $\alpha$, para la configuración $\mathrm{JWC}_{51}$, (a) y la configuración $\mathrm{JWC}_{15}$, (b). Número de Reynolds de la corriente libre $\mathrm{Re}=250000 \ldots$. . . . . . . . . . .

4.25. Variación del coeficiente de sustentación, $\overline{C_{L}}$, en función del ángulo de flecha del ala trasera,$\varphi_{R}$, y del ángulo diedro del ala trasera, $\delta_{R}$, para cada ángulo de ataque, $\alpha$. Número de Reynolds de la corriente libre $\operatorname{Re}=250000 \ldots \ldots \ldots \ldots \ldots \ldots \ldots$ 
4.26. Variación del coeficientes de resistencia, $\overline{C_{D}}$, en función del ángulo de flecha del ala trasera, $\varphi_{R}$, y del ángulo diedro del ala trasera, $\delta_{R}$, para cada ángulo de ataque, $\alpha$. Número de Reynolds de la corriente libre $\operatorname{Re}=250000$.

4.27. Variación del coeficiente de resistencia inducida, $\overline{C_{D i}}$, en función del ángulo de flecha del ala trasera, $\varphi_{R}$, y del ángulo diedro del ala trasera, $\delta_{R}$, para cada ángulo de ataque, $\alpha$. Número de Reynolds de la corriente libre $\mathrm{Re}=250000$.

4.28. Variación de la eficiencia aerodinámica, $E$, en función del ángulo de flecha del ala trasera, $\varphi_{R}$, y del ángulo diedro del ala trasera, $\delta_{R}$, para cada ángulo de ataque, $\alpha$. Número de Reynolds de la corriente libre $R e=250000.72$

4.29. Variación del factor para potencia mínima requerida, $C_{L}^{3 / 2} / C_{D}$ (representado en el gráfico con el valor $P$ ), en función del ángulo de flecha del ala trasera, $\varphi_{R}$, y del ángulo diedro del ala trasera , $\delta_{R}$, para cada ángulo de ataque, $\alpha$. Número de Reynolds de la corriente libre $\operatorname{Re}=250000$.

4.30. Variación de la relación de coeficientes, $\overline{C_{D i} / C_{L}^{2}}$, en función del ángulo de flecha del ala trasera, $\varphi_{R}$, y del ángulo diedro del ala trasera , $\delta_{R}$. Número de Reynolds de la corriente libre $R e=250000$. . . . . . . . . .

4.31. Variación del valor del coeficiente de sustentación máximo, $C_{L_{\max }}$, y del valor del coeficiente de resistencia mínimo, $C_{D_{\min }}$, en función del ángulo de flecha del ala trasera, $\varphi_{R}$, y del ángulo diedro del ala trasera, $\delta_{R}$. Número de Reynolds de la corriente libre $R e=250000$. . . . . . . . . . 74

4.32. Variación del valor de la eficiencia aerodinámica máxima, $E_{\max }$, y del valor máximo del factor para potencia mínima requerida, $C_{L}^{3 / 2} / C_{D}$, en función del ángulo de flecha del ala trasera, $\varphi_{R}$, y del ángulo diedro del ala trasera, $\delta_{R}$. Número de Reynolds de la corriente libre $R e=250000$.

5.1. Esquema del modelo de linea sustentadora para la configuración de alas

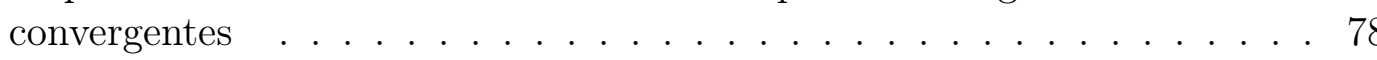

5.2. Modelo geométrico de línea sustentadora para la configuración de alas convergentes

5.3. Vistas normalizadas de la definición geométrica del modelo de linea sustentadora para la configuración de alas convergentes, donde los puntos representan las 25 configuraciones de ensayo.

4. Vistas normalizadas de la definición geométrica adimensional del modelo de línea sustentadora para la configuración de alas convergentes, donde los puntos representan las 25 configuraciones de ensayo. . . . . . . . . . 85

5.5. Esquema de fuerzas sobre cualquier sección arbitraria del ala. . . . . . . 88

5.6. Valores de la circulación adimensional del ala delantera, $G$, y del ala trasera, $g$, en función del ángulo de ataque, $\alpha$, para las configuraciones de ensayo $\operatorname{JWC}_{15}\left(\varphi_{R}=-30^{\circ} \mathrm{y}, \delta_{R}=-25^{\circ}\right)$ y $\operatorname{JWC}_{51}\left(\varphi_{R}=-10^{\circ} \mathrm{y}\right.$,

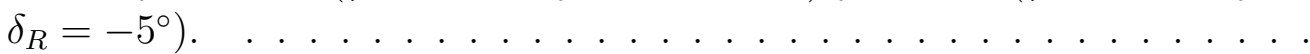

5.7. Variación del valor de la circulación adimensional normalizada, $\bar{G}$, en función del ángulo de flecha del ala trasera,$\varphi_{R}$, y del ángulo diedro del ala trasera,$\delta_{R}$, para cada ángulo de ataque, $\alpha$.

5.8. Variación del valor de la circulación adimensional normalizada, $\bar{g}$, en función del ángulo de flecha del ala trasera , $\varphi_{R}$, y del ángulo diedro del ala trasera,$\delta_{R}$, para cada ángulo de ataque, $\alpha$. 
5.9. Valores de la componentes de la velocidad inducida adimensional en ejes cuerpo para las configuraciones de ensayo $\operatorname{JWC}_{15}\left(\varphi_{R}=-30^{\circ} \mathrm{y}\right.$ , $\left.\delta_{R}=-25^{\circ}\right)$ y $\mathrm{JWC}_{51}\left(\varphi_{R}=-10^{\circ} \mathrm{y}, \delta_{R}=-5^{\circ}\right)$, para un ángulo de ataque $\alpha=5^{\circ}$.

5.10. Valores de la componentes de la velocidad inducida adimensional en ejes cuerpo para las configuraciones de ensayo $\operatorname{JWC}_{15}\left(\varphi_{R}=-30^{\circ} \mathrm{y}\right.$ , $\left.\delta_{R}=-25^{\circ}\right)$ y $\mathrm{JWC}_{51}\left(\varphi_{R}=-10^{\circ} \mathrm{y}, \delta_{R}=-5^{\circ}\right)$, para un ángulo de ataque $\alpha=10^{\circ}$.

5.11. Valores de la componentes de la velocidad inducida adimensional en ejes cuerpo para las configuraciones de ensayo $\operatorname{JWC}_{15}\left(\varphi_{R}=-30^{\circ} \mathrm{y}\right.$ , $\left.\delta_{R}=-25^{\circ}\right)$ y $\mathrm{JWC}_{51}\left(\varphi_{R}=-10^{\circ} \mathrm{y}, \delta_{R}=-5^{\circ}\right)$, para un ángulo de ataque $\alpha=15^{\circ}$.

5.12. Valores del ángulo de ataque efectivo, $\alpha_{e}$, para las configuraciones de ensayo $\mathrm{JWC}_{15}\left(\varphi_{R}=-30^{\circ}\right.$ y $\left.\delta_{R}=-25^{\circ}\right)$ y $\operatorname{JWC}_{51}\left(\varphi_{R}=-10^{\circ} \mathrm{y}\right.$ $\delta_{R}=-5^{\circ}$ ), para un ángulo de ataque $\alpha=5^{\circ}$.

5.13. Valores del ángulo de ataque efectivo, $\alpha_{e}$, para las configuraciones de ensayo $\operatorname{JWC}_{15}\left(\varphi_{R}=-30^{\circ}\right.$ y $\left.\delta_{R}=-25^{\circ}\right)$ y $\operatorname{JWC}_{51}\left(\varphi_{R}=-10^{\circ} \mathrm{y}\right.$ $\delta_{R}=-5^{\circ}$ ), para un ángulo de ataque $\alpha=10^{\circ}$.

5.14. Valores del ángulo de ataque efectivo, $\alpha_{e}$, para las configuraciones de ensayo $\mathrm{JWC}_{15}\left(\varphi_{R}=-30^{\circ}\right.$ y $\left.\delta_{R}=-25^{\circ}\right)$ y $\operatorname{JWC}_{51}\left(\varphi_{R}=-10^{\circ} \mathrm{y}\right.$ $\delta_{R}=-5^{\circ}$ ), para un ángulo de ataque $\alpha=15^{\circ}$.

5.15. Valores de las componentes en dirección del eje $x$ y $z$ de las fuerzas aerodinámicas adimensionales proyectadas sobre ejes cuerpo, $\overline{F_{a}}$, para las configuraciones de ensayo $\mathrm{JWC}_{15}\left(\varphi_{R}=-30^{\circ} \mathrm{y}, \delta_{R}=-25^{\circ}\right) \mathrm{y}$ $\mathrm{JWC}_{51}\left(\varphi_{R}=-10^{\circ}\right.$ y $\left.\delta_{R}=-5^{\circ}\right)$, para un ángulo de ataque $\alpha=5^{\circ} . .100$

5.16. Valores de las componentes en dirección del eje $x$ y $z$ de las fuerzas aerodinámicas adimensionales proyectadas sobre ejes cuerpo, $\overline{F_{a}}$, para las configuraciones de ensayo $\mathrm{JWC}_{15}\left(\varphi_{R}=-30^{\circ} \mathrm{y}, \delta_{R}=-25^{\circ}\right)$ y $\mathrm{JWC}_{51}\left(\varphi_{R}=-10^{\circ}\right.$ y $\left.\delta_{R}=-5^{\circ}\right)$, para un ángulo de ataque $\alpha=10^{\circ}$.

5.17. Valores de las componentes en dirección del eje $x$ y $z$ de las fuerzas aerodinámicas adimensionales proyectadas sobre ejes cuerpo, $\overline{F_{a}}$, para las configuraciones de ensayo $\mathrm{JWC}_{15}\left(\varphi_{R}=-30^{\circ} \mathrm{y}, \delta_{R}=-25^{\circ}\right) \mathrm{y}$ $\mathrm{JWC}_{51}\left(\varphi_{R}=-10^{\circ}\right.$ y $\left.\delta_{R}=-5^{\circ}\right)$, para un ángulo de ataque $\alpha=15^{\circ}$.

5.18. Variación de los valores calculados por el modelo numérico de los coeficientes de sustentación, $C_{L}$, de resistencia inducida, $C_{D i}$, de resistencia, $C_{D}$ y de eficiencia aerodinámica, $E$, en función del ángulo de ataque, $\alpha$, para la configuración de referencia de ensayo $\mathrm{JWC}_{15}$

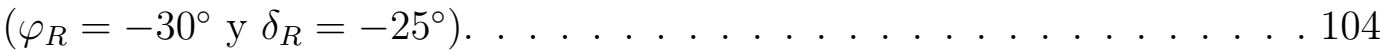

5.19. Variación de los valores de los coeficientes de sustentación, $C_{L}$, de resistencia inducida, $C_{D i}$, de resistencia, $C_{D}$ y de eficiencia aerodinámica, $E$, en función del ángulo de ataque, $\alpha$, para la configuración de referencia de ensayo $\operatorname{JWC}_{15}\left(\varphi_{R}=-30^{\circ}\right.$ y $\left.\delta_{R}=-25^{\circ}\right)$. Comparación entre el modelo numérico y los resultados experimentales. . . . . . . . . . . . . 105

6.1. Esquema de velocidades inducidas y de ángulo de ataque efectivo para una sección transversal genérica en ala delantera y en ala trasera. . . . . 108

6.2. Valores del ángulo de ataque efectivo, $\alpha_{e}$, para las configuraciones de ensayo $\mathrm{JWC}_{15}\left(\varphi_{R}=-30^{\circ} \mathrm{y}, \delta_{R}=-25^{\circ}\right)$ y $\mathrm{JWC}_{51}\left(\varphi_{R}=-10^{\circ} \mathrm{y}\right.$, $\left.\delta_{R}=-5^{\circ}\right)$, para un ángulo de ataque $\alpha=10^{\circ}$. 
6.3. Factor de eficiencia $e$ para el modelo de alas convergentes en función del ángulo diedro del ala trasera, $\delta_{R} \ldots \ldots$. . . . . . . . . . . . . . . 109

6.4. Variación de la relación de coeficientes, $\overline{C_{D i} / C_{L}^{2}}$, en función del ángulo de flecha del ala trasera, $\varphi_{R}$, y del ángulo diedro del ala trasera , $\delta_{R}$. Número de Reynolds de la corriente libre Re $=250000$. . . . . . . . . 110

6.5. Esquema de la tendencia de variación de los coeficientes aerodinámicos de sustentación, $C_{L}$, resistencia, $C_{D}$, y resistencia inducida, $C_{D i}$, al modificar la altura y la separación del ala trasera. . . . . . . . . . . . . . . . . 112

6.6. Imagen que muestra las configuraciones que presentan los mejores valores en los coeficientes aerodinámicos. . . . . . . . . . . . . . . 112

A.1. Esquema de transformación para el modelo de alas en forma de diamante.127

A.2. Esquema de transformación Schwarz-Christoffel para el modelo de alas en forma de diamante. El parámetro a representa el lado del polígono. . . 128

A.3. Esquema de parámetros geométricos: (a) Modelo de alas en forma de diamante; (b) Modelo de alas convergentes . . . . . . . . . . . . . . . . 129

A.4. Modelo de Letcher del factor de eficiencia aerodinámica $e$ en función del ángulo diedro $\delta$ para alas en forma de diamante [Letcher 1972]. . . . . . 130

A.5. Modelo de DeYoung del factor de eficiencia aerodinámica $e$ en función del ángulo diedro $\delta$ para alas en forma de diamante . . . . . . . . . . . 132

A.6. Esquema de transformación Schwarz-Christoffel para el modelo de alas convergentes. El parámetro $L_{F_{t}}$ representa la longitud proyectada en el plano de Trefftz de la semiala delantera, $L_{R_{t}}$ es la longitud proyectada de la semiala trasera y $L_{F 1_{t}}$ corresponde a la longitud proyectada de la parte de la semiala delantera después del punto de unión de ambas alas.

A.7. Modelo de Letcher del factor de eficiencia de Oswald, e, para el modelo de alas convergentes en función del ángulo diedro del ala trasera $\delta_{R}$. . . 135

A.8. Modelo de DeYoung del factor de eficiencia e para el modelo de alas convergentes: (a) en función del ángulo diedro del ala trasera $\delta_{R}, \mathrm{y}$ (b) en función de la separación vertical entre las dos alas $H$. . . . . . . . 137

A.9. Modelo de DeYoung. Factor de eficiencia aerodinámica $\bar{e}=e / e_{12525}$, en función del ángulo diedro del ala delantera $\delta_{F}$ y del ala trasera $\delta_{R}$, para varios valores de $B$ en el modelo de alas convergentes. . . . . . . . . . . 138

B.1. Coordenadas de la matriz de puntos de medida y sistema de referencia para la determinación de la intensidad de turbulencia, $I_{T}$. Dimensiones en mm. . . . . . . . . . . . . . . . . . . . . . . . . . . . . . . 140

B.2. Valores de la convergencia del valor de la media de la velocidad, $\overline{U_{x}}$ (a) y de la desviación estándar de la velocidad, $\sigma_{U_{x}}$ (b) en función del tiempo de medida, $t$, para un punto situado en la fila 5 y columna 6 . . . . . . 141

B.3. Distribución de velocidad en la cámara de ensayo medida con sonda de hilo caliente, $\overline{U_{x h w}}$, en la cámara de ensayo del túnel A9. . . . . . . . . . 141

B.4. Distribución de velocidad en la cámara de ensayo medida con sonda de hilo caliente, $\bar{U}_{x h w}$, comparada con la velocidad medida por tubo Pitot, $\bar{U}_{x p t}$, en la cámara de ensayo del túnel A9. . . . . . . . . . . . . . . . . 142

B.5. Distribución de la intensidad de turbulencia en el plano transversal $y_{T} z_{T}$ de la cámara de ensayo del túnel A9. . . . . . . . . . . . . . . . . . . . 142 
C.1. Variación del coeficiente de sustentación, $C_{L}$, en función del ángulo de ataque, $\alpha$, para ángulos de flecha del ala trasera $\varphi_{R}=-10^{\circ}, \varphi_{R}=-15^{\circ}$, $\varphi_{R}=-20^{\circ}, \varphi_{R}=-25^{\circ}$ y $\varphi_{R}=-30^{\circ}$ y para ángulos diedro del ala trasera $\delta_{R}=-5, \delta_{R}=-10, \delta_{R}=-15, \delta_{R}=-20$ y $\delta_{R}=-25$. Número de Reynolds de la corriente libre $R e=250000$. . . . . . . . . . . . . 145

C.2. Variación del coeficiente de sustentación, $C_{D}$, en función del ángulo de ataque, $\alpha$, para ángulos de flecha del ala trasera $\varphi_{R}=-10^{\circ}, \varphi_{R}=-15^{\circ}$, $\varphi_{R}=-20^{\circ}, \varphi_{R}=-25^{\circ}$ y $\varphi_{R}=-30^{\circ}$ y para ángulos diedro del ala trasera $\delta_{R}=-5, \delta_{R}=-10, \delta_{R}=-15, \delta_{R}=-20$ y $\delta_{R}=-25$. Número de Reynolds de la corriente libre $R e=250000$. . . . . . . . . . . . 146

C.3. Variación del coeficiente de resistencia inducida, $C_{D i}$, en función del ángulo de ataque, $\alpha$, para ángulos de flecha del ala trasera $\varphi_{R}=-10^{\circ}$, $\varphi_{R}=-15^{\circ}, \varphi_{R}=-20^{\circ}, \varphi_{R}=-25^{\circ}$ y $\varphi_{R}=-30^{\circ}$ y para ángulos diedro del ala trasera $\delta_{R}=-5, \delta_{R}=-10, \delta_{R}=-15, \delta_{R}=-20$ у $\delta_{R}=-25$.

Número de Reynolds de la corriente libre $R e=250000$. . . . . . . . . 147

C.4. Variación de la eficiencia aerodinámica, $E=C_{L} / C_{D}$, en función del ángulo de ataque, $\alpha$, para ángulos de flecha del ala trasera $\varphi_{R}=-10^{\circ}$, $\varphi_{R}=-15^{\circ}, \varphi_{R}=-20^{\circ}, \varphi_{R}=-25^{\circ}$ y $\varphi_{R}=-30^{\circ}$ y para ángulos diedro del ala trasera $\delta_{R}=-5, \delta_{R}=-10, \delta_{R}=-15, \delta_{R}=-20$ y $\delta_{R}=-25$. Número de Reynolds de la corriente libre $R e=250000$. . . . . . . . . 148

C.5. Variación del coeficiente de momento de cabeceo, $C_{M y}$, en función del ángulo de ataque, $\alpha$, para ángulos de flecha del ala trasera $\varphi_{R}=-10^{\circ}$, $\varphi_{R}=-15^{\circ}, \varphi_{R}=-20^{\circ}, \varphi_{R}=-25^{\circ}$ y $\varphi_{R}=-30^{\circ} \mathrm{y}$ para ángulos diedro del ala trasera $\delta_{R}=-5, \delta_{R}=-10, \delta_{R}=-15, \delta_{R}=-20$ y $\delta_{R}=-25$. Número de Reynolds de la corriente libre $R e=250000$

C.6. Variación de la relación de coeficientes, $C_{L}^{3 / 2} / C_{D}$, en función del ángulo de ataque, $\alpha$, para ángulos de flecha del ala trasera $\varphi_{R}=-10^{\circ}, \varphi_{R}=-15^{\circ}$, $\varphi_{R}=-20^{\circ}, \varphi_{R}=-25^{\circ}$ y $\varphi_{R}=-30^{\circ} \mathrm{y}$ para ángulos diedro del ala trasera $\delta_{R}=-5, \delta_{R}=-10, \delta_{R}=-15, \delta_{R}=-20$ y $\delta_{R}=-25$. Número de Reynolds de la corriente libre $\mathrm{Re}=250000$.

C.7. Variación del coeficiente de resistencia, $C_{D}$, en función del ángulo de ataque, $\alpha$, para ángulos de flecha del ala trasera $\varphi_{R}=-10^{\circ}, \varphi_{R}=-15^{\circ}$, $\varphi_{R}=-20^{\circ}, \varphi_{R}=-25^{\circ}$ y $\varphi_{R}=-30^{\circ}$ y para ángulos diedro del ala trasera $\delta_{R}=-5, \delta_{R}=-10, \delta_{R}=-15, \delta_{R}=-20$ y $\delta_{R}=-25$. Número de Reynolds de la corriente libre $R e=250000$. . . . . . . . . . . . . 151

C.8. Variación del coeficiente de resistencia inducida, $C_{D i}$, en función del ángulo de ataque, $\alpha$, para ángulos de flecha del ala trasera $\varphi_{R}=-10^{\circ}$, $\varphi_{R}=-15^{\circ}, \varphi_{R}=-20^{\circ}, \varphi_{R}=-25^{\circ}$ y $\varphi_{R}=-30^{\circ}$ y para ángulos diedro del ala trasera $\delta_{R}=-5, \delta_{R}=-10, \delta_{R}=-15, \delta_{R}=-20$ y $\delta_{R}=-25$. Número de Reynolds de la corriente libre $R e=250000$. . . . . . . . . . 152

C.9. Variación de la eficiencia aerodinámica, $E=C_{L} / C_{D}$, en función del ángulo de ataque, $\alpha$, para ángulos de flecha del ala trasera $\varphi_{R}=-10^{\circ}$, $\varphi_{R}=-15^{\circ}, \varphi_{R}=-20^{\circ}, \varphi_{R}=-25^{\circ}$ y $\varphi_{R}=-30^{\circ}$ y para ángulos diedro del ala trasera $\delta_{R}=-5, \delta_{R}=-10, \delta_{R}=-15, \delta_{R}=-20$ y $\delta_{R}=-25$. Número de Reynolds de la corriente libre $R e=250000$. . . . . . . . . . 153 
C.10.Variación de la relación de coeficientes, $C_{L}^{3 / 2} / C_{D}$, en función del ángulo de ataque, $\alpha$, para ángulos de flecha del ala trasera $\varphi_{R}=-10^{\circ}, \varphi_{R}=-15^{\circ}$, $\varphi_{R}=-20^{\circ}, \varphi_{R}=-25^{\circ}$ y $\varphi_{R}=-30^{\circ}$ y para ángulos diedro del ala trasera $\delta_{R}=-5, \delta_{R}=-10, \delta_{R}=-15, \delta_{R}=-20$ y $\delta_{R}=-25$. Número de Reynolds de la corriente libre $\mathrm{Re}=250000$.

C.11.Variación del coeficiente de sustentación, $C_{L}$, en función del coeficiente de resistencia, $C_{D}$, para ángulos de flecha del ala trasera $\varphi_{R}=-10^{\circ}$, $\varphi_{R}=-15^{\circ}, \varphi_{R}=-20^{\circ}, \varphi_{R}=-25^{\circ}$ y $\varphi_{R}=-30^{\circ}$ y para ángulos diedro del ala trasera $\delta_{R}=-5, \delta_{R}=-10, \delta_{R}=-15, \delta_{R}=-20$ y $\delta_{R}=-25$. Número de Reynolds de la corriente libre $R e=250000$. . . . . . . . . . 155

C.12.Variación del cuadrado del coeficiente de sustentación, $C_{L}^{2}$, en función del coeficiente de resistencia, $C_{D}$, para ángulos de flecha del ala trasera $\varphi_{R}=-10^{\circ}, \varphi_{R}=-15^{\circ}, \varphi_{R}=-20^{\circ}, \varphi_{R}=-25^{\circ}$ y $\varphi_{R}=-30^{\circ} \mathrm{y}$ para ángulos diedro del ala trasera $\delta_{R}=-5, \delta_{R}=-10, \delta_{R}=-15, \delta_{R}=-20$ y $\delta_{R}=-25$. Número de Reynolds de la corriente libre $R e=250000$. . . 156

C.13.Variación del coeficiente de sustentación, $C_{L}$, en función del coeficiente de resistencia, $C_{D}$, para ángulos de flecha del ala trasera $\varphi_{R}=-10^{\circ}$, $\varphi_{R}=-15^{\circ}, \varphi_{R}=-20^{\circ}, \varphi_{R}=-25^{\circ}$ y $\varphi_{R}=-30^{\circ} \mathrm{y}$ para ángulos diedro del ala trasera $\delta_{R}=-5, \delta_{R}=-10, \delta_{R}=-15, \delta_{R}=-20$ у $\delta_{R}=-25$. Número de Reynolds de la corriente libre Re $=250000$. . . . . . . . . 157

C.14.Variación de la eficiencia aerodinámica, $E=C_{L} / C_{D}$, en función del coeficiente de sustentación, $C_{L}$, para ángulos de flecha del ala trasera $\varphi_{R}=-10^{\circ}, \varphi_{R}=-15^{\circ}, \varphi_{R}=-20^{\circ}, \varphi_{R}=-25^{\circ}$ y $\varphi_{R}=-30^{\circ} \mathrm{y}$ para ángulos diedro del ala trasera $\delta_{R}=-5, \delta_{R}=-10, \delta_{R}=-15, \delta_{R}=-20$ y $\delta_{R}=-25$. Número de Reynolds de la corriente libre $R e=250000$. . . 158

C.15.Variación del coeficiente de momento de cabeceo, $C_{M y}$, en función del coeficiente de sustentación, $C_{L}$, para ángulos de flecha del ala trasera $\varphi_{R}=-10^{\circ}, \varphi_{R}=-15^{\circ}, \varphi_{R}=-20^{\circ}, \varphi_{R}=-25^{\circ}$ y $\varphi_{R}=-30^{\circ} \mathrm{y}$ para ángulos diedro del ala trasera $\delta_{R}=-5, \delta_{R}=-10, \delta_{R}=-15, \delta_{R}=-20$ y $\delta_{R}=-25$. Número de Reynolds de la corriente libre $R e=250000$.

D.1. Variación del coeficiente de sustentación, $\overline{C_{L}}$, en función del ángulo de flecha del ala trasera,$\varphi_{R}$, y del ángulo diedro del ala trasera , $\delta_{R}$, para cada ángulo de ataque, $\alpha$. Número de Reynolds de la corriente libre $\operatorname{Re}=250000$.

D.2. Variación del coeficiente de sustentación, $\overline{C_{L}}$, en función del ángulo de flecha del ala trasera , $\varphi_{R}$, y del ángulo diedro del ala trasera , $\delta_{R}$, para cada ángulo de ataque, $\alpha$. Número de Reynolds de la corriente libre $\operatorname{Re}=250000$.

D.3. Variación del coeficiente de sustentación, $\overline{C_{L}}$, en función del ángulo de flecha del ala trasera , $\varphi_{R}$, y del ángulo diedro del ala trasera , $\delta_{R}$, para cada ángulo de ataque, $\alpha$. Número de Reynolds de la corriente libre $\operatorname{Re}=250000$.

D.4. Variación del coeficiente de sustentación, $\overline{C_{L}}$, en función del ángulo de flecha del ala trasera,$\varphi_{R}$, y del ángulo diedro del ala trasera , $\delta_{R}$, para cada ángulo de ataque, $\alpha$. Número de Reynolds de la corriente libre $\operatorname{Re}=250000$. 
D.5. Variación del coeficiente de sustentación, $\overline{C_{L}}$, en función del ángulo de flecha del ala trasera , $\varphi_{R}$, y del ángulo diedro del ala trasera , $\delta_{R}$, para cada ángulo de ataque, $\alpha$. Número de Reynolds de la corriente libre $\mathrm{Re}=250000$.

D.6. Variación del coeficiente de resistencia, $\overline{C_{D}}$, en función del ángulo de flecha del ala trasera , $\varphi_{R}$, y del ángulo diedro del ala trasera , $\delta_{R}$, para cada ángulo de ataque, $\alpha$. Número de Reynolds de la corriente libre $\mathrm{Re}=250000$.

D.7. Variación del coeficiente de resistencia, $\overline{C_{D}}$, en función del ángulo de flecha del ala trasera,$\varphi_{R}$, y del ángulo diedro del ala trasera , $\delta_{R}$, para cada ángulo de ataque, $\alpha$. Número de Reynolds de la corriente libre $\mathrm{Re}=250000$.

D.8. Variación del coeficiente de resistencia, $\overline{C_{D}}$, en función del ángulo de flecha del ala trasera , $\varphi_{R}$, y del ángulo diedro del ala trasera , $\delta_{R}$, para cada ángulo de ataque, $\alpha$. Número de Reynolds de la corriente libre $\operatorname{Re}=250000$.

D.9. Variación del coeficiente de resistencia, $\overline{C_{D}}$, en función del ángulo de flecha del ala trasera,$\varphi_{R}$, y del ángulo diedro del ala trasera , $\delta_{R}$, para cada ángulo de ataque, $\alpha$. Número de Reynolds de la corriente libre $\mathrm{Re}=250000$.

D.10.Variación del coeficiente de resistencia, $\overline{C_{D}}$, en función del ángulo de flecha del ala trasera,$\varphi_{R}$, y del ángulo diedro del ala trasera , $\delta_{R}$, para cada ángulo de ataque, $\alpha$. Número de Reynolds de la corriente libre $\operatorname{Re}=250000$.

D.11.Variación del coeficiente de resistencia inducida, $\overline{C_{D i}}$, en función del ángulo de flecha del ala trasera , $\varphi_{R}$, y del ángulo diedro del ala trasera , $\delta_{R}$, para cada ángulo de ataque, $\alpha$. Número de Reynolds de la corriente libre $\operatorname{Re}=250000$.

D.12.Variación del coeficiente de resistencia inducida, $\overline{C_{D i}}$, en función del ángulo de flecha del ala trasera , $\varphi_{R}$, y del ángulo diedro del ala trasera , $\delta_{R}$, para cada ángulo de ataque, $\alpha$. Número de Reynolds de la corriente libre $\mathrm{Re}=250000$.

D.13.Variación del coeficiente de resistencia inducida, $\overline{C_{D i}}$, en función del ángulo de flecha del ala trasera , $\varphi_{R}$, y del ángulo diedro del ala trasera , $\delta_{R}$, para cada ángulo de ataque, $\alpha$. Número de Reynolds de la corriente libre $\operatorname{Re}=250000$.

D.14.Variación del coeficiente de resistencia inducida, $\overline{C_{D i}}$, en función del ángulo de flecha del ala trasera , $\varphi_{R}$, y del ángulo diedro del ala trasera , $\delta_{R}$, para cada ángulo de ataque, $\alpha$. Número de Reynolds de la corriente libre $\mathrm{Re}=250000$.

D.15.Variación del coeficiente de resistencia inducida, $\overline{C_{D i}}$, en función del ángulo de flecha del ala trasera , $\varphi_{R}$, y del ángulo diedro del ala trasera , $\delta_{R}$, para cada ángulo de ataque, $\alpha$. Número de Reynolds de la corriente libre $\mathrm{Re}=250000$.

D.16.Variación del coeficiente de eficiencia aerodinámica, $\bar{E}$, en función del ángulo de flecha del ala trasera,$\varphi_{R}$, y del ángulo diedro del ala trasera , $\delta_{R}$, para cada ángulo de ataque, $\alpha$. Número de Reynolds de la corriente libre $\operatorname{Re}=250000$. 
D.17.Variación del coeficiente de eficiencia aerodinámica, $\bar{E}$, en función del ángulo de flecha del ala trasera,$\varphi_{R}$, y del ángulo diedro del ala trasera , $\delta_{R}$, para cada ángulo de ataque, $\alpha$. Número de Reynolds de la corriente libre $\operatorname{Re}=250000$.

D.18.Variación del coeficiente de eficiencia aerodinámica, $\bar{E}$, en función del ángulo de flecha del ala trasera,$\varphi_{R}$, y del ángulo diedro del ala trasera , $\delta_{R}$, para cada ángulo de ataque, $\alpha$. Número de Reynolds de la corriente libre $\operatorname{Re}=250000$.

D.19.Variación del coeficiente de eficiencia aerodinámica, $\bar{E}$, en función del ángulo de flecha del ala trasera , $\varphi_{R}$, y del ángulo diedro del ala trasera , $\delta_{R}$, para cada ángulo de ataque, $\alpha$. Número de Reynolds de la corriente libre $\operatorname{Re}=250000$.

D.20.Variación del coeficiente de eficiencia aerodinámica, $\bar{E}$, en función del ángulo de flecha del ala trasera,$\varphi_{R}$, y del ángulo diedro del ala trasera , $\delta_{R}$, para cada ángulo de ataque, $\alpha$. Número de Reynolds de la corriente libre $\operatorname{Re}=250000$.

D.21.Variación del coeficiente para potencia mínima requerida normalizado, $\bar{P}=\overline{C_{L}^{3 / 2} / C_{D}}$, en función del ángulo de flecha del ala trasera , $\varphi_{R}, \mathrm{y}$ del ángulo diedro del ala trasera , $\delta_{R}$, para cada ángulo de ataque, $\alpha$. Número de Reynolds de la corriente libre Re $=250000$. . . . . . . . . . 182

D.22.Variación del coeficiente para potencia mínima requerida normalizado, $\bar{P}=\overline{C_{L}^{3 / 2} / C_{D}}$, en función del ángulo de flecha del ala trasera , $\varphi_{R}, \mathrm{y}$ del ángulo diedro del ala trasera,$\delta_{R}$, para cada ángulo de ataque, $\alpha$. Número de Reynolds de la corriente libre Re $=250000$. . . . . . . . . 183

D.23.Variación del coeficiente para potencia mínima requerida normalizado, $\bar{P}=\overline{C_{L}^{3 / 2} / C_{D}}$, en función del ángulo de flecha del ala trasera , $\varphi_{R}, \mathrm{y}$ del ángulo diedro del ala trasera , $\delta_{R}$, para cada ángulo de ataque, $\alpha$. Número de Reynolds de la corriente libre Re $=250000$. . . . . . . . . . . 184

E.1. Variación de los valores de los coeficientes de sustentación, $C_{L}$, de resistencia inducida, $C_{D i}$, de resistencia, $C_{D}$, y de eficiencia aerodinámica, $E$, en función del ángulo de ataque, $\alpha$, para la configuración de ensayo $\mathrm{JWC}_{11}\left(\varphi_{R}=-10^{\circ}\right.$ y $\left.\delta_{R}=-5^{\circ}\right)$. Comparación entre el modelo numérico y los resultados experimentales.

E.2. Variación de los valores de los coeficientes de sustentación, $C_{L}$, de resistencia inducida, $C_{D i}$, de resistencia, $C_{D}$, y de eficiencia aerodinámica, $E$, en función del ángulo de ataque, $\alpha$, para la configuración de ensayo $\mathrm{JWC}_{21}\left(\varphi_{R}=-15^{\circ}\right.$ y $\left.\delta_{R}=-5^{\circ}\right)$. Comparación entre el modelo numérico y los resultados experimentales.

E.3. Variación de los valores de los coeficientes de sustentación, $C_{L}$, de resistencia inducida, $C_{D i}$, de resistencia, $C_{D}$ y de eficiencia aerodinámica, $E$, en función del ángulo de ataque, $\alpha$, para la configuración de ensayo $\mathrm{JWC}_{31}\left(\varphi_{R}=-20^{\circ}\right.$ y $\left.\delta_{R}=-5^{\circ}\right)$. Comparación entre el modelo numérico y los resultados experimentales. 
E.4. Variación de los valores de los coeficientes de sustentación, $C_{L}$, de resistencia inducida, $C_{D i}$, de resistencia, $C_{D}$ y de eficiencia aerodinámica, $E$, en función del ángulo de ataque, $\alpha$, para la configuración de ensayo $\mathrm{JWC}_{41}\left(\varphi_{R}=-25^{\circ}\right.$ y $\left.\delta_{R}=-5^{\circ}\right)$. Comparación entre el modelo numérico y los resultados experimentales. . . . . . . . . . . . . . . . . . . 190

E.5. Variación de los valores de los coeficientes de sustentación, $C_{L}$, de resistencia inducida, $C_{D i}$, de resistencia, $C_{D}$, y de eficiencia aerodinámica, $E$, en función del ángulo de ataque, $\alpha$, para la configuración de ensayo $\mathrm{JWC}_{51}\left(\varphi_{R}=-30^{\circ}\right.$ y $\left.\delta_{R}=-5^{\circ}\right)$. Comparación entre el modelo numérico y los resultados experimentales. . . . . . . . . . . . . . . . . 191

E.6. Variación de los valores de los coeficientes de sustentación, $C_{L}$, de resistencia inducida, $C_{D i}$, de resistencia, $C_{D}$, y de eficiencia aerodinámica, $E$, en función del ángulo de ataque, $\alpha$, para la configuración de ensayo $\mathrm{JWC}_{12}\left(\varphi_{R}=-10^{\circ}\right.$ y $\left.\delta_{R}=-10^{\circ}\right)$. Comparación entre el modelo numérico y los resultados experimentales. . . . . . . . . . . . . . . . . 192

E.7. Variación de los valores de los coeficientes de sustentación, $C_{L}$, de resistencia inducida, $C_{D i}$, de resistencia, $C_{D}$, y de eficiencia aerodinámica, $E$, en función del ángulo de ataque, $\alpha$, para la configuración de ensayo $\mathrm{JWC}_{22}\left(\varphi_{R}=-15^{\circ}\right.$ y $\left.\delta_{R}=-10^{\circ}\right)$. Comparación entre el modelo numérico y los resultados experimentales. . . . . . . . . . . . . . . 193

E.8. Variación de los valores de los coeficientes de sustentación, $C_{L}$, de resistencia inducida, $C_{D i}$, de resistencia, $C_{D}$, y de eficiencia aerodinámica, $E$, en función del ángulo de ataque, $\alpha$, para la configuración de ensayo $\mathrm{JWC}_{32}\left(\varphi_{R}=-20^{\circ}\right.$ y $\left.\delta_{R}=-10^{\circ}\right)$. Comparación entre el modelo numérico y los resultados experimentales. . . . . . . . . . . . . . . . . 194

E.9. Variación de los valores de los coeficientes de sustentación, $C_{L}$, de resistencia inducida, $C_{D i}$, de resistencia, $C_{D}$, y de eficiencia aerodinámica, $E$, en función del ángulo de ataque, $\alpha$, para la configuración de ensayo $\mathrm{JWC}_{42}\left(\varphi_{R}=-25^{\circ}\right.$ y $\left.\delta_{R}=-10^{\circ}\right)$. Comparación entre el modelo numérico y los resultados experimentales. . . . . . . . . . . . . . . 195

E.10.Variación de los valores de los coeficientes de sustentación, $C_{L}$, de resistencia inducida, $C_{D i}$, de resistencia, $C_{D}$, y de eficiencia aerodinámica, $E$, en función del ángulo de ataque, $\alpha$, para la configuración de ensayo $\mathrm{JWC}_{52}\left(\varphi_{R}=-30^{\circ}\right.$ y $\left.\delta_{R}=-10^{\circ}\right)$. Comparación entre el modelo numérico y los resultados experimentales. . . . . . . . . . . . . . 196

E.11.Variación de los valores de los coeficientes de sustentación, $C_{L}$, de resistencia inducida, $C_{D i}$, de resistencia, $C_{D}$, y de eficiencia aerodinámica, $E$, en función del ángulo de ataque, $\alpha$, para la configuración de ensayo $\mathrm{JWC}_{13}\left(\varphi_{R}=-10^{\circ}\right.$ y $\left.\delta_{R}=-15^{\circ}\right)$. Comparación entre el modelo numérico y los resultados experimentales. . . . . . . . . . . . . 197

E.12. Variación de los valores de los coeficientes de sustentación, $C_{L}$, de resistencia inducida, $C_{D i}$, de resistencia, $C_{D}$, y de eficiencia aerodinámica, $E$, en función del ángulo de ataque, $\alpha$, para la configuración de ensayo $\mathrm{JWC}_{23}\left(\varphi_{R}=-15^{\circ}\right.$ y $\left.\delta_{R}=-15^{\circ}\right)$. Comparación entre el modelo numérico y los resultados experimentales. . . . . . . . . . . . . . . 198 
E.13. Variación de los valores de los coeficientes de sustentación, $C_{L}$, de resistencia inducida, $C_{D i}$, de resistencia, $C_{D}$, y de eficiencia aerodinámica, $E$, en función del ángulo de ataque, $\alpha$, para la configuración de ensayo $\mathrm{JWC}_{33}\left(\varphi_{R}=-20^{\circ}\right.$ y $\left.\delta_{R}=-15^{\circ}\right)$. Comparación entre el modelo numérico y los resultados experimentales. . . . . . . . . . . . . . . . 199

E.14.Variación de los valores de los coeficientes de sustentación, $C_{L}$, de resistencia inducida, $C_{D i}$, de resistencia, $C_{D}$, y de eficiencia aerodinámica, $E$, en función del ángulo de ataque, $\alpha$, para la configuración de ensayo $\mathrm{JWC}_{43}\left(\varphi_{R}=-25^{\circ}\right.$ y $\left.\delta_{R}=-15^{\circ}\right)$. Comparación entre el modelo numérico y los resultados experimentales. . . . . . . . . . . . . . . . . 200

E.15. Variación de los valores de los coeficientes de sustentación, $C_{L}$, de resistencia inducida, $C_{D i}$, de resistencia, $C_{D}$, y de eficiencia aerodinámica, $E$, en función del ángulo de ataque, $\alpha$, para la configuración de ensayo $\mathrm{JWC}_{53}\left(\varphi_{R}=-30^{\circ}\right.$ y $\left.\delta_{R}=-15^{\circ}\right)$. Comparación entre el modelo numérico y los resultados experimentales. . . . . . . . . . . . . .

E.16. Variación de los valores de los coeficientes de sustentación, $C_{L}$, de resistencia inducida, $C_{D i}$, de resistencia, $C_{D}$, y de eficiencia aerodinámica, $E$, en función del ángulo de ataque, $\alpha$, para la configuración de ensayo $\mathrm{JWC}_{14}\left(\varphi_{R}=-10^{\circ}\right.$ y $\left.\delta_{R}=-20^{\circ}\right)$. Comparación entre el modelo numérico y los resultados experimentales. . . . . . . . . . . . . . . . 202

E.17.Variación de los valores de los coeficientes de sustentación, $C_{L}$, de resistencia inducida, $C_{D i}$, de resistencia, $C_{D}$, y de eficiencia aerodinámica, $E$, en función del ángulo de ataque, $\alpha$, para la configuración de ensayo $\mathrm{JWC}_{24}\left(\varphi_{R}=-15^{\circ}\right.$ y $\left.\delta_{R}=-20^{\circ}\right)$. Comparación entre el modelo numérico y los resultados experimentales. . . . . . . . . . . . . . . . 203

E.18. Variación de los valores de los coeficientes de sustentación, $C_{L}$, de resistencia inducida, $C_{D i}$, de resistencia, $C_{D}$, y de eficiencia aerodinámica, $E$, en función del ángulo de ataque, $\alpha$, para la configuración de ensayo $\mathrm{JWC}_{34}\left(\varphi_{R}=-20^{\circ}\right.$ y $\left.\delta_{R}=-20^{\circ}\right)$. Comparación entre el modelo numérico y los resultados experimentales. . . . . . . . . . . . . . . . . . 204

E.19. Variación de los valores de los coeficientes de sustentación, $C_{L}$, de resistencia inducida, $C_{D i}$, de resistencia, $C_{D}$, y de eficiencia aerodinámica, $E$, en función del ángulo de ataque, $\alpha$, para la configuración de ensayo $\mathrm{JWC}_{44}\left(\varphi_{R}=-25^{\circ}\right.$ y $\left.\delta_{R}=-20^{\circ}\right)$. Comparación entre el modelo numérico y los resultados experimentales. . . . . . . . . . . . . . 205

E.20. Variación de los valores de los coeficientes de sustentación, $C_{L}$, de resistencia inducida, $C_{D i}$, de resistencia, $C_{D}$, y de eficiencia aerodinámica, $E$, en función del ángulo de ataque, $\alpha$, para la configuración de ensayo $\mathrm{JWC}_{54}\left(\varphi_{R}=-30^{\circ}\right.$ y $\left.\delta_{R}=-20^{\circ}\right)$. Comparación entre el modelo numérico y los resultados experimentales. . . . . . . . . . . . . 206

E.21. Variación de los valores de los coeficientes de sustentación, $C_{L}$, de resistencia inducida, $C_{D i}$, de resistencia, $C_{D}$, y de eficiencia aerodinámica, $E$, en función del ángulo de ataque, $\alpha$, para la configuración de ensayo $\mathrm{JWC}_{15}\left(\varphi_{R}=-10^{\circ}\right.$ y $\left.\delta_{R}=-25^{\circ}\right)$. Comparación entre el modelo numérico y los resultados experimentales. . . . . . . . . . . . . 207 
E.22. Variación de los valores de los coeficientes de sustentación, $C_{L}$, de resistencia inducida, $C_{D i}$, de resistencia, $C_{D}$, y de eficiencia aerodinámica, $E$, en función del ángulo de ataque, $\alpha$, para la configuración de ensayo $\mathrm{JWC}_{25}\left(\varphi_{R}=-15^{\circ}\right.$ y $\left.\delta_{R}=-25^{\circ}\right)$. Comparación entre el modelo numérico y los resultados experimentales. . . . . . . . . . . . . . . 208

E.23. Variación de los valores de los coeficientes de sustentación, $C_{L}$, de resistencia inducida, $C_{D i}$, de resistencia, $C_{D}$, y de eficiencia aerodinámica, $E$, en función del ángulo de ataque, $\alpha$, para la configuración de ensayo $\mathrm{JWC}_{35}\left(\varphi_{R}=-20^{\circ}\right.$ y $\left.\delta_{R}=-25^{\circ}\right)$. Comparación entre el modelo numérico y los resultados experimentales. . . . . . . . . . . . . . . 209

E.24. Variación de los valores de los coeficientes de sustentación, $C_{L}$, de resistencia inducida, $C_{D i}$, de resistencia, $C_{D}$, y de eficiencia aerodinámica, $E$, en función del ángulo de ataque, $\alpha$, para la configuración de ensayo $\mathrm{JWC}_{45}\left(\varphi_{R}=-25^{\circ}\right.$ y $\left.\delta_{R}=-25^{\circ}\right)$. Comparación entre el modelo numérico y los resultados experimentales. . . . . . . . . . . . . . . 210

E.25. Variación de los valores de los coeficientes de sustentación, $C_{L}$, de resistencia inducida, $C_{D i}$, de resistencia, $C_{D}$, y de eficiencia aerodinámica, $E$, en función del ángulo de ataque, $\alpha$, para la configuración de ensayo $\mathrm{JWC}_{55}\left(\varphi_{R}=-30^{\circ}\right.$ y $\left.\delta_{R}=-25^{\circ}\right)$. Comparación entre el modelo numérico y los resultados experimentales. . . . . . . . . . . . . . 211 


\section{Índice de tablas}

4.1. Parámetros geométricos para la definición del modelo de ensayo. . . . . 46

4.2. Valor de los parámetros geométricos para la definición del modelo de ensayo. . . . . . . . . . . . . . . . . . . . . . . . . . . . 49

4.3. Configuraciones de estudio en función del ángulo de flecha, $\varphi_{R}$, y del ángulo diedro, $\delta_{R}$, del ala trasera. . . . . . . . . . . . . . . . . 49

4.4. Valores de la distancia adimensional $\overline{l_{t}}$ en función del ángulo de flecha, $\varphi_{R}$ del ala trasera. . . . . . . . . . . . . . . . . . . 52

4.5. Valores de la altura adimensional $\overline{h_{t}}$ en función del ángulo diedro, $\delta_{R}$ del ala trasera. . . . . . . . . . . . . . . . . . . 52

4.6. Valores del rango de medida y de resolución del sensor Delta SI-330-30. 55

4.7. Valor del factor de eficiencia, $e$, para calcular la resistencia inducida, en función del ángulo de flecha, $\varphi_{R}$ y del ángulo diedro, $\delta_{R}$ del ala trasera. . 63

6.1. Resumen de la variación de los coeficientes aerodinámicos con la altura y la separación del ala trasera. . . . . . . . . . . . . . . . . . . . 111

6.2. Tabla resumen donde se muestra en que configuraciones se presentan los valores máximo y mínimo de los coeficientes aerodinámicos . . . . . . . . 111

6.3. Valores de los coeficientes aerodinámicos obtenidos numéricamente comparados con respecto a los obtenidos experimentalmente de la configuración de referencia $\mathrm{JWC}_{15}\left(\delta_{R}=-25^{\circ}, \varphi_{R}=-30^{\circ}\right) \ldots \ldots 114$

A.1. Valores del factor de eficiencia según el modelo de Letcher y el modelo de DeYoung . . . . . . . . . . . . . . . . . . . . . 132 



\section{Nomenclatura}

\section{Acrónimos}

$\mathrm{CO}_{2} \quad$ Dióxido de carbono

AFRL Air Force Research Laboratory

ANA All Nippon Airways

ASTM American Society for Testing and Materials

BWS Best Wing System

CIRA Centro Italiano Ricerche Aerospaziali

CNG Carbon Neutral Growth

DOC Direct Operating Cost

FDM Fused Deposition Modeling

HAPD High Altitude Performance Demostrator

IEEE Instituto Español de Estudios Estratégicos

ISR Intelligence, Surveillance and Reconnaissance

KLM Koninklijke Luchtvaart Maatschappij

LALE Low Altitude Long Endurance

MALE Medium Altitude Long Endurance

OACI Aviación Civil Internacional

PAE Plataforma Aeroespacial Española

SIP Isoparafina de azúcar fermentada hidroprocesada

UAS Unmanned Aircraft Systems

UAV Unmanned Aerial Vehicle 


\section{Letras Griegas}

$\alpha$

$\alpha_{e}$

$\alpha_{i}$

$\delta$

$\delta_{F}$

$\delta_{R}$

$\eta_{c}$

$\eta_{t}$

$\gamma$

$\Lambda$

$\Lambda_{F}$

$\lambda_{F}$

$\Lambda_{L}$

$\lambda_{L}$

$\Lambda_{R}$

$\lambda_{R}$

$\Phi$

$\rho$

$\sigma$

$\sigma_{L}$

$\sigma_{U_{x}}$

$\Gamma$

$\varphi_{F}$

$\varphi_{R}$

\section{Letras Romanas}

$\mathbf{v}_{i}$

V

$\overline{C_{D i}}$

Ángulo de ataque

Ángulo de ataque efectivo

Ángulo de ataque inducido

Ángulo de decalaje

Ángulo diedro del ala delantera

Ángulo diedro del ala trasera

Función de curvatura del perfil

Función de espesor del perfil

Circulación ala trasera

Alargamiento del ala

Alargamiento del ala delantera

Estrechamiento ala delantera

Coordenada del vector de la línea sustentadora sobre el ala delantera

Coordenada del vector de la línea sustentadora sobre el ala trasera

Alargamiento del ala trasera

Estrechamiento ala trasera

Campo potencial de velocidades

Densidad del aire de la corriente libre

Valor de interferencia de Prandtl

Coordenada del vector de las líneas de torbellinos libres

Desviación estándar de la velocidad

Circulación ala delantera

Ángulo de flecha del ala delantera

Ángulo de flecha del ala trasera

Vector velocidad inducida

Vector velocidad local

Coeficiente de resistencia inducida normalizado con la configuración de referencia 
$\overline{C_{D}} \quad$ Coeficiente de resistencia normalizado con la configuración de referencia

$\overline{C_{L}} \quad$ Coeficiente de sustentación normalizado con la configuración de referencia

Cuerda media aerodinámica efectiva

Fuerza aerodinámica adimensional

Circulación adimensional normalizada ala delantera

Circulación adimensional normalizada ala trasera

$\overline{h_{t}}$

Altura relativa del ala trasera adimensional

Posición longitudinal relativa del ala trasera adimensional

$\widehat{C_{D i}}$

Coeficiente de resistencia inducida adimensionalizado con respecto a la longitud no proyectada del ala delantera

$\widehat{C_{D}} \quad$ Coeficiente de resistencia adimensionalizado con respecto a la longitud no proyectada del ala delantera

$\widehat{C_{L}} \quad$ Coeficiente de sustentación adimensionalizado con respecto a la longitud no proyectada del ala delantera

$B \quad$ Parámetro de relación de envergaduras

$b$

Envergadura efectiva

$b_{F} \quad$ Envergadura ala delantera

$b_{R} \quad$ Envergadura ala trasera

$C_{D e} \quad$ Coeficiente de resistencia experimental

$C_{\text {Die }} \quad$ Coeficiente de resistencia inducida experimental

$C_{\text {Din }} \quad$ Coeficiente de resistencia inducida numérico

$C_{D i} \quad$ Coeficiente de resistencia inducida

$C_{D n} \quad$ Coeficiente de resistencia numérico

$C_{D} \quad$ Coeficiente de resistencia

$c_{d} \quad$ Coeficiente de resistencia del perfil

$C_{L e} \quad$ Coeficiente de sustentación experimental

$C_{L n} \quad$ Coeficiente de sustentación numérico

$C_{L} \quad$ Coeficiente de sustentación

$c_{l} \quad$ Coeficiente de sustentación del perfil

$C_{M y} \quad$ Coeficiente de momento de cabeceo 


\begin{tabular}{|c|c|}
\hline$c_{r F}$ & Cuerda en el encastre del ala delantera \\
\hline$c_{r R}$ & Cuerda en el encastre del ala trasera \\
\hline$c_{t F}$ & Cuerda en punta del ala delantera \\
\hline$c_{t R}$ & Cuerda en punta del ala trasera \\
\hline$d$ & Cuerda promedio \\
\hline$E$ & Coeficiente de eficiencia aerodinámica \\
\hline$e$ & Factor de Oswald \\
\hline$e_{t}$ & Factor de Oswald teórico \\
\hline$F_{a}$ & Fuerza aerodinámica \\
\hline$f_{T}$ & Frecuencia de medida \\
\hline$G$ & Circulación adimensional ala delantera \\
\hline$g$ & Circulación adimensional ala trasera \\
\hline$G_{B}$ & Parámetro de gap \\
\hline$H$ & Valor adimensional de la separación vertical entre alas \\
\hline$h_{t}$ & Altura relativa del ala trasera \\
\hline$I_{T}$ & Intensidad de turbulencia \\
\hline$k$ & Factor de envergadura del monoplano equivalente \\
\hline$L$ & Fuerza de sustentación total \\
\hline$L / D$ & Eficiencia aerodinásmica \\
\hline$L_{D}$ & Longitud no proyectada de la semiala delantera \\
\hline$L_{F 1_{t}}$ & $\begin{array}{l}\text { Longitud proyectada plano de Trefftz de la parte de la semiala } \\
\text { delantera después del punto de unión de ambas alas }\end{array}$ \\
\hline$L_{F_{t}}$ & Longitud proyectada en el plano de Trefftz de la semiala delantera \\
\hline$L_{F}$ & Fuerza de sustentación ala delantera \\
\hline$L_{R_{t}}$ & Longitud proyectada en el plano de Trefftz de la semiala trasera \\
\hline$L_{R}$ & Fuerza de sustentación ala trasera \\
\hline$L_{T}$ & Longitud no proyectada de la semiala trasera \\
\hline$l_{t}$ & Posición longitudinal relativa del ala trasera \\
\hline$M$ & Masa añadida \\
\hline$M_{T}$ & Masa por unidad de tiempo \\
\hline
\end{tabular}




$\begin{array}{ll}M F & \text { Factor de Munk } \\ p & \text { Presión } \\ q & \text { Presión dinámica } \\ R & \text { Parámetro de Munk } \\ r & \text { Relación de sustentaciones } \\ S_{F} & \text { Superficie alar del ala delantera } \\ S_{R} & \text { Superficie alar del ala trasera } \\ t_{T} & \text { Tiempo de medida } \\ U_{\infty} & \text { Velocidad de la corriente libre }\end{array}$

\section{Símbolos Matemáticos}

$\Gamma \quad$ Función Gamma

\section{Configuraciones de Ensayo}

$\mathrm{JWC}_{15} \quad$ Configuración de referencia

\section{Otros Símbolos}

$$
\begin{aligned}
& (x, y, z) \quad \text { Sistema de referencia ejes cuerpo } \\
& \left(x_{F L}, y_{F L}, z_{F L}\right) \text { Sistema de referencia ejes ala delantera izquierda } \\
& \left(x_{F R}, y_{F R}, z_{F R}\right) \text { Sistema de referencia ejes ala delantera derecha } \\
& \left(x_{R L}, y_{R L}, z_{R L}\right) \text { Sistema de referencia ejes ala trasera izquierda } \\
& \left(x_{R R}, y_{R R}, z_{R R}\right) \text { Sistema de referencia ejes ala trasera derecha }
\end{aligned}
$$





\section{Capítulo 1}

\section{Introducción}

En los últimos 50 años una de las principales áreas de innovación en el campo del diseño aeronáutico es la búsqueda de mejoras que optimicen la eficiencia aerodinámica, la eficiencia estructural y el peso de la aeronave [Chambers 2005]. Aunque el objetivo de mejorar las características aerodinámicas, y como consecuencia las actuaciones de la aeronave, ha sugerido a los diseñadores la idea de usar configuraciones basadas en alas de gran alargamiento, los problemas del peso de la estructura, incrementados por el aumento de rigidez estructural necesario para cumplir los requisitos exigidos, han limitado la geometría a un diseño tradicional y conservador.

Como es conocido, factores como el incremento en el coste del petróleo y sus derivados (el cual parece estabilizado desde el 2012) [Morrison et al. 2010] y el continuo crecimiento en el mercado de la aviación comercial [Mac 2016], está produciendo un constante impacto en los Costes Directos de Operación (Direct Operating Cost, DOC) incrementándolos entre un $30 \%$ y un $40 \%$, provocando la búsqueda de soluciones innovadoras en el diseño, tanto de las aeronaves como de los sistemas motopropulsores, que permitan una reducción de los costes por medio del uso de sistemas energéticamente mas eficientes [Cuerno-Rejado et al. 2010].

Sin lugar a dudas, una de las principales condiciones para el futuro diseño de aeronaves, es el precio del combustible (Figura 1.1). En un futuro previsible, el queroseno será el único combustible viable para los aviones de pasajeros, debido a su excelente relación entre energía por volumen y peso. Es probable que, en al menos 10 a 20 años vista, e independientemente que la producción de queroseno se realice a partir de combustibles fósiles, biomasa o productos de desecho, el impacto efectivo que el precio del combustible tendrá en los DOC seguro aumentará considerablemente [Jupp 2016].

En la actualidad no existen tecnologías que puedan sustituir al motor a reacción para los viajes de largo recorrido. Según lo indicado por la Organización de Aviación Civil Internacional (OACI), durante 2014 el mayor desarrollo realizado en el estudio de combustibles alternativos de aviación ha sido la aprobación, por la American Society for Testing and Materials (ASTM), de un proceso llamado 
síntesis de isoparafina de azúcar fermentada hidroprocesada (SIP), que fue conocido anteriormente como conversión directa de azúcar a hidrocarbono [Mac 2016].

Durante el 2014 los fabricantes de aeronaves más significativos, conjuntamente con algunas compañías aéreas, han desarrollado iniciativas en la obtención de combustible de avión que minimizarán el uso de combustible tradicional. Las más importantes de ellas han sido, en primer lugar el vuelo del 787 ecoDemonstrator de Boeing, que voló con una mezcla de diésel verde $15 \%$, que es considerablemente utilizado en el transporte terrestre y en segundo, las 10 horas de vuelo que la Koninklijke Luchtvaart Maatschappij (usualmente llamada Royal Dutch Airlines, literalmente del neerlandés Compañía Real de Aviación, KLM) realizó, utilizando un Airbus A330-200 usando una mezcla de $20 \%$ de combustible de aviación alternativo producido a partir de aceite de cocina [Mac 2016].

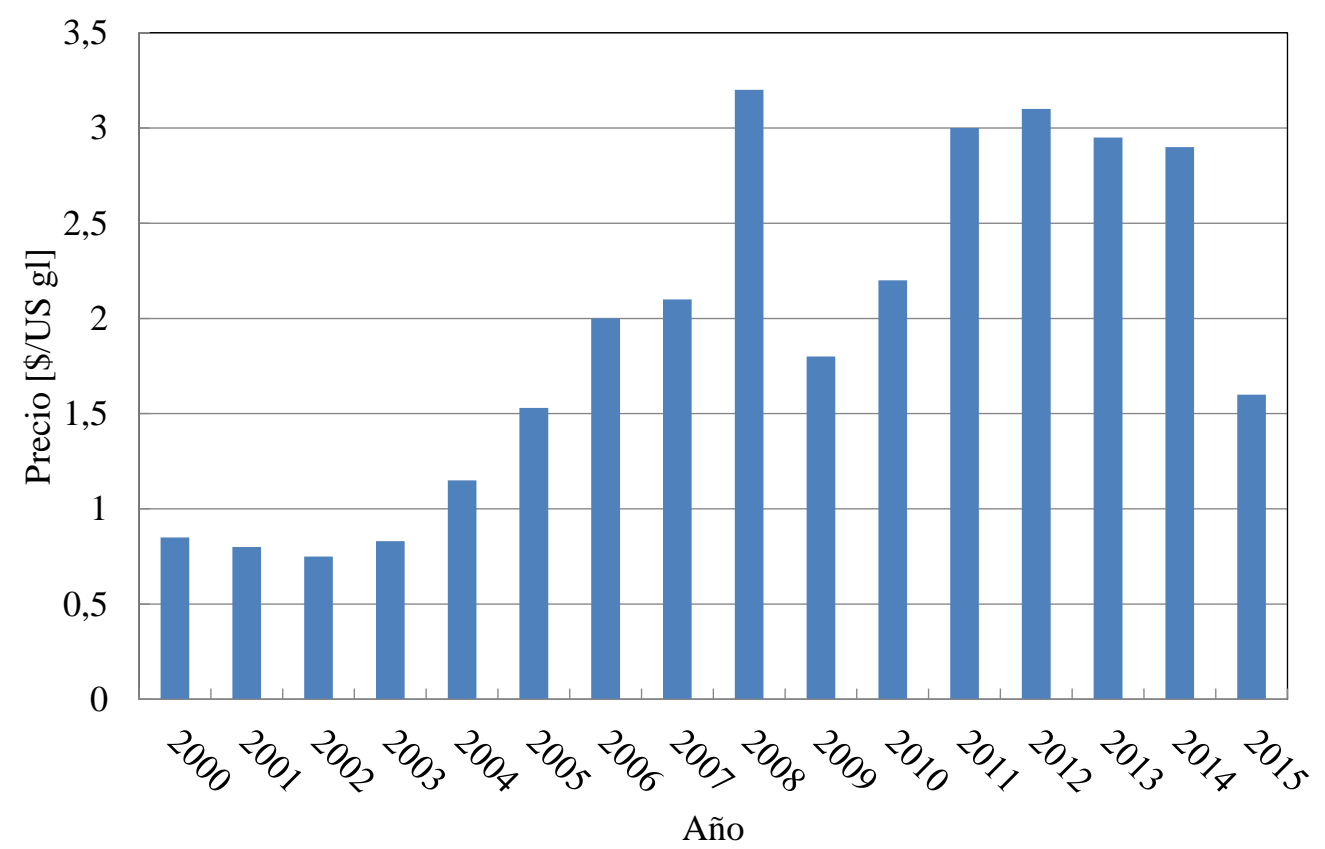

Figura 1.1: Variación del precio del combustible de aviación [Jupp 2016].

Por otro lado existe en la actualidad una creciente preocupación social por el impacto que produce la aviación comercial en el cambio climático, aunque el consenso actual es que la aviación comercial contribuye actualmente alrededor del $2 \%$ al $3 \%$ del dióxido de carbono $\left(\mathrm{CO}_{2}\right)$ producido por el hombre, predicciones futuras señalan un aumento de estos valores de un $15 \%$ a un $30 \%$ dependiendo del crecimiento continuo del tráfico aéreo que se espera, a la vista de la evolución del mercado, supere el doble de pasajeros-kilómetros de hoy en día [Mac 2016], por ejemplo, entre 2009 y 2014, el número de pasajeros en vuelos directos creció a un ritmo del 6,6\% anual, mientras que los pasajeros de conexión crecieron a un ritmo del $3,0 \%$, con una reducción de la cuota de conexión mundial que fue del orden 14,4\% en 2009 al 12,2\% en 2014. Esto se debe tanto a una mayor conectividad directa proporcionada por aerolíneas de bajo coste, y debido a nuevos servicios directos de larga distancia gracias a aeronaves como el Boeing 787 y Airbus A350.

Para minimizar este efecto, en 2009, la industria de la aviación, que comprende tanto aerolíneas y demás empresas relacionadas con la aviación como 
aeropuertos, fabricantes de aviones y proveedores de servicios de navegación aérea, establecieron un compromiso con un enfoque unificado en la reducción de emisiones que abarca tres objetivos [Tyler 2015]:

1. Mejora de la eficiencia de combustible en un promedio de 1,5\% anual hasta 2020 .

2. Limitar las emisiones netas a través del crecimiento neutro en carbono a partir de 2020 (Carbon Neutral Growth 2020, CNG 2020).

3. Reducir las emisiones netas a la mitad para el año 2050, en comparación a 2005.

Estos objetivos tan ambiciosos colocan a la aviación comercial en la vanguardia de las industrias a nivel mundial en la gestión de su impacto sobre el cambio climático.

Para lograr estos tres objetivos, en la reducción de las emisiones de carbono, la industria ha adoptado una estrategia basada en cuatro pilares que comprenden: mejoras en la tecnología, las operaciones aéreas, las infraestructura y medidas económicas del mercado de la aviación comercial. La inversión continua en nuevas aeronaves y mejoras en su eficiencia está ayudando a la industria aeronáutica a cumplir el primer objetivo (Figura 1.2). El segundo y tercer objetivo, sin embargo, son tan significativos que no es posible alcanzarlos sólo por medio del sector de la fabricación de aeronaves y sin la ayuda del resto de los agentes implicados como son las compañías aéreas y los aeropuertos.

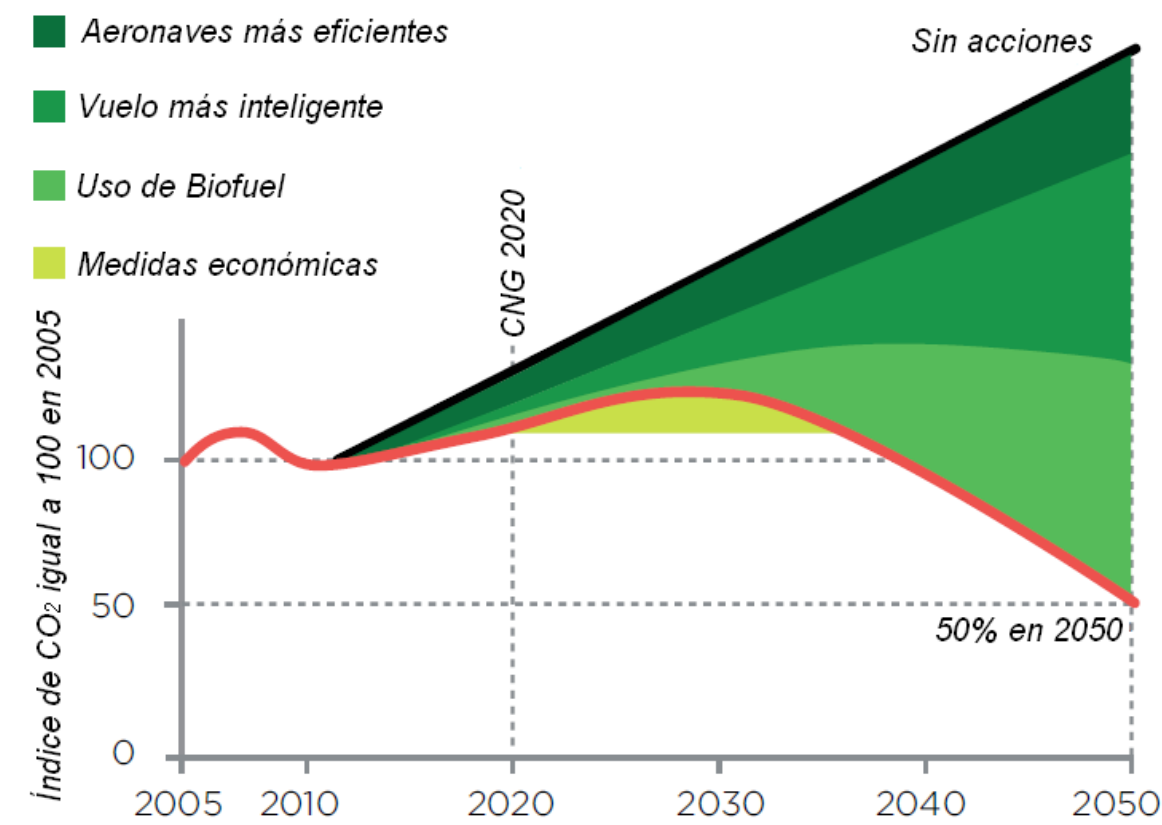

Figura 1.2: Previsión temporal de la reducción de emisiones de $\mathrm{CO}_{2}$ en función de acciones ejecutadas [Tyler 2015].

La eficiencia y por lo tanto la reducción de las emisiones de $\mathrm{CO}_{2}$ de las aeronaves es un área donde se han realizado las mayores mejoras. Con el tiempo, los 
aviones antiguos, menos eficientes se retiran, y aviones nuevos y más eficientes entran en servicio, provocando un aumento en la eficiencia de la flota de las compañías aéreas. Durante 2014 se han producido una serie de avances en este ámbito; cuatro ejemplos de estos avances son:

- Airbus entregó su primer A350 XWB a las líneas aéreas de Qatar el 23 de diciembre de 2014. Este nuevo diseño ofrece una mejora en la eficiencia de aproximadamente el $25 \%$ de las aeronaves de tamaño similar.

- El E-Fan de Airbus hizo su primer vuelo en abril. El avión es un avión eléctrico demostrador libre de emisiones de $\mathrm{CO}_{2}$. El programa tiene el objetivo a largo plazo de la producción de una línea aérea regional híbrida.

- La aeronave Solar Impulse II, propulsada únicamente por energía solar, completó la vuelta al mundo en 2015-2016.

- Boeing entregó el primer 787-9 a ANA (All Nippon Airways) el 29 de julio de 2014. El 787-9 tiene más alcance y puede transportar más pasajeros que el 787-8 que entró en servicio en 2011; ambas aeronaves son $20 \%$ más eficientes que los aviones de tamaño similar.

Con estas ideas los fabricantes de aeronaves tienen que compaginar los requisitos sociales y los requisitos de las compañías aéreas, que como potenciales clientes establecen en el diseño de misiones con una reducción en los DOC.

Hasta la fecha, todos los avances destinados a mejorar la eficiencia de las aeronaves, en la aviación comercial, se han realizado conservando la configuración convencional, es decir, aeronaves de fuselaje esbelto, alas en situación media o baja, estabilizadores vertical y horizontal unidos al cono de cola y motores montados bajo ala o unidos en la parte trasera del fuselaje, aunque todo indica que los niveles de desarrollo tecnológico, donde la inversión en los avances compensa los resultados obtenidos, están alcanzando sus propios límites de productividad significativa. Una prueba clara de ello se puede encontrar en los últimos desarrollos de la empresa Boeing, donde su último modelo el Boeing 787 Dreamliner está diseñado con el objetivo de que el $50 \%$ de la estructura primaria, incluyendo fuselaje y alas, están fabricadas en materiales compuestos, lo que supone, según la empresa, una utilización de un $20 \%$ menos de combustible que cualquier otro avión de su tamaño en misiones similares.

Por otro lado existe una alternativa más clara que pasa por apostar por el desarrollo de configuraciones geométricas no convencionales, las cuales ya son consideradas como opciones reales por las grandes empresas aeronáuticas. El concepto de avión de configuración no convencional de Airbus integra lo que podría ser el transporte aéreo en 2050, o incluso en 2030 si los avances en las tecnologías ya existentes siguen a buen ritmo, como son: alas extremadamente largas y delgadas, motores semiencastrados, colas en forma de U [Mannings 2011]. El resultado es un menor consumo de combustible y una significativa reducción de las emisiones de gases contaminantes.

Uno de los objetivos pasa por modificar aquellos parámetros ligados a las superficies sustentadoras y al propio fuselaje de la aeronave, con la idea 
de maximizar la relación sustentación/resistencia, $L / D$, (eficiencia aerodinámica), como por ejemplo:

- La envergadura: La envergadura de la aeronave debe ser lo más grande posible, aunque si la envergadura aumenta, el peso del ala también aumenta, especialmente si esto se hace sin aumentar las longitudes de cuerda, para mantener el área de la superficie alar al mínimo.

- La resistencia inducida: Para una buena eficiencia es necesario un diseño de aeronave con el objetivo de minimizar este parámetro.

- El borde marginal del ala: Es necesario un buen diseño de punta del ala. Es conocido que el uso de winglets u otros dispositivos aerodinámicos de punta de ala pueden reducir el factor de resistencia inducida aparente; hay autores que relacionan la altura de estos dispositivos con el valor de la envergadura, asegurando que un aumento de esta última tiene el mismo resultado que un ala con envergadura más reducida con winglets [Jones and Lasinski 1980].

- Capa límite laminar: El mantenimiento de flujo laminar sobre las superficies de la aeronave puede ser de gran utilidad para reducir el valor de la resistencia aerodinámica, incluso si el aumento se produce en una proporción relativamente pequeña de estas.

En las últimas décadas se han realizado pruebas de vuelo con éxito para demostrar la posibilidad de mantener mayores áreas de flujo laminar por succión, a través de las superficies, para eliminar el flujo turbulento de baja energía que se produce cerca de la superficie.

Siguiendo esta línea se han realizado varias propuestas en el pasado basadas en dos parámetros principales: el número y posición de las superficies sustentadoras (canard, en tándem, clásica, triplanos, unidas en punta, sin cola) y las soluciones pensadas para la disposición de la carga útil (uno o dos fuselajes, parcialmente en el interior del fuselaje y el ala, completamente en el ala) [Cuerno-Rejado et al. 2010]. Desde el punto de vista de las compañías aéreas muchas de estas configuraciones han sido rechazadas por considerarlas poco atractivas para los pasajeros.

Por otro lado existen modelos de configuración no convencional denominados nonplanar wings que incluyen configuraciones tales como biplanos, box-wing y ring-wing (denominados sistemas cerrados), alas convergentes y alas con winglets [Kroo et al. 1995].

Tales diseños pueden ser de interés debido a su potencial en lo referente a una posible reducción en el valor de la resistencia inducida, que es una limitación clave para muchos aviones, especialmente para el transporte comercial. Sin embargo, varias características son desfavorables, como los efectos sobre la estabilidad y el control, las características de la estela y las implicaciones estructurales del diseño en sí.

La idea original sobre el problema de encontrar configuraciones cuyas prestaciones aerodinámicas mejoren las de las aeronaves convencionales (sobre todo en lo referente a las condiciones para minimizar el valor de resistencia inducida), se atribuye a Prandtl [Prandtl 1924] que introdujo la terminología del Best Wing 


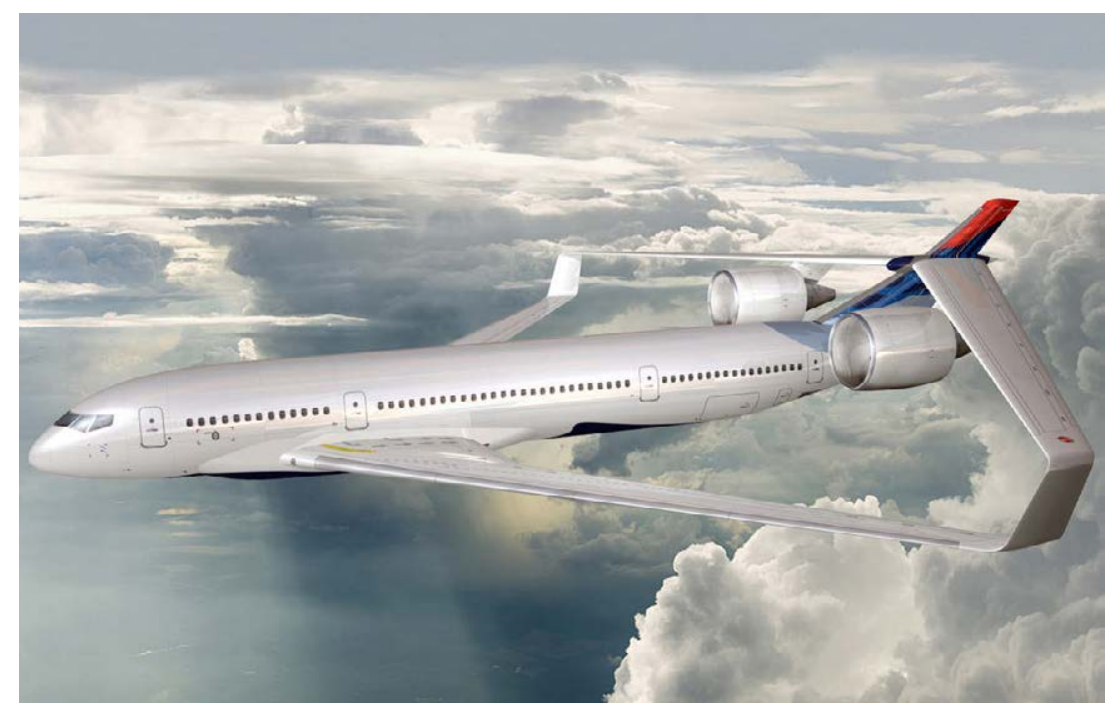

Figura 1.3: Representación artística del concepto de aeronave con configuración box wing de Lockheed Martin (CNASA/Lockheed Martin).

System (BWS) para referirse al modelo en configuración box-wing (Figura 1.3) y para hacer hincapié en sus mejores propiedades en el rendimiento aerodinámico, como la posible reducción de la resistencia inducida y la reducción de peso debido al concepto de ala cerrada.

Prantdl, determinó expresiones aproximadas para cuantificar el valor de la resistencia inducida para este tipo de configuraciones.

Desde ese momento, las propuestas de Prantdl, han servido de punto de partida para el desarrollo de trabajos realizados por diferentes investigadores, con el objetivo de encontrar configuraciones no convencionales que, de la misma manera, presentan ventajas similares, como es el caso de un modelo de configuración no convencional, denominado avión de alas convergentes, que consiste en un diseño que utiliza dos alas, una delantera y otra trasera ambas unidas en tándem de tal manera que tienen forma de diamante tanto vistas en planta como en alzado. En la Figura 1.4 se muestra una representación esquemática de este tipo de configuración.

En la actualidad varios autores han retomado el interés por este tipo de configuración, aunque centrándose en configuraciones de tipo box-wing [Demasi 2007; Demasi et al. 2013; 2015a;b], postulando que su uso en aeronaves actuales podría suponer reducciones en el valor de la resistencia inducida del orden del $20 \%$ al $30 \%$ [Frediani 2005].

A juicio del autor, es y seguirá siendo un reto conseguir que las compañías aéreas sean partícipes en favorecer el cambio social y hacer atractivos, para los pasajeros, cambios tan significativos en la morfología de las aeronaves. Sin embargo existe una alternativa con mayor predisposición a la implantación de estos cambios como es el caso de los sistemas aéreos no tripulados (UAS) donde, como puede observarse en la Figura 1.5, existe ya una gran variabilidad de configuraciones. 


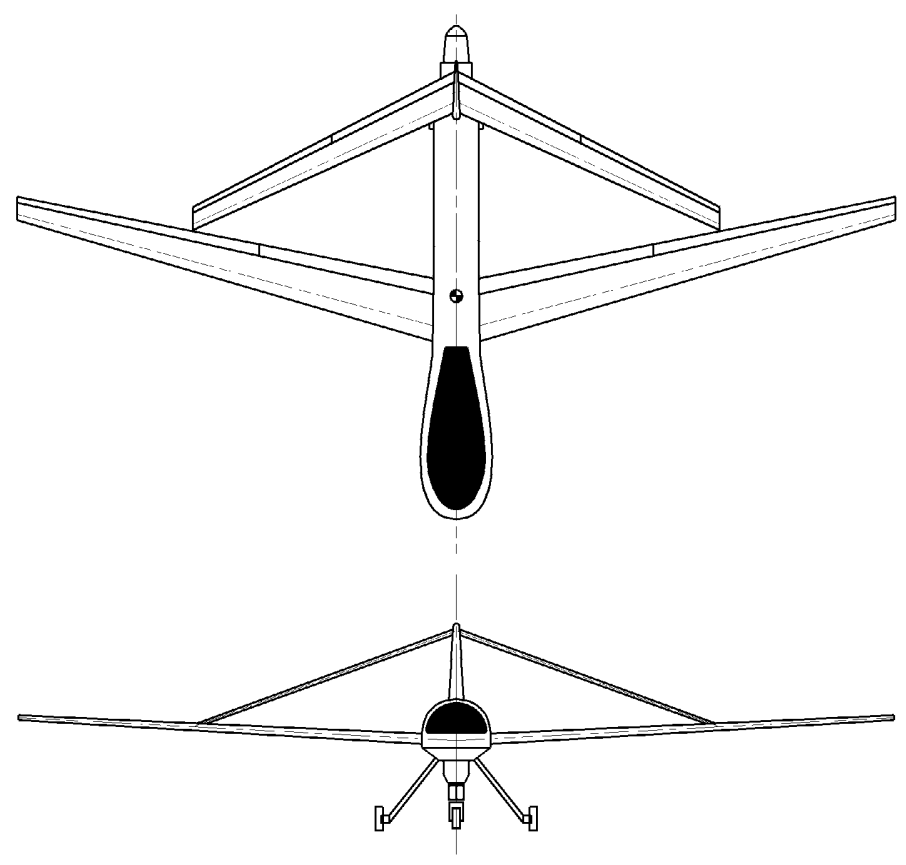

Figura 1.4: Representación esquemática de aeronave con configuración de alas convergentes.

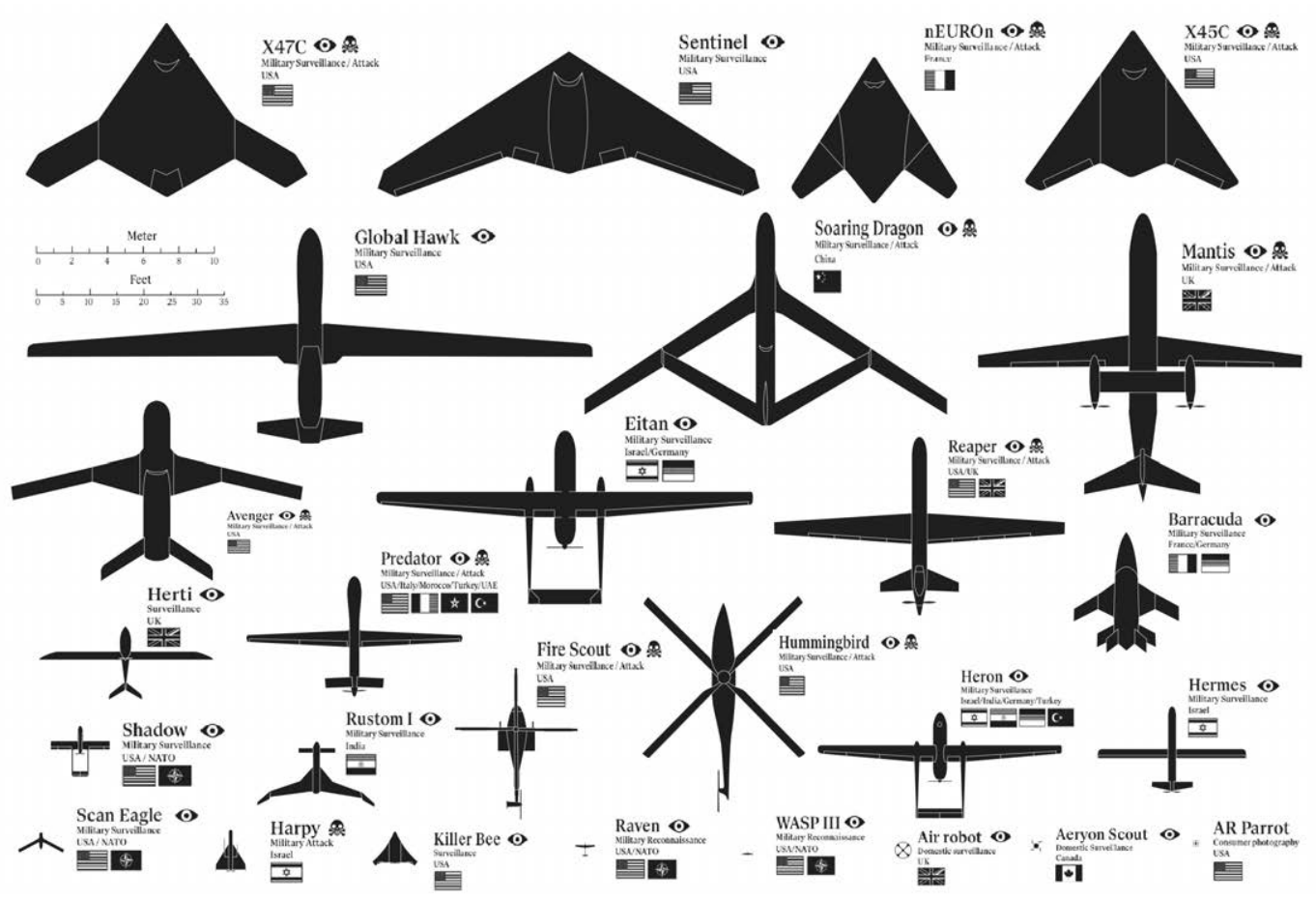

Figura 1.5: Representación esquemática de diferentes formas en planta de UAS.

El sector de los UAS es el más dinámico dentro de la industria aeroespacial, y se prevé que el mercado sea el doble en una década debido, esencialmente, al enorme interés que está suscitando esta tecnología. Son clave en el ámbito militar para actividades ISR (Inteligencia, Vigilancia y Reconocimiento), además de para otras muchas misiones. Todo esto requiere del desarrollo de tecnologías clave como sistemas de sensores, sistemas de comunicaciones, etc. 
Esta situación hace de estos sistemas un marco excelente para la investigación, desarrollo y ejecución de nuevas configuraciones, no convencionales, más fáciles de implementar que en la aviación comercial, donde aparecen mayores dificultades legales (certificación de aeronaves) e incluso, como se ha dicho, problemas de aceptación social. Además las exigencias en las condiciones de ensayo son más fácilmente abordables como condiciones geométricas (dimensiones de la aeronave), similitud del número de Reynolds, entre otras.

Según el Instituto Español de Estudios Estratégicos (IEEE) [Gómez 2001], los Sistemas Aéreos No Tripulados (UAS) representan el nuevo paso en la evolución aeronáutica que permitirá reducir el coste de la gestión de seguridad europea e impulsar el surgimiento de nuevas oportunidades para explotar tecnologías que se encuentran dispersas en este y en otros campos.

Se espera que las operaciones de UAS superen las operaciones de aeronaves tripuladas, tanto para el sector militar como para el comercial, en 2035. Las tecnologías necesarias para apoyar esta transformación se están desarrollando rápidamente, los costes están disminuyendo, y las aplicaciones están aumentando.

La clave para el desarrollo del mercado de los UAS es la capacidad de avanzar, habilitar y unir tecnologías para la identificación y creación nuevas aplicaciones, definir nuevas misiones y generar nuevos mercados. Esto a su vez fuerza a desarrollar y mejorar las capacidades tecnológicas más recientes, que reduzcan los costes y amplíen las capacidades. Según la Plataforma Aeroespacial Española [PAE 2014] se han identificado diez áreas prioritarias de tecnología de subsistema UAS claves que jugarán un papel activo en las necesidades actuales y en la creación de nuevos mercados para satisfacer las nuevas demandas de los UAS. Estos son:

- Estructuras y materiales

- Procesos de fabricación.

- Simulación operativa.

- Aerodinámica.

- Sistemas de control.

- Equipos y sistemas embarcados.

- Equipos y sistemas de tierra.

- Técnicas de ensayo.

- Gestión del tráfico aéreo.

- Integración de sistemas.

Teniendo en cuenta lo anterior y siempre desde el punto de vista de la plataforma, uno de los objetivos principales en los aviones no tripulados se centra en maximizar la capacidad de carga útil, la autonomía y el alcance, por lo que es ahí donde este tipo de configuraciones particulares pueden proporcionar grandes ventajas con respecto a configuraciones convencionales. 
Para conseguir este objetivo, un aspecto importante, dentro del área tecnológica de aerodinámica, es la idea recursiva de reducir la resistencia aerodinámica. En este punto influye mucho el tipo de configuración. Dependiendo del uso que se le vaya a dar al aparato, la aerodinámica puede ser muy básica o muy elaborada. Por ejemplo un UAS militar que debe cumplir su misión en un entorno hostil, en poco tiempo tiene unos requerimientos aerodinámicos diferentes a un avión civil que ha de sobrevolar una zona para realizar algún tipo de estudio.

Desde hace unos pocos años hasta la fecha, existe un interés creciente en el uso de configuraciones no convencionales y por supuesto de configuraciones de alas convergentes en plataformas de UAS, como lo demuestran las recientes publicaciones e investigaciones al respecto.

Tales como los trabajos iniciados en el 2006 por el Centro de Investigación Aeroespacial Italiano (CIRA) en el estudio, diseño y construcción de un modelo demostrador de UAV con configuración de alas convergentes para vuelos a gran altitud (High Altitude Performance Demostrator, HAPD) [Belardo et al. 2014], o el estudio para el desarrollo de un modelo UAV con capacidades ISR definido por el Air Force Research Laboratory (AFRL) y materializado por Boeing en el diseño, desarrollo y construcción del modelo Sersor Craft [Roberts et al. 2003; Nangia et al. 2003; Nangia and Palmer 2006; Rasmussen et al. 2006; 2009], o otros como los desarrollos realizados por Zafirov en la Universidad Técnica de Sofia [Zafirov 2013].

\subsection{Objetivos de la Tesis}

El contenido de la Tesis Doctoral tiene como punto de partida, en primer lugar, los trabajos realizados por la Dr. ${ }^{a}$ Ingeniero Aeronáutico D. a Cristina Cuerno Rejado y en segundo lugar, los resultados y conclusiones obtenidos en el trabajo de investigación previo realizado por el autor (Optimización de Avión No Convencional: Joined-Wing) Pérez-Álvarez [2010].

El principal objetivo es la optimización de una configuración geométrica no convencional del tipo de alas convergentes (Figura 1.4), realizada por medio del estudio aerodinámico paramétrico de diferentes configuraciones de modelos físicos de ensayos. Cada modelo de ensayo está caracterizado por parámetros geométricos que definen la posición relativa del ala trasera con respecto a la delantera $\left(l_{t}, h_{t}\right)$, los cuales pueden sustituirse por valores del ángulo diedro, $\delta_{R}$, y ángulo de flecha, $\varphi_{R}$, de dicha ala.

Con tal propósito se analizan los datos de las fuerzas aerodinámicas obtenidos en ensayos en túnel aerodinámico mediante célula de carga, representando diferentes gráficos y mapas de configuraciones tipo de los diversos coeficientes aerodinámicos $\left(C_{L}, C_{D}\right.$ y $\left.C_{M y}\right)$ y de parámetros asociados $\left(C_{D i}, E, C_{L}^{3 / 2} / C_{D}\right)$, mostrando sus valores máximos y mínimos.

Por su importancia para el cálculo particular del coeficiente de resistencia inducida, $C_{D i}$, es necesaria la realización de un modelo numérico para la determinación del factor de eficiencia, e, (parámetro de Oswald) cuyos cálculos realizados para modelos convencionales no deben considerarse válidos para su aplicación en este tipo de configuración. 
Por otro lado el gran número de datos obtenidos permite la definición de un modelo numérico simple que proporcione resultados rápidos sea cual sea el valor de las variables de estudio y para resultados propios de fases iniciales de diseño conceptual en un proyecto de aeronave no convencional.

De esta forma, una primera parte del estudio realizado en el trabajo es de carácter experimental, mientras que el resto es de carácter teórico y numérico. La parte experimental de la investigación consiste en el estudio del comportamiento de un modelo paramétrico en túnel aerodinámico, donde se realiza el análisis del efecto de la interacción de las dos alas, analizando su comportamiento.

En la parte numérica se realiza la correlación de los datos experimentales con los resultados proporcionados mediante la simulación por medio de modelos numéricos básicos.

Para tal propósito se definen y construyen un número significativo de modelos de ensayo para túnel aerodinámico modulares por medio de tecnología tipo aditiva propia de impresora de modelos 3D.

De esta manera las dimensiones de los modelos de ensayo se calcularán para tener en cuenta un segundo objetivo de usar los resultados del trabajo para su aplicación al entorno de los UAS tipo MALE (Medium Altitude Long Endurance) o LALE (Low Altitude Long Endurance).

\subsection{Organización de la Tesis}

Este documento de Tesis está divido en cuatro capítulos principales, que describen las características fundamentales relacionadas con la influencia de la configuraciones en diferentes parámetros aerodinámicos del modelo.

Con el ánimo de facilitar la lectura lo máximo posible, se han incluido tres apéndices, que se encuentran ligados a cada capítulo y cuya finalidad es la de aclarar algún concepto necesario o incluir colecciones de gráficas de los diferentes resultados.

Capítulo 1: En este capítulo se presenta una introducción y dónde se muestra la situación actual en el sector aeronáutico donde cómo y por qué es posible el desarrollo de este tipo de configuraciones no convencionales de aeronaves.

Capítulo 2: En este capítulo se presenta los antecedentes, en forma de revisión bibliográfica, de los estudios e investigaciones realizados hasta la fecha y relacionados con la configuración del sistema de alas múltiples y de alas convergentes. Se analiza la evolución histórica en el desarrollo de la investigación de la configuración, describiendo el concepto de aeronave de alas convergentes y su influencia, desde un punto de vista general, en la aerodinámica y en la estructura (especialmente en el peso) en aquellos estudios considerados más significativos y cuyos resultados son de especial importancia.

Capítulo 3: En este capítulo se analiza, de manera más exhaustiva, la influencia que presenta esta configuración en los coeficientes aerodinámicos de sustentación, $C_{L}$, y de resistencia inducida, $C_{D i}$. La revisión de los teoremas de Prandtl y Munk permiten entender mejor el comportamiento del modelo de alas 
convergentes y cómo cuantificar la influencia que esta disposición de las alas produce en los coeficientes. Es de especial interés estudiar un método generalizado para determinar el factor de eficiencia de Oswald para modelos de aeronaves de geometría de alas convergentes.

Anexo A: Para un correcta determinación del coeficiente de resistencia inducida, es necesario el estudio de modelos de cálculo del factor de eficiencia de Oswald en sistemas de alas múltiples, y cuyo objetivo es su adaptación en el modelo de alas convergentes. Con tal propósito se estudian los modelos diseñados por Letcher y DeYoung, obteniendo un resultado en función del ángulo diedro del ala trasera como parámetro, aplicable al modelo de aeronave de alas convergentes.

Capítulo 4: Este capítulo tiene como objetivo la presentación y el análisis de los ensayos realizados de un modelo físico en túnel aerodinámico por medio de un sensor de fuerza/par. En primer lugar se muestran las características del modelo físico de ensayo, dimensiones de las diferentes configuraciones, características geométricas así como los criterios considerados en su elección y construcción. En segundo lugar se analizan los aspectos técnicos relacionados con los medios físicos (equipos de medida) necesarios para la realización de los ensayos. Por último se presentan los diferentes parámetros de ensayo así como los diferentes resultados, mostrando los corrspondientes a la configuración de referencia $\left(\mathrm{JWC}_{15}\right)$ y los datos característicos en función de los parámetros de estudio.

Anexo B: Para la realización de los ensayos es necesario la caracterización de los diferentes equipos y en especial de la cámara de ensayo del túnel aerodinámico con el fin de estudiar las características de la vena fluida y de su validez par el tipo de ensayo realizado.

Anexo C: Se presenta en este anexo un conjunto de gráficas que permiten analizar todos los resultados obtenidos en la realización de los ensayos, dónde están representados los valores de los coeficientes aerodinámicos en función del ángulo de ataque, $\alpha$, agrupados en función del ángulo diedro y del ángulo de flecha del ala trasera. Los datos presentados corresponden a todas las configuraciones y a comparaciones realizadas con respecto a la configuración de referencia.

Anexo D: Se presenta en este anexo un conjunto de gráficas que permiten analizar todos los resultados obtenidos en la realización de los ensayos, donde se presenta los resultados completos de los valores de los coeficientes aerodinámicos del modelo de alas convergentes en formato de mapa de color con líneas de isovalores en función del ángulo diedro, $\delta_{R}=$ $\left[-5^{\circ},-25^{\circ}\right]$, del ala trasera y del ángulo de flecha, $\varphi_{R}=\left[-10^{\circ},-30^{\circ}\right]$, del ala trasera, para ángulos de ataque, $\alpha=\left[-14^{\circ}, 15^{\circ}\right]$. Los datos presentados corresponden a todas las configuraciones y a comparaciones realizadas con respecto a la configuración de referencia.

Capítulo 5: En este capítulo se recoge los cálculos realizados para la definición de un modelo numérico simple que reproduzca los resultados de ensayo. Este modelo numérico está basado en aproximar las características aerodinámicas 
del ala por medio de una linea de torbellinos, siendo el modelo más simple que se puede proponer para resolver este problema la sustitución de la circulación, en cualquier estación a lo largo de la envergadura del ala, y reemplazarla por un único torbellino constante concentrado, teniendo en cuenta tanto el ángulo de flecha del ala delantera, $\varphi_{F}$, el ángulo de flecha del ala trasera, $\varphi_{R}$, así como el ángulo diedro del ala delantera, $\delta_{F}$, y el ángulo diedro del ala trasera, $\delta_{R}$.

Anexo E: Se presenta en este anexo un conjunto de gráficas donde se comparan los resultados obtenidos mediante el modelo numérico propuesto con los resultados obtenidos experimentalmente. Los datos representados corresponden a los valores de los coeficientes de sustentación, $C_{L}$, resistencia inducida, $C_{D i}$, resistencia, $C_{D}$, y eficiencia aerodinámica, $E$, en función del ángulo de ataque, $\alpha$, para valores del ángulo diedro, $\delta_{R}=\left[-5^{\circ},-25^{\circ}\right]$, del ángulo de flecha, $\varphi_{R}=\left[-10^{\circ},-30^{\circ}\right]$, del ala trasera.

Capítulo 6: En este capítulo se presenta una memoria resumen con las conclusiones sobre los aspectos relacionados con la determinación experimental de los coeficientes aerodinámicos, así como de su determinación por medio del modelo numérico presentado.

\subsection{Publicaciones realizadas}

Como es de entender, en todo trabajo de carácter experimental es necesario un conocimiento exhaustivo de las diferentes instalaciones y equipos involucrados en los ensayos que se desean realizar. En muchas ocasiones estas instalaciones y equipos tiene un elevado coste y suelen estar dedicadas a ensayos de proyectos en curso.

Para la realización de la Tesis Doctoral ha sido necesario conocer el uso y funcionamiento de las diversas instalaciones utilizadas para la ejecución de los ensayos, lo que ha implicado la participación en proyectos que permitiera una formación de primera mano, dando como resultado la colaboración en publicaciones, que si bien sus contenidos no están relacionados directamente con con la Tesis Doctoral, si el hecho del aprendizaje adquirido. En este sentido se han realizado las publicaciones siguientes:

- Autores: Yeow, T.S., Cuerva-Tejero A. \& Pérez-Alvarez, J.

Título: Reproducing the Bolund experiment in wind tunnel

Revista Internacional: Wind Energy

Fecha: 2014

Editorial: Published online in Wiley Online Library

Índice de impacto en JCR: 3.069

- Autores: S. Pindado, J. Pérez-Alvarez \& S. Ávila.

Título: On Cup Anemometer Rotor Aerodynamics

Revista Internacional: Sensors, V. 12, pp. 6198-6117

Fecha: 2012 
Editorial: Sensors Editorial

Índice de impacto en JCR: 1.953

Por otro lado y directamente relacionado con el contenido del trabajo, se ha realizado una publicación donde se presenta un análisis de los resultados obtenidos en los ensayos sobre el modelo de alas convergentes.

- Autores: Javier Perez-Alvarez; Cristina Cuerno Rejado; José Meseguer Ruiz

Título: Aerodynamic parametric analysis of an unconventional joined-wing aircraft configuration.

Revista Internacional: Proceedings of the Institution of Mechanical Engineers Part G Journal of Aerospace Engineering. pp. 1 - 17. 04/2015. ISSN 0954-4100

Fecha: 2015

Editorial: SAGE

Índice de impacto en JCR: 0.653 



\section{Capítulo 2}

\section{Antecedentes}

\subsection{Introducción.}

Han sido varios los autores que han dedicado sus esfuerzos a la investigación de las características de la configuración de alas convergentes, especialmente desde un punto de vista geométrico, estructural y aerodinámico. A continuación se expone una descripción de la evolución de la idea conceptual del modelo así como aquellos trabajos de investigación cuyos resultados muestran los aspectos mas significativos y relevantes de este tipo de configuraciones.

\subsection{El concepto de aeronave de alas convergentes.}

Entre los primeros diseños que estudiaron configuraciones de aeronaves de alas convergentes cabe destacar el planeador diseñado y construido por Reinhold Platz [Platz 1924] en Europa en 1920 en dos versiones distintas. Platz también construyó una versión moderna en 1963. El planeador estaba diseñado con una estructura en forma de cruz, consistente en un larguero transversal, el cual formaba el borde de ataque del ala trasera, unido a un larguero longitudinal que servía de fuselaje. A estos largueros se unían una vela trasera y una delantera para formar las alas en tándem (Figura 2.1).

En 1932, Ben Brown de la Universidad de Kansas diseño y construyó el rudimentario modelo de alas múltiples convergentes (Figura 2.2) [Wolkovitch 1982]. Constaba de tres alas, un ala alta delantera con flecha positiva, otra ala alta trasera con flecha regresiva y una tercera baja sin flecha pero con ángulo diedro positivo. Aunque realizó algunos vuelos de exhibición no tuvo gran repercusión tecnológica en los diseños de la época.

Durante los años 70, Julian Wolkovitch de ACA Industries [Chambers 2005] realizó y presentó su primer diseño de aeronave de alas convergentes. La principal característica fue la disposición del encastre del ala trasera intencionadamente elevado con respecto al ala delantera. La combinación de ala 


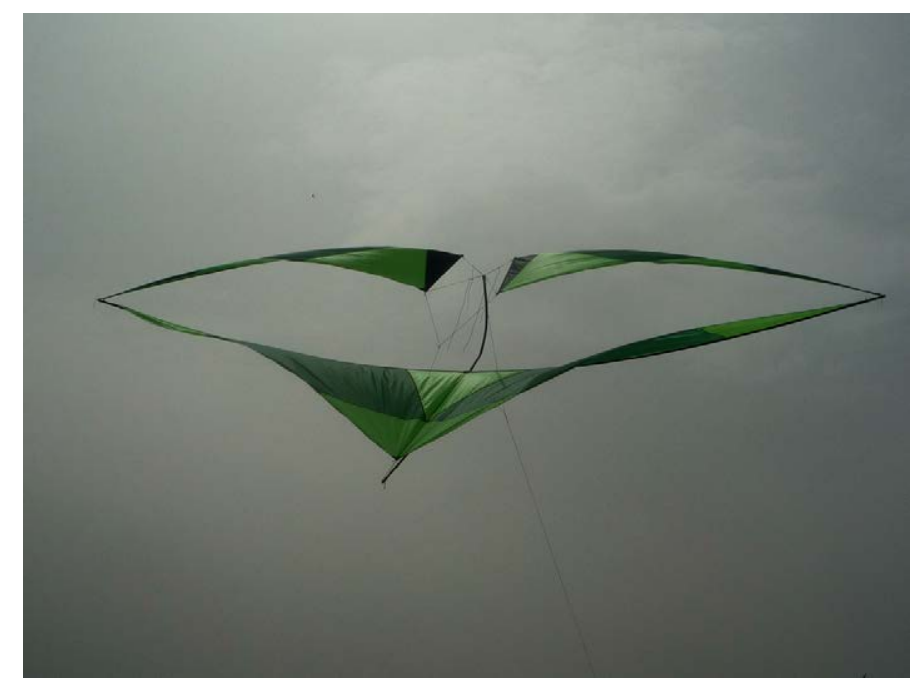

Figura 2.1: Foto de reconstrucción del planeador diseñado por Reinhold Platz en 1920, donde se pueden observar los dos largueros en cruz y las alas delantera y trasera [Platz 1924].

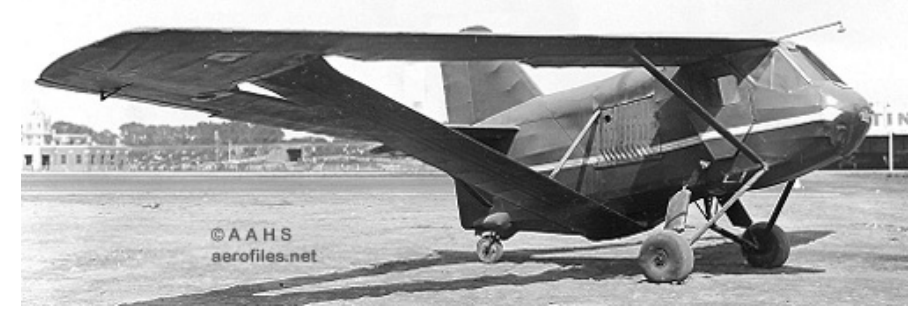

Figura 2.2: Aeronave de alas múltiples diseñada y construida por Ben Brown en 1932 [Wolkovitch 1982].

delantera-ala trasera forma un entramado estructural en celosía que libera parte de la carga del ala delantera haciendo la estructura más resistente y ligera.

En 1979, Julian Wolkovitch presentó a J. L. Johnson, subdirector del Departamento de Dinámica y Estabilidad, una propuesta para la realización de ensayos en túnel aerodinámico de un modelo de aeronave en configuración de alas convergentes de aplicaciones agrícolas. La configuración, que el constructor de prototipos Elbert L. Rutan (fundador de Scaled Composites, Inc.) había diseñado, conocida con el nombre de "Model 58 Predator", había surgido de un estudio de viabilidad de la Royal Air Force realizado por David Record de Advanced Technology Aircraft Co., Ltd. (ATAC) y consistía en una aeronave propulsada por hélice, con la unión del ala trasera en el ala delantera a media envergadura y winglets (Figura 2.3). Tanto Wolkovitch como Rutan estaban convencidos que su modelo propuesto presentaba mejoras significativas sobre los modelos convencionales [Chambers 2005].

Debido al interés generado y la necesidad en proporcionar datos sobre esta configuración, NASA fabricó un modelo a escala (Figura 2.3) para la realización de ensayos en el túnel subsónico del NASA Langley Research Center. Aunque los ensayos fueron, en principio, muy básicos y limitados, debido a las condiciones de ensayo (bajos números de Reynolds), estos fueron considerados de gran interés para la comunidad aeronáutica. 


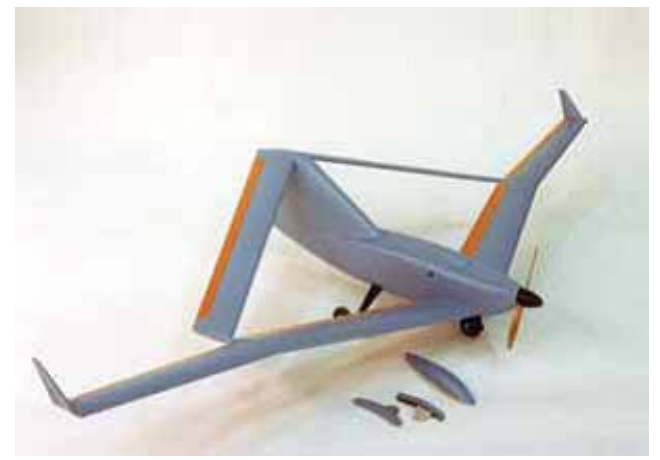

Figura 2.3: Modelo de ensayo de aplicaciones agrícolas de Elbert L. Rutan medido en Langley [Chambers 2005].

Un año más tarde Wolkovitch inició el proceso para patentar su diseño cuya presentación final se realizó en 1982 [Wolkovitch 1982].

En 1984, Michael H. Durham y Rodney H. Ricketts del departamento de aeroelasticidad del NASA Langley Resech Center, se unieron para un estudio analítico y experimental de los beneficios de este tipo de configuración sobre las características de aeroelasticidad dinámica (flameo) en vehículos con alas de gran alargamiento (Figura 2.4).

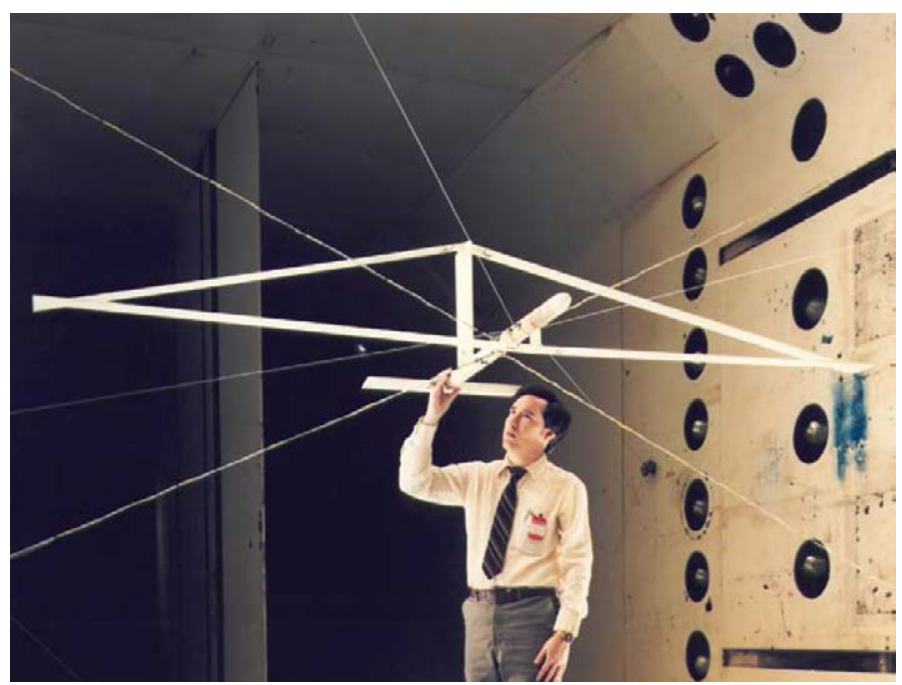

Figura 2.4: El investigador Mike Durham con el modelo de configuración de alas convergentes para el estudio de flameo en vehículos con alas de gran alargamiento [Chambers 2005].

En 1986 mientras que el NASA Langley Research Center estaba comprometido en evaluar los beneficios de esta configuración para aplicaciones civiles y militares, el NASA Ames Research Center realizaba esfuerzos similares incluyendo estudios para su aplicación en transporte aéreo.

Los investigadores del NASA Ames Research Center, que comenzaron a estudiar el concepto de avión de alas convergentes, reconocieron la necesidad de herramientas de diseño y análisis más sofisticadas para poder evaluar adecuadamente los beneficios relacionados con el ahorro de peso estructural y la reducción de la resistencia aerodinámica. 
En 1986, se comenzó a trabajar en un código que combinaba análisis estructural y cálculo aerodinámico que sería apropiado para el diseño conceptual del modelo. Stephen C. Smith en el NASA Ames Research Center e Ilan M. Kroo y John W. Gallman en la Universidad de Stanford colaboraron en su desarrollo. El modelo aerodinámico se basaba en un código vortex-lattice que incluía una rutina de optimización en la definición de unión óptima de las alas para minimizar la resistencia inducida [Gallman et al. 1990].

En 1989 las teorías planteadas por ACA Industries, y con la ayuda del NASA Ames Research Center, fueron estudiadas en túnel aerodinámico para medir las características aerodinámicas del modelo de alas convergentes. Los ensayos se realizaron modificando la aeronave NASA-AD1, conservando el fuselaje y los motores en dos versiones diferentes (Figura 2.5) [Smith and Stonum 1989].
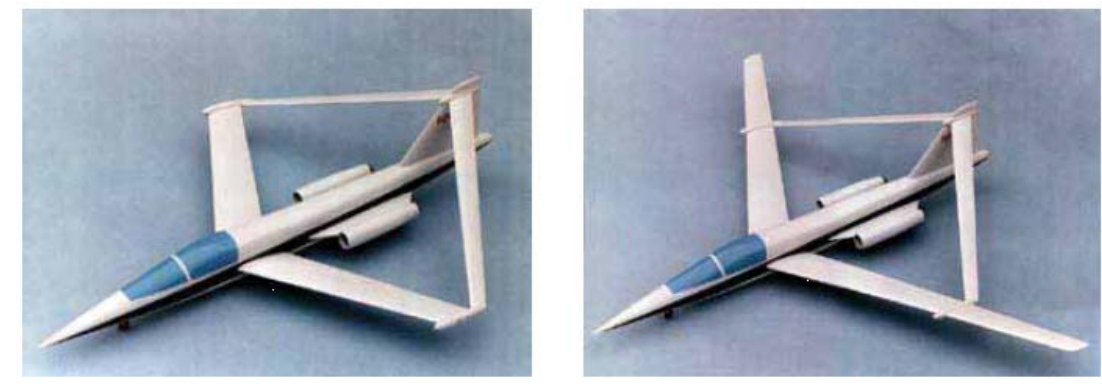

Figura 2.5: NASA-AD1 modificado en dos modelos de alas convergentes de ensayo en túnel [Smith and Stonum 1989].

A principios de los años 90 la armada de EE.UU. desarrolló el programa E-2C solicitando a la oficina de NASA Langley el estudio de la configuración EX (Figura 2.6) en túnel transónico. Acorde con el plan de la NASA de explorar configuraciones no convencionales de interés y en ese marco de cooperación, en julio de 1993, se realizaron los ensayos para evaluar las características, aerodinámica longitudinal y lateral así como la efectividad de las superficies de control.

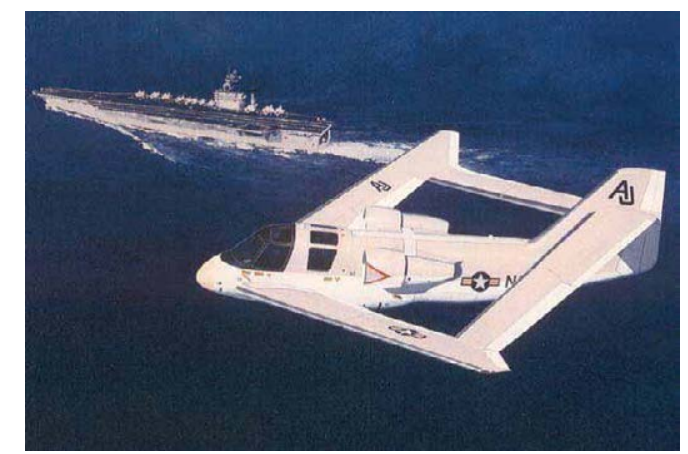

Figura 2.6: Imagen artística del modelo de aeronave de alas convergentes Boeing EX [Chambers 2005].

A principios de los años 2000 Boeing desarrolló el modelo alas convergentes SensorCraft [Nangia and Palmer 2006; Rasmussen et al. 2006] con el propósito de cubrir las necesidades de las fuerzas aéreas de Estados Unidos para el diseño de una aeronave con mayores y mejores capacidades de vigilancia. Los factores que impulsa 
el diseño de la aeronave SensorCraft son la necesidad de mantener una cobertura de 360 grados de radar sobre la zona objetivo y ampliar la capacidad actual de los aviones no tripulados (Figura 2.7).

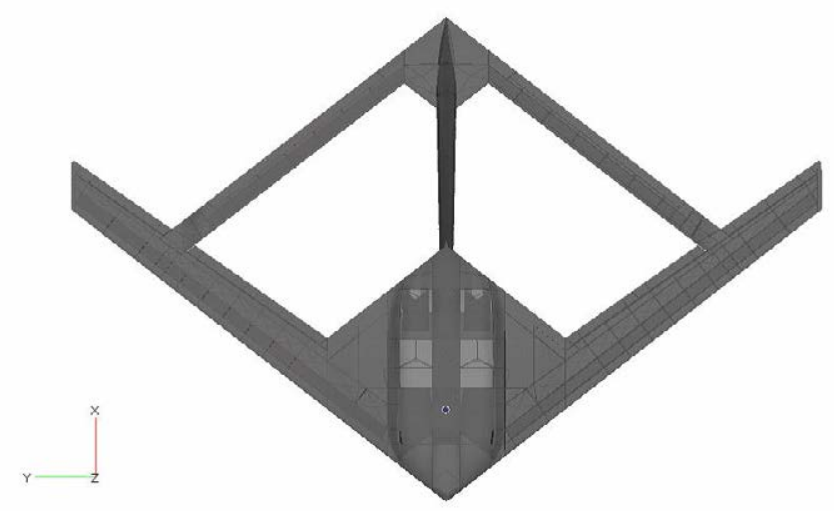

Figura 2.7: Vista superior de la aeronave de alas convergentes Boeing SensorCraft [Nangia and Palmer 2006].

A finales de la primera década del siglo XXI el Air Force Research Laboratories (AFRL) encomendó al Departamento de Aeronáutica de la United States Air Force Academy (Academia de la USAF) la investigación de las características aerodinámicas del modelo de alas convergentes denominado Houck [Oligney et al. 2008]. El modelo de estudio se basaba en el concepto LRLE (Long Range, Long Endurance) dentro del entorno de las aeronaves no tripuladas (UAS).

En octubre de 2011, utilizando como plataforma el modelo Boeing SensorCraft y con fondos del AFRL, el instituto tecnológico de Virginia (VT) y la universidad de Victoria (UVic) realizaron con éxito el primer vuelo de pruebas de un modelo de avión no tripulado tipo HALE (High Altitude Long Endurance) de alas convergentes de $5 \mathrm{~m}$ de envergadura [Aarons et al. 2011].

Por otro lado, a principios de 2014 se hicieron públicas las primeras imágenes del modelo "Guizhou Soar Dragon" (Figura 2.8), UAV en configuración de alas convergentes tipo HALE cuya desarrollo se inició en 2011 por las fuerzas aéreas del ejército de liberación popular de la República de China. El modelo, de aplicaciones de reconocimiento (por determinar), recuerda en su fuselaje al Northrop Grumman Global Hawk.

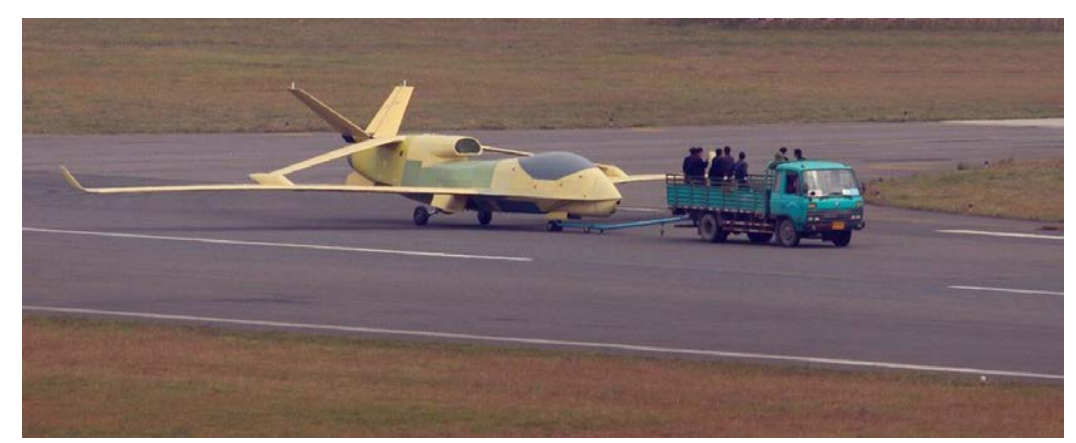

Figura 2.8: Modelo de alas convergentes "Guizhou Soar Dragon" (C)www.MilitaryFactory.com). 
Esta situación hace que el modelo de alas convergentes sea un candidato idóneo para su investigación y desarrollo con vistas a su aplicación futura al transporte aéreo, siempre y cuando se superen los obstáculos sociales que implica introducir cambios tan significativos en la geometría de la aeronave civil. Por otro lado y de una forma más inmediata este tipo de configuraciones son perfectas para su aplicación al campo de los sistemas de vehículos aéreos no tripulados UAS (Unmanned Aerial Systems) donde las particulares características aerodinámicas de esta configuración pueden suponer una gran ventaja [Nangia and Palmer 2006; Rasmussen et al. 2009].

\subsection{Influencia en la estructura de las alas}

Uno de los estudios significativos en relación a la estructura en el modelo de aeronave de alas convergentes, ha sido y es la posibilidad de reducción de peso de la estructura del sistema de alas. Son muchos los estudios realizados donde se muestra que, para las mismas condiciones aerodinámicas que en una aeronave convencional, la configuración del modelo de alas convergentes produce una reducción significativa en el peso de la estructura en comparación de un modelo de avión convencional. La particular unión de las dos alas, donde el ala trasera se comporta como soporte del ala delantera, implica una reducción de las cargas que sufre ésta por causa del momento flector inducido por la sustentación. Esta característica se traduce en una reducción de peso de la estructura y una reducción de la deflexión de las alas, permitiendo un aumento de su envergadura y por consiguiente un aumento de su alargamiento.

Samuels [Samuels 1982] comparó el peso de la estructura entre dos aeronaves, una de referencia (Boeing 727) con configuración convencional, ala más estabilizador horizontal-vertical y otra con configuración de alas convergentes. Tanto para la aeronave de referencia como para la configuración de alas convergentes, Samuels generó modelos de cálculo de elementos finitos de las superficies sustentadoras, optimizados con el criterio de Von Mises y bajo las condiciones de igualdad de sustentación y de resistencia aerodinámica.

El resultado de los cálculos mostró la existencia de diferencias significativas entre la distribución de espesores del ala de ambos modelos. En el caso del modelo de referencia la distribución de espesores de pared fue la esperada para esta configuración; para el caso del modelo de alas convergentes los resultados de los cálculos mostraron dos diferencias con respecto al modelo de referencia. La primera fue la existencia de dos espesores de pared máximos distintos en el extradós e intradós de cada superficie alar; la segunda fue que la distribución de espesores de pared tiende a no ser simétrica en cuanto a la forma en la superficie de extradós y de intradós (Figura 2.9). Esto es debido a que la dirección del eje de flexión o de momentos flectores no es paralela al eje longitudinal de la aeronave, sino que sigue la dirección resultante de unir los puntos $1 / 4$ de las secciones de encastre del ala delantera con el ala trasera (Figura 2.10). Este resultados es acorde con los obtenidos por [Wolkovitch 1985; 1986] que determinó que la necesidad de un cajón de torsión no simétrico se debe a que las cargas que actúan sobre cada ala se descomponen en cargas paralelas al plano del eje de flexión y cargas normales al plano del eje de flexión. Las cargas paralelas al eje de flexión son adecuadamente soportadas por 


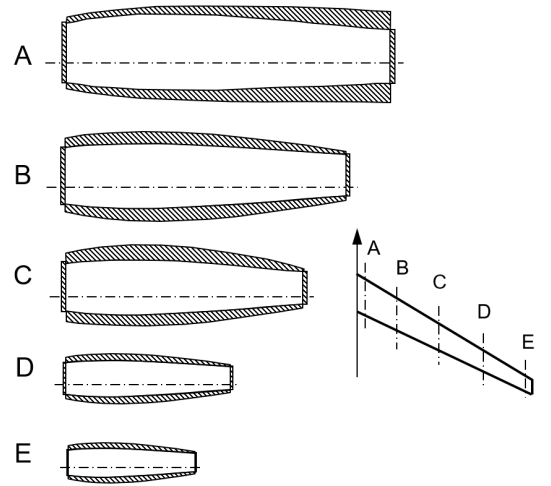

(a)

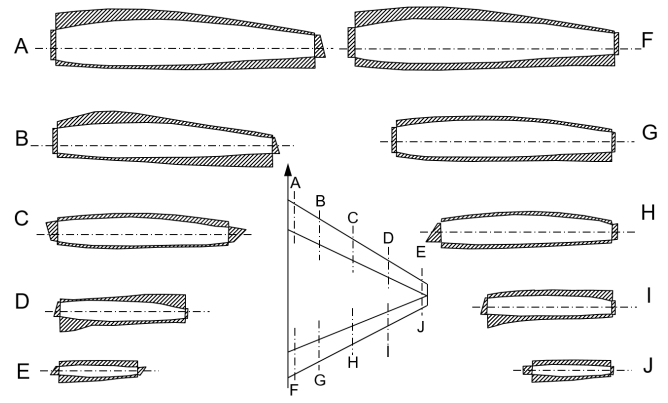

(b)

Figura 2.9: Distribución de espesores del cajón de torsión según Samuels [Samuels 1982]: (a) modelo de referencia; (b) modelo de alas convergentes. Las zonas sombreadas representan la distribución de esfuerzos sobre el cajón de torsión del ala

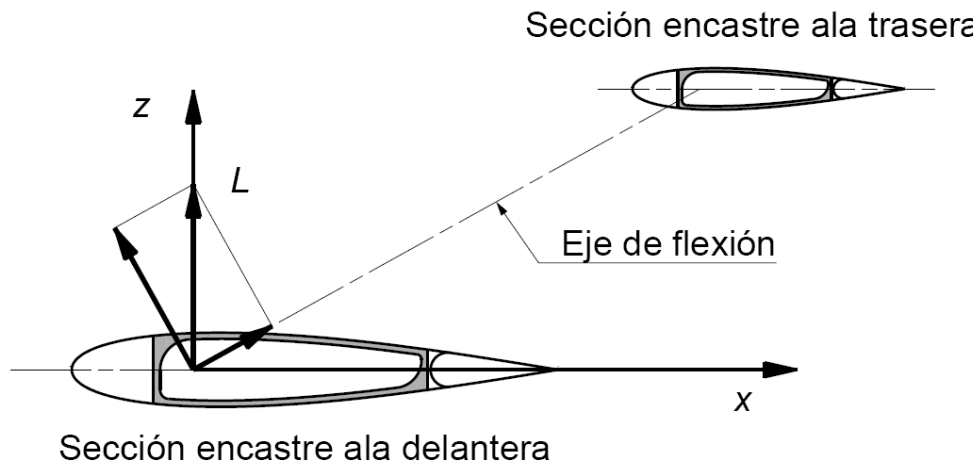

Figura 2.10: Esquema de descomposición de la fuerza de sustentación, $L$, en fuerzas paralelas y normales al plano del eje de flexión definido por la unión de los puntos $1 / 4$ de las secciones en el encastre del ala delantera y del ala trasera.

la estructura de ambas alas; en cambio, las cargas normales producen flexión en las alas alrededor del eje, haciendo necesario incrementar la masa estructural en la estructura del cajón de torsión cerca de dicho eje [Wolkovitch 1986].

Como consecuencia de estos estudios, según Samuels, el peso de la estructura de las alas en la configuración de alas convergentes se reduce entre un $12 \%$ y un $20 \%$ con respecto al modelo de referencia [Samuels 1982].

Poco tiempo después Wolkovitch, al igual que Samuels, comparó el peso de dos aeronaves, entre una configuración convencional y otra con configuración de alas convergentes. Ambas aeronaves se configuran para que tengan un comportamiento aerodinámico equivalente. El estudio mostró que el peso de la estructura, en el modelo de alas convergentes, presentaba reducciones de peso del mismo orden que las obtenidas por Samuels (Figura 2.11) [Wolkovitch 1986]. 


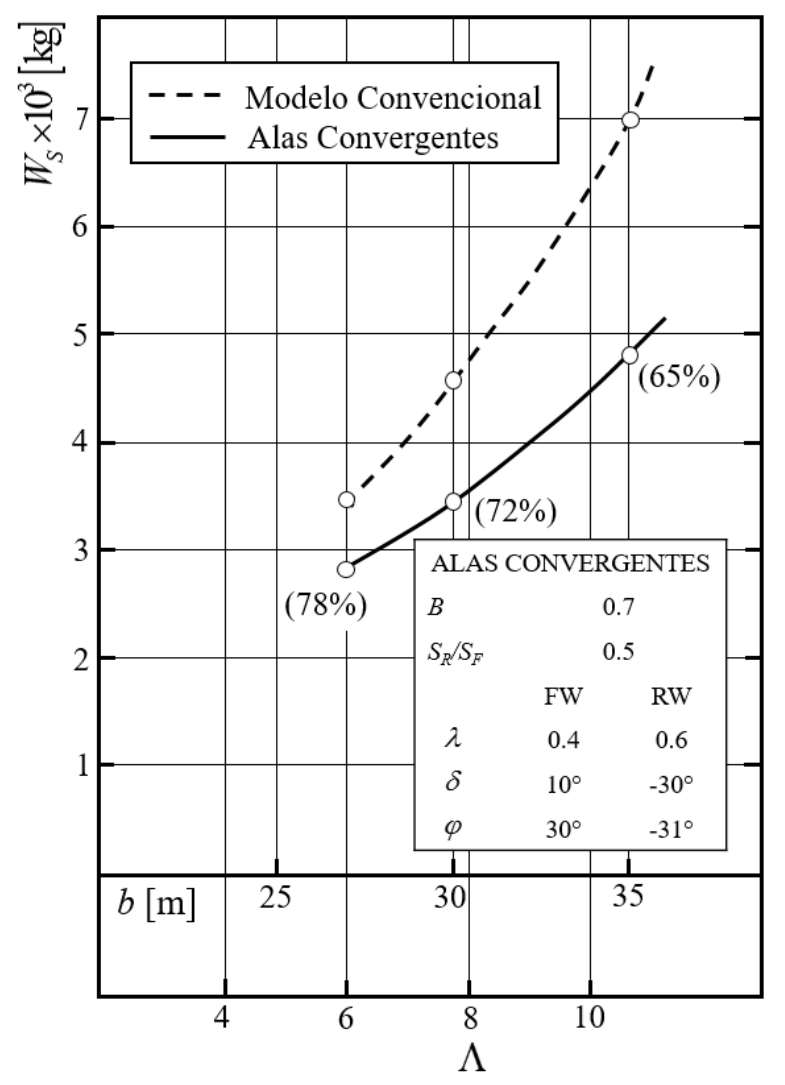

Figura 2.11: Peso de la estructura de las superficies sustentadoras, $W_{S}$, en función del alargamiento, $\Lambda$, y de la envergadura efectiva, $b$, para el modelo de ala convergentes y para el modelo convencional [Wolkovitch 1986].

Además y a diferencia de Samuels, Wolkovitch cuantificó el porcentaje de las variaciones del peso de la estructura en función de parámetros característicos del modelo de alas convergentes, en especial, por su importancia, la situación del punto de unión de ala trasera con respecto al ala delantera. Los estudios dieron como resultado que el valor óptimo para peso mínimo se produce cuando el parámetro de relación de envergaduras, $B$, es del orden del 0.7 (Figura 2.12), definido como:

$$
B=\frac{b_{R}}{b_{F}}
$$

donde $b_{R}$, es la envergadura del ala trasera, $b_{F}$, la envergadura del ala delantera.

Por otro lado Wolkovitch definió nuevos parámetros geométricos para caracterizar la influencia en el peso de la estructura. En los modelos convencionales el peso de la estructura es frecuentemente relacionado con parámetros geométricos en términos de envergadura, espesor máximo del perfil en encastre, etc. Desde el punto de vista del modelo de alas convergentes existen dos parámetros característicos; la cuerda promedio, $d$, (Figura 2.13) y la envergadura efectiva, $b$. La envergadura efectiva se define como el valor medio de las longitudes verdaderas (no proyectadas) de la línea $1 / 4$ del ala delantera y trasera,

$$
b=\frac{1}{2}\left[\left(\frac{b_{F}}{\cos \delta_{F} \cos \varphi_{F}}\right)+\left(\frac{b_{R}}{\cos \delta_{R} \cos \varphi_{R}}\right)\right]
$$




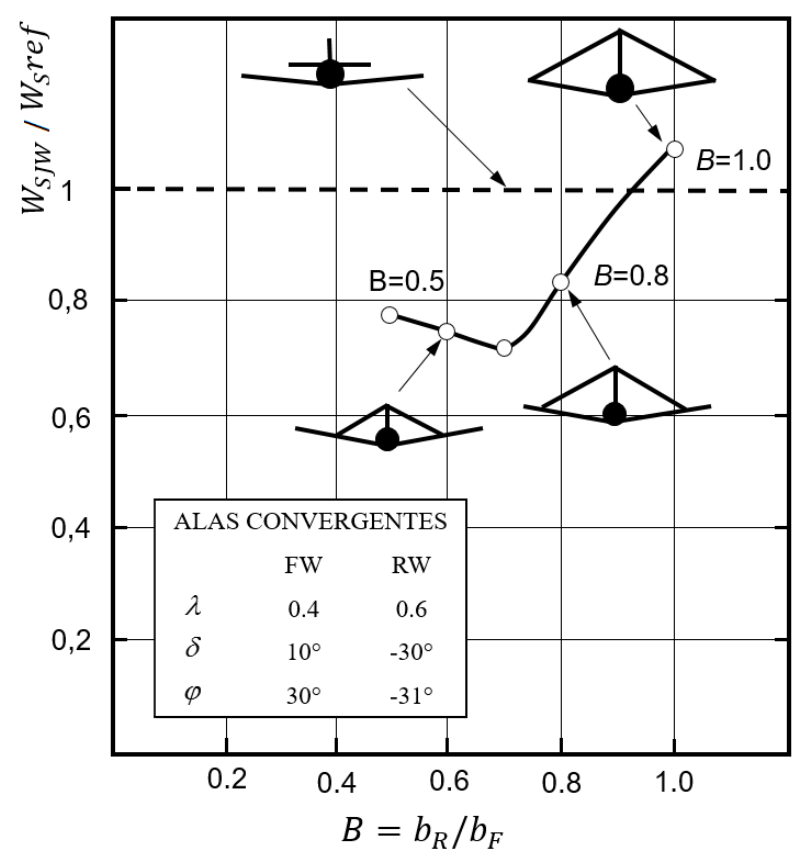

Figura 2.12: Relación entre el peso de la estructura de las superficies sustentadoras, $W_{s j w} / W_{\text {sref }}$ en función de la relación de envergaduras, $B$, para el modelo de alas convergentes y para el modelo convencional [Wolkovitch 1986].

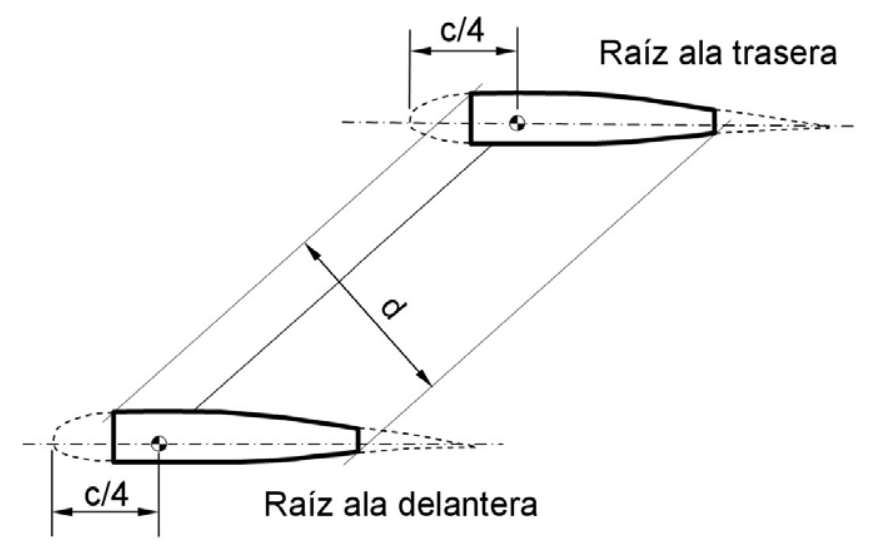

Figura 2.13: Esquema general de la definición geométrica del parámetro cuerda promedio $d$, [Wolkovitch 1986].

donde $\delta_{F}, \varphi_{F}$, son el ángulo diedro y ángulo de flecha del ala delantera y $\delta_{R}, \varphi_{R}$, el ángulo diedro y ángulo de flecha del ala trasera.

Con el propósito de reducir el número de ensayos necesarios para la determinación del peso de la estructura, a finales de los años 80, en el NASA Ames Research Center, Shyu y Miura [Miura et al. 1988], realizaron diferentes análisis estructurales cuyo objetivo era la definición de un procedimiento paramétrico para la estimación del peso de la estructura que hasta la fecha se realizaba por medio de procedimientos estadísticos basados en diseños anteriores.

Por un lado, además de los parámetros ya estudiados por Wolkovitch y Samuels, es decir, la localización del punto de unión de las dos alas, la envergadura efectiva y el ángulo diedro, Shyu y Miura estudiaron como esta configuración influye 


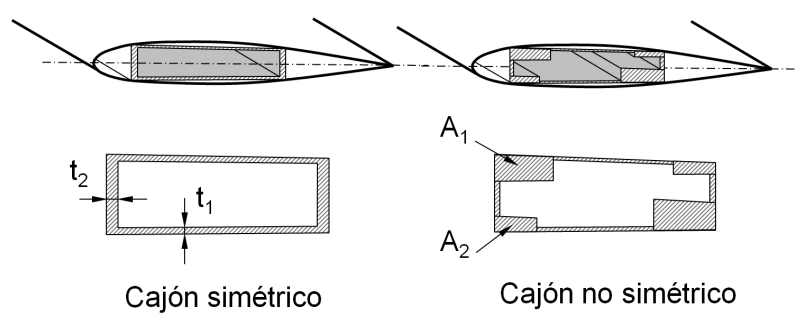

Figura 2.14: Esquema de cajón de torsión simétrico y no simétrico [Kroo et al. 1991].

en la geometría y dimensiones del cajón de torsión de cada ala, el ángulo de flecha, el ángulo diedro, la envergadura y el factor de estrechamiento. Según sus conclusiones y después de estudiar más de 50 casos diferentes, todas las ventajas desde el punto de vista de ahorro de peso aparecen, una vez más, cuando el parámetro de relación de envergaduras, $B$, se encuentra en valores entre 0.6 y 0.7 [Miura et al. 1988].

De forma similar, Hajela y Chen [Hajela and Chen 1988] desarrollaron un método denominado de viga equivalente para la realización de cálculos preliminares para la optimización del peso de la estructura del ala y con el propósito de reducir lo que en 1988 eran costosos cálculos de modelos de elementos finitos de gran número de grados de libertad.

Puede parecer, como conclusión inmediata, que la mayoría de los autores coinciden en la idea de que la configuración del modelo de alas convergentes reduce significativamente el peso de la estructura de las superficies sustentadoras entre un $12 \%$ y un $20 \%$, con una fuerte dependencia de la selección del punto de unión de las dos alas, la distancia relativa, la altura relativa entre ambas y de la definición de la geometría interna del cajón de torsión, aunque otros autores se muestran claramente contrarios a esta idea.

Siguiendo esta línea, Kroo, Gallman y Smith [Kroo et al. 1991], desarrollaron una herramienta de análisis sistemático que pudiera usarse en las primeras fases de diseño de aeronaves de tipo de alas convergentes, cuantificando las ventajas e inconvenientes en este tipo de avión. Para tal propósito, la investigación se centró en la optimización aerodinámica y estructural.

Para la optimización estructural, analizaron diferentes configuraciones del modelo de alas convergentes, con valores de la variable geométrica $B$ entre 0.4 y 1.0, y posiciones relativas del perfil en el encastre del ala trasera con respecto a la delantera variando entre $8.5 \mathrm{~m}$ y $12.0 \mathrm{~m}$. Todas las configuraciones fueron analizadas usando dos tipos de cajón de torsión; uno simétrico y otro no simétrico (Figura 2.14), similares a los estudiados por Wolkovitch, Samuels, Hajela y Shyu.

Los estudios mostraron resultados similares a los ya obtenidos por Wolkovitch, en cuanto a la posición óptima relativa de la unión de las dos alas, aunque sólo para el caso de cajón de torsión no simétrico (Figura 2.15).

Por otro lado, y a la vista de los resultados obtenidos, Kroo, Gallman y Smith [Gallman et al. 1993] se mostraron contrarios a la afirmación de que el modelo de alas convergentes presenta grandes ventajas desde el punto del ahorro de peso, con respecto a una aeronave convencional. Esta teoría se basa en que las alas en esta configuración soportan cargas muy diferentes a las de las alas del modelo 


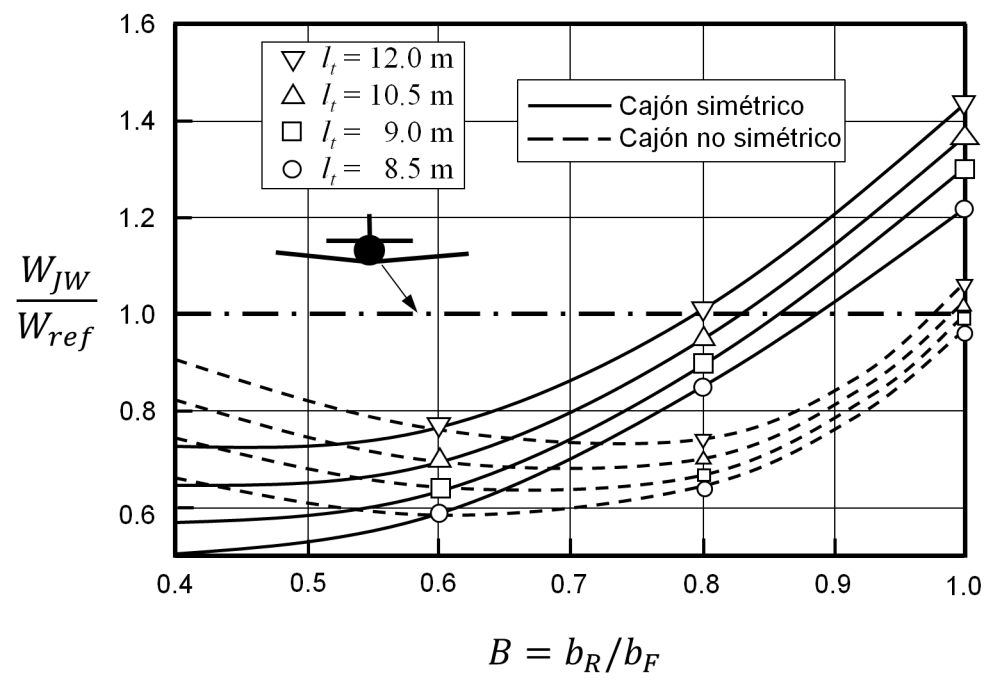

Figura 2.15: Relación entre el peso del modelo de alas convergentes y el modelo de referencia (aeronave convencional equivalente) con la posición de unión de las dos alas, para cada caso de cajón de torsión (simétrico y no simétrico) y para diferentes posiciones relativas de ala trasera con respecto a delantera, $l_{t}$, [Kroo et al. 1991].

convencional. La unión del ala trasera con el empenaje vertical presenta fuerzas de reacción que alivian el momento flector del ala, permitiendo en un principio reducir el peso de la estructura pero, por otro lado, esta misma unión presenta problemas de pandeo en el ala trasera, lo que implica un aumento del peso de la estructura de un $13 \%$ para minimizar el problema [Gallman and Kroo 1996].

Más recientemente Maxwell Blair y Robert A. Canfield [Blair and Canfield 2002; Blair et al. 2005] han desarrollado una herramienta analítica más precisa, para la estimación del peso, que el método desarrollado por Kroo, Gallman y Smith. Blair y Canfield defienden que los estudios que rechazan las ventajas del modelo de alas convergentes, desde el punto de vista del ahorro de peso estructural, lo hacen porque se basan en resultados de modelos simplificados que no tienen en cuenta los beneficios de modelos de cálculo aeroelástico no lineal.

Por otro lado las características especiales de la estructura del ala, tanto en su geometría externa (alas convergentes) como interna (distribución de masa estructural no simétrica), producen un comportamiento diferente en ala trasera. Teniendo en cuenta factores de carga positivo el ala trasera trabaja con un estado de cargas a compresión debido, en primer lugar, a su propia carga alar y en segundo a las cargas transmitidas por el ala delantera a través de su unión, lo que implica que las posibilidades de pandeo local de la estructura aumenten [Wolkovitch 1986].

Según Samuels [Samuels 1982] el desplazamiento vertical de las alas, en dirección del eje $z$, en el modelo de alas convergentes es mayor que en el caso de un ala clásica en la zona desde el encastre hasta el punto donde se realiza la unión de ambas, haciéndose menor en las proximidades del punto y disminuyendo en comparación con el desplazamiento de las alas clásicas desde ese punto. Como ya se ha comentado Kroo, Gallman y Smith [Kroo et al. 1991] llegaron a la misma conclusión, afirmando que el ala trasera tenía que soportar fuertes cargas de compresión debido a la reacción conjunta de las dos alas en la unión. En 1996, Kroo y Gallman [Gallman and Kroo 1996] realizaron un exhaustivo estudio evaluando varios métodos de cálculo para 
tener en cuenta estos efectos.

Por otro lado y de forma similar Mayuresh [Patil 2003] también afirma que el ala trasera soporta cargas de compresión elevadas producidas, en parte, por restringir el movimiento vertical del ala delantera en el punto de unión. La carga de compresión puede conducir al pandeo del ala trasera, aumentando la probabilidad de pandeo según disminuye el espesor del ala trasera, lo que implica la necesidad de reforzar la estructura del ala trasera aumentando por consiguiente su peso

\subsection{Aerodinámica de la aeronave de alas convergentes.}

Desde un punto de vista aerodinámico, todos los estudios realizados sobre este tipo de configuración particular respecto a la disposición de las superficies sustentadoras, presentan que la presencia del ala trasera, en sus diferentes configuraciones, tiene una influencia directa en los valores de las fuerzas aerodinámicas de sustentación y resistencia, y por consiguiente en los coeficientes aerodinámicos de sustentación, $C_{L}$, y resistencia, $C_{D}$, del sistema [Wolkovitch 1986; Kroo et al. 1991; Wai et al. 1996; Gallman et al. 1993; Nangia and Palmer 2006; Cuerno-Rejado et al. 2010].

los estudios realizados durante las décadas de los 80 y de los 90 sobre el modelo de alas convergentes desde el punto de vista de su aerodinámica, han establecido comparaciones entre configuraciones de alas convergentes y configuraciones tradicionales equivalentes, es decir con igual superficie alar y envergadura. Además, también se han realizado estudios donde se han analizado las posiciones en la unión entre el ala delantera y el ala trasera variando la separación, $l_{t}$, y altura relativa, $h_{t}$, entre las mismas manteniendo, en algunos casos, y modificando en otros los valores de ángulo diedro y ángulo de flecha de ambas alas (Figura 2.16) [Hirose et al. 1990; Kroo et al. 1991]. Estos estudios han dado como resultado, en primer lugar, que el valor del coeficiente de resistencia, $C_{D}$, es menor que para el caso del modelo de avión convencional y que esta diferencia aumenta según aumenta la separación longitudinal entre alas, $l_{t}$, y el parámetro de relación de envergaduras, $B$, [Wolkovitch 1986; Kroo et al. 1991], (ver Figura 2.17).

En segundo lugar, al comparar esta configuración con configuraciones tradicionales equivalentes, se ha comprobado que se produce un aumento en el valor del coeficiente de sustentación, $C_{L}$, del orden del 4 al $7 \%$ [Wolkovitch 1986; Mamla and Galinski 2009], aunque estos autores sugieren que este aumento se produce más por la contribución del ala trasera como elemento sustentador, siendo incluso menor que el esperado debido a motivos de interacción aerodinámica entre ambas alas. Tal y como se explicará en el capítulo siguiente, este aumento decrece con la separación longitudinal, $l_{t}$, y vertical, $h_{t}$, entre las dos alas, a pesar de que, para un mismo valor de la relación de envergaduras, $B$, el aumento de estos parámetros implica mayor superficie alar y por consiguiente mayor fuerza de sustentación esperada en el ala trasera.

Además, ya en la primera década de los años 2000, con la explosión del mercado de las aeronaves no tripuladas, los estudios sobre la aerodinámica del modelo de alas convergentes continuaron como aplicación orientada al entorno de 


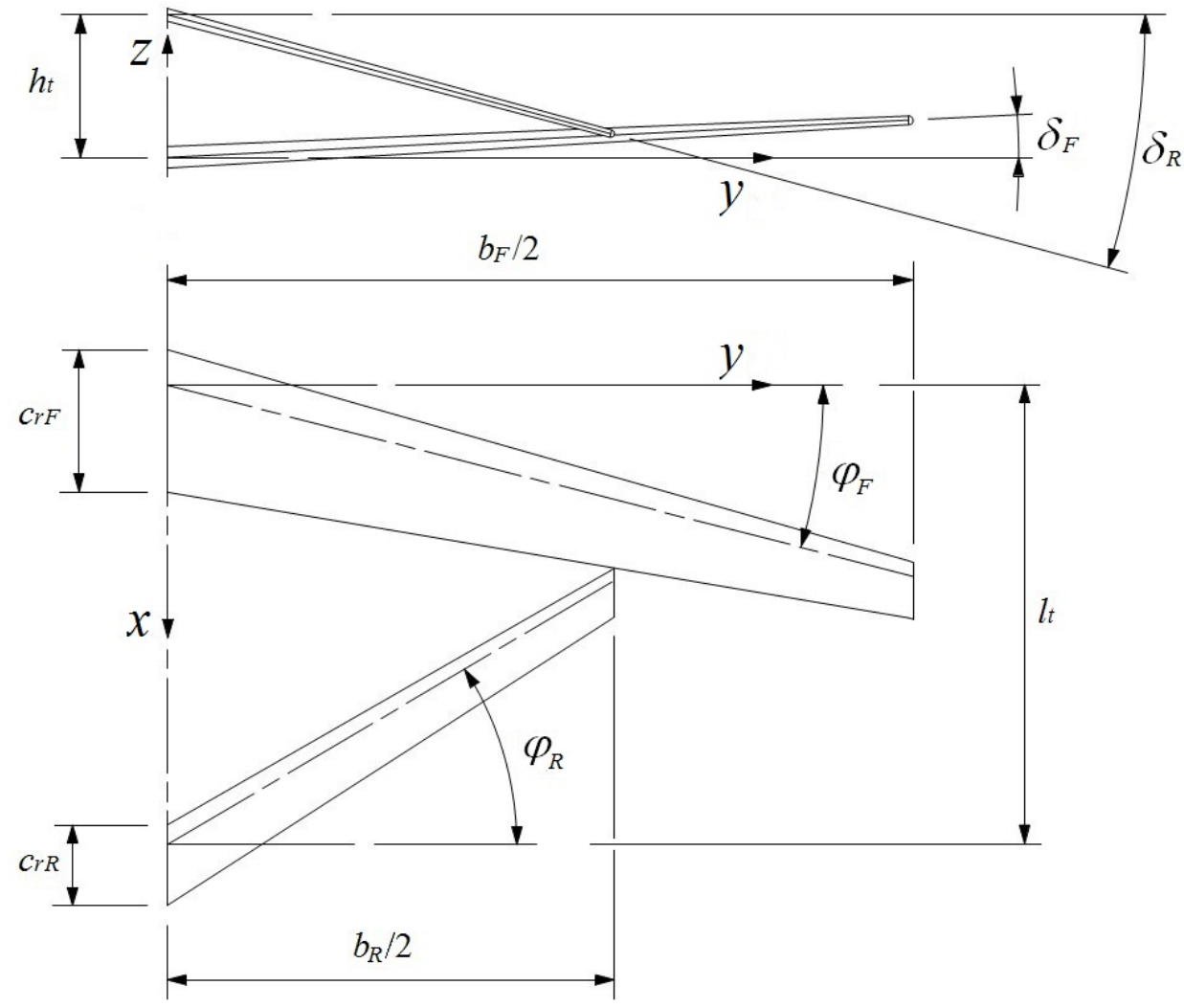

Figura 2.16: Vista en alzado y planta de la representación esquemática del modelo de alas convergentes con los principales parámetros geométricos.

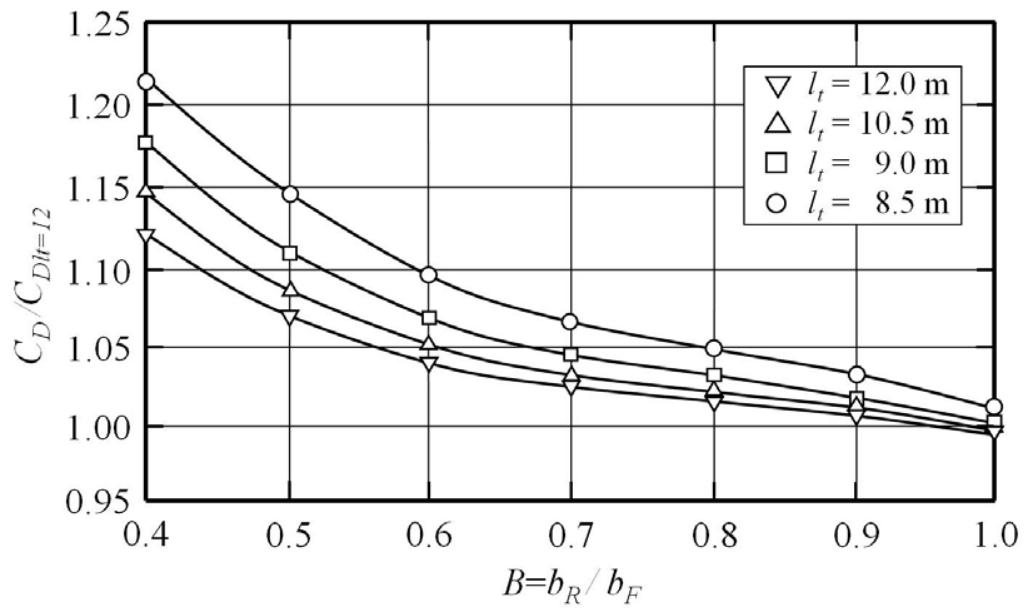

Figura 2.17: Relación entre el coeficiente de resistencia y el parámetro de relación de envergaduras, $B$, para diferentes posiciones relativas de ala trasera con respecto a delantera [Kroo et al. 1991]. Variación del valor del coeficiente de resistencia normalizado con el valor del coeficiente de resistencia para $l_{t}=12 \mathrm{~m}$ y $B=1$. 
los UAS (Unmanned Air System), como el caso de los estudios de optimización aerodinámica realizados por Rasmussen, Canfield y Blair [Rasmussen et al. 2004] con el objetivo de diseñar modelos de UAS para misiones de gran autonomía LE (long-endurance), o como el caso de Oligney, Frash y Yechout [Oligney et al. 2008], que estudiaron y ensayaron el modelo Houck de alas convergentes con el propósito de determinar los valores de resistencia (inducida, presión y fricción) para aeronaves en configuración de alas convergentes y en régimen subsónico bajo (Figura 2.18).

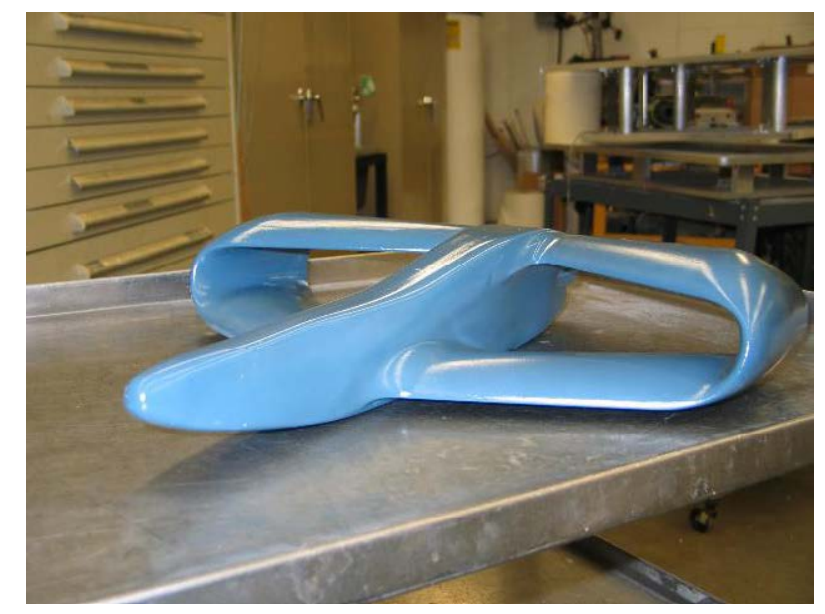

Figura 2.18: Modelo de alas convergentes Houck [Oligney et al. 2008].

Como conclusión parece que se puede establecer que en general todos los estudios presentan en sus resultados un aumento en el coeficiente de sustentación, $C_{L}$, y una reducción significativa del coeficiente de resistencia inducida, $C_{D i}$, en el modelo de alas convergentes, aunque no todos los autores presentan como la principal causa aspectos únicamente aerodinámicos debidos a la influencia entre asmbas alas, sino más bien causas debidas a la propia configuración geométrica del modelo como el aumento de la superficie sustentadora y el aumento del alargamiento del sistema de alas convergentes debido a la capacidad estructural de esta configuración de soportar alas con mayos enbergadura.

Por otro lado no todos los autores defienden el modelo clásico de las convergentes, es decir ala delantera baja y ala trasera alta, como la forma geométrica más optima de situar y unir ambas alas. Ya en 1986 Selberg y Cronin [Selberg and Cronin 1986] analizaron, desde el punto de vista aerodinámico, dos modelos de aeronave de alas convergentes; uno, denominado stagger positivo, con el ala delantera situada por encima del ala trasera y otro, denominado stagger negativo, con el ala delantera situada por debajo del ala trasera, llegando a la conclusión de que el modelo denominado de stagger positivo presentaba valores del coeficiente de resistencia del orden del $12 \%$ menores que en el caso del modelo de alas convergentes clásico.

De forma similar Bagwill y Selberg [Bagwill and Selberg 1996a; 1997] llegaron a la misma conclusión aunque los márgenes de los valores de los coeficientes que presentaban eran menores, del orden del $2 \%$ al $3 \%$. 


\section{Capítulo 3}

\section{Influencia de la configuración sobre los coeficientes aerodinámicos.}

\subsection{Introducción.}

En general, las teorías introducidas por Prandt [Prandtl 1920a;b], para el caso de sistemas de alas múltiples, y Munk [Munk 1923], para el caso de sistemas biplanos, son de gran utilidad para entender cómo es el efecto que produce la presencia de dos alas y proporcionar una explicación a como esta configuración influye en los coeficientes aerodinámicos de sustentación, $C_{L}$, y de resistencia inducida, $C_{D i}$.

\subsection{Influencia en el coeficiente de sustentación.}

Como ya se ha introducido en el capítulo anterior, una de las ventajas que presenta el modelo de alas convergentes es el aumento del coeficiente de sustentación, $C_{L}$, y de su valor máximo, $C_{L_{\max }}$, del orden de entre un $4 \%$ y un $7 \%$ para vuelo horizontal equilibrado [Wolkovitch 1986; Mamla and Galinski 2009].

De forma general es posible aplicar la teoría general de biplanos [Munk 1923] y adaptarla para el modelo de alas convergentes, por lo que hay que tener en cuenta ciertos parámetros geométricos particulares de esta configuración. En la Figura 3.1 está representada un sección transversal típica (plano $x z$ ) donde se muestra el ángulo de decalaje, $\delta$, entre las dos alas, que es considerado positivo cuando el ala superior tiene mayor incidencia que el ala inferior.

El valor gap, $G_{B}$, se define como la separación vertical entre las dos alas medida desde los punto $1 / 4$ de las cuerda de cada ala en la dirección del eje $z$ y el parámetro de stagger, $s$, se define como la separación longitudinal entre las dos alas, medida desde el punto $1 / 4$ de las cuerda del ala superior hasta el punto $1 / 4$ de la cuerda del ala inferior en la dirección del eje $x$. El parámetro de stagger también puede definirse como el valor del ángulo $\sigma$, entre el eje z y la línea que une el punto $1 / 4$ de la cuerda de cada ala, expresado como $\sigma=\arctan \left(s / G_{B}\right)$. 


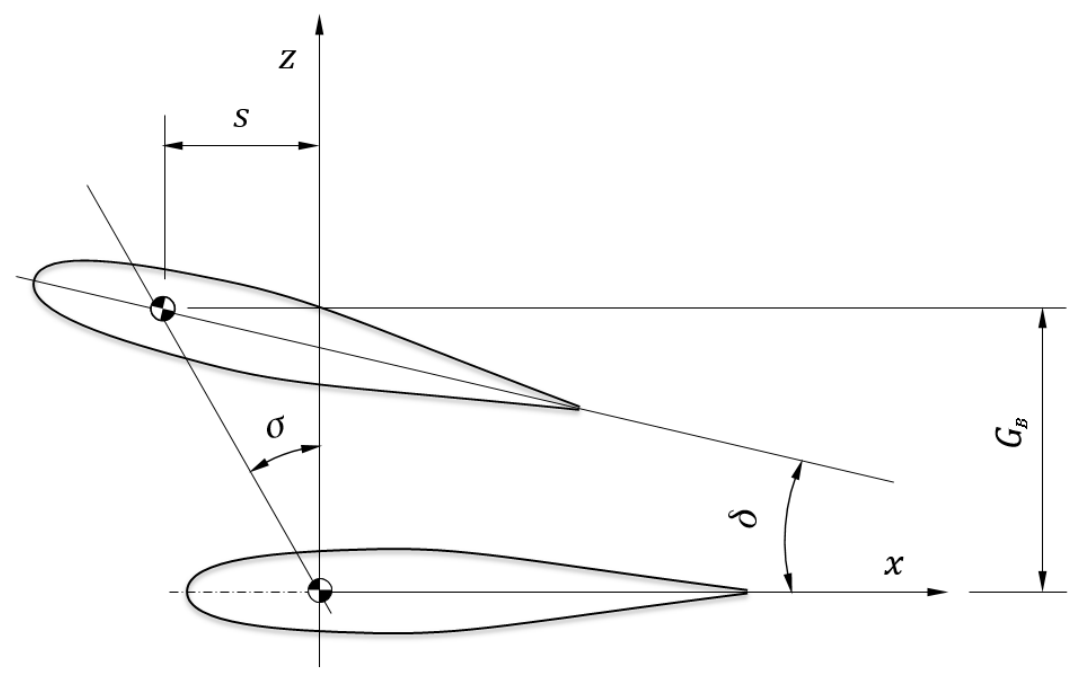

Figura 3.1: Sección transversal típica del modelo biplano donde se muestra un esquema de los parámetros geométricos característicos: Decalaje $(\delta)$, separación longitudinal (stagger, $s)$ y separación vertical (gap, $\left.G_{B}\right)$.

El valor del parámetro $s$, se considera positivo cuando la medida sigue el sentido del eje $x$ negativo, es decir, cuando el punto $1 / 4$ de la cuerda del ala alta se encuentra por delante del punto $1 / 4$ de la cuerda del ala baja, como en el caso del esquema de la Figura3.1.

De esta manera, si se tiene en cuenta cualquier sección transversal en planos paralelos al plano $x z$, el modelo de alas convergentes puede considerarse como un biplano con stagger negativo, $s$ (separación longitudinal entre alas en dirección del eje $x$ ), es decir, ala superior situada por detrás del ala inferior y gap,$G_{B}$ (separación vertical entre alas en dirección del eje $z$ ), es decir, ala trasera situada por encima del ala delantera. A diferencia del modelo biplano clásico, donde los parámetros de separación longitudinal y vertical son constantes, en el modelo de alas convergentes estos términos son variables en función de la distancia en el eje $y$, tomando valores desde una configuración en biplano hasta una configuración de alas en tandem en el punto de unión de ambas alas (Figura 3.2), de forma que sus valores geométricos característicos quedan definidos por las expresiones siguientes:

$$
\begin{gathered}
s(y)=l_{t}+y\left(\tan \varphi_{R}-\tan \varphi_{F}\right) \\
G_{B}(y)=h_{t}+y\left(\tan \delta_{R}-\tan \delta_{F}\right) \\
s(0)=l_{t}, s\left(b_{R}\right)=\frac{1}{4} c_{t R}+\frac{3}{4} c_{r F}\left[B\left(\lambda_{F}-1\right)+1\right] \\
G_{B}(0)=h_{t}, G_{B}\left(b_{R}\right)=0 \\
l_{t}=\frac{b_{R}}{2}\left(\tan \varphi_{F}-\tan \varphi_{R}\right)+\frac{1}{4} c_{t R}+\frac{3}{4} c_{r F}\left[B\left(\lambda_{F}-1\right)+1\right]
\end{gathered}
$$




$$
\begin{gathered}
h_{t}=\frac{b_{R}}{2}\left(\tan \delta_{F}-\tan \delta_{R}\right) \\
\theta(y)=\frac{\pi}{2}+\arctan \frac{G_{B}(y)}{s(y)},
\end{gathered}
$$

donde $c_{t R}, \mathrm{y} c_{r F}$, corresponden a los valores de la cuerda en punta del ala trasera y de la cuerda en raíz de el ala delantera respectivamente y $\lambda_{F}=c_{t F} / c_{r F}$, al valor del parámetro de estrechamiento del ala delantera.

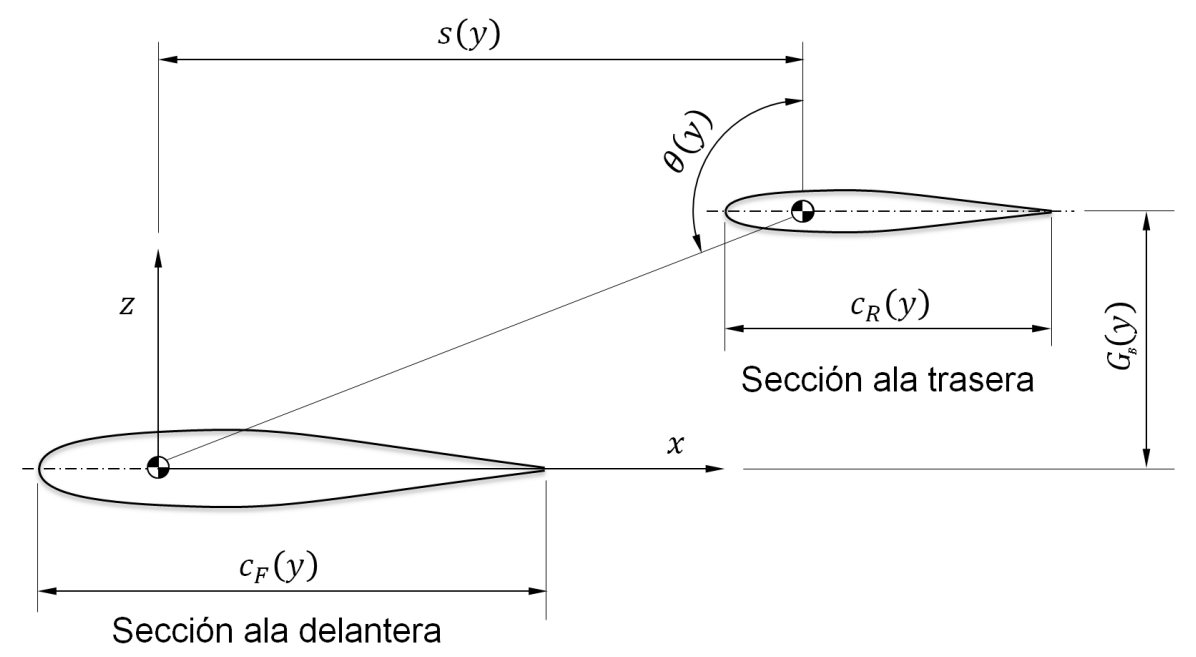

Figura 3.2: Sección transversal típica del modelo de alas convergentes donde se muestra un esquema de los parámetros geométricos de cada ala.

En principio y como es conocido, los coeficientes de sustentación de las alas individuales de un biplano son proporcionados por [Mock 1927]:

$$
\begin{aligned}
& C_{L_{U}}=C_{L}+\triangle C_{L_{U}} \\
& C_{L_{L}}=C_{L}+\triangle C_{L_{L}},
\end{aligned}
$$

donde $C_{L_{U}}, C_{L_{L}}$ y $C_{L}$, corresponden a los coeficientes de sustentación del ala superior, ala inferior y biplano respectivamente. Además el valor del incremento de los coeficientes de sustentación del ala superior e inferior están relacionados en la forma:

$$
\triangle C_{L_{U}}=-\triangle C_{L_{L}} \frac{S_{F}}{S_{R}}
$$

Diehl [Diehl 1934] calculó que el valor del incremento de sustentación del ala superior podía expresarse en función de dos constantes $k_{1}$ y $k_{2}$, dependientes de la separación longitudinal y vertical de las dos alas como:

$$
\triangle C_{L_{U}}=k_{1}+C_{L} k_{2}
$$


Además, la teoría generalizada de biplanos de Munk [Munk 1923] ofrece formulas, por medio de datos teóricos y experimentales para el estudio de las propiedades aerodinámicas de los sistemas biplanos. Munk define que el sistema experimenta un variación en el valor del coeficiente de sustentación para el caso de alas con separación longitudinal (stagger) en la forma:

$$
\triangle C_{L}= \pm 2 C_{L} \frac{S}{b^{2}}\left(\frac{1}{k^{2}}-\frac{1}{2}\right) \frac{b}{R} \frac{s}{b}
$$

donde el signo positivo corresponde al valor de incremento para el ala alta y el signo negativo para el ala baja, $S$ es la superficie alar total, $b$ la envergadura mayor, $s$ la separación longitudinal entre el ala delantera y trasera, y $k$ es el factor de envergadura del monoplano equivalente que tiene la misma superficie alar y la misma resistencia inducida. Para un avión monoplano $k=1$, pero para un biplano este valor varía con la relación entre la separación vertical y la envergadura media, aumentando según crece la separación vertical de las dos alas (Figura 3.3) [Stinton 1983; Diehl 1986; Roedts 2009].

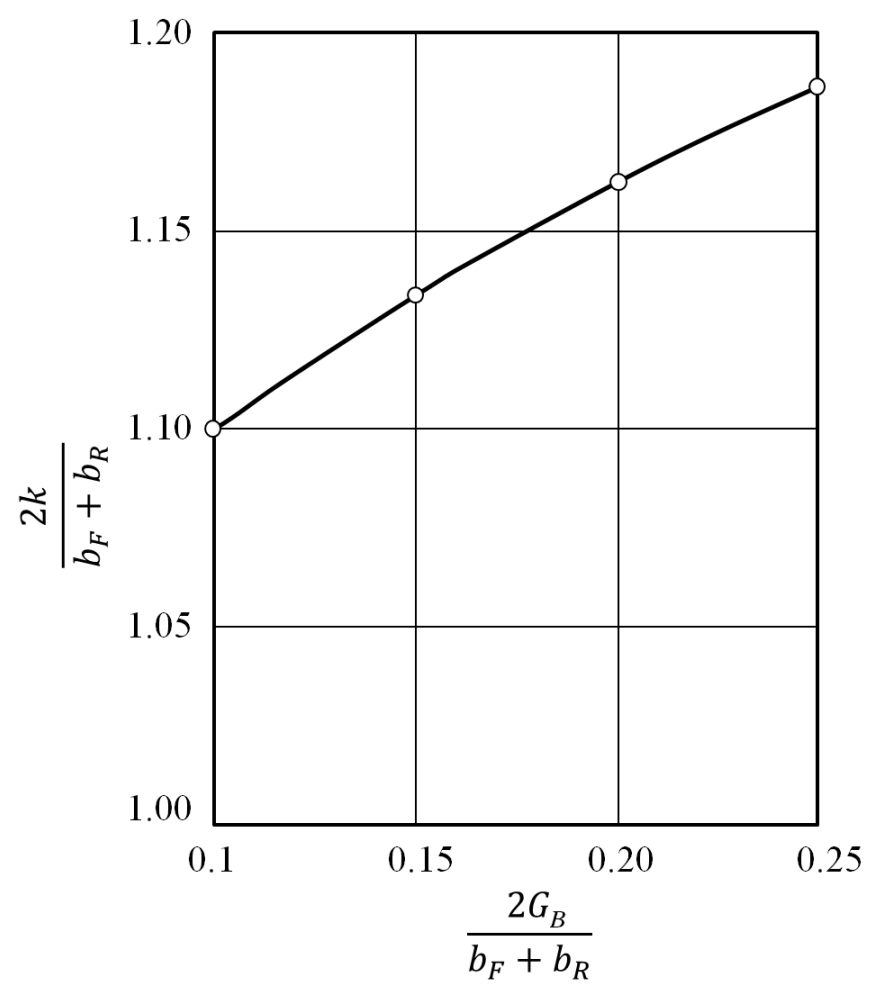

Figura 3.3: Variación del factor de envergadura del monoplano equivalente, $k$, en función de la separación vertical entre el ala delantera y el ala trasera, $G_{B}$, [Stinton 1983; Roedts 2009].

Por otro lado Munk definió el parámetro $R$, como la distancia $\sqrt{y^{2}+G_{B}^{2}}$, usada para el cálculo de la deflexión de la estela inducida por las alas y medida desde el punto medio de cada ala y el origen de cada torbellino longitudinal, donde $G_{B}$, representa la separación vertical e $y$, la coordenada lateral, siendo la intensidad del torbellino la correspondiente a una distribución de sustentación elíptica en cada ala. El valor de $R$, queda definido por: 


$$
R=\frac{b}{2} \frac{\int_{0}^{1} \frac{y^{2} \mathrm{~d} y}{\sqrt{\left(1-y^{2}\right)\left(y^{2}+G_{B}^{2}\right)^{2}}}}{\int_{0}^{1} \frac{y^{2} \mathrm{~d} y}{\sqrt{\left(1-y^{2}\right)\left(y^{2}+G_{B}^{2}\right)^{3}}}},
$$

siendo la integral del numerador $\frac{\pi}{2}\left(1+\frac{G_{B}}{\sqrt{1-G_{B}^{2}}}\right)$, y la integral del denominador $\frac{F(p)-E(p)}{\sqrt{1+G_{B}^{2}}}$, donde $F(p)$, y $E(p)$, son las integrales elípticas completas de primera y segunda especie respectivamente, de módulo $p=1 / \sqrt{1+G_{B}^{2}}$.

Este parámetro, relacionado con el factor de envergadura del monoplano equivalente, define lo que formalmente se denomina factor de Munk, $F_{M}$, función de la separación vertical de las dos alas, $G_{B}$,

$$
F_{M}=\left(\frac{1}{k^{2}}-\frac{1}{2}\right) \frac{b}{R}
$$

En un modelo biplano, el valor del factor de Munk crece cuando la separación vertical de las alas, $G_{B}$, disminuye, y por consiguiente el coeficiente de sustentación también aumenta [Kang et al. 2009]. Así en la Figura 3.4 se puede apreciar cómo el valor de este factor disminuye según aumenta el valor del ángulo diedro, $\delta_{R}$, del ala trasera (en valor absoluto).

Como es conocido, la presencia del ala superior modifica las condiciones aerodinámicas del ala inferior y viceversa, siendo evidente que cuanto mayor sea la separación longitudinal positiva o la separación vertical positiva menor será el efecto de interferencia igualando las cargas de ambas alas y por consiguiente, como se ha citado anteriormente, la sustentación también será mayor, tal como muestran los resultados obtenidos por Norton [Norton 1921], o por Montgomery y Noyes [Knight and Noyes 1929]. Además, en un modelo biplano clásico, donde el ala más elevada se encuentra por delante del ala más baja, es este ala la que experimenta valores mayores de sustentación, debido a la circulación del ala inferior, aumentando la velocidad del viento en el ala superior y la circulación del ala superior, disminuyendo la velocidad del viento del ala inferior. Sin embargo, en el modelo de configuración de alas convergentes, donde el ala alta se encuentra situada por detrás del ala baja, el efecto producido es el contrario siendo los cambios en los valores de la sustentación del ala delantera y del ala trasera más pronunciados con la separación longitudinal de las alas.

Por otra parte, Julian Wolkovitch [Wolkovitch 1986] y posteriormente Demasi [Demasi et al. 2015a], comprobaron que en este tipo de configuración, el ala delantera produce el $80 \%$ de la sustentación total del sistema. Además Wolkovitch, determinó que este ala muestra la tendencia a entrar en pérdida a ángulos de ataque inferiores a los que lo hace el ala trasera. Esta condición aporta al modelo excelentes capacidades de recuperación ante la entrada en pérdida proporcionando buenas características de estabilidad, pero en cambio no aprovecha todo el potencial sustentador del ala trasera. El hecho de que el ala trasera no alcance los valores máximos del coeficiente de sustentación, implica que el ala se encuentra sobredimensionada teniendo más peso que el necesario siendo, además, su carga alar del orden del 50-60\% del ala delantera. 


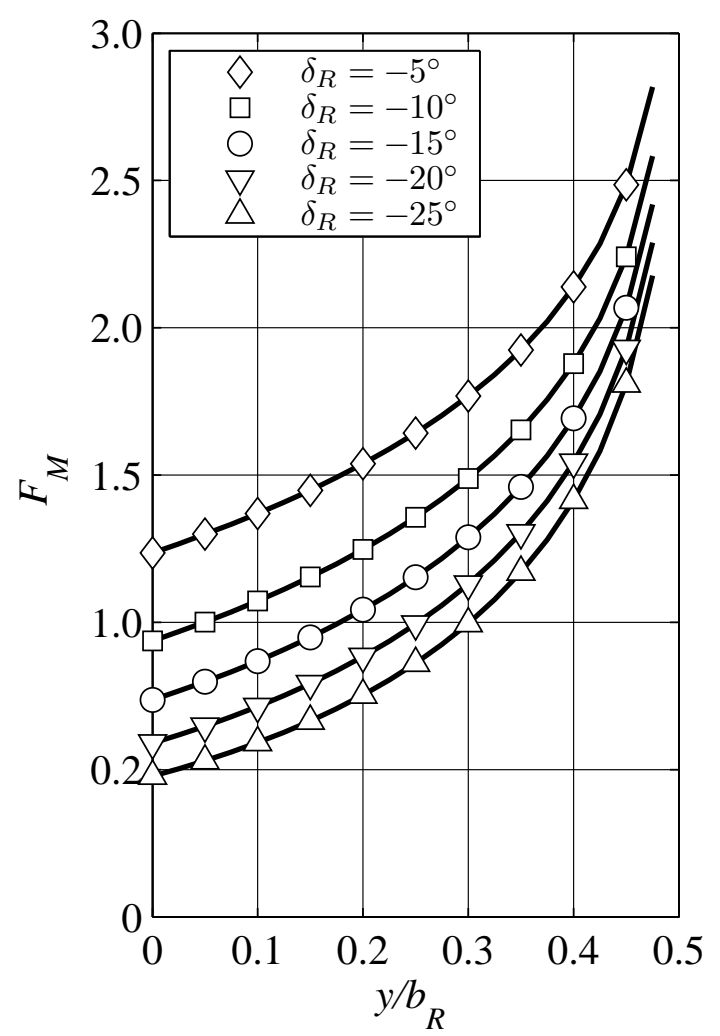

Figura 3.4: Variación del factor de Munk, $F_{M}$, a lo largo de la envergadura del ala trasera, $b_{R}$, y en función del ángulo diedro de la misma, $\delta_{R}$.

Además, según Wolkovitch [Wolkovitch 1986], la razón que justifica que el ala delantera entre en pérdida antes que el ala trasera es que el ala trasera experimenta una reducción del ángulo de ataque efectivo inducido por el ala delantera (Figura 3.5). Una buena elección de la geometría del ala trasera, en términos de su ángulo de flecha y diedro, y de los perfiles aerodinámicos de ambas alas pueden proporcionar mejoras en el aprovechamiento de la sustentación local del ala trasera y por consiguiente de la sustentación global del sistema sin pérdidas de prestaciones en su estabilidad [Selberg and Cronin 1986; Addoms and Spaid 1975].

Sin embargo y a pesar de lo establecido por Prandtl [Prandtl 1924], la sustentación total del modelo de alas convergentes presenta valores inferiores que el resultado de la simple suma de la sustentación producida por el ala delantera, $L_{F}$, y el ala trasera, $L_{R}$, de forma individual. Esta reducción se produce debido a la influencia que causa la presencia del ala delantera en el ala trasera y viceversa [Hirose et al. 1990], especialmente en el punto de unión de las dos alas. La principal causa es un descenso en la sustentación del ala trasera debido a la velocidad inducida por el ala delantera. Este descenso se minimiza y resulta aerodinámicamente más efectivo al cambiar la posición del ala delantera con respecto al ala trasera, es decir, ala delantera por encima del ala trasera [Mamla and Galinski 2009], aunque reduce las prestaciones estructurales y presenta mayores complicaciones constructivas.

Por todo lo anterior se puede establecer que, en un modelo de alas 


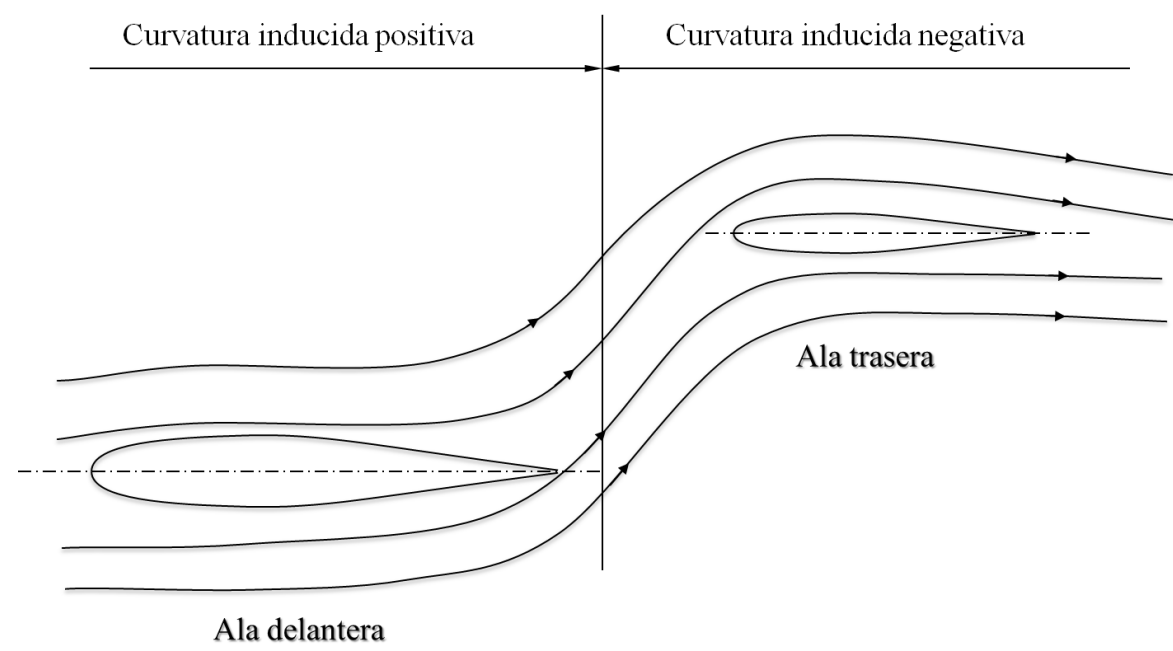

Figura 3.5: Sección transversal típica del modelo de alas convergentes donde se muestra, esquemáticamente, el efecto de la presencia de las dos alas [Wolkovitch 1985].

convergentes, el valor del coeficiente de sustentación aumenta según disminuye la distancia longitudinal $l_{t}$, y la distancia vertical $h_{t}$, entre el ala delantera y el ala trasera, o lo que es equivalente según disminuye el ángulo diedro $\delta_{R}$, y el ángulo de flecha $\varphi_{R}$, del ala trasera en valor absoluto.

\subsection{Influencia en el coeficiente de resistencia inducida.}

En general, una de las ventajas más importantes del modelo de avión de alas convergentes, es una reducción significativa en el valor del coeficiente de resistencia inducida, $C_{D i}$.

Para el caso de una configuración de avión monoplano con ala sin torsión y distribución de cuerda y sustentación elípticas, el resultado clásico de la teoría de la línea de sustentadora de Prandtl [Prandtl 1920a;b] para el coeficiente de resistencia inducida es:

$$
C_{D i}=\frac{C_{L}^{2}}{\pi \Lambda}
$$

donde $\Lambda$ es el valor del alargamiento del ala, definido como $\Lambda=b^{2} / S$, siendo $b$, el valor de la envergadura del ala y $S$, la superficie alar. La ecuación (3.15) representa el valor teórico de la resistencia inducida mínima y es considerado como valor de referencia para el diseño aerodinámico de alas. Como la distribución de sustentación de la aeronave en su conjunto se desvía de la distribución elíptica, el coeficiente de resistencia inducida se suele escribir de la siguiente manera:

$$
C_{D i}=\frac{C_{L}^{2}}{\pi \Lambda e}
$$

siendo e, el factor de eficiencia de Oswald cuyo valor depende de la distribución de circulación a lo largo de la envergadura del ala. El valor teórico del factor de eficiencia, para el caso de avión monoplano, es menor o igual que la unidad, aunque 
para configuraciones de alas múltiples este factor puede alcanzar valores mayores que uno, lo que implica valores de la resistencia inducida menores que el mínimo proporcionado por la ecuación (3.15).

El problema de reducir la resistencia inducida de sistemas de alas múltiples (incluyendo la configuración de alas convergentes) fue estudiado por Prandtl [Prandtl 1924], que proporcionó fórmulas para el cálculo teórico del factor de eficiencia de Oswald usando las leyes generales establecidas por Munk [Munk 1923]. Investigaciones numéricas recientes han demostrado que los teoremas de resistencia inducida mínima de Munk también son aplicables a modelos de alas convergentes así como para modelos de alas dobles en general [Demasi et al. 2014; 2015a;b].

Según Prandtl la determinación de la resistencia inducida para el caso de configuraciones de ala múltiple, consiste en el cálculo de la resistencia inducida de cada ala que define el sistema, añadiendo la resistencia inducida debida a la mutua influencia entre cada ala. Para el caso particular de un sistema de dos alas, como en el modelo de alas convergentes, el término de resistencia inducida, $D_{i}$, puede escribirse como [Prandtl 1924]:

$$
D_{i}=\frac{1}{\pi q}\left(\frac{L_{F}^{2}}{b_{F}^{2}}+2 \sigma \frac{L_{F} L_{R}}{b_{F} b_{R}}+\frac{L_{R}^{2}}{b_{R}^{2}}\right),
$$

donde $b_{F}$ y $b_{R}$ corresponden a los valores de envergadura del ala delantera y trasera respectivamente, $L_{F}$, y $L_{R}$, son los valores de sustentación de las alas y $\sigma$, el valor de interferencia de Prandtl para sistemas biplanos. Este valor depende de la relación entre envergaduras y la separación vertical de las dos alas, $h_{t}$, tal como muestra la Figura 3.6. Este valor puede aproximarse por la ecuación proporcionada por Laitone [Laitone 1990]:

$$
\frac{\sigma}{B}=1-\sqrt{\left[1+\left(\frac{b_{F}}{2 h_{t}}\right)^{2}\right]} .
$$

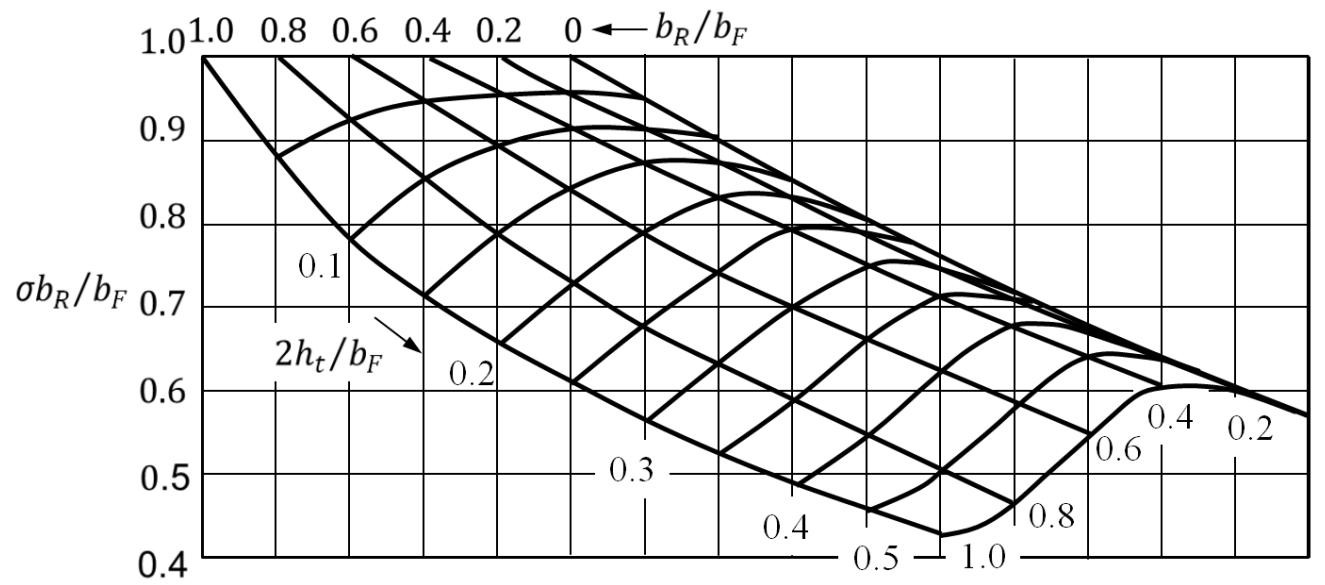

Figura 3.6: Aproximación de Laitone para el valor de interferencia de Prandtl $\sigma$, [Laitone 1990].

Por otra parte, Prandtl estableció que la sustentación del sistema completo, $L$, era la suma de la sustentación del cada ala, estableciendo la relación de sustentaciones entre el ala delantera y trasera, $r$, como: 


$$
r=\frac{L_{R}}{L_{F}}=\frac{x}{(1-x)}
$$

donde $x$, representa el valor proporcional de la contribución de la sustentación del ala delantera a la sustentación total del sistema, definido como:

$$
x=1-\frac{L_{F}}{L}
$$

Sustituyendo entonces (3.20) en (3.17), el valor de la resistencia inducida, $D_{i}$, puede escribirse como:

$$
D_{i}=\frac{L^{2}}{\pi q b_{F}^{2}} f(x)
$$

siendo $f(x)$ :

$$
f(x)=(1-x)^{2}+2 \sigma \frac{x(1-x)}{B}+\frac{x^{2}}{B^{2}}
$$

El valor de la resistencia inducida puede hacerse mínimo buscando el valor de $x$, que hace que la derivada de la función $f(x)$, se anule, de forma que:

$$
\frac{\mathrm{d} f(x)}{\mathrm{d} x}=0 \rightarrow x=\frac{(B-\sigma)}{\left(\frac{1}{B}-2 \sigma+B\right)} \rightarrow f(x)=\frac{\left(1-\sigma^{2}\right)}{\left(1-2 \sigma B+B^{2}\right)}
$$

Sustituyendo, ahora (3.23) en (3.21) se obtiene el valor de la resistencia inducida mínima [Prandtl 1923].

$$
D_{\text {imin }}=\frac{L^{2}}{\pi q b_{F}^{2}} \frac{\left(1-\sigma^{2}\right)}{\left(1-2 \sigma B+B^{2}\right)}
$$

Además, y de forma general, sustituyendo (3.20) en (3.17), el valor del coeficiente de resistencia inducida, $C_{D i}$ puede escribirse como:

$$
C_{D i}=\frac{C_{L}^{2}}{\pi \Lambda}\left(\frac{B^{2}+2 \sigma r B+r^{2}}{(1+r)^{2} B^{2}}\right)
$$

Comparando con (3.16), el factor de eficiencia de Oswald queda:

$$
e=\frac{B^{2}\left(1+r^{2}\right)}{B^{2}+2 \sigma B r+r^{2}}
$$

Las teorías de Prandtl-Munk predicen que los sistemas con configuración de alas múltiples presentan menos resistencia inducida que sistemas con configuraciones convencionales con valores de envergadura y sustentación total similares.

En este contexto, Prandtl estableció que la idea principal para reducir la resistencia inducida es reducir, todo lo posible, los gradientes de circulación. 
Con esta idea, Wolkovitch [Wolkovitch 1979; 1986] realizó varios estudios donde mostraba las ventajas en el uso de sistemas con configuración de ala múltiple.

En primer lugar una de las ventajas, que él demostró, fue que (en configuraciones de alas en tándem) la resistencia inducida es menor si la separación vertical entre el ala delantera y el ala trasera aumentaba.

En segundo lugar, Wolkovitch [Wolkovitch 1986] comprobó, en ensayos realizados en túnel aerodinámico, que el valor del factor de eficiencia de Oswald, $e$, resultaba mayor que el que predice la teoría de biplanos de Prandtl-Munk [Munk 1923], (Figura 3.7).

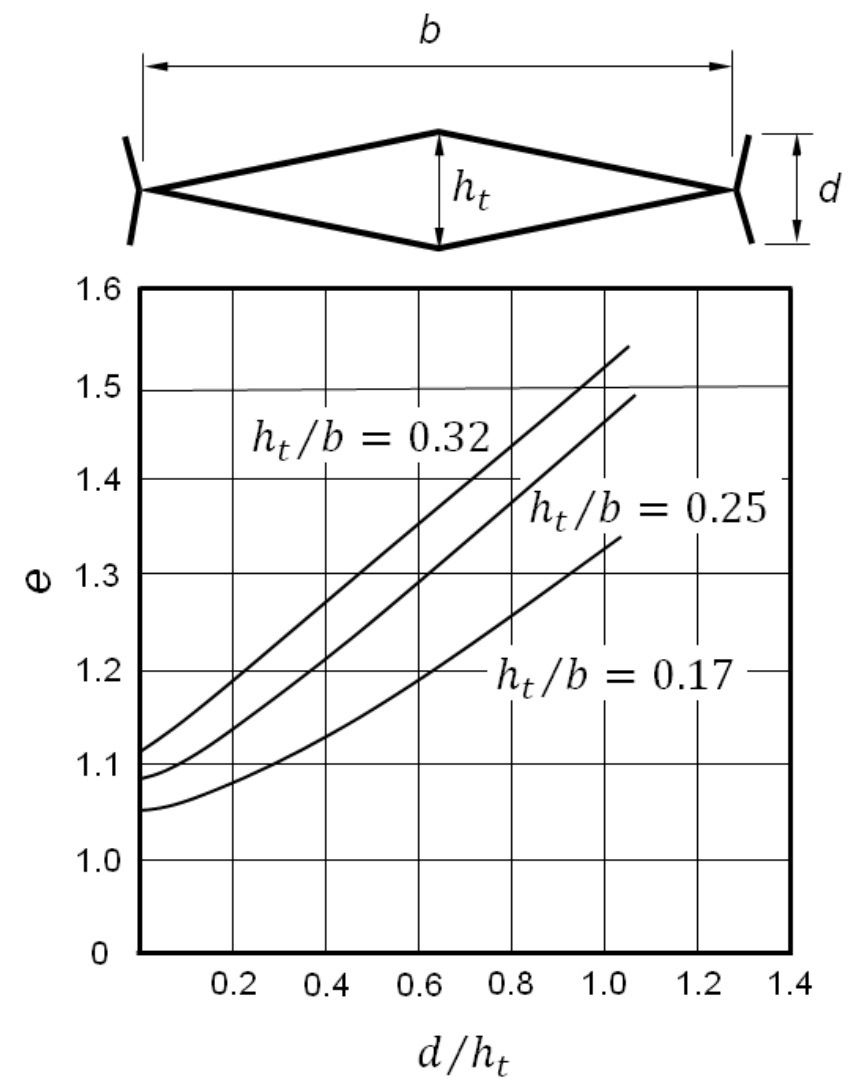

Figura 3.7: Valor teórico del factor de eficiencia de Oswald, e, para la configuración de alas convergentes con álulas (winglet) simétricos e inclinados un ángulo de valor $\pi / 2$ con respecto al ala [Wolkovitch 1986].

El método analítico de Prandtl-Munk daba como resultado que la relación del parámetro de eficiencia de Oswald, e, de la configuración de alas convergentes con respecto al monoplano equivalente es del orden de 1.05, mientras que los ensayos realizados por Wolkovitch mostraron que ese valor es de 1.09, es decir, un $4 \%$ mayor que lo que predice la teoría de Prandtl-Munk. Wolkovitch justificó este resultado alegando que la teoría clásica no tenía en cuenta la deflexión de la estela sobre el ala delantera, ni la influencia de los valores de los ángulos de flecha de ambas alas, cuantificando esta variación como:

$$
e=e_{t}\left[1+\frac{\left(\tan \varphi_{F}+\tan \varphi_{R}\right)}{\left(\frac{3}{2} \pi\right)^{2}+1}\right]
$$


donde $\varphi_{F}, \mathrm{y} \varphi_{R}$, son los ángulos de flecha en la línea de puntos $1 / 4$ del ala delantera y trasera respectivamente y $e_{t}$, es el valor teórico del factor de eficiencia de Oswald.

Las teorías de Prandtl y Munk, al considerar solo la huella o contorno que dejan las alas en el plano de Trefftz, no pueden tener en cuenta los efectos de la separación entre ambas alas en la resistencia inducida total del sistema, tal y como establece el primer teorema de Munk o como normalmente se le conoce como el teorema de separación de Munk [Munk 1921]:

\section{"The total induced drag of any multiplane lifting system is unaltered if any of the lifting elements are moved in the direction of the motion provided that the attitude of the elements is adjusted to maintain the same lift distribution of lift among them."}

Sin embargo Wolkovitch concluyó, con los datos obtenidos en ensayos en túnel aerodinámico, que la influencia que produce la separación longitudinal de las dos alas en la resistencia inducida es despreciable cuando esta es menor que la envergadura y del orden de la CMA (cuerda media aerodinámica) para el caso de un biplano, mientras que para la configuración de alas convergentes esta separación es mucho mayor, es decir, que en el modelo de alas convergentes existe una influencia clara de los ángulo de flecha de las respectivas superficies sustentadoras [Wolkovitch 1986].

Este aumento del valor del factor de eficiencia produce una reducción del coeficiente de resistencia inducida, $C_{D i}$, del orden del $5 \%$.

Anteriormente, el valor teórico del factor de eficiencia de Oswald había sido estimado por Cone [Cone 1962] y Letcher [Letcher 1972] para varios tipos de formas y configuraciones de sistemas de ala múltiple, aunque fue DeYoung [DeYoung 1980], varios años después, quien desarrollo una ecuación generalizada para el cálculo del factor de eficiencia para cualquier tipo de forma en la sección transversal (plano $x-z)$ de las alas incluyendo la configuración de alas convergentes.

Letcher estableció, haciendo uso de los teoremas Munk [Munk 1921], que la resistencia inducida mínima se produce cuando la componente normal de la velocidad inducida es proporcional al $\cos \delta$, siendo $\delta$, el ángulo diedro de cada elemento sustentador, que para el caso de estudio este valor es constante a lo largo de la envergadura del ala delantera y del ala trasera.

Como es conocido, en puntos muy alejados corriente abajo del ala (plano de Trefftz), el movimiento del fluido inducido por los torbellinos del ala es bidimensional en el plano transversal [DeYoung 1979; Smith 1996] y la estela, en primera aproximación, puede considerarse plana. Aunque esta afirmación no es del todo exacta ya que existe enrollamiento de la estela y la velocidad inducida presenta, en el plano de Trefftz, una componente $u$, en dirección del eje $x$, negativo [Smith 1996] (ver Figura 3.8). Sin embargo, como el enrollamiento de la estela se produce lo suficientemente lejos, la mayor parte de la misma que afecta al ala puede considerarse plana (Figura 3.9).

Por otro lado la estela no permanece contenida en el plano $z=0$, sino que tiene una pequeña inclinación asintótica cuyo ángulo puede expresarse con la relación $2 w / U_{\infty}$, siendo $U_{\infty}$ la velocidad de la corriente libre y $w$ la velocidad inducida sobre el ala. 


\section{Estela alineada con la corriente libre}

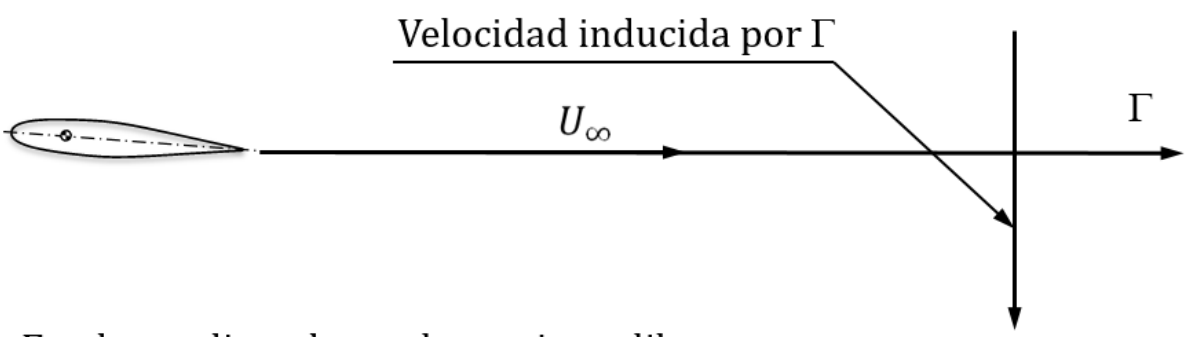

Estela no alineada con la corriente libre

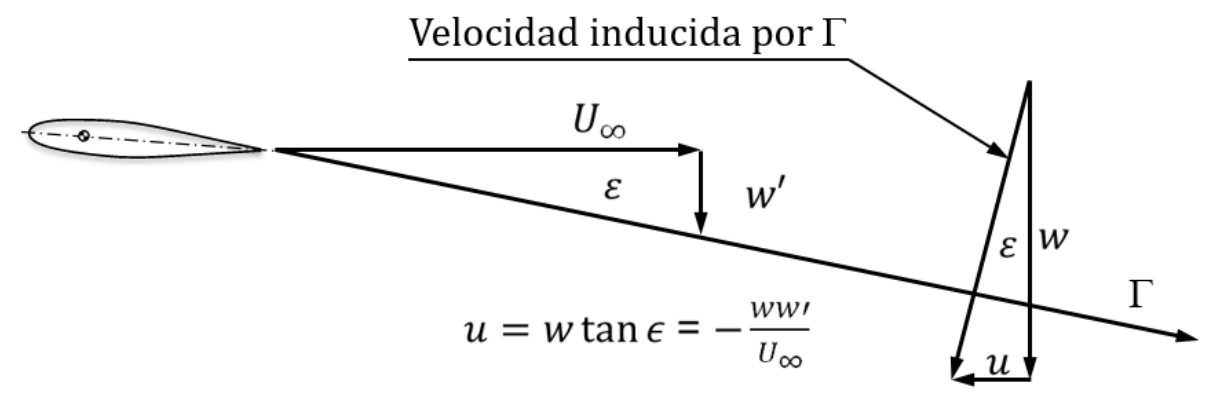

Figura 3.8: Esquema de la perturbación de la velocidad $U_{\infty}$, cuando la estela se encuentra y no se encuentra alineada con la corriente libre.

En ciertas situaciones cuando un cuerpo es acelerado en un fluido, es posible utilizar la segunda ley de Newton para calcular la fuerza que actúa sobre el cuerpo. En lugar de considerar la distribución de velocidades en la estela, se puede asumir que la acción del ala sobre el aire está toda concentrada en la parte de aire que pasa a través de cierta superficie $S_{T}$, perpendicular a la dirección del movimiento, al pasar por esta superficie el aire experimenta una reacción en dirección del eje $z$, y como consecuencia se comunica una cantidad de movimiento sobre este. La masa de aire que pasa a través de la superficie $S_{T}$, por unidad de tiempo está definida como $M_{T}=\rho S_{T} U_{\infty}$, por lo que en vuelo estacionario, la sustentación del ala puede expresarse en términos de conservación de la cantidad de movimiento en la forma [Durand 1963]:

$$
L=M U_{\infty} \cdot 2 w,
$$

donde $M$, es conocida con el nombre masa añadida, y es la masa inducida [Bos 1987] por unidad de longitud del perfil bidimensional $P$, definido como la sección transversal del ala proyectada sobre el plano de Trefftz. Esta masa inducida es una propiedad del perfil $P$ y es proporcional a la densidad de la corriente libre $\rho$ y un área de referencia [Katz and Plotkin 2001].

Este movimiento implica que al aire se le comunique una cantidad de energía cinético de valor:

$$
E=\frac{1}{2} M U_{\infty}(2 w)^{2}
$$

Por otro lado el trabajo para generar esta energía es debido a la resistencia inducida cuyo valor es $E=D_{i} U_{\infty}$, por lo que la resistencia inducida, en sí, puede escribirso como: 


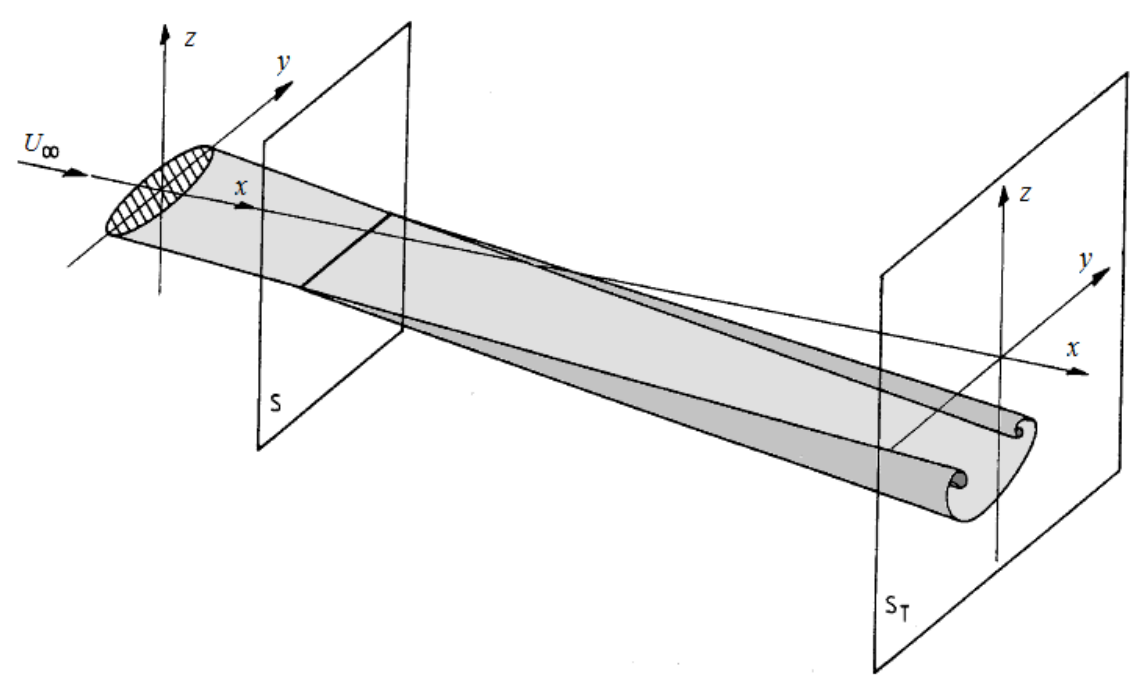

(a)

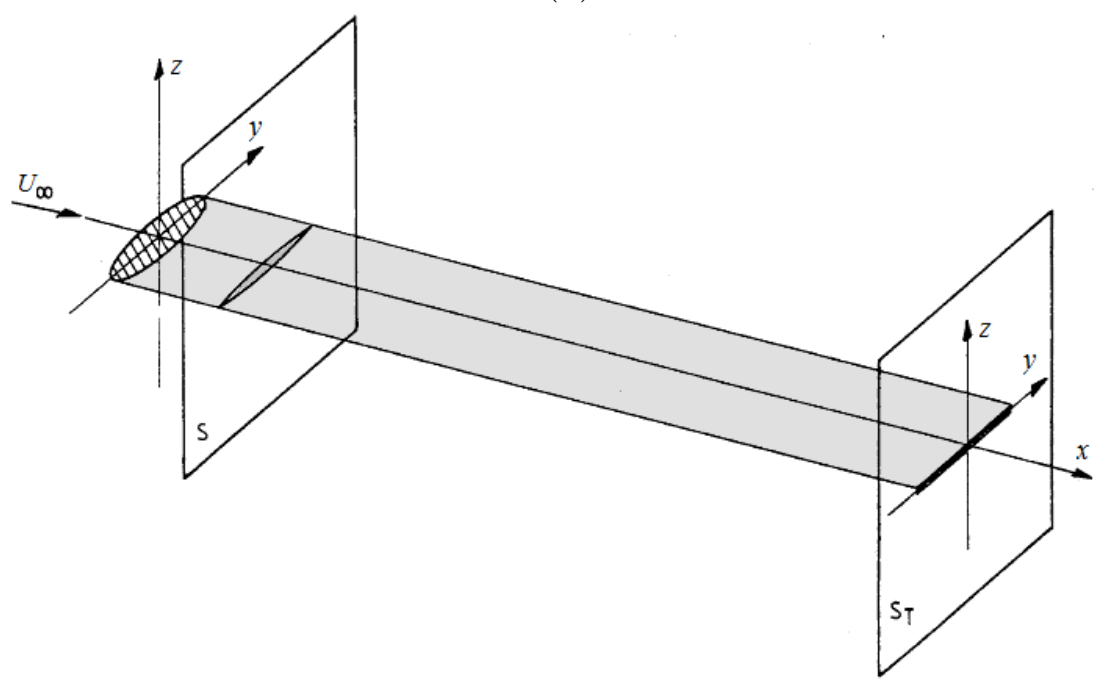

(b)

Figura 3.9: Esquema del movimiento del fluido inducido por los torbellinos del ala. (a) Esquema con enrollamiento de estela y (b) esquema de estela plana [Bos 1987].

$$
2 D_{i}=M(2 w)^{2}
$$

donde $M$, es también denominada con el nombre masa añadida, y es la masa inducida en el movimiento [Bos 1987] por unidad de longitud del perfil bidimensional $P$, definido como la sección transversal del ala proyectada sobre el plano de Trefftz. Esta masa inducida es una propiedad del perfil $P$ y es proporcional a la densidad de la corriente libre $\rho$ y un área de referencia [Katz and Plotkin 2001].

Como es fácil de ver, la velocidad inducida puede eliminarse expresando la resistencia inducida en términos de su coeficiente y del coeficiente de sustentación.

$$
C_{D i}=\frac{1}{4}\left(\frac{\rho}{S M}\right) C_{L}^{2}
$$


siendo, $\rho$, la densidad de la corriente libre y $S$, es la superficie alar a la que los coeficientes están referenciados.

El problema radica en la determinación del valor de la masa inducida, $M$, para cada forma de la sección transversal proyectada $P$. Para calcularla es posible determinar el potencial complejo que satisface la condición de contorno y presenta circulación nula alrededor del cuerpo, por medio de la función analítica regular [Bos 1987]:

$$
f(z)=2 w\left(z+\sum_{n=1}^{\infty} A_{n} z^{-n}\right)
$$

donde la variable compleja $z=x+$ i $y$ está definida en el plano de Trefftz, siendo los términos, $A_{n}$, valores reales.

La masa añadida, $M$, puede identificarse con el primer término, $A_{1}$, [Letcher 1972; Bos 1987] como:

$$
M=2 \pi \rho A_{1},
$$

siendo el término, $A_{1}$, equivalente al residuo de la función $g(z)=z+\sum_{n=1}^{\infty} A_{n} z^{-n}$, (ver anexo A). Este valor puede obtenerse analíticamente haciendo uso del teorema global de la función inversa y de la transformación compleja de Schwarz-Christoffel [Churchill and Brown 1992] que permite transformas un polígono cerrado (sección transversal del ala, $P$ ) en el eje real, $\xi$, (ver Figura 3.10). Por convenio con respecto a las funciones de variable compleja se han transformado los ejes cuerpo $y-z$ de la sección transversal del ala en los ejes $x-$ i $y$ en el plano de Trefftz.

En general, este modelo analítico ha sido estudiado y aplicado a geometrías de sistemas de alas múltiples y sistemas de alas convergentes, aunque en este último caso limitadas al modelo de alas en forma de diamante, es decir, alas delantera y trasera unidas en punta de ala y con ángulos diedros del ala delantera $\delta_{F}$, y del ala trasera, $\delta_{R}$, iguales y de signo contrario, lo que proporciona como resultado formas de diamante (rombo) en la sección transversal del ala, $P$ (Figura 3.10-a).

Luego, aplicando el modelo de Letcher para el caso de alas en forma de diamante (ver anexo A), el factor de eficiencia puede expresarse como:

$$
e=\frac{\pi\left(1+H^{2}\right)\left(1-\frac{2}{\pi} \tan ^{-1} H\right)}{\left[\Gamma\left(\frac{1}{2}+\frac{1}{\pi} \tan ^{-1} H\right) \Gamma\left(1-\frac{1}{\pi} \tan ^{-1} H\right)\right]^{2}},
$$

donde, $\Gamma$, es la aplicación matemática función gamma y el parámetro, $H$, representa el valor adimensional de la separación vertical entre las dos alas, $h_{t}$, y cuyo valor, para el caso de alas en forma de diamante, puede ser calculado en función del valor del ángulo diedro común, $\delta$, como:

$$
H=\frac{h_{t}}{b}=\tan \delta
$$

Sin embargo DeYoung [DeYoung 1980] desarrolló un método para realizar la estimación del factor de eficiencia aplicable a cualquier tipo de configuración de 


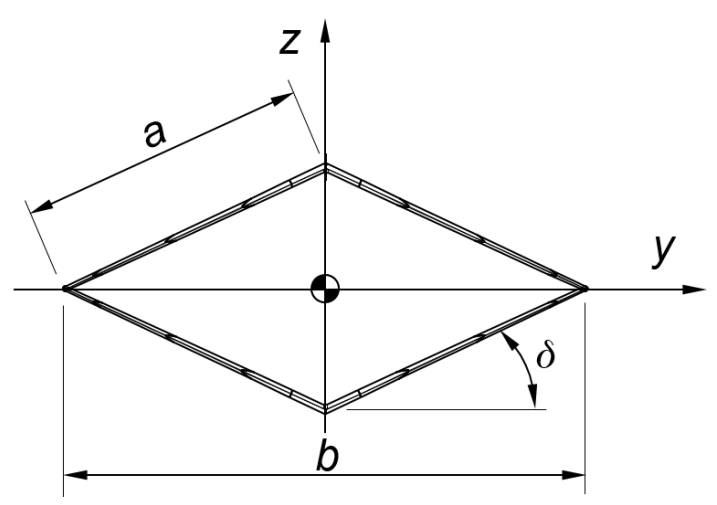

(a)

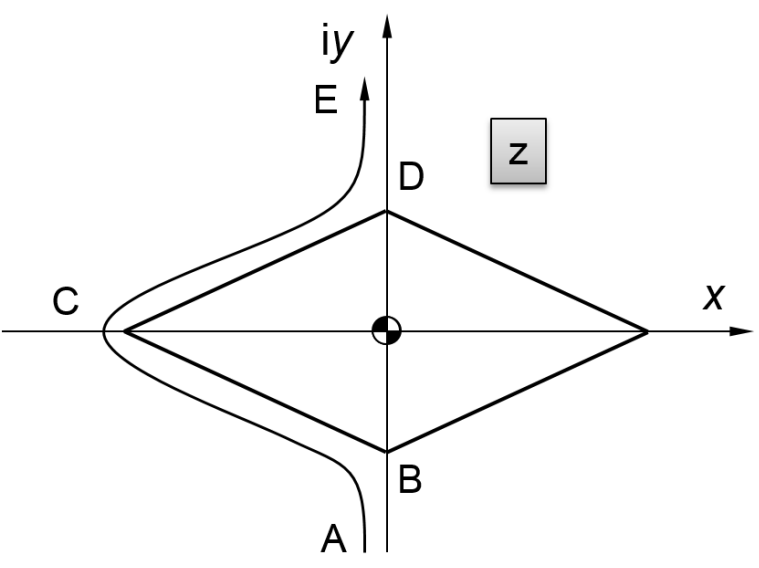

(b1)

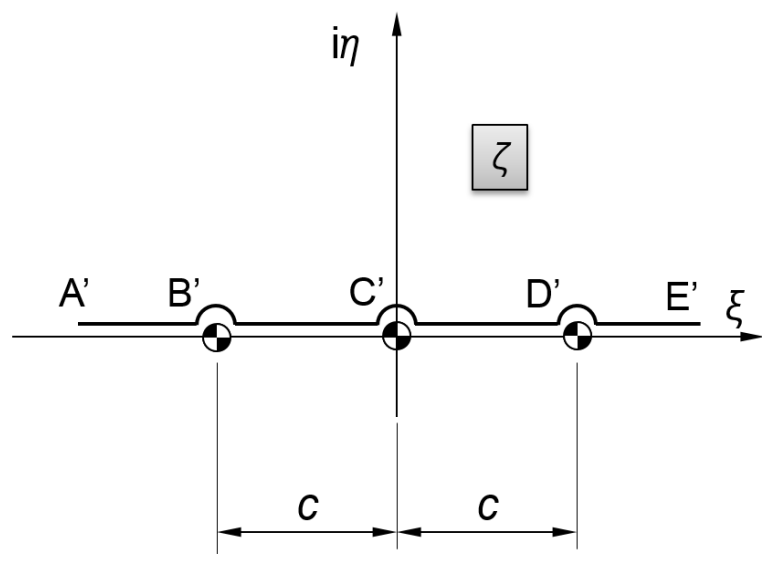

(b2)

Figura 3.10: Esquema de transformación de Schwarz-Christoffel para el modelo de alas en forma de diamante. (a) Vista frontal (plano $y-z$ ) del sistema de alas en forma de diamante; (b1) esquema del perfil proyectado, $P$, sobre el plano de Trefftz ; (b2) esquema del perfil transformado sobre el eje real $\xi$.

sistemas de alas múltiples y sistemas de alas convergentes. Este método define una ecuación generalizada basada en ocho tipos de configuraciones de alas múltiples y de alas convergentes, los cuales presentan solución analítica exacta al cálculo del factor de eficiencia de Oswald, e. La ecuación general se desarrolló por medio de métodos estadísticos teniendo en cuenta cada configuración en la que está basada el método, tanto desde el punto de vista aerodinámico como geométrico.

Este método proporciona una función de cálculo sencilla cuyos resultados presentan mínimas desviaciones con respecto a los valores que proporcionan los métodos analíticos, tal y como se puede apreciar en la Figura 3.11 donde se muestra el valor de, e, en función de la separación vertical adimensional, $H$. 


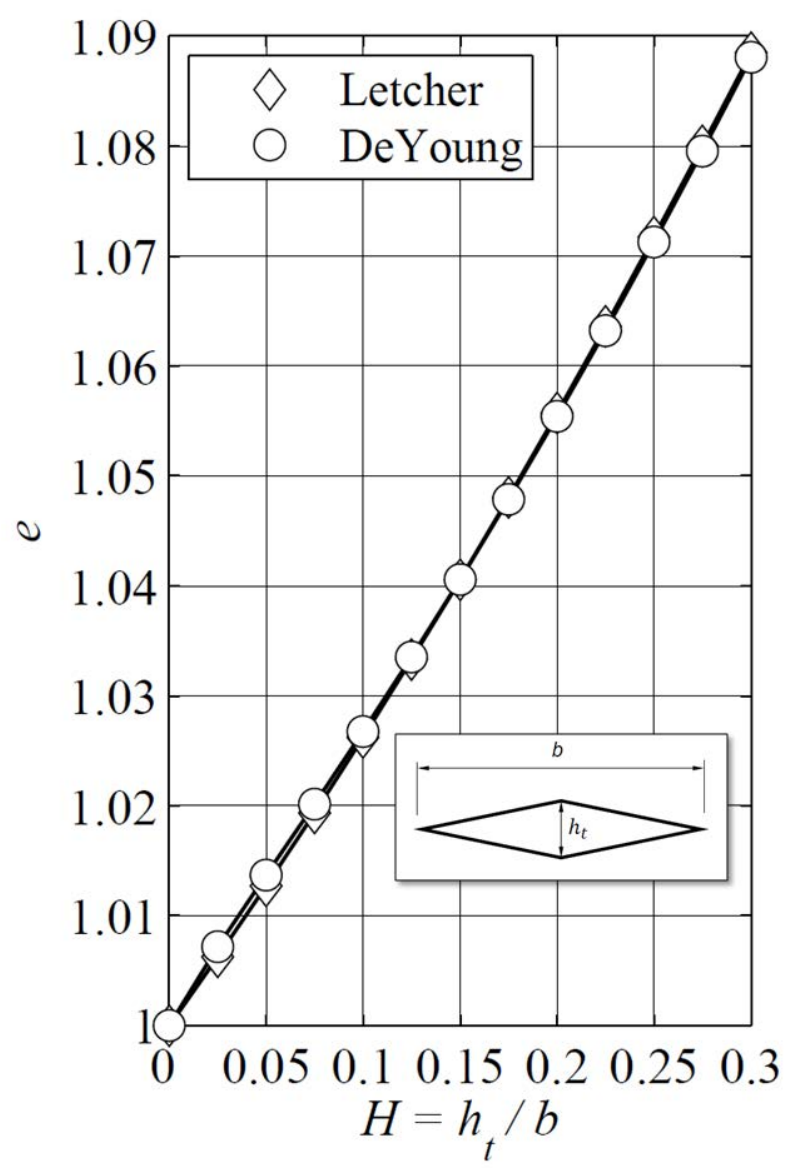

Figura 3.11: Valor teórico del factor de eficiencia de Oswald, e, para la configuración de alas convergentes en forma de diamante calculado con el método analítico de Letcher y el predictivo de DeYoung [DeYoung 1980; Letcher 1972]. 


\section{Capítulo 4}

\section{Ensayos para la determinación de los coeficientes aerodinámicos}

\subsection{Introducción.}

Para la determinación de los coeficientes aerodinámicos del modelo de alas convergentes es necesario la realización de ensayos en túnel aerodinámico. A tal efecto es necesario definir los parámetros de la configuración geométrica de ensayo, así como los métodos de fabricación de los componentes, la construcción de los modelos físicos, su posterior instalación en el túnel, la definición de los parámetros de ensayo y el postprocesado de los resultados.

Con los datos obtenidos es posible la realización de estudios comparativos de los resultados de las medidas en los ensayos, entre diferentes configuraciones geométricas del modelo.

Por todo ello a continuación se exponen y describen la definición del modelo de ensayo y los diferentes equipos utilizados para la realización de las medidas necesarias durante la caracterización y el ensayo. Además se presentan diversos procedimientos en la realización de las medidas y la calibración de los equipos en los casos necesarios.

Una vez elegidos, analizados y construidos todos los elementos necesarios se miden las fuerzas aerodinámicas generadas en el modelo de ensayo (sustentación y resistencia), la presión dinámica y la velocidad en la cámara de ensayo del túnel. Los equipos utilizados son los siguientes:

- Túnel aerodinámico Túnel A9.

- Sensor Fuerza/Par Delta SI-330-30.

- Equipo de anemometría de hilo caliente DANTEC.

En general los criterios para la elección de los equipos han sido técnicos, aunque en algunos casos la disponibilidad de los mismos ha condicionado la elección entre 
varias alternativas. A su vez esta elección ha influido en parámetros geométricos del modelo y en parámetros de operación en el ensayo.

\subsection{Modelo físico de ensayo}

Para la realización del estudio comparativo, es necesaria la construcción de un modelo físico de ensayo que permita adoptar, de forma sencilla, la geometría de las diferentes configuraciones.

Este modelo físico de ensayo está constituido por la semiala delantera, la semiala trasera y una placa de unión para el encastre de ambas, siendo la configuración básica la representada, con sus parámetros geométricos, en planta y alzado, en la Figura 2.16.

La geometría del modelo de ensayo está completamente determinada con los parámetros definidos en la Tabla 4.1

Tabla 4.1: Parámetros geométricos para la definición del modelo de ensayo.

\begin{tabular}{cl}
\hline Parámetro & \multicolumn{1}{c}{ Definición } \\
\hline$b_{F}$ & Envergadura ala delantera \\
$\lambda_{F}$ & Estrechamiento ala delantera \\
$c_{r F}$ & Cuerda en raíz ala delantera \\
$\varphi_{F}$ & Ángulo de flecha ala delantera \\
$\delta_{F}$ & Ángulo diedro ala delantera \\
$\lambda_{R}$ & Estrechamiento ala trasera \\
$c_{r}$ & Cuerda en raíz ala trasera \\
$\varphi_{R}$ & Ángulo de flecha ala trasera \\
$\delta_{R}$ & Ángulo diedro ala trasera \\
$B$ & Relación entre envergaduras \\
$l_{t}$ & Distancia entre alas (ver expresión 3.5) \\
$h_{t}$ & Altura entre alas (ver expresión 3.6) \\
\hline
\end{tabular}

El resto de los parámetros geométricos necesarios para la completa definición del modelo de ensayos son funciones de los anteriores relacionados por medio de expresiones que permiten determinar la cuerda en punta del ala delantera, $c_{t F}=\lambda_{F} c_{r F}$; la envergadura del ala trasera, $b_{R}=B b_{F} ;$ y la cuerda en punta del ala trasera, $c_{t R}=\lambda_{R} c_{r R}$.

Para el establecimiento de los valores de los parámetros geométricos se han tenido en cuenta varias consideraciones de aspecto técnico y funcional, como:

- El valor de la envergadura del ala delantera, y de la cuerda en la raíz, han sido determinados para evitar correcciones por bloqueo geométrico en la cámara de ensayo del túnel aerodinámico. Como es bien sabido, para que esto suceda, la relación entre el área frontal del modelo de ensayo y el área de la sección transversal de la cámara del túnel ha de ser menor que 7.5\% [Barlow et al. 1999]. Para el modelo de ensayo, con las dimensiones determinadas y para el caso más desfavorable, es decir, el ala trasera en su posición de altura más baja y más separada y el conjunto en ángulo de ataque, a un valor de $15^{\mathrm{O}}$, esta relación es del orden del $4 \%$. 
- Otra condición que limita del valor de la envergadura del ala delantera ha sido el proceso de fabricación. Para obtener una buena precisión en la definición del modelo de ensayo, la fabricación de las alas ha sido realizada en material ABSplus empleado técnicas FDM (Fused Deposition Modeling) de impresión 3D [Wong and Hernandez 2012], lo que implica limitar el tamaño de las mismas a la capacidad de volumen de trabajo de la cámara de la impresora 3D.

- Para la selección del valor del parámetro de relación de envergaduras, $B$, se han tenido en cuenta los estudios realizados por diferentes autores, los cuales, tras analizar diferentes configuraciones, sugieren que los valores óptimos de este parámetro se encuentran entre 0.6 y 0.75 [Wolkovitch 1986; Kroo et al. 1991].

- El valor asignado a la flecha del ala delantera, $\varphi_{F}=15^{\circ}$, difiere del que, en general, ha sido definido en la literatura [Smith and Stonum 1989]. Si bien la mayoría de los autores estudian configuraciones próximas a los $30^{\mathrm{O}} \mathrm{deg}$ se ha decidido estudiar valores más pequeños; primero para buscar situaciones más acordes con el propósito del trabajo, y segundo por los condicionantes geométricos y de velocidad en la cámara de ensayo.

- Para la unión de las dos alas se ha considerado una unión rígida, la cual ha sido elegida por ser la que presenta mejor relación entre resistencia y rigidez [O'Banion et al. 1982; Stearman et al. 1990] (Figura 4.1).

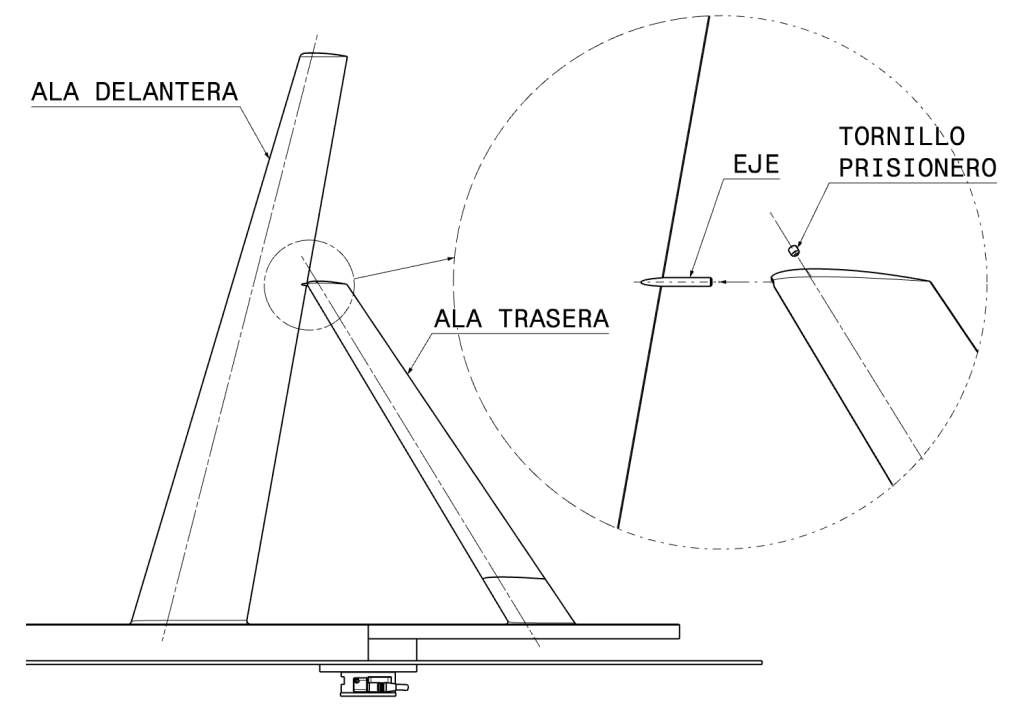

Figura 4.1: Esquema donde se muestra la unión rígida entre el ala delantera y el ala trasera. El detalle muestra la unión fija del eje al ala delantera y la unión con el ala trasera haciendo uso de un tornillo prisionero para el bloqueo del eje.

- Por último para la elección del perfil se han considerado aspectos constructivos, de resistencia y aerodinámicos [Bertagnolio 2008]. La elección de un perfil simétrico simplifica la fabricación de las alas. El espesor es relativamente elevado y proporciona mayor rigidez al conjunto, y desde el punto de vista aerodinámico, como es conocido, espesores relativos entre el $12 \%$ y el $15 \%$ proporcionan mayor valor del coeficiente de sustentación máximo [Bertin and Smith 1989]. 
Desde otro punto de vista, el perfil NACA0015 muestra un comportamiento aceptable para bajos números de Reynolds, tal como se muestra en la Figuras 4.2 y 4.3 .

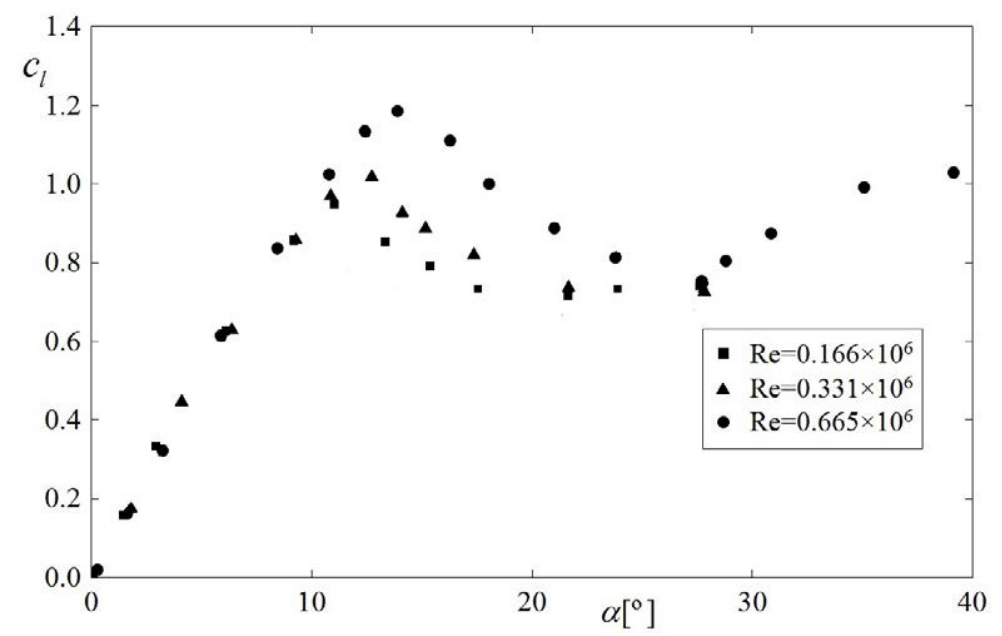

Figura 4.2: Variación del coeficiente de sustentación, $c_{l}$, con el ángulo de ataque, $\alpha$, para el perfil NACA0015 y para diferentes valores de número de Reynolds [Cuerva et al. 2006].

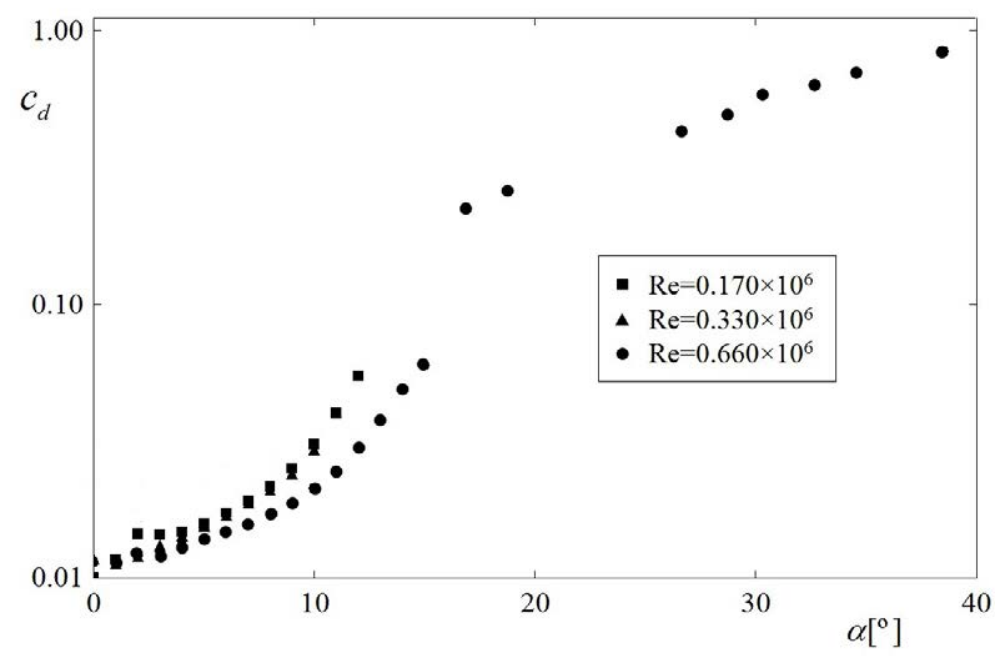

Figura 4.3: Variación del coeficiente de resistencia, $c_{d}$, con el ángulo de ataque, $\alpha$, para el perfil NACA0015 y para diferentes valores de número de Reynolds [Cuerva et al. 2006].

Teniendo en cuenta estos criterios es posible asignar dimensiones a los diferentes parámetros geométricos del ala delantera y del ala trasera (Tabla 4.2).

Todas las configuraciones de estudio se obtienen manteniendo todos los parámetros del ala delantera constantes y modificando dos parámetros geométricos del ala trasera: el ángulo diedro, $\delta_{R}$ y el ángulo de flecha, $\varphi_{R}$. Como resultado, se obtienen 25 configuraciones de ensayo diferentes (Tabla 4.3).

Debido a la gran variabilidad de configuraciones de ensayo es necesario la construcción de un modelo versátil que permita el cambio de cada configuración 
Tabla 4.2: Valor de los parámetros geométricos para la definición del modelo de ensayo.

\begin{tabular}{cl}
\hline Parámetro & \multicolumn{1}{c}{ valor } \\
\hline$b_{F}$ & $1.56 \mathrm{~m}$ \\
$\lambda_{F}$ & 0.4 \\
$c_{r F}$ & $0.158 \mathrm{~m}$ \\
$\varphi_{F}$ & $15^{\mathrm{O}}$ \\
$\delta_{F}$ & $3^{\mathrm{o}}$ \\
$B$ & 0.6 \\
$b_{R}$ & $0.93 \mathrm{~m}$ \\
$\lambda_{R}$ & 0.6 \\
$c_{r}$ & 0.09 \\
$\varphi_{R}$ & Ver Tabla 4.3 \\
$\delta_{R}$ & Ver Tabla 4.3 \\
\hline
\end{tabular}

Tabla 4.3: Configuraciones de estudio en función del ángulo de flecha, $\varphi_{R}, \mathrm{y}$ del ángulo diedro, $\delta_{R}$, del ala trasera.

\begin{tabular}{c|ccccc}
\hline \multirow{2}{*}{$\begin{array}{c}\text { Ángulo diedro del ala } \\
\text { trasera, } \delta_{R}\left[^{\circ}\right]\end{array}$} & \multicolumn{5}{|c}{ Ángulo de flecha del ala trasera, $\varphi_{R}\left[^{\circ}\right]$} \\
\cline { 2 - 6 } & -10 & -15 & -20 & -25 & -30 \\
\hline-25 & $\mathrm{JWC}_{11}$ & $\mathrm{JWC}_{12}$ & $\mathrm{JWC}_{13}$ & $\mathrm{JWC}_{14}$ & $\mathrm{JWC}_{15}$ \\
-20 & $\mathrm{JWC}_{21}$ & $\mathrm{JWC}_{22}$ & $\mathrm{JWC}_{23}$ & $\mathrm{JWC}_{24}$ & $\mathrm{JWC}_{25}$ \\
-15 & $\mathrm{JWC}_{31}$ & $\mathrm{JWC}_{32}$ & $\mathrm{JWC}_{33}$ & $\mathrm{JWC}_{34}$ & $\mathrm{JWC}_{35}$ \\
-10 & $\mathrm{JWC}_{41}$ & $\mathrm{JWC}_{42}$ & $\mathrm{JWC}_{43}$ & $\mathrm{JWC}_{44}$ & $\mathrm{JWC}_{45}$ \\
-5 & $\mathrm{JWC}_{51}$ & $\mathrm{JWC}_{52}$ & $\mathrm{JWC}_{53}$ & $\mathrm{JWC}_{54}$ & $\mathrm{JWC}_{55}$ \\
\hline
\end{tabular}

de forma cómoda, sencilla y rápida. Para tal propósito, la construcción del modelo físico de ensayo se ha realizado, como ya se ha comentado, mediante tecnología FDM de impresión 3D. Los procesos FDM crean piezas añadiendo capa por capa de abajo a arriba calentando y extruyendo un filamento termoplástico de material ABSplus [Latiff et al. 2014]. El proceso es sencillo:

- Procesado previo: El software de preparación de bandeja lamina y coloca un archivo CAD 3D. A continuación, calcula la trayectoria para extrudir el material termoplástico y cualquier material de soporte necesario.

- Producción: La impresora 3D calienta el material termoplástico hasta que alcanza un estado semilíquido y lo deposita en gotas ultrafinas a lo largo de la trayectoria de extrusión. En los casos en los que se precisa un soporte o apoyo, la impresora 3D deposita un material eliminable que hace las veces de andamiaje.

- Posprocesado: Se retira el material de soporte o se disuelve en agua y detergente y, a continuación, la pieza ya se puede utilizar.

En la Figura 4.4 se muestra el modelo de impresora 3D utilizado para la construcción de los modelos de ensayo.

Cada configuración de ensayo el ala trasera está compuesta de dos piezas: una pieza básica (marca 3 en Figura 4.5) que define el ángulo diedro y el ángulo 


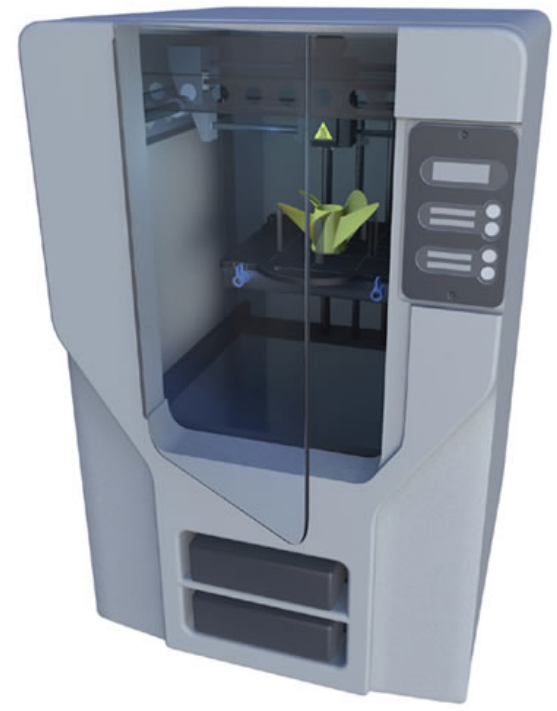

Figura 4.4: Máquina de impresión 3D con tecnología FDM.

de flecha, y una pieza de ala parcial que completa y define la envergadura del ala trasera (marca 2 en Figura 4.5)

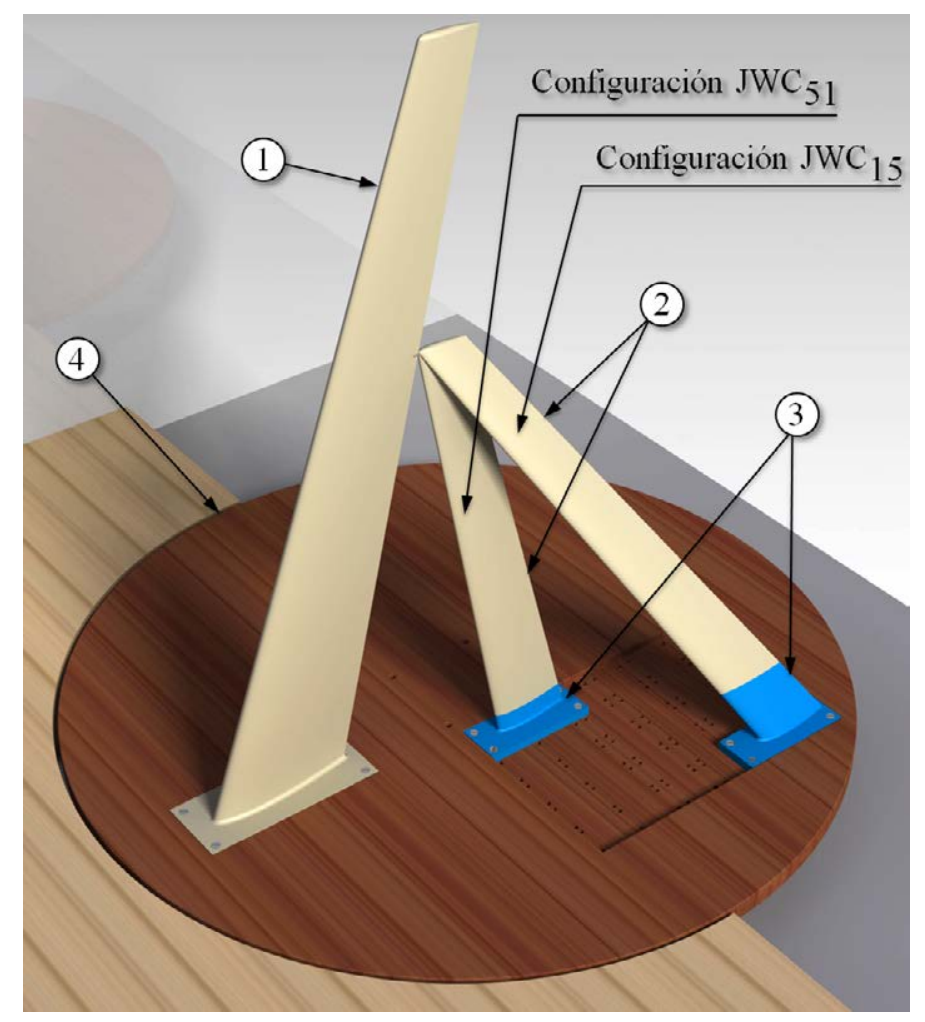

Figura 4.5: Esquema del modelo de ensayo donde se muestra la unión del ala delantera (1) con el ala trasera $(2,3)$ y fijada en la mesa giratoria (4). La figura muestra dos configuraciones diferentes: $J \mathrm{WC}_{51} \mathrm{y}$ $\mathrm{JWC}_{15}$.

La unión con el ala delantera (marca 1 en Figura 4.5) está definida en un punto único para todas las configuraciones, por lo que la longitud de cada cuerpo de ala trasera es diferente en función de cada posición en la mesa giratoria (marca 4 en Figura 4.5), siendo el parámetro de relación de envergaduras, $B$, constante. 
En la Figura 4.6 se muestra los modelos construidos en impresión 3D de la pieza básica correspondientes al ángulo de flecha del ala trasera, $\varphi_{R}=-10^{\circ}$, y para todos los ángulos diedros del ala trasera, $\delta_{R}=\left[-5^{\circ},-10^{\circ},-15^{\circ},-20^{\circ},-25^{\circ}\right]$.

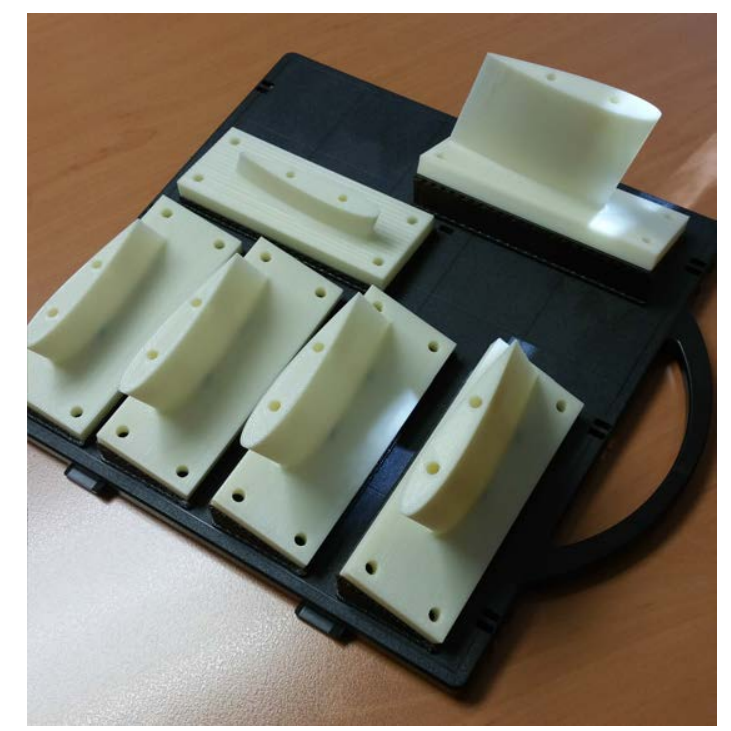

Figura 4.6: Bandeja de impresora 3D con los modelos construidos de la pieza básica para ángulo de flecha del ala trasera, $\varphi_{R}=$ $-10^{\circ}$ y para todo los ángulos diedros del ala trasera, $\delta_{R}=$ $\left[-5^{\circ},-10^{\circ},-15^{\circ},-20^{\circ},-25^{\circ}\right]$.

La variación de estos parámetros (ángulo diedro y ángulo de flecha del ala trasera) implican cambios en la posición del ala trasera con respecto al ala delantera (Figura 4.7). Por un lado, la variación del ángulo diedro implica cambios en la posición de la cuerda en la raíz del ala trasera en dirección del eje $z, h_{t}$. decreciendo si el ángulo diedro decrece en valor absoluto. Por otro lado, un cambio en el valor del ángulo de flecha modifica la posición de la cuerda en la raíz del ala trasera en dirección del eje $x, l_{t}$, reduciendo esta distancia si el ángulo de flecha decrece en valor absoluto.

Como ya ha definido en el punto 3.2 del capítulo 3, y ahora se recuerda, la variación de la distancia y de la altura entre el ala delantera y el ala trasera está relacionada con el resto de los parámetros geométricos como:

$$
\begin{gathered}
l_{t}=\frac{b_{R}}{2}\left(\tan \varphi_{F}-\tan \varphi_{R}\right)+\frac{1}{4} c_{t R}+\frac{3}{4} c_{r F}\left[B\left(\lambda_{F}-1\right)+1\right] \\
h_{t}=\frac{b_{R}}{2}\left(\tan \delta_{F}-\tan \delta_{R}\right)
\end{gathered}
$$

De forma general y para el resto de los cálculos, los valores de la variación de distancia y altura entre alas se presentan adimensionalizados con respecto a los valores de la configuración $\mathrm{JWC}_{15}$ (ver Tabla 4.3). Estos valores de referencia corresponde al valor del ángulo diedro del ala trasera, $\delta_{R}=-25^{\circ}$, y al valor del ángulo de flecha del ala trasera, $\varphi_{R}=-30^{\circ}$. Los valores adimensionales quedan en la forma: 


$$
\begin{gathered}
\overline{l_{t}}=\frac{l_{t}}{l_{t J W C_{15}}} \\
\overline{h_{t}}=\frac{h_{t}}{h_{t J W C_{15}}}
\end{gathered}
$$

De esta forma y haciendo uso de las expresiones (4.1) y (4.2) los valores de la distancia adimensional, $\overline{l_{t}}$, y de la altura adimensional, $\overline{h_{t}}$, están representados en la Tabla 4.4 y en la Tabla 4.5.

Tabla 4.4: Valores de la distancia adimensional $\overline{l_{t}}$ en función del ángulo de flecha, $\varphi_{R}$ del ala trasera.

\begin{tabular}{cccccc} 
& \multicolumn{6}{c}{ Ángulo de flecha del ala trasera, $\varphi_{R}\left[^{\circ}\right]$} \\
\cline { 2 - 6 } & -10 & -15 & -20 & -25 & -30 \\
\hline$\overline{l_{t}}$ & 0.6 & 0.7 & 0.8 & 0.9 & 1.0 \\
\hline
\end{tabular}

Tabla 4.5: Valores de la altura adimensional $\overline{h_{t}}$ en función del ángulo diedro, $\delta_{R}$ del ala trasera.

\begin{tabular}{cccccc}
\cline { 2 - 6 } & \multicolumn{5}{c}{ Ángulo diedro del ala trasera, $\delta_{R}\left[^{\circ}\right]$} \\
\cline { 2 - 6 } & -5 & -10 & -15 & -20 & -25 \\
\hline$\overline{h_{t}}$ & 0.3 & 0.4 & 0.6 & 0.8 & 1.0 \\
\hline
\end{tabular}

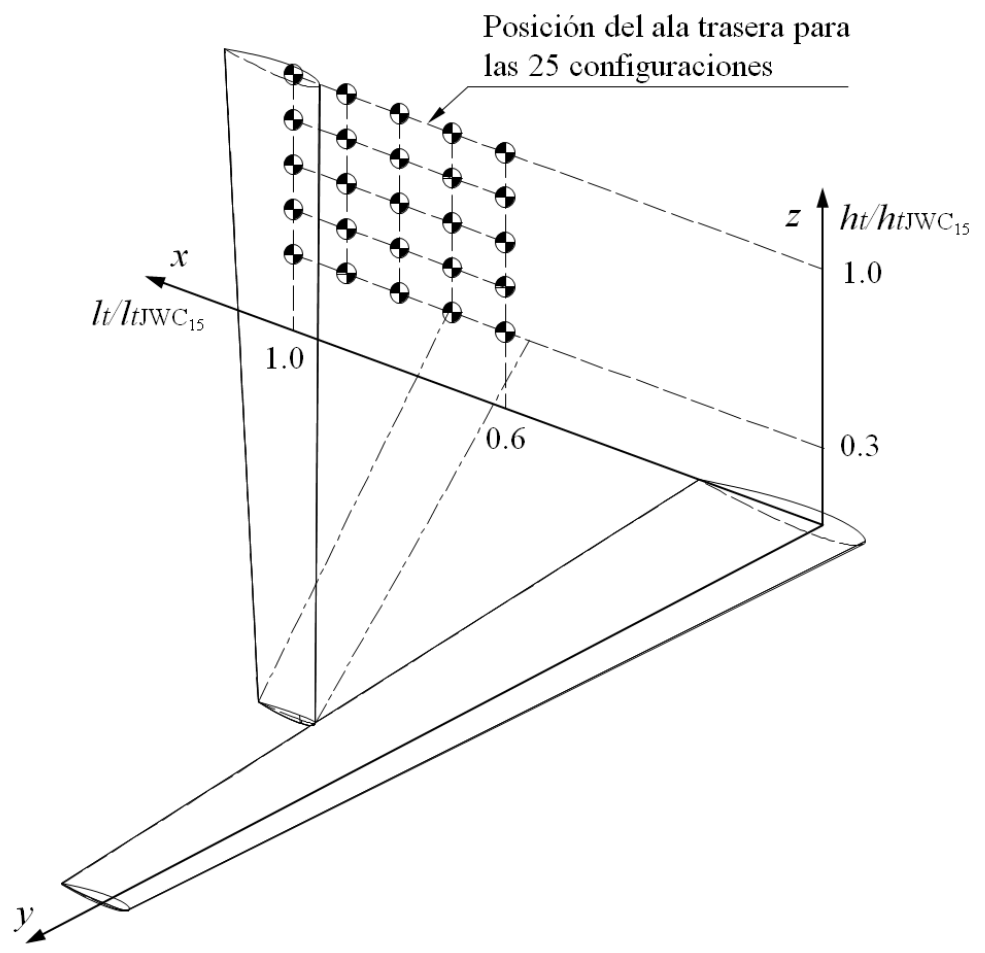

Figura 4.7: Esquema donde se muestra las posiciones del ala trasera con respecto al ala delantera.

Como resultado final, en la Figura 4.8 se muestra el modelo de ensayo correspondiente a la configuración $\mathrm{JWC}_{15}$, instalado en la cámara de ensayo del túnel de viento A9. 


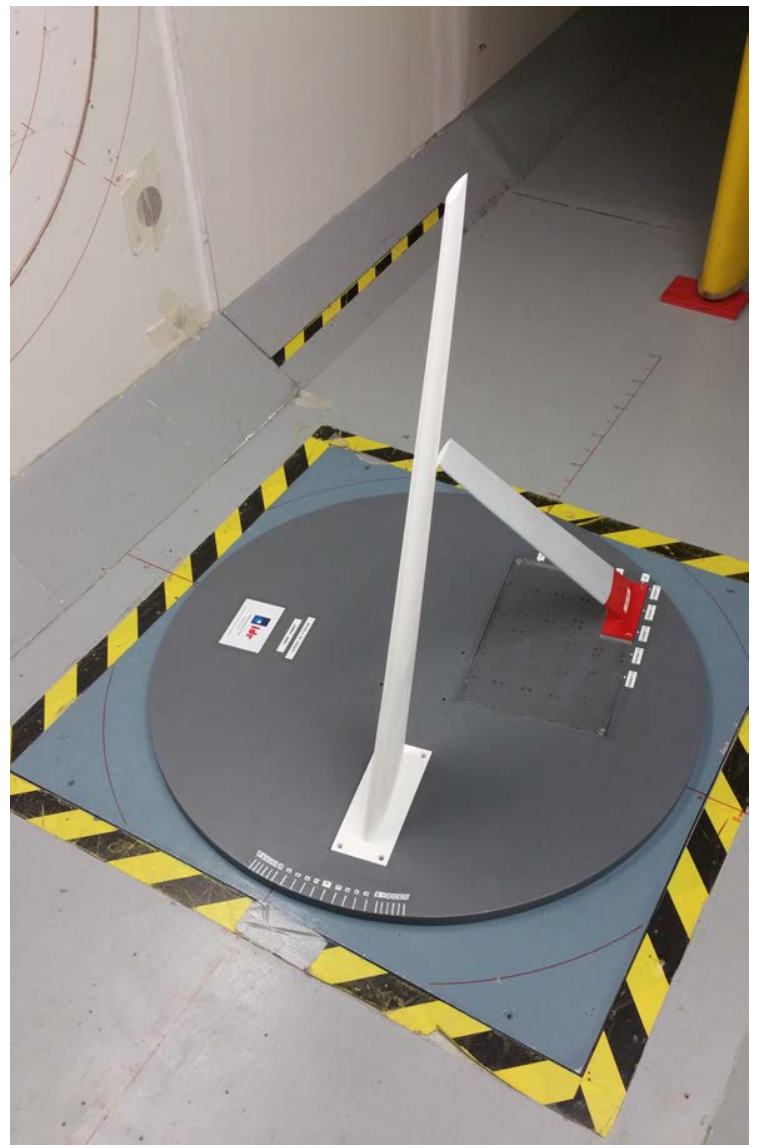

Figura 4.8: Modelo de ensayo correspondiente a la configuración $\mathrm{JWC}_{15}$, instalado en la cámara de ensayo del túnel A9.

\subsection{Equipos de medida}

Todos los ensayos presentados en el documento de tesis han sido realizados en las instalaciones pertenecientes al Instituto Universitario de Microgravedad "Ignacio Da Riva" (IDR) adscrito a la Universidad Politécnica de Madrid (UPM).

La aptitud de estos equipos, para trabajos de carácter aeronáutico, ha sido sobradamente probada en numerosos experimentos y ensayos realizados en el IDR, no obstante es necesario realizar una breve descripción técnica de los mismos.

\subsubsection{Túnel aerodinámico A9}

Como la naturaleza de la configuración del modelo de ensayo es tridimensional se impone la necesidad del uso de un túnel para tal propósito.

Para tales aplicaciones el IDR dispone de varios túneles aunque debido a la geometría del modelo se ha considerado el Túnel A9 el más adecuado para el ensayo.

El Túnel A9 del Instituto IDR/UPM es de corriente aspirada, cámara de ensayos cerrada y circuito fluido abierto (tipo Eiffel) [Meseguer et al. 2007]. Según se muestra esquemáticamente en la Figura 4.9, los elementos que componen el Túnel A9 son: la contracción de entrada (1), la cámara de ensayos (2), el difusor (3) que actúa como adaptador a la sección de ventiladores y los propios ventiladores (4). La 
contracción es bidimensional: la corriente sólo se contrae en uno de los planos de simetría del túnel, de forma que el techo y el suelo de la contracción son paralelos al techo y al suelo del Laboratorio mientras que las paredes verticales de la contracción describen una curva suave que adapta la sección de entrada a la cámara de ensayos. La contracción tiene una sección de entrada de $4.8 \mathrm{~m}$ de ancho y $1.8 \mathrm{~m}$ de alto, con una longitud de $5.34 \mathrm{~m}$.
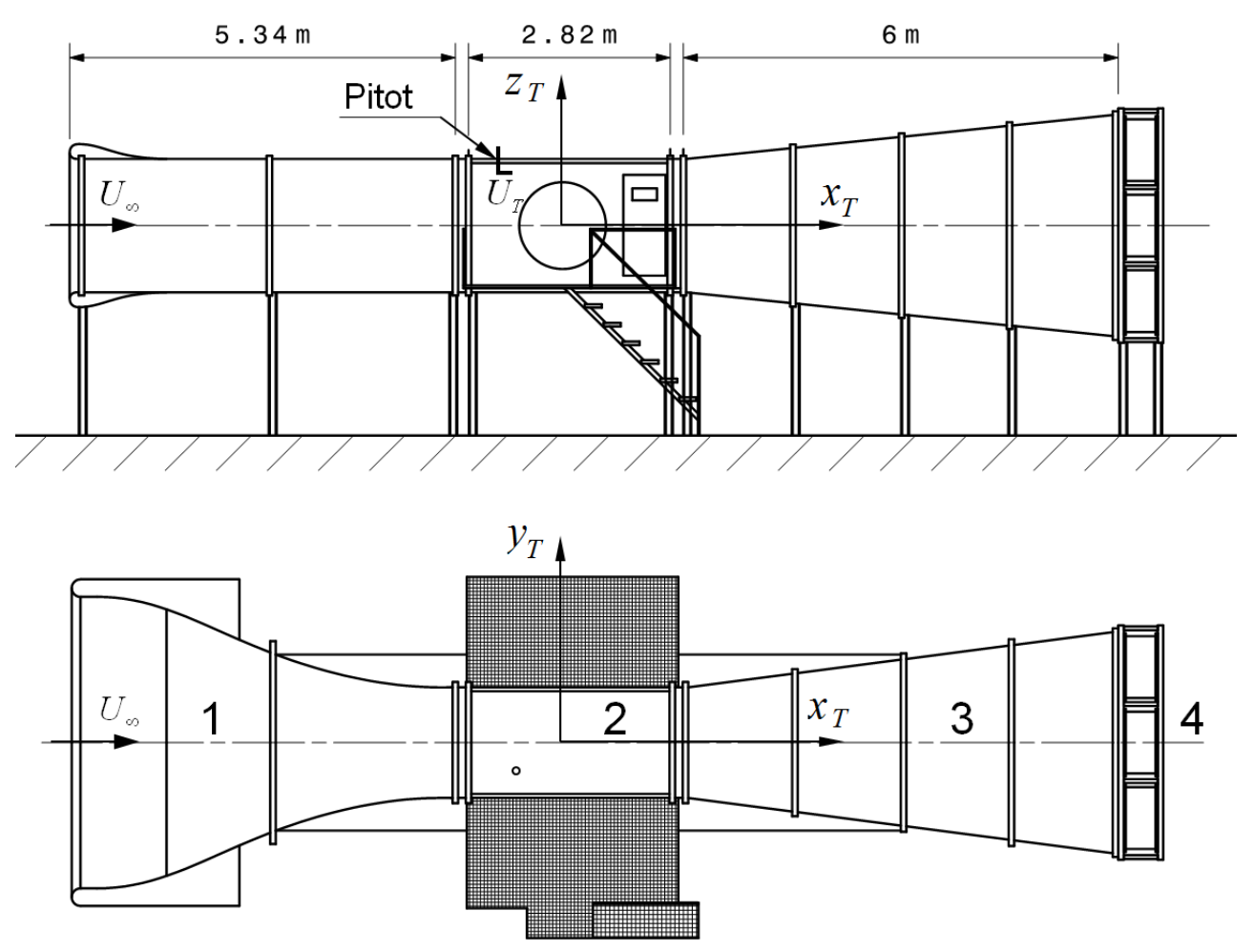

Figura 4.9: Esquema en planta y alzado del Túnel A9: (1) contracción, (2) cámara de ensayos, (3) adaptador y (4) ventiladores.

La cámara de ensayos tiene aproximadamente $3 \mathrm{~m}$ de longitud y una sección de $1.8 \mathrm{~m}$ de alto y $1.5 \mathrm{~m}$ de ancho; a la cámara de ensayos se accede desde dos plataformas, una a cada lado del túnel, mediante sendas puertas. Debajo de la cámara de ensayos está la balanza para la medida de cargas aerodinámicas globales, diseñada y construida en el IDR, y en el techo, además del sistema de iluminación de la cámara y los tubos de Pitot que miden la velocidad de referencia, hay una cámara de vídeo que permite al operador observar la ejecución de los ensayos a través del correspondiente monitor situado en la sala de control.

Corriente abajo de la cámara de ensayos está el adaptador a la sección de ventiladores, cuya longitud es de $6 \mathrm{~m}$. El Túnel A9 está impulsado por nueve ventiladores SODECA serie HTC 90, de ocho palas, con una potencia nominal de $10 \mathrm{~kW}$ y un diámetro interior de $0.9 \mathrm{~m}$ cada uno. Los ventiladores, de velocidad regulable, están dispuestos según una matriz de 3x3 y descargan directamente al local. La velocidad máxima en la cámara de ensayos del Túnel A9 no superar los $28 \mathrm{~m} / \mathrm{s}$, lo que arroja valores del número de Reynolds del orden de $10^{6}$ para las longitudes características habituales de las maquetas. 
Al mismo tiempo la presión dinámica en la cámara de ensayos se mide con un tubo de Pitot, marca Airflow modelo 3.3.311, para, a partir de la presión, calcular la velocidad del aire en la cámara de ensayos de la corriente libre sin perturbar.

Todas las señales diferenciales de presión se miden con cápsula Druck modelo PDCR 22, conectada a la señal de presión dinámica.

La señal eléctrica generada en las cápsulas de presión pasa a sendos acondicionadores de señal, Druck modelo DPI 260, que además proporcionan una indicación numérica visual del valor de la presión que se está midiendo. Las señales eléctricas analógicas, una vez filtradas, son medidas por un sistema de adquisición de datos IOTech modelo ADC 488, conectado a un bus IEEE 488 controlado por un ordenador. Este equipo de adquisición de datos posee también entradas y salidas digitales que permiten controlar y comprobar, a través de la correspondiente interfaz, la posición de lectura de los lectores secuenciales.

\subsubsection{Sensor célula de carga Fuerza/Par Delta SI-330-30}

La medida de cargas globales sobre el modelo de ensayo montado en el Túnel A9 se realiza mediante el célula de carga Delta SI-330-30 de seis componentes $\left(F_{x}, F_{y}, F_{z}, M_{x}, M_{y}\right.$ y $\left.M_{z}\right)$, lo que posibilita la medida simultánea de las fuerzas aerodinámicas de sustentación, $L$, resistencia, $D$ y momento de cabeceo, $M_{y}$.

Para la elección del sensor de medida se han tenido en cuenta las fuerzas máximas que el modelo de ensayo genera, el rango de medidas que puede realizar el sensor (Tabla 4.6) y las dimensiones para su fácil instalación.

Tabla 4.6: Valores del rango de medida y de resolución del sensor Delta SI-33030.

\begin{tabular}{ccc|ccc}
\hline \multicolumn{3}{c|}{ Rango de medida } & \multicolumn{3}{c}{ Resolución } \\
\hline$F_{x}, F_{y}$ & $F_{z}$ & $M_{x}, M_{y}, M_{z}$ & $F_{x}, F_{y}$ & $F_{z}$ & $M_{x}, M_{y}, M_{z}$ \\
$330 \mathrm{~N}$ & $990 \mathrm{~N}$ & $30 \mathrm{Nm}$ & $1 / 16 \mathrm{~N}$ & $1 / 8 \mathrm{~N}$ & $5 / 1333 \mathrm{Nm}$ \\
\hline
\end{tabular}

El sistema consiste en transductor, cable flexible recubierto y sistema inteligente de adquisición de datos con interfaz Ethernet/DeviceNet o controlador de FT (Figura 4.10).

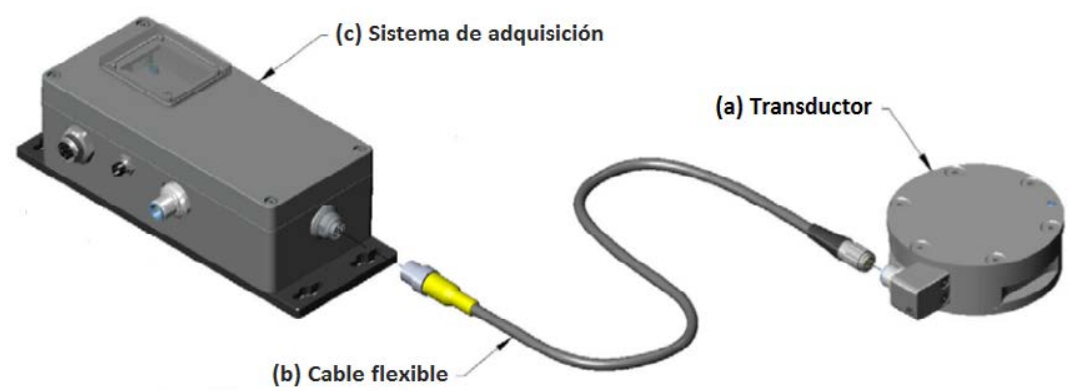

Figura 4.10: Célula de carga Delta SI-330-30. Transductor (a), cable flexible (b) y sistema de adquisición de datos (c).

La célula de carga está situada en el exterior de la cámara de ensayos del túnel A9 en el suelo en un espacio reservado para su fácil acceso. En la Figura 
4.11 se muestra un esquema de la sección transversal de la cámara de ensayo junto con un modelo genérico, el motor entre balanza y modelo y la propia balanza. El motor es controlado para permitir variar el ángulo de ataque del modelo de forma automática.

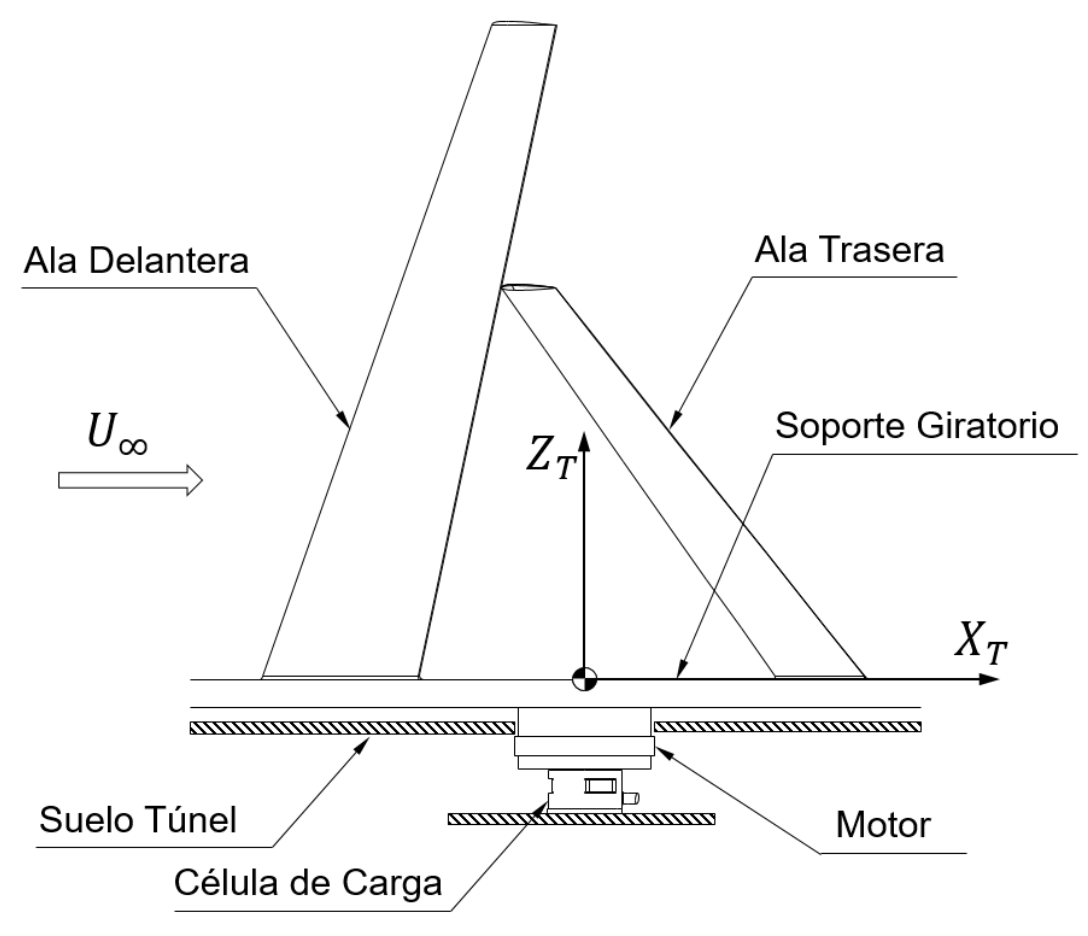

Figura 4.11: Esquema de la sección transversal de la cámara de ensayo donde se muestra la posición del modelo, célula de carga y motor.

Las fuerzas que actúan en el modelo son transmitidas a las células de carga del sensor. La salida de cada célula de carga se toma a través de un amplificador a un sistema de adquisición de datos instalado en un ordenador proporcionando las series temporales de las fuerzas en cada sensor.

Para determinar el valor de las fuerzas aerodinámicas de sustentación, resistencia y momento de cabeceo, a partir de las fuerzas en los sensores, es necesaria la calibración de la célula de carga, mediante el procedimiento expuesto en la guía de usuario, por medio de su correspondiente matriz de transformación.

\subsubsection{Equipo de anemometría de hilo caliente}

Para la medida de intensidad de turbulencia en el túnel (ver anexo B) se utiliza un equipo de anemometría de hilo caliente de temperatura constante (CTA) DANTEC que consta: de una sonda de una componente, modelo 55P16 con su soporte y cableado; un cuadro CTA module 90C10, con una capacidad total de 6 canales; un ordenador y un sistema de guías con orientación programable para la definición de áreas de medida.

El principio de funcionamiento de la anemometría de hilo caliente se basa en la transferencia de calor por convección entre el hilo calentado y el flujo de aire que pasa a su alrededor, existe una relación entre la velocidad del flujo de aire y la tensión de salida del sensor. El sensor es calentado por una corriente eléctrica 
y enfriado proporcionalmente, de acuerdo a la componente de la velocidad del aire que incide perpendicular en el elemento sensible del sensor.

Con una sonda de una componente es posible medir la componente longitudinal de la velocidad del flujo [Rodríguez et al. 2014]. El esquema de la instalación está representado en la Figura 4.12.

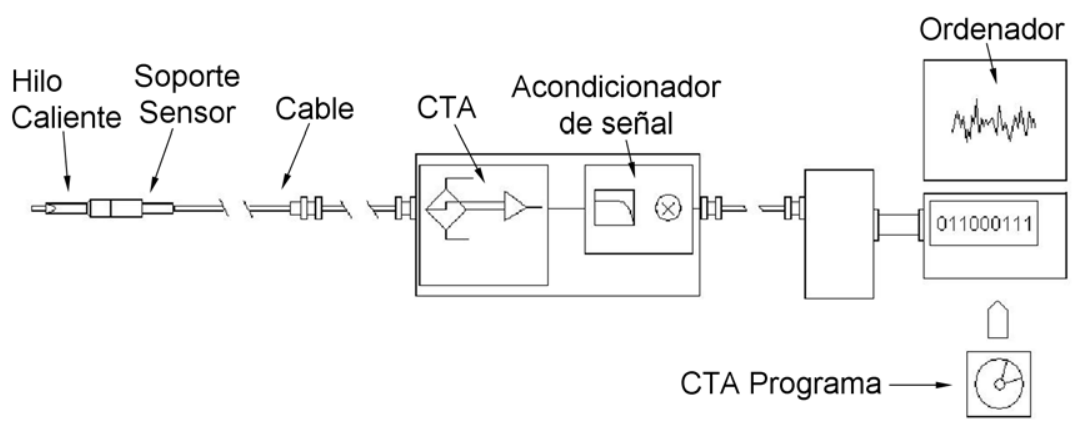

Figura 4.12: Esquema de instalación del equipo de anemometría de hilo caliente DANTEC.

\subsection{Disposición del modelo en la cámara de ensayo del túnel A9 y parámetros de ensayo}

Una vez elegidos los equipos para la realización de las diferentes medidas en el túnel y la calibración del mismo (ver anexo B), se procede al montaje del modelo de ensayo en el túnel y a la selección de los parámetros de ensayo.

\subsubsection{Montaje del modelo de ensayo en la cámara del túnel A9}

En la cámara de ensayo del túnel A9 existen varias posibilidades de montaje: suelo y paredes laterales. La elección más optima es el suelo del túnel, primero por facilidad de montaje de la balanza donde ya se dispone de un espacio para su instalación, y segundo porque a la vista del diagrama de intensidad de turbulencia (Figura B.5) es la que presenta valores de intensidad de turbulencia aceptables. Esta posición está representada en la Figura 4.13.

\subsubsection{Parámetros de ensayo}

Para obtener las fuerzas aerodinámicas sobre el modelo de ensayo los parámetros son:

- Velocidad de la corriente libre en el túnel: $U=25 \mathrm{~m} / \mathrm{s}$.

- Rango de ángulos de ataque: $\alpha=\left[-15^{\circ}, 15^{\circ}\right]$, con intervalos de $1^{\circ}$.

- Frecuencia de medida: $f=1000 \mathrm{~Hz}$.

- Tiempo de medida: $t=120 \mathrm{~s}$ 


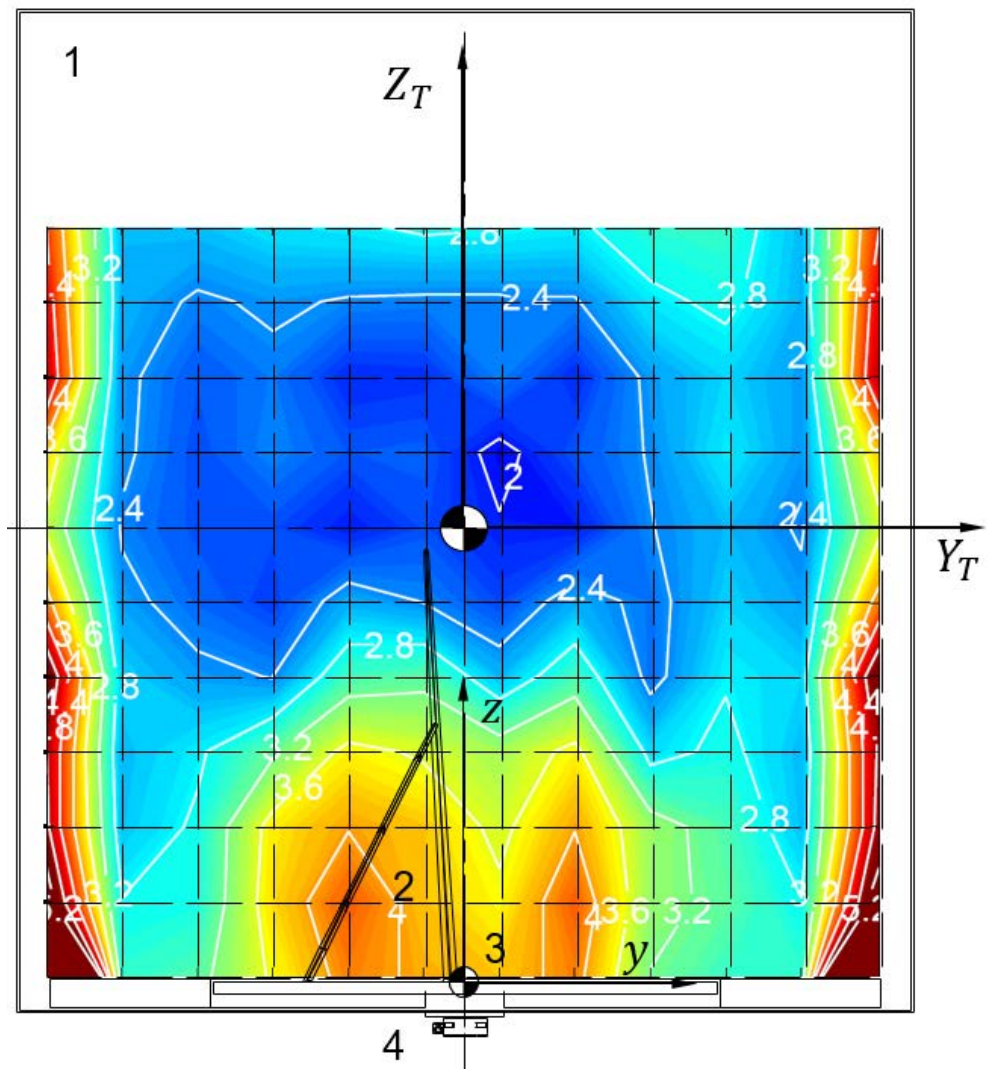

Figura 4.13: Esquema del montaje del modelo en la cámara de ensayo del túnel A9, donde se muestra la distribución de intensidad de turbulencia. (1) Cámara de ensayo, (2) modelo de ensayo, (3) motor giratorio y (4) balanza.

- Número de Reynolds basado en la cuerda media aerodinámica del ala delantera: $\operatorname{Re}=250000$.

La elección de la velocidad de ensayo está determinada por el número de Reynolds (que es aceptable) y por las fuerzas producidas por el modelo en el sensor célula de carga, lo que implica, que en su selección se debe evitar problemas de saturación de la medida. Valores mayores de velocidad producen, para ángulos de ataque elevados, saturación en la célula de carga.

Al realizar las medidas a esta velocidad y teniendo en cuenta las dimensiones del modelo de ensayo, el número de Reynolds basado en la cuerda media aerodinámica que se alcanza es aceptable para el entorno de la categoría de UAS al que está orientado el presente estudio.

Por otro lado, y a la vista de la Figura 4.13, los valores de intensidad de turbulencia son del orden del $3 \%$ en la zona central de la cámara de ensayo del túnel (ver apéndice B), estos valores son mayores que los requeridos para ensayos de aerodinámica de aviones $(<0.5 \%)$ [Barlow et al. 1999], aunque se consideran aceptables para su aplicación en el campo de los sistemas aéreos no tripulados (UAS).

La altitud normal de operación para el caso de los UAS de clase I de categorías micro, mini y pequeña, es inferior a los 1500 m [M.O.D. 2011; PAE 2014] donde la configuración de la atmósfera se debe a la capa límite atmosférica (ABL). La ABL es causada por la interacción del viento geostrófico con los obstáculos de la 
superficie de la Tierra. La interacción se produce en dos formas primarias: mecánica y térmica. La interacción mecánica se produce a partir de la fricción ejercida por el viento contra la superficie del suelo que da lugar a un perfil de velocidad media (10 a $20 \mathrm{~m} / \mathrm{s}$ ) que varía con la altura. En la atmósfera más baja el nivel de intensidad de turbulencia (basado en la velocidad media del viento) está íntimamente relacionada con la velocidad.

En el caso de UAS, la velocidad de referencia es la velocidad de vuelo (20 a $50 \mathrm{~m} / \mathrm{s}$ ), de modo que la intensidad de la turbulencia es de entre 5 a $15 \%$, siendo estos niveles mayores que los de la aviación comercial convencional.

Para la elección de los parámetros de frecuencia y tiempo de medida es necesario realizar un análisis del valor medio y de la desviación estándar de las fuerzas obtenidas en el sensor de célula de carga, con el objetivo de analizar si se obtienen valores representativos con el tiempo de medida elegido. Para tal efecto se han considerado los valores de las fuerzas medidos en la dirección del eje $x$ del sensor, $F_{x}$. Cada valor es medido en un tiempo $t$, con incrementos de $t=5 \mathrm{~s}$, hasta llegar al tiempo final de $t=120 \mathrm{~s}$, y es comparado con el valor medido para el tiempo $t=120$ s de la configuración $\mathrm{JWC}_{15}$ (configuración de referencia) y para tres ángulos de ataque diferentes de $\alpha=0^{\circ}, \alpha=4^{\circ}$ y $\alpha=8^{\circ}$.

En las Figuras 4.14, 4.15 y 4.16 se muestra que el valor medio de la medida de la fuerza para cada instante $t$, normalizado con respecto al valor medio de la fuerza para $t=120 \mathrm{~s},\left.\overline{F_{x}}\right|_{t} /\left.\overline{F_{x}}\right|_{t=120 \mathrm{~s}}$, converge rápidamente al valor del tiempo de muestreo para $t=120 \mathrm{~s}$, con un error menor de $0.1 \%$, a los $60 \mathrm{~s}$ y que el valor de la desviación estándar de la fuerza, $\left.\sigma_{F_{x}}\right|_{t} /\left.\sigma_{F_{x}}\right|_{t=120 \mathrm{~s}}$, converge con una desviación menor de $2.5 \%$, a los $60 \mathrm{~s}$.

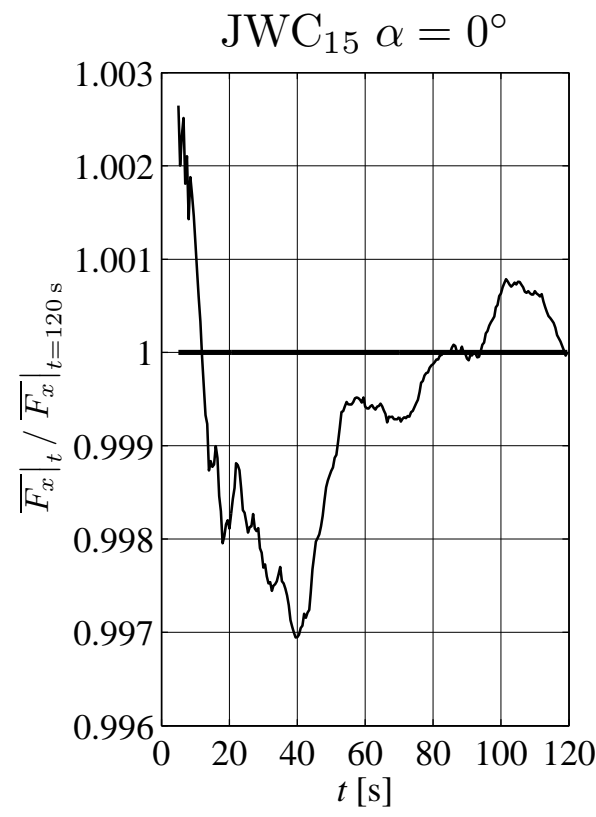

(a)

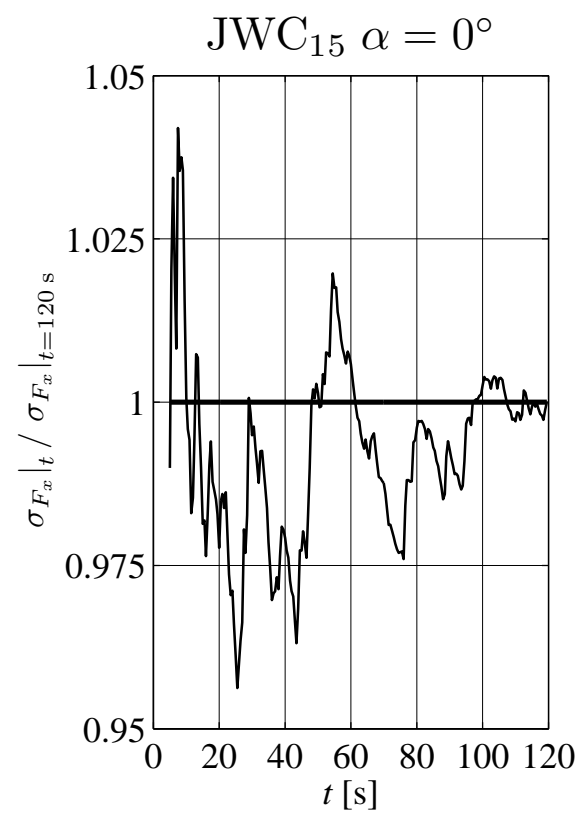

(b)

Figura 4.14: Valores de la convergencia en cada instante en función del tiempo de medida, $t$, para la configuración $\mathrm{JWC}_{15}$ y para un ángulo de ataque $\alpha=0^{\circ}$ : (a) valor medio, $\overline{F_{x}}$, de la fuerza $F_{x}$, (b) valor de la desviación estándar, $\sigma_{F_{x}}$, de la fuerzas $F_{x}$. 


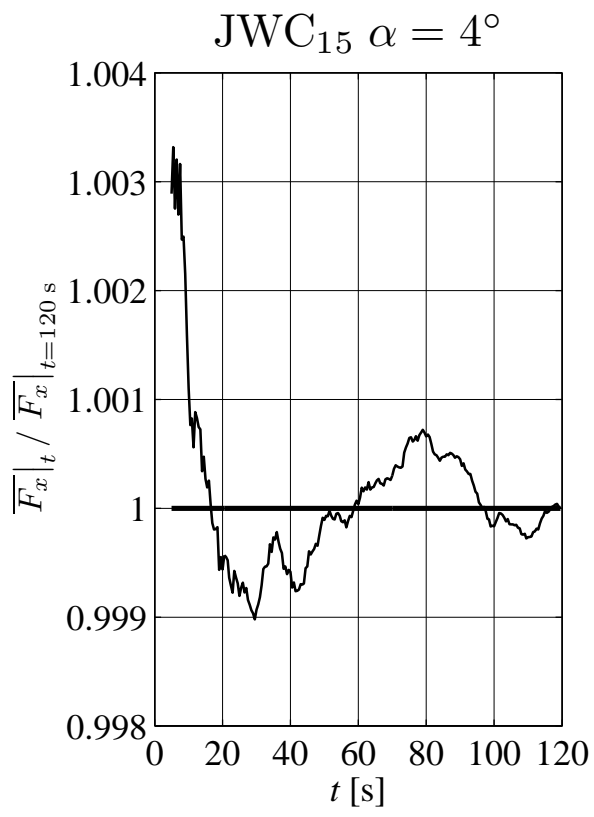

(a)

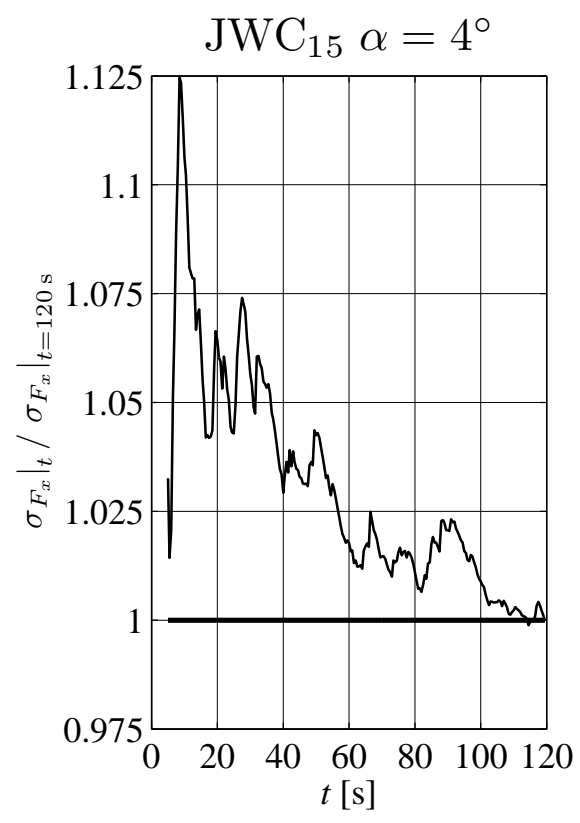

(b)

Figura 4.15: Valores de la convergencia en cada instante en función del tiempo de medida, $t$, para la configuración $\mathrm{JWC}_{15}$ y para un ángulo de ataque $\alpha=4^{\circ}$ : (a) valor medio, $\overline{F_{x}}$, de la fuerza $F_{x}$, (b) valor de la desviación estándar, $\sigma_{F_{x}}$, de la fuerzas $F_{x}$.

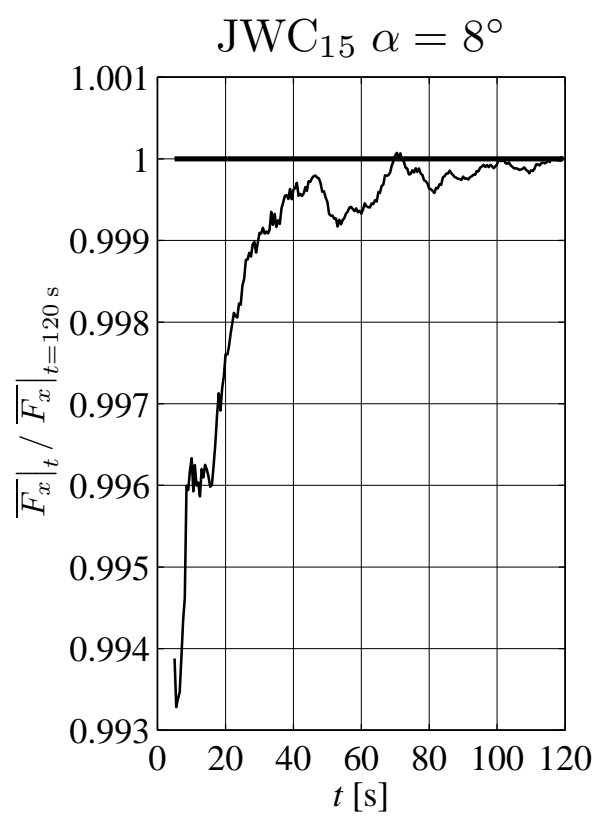

(a)

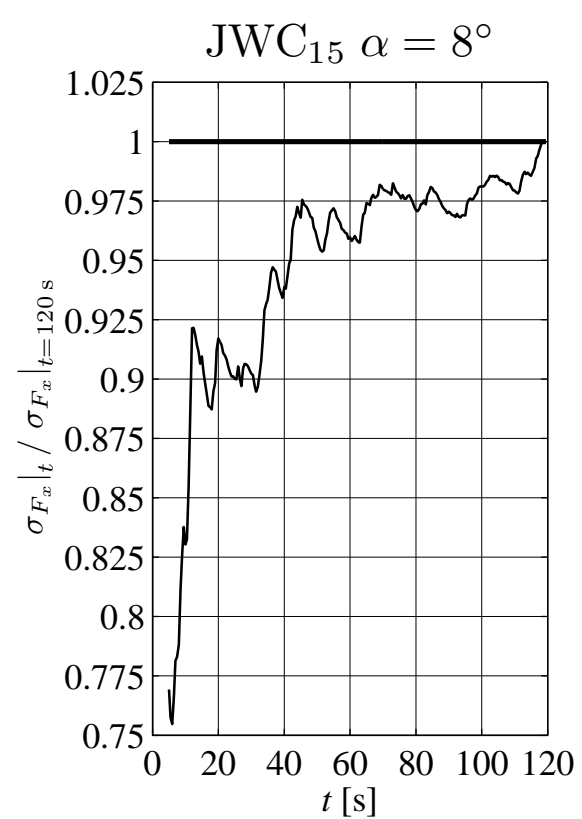

(b)

Figura 4.16: Valores de la convergencia en cada instante en función del tiempo de medida, $t$, para la configuración $\mathrm{JWC}_{15}$ y para un ángulo de ataque $\alpha=8^{\circ}$ : (a) valor medio, $\overline{F_{x}}$, de la fuerza $F_{x}$, (b) valor de la desviación estándar, $\sigma_{F_{x}}$, de la fuerzas $F_{x}$.

A pesar de que la desviación entre el valor de la media y de la desviación estándar de la fuerza medidas de $t=5 \mathrm{~s}$, y las medidas en $t=120 \mathrm{~s}$ es relativamente 
pequeña se ha optado por la elección de este último tiempo de medida.

Una vez establecidos los parámetros de ensayo el proceso de medida es el siguiente:

1. Unido el modelo al sensor de célula de carga, se sitúa al ángulo de ataque requerido haciendo uso del posicionador angular Newport RV80PP con precisión de $0,01^{\circ}$.

2. Se realiza una primera medida con la balanza y con el túnel apagado para que de esta manera sea posible eliminar la fuerza producida por el peso propio del modelo.

3. Se pone en marcha el túnel a la velocidad deseada y se espera un tiempo, del orden del tiempo de muestreo, hasta que la velocidad de la corriente sea estable.

4. Se toman las medidas de las fuerzas transmitidas a las tres células de carga durante el tiempo de muestreo y a la frecuencia de muestreo. Al mismo tiempo se mide la presión dinámica en la cámara de ensayos con un tubo de Pitot.

5. Una vez obtenidas las series temporales correspondientes a las fuerzas aerodinámicas, se calcula su media y desviación estándar, y por medio del valor medio de la presión dinámica, sus correspondientes coeficientes.

Este proceso está representado de forma esquemática por medio de un diagrama de bloques en la Figura 4.17.

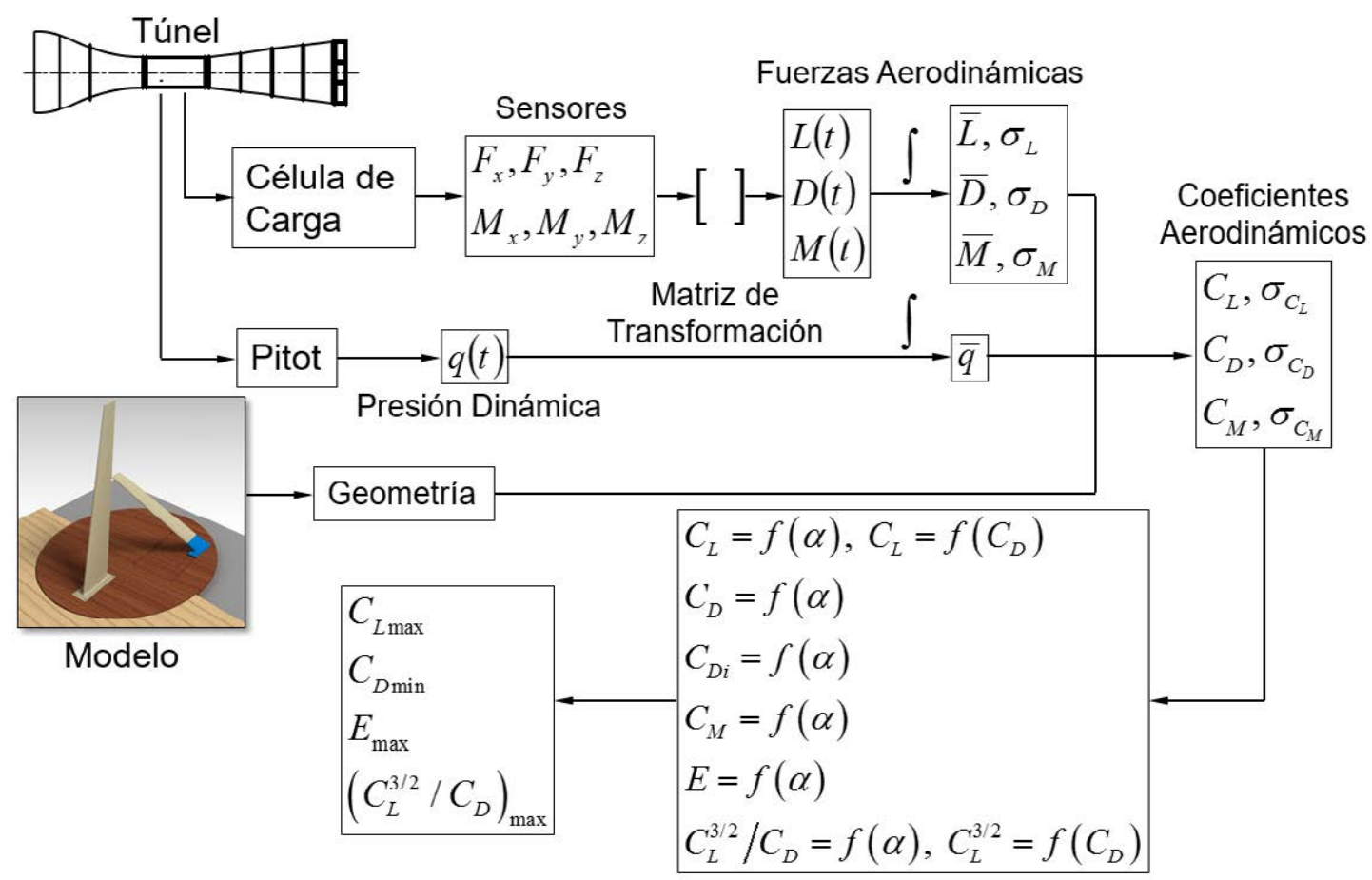

Parámetros Aerodinámicos

Figura 4.17: Esquema del proceso de ensayo para la obtención de fuerzas, coeficientes y parámetros aerodinámicos. 


\subsection{Resultados de ensayo}

Realizando un análisis aerodinámico comparativo, es posible optimizar la configuración del modelo de alas convergentes desde el punto de vista de la posición del ala trasera, con el objetivo de determinar las mejores características aerodinámicas en una fase preliminar de diseño.

Específicamente el análisis experimental está orientado al cálculo de los coeficientes aerodinámicos de sustentación, $C_{L}$, resistencia, $C_{D}$ y momento de cabeceo, $C_{M_{y}}$ evaluados de las series temporales de las fuerzas de sustentación, $L$, resistencia, $D$ y momento, $M_{y}$, medidas por el sensor célula de carga, según las expresiones:

$$
\begin{gathered}
C_{L}=\frac{L}{q b \bar{c}}, \\
C_{D}=\frac{D}{q b \bar{c}}, \\
C_{M_{y}}=\frac{M_{y}}{q b^{2} \bar{c}},
\end{gathered}
$$

donde, $q$, es la presión dinámica, $b$, es la envergadura efectiva [Wolkovitch 1986] y, $\bar{c}$, es la cuerda media aerodinámica efectiva del conjunto ala delantera y ala trasera, acorde con las expresiones:

$$
\begin{gathered}
b=\frac{1}{2}\left[\left(\frac{b_{F}}{\cos \delta_{F} \cos \varphi_{F}}\right)+\left(\frac{b_{R}}{\cos \delta_{R} \cos \varphi_{R}}\right)\right], \\
\Lambda=\frac{\Lambda_{F}\left(\frac{S_{F}}{S_{R}}\right)+\Lambda_{R}\left(\frac{S_{R}}{S_{F}}\right)}{\left(\frac{S_{F}}{S_{R}}+1\right)+\left(\frac{S_{R}}{S_{F}}+1\right)}, \\
\bar{c}=\frac{b}{\Lambda},
\end{gathered}
$$

donde, $S_{F}$, es la superficie alar del ala delantera, $S_{R}$, es la superficie alas del ala trasera y $\Lambda$, es el alargamiento del modelo de alas convergentes [Wolkovitch 1986; Bagwill and Selberg 1996b].

La envergadura efectiva, $b$, está definida como el valor medio de la longitud no proyectada de la línea de puntos $1 / 4$ del ala delantera y del ala trasera. El alargamiento, $\Lambda$, es parámetro geométrico que tiene en consideración la relación entre la superficie alar del ala delantera y del ala trasera, pero sin tener en cuenta ninguna consideración de acoplamiento aerodinámico causado por la unión de las dos alas.

Por otro lado se han determinado también los coeficientes aerodinámicos asociados como la resistencia inducida, $C_{D i}$, y la eficiencia aerodinámica, $E$, calculados por las expresiones: 


$$
\begin{gathered}
C_{D i}=\frac{C_{L}^{2}}{\pi \Lambda e}, \\
E=\frac{C_{L}}{C_{D}},
\end{gathered}
$$

siendo, e, el parámetro de eficiencia de Oswald y cuyo valor aumenta según aumenta el ángulo diedro del ala trasera, $\delta_{R}$, en valor absoluto (ver apéndice A).

Para tener en cuenta la influencia del ángulo de flecha del ala delantera, $\varphi_{F}$, y del ala trasera, $\varphi_{R}$, este valor se corrige según la expresión 3.27 [Wolkovitch 1986] (ver Tabla 4.7).

Tabla 4.7: Valor del factor de eficiencia, $e$, para calcular la resistencia inducida, en función del ángulo de flecha, $\varphi_{R}$ y del ángulo diedro, $\delta_{R}$ del ala trasera.

\begin{tabular}{c|ccccc}
\hline \multirow{2}{*}{$\begin{array}{c}\text { Ángulo diedro del ala } \\
\text { trasera, } \delta_{R}\left[^{\circ}\right]\end{array}$} & \multicolumn{5}{|c}{ Ángulo de flecha del ala trasera, $\varphi_{R}\left[^{\circ}\right]$} \\
\cline { 2 - 6 } & -10 & -15 & -20 & -25 & -30 \\
\hline-25 & 1.038 & 1.042 & 1.046 & 1.051 & 1.056 \\
-20 & 1.033 & 1.037 & 1.042 & 1.046 & 1.051 \\
-15 & 1.029 & 1.033 & 1.037 & 1.042 & 1.047 \\
-10 & 1.026 & 1.030 & 1.034 & 1.038 & 1.043 \\
-5 & 1.023 & 1.027 & 1.031 & 1.036 & 1.040 \\
\hline
\end{tabular}

Para la presentación de los datos comparativos entre las diferentes configuraciones (ver apéndice $\mathrm{C}$ ) se define la configuración $\mathrm{JWC}_{15}$ como configuración de referencia. Para esta configuración, los valores de los coeficientes de sustentación, $C_{L}$, de resistencia, $C_{D}$, de momento de cabeceo, $C_{M y}$, con respecto al punto $1 / 4$ de la cuerda en el encastre del ala delantera, y de la eficiencia aerodinámica, $E$, en función del ángulo de ataque, $\alpha$, están representados en la Figuras 4.18 y 4.19, donde además del valor de los coeficientes se muestra el valor de la desviación estándar de los mismos.

De igual manera en la Figura 4.20 se presentan los valores del coeficiente de resistencia inducida, $C_{D i}$, y del parámetro $C_{L}^{3 / 2} / C_{D}$, para potencia mínima requerida, en función del ángulo de ataque, $\alpha$.

Por otro lado en la Figura 4.21 se presentan los valores del coeficiente de sustentación, $C_{L}$, y del parámetro $C_{L}^{3 / 2}$ en función del coeficiente de resistencia, $C_{D}$. Donde la línea de trazos es la tangente a la curva, siendo el punto de contacto el valor máximo de la relación $C_{L}^{3 / 2} / C_{D}$.

En la Figura 4.22 se presentan los valores de la eficiencia aerodinámica, $E$, y de momento de cabeceo, $C_{M y}$, en función del coeficiente de sustentación, $C_{L}$.

Finalmente en la Figura 4.23 se presenta el valor del coeficiente de resistencia inducida, $C_{D i}$, en función del cuadrado del coeficiente de sustentación, $C_{L}$, que de acuerdo con (4.11) debería ser una recta con un valor de la pendiente, $m$, definida por:

$$
m=\frac{C_{D i}}{C_{L}^{2}}=\frac{1}{\pi \Lambda e}
$$



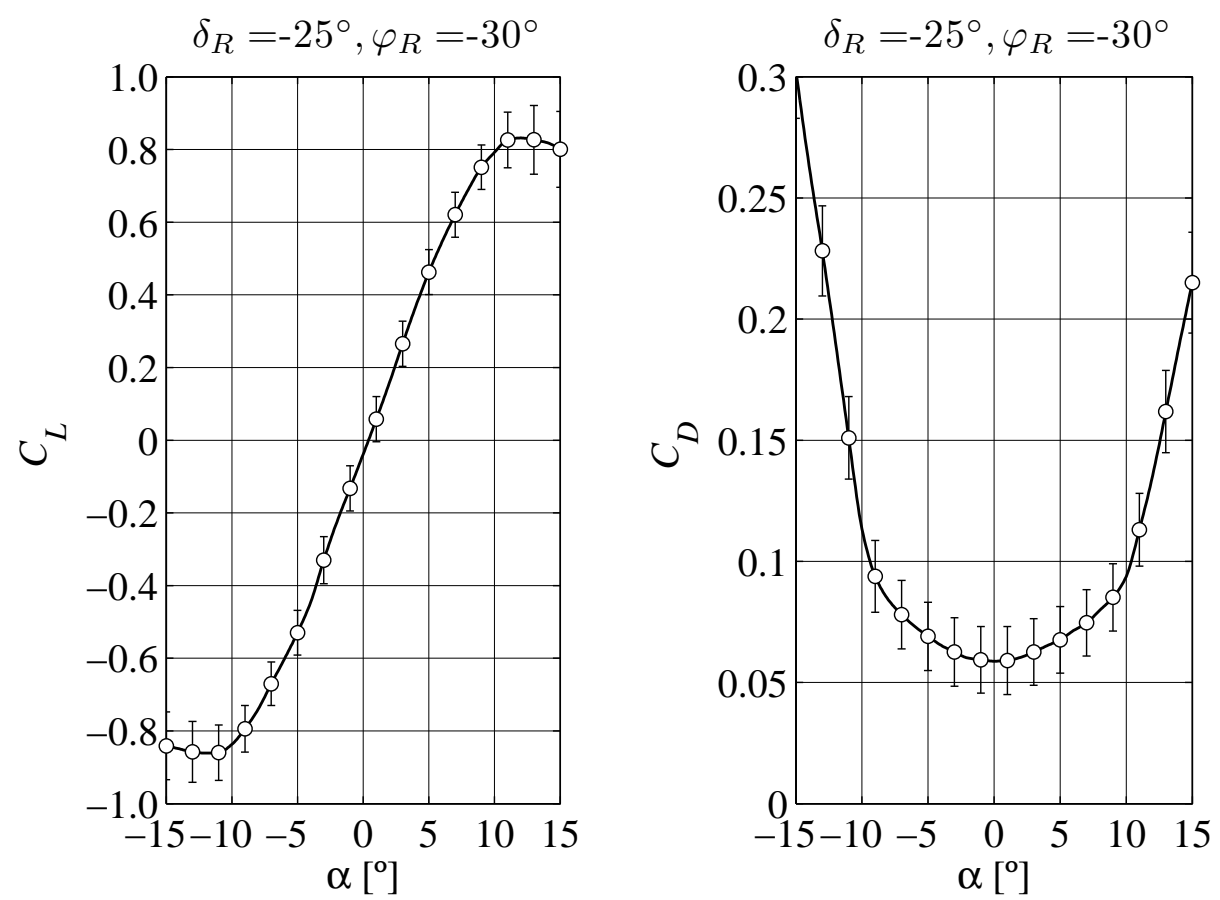

Figura 4.18: Variación de los coeficientes de sustentación, $C_{L}$, y de resistencia, $C_{D}$, en función del ángulo de ataque, $\alpha$, para el ángulo de flecha del ala trasera, $\varphi_{R}=-30^{\circ}$ y para el ángulo diedro del ala trasera, $\delta_{R}=-25^{\circ}$. Número de Reynolds de la corriente libre $\mathrm{Re}=250000$. Configuración de referencia $\mathrm{JWC}_{15}$. Las barras verticales representan el valor de la desviación estándar, $\pm \sigma_{C_{L}}$ y $\pm \sigma_{C_{D}}$, para cada coeficiente respectivamente.

Para la presentación y análisis del efecto combinado del ángulo de flecha y ángulo diedro del ala trasera, en la variación de los coeficientes aerodinámicos $\left(C_{L}\right.$, $\left.C_{D}, C_{D i}\right)$, de las diferentes configuraciones, se representan los valores, $\overline{C_{L}}, \overline{C_{D}}, \overline{C_{D i}}$, normalizados con respecto a los valores de la configuración de referencia, $\mathrm{JWC}_{15}$, $\left(C_{L_{15}}, C_{D_{15}}, C_{D i_{15}}\right)$. Por claridad sólo se muestran en este capítulo los resultados correspondientes a los ángulos de ataque $\alpha=\left[4^{\circ}, 14^{\circ}\right]$, con incrementos de $2^{\circ}$. En el apéndice $\mathrm{E}$ se encuentran representados todos valores para cada ángulo de ataque $\alpha=\left[-14^{\circ}, 15^{\circ}\right]$.

En primer lugar, en la Figura 4.25 se representa la variación del coeficiente de sustentación normalizado, $\overline{C_{L}}$, del modelo completo. Como puede observarse, el valor del coeficiente de sustentación, $C_{L}$, aumenta según se reduce la distancia entre el ala trasera y el ala delantera [Hirose et al. 1990]; este resultado es acorde con lo expuesto en el apartado 3.2 y es debido a que el ala delantera induce una deflexión negativa de la estela (downwash) sobre el ala trasera, mientras que el ala trasera induce una deflexión de la estela positiva (upwash) sobre el ala delantera [Wolkovitch 1986]. Debido a esto la sustentación total del modelo de alas convergentes es menor que la simple suma de la sustentación de las dos alas (ver Figura 4.24).

En la Figura 4.26 se representa la variación del coeficiente de resistencia normalizado, $\overline{C_{D}}$, del modelo completo. A la vista de los resultados se puede afirmar que el valor del coeficiente presenta poca dependencia con el ángulo diedro del ala trasera, donde el valor del coeficiente decrece si la altura, $h_{t}$, del ala trasera con respecto al ala delantera también decrece. Por otro lado este valor si muestra una 

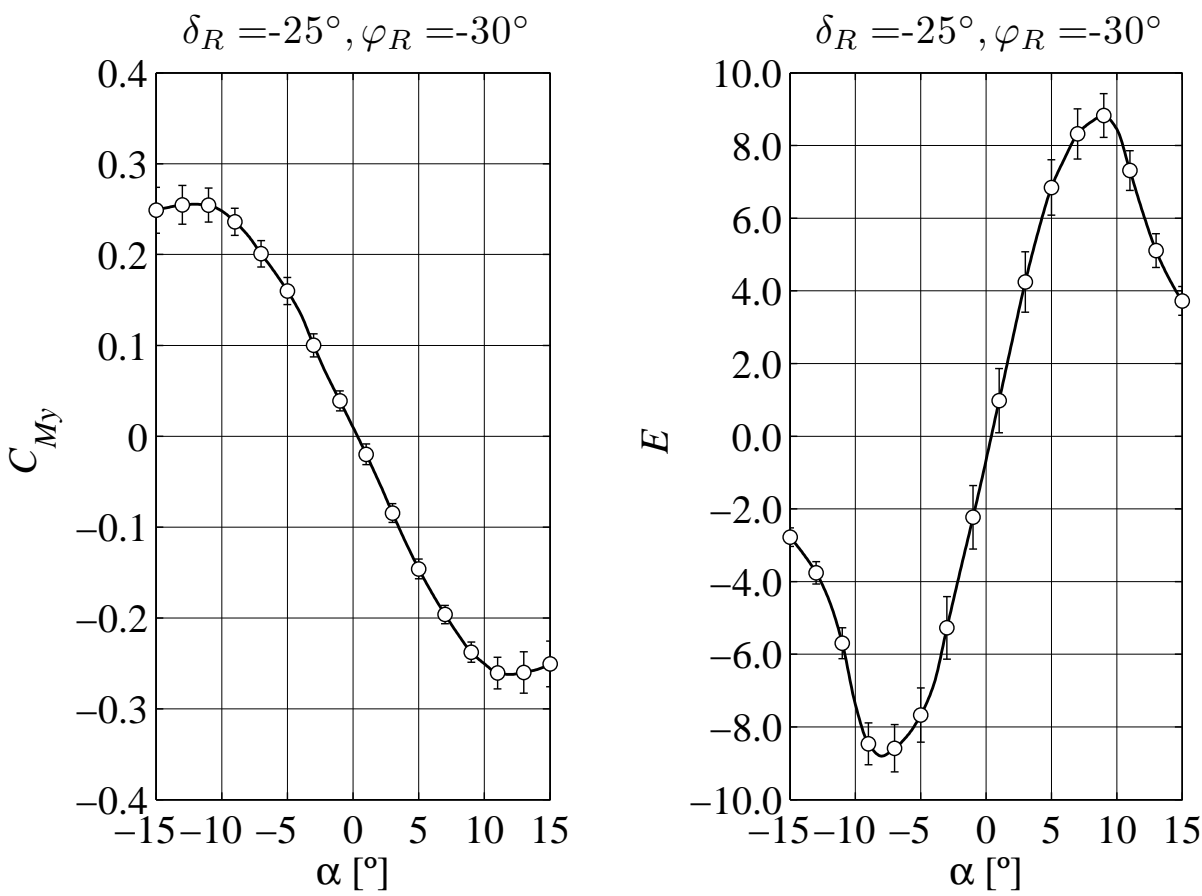

Figura 4.19: Variación del coeficientes de momento, $C_{M y}$, y de la eficiencia aerodinámica, $E$,en función del ángulo de ataque, $\alpha$, para el ángulo de flecha del ala trasera, $\varphi_{R}=-30^{\circ}$ y para el ángulo diedro del ala trasera, $\delta_{R}=-25^{\circ}$. Número de Reynolds de la corriente libre $\mathrm{Re}=250000$. Configuración de referencia $\mathrm{JWC}_{15}$. Las barras verticales representan el valor de la desviación estándar, $\pm \sigma_{C_{M y}}$ y $\pm \sigma_{E}$, para cada coeficiente respectivamente.

fuerte dependencia con la separación, $l_{t}$, disminuyendo si la separación aumenta [Bagwill and Selberg 1996b]. Este resultado es debido a la interferencia entre ambas alas, que disminuye según aumenta el ángulo de ataque y la separación entre ambas alas.

En la Figura 4.27 se representa la variación del coeficiente de resistencia inducida normalizado, $\overline{C_{D i}}$, del modelo completo que, como se puede observar, aumenta si la separación y altura del ala trasera disminuyen [Bagwill and Selberg 1997]. Además el valor del coeficiente es inversamente proporcional al valor del factor de eficiencia, e, (ver Tabla4.7 y Figura A.9), que aumenta si la separación y la altura entre ambas alas aumenta.

Seguidamente, en la Figura 4.28 se representa la variación de la eficiencia aerodinámica, $E$, y en la Figura 4.29 se representa el valor del factor para potencia mínima requerida, $C_{L}^{3 / 2} / C_{D}$. El comportamiento de ambos parámetros es en líneas generales similar aumentando si la separación longitudinal del ala trasera aumenta y la separación vertical disminuye.

Por último en la Figura 4.30 se representa la variación de la pendiente, $m$, ( ver ecuación 4.13). Este valor es constante para todo ángulo de ataque y sólo depende del alargamiento efectivo, $\Lambda$, y del factor de eficiencia de Owswald, $e$.

En cada figura, y por cada ángulo de ataque representado, se muestran los valores máximo y mínimo de cada coeficiente, así como los valores de los coeficientes correspondientes de la configuración de referencia en cada caso. 

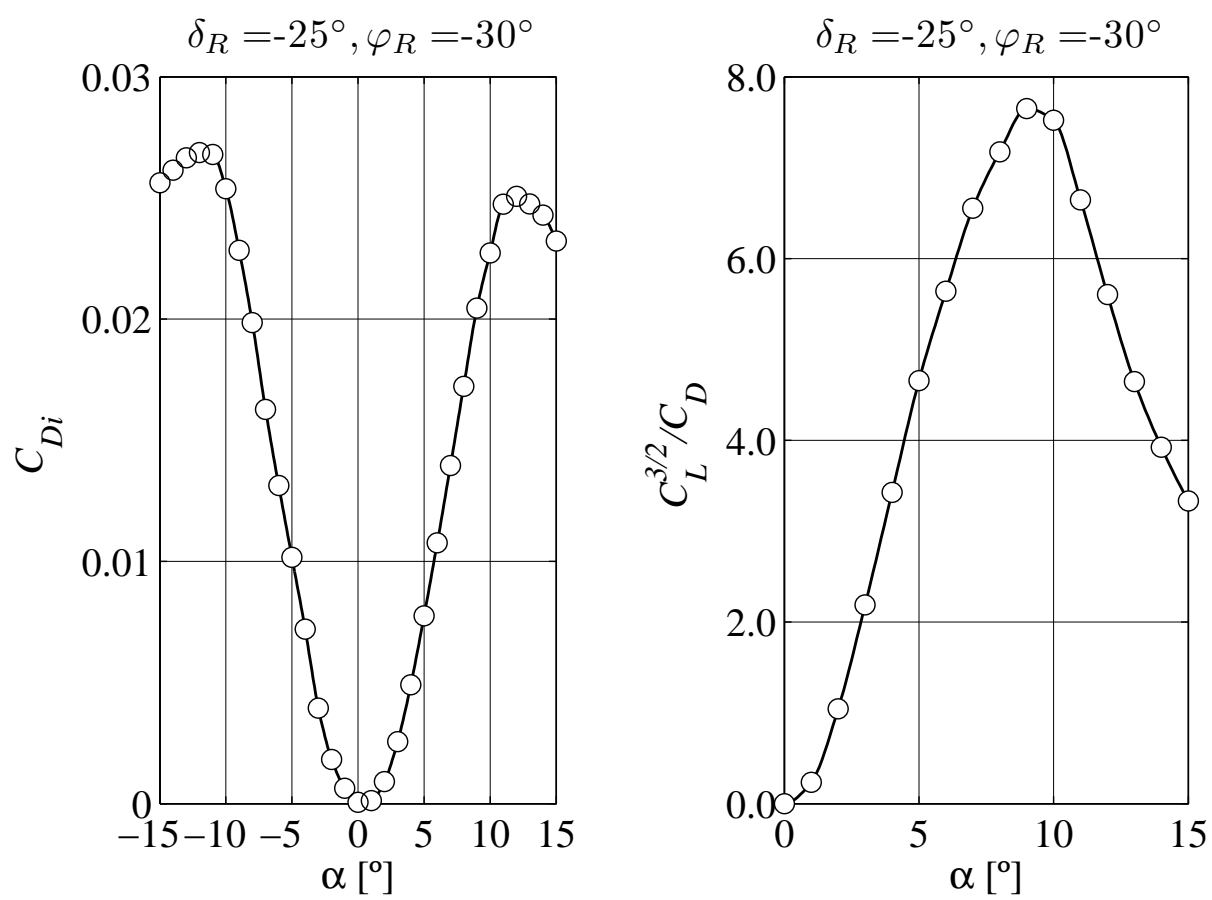

Figura 4.20: Variación de los coeficientes de resistencia inducida, $C_{D i}$, y del factor para potencia mínima requerida, $C_{L}^{3 / 2} / C_{D}$, en función del ángulo de ataque, $\alpha$, para el ángulo de flecha del ala trasera, $\varphi_{R}=$ $-30^{\circ}$ y para el ángulo diedro del ala trasera, $\delta_{R}=-25^{\circ}$. Número de Reynolds de la corriente libre $\mathrm{Re}=250000$. Configuración de referencia $\mathrm{JWC}_{15}$.

Por último es importante representar los resultados de los valores extremos, máximos o mínimos en cada caso, de los coeficiente, así en la Figura 4.31 se representan el valor máximo del coeficiente de sustentación, $C_{L_{\max }}$, y el coeficiente de resistencia mínimo, $C_{D_{m i n}}$, y en la Figura 4.32 el valor la eficiencia aerodinámica máxima,$E_{m a x}$ y del valor máximo del factor para potencia mínima requerida, $C_{L}^{3 / 2} / C_{D}$

Para el caso del coeficiente de sustentación, el valor máximo se produce a ángulos de ataque de $\alpha=12^{\circ}$, y en el caso del coeficiente de resistencia mínimo, el valor se producen a ángulos de ataque de $\alpha=0^{\circ}$ (ver apéndice $\mathrm{C}$ ), sin embargo, para el valor de la eficiencia aerodinámica máxima y para el valor mínimo del parámetro de potencia, estos valores se producen a ángulos de ataque de $\alpha=9^{\circ}$ en ambos casos (ver apéndice C). En estas figuras se muestra la variación del valor de los coeficientes en función del ángulo diedro y del ángulo de flecha del ala trasera.

Estos resultados se repiten, de forma similar, para todas las configuraciones de ensayo y a la vista de los mismos parece que se puede afimar, que es la configuración de referencia, $\mathrm{JWC}_{15}$, la que presenta mejores valores. 

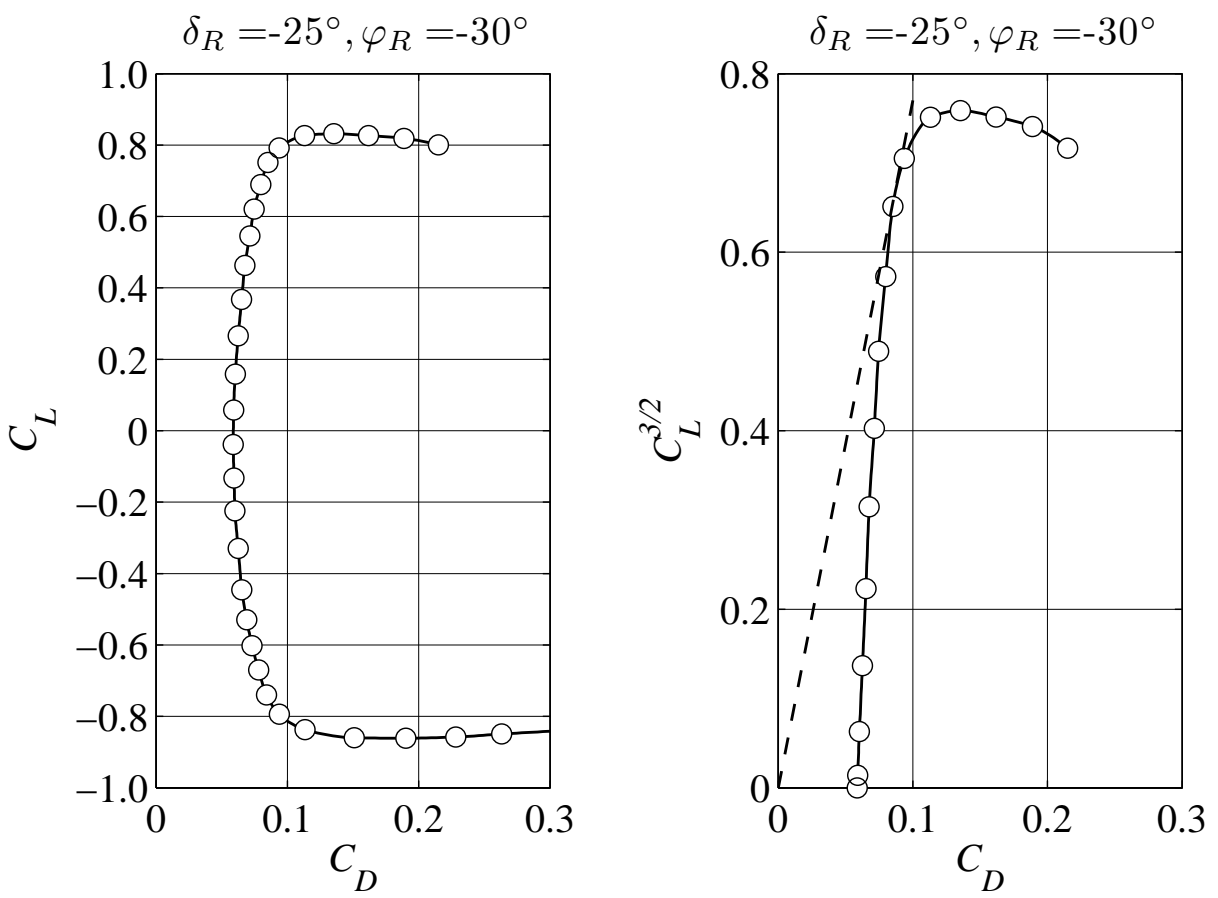

Figura 4.21: Variación del coeficiente de sustentación, $C_{L}$, y del coeficiente $C_{L}^{3 / 2}$, en función del coeficiente de resistencia, $C_{D}$, para el ángulo de flecha del ala trasera, $\varphi_{R}=-30^{\circ}$ y para el ángulo diedro del ala trasera, $\delta_{R}=-25^{\circ}$. La pendiente de la línea de trazos es el valor máximo de $C_{L}^{3 / 2} / C_{D}$. Número de Reynolds de la corriente libre $\mathrm{Re}=250000$. Configuración de referencia $\mathrm{JWC}_{15}$.
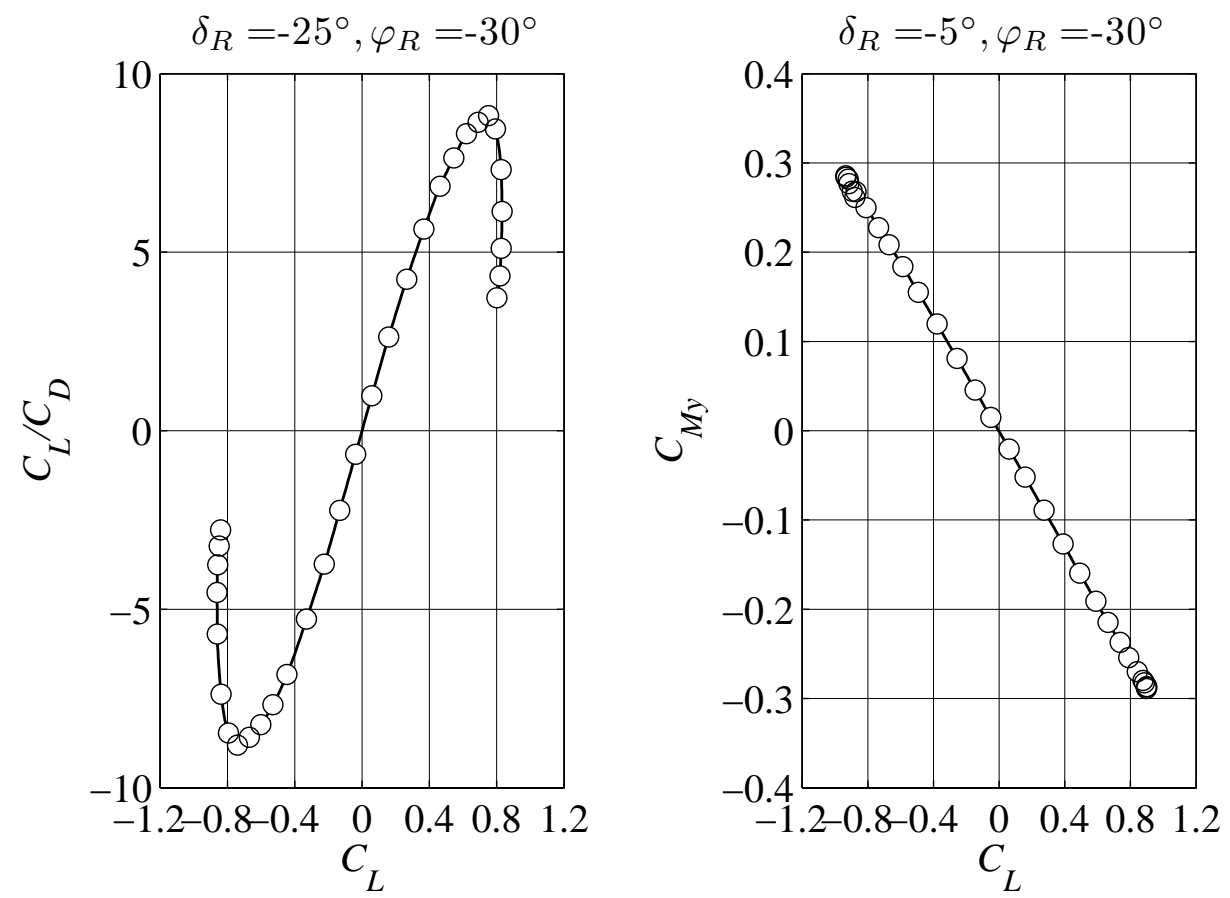

Figura 4.22: Variación de la eficiencia aerodinámica, $E$, y del coeficiente de momento, $C_{M y}$, en función del coeficiente de sustentación, $C_{L}$, para el ángulo de flecha del ala trasera, $\varphi_{R}=-30^{\circ}$ y para el ángulo diedro del ala trasera,$\delta_{R}=-25^{\circ}$. Número de Reynolds de la corriente libre $\mathrm{Re}=250000$. Configuración de referencia $\mathrm{JWC}_{15}$. 


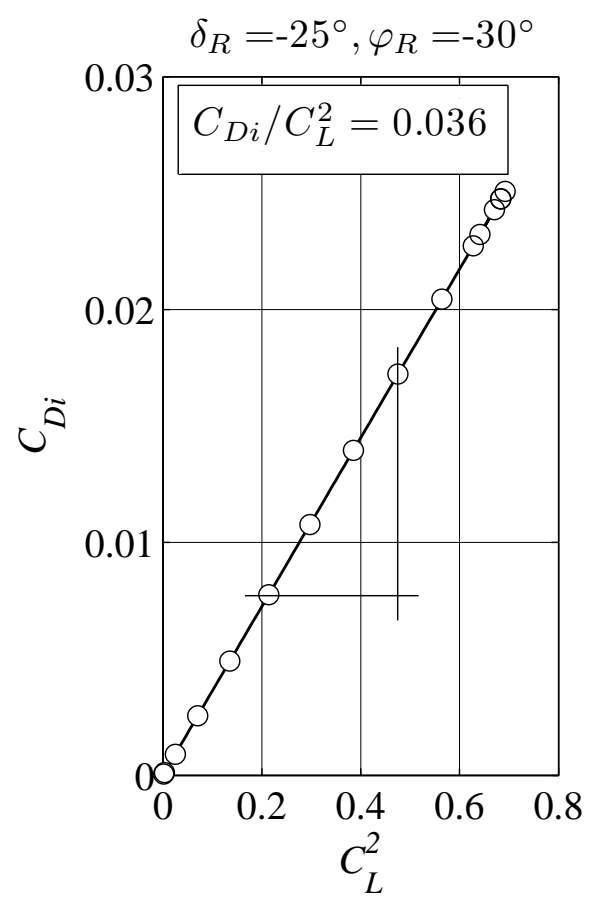

Figura 4.23: Variación de los coeficientes de resistencia inducida, $C_{D i}$, en función del cuadrado del coeficiente de sustentación, $C_{L}$, para el ángulo de flecha del ala trasera, $\varphi_{R}=-30^{\circ}$ y para el ángulo diedro del ala trasera, $\delta_{R}=-25^{\circ}$. Número de Reynolds de la corriente libre $\mathrm{Re}=250000$. Configuración de referencia $\mathrm{JWC}_{15}$.

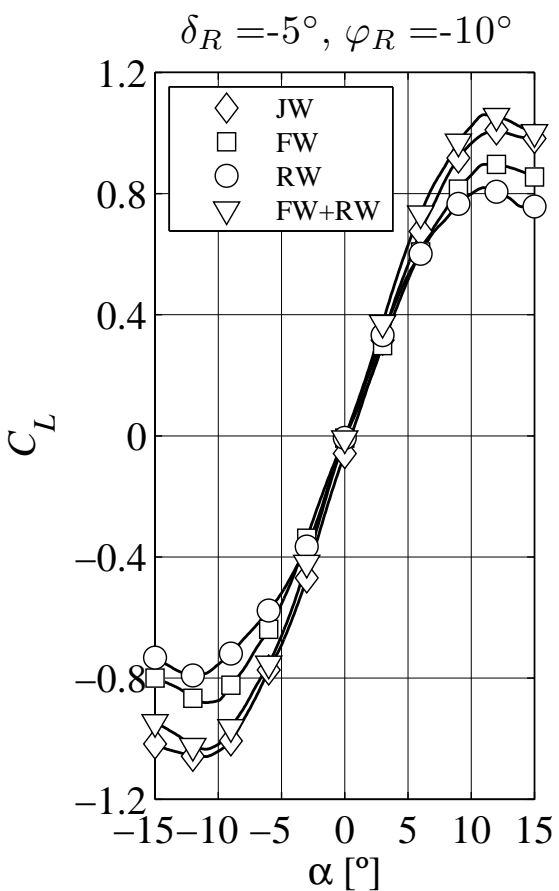

(a)

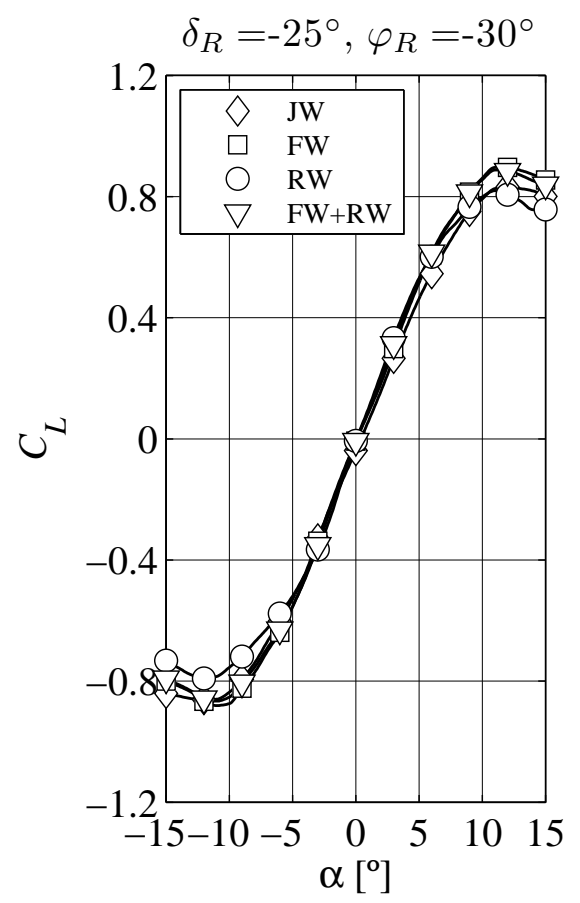

(b)

Figura 4.24: Comparación del coeficiente de sustentación, $C_{L}$, del modelo de alas convergentes $(\mathrm{JW})$, de el ala delantera $(\mathrm{FW})$, del ala trasera (RW) y de las suma entre ambas alas $(\mathrm{FW}+\mathrm{RW})$, en función del ángulo de ataque, $\alpha$, para la configuración $\mathrm{JWC}_{51}$, (a) y la configuración $\mathrm{JWC}_{15}$, (b). Número de Reynolds de la corriente libre $\mathrm{Re}=250000$. 



Figura 4.25: Variación del coeficiente de sustentación, $\overline{C_{L}}$, en función del ángulo de flecha del ala trasera,$\varphi_{R}$, y del ángulo diedro del ala trasera, $\delta_{R}$, para cada ángulo de ataque, $\alpha$. Número de Reynolds de la corriente libre $\mathrm{Re}=250000$. 

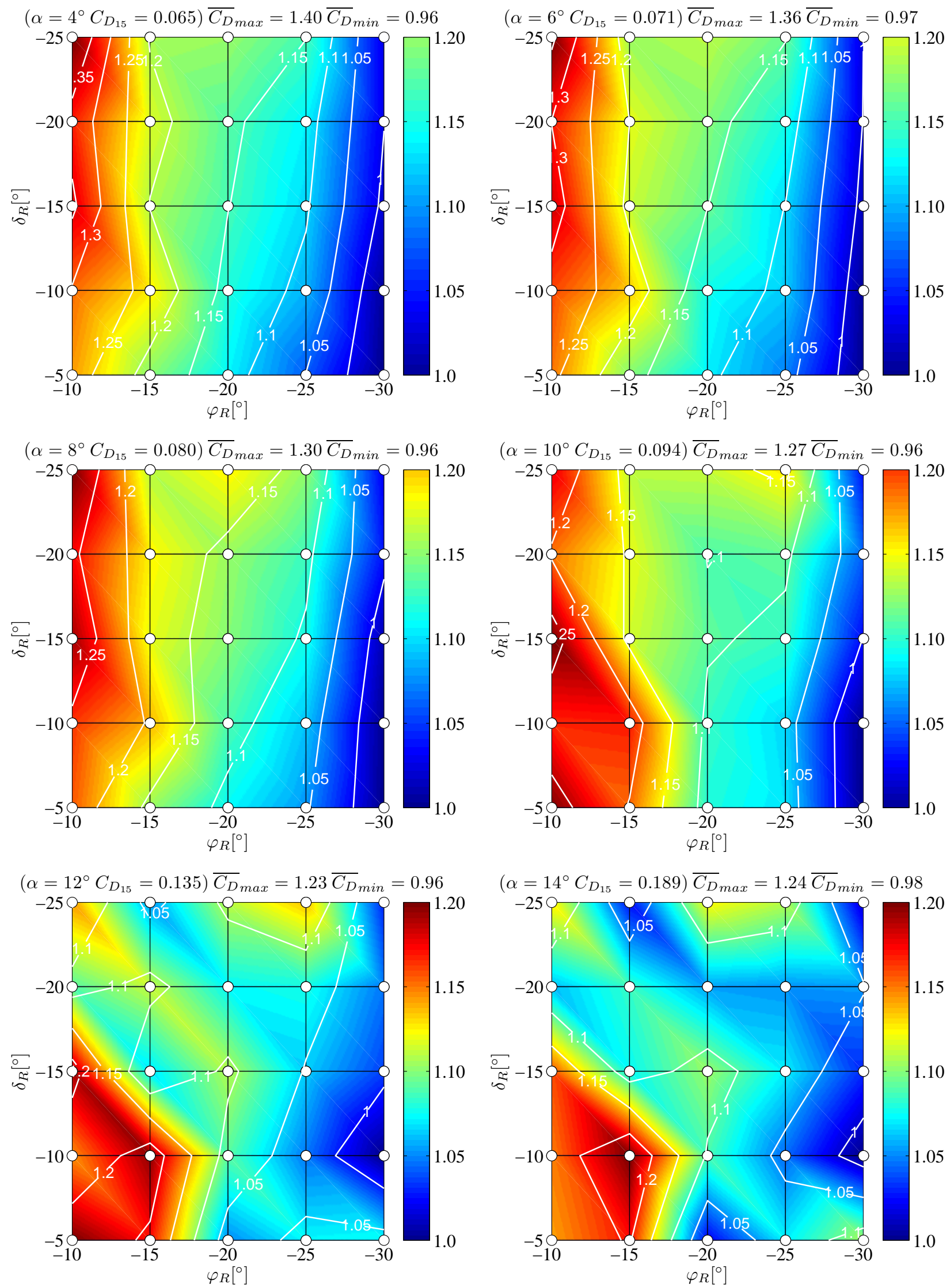

Figura 4.26: Variación del coeficientes de resistencia, $\overline{C_{D}}$, en función del ángulo de flecha del ala trasera, $\varphi_{R}$, y del ángulo diedro del ala trasera, $\delta_{R}$, para cada ángulo de ataque, $\alpha$. Número de Reynolds de la corriente libre $\mathrm{Re}=250000$. 

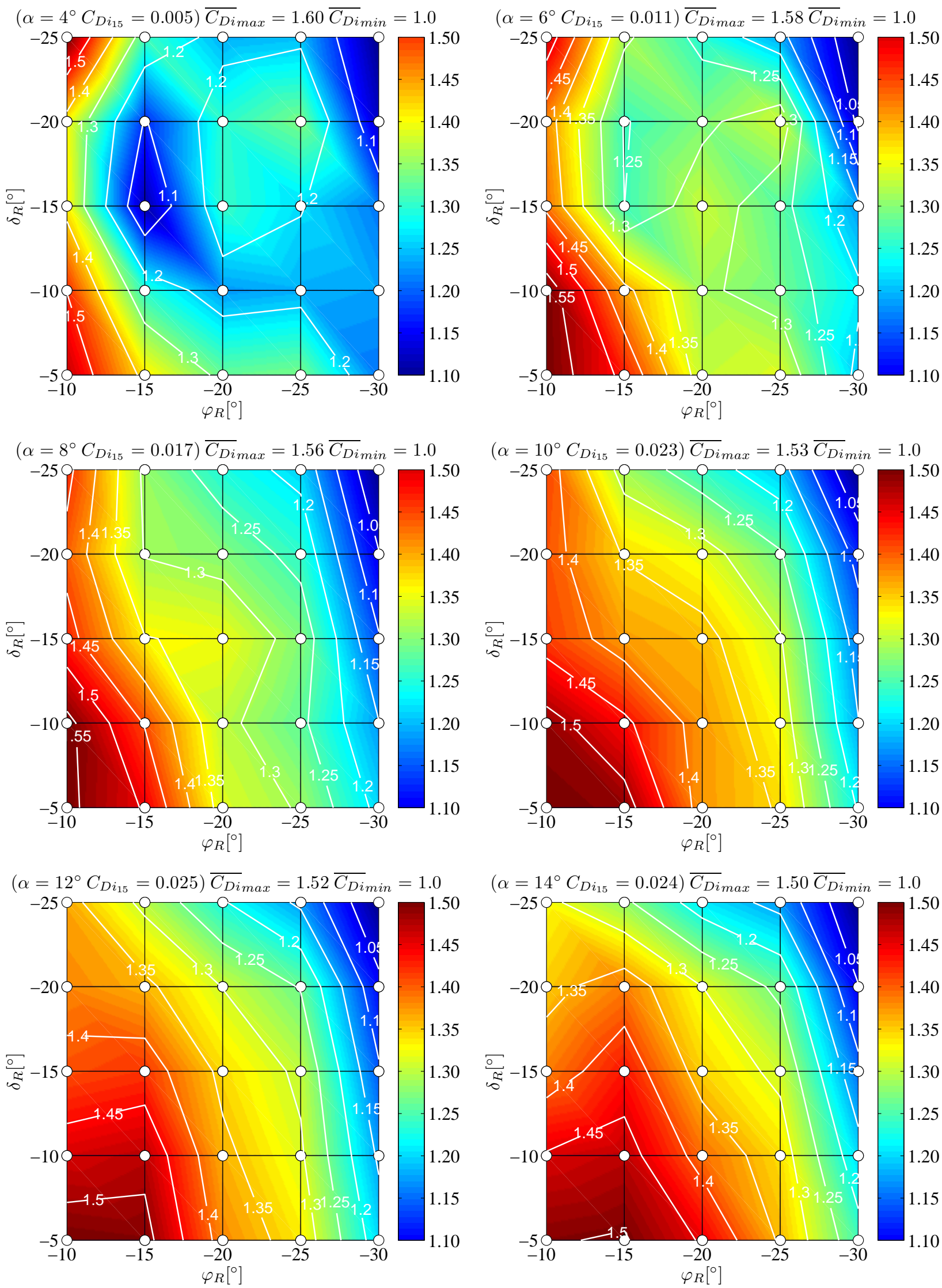

Figura 4.27: Variación del coeficiente de resistencia inducida, $\overline{C_{D i}}$, en función del ángulo de flecha del ala trasera, $\varphi_{R}$, y del ángulo diedro del ala trasera, $\delta_{R}$, para cada ángulo de ataque, $\alpha$. Número de Reynolds de la corriente libre $\mathrm{Re}=250000$. 

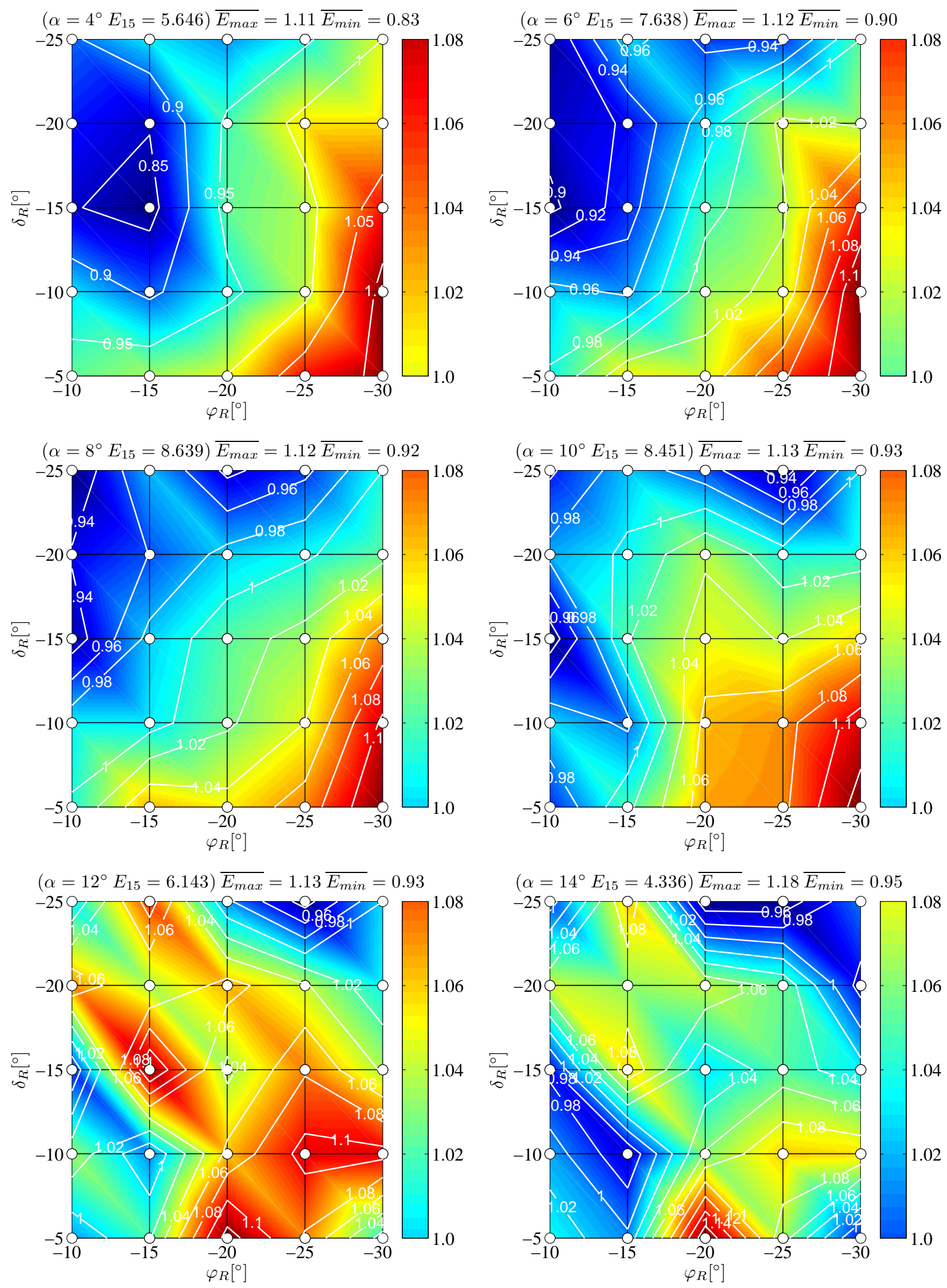

Figura 4.28: Variación de la eficiencia aerodinámica, $E$, en función del ángulo de flecha del ala trasera, $\varphi_{R}$, y del ángulo diedro del ala trasera, $\delta_{R}$, para cada ángulo de ataque, $\alpha$. Número de Reynolds de la corriente libre $R e=250000$. 



Figura 4.29: Variación del factor para potencia mínima requerida, $C_{L}^{3 / 2} / C_{D}$ (representado en el gráfico con el valor $P$ ), en función del ángulo de flecha del ala trasera, $\varphi_{R}$, y del ángulo diedro del ala trasera , $\delta_{R}$, para cada ángulo de ataque, $\alpha$. Número de Reynolds de la corriente libre $\mathrm{Re}=250000$. 


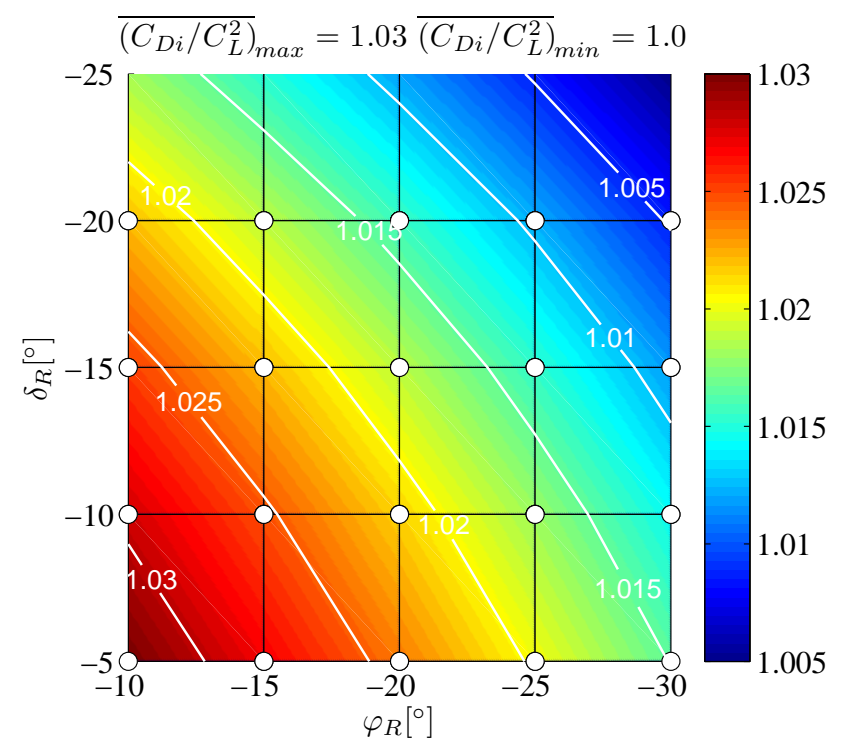

Figura 4.30: Variación de la relación de coeficientes, $\overline{C_{D i} / C_{L}^{2}}$, en función del ángulo de flecha del ala trasera, $\varphi_{R}$, y del ángulo diedro del ala trasera , $\delta_{R}$. Número de Reynolds de la corriente libre $\mathrm{Re}=$ 250000 .

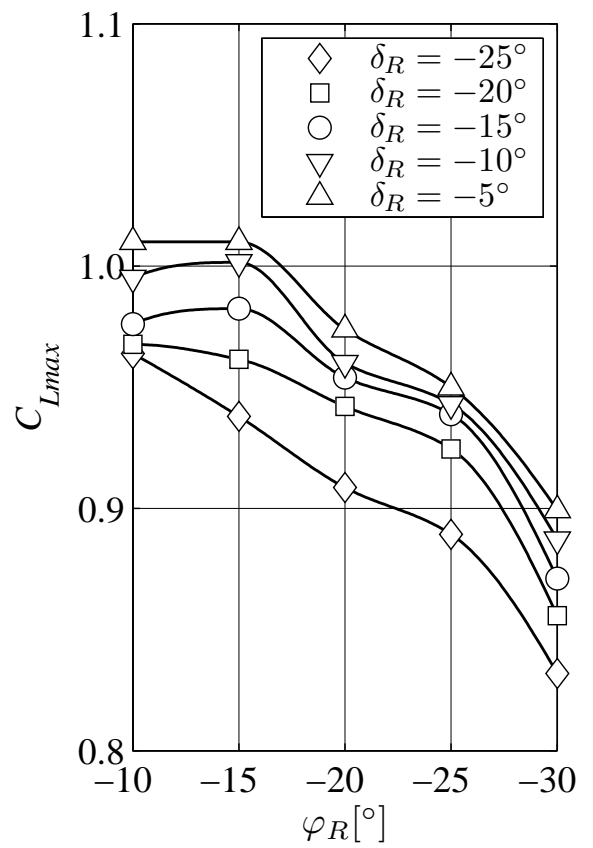

(a)

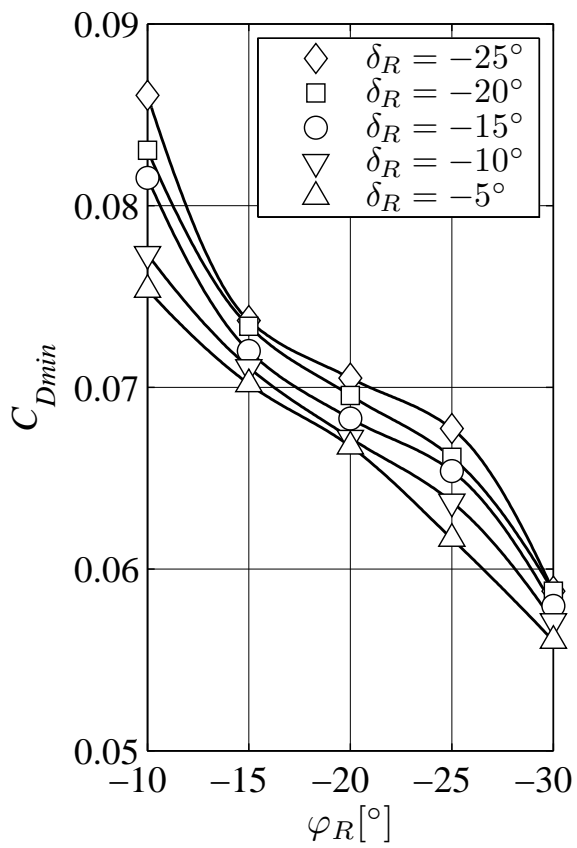

(b)

Figura 4.31: Variación del valor del coeficiente de sustentación máximo, $C_{L_{\max }}$, y del valor del coeficiente de resistencia mínimo, $C_{D_{\min }}$, en función del ángulo de flecha del ala trasera, $\varphi_{R}$, y del ángulo diedro del ala trasera, $\delta_{R}$. Número de Reynolds de la corriente libre Re $=250000$. 


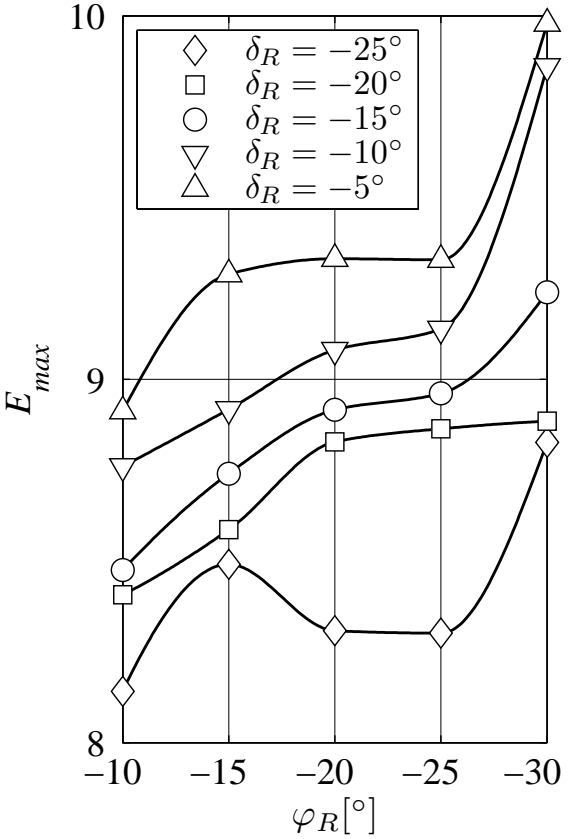

(a)

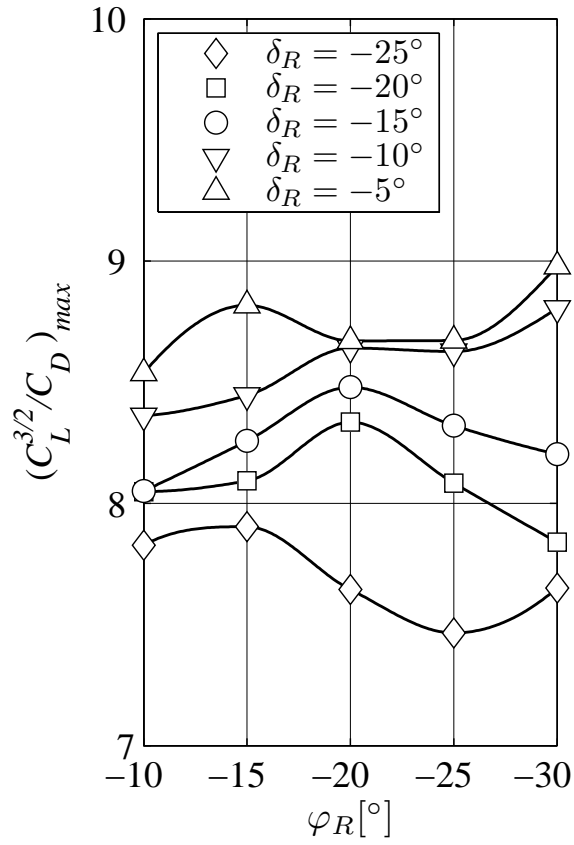

(b)

Figura 4.32: Variación del valor de la eficiencia aerodinámica máxima, $E_{\max }$, y del valor máximo del factor para potencia mínima requerida, $C_{L}^{3 / 2} / C_{D}$, en función del ángulo de flecha del ala trasera, $\varphi_{R}, \mathrm{y}$ del ángulo diedro del ala trasera, $\delta_{R}$. Número de Reynolds de la corriente libre $\operatorname{Re}=250000$. 



\section{Capítulo 5}

\section{Modelo numérico simplificado}

\subsection{Introducción}

Se considera el modelo de alas convergentes, que se muestra en la Figura 5.1 , moviéndose a velocidad constante $U$, y con un ángulos de ataque $\alpha$, en el seno de un fluido no perturbado. El ángulo de ataque $\alpha$, está definido como el ángulo entre la dirección de la velocidad de la corriente libre $U_{\infty}$, de componentes $u, v$ y $w$, respectivamente, y el eje $x$ del sistema de referencia de coordenadas ejes-cuerpo $(x, y, z)$ con origen en el punto $1 / 4$ de la cuerda en el encastre del ala delantera. Por criterios de simplicidad no se ha tenido en cuenta en el modelo la componente lateral $v$, de la velocidad de la corriente libre, por lo que el ángulo de ataque $\alpha$, puede expresarse por:

$$
\alpha=\tan ^{-1} \frac{w}{u}
$$

Se asume que el fluido alrededor de las alas y en la estela es no viscoso, incompresible e irrotacional, donde el campo de velocidades resultante, debido al movimiento de las alas en el seno del fluido, puede obtenerse resolviendo la ecuación de continuidad [Katz and Plotkin 2001].

$$
\nabla^{2} \Phi=0
$$

donde $\Phi$, es el campo potencial de velocidades definido en el sistema de referencia ejes-cuerpo. Por otro lado, las condiciones de contorno exigen que la perturbación inducida en el fluido por las alas sea nula a gran distancia (corriente arriba) lejos de estas.

$$
\lim _{x \rightarrow \infty} \nabla \Phi=U_{\infty}
$$




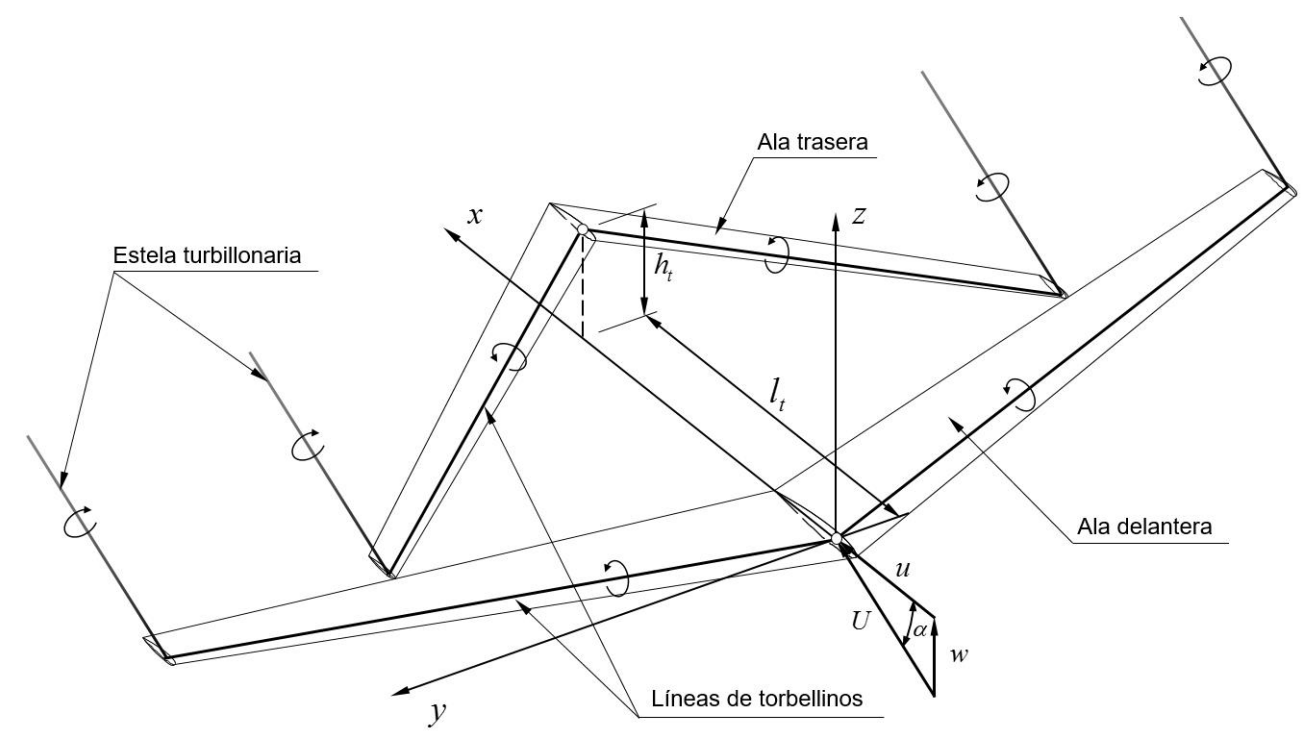

Figura 5.1: Esquema del modelo de linea sustentadora para la configuración de alas convergentes

De igual manera, la componente normal de la velocidad sobre las superficie de las alas debe ser nula.

$$
\nabla \Phi \cdot \mathbf{n}=0
$$

donde $\mathbf{n}$ define el vector normal a la superficie de cada ala.

De esta manera la solución se reduce a encontrar una distribución de singularidades que satisfagan la ecuación 5.4, conocida esta distribución el vector velocidad $\mathbf{V}$, en cada punto es conocido y por lo tanto la presión $p$ en cada punto puede calcularse haciendo uso de la ecuación de Bermoulli,

$$
p_{\infty}+\frac{\rho}{2} U_{\infty}^{2}=p+\frac{\rho}{2} q^{2}
$$

La solución analítica de este problema, no existe en general para cualquier forma y configuración de alas, y resulta complicada por la propia dificultad de definir la condición de contorno requerida en (5.4) sobre la superficie de las alas, lo que implica simplificar el problema empleando modelos más sencillos.

\subsection{Modelo de líneas sustentadoras}

Como se ha descrito en el punto anterior, encontrar una solución analítica del problema resulta complejo, aunque, por otro lado es posible plantear simplificaciones que proporcionen soluciones aproximadas aceptables. Una de las posibles simplificaciones es aproximar las características aerodinámicas de cada ala por medio de una línea o herradura de torbellinos.

\subsubsection{Definición del modelo de linea sustentadora}

Para la resolución del problema se define, el modelo de alas convergentes, como una combinación de dos herraduras de torbellinos, que sustituyen al ala 


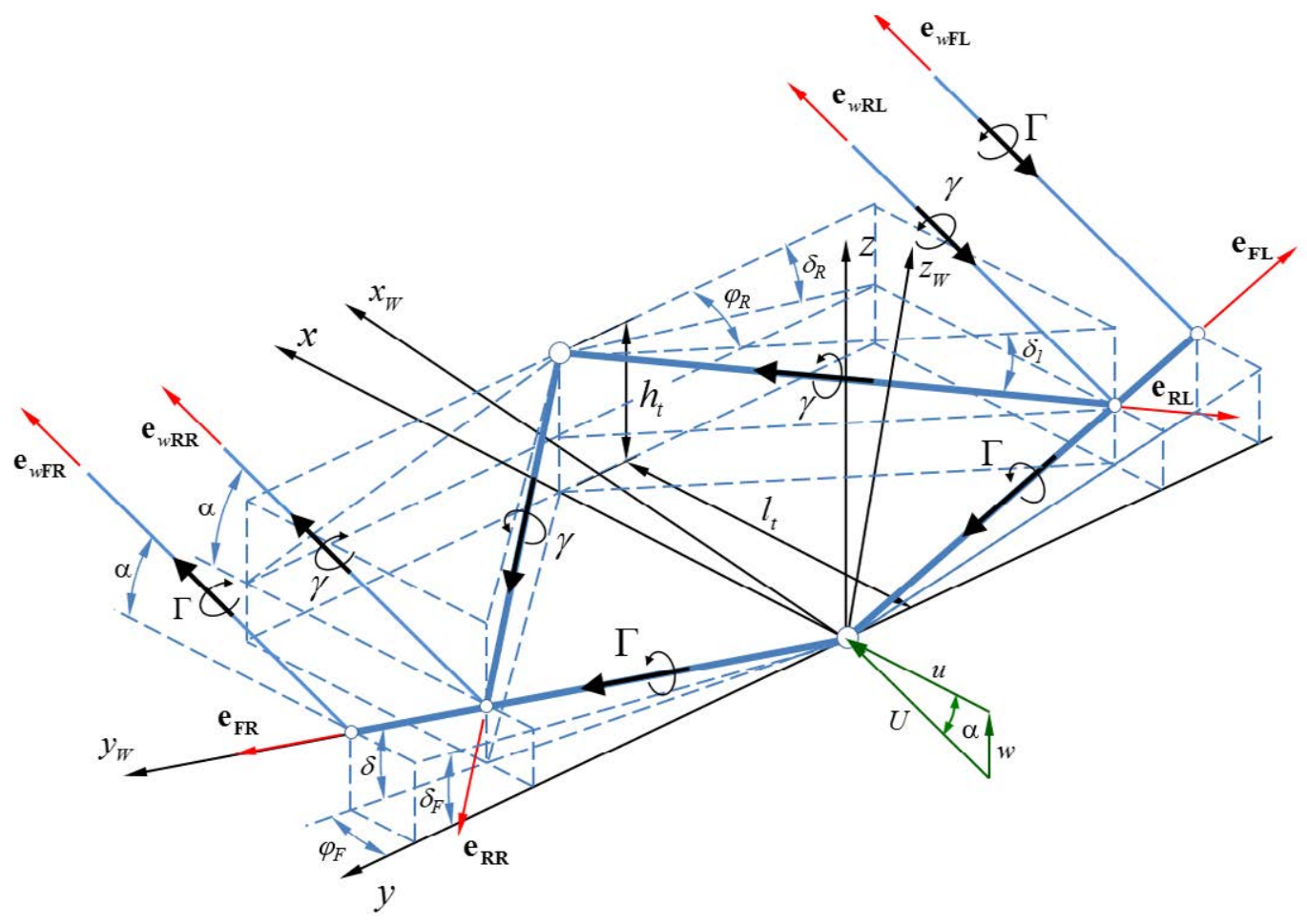

Figura 5.2: Modelo geométrico de línea sustentadora para la configuración de alas convergentes

delantera y al ala trasera respectivamente, tal y como se muestra en la Figura 5.2. Estas herraduras de torbellinos están formadas por líneas que siguen la línea de puntos $1 / 4$ de cada sección de ala y por dos líneas de torbellinos libres que escapan de la superficies sustentadoras cerca de los bordes marginales y que son arrastrados por la corriente para formar la estela turbillonaria corriente abajo del ala [Küchemann 1938; Phillips and Snyder 2000].

Como es conocido y tal y como se muestra en la ecuación 5.2, el campo de velocidades puede calcularse resolviendo la ecuación de Laplace para el campo potencial perturbado, siendo la condición de contorno evaluada en $z=0$ determinada por:

$$
\frac{\partial \Phi}{\partial z}(x, y, \pm 0)=Q_{\infty}\left(\frac{\partial \eta_{c}}{\partial x} \pm \frac{\partial \eta_{t}}{\partial x}-\alpha\right)
$$

siendo $\eta_{c}$, la función de curvatura del perfil de cada ala y $\eta_{t}$, la función de espesor. Estas funciones quedan definidas por medio de las funciones geométricas del contorno de intradós, $\eta_{l}$, y estradós, $\eta_{u}$, de la superficie del ala:

$$
\begin{aligned}
& \eta_{c}=\frac{1}{2}\left(\eta_{u}+\eta_{l}\right) \\
& \eta_{t}=\frac{1}{2}\left(\eta_{u}-\eta_{l}\right)
\end{aligned}
$$


Para la resolución del problema de línea sustentadora se calcula la velocidad inducida $\Delta \mathbf{v}_{i}$, producida por cada elemento diferencial dl, de cada línea de torbellinos por medio de la ley de Biot-Savart,

$$
\triangle \mathbf{v}_{i}=-\frac{1}{4 \pi} \frac{\triangle \Gamma \cdot \mathbf{r} \times \mathrm{d} \mathbf{l}}{r^{3}}
$$

El modelo más simple que se puede proponer para resolver este problema para su aplicación al modelo de alas convergentes es sustituir la circulación, en cualquier estación a lo largo de la envergadura del ala, y reemplazarla por un único torbellino constante concentrado, pero tenido en cuenta tanto el ángulo de flecha del ala delantera, $\varphi_{F}$, el ángulo de flecha del ala trasera, $\varphi_{R}$, así como el ángulo diedro del ala delantera, $\delta_{F}$, y el ángulo diedro del ala trasera, $\delta_{R}$, [Brebner and Wyatt 1970]. Además, los torbellinos locales de circulación en el ala delantera, $\Gamma$, y en el ala trasera, $\gamma$, estarán situados a lo largo de una única línea de torbellinos por cada ala, siendo la dirección de las líneas de torbellinos de la estela coincidentes con la dirección de la corriente libre, como se muestra en la Figura 5.2.

Claramente, en el caso real, la estela de torbellinos no está alineada con la corriente libre. Este último caso es mucho más complicado [Mortara et al. 1991] porque la posición final de la estela no es conocida y tiene que ser determinada [Smith 1996]. Por lo tanto, el problema se vuelve no lineal incluso si las ecuaciones que gobiernan el flujo son lineales.

\subsubsection{Parámetros geométricos y sistemas de referencia}

El modelo de alas convergentes queda determinado, desde el punto de vista geométrico, con los parámetros definidos en la Tabla 4.2 .

Para poder establecer relaciones entre las variables locales y globales, el modelo está compuesto de cinco sistemas de referencia: el sistema de referencia ejes cuerpo $(x, y, z)$, y otros cuatro sistemas de referencia: ejes ala fijos al ala delantera derecha $\left(x_{F R}, y_{F R}, z_{F R}\right)$, al ala delantera izquierda $\left(x_{F L}, y_{F L}, z_{F L}\right)$, al ala trasera derecha $\left(x_{R R}, y_{R R}, z_{R R}\right)$ y al ala trasera izquierda $\left(x_{R L}, y_{R L}, z_{R L}\right)$. Todos los sistemas de coordenadas tienen su origen en el punto $1 / 4$ de la cuerda en el encastre del ala delantera.

Por otro lado las componentes del vector de cada línea sustentadora proyectadas sobre cada sistema de coordenadas ejes ala están definidas como:

$$
\mathbf{e}_{F R_{W}}=\mathbf{e}_{F L_{W}}=\mathbf{e}_{R R_{W}}=\mathbf{e}_{R L_{W}}=\left[\begin{array}{l}
0 \\
1 \\
0
\end{array}\right]
$$

Estos vectores se proyectan sobre el sistema de referencia ejes cuerpo usando las correspondientes matrices de transformación,

$$
\mathbf{e}_{F R_{B}}=\mathbf{M}_{F R W B} \cdot \mathbf{e}_{F R_{W}}=\left[\begin{array}{c}
\cos \delta \sin \varphi_{F} \\
\cos \delta \cos \varphi_{F} \\
\sin \delta
\end{array}\right]
$$




$$
\begin{gathered}
\mathbf{e}_{F L_{B}}=\mathbf{M}_{F L W B} \cdot \mathbf{e}_{F L_{W}}=\left[\begin{array}{c}
\cos \delta \sin \varphi_{F} \\
-\cos \delta \cos \varphi_{F} \\
\sin \delta
\end{array}\right] \\
\mathbf{e}_{R R_{B}}=\mathbf{M}_{R R W B} \cdot \mathbf{e}_{R R_{W}}=\left[\begin{array}{c}
\cos \delta_{1} \sin \varphi_{R} \\
\cos \delta_{1} \cos \varphi_{R} \\
\sin \delta_{1}
\end{array}\right] \\
\mathbf{e}_{R L_{B}}=\mathbf{M}_{R L W B} \cdot \mathbf{e}_{R L_{W}}=\left[\begin{array}{c}
\cos \delta_{1} \sin \varphi_{R} \\
-\cos \delta_{1} \cos \varphi_{R} \\
\sin \delta_{1}
\end{array}\right],
\end{gathered}
$$

donde el ángulo $\delta$ y él ángulo $\delta_{1}$ corresponden al ángulo diedro del ala delantera y del ala trasera respectivamente, proyectados sobre los sistemas ejes ala del ala delantera y trasera. Por otro lado las matrices $\mathbf{M}_{F R W B}, \mathbf{M}_{F l W B}, \mathbf{M}_{R R W B}$ y $\mathbf{M}_{R L W B}$ son las correspondientes al cambio de ejes ala a ejes cuerpo para el ala delantera derecha, ala delantera izquierda, ala trasera derecha y ala trasera izquierda respectivamente y definidas como:

$$
\begin{gathered}
\mathbf{M}_{F R W B}=\left[\begin{array}{ccc}
\cos \varphi_{F} & \cos \delta \sin \varphi_{F} & -\sin \delta \sin \varphi_{F} \\
-\sin \varphi_{F} & \cos \delta \cos \varphi_{F} & -\sin \delta \cos \varphi_{F} \\
0 & \sin \delta & \cos \delta
\end{array}\right] \\
\mathbf{M}_{F L W B}=\left[\begin{array}{ccc}
\cos \varphi_{F} & \cos \delta \sin \varphi_{F} & -\sin \delta \sin \varphi_{F} \\
\sin \varphi_{F} & -\cos \delta \cos \varphi_{F} & \sin \delta \cos \varphi_{F} \\
0 & \sin \delta & \cos \delta
\end{array}\right] \\
\mathbf{M}_{R R W B}=\left[\begin{array}{ccc}
\cos \varphi_{R} & \cos \delta_{1} \sin \varphi_{R} & -\sin \delta_{1} \sin \varphi_{R} \\
-\sin \varphi_{F} & \cos \delta_{1} \cos \varphi_{R} & -\sin \delta_{1} \cos \varphi_{R} \\
0 & \sin \delta_{1} & \cos \delta_{1}
\end{array}\right] \\
\mathbf{M}_{R L W B}=\left[\begin{array}{ccc}
\cos \varphi_{R} & \cos \delta_{1} \sin \varphi_{R} & -\sin \delta_{1} \sin \varphi_{R} \\
\sin \varphi_{F} & -\cos \delta_{1} \cos \varphi_{R} & \sin \delta_{1} \cos \varphi_{R} \\
0 & \sin \delta_{1} & \cos \delta_{1}
\end{array}\right]
\end{gathered}
$$

De igual manera, las componentes del vector de cada línea de estela proyectadas en el sistema de referencia de ejes cuerpo, quedan definidas por:

$$
\mathbf{e}_{w F R_{B}}=\mathbf{e}_{w F L_{B}}=\mathbf{e}_{w R R_{B}}=\mathbf{e}_{w R L_{B}}=\left[\begin{array}{c}
\cos \alpha \\
0 \\
\sin \alpha
\end{array}\right]
$$

donde $\alpha$ corresponde al ángulo de ataque de la corriente libre.

Por otro lado, y una vez determinados los vectores de cada línea sustentadora del ala en el sistema de referencia ejes cuerpo, se puede determinar las coordenadas de un punto genérico de cada ala delantera, en el citado sistema de referencia, por medio de: 


$$
\begin{aligned}
& \mathbf{x}_{F R_{B}}=\Lambda_{L} \mathbf{e}_{F R_{B}}, \\
& \mathbf{x}_{F L_{B}}=\Lambda_{L} \mathbf{e}_{F L_{B}},
\end{aligned}
$$

donde $\Lambda_{L} \in\left[0, L_{D}\right]$ es la variación de la coordenada del vector de la línea sustentadora sobre el ala delantera, siendo $L_{D}$ la longitud no proyectada de la semiala delantera que queda definido por:

$$
L_{D}=\frac{b_{F}}{2 \cos \delta \cos \varphi_{F}}
$$

Así mismo, las coordenadas de un punto genérico de cada ala trasera, en el sistema de referencia ejes cuerpo queda determinado por medio de:

$$
\begin{gathered}
\mathbf{x}_{R R_{B}}=\mathbf{x}_{R 0_{B}}+\lambda_{L} \mathbf{e}_{R R_{B}}, \\
\mathbf{x}_{R L_{B}}=\mathbf{x}_{R 0_{B}}+\lambda_{L} \mathbf{e}_{R L_{B}},
\end{gathered}
$$

donde $\lambda_{L} \in\left[0, L_{T}\right]$ es la variación de la coordenada del vector de la linea sustentadora sobre el ala trasera, y $L_{R}$, es la longitud no proyectada de la semiala trasera que queda definido por:

$$
L_{T}=\frac{b_{R}}{2 \cos \delta_{1} \cos \varphi_{R}}
$$

Por otro lado, $\mathbf{x}_{R 0_{B}}$ establece las coordenadas de la posición del punto $1 / 4$ de la cuerda en el encastre de el ala trasera, definidas por:

$$
\mathbf{x}_{R 0_{B}}=\left[\begin{array}{c}
l_{t} \\
0 \\
h_{t}
\end{array}\right],
$$

donde $l_{t} \mathrm{y} h_{t}$, son la separación longitudinal y separación vertical del ala trasera, respectivamente, y quedan definidos por:

$$
\begin{gathered}
l_{t}=B L_{D} \cos \delta_{F} \cos \varphi_{F}\left(\tan \varphi_{F}-\tan \varphi_{R}\right)+\frac{1}{4} c_{t R}+\frac{3}{4} c_{r F}\left[B\left(\lambda_{F}-1\right)+1\right], \\
h_{t}=B L_{D} \cos \delta_{F} \cos \varphi_{F}\left(\tan \delta_{F}-\tan \delta_{R}\right),
\end{gathered}
$$

siendo $c_{t R}$, la cuerda en punta del ala trasera, $c_{r F}$, la cuerda en el encastre del ala delantera, $\lambda_{F}$, el estrechamiento del ala delantera y $B$, la relación entre envergaduras del ala trasera y delantera, definida por:

$$
B=\frac{b_{R}}{b_{F}}
$$


De forma similar, pero ahora para la posición genérica de un punto en cada línea de estela del ala delantera referida al sistema de ejes cuerpo es:

$$
\begin{gathered}
\mathbf{x}_{w F R_{B}}=\sigma_{L} \mathbf{e}_{w F R_{B}}+L_{D} \mathbf{e}_{F R_{B}} \\
\mathbf{x}_{w F L_{B}}=\sigma_{L} \mathbf{e}_{w F L_{B}}+L_{D} \mathbf{e}_{F L_{B}}
\end{gathered}
$$

Finalmente, la posición genérica de un punto en cada línea de estela del ala trasera referida al sistema de ejes cuerpo es:

$$
\begin{gathered}
\mathbf{x}_{w R R_{B}}=\mathbf{x}_{R 0_{B}}+\sigma_{L} \mathbf{e}_{w R R_{B}}+L_{T} \mathbf{e}_{R R_{B}} \\
\mathbf{x}_{w R L_{B}}=\mathbf{x}_{R 0_{B}}+\sigma_{L} \mathbf{e}_{w R L_{B}}+L_{T} \mathbf{e}_{R L_{B}},
\end{gathered}
$$

donde $\sigma_{L} \in[0, \infty]$ es la variación de la coordenada del vector de las líneas de torbellinos libres que escapan de la superficies sustentadoras.

Definidos todos los parámetros geométricos es posible analizar cualquier combinación de geometría, que en el caso de estudio se obtienen modificando los parámetros geométricos del ala trasera correspondientes al ángulo diedro, $\delta_{R}$ y el ángulo de flecha, $\varphi_{R}$. Como resultado, se obtienen 25 configuraciones de ensayo diferentes (Tabla 4.3), tal como se muestra en la Figura 5.3.

Para la realización de los cálculos todos los parámetros geométricos relativos a valores de longitud se han adimensionalizado usando como referencia el parámetro de la longitud no proyectada de la semiala delantera, $L_{D}$, quedando el resto de parámetros en la forma: $\overline{b_{F}}=b_{F} / L_{D}, \overline{b_{R}}=b_{R} / L_{D}, \overline{L_{T}}=L_{T} / L_{D}$, $\overline{l_{t}}=l_{t} / L_{D}, \mathrm{y} \overline{h_{t}}=h_{t} / L_{D}$.

Debido a esto los parámetros utilizados para la determinación de puntos sobre las alas, $\Lambda_{L}$ y $\lambda_{L}$, varían en la forma: $\Lambda_{L} \in[0,1]$, y $\lambda_{L} \in\left[0, \overline{L_{T}}\right]$, y la coordenada de la posición del punto $1 / 4$ de la cuerda en el encastre de el ala trasera, $\mathbf{x}_{R 0_{B}}$, queda ahora definida por:

$$
\mathbf{x}_{R 0_{B}}=\left[\begin{array}{c}
\overline{l_{t}} \\
0 \\
\overline{h_{t}}
\end{array}\right]
$$

De esta forma en la Figura 5.4 se representan los datos geométricos del modelo de alas convergentes de forma similar a la Figura 5.3 pero ahora adimensionalizados.

\subsubsection{Cálculo de velocidades inducidas}

Todas las velocidades inducidas por cada línea de torbellinos se calculan por medio de la ley de Biot-Savart

$$
\mathrm{d} \mathbf{v}_{i}=\frac{\Gamma}{4 \pi} \frac{\mathrm{d} \mathbf{l} \times\left(\mathbf{r}_{0}-\mathbf{r}_{1}\right)}{\left|\mathbf{r}_{0}-\mathbf{r}_{1}\right|^{3}}
$$



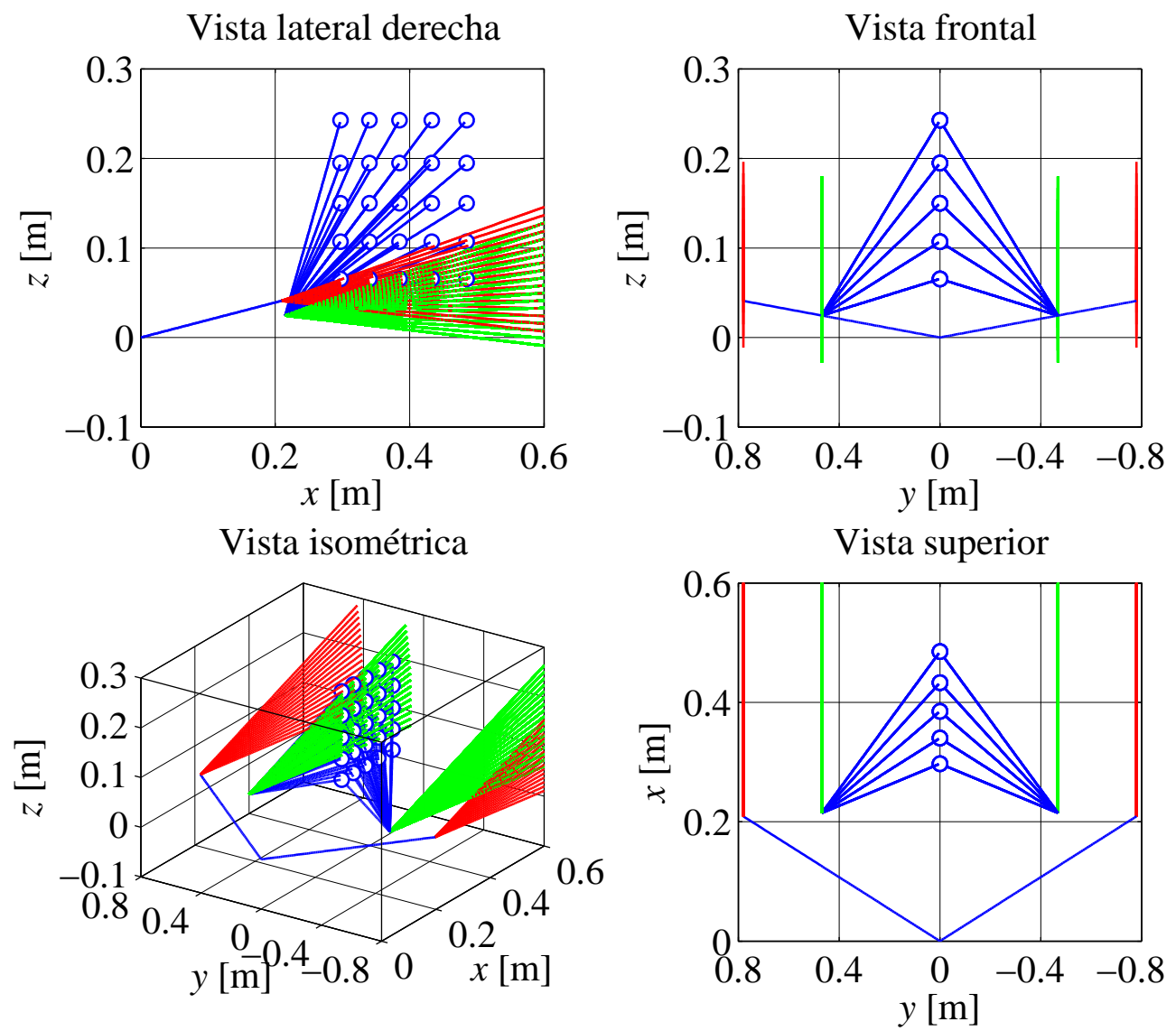

Figura 5.3: Vistas normalizadas de la definición geométrica del modelo de linea sustentadora para la configuración de alas convergentes, donde los puntos representan las 25 configuraciones de ensayo.

La velocidad inducida en la línea de torbellinos de cada ala está compuesta por la influencia de las otras siete líneas de torbellinos. Por ejemplo, la velocidad inducida (en el sistema de coordenadas ejes cuerpo) en el semiala derecha delantera, debido a las otras siete líneas de torbellinos es [Brebner and Wyatt 1970]:

- Velocidad inducida de la semiala delantera izquierda sobre la semiala delantera derecha:

$$
\mathbf{v}_{i F L F R_{B}}=\frac{-\Gamma}{4 \pi} \int \frac{\mathbf{e}_{F L_{B}} \times\left(\mathbf{x}_{F R_{B}}-\mathbf{x}_{F L_{B}}\right)}{\left|\mathbf{x}_{F R_{B}}-\mathbf{x}_{F L_{B}}\right|^{3}} \mathrm{~d} \mathbf{l}
$$

- Velocidad inducida de la estela de la semiala delantera derecha sobre la semiala delantera derecha:

$$
\mathbf{v}_{i F R w F R_{B}}=\frac{\Gamma}{4 \pi} \int \frac{\mathbf{e}_{w F R_{B}} \times\left(\mathbf{x}_{F R_{B}}-\mathbf{x}_{w F R_{B}}\right)}{\left|\mathbf{x}_{F R_{B}}-\mathbf{x}_{w F R_{B}}\right|^{3}} \mathrm{~d} \mathbf{l}
$$

- Velocidad inducida de la estela de la semiala delantera izquierda sobre la semiala delantera derecha:

$$
\mathbf{v}_{i F L w F R_{B}}=\frac{-\Gamma}{4 \pi} \int \frac{\mathbf{e}_{w F L_{B}} \times\left(\mathbf{x}_{F R_{B}}-\mathbf{x}_{w F L_{B}}\right)}{\left|\mathbf{x}_{F R_{B}}-\mathbf{x}_{w F L_{B}}\right|^{3}} \mathrm{~d} \mathbf{l}
$$




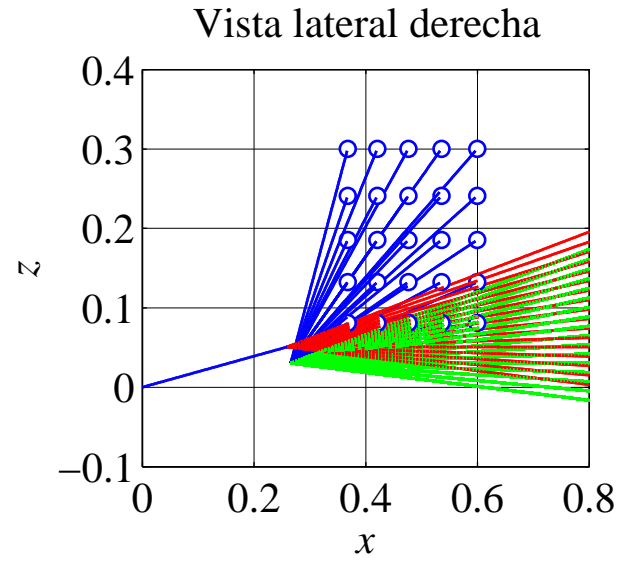

Vista isométrica

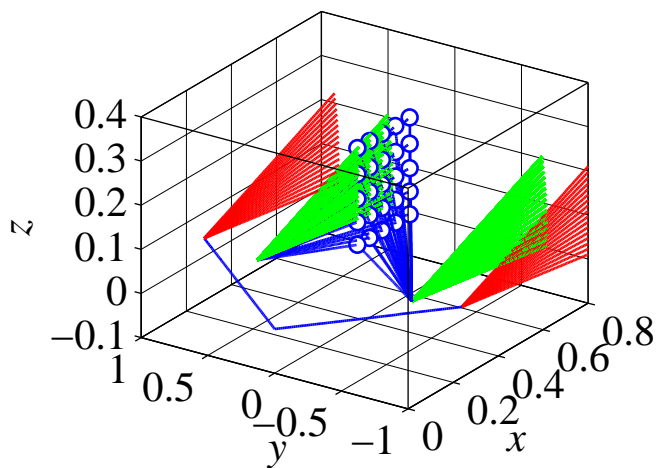

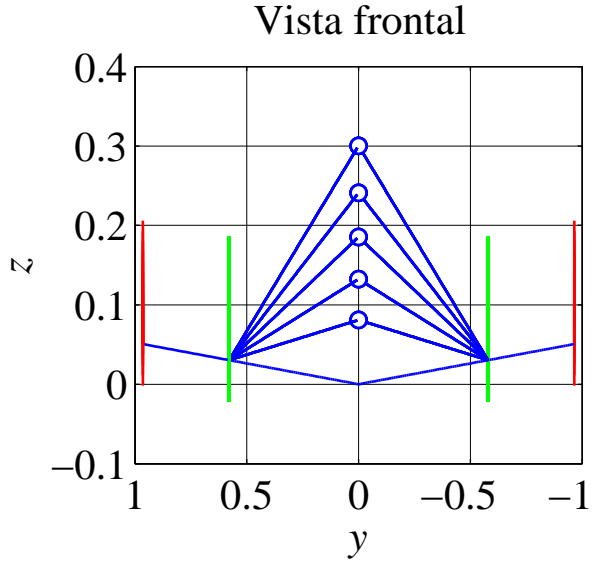

Vista superior

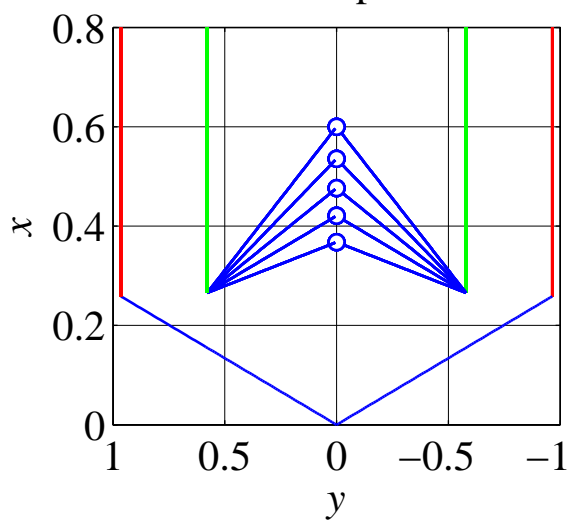

Figura 5.4: Vistas normalizadas de la definición geométrica adimensional del modelo de línea sustentadora para la configuración de alas convergentes, donde los puntos representan las 25 configuraciones de ensayo.

- Velocidad inducida de la semiala trasera derecha sobre la semiala delantera derecha:

$$
\mathbf{v}_{i R R F R_{B}}=\frac{\gamma}{4 \pi} \int \frac{\mathbf{e}_{R R_{B}} \times\left(\mathbf{x}_{F R_{B}}-\mathbf{x}_{R R_{B}}\right)}{\left|\mathbf{x}_{F R_{B}}-\mathbf{x}_{R R_{B}}\right|^{3}} \mathrm{~d} \mathbf{l}
$$

- Velocidad inducida de la semiala trasera izquierda sobre la semiala delantera derecha:

$$
\mathbf{v}_{i R L F R_{B}}=\frac{-\gamma}{4 \pi} \int \frac{\mathbf{e}_{R L_{B}} \times\left(\mathbf{x}_{F R_{B}}-\mathbf{x}_{R L_{B}}\right)}{\left|\mathbf{x}_{F R_{B}}-\mathbf{x}_{R L_{B}}\right|^{3}} \mathrm{~d} \mathbf{l}
$$

- Velocidad inducida de la estela de la semiala trasera derecha sobre la semiala delantera derecha:

$$
\mathbf{v}_{i R R w F R_{B}}=\frac{\gamma}{4 \pi} \int \frac{\mathbf{e}_{w R R_{B}} \times\left(\mathbf{x}_{F R_{B}}-\mathbf{x}_{w R R_{B}}\right)}{\left|\mathbf{x}_{F R_{B}}-\mathbf{x}_{w R R_{B}}\right|^{3}} \mathrm{~d} \mathbf{l}
$$

- Velocidad inducida de la estela de la semiala trasera izquierda sobre la semiala delantera derecha:

$$
\mathbf{v}_{i R L w F R_{B}}=\frac{-\gamma}{4 \pi} \int \frac{\mathbf{e}_{w R L_{B}} \times\left(\mathbf{x}_{F R_{B}}-\mathbf{x}_{w R L_{B}}\right)}{\left|\mathbf{x}_{F R_{B}}-\mathbf{x}_{w R L_{B}}\right|^{3}} \mathrm{~d} \mathbf{l}
$$


Como resultado la velocidad inducida total en la semiala delantera derecha queda:

$$
\begin{aligned}
\mathbf{v}_{i F R_{B}} & =\mathbf{v}_{i F L F R_{B}}+\mathbf{v}_{i F R w F R_{B}}+\mathbf{v}_{i F L w F R_{B}}+\mathbf{v}_{i R R F R_{B}} \\
& +\mathbf{v}_{i R L F R_{B}}+\mathbf{v}_{i R R w F R_{B}}+\mathbf{v}_{i R L w F R_{B}}
\end{aligned}
$$

De igual manera, para el resto de las lineas, la velocidad inducida total queda:

$$
\begin{aligned}
\mathbf{v}_{i F L_{B}} & =\mathbf{v}_{i F R F L_{B}}+\mathbf{v}_{i F R w F L_{B}}+\mathbf{v}_{i F L w F L_{B}}+\mathbf{v}_{i R R F L_{B}} \\
& +\mathbf{v}_{i R L F L_{B}}+\mathbf{v}_{i R R w F L_{B}}+\mathbf{v}_{i R L w F L_{B}} \\
& \\
\mathbf{v}_{i R R_{B}} & =\mathbf{v}_{i F L R R_{B}}+\mathbf{v}_{i F R w R R_{B}}+\mathbf{v}_{i F L w R R_{B}}+\mathbf{v}_{i F R R R_{B}} \\
& +\mathbf{v}_{i R L R R_{B}}+\mathbf{v}_{i R R w R R_{B}}+\mathbf{v}_{i R L w R R_{B}} \\
& \\
\mathbf{v}_{i R L_{B}} & =\mathbf{v}_{i F L R L_{B}}+\mathbf{v}_{i F R w R L_{B}}+\mathbf{v}_{i F L w R L_{B}}+\mathbf{v}_{i F R R L_{B}} \\
& +\mathbf{v}_{i R R R L_{B}}+\mathbf{v}_{i R R w R L_{B}}+\mathbf{v}_{i R L w R L_{B}}
\end{aligned}
$$

Para calcular los valores de las velocidades inducidas es necesario obtener los valores de la circulación local, $\Gamma$ para el ala delantera y $\gamma$, para el ala trasera utilizando el teorema de Kutta-Joukowski [Katz and Plotkin 2001]. Cuando el valor de la circulación es conocida, el valor de las fuerzas aerodinámicas por unidad de longitud de envergadura se puede calcular utilizando la siguiente expresión:

$$
\mathrm{d} \mathbf{F}_{\mathbf{a} B}=\rho \Gamma \mathbf{V}_{B} \times \mathrm{d} \mathbf{l},
$$

siendo $\mathbf{V}_{B}$ el vector de la velocidad local en ejes cuerpo en cada punto del ala y definido por:

$$
\mathbf{V}_{B}=\left[\begin{array}{c}
u \\
0 \\
w
\end{array}\right]+\mathbf{v}_{i W_{B}}
$$

donde $\mathbf{v}_{i W_{B}}$ corresponde a la velocidad inducida de cada ala en ejes cuerpo.

De esta manera el módulo del vector velocidad en cualquier punto de la línea del ala delantera en ejes cuerpo queda:

$$
V_{F R_{B}}=\sqrt{\left(u+v_{i x F R_{B}}\right)^{2}+\left(w+v_{i z F R_{B}}\right)^{2}}
$$

De forma similar se procede con el resto de las alas

$$
V_{F L_{B}}=\sqrt{\left(u+v_{i x F L_{B}}\right)^{2}+\left(w+v_{i z F L_{B}}\right)^{2}}
$$




$$
\begin{aligned}
& V_{R R_{B}}=\sqrt{\left(u+v_{i x R R_{B}}\right)^{2}+\left(w+v_{i z R R_{B}}\right)^{2}}, \\
& V_{R L_{B}}=\sqrt{\left(u+v_{i x R L_{B}}\right)^{2}+\left(w+v_{i z R L_{B}}\right)^{2}},
\end{aligned}
$$

quedando la orientación del vector velocidad definida por el ángulo de ataque efectivo, $\alpha_{e}$, en cada sección, calculado como la diferencia entre el ángulo de ataque, $\alpha$, y el ángulo de ataque inducido, $\alpha_{i}$, de ala, en la forma:

$$
\begin{aligned}
& \alpha_{e F R}=\tan ^{-1}\left(\frac{w+v_{i z F R_{B}}}{u+v_{i x F R_{B}}}\right) \\
& \alpha_{e F L}=\tan ^{-1}\left(\frac{w+v_{i z F L_{B}}}{u+v_{i x F L_{B}}}\right) \\
& \alpha_{e R R}=\tan ^{-1}\left(\frac{w+v_{i z R R_{B}}}{u+v i x_{R R_{B}}}\right) \\
& \alpha_{e R L}=\tan ^{-1}\left(\frac{w+v_{i z R L_{B}}}{u+v_{i x R L_{B}}}\right),
\end{aligned}
$$

siendo $\alpha_{e F R}, \alpha_{e F L}, \alpha_{e R R}$ y $\alpha_{e R L}$ los ángulos de ataque efectivo para el ala delantera derecha, ala delantera izquierda, ala trasera derecha y ala trasera izquierda respectivamente.

Para determinar el valor de la circulación de cada ala y como consecuencia el valor de la velocidad inducida y el valor del ángulo de ataque efectivo, es necesario comparar el valor de la fuerzas aerodinámicas en dirección perpendicular a la corriente incidente libre con el valor de la sustentación local en cada sección del ala (ver Figura 5.5), estableciendo la igualdad entre ambas en la forma:

$$
\begin{aligned}
\frac{1}{2} \rho V_{F R_{W}}^{2}\left(y_{F R}\right) c\left(y_{F R}\right) c_{l}\left(\alpha_{e F R}, y_{F R}\right) \mathrm{d} y_{F R} & - \\
\rho \Gamma V_{F R_{W}}\left(y_{F R}\right) \cos \alpha_{i F R}\left(y_{F R}\right) \mathrm{d} y_{F R} & =0 \\
\frac{1}{2} \rho V_{F L_{W}}^{2}\left(y_{F L}\right) c\left(y_{F L}\right) c_{l}\left(\alpha_{e F L}, y_{F L}\right) \mathrm{d} y_{F L} & - \\
\rho \Gamma V_{F L_{W}}\left(y_{F L}\right) \cos \alpha_{i F L}\left(y_{F L}\right) \mathrm{d} y_{F L} & =0 \\
\frac{1}{2} \rho V_{R R_{W}}^{2}\left(y_{R R}\right) c\left(y_{R R}\right) c_{l}\left(\alpha_{e R R}, y_{R R}\right) \mathrm{d} y_{R R} & - \\
\rho \gamma V_{R R_{W}}\left(y_{R R}\right) \cos \alpha_{i R R}\left(y_{R R}\right) \mathrm{d} y_{R R} & =0 \\
\frac{1}{2} \rho V_{R L_{W}}^{2}\left(y_{R L}\right) c\left(y_{R L}\right) c_{l}\left(\alpha_{e R L}, y_{R L}\right) \mathrm{d} y_{R L} & - \\
\rho \gamma V_{R L_{W}}\left(y_{R L}\right) \cos \alpha_{i R L}\left(y_{R L}\right) \mathrm{d} y_{R L} & =0
\end{aligned}
$$

siendo $\alpha_{i F R}, \alpha_{i F L}, \alpha_{i R R}$ y $\alpha_{i R L}$, los ángulos de ataque inducidos para el ala delantera derecha, ala delantera izquierda, ala trasera derecha y ala trasera izquierda respectivamente $\mathrm{y}, c_{l}$, el valor del coeficiente de sustentación local en cada sección de ala en función del ángulo de ataque efectivo. 


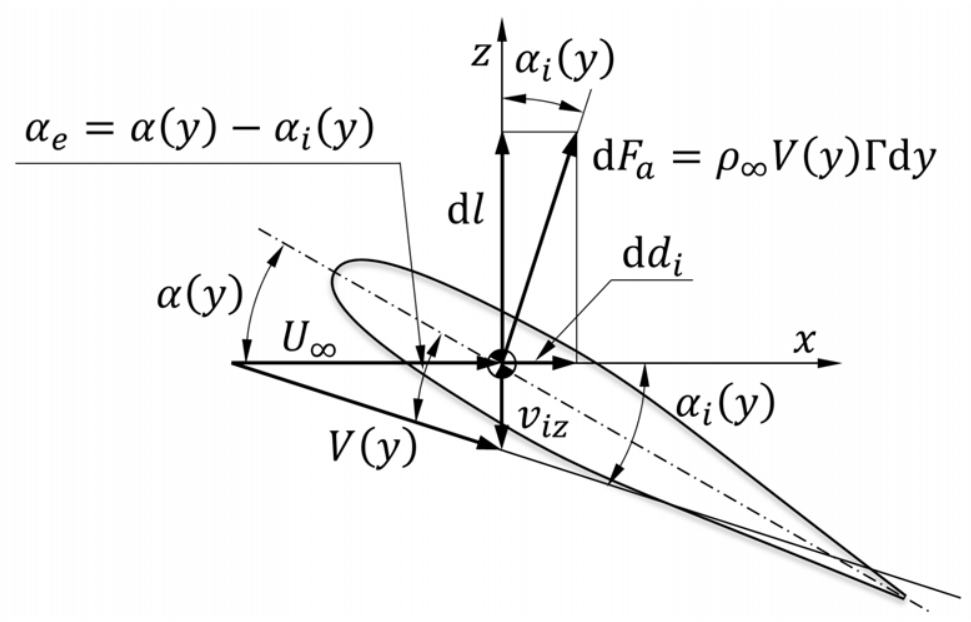

Figura 5.5: Esquema de fuerzas sobre cualquier sección arbitraria del ala.

De manera similar al punto anterior, para la realización de los cálculos, todos los parámetros relativos a valores de longitud, valores de velocidad y valores de circulación se han adimensionalizado usando como referencia el parámetro de la longitud no proyectada de la semiala delantera, $L_{D}$, y la velocidad de la corriente libre, $U_{\infty}$, quedando el resto de parámetros en la forma: $\overline{\mathbf{V}_{B}}=\mathbf{V}_{B} / U_{\infty}$, $G=\Gamma /\left(L_{D} U_{\infty}\right)$ y $g=\gamma /\left(L_{D} U_{\infty}\right)$.

De esta forma integrando las expresiones definidas por las ecuaciones (5.57), (5.58), (5.59) y (5.60), a lo largo de la envergadura adimensional del ala delantera y del ala trasera es posible, utilizando el método Newton-Raphson [Botero Arango et al. 2005; Powell 1968], resolver el sistema de ecuaciones no lineales para cada ala y obtener los valores de la circulación adimensional, $G$ y $g$, en función del ángulo de ataque y para cada configuración de estudio (ver Tabla 4.3).

Así, en la Figura 5.6 están representados los resultados del cálculo de los valores de la circulación adimensional del ala delantera, $G$, y del ala trasera, $g$, en función del ángulo de ataque, $\alpha$, para las configuraciones de ensayo $\mathrm{JWC}_{15}$ $\left(\varphi_{R}=-30^{\circ} \mathrm{y}, \delta_{R}=-25^{\circ}\right)$ y $\mathrm{JWC}_{51}\left(\varphi_{R}=-10^{\circ}\right.$ y $\left.\delta_{R}=-5^{\circ}\right)$.

De igual manera que en el capítulo anterior, para la presentación y análisis del efecto combinado del ángulo de flecha y del ángulo diedro del ala trasera, en la variación de la circulación, todos los valores adimensionales de, $G$ y $g$, de las diferentes configuraciones se representan normalizados con respecto a los valores de la configuración de referencia, $J W C_{15}$ como los valores, $\bar{G}=G / G_{15}$ y $\bar{g}=g / g_{15}$.

De esta forma y en primer lugar en la Figura 5.7 se representa la variación del valor de la circulación adimensional normalizada, $\bar{G}$, del ala delantera en función del ángulo de flecha del ala trasera, $\varphi_{R}$, y del ángulo diedro del ala trasera, $\delta_{R}$, para ángulos de ataque $\alpha=\left[2^{\circ}, 12^{\circ}\right]$, con incrementos de $2^{\circ}$. Como puede observarse, el valor de la circulación aumenta según se reduce la distancia entre el ala trasera y el ala delantera, es decir según disminuye el ángulo de flecha y en ángulo diedro del ala trasera en valor absoluto.

En segundo lugar, en la Figura 5.8 se representa la variación del valor de la circulación adimensional normalizada, $\bar{g}$, del ala trasera en función del ángulo de flecha del ala trasera, $\varphi_{R}$, y del ángulo diedro del ala trasera, $\delta_{R}$, para ángulos 

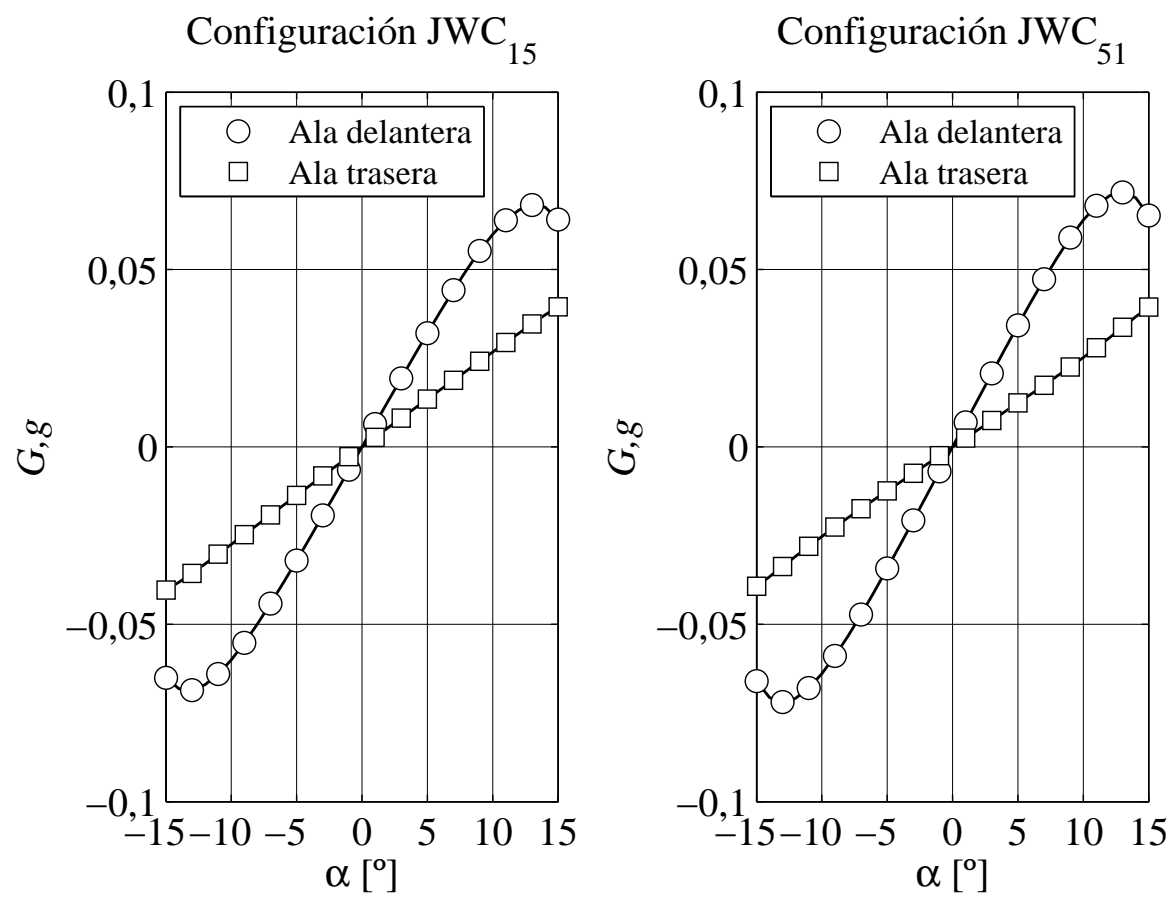

Figura 5.6: Valores de la circulación adimensional del ala delantera, $G$, y del ala trasera, $g$, en función del ángulo de ataque, $\alpha$, para las configuraciones de ensayo $\mathrm{JWC}_{15}\left(\varphi_{R}=-30^{\circ} \mathrm{y}, \delta_{R}=-25^{\circ}\right)$ y $\mathrm{JWC}_{51}\left(\varphi_{R}=-10^{\circ} \mathrm{y}, \delta_{R}=-5^{\circ}\right)$.

de ataque $\alpha=\left[2^{\circ}, 12^{\circ}\right.$, con incrementos de $2^{\circ}$. Como puede observarse, el valor de la circulación, en este caso, aumenta según aumenta la distancia longitudinal y disminuye la altura entre ambas alas.

Una vez determinado el valor de la circulación, es posible calcular los valores de las componentes de velocidad inducida para ambas alas. En la Figuras $5.9,5.10$ y 5.11 están representadas las componentes de la velocidad inducida adimensional a lo largo de la envergadura adimensional de ambas alas en ejes cuerpo, para las configuraciones $\mathrm{JWC}_{15}\left(\varphi_{R}=-30^{\circ} \mathrm{y}, \delta_{R}=-25^{\circ}\right)$ y $\mathrm{JWC}_{51}\left(\varphi_{R}=-10^{\circ} \mathrm{y}\right.$ , $\left.\delta_{R}=-5^{\circ}\right)$ y para ángulos de ataque $\alpha=5^{\circ}, \alpha=10^{\circ}$ y $\alpha=15^{\circ}$, respectivamente.

Como puede observarse, existe influencia del ala trasera sobre la delantera, que es significativa en el tramo de envergadura donde esta está presente, perturbando la velocidad inducida y modificando las tendencias de las curvas, especialmente en la componentes en dirección del eje $x, v_{i x}$ y en dirección del eje $z, v_{i z}$, además, como se puede comprobar, cuanto más cerca se encuentra el ala trasera del ala delantera, las perturbaciones sobre las velocidades inducidas, en la primera, son menores.

En efecto, esta perturbación existe y es más acentuada según aumenta el valor del ángulo de ataque $\alpha$, y es más significativa en la configuración de referencia $\mathrm{JWC}_{15}\left(\varphi_{R}=-30^{\circ} \mathrm{y}, \delta_{R}=-25^{\circ}\right)$. Comparando ambas configuraciones, el valor de las componente de la velocidad inducida en dirección del eje $z$ y del eje $x$ en el ala delantera, muestran valores mayores en la configuración $\mathrm{JWC}_{51}\left(\varphi_{R}=-10^{\circ} \mathrm{y}\right.$, $\left.\delta_{R}=-5^{\circ}\right)$. Este efecto es especialmente claro en la componente de la velocidad en dirección del eje $z$.

Por otro lado, también las componentes de la velocidad inducida en el ala trasera se ven influidas por la presencia del ala delantera, modificando sus valores, 

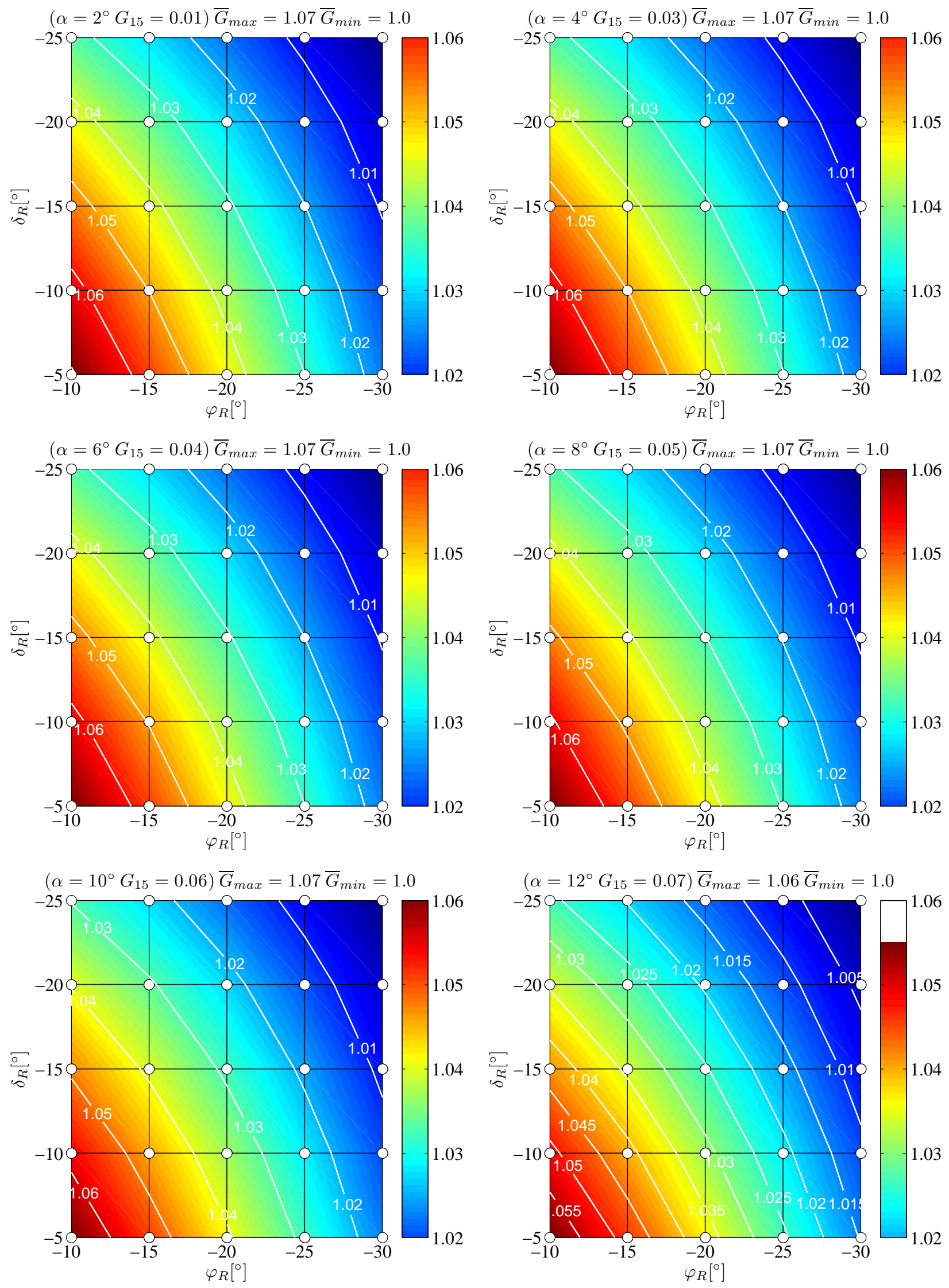

Figura 5.7: Variación del valor de la circulación adimensional normalizada, $\bar{G}$, en función del ángulo de flecha del ala trasera, $\varphi_{R}$, y del ángulo diedro del ala trasera,$\delta_{R}$, para cada ángulo de ataque, $\alpha$. 

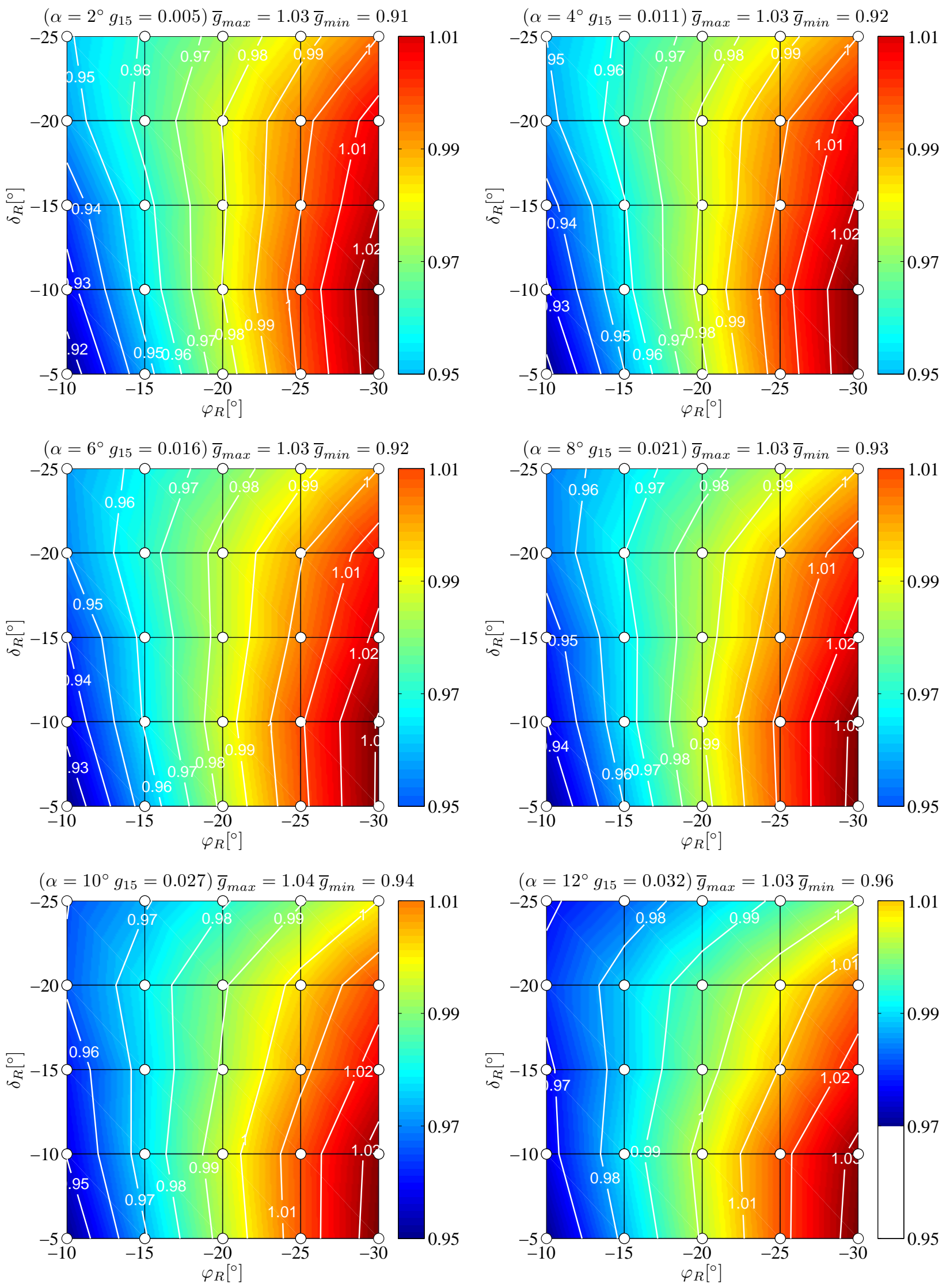

Figura 5.8: Variación del valor de la circulación adimensional normalizada, $\bar{g}$, en función del ángulo de flecha del ala trasera, $\varphi_{R}$, y del ángulo diedro del ala trasera,$\delta_{R}$, para cada ángulo de ataque, $\alpha$. 


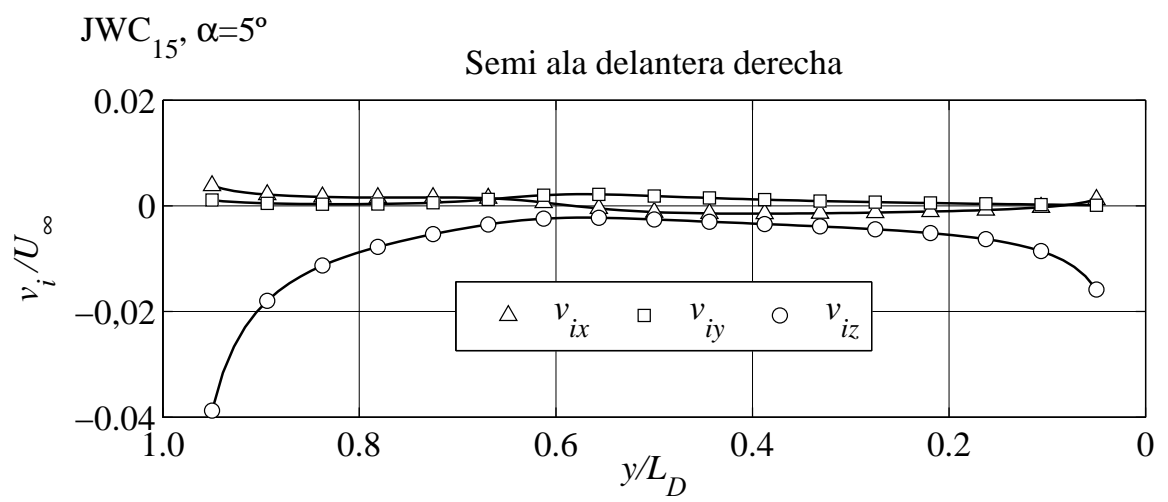

Semi ala trasera derecha
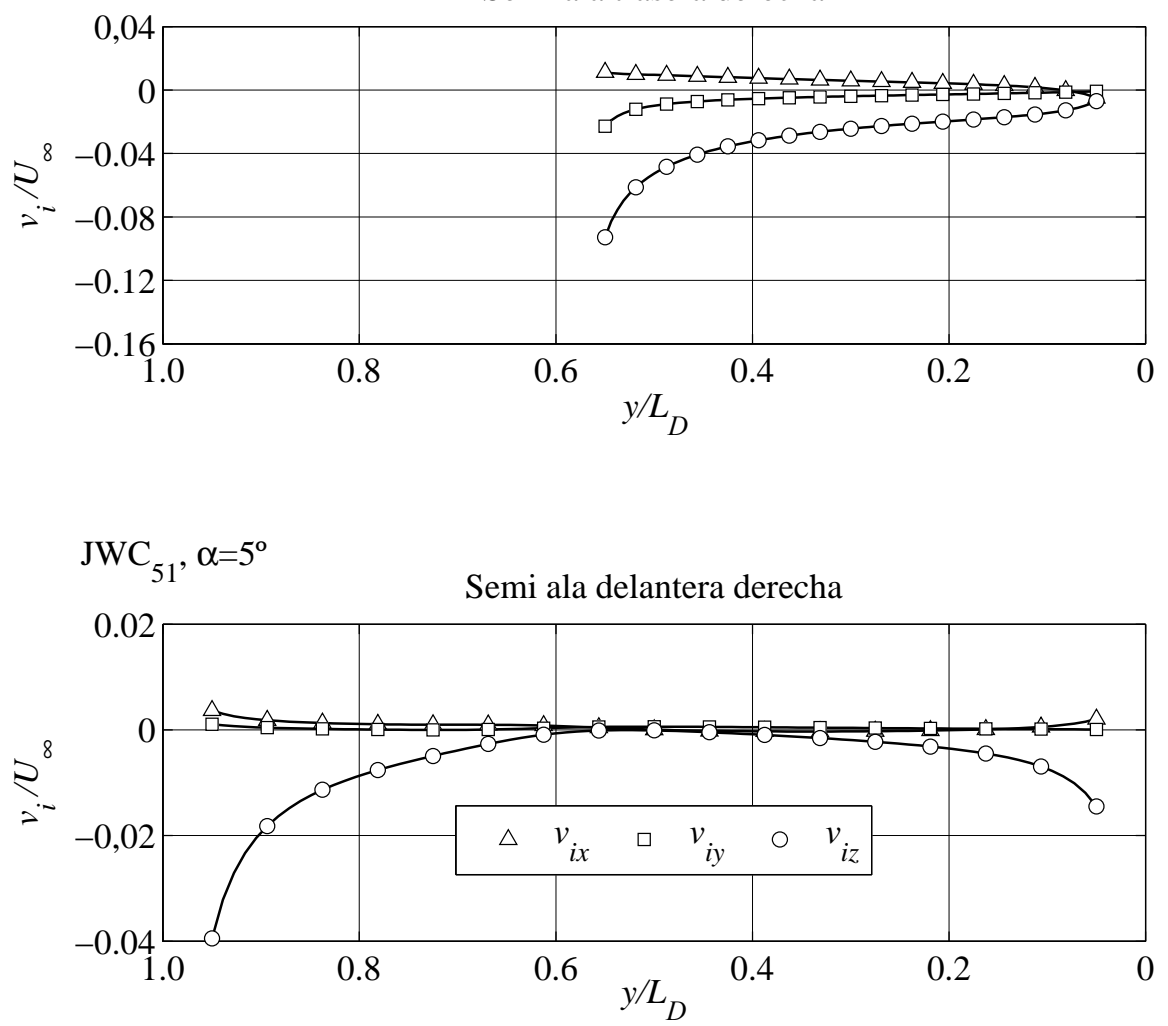

Semi ala trasera derecha

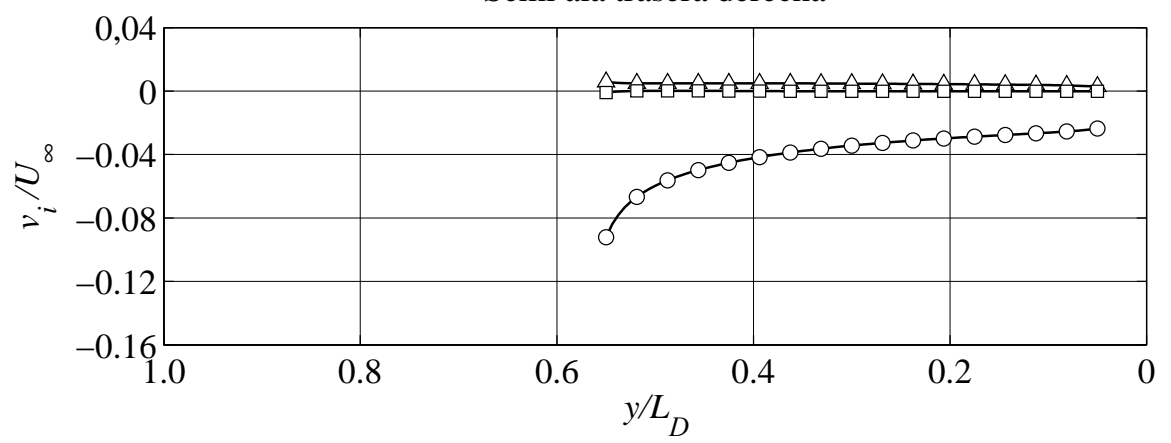

Figura 5.9: Valores de la componentes de la velocidad inducida adimensional en ejes cuerpo para las configuraciones de ensayo $\mathrm{JWC}_{15}\left(\varphi_{R}=-30^{\circ}\right.$ $\left.\mathrm{y}, \delta_{R}=-25^{\circ}\right)$ y $\mathrm{JWC}_{51}\left(\varphi_{R}=-10^{\circ} \mathrm{y}, \delta_{R}=-5^{\circ}\right)$, para un ángulo de ataque $\alpha=5^{\circ}$. 


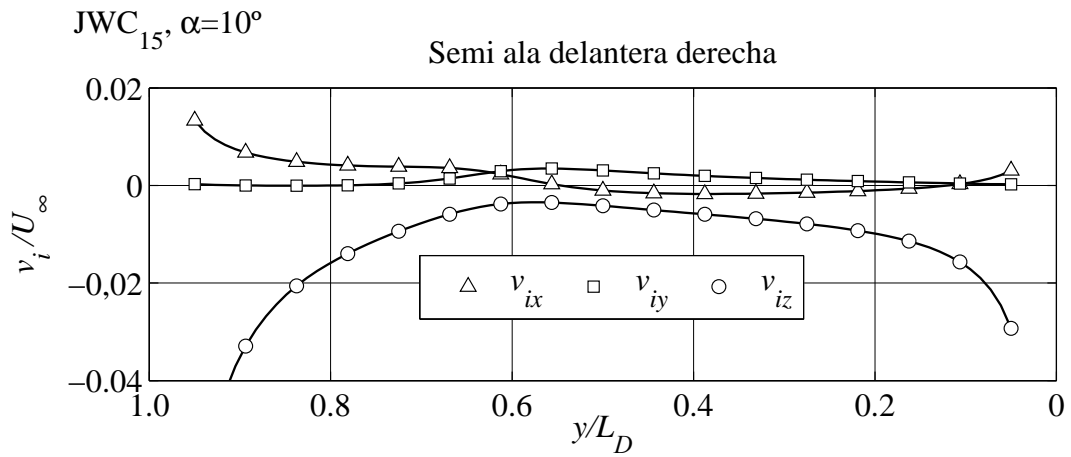

Semi ala trasera derecha

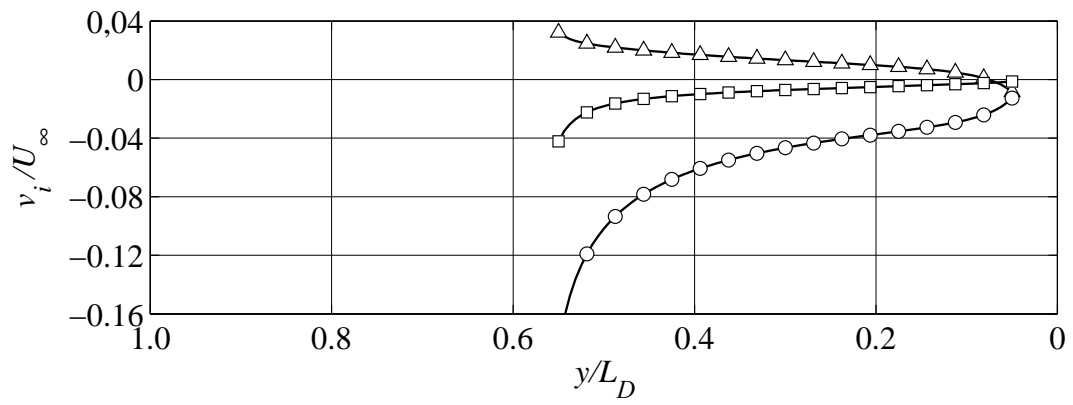

$\mathrm{JWC}_{51}, \alpha=10^{\circ}$

Semi ala delantera derecha

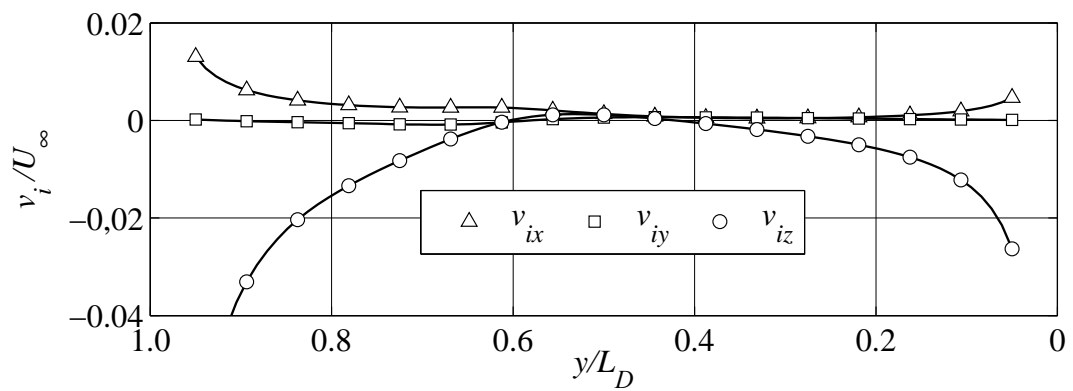

Semi ala trasera derecha

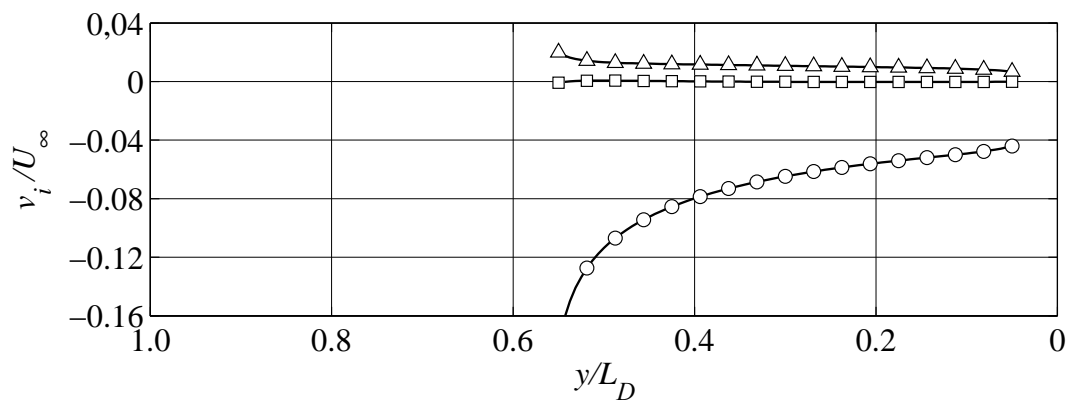

Figura 5.10: Valores de la componentes de la velocidad inducida adimensional en ejes cuerpo para las configuraciones de ensayo $\operatorname{JWC}_{15}\left(\varphi_{R}=\right.$ $\left.-30^{\circ} \mathrm{y}, \delta_{R}=-25^{\circ}\right)$ y $\mathrm{JWC}_{51}\left(\varphi_{R}=-10^{\circ} \mathrm{y}, \delta_{R}=-5^{\circ}\right)$, para un ángulo de ataque $\alpha=10^{\circ}$. 


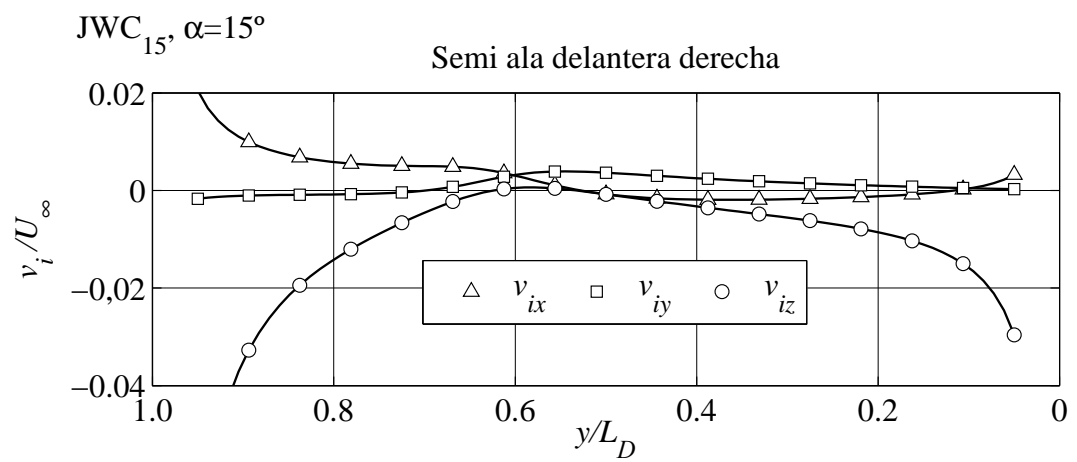

Semi ala trasera derecha

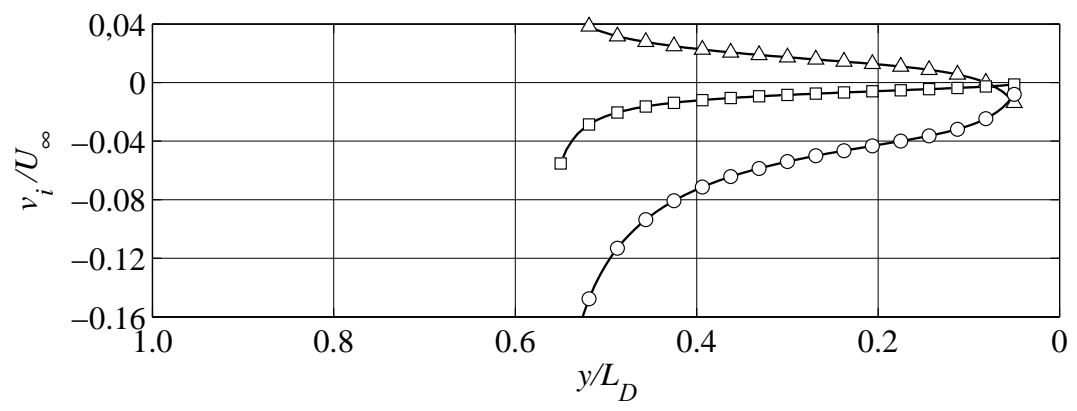

$\mathrm{JWC}_{51}, \alpha=15^{\circ}$

Semi ala delantera derecha

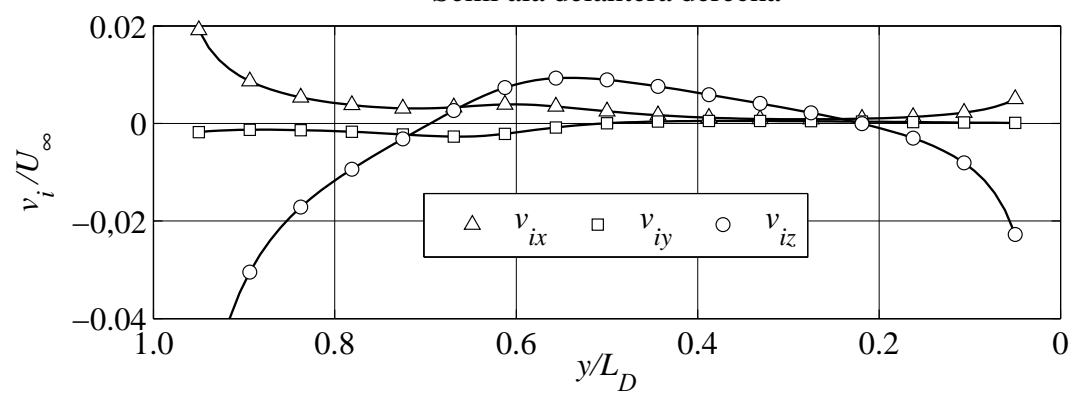

Semi ala trasera derecha

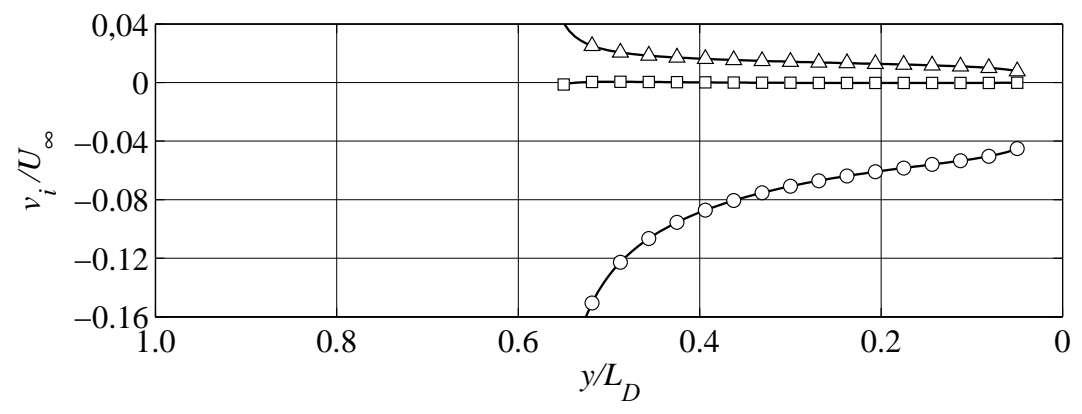

Figura 5.11: Valores de la componentes de la velocidad inducida adimensional en ejes cuerpo para las configuraciones de ensayo $\operatorname{JWC}_{15}\left(\varphi_{R}=\right.$ $\left.-30^{\circ} \mathrm{y}, \delta_{R}=-25^{\circ}\right)$ y $\mathrm{JWC}_{51}\left(\varphi_{R}=-10^{\circ} \mathrm{y}, \delta_{R}=-5^{\circ}\right)$, para un ángulo de ataque $\alpha=15^{\circ}$. 
que aumentan en función del ángulo de ataque en el caso de la configuración de referencia $\mathrm{JWC}_{15}\left(\varphi_{R}=-30^{\circ}\right.$ y $\left.\delta_{R}=-25^{\circ}\right)$.

Finalmente, determinados los valores de las componentes de la velocidad inducida en ambas alas es fácil calcular el ángulo de ataque efectivo resultante en cada sección del ala delantera y del ala trasera, donde este valor queda determinado por la diferencia entre el ángulo de ataque de la corriente libre, $\alpha$, y el ángulo de ataque inducido, $\alpha_{i}$, calculado mediante las ecuaciones (5.53), (5.54), (5.55) y (5.56).

De esta manera, en la Figuras 5.12, 5.13 y 5.14 están representados los valores del ángulo de ataque efectivo, $\alpha_{e}$, a lo largo de la envergadura adimensional de ambas alas para las configuraciones $\mathrm{JWC}_{15}\left(\varphi_{R}=-30^{\circ}\right.$ y $\left.\delta_{R}=-25^{\circ}\right)$ y $\mathrm{JWC}_{51}$ $\left(\varphi_{R}=-10^{\circ}\right.$ y $\left.\delta_{R}=-5^{\circ}\right)$ y para ángulos de ataque $\alpha=5^{\circ}, \alpha=10^{\circ}$ y $\alpha=15^{\circ}$, respectivamente.

De forma similar a lo que ocurre con las velocidades inducidas, la distribución del ángulo de ataque efectivo a lo largo de la envergadura del ala delantera se encuentra influido por la presencia del ala trasera disminuyendo su valor.

\subsubsection{Cálculo de las fuerzas aerodinámicas}

Una vez determinados los valores de la circulación y de las velocidades incidentes en cada sección del ala, tanto delantera como trasera, es sencillo determinar el valor de las componentes de las fuerzas aerodinámicas y como consecuencia el valor de las fuerzas de sustentación y de resistencia inducida.

El cálculo de las componentes de las fuerzas aerodinámicas en ejes ala se realiza proyectando el valor de las fuerzas de sustentación, $l$, y resistencia aerodinámica, $d$, local de cada sección del ala delantera y del ala trasera en las direcciones $x$ y $z$ en ejes ala. De esta manera para el caso del ala derecha delantera las componentes de las fuerzas aerodinámicas se calculan según:

$$
\begin{aligned}
& \mathrm{d} F a_{x F R}=\mathrm{d} F a_{x F R}(l)+\mathrm{d} F a_{x F R}(d), \\
& \mathrm{d} F a_{z F R}=\mathrm{d} F a_{z F R}(l)+\mathrm{d} F a_{z F R}(d),
\end{aligned}
$$

donde $\mathrm{d} F a_{x F R}(l)$ y $\mathrm{d} F a_{z F R}(l)$ son las componentes de las fuerzas aerodinámicas derivadas de la sustentación local y definidas en la forma:

$$
\begin{gathered}
\mathrm{d} F a_{x F R}(l)=\frac{1}{2} \rho V_{F R_{W}}^{2}\left(y_{F R}\right) c\left(y_{F R}\right) c_{l}\left(\alpha_{e F R}, y_{F R}\right)\left(-\sin \alpha\left(y_{F R}\right)\right) \mathrm{d} y_{F R}, \\
\mathrm{~d} F a_{z F R}(l)=\frac{1}{2} \rho V_{F R_{W}}^{2}\left(y_{F R}\right) c\left(y_{F R}\right) c_{l}\left(\alpha_{e F R}, y_{F R}\right) \cos \alpha\left(y_{F R}\right) \mathrm{d} y_{F R}
\end{gathered}
$$

y $\mathrm{d} F a_{x F R}(d)$ y $\mathrm{d} F a_{z F R}(d)$ son las componentes de las fuerzas aerodinámicas derivadas de la resistencia local y definidas en la forma: 


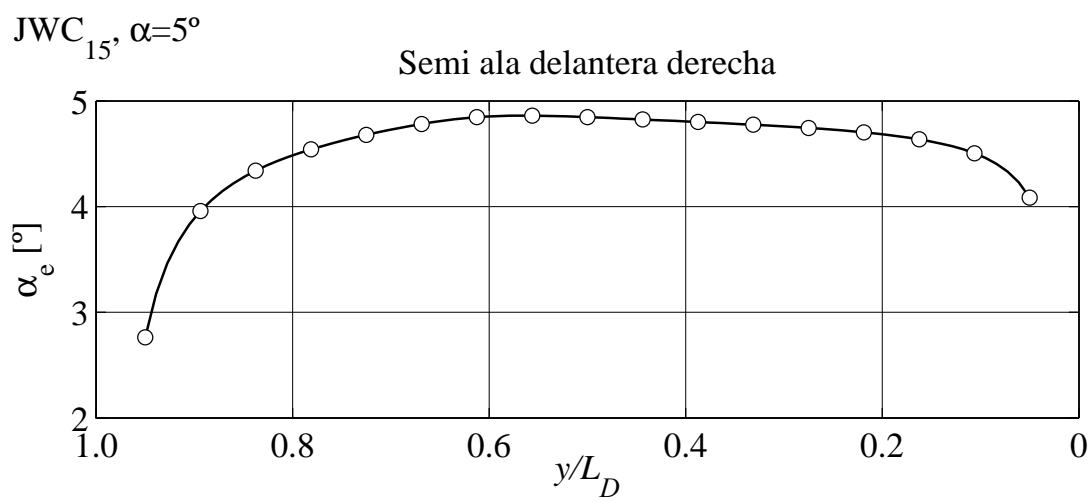

Semi ala trasera derecha

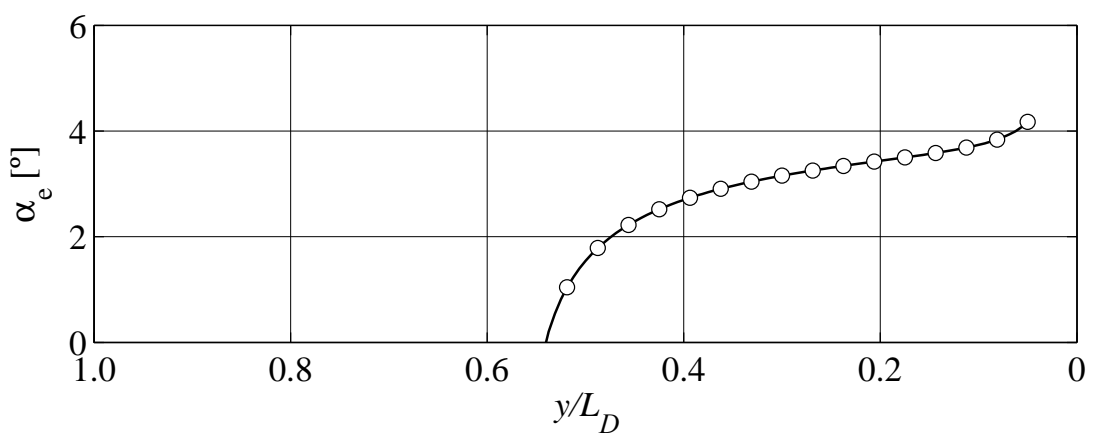

$\mathrm{JWC}_{51}, \alpha=5^{\mathrm{o}}$

Semi ala delantera derecha

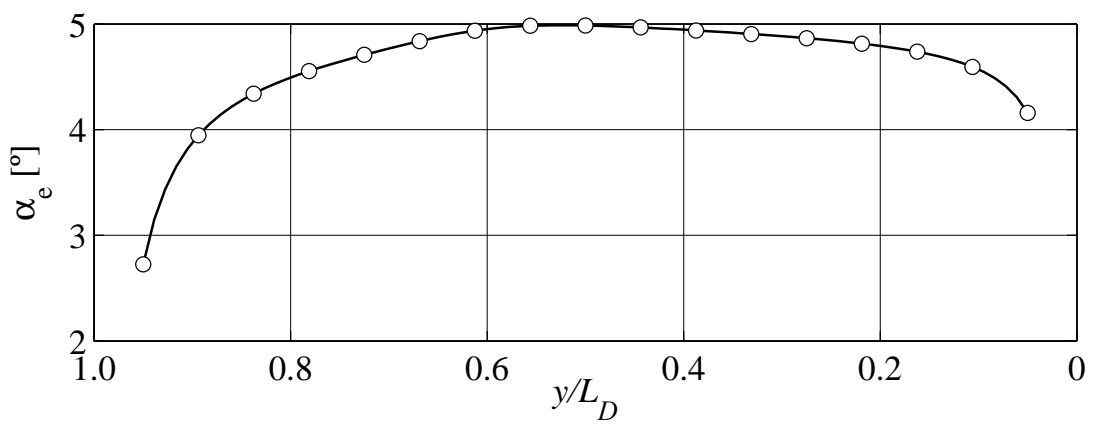

Semi ala trasera derecha

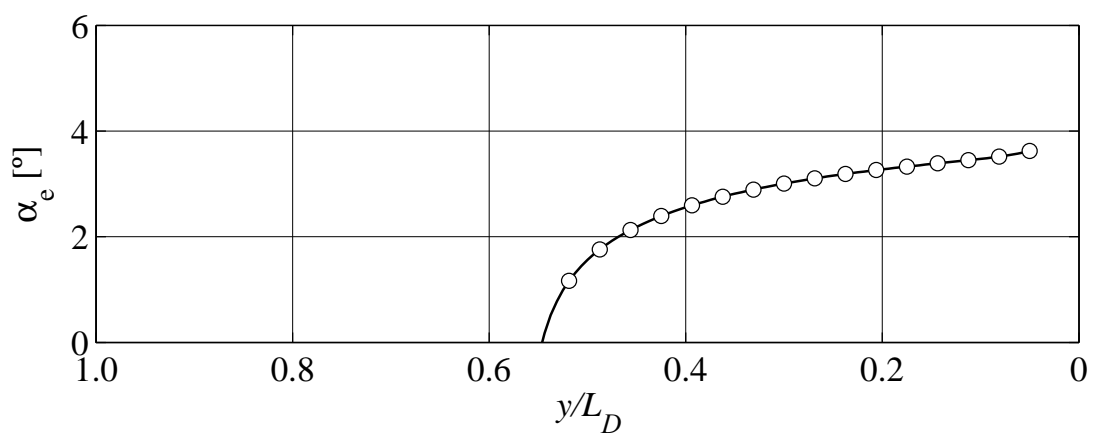

Figura 5.12: Valores del ángulo de ataque efectivo, $\alpha_{e}$, para las configuraciones de ensayo $\mathrm{JWC}_{15}\left(\varphi_{R}=-30^{\circ}\right.$ y $\left.\delta_{R}=-25^{\circ}\right)$ y $\mathrm{JWC}_{51}\left(\varphi_{R}=-10^{\circ}\right.$ y $\delta_{R}=-5^{\circ}$ ), para un ángulo de ataque $\alpha=5^{\circ}$. 


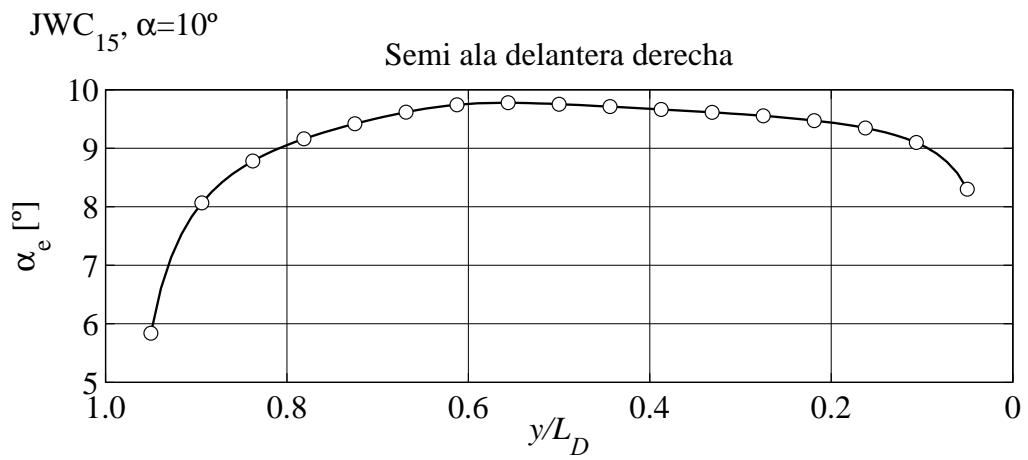

Semi ala trasera derecha

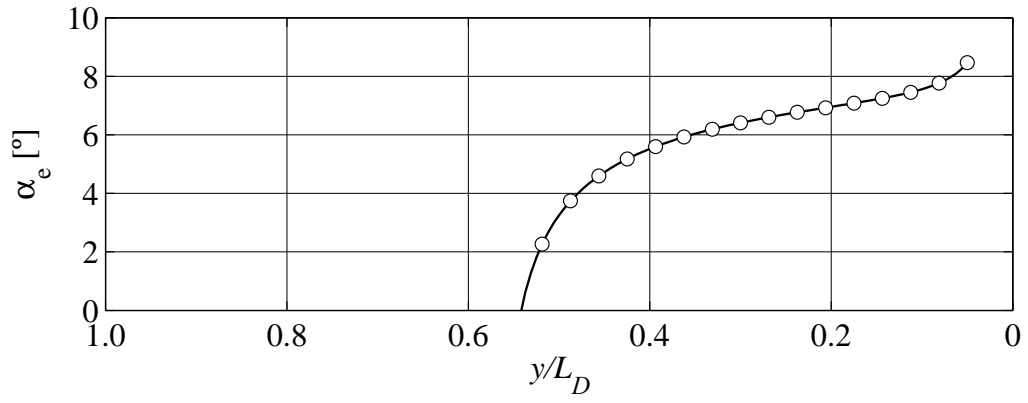

$\mathrm{JWC}_{51}, \alpha=10^{\circ}$

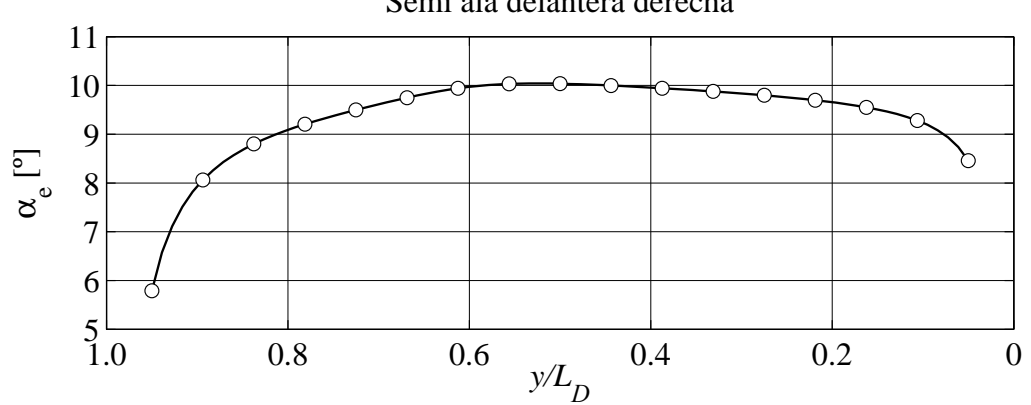

Semi ala trasera derecha

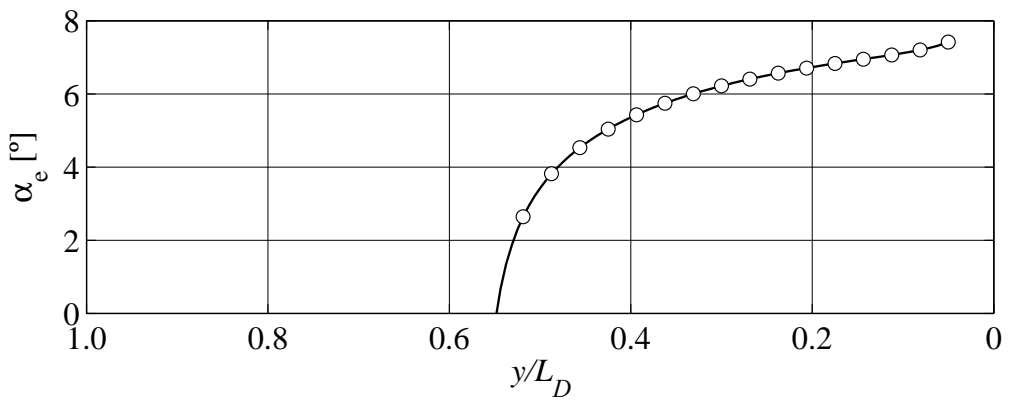

Figura 5.13: Valores del ángulo de ataque efectivo, $\alpha_{e}$, para las configuraciones de ensayo $\mathrm{JWC}_{15}\left(\varphi_{R}=-30^{\circ}\right.$ y $\left.\delta_{R}=-25^{\circ}\right)$ y $\operatorname{JWC}_{51}\left(\varphi_{R}=-10^{\circ}\right.$ y $\left.\delta_{R}=-5^{\circ}\right)$, para un ángulo de ataque $\alpha=10^{\circ}$. 


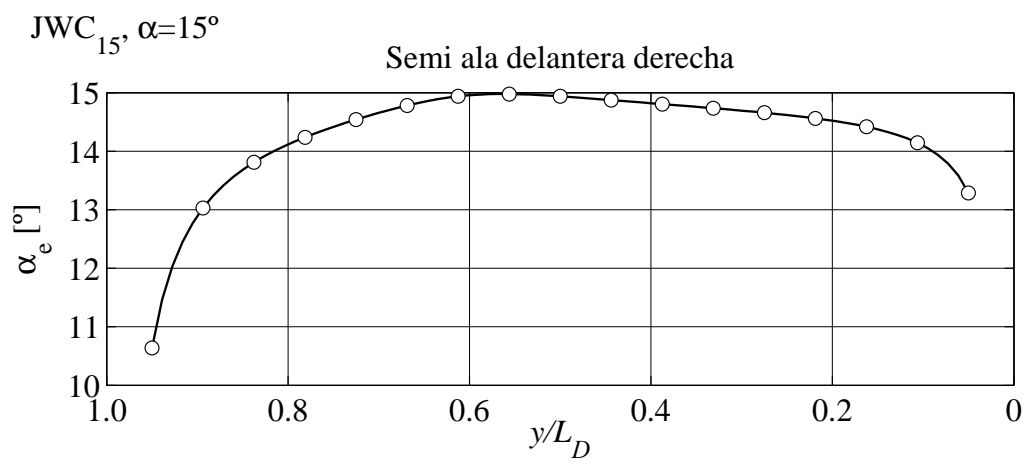

Semi ala trasera derecha
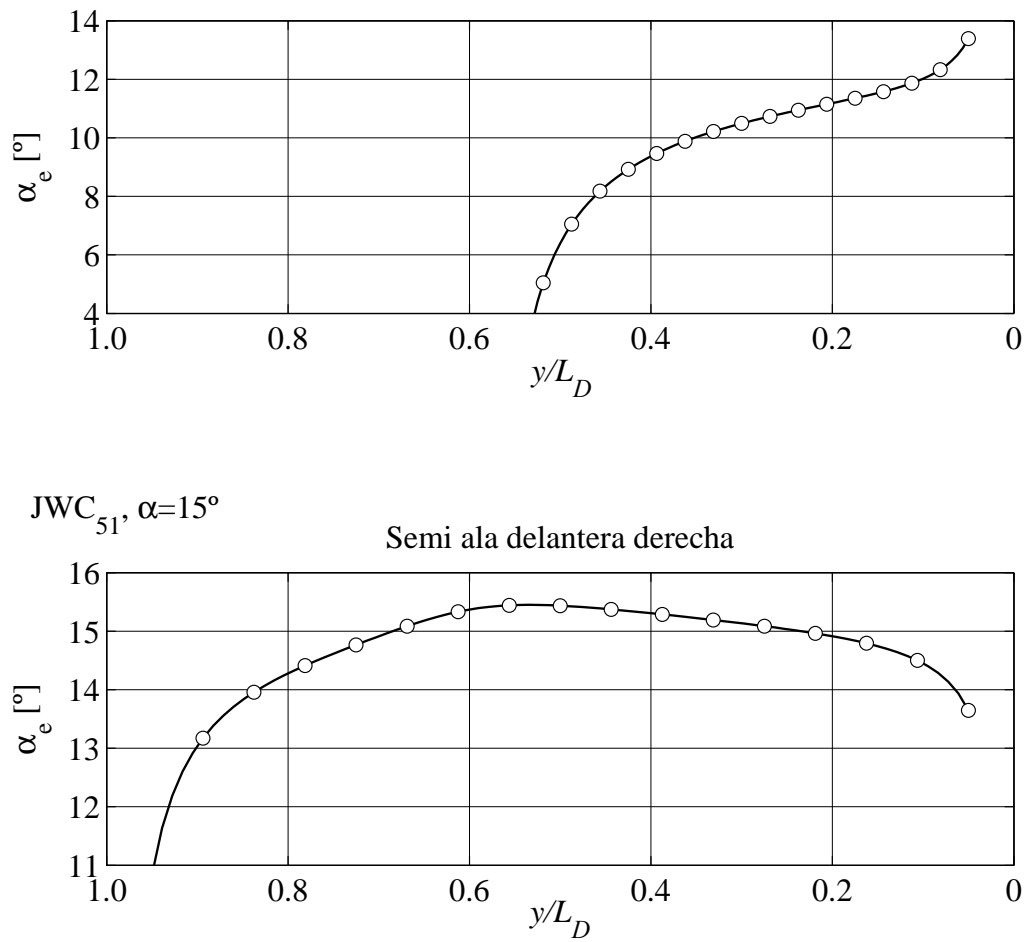

Semi ala trasera derecha

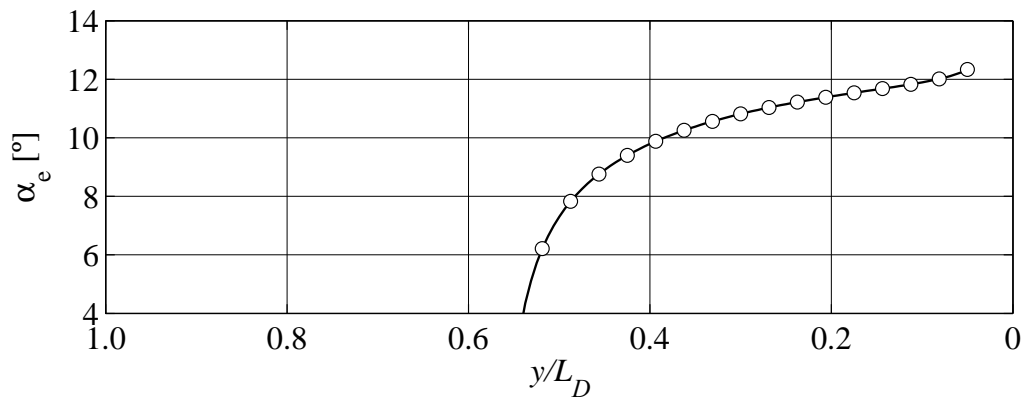

Figura 5.14: Valores del ángulo de ataque efectivo, $\alpha_{e}$, para las configuraciones de ensayo $\mathrm{JWC}_{15}\left(\varphi_{R}=-30^{\circ}\right.$ y $\left.\delta_{R}=-25^{\circ}\right)$ y $\mathrm{JWC}_{51}\left(\varphi_{R}=-10^{\circ}\right.$ y $\left.\delta_{R}=-5^{\circ}\right)$, para un ángulo de ataque $\alpha=15^{\circ}$. 


$$
\begin{aligned}
& \mathrm{d} F a_{x F R}(d)=\frac{1}{2} \rho V_{F R_{W}}^{2}\left(y_{F R}\right) c\left(y_{F R}\right) c_{d}\left(\alpha_{e F R}, y_{F R}\right) \cos \alpha\left(y_{F R}\right) \mathrm{d} y_{F R}, \\
& \mathrm{~d} F a_{z F R}(d)=\frac{1}{2} \rho V_{F R_{W}}^{2}\left(y_{F R}\right) c\left(y_{F R}\right) c_{d}\left(\alpha_{e F R}, y_{F R}\right) \sin \alpha\left(y_{F R}\right) \mathrm{d} y_{F R},
\end{aligned}
$$

De forma similar se procede para el cálculo de las fuerzas aerodinámicas para el resto de alas.

Para la realización de los cálculos, todos los parámetros relativos a valores de fuerza se han adimensionalizado usando como referencia el parámetro de la longitud no proyectada de la semiala delantera, $L_{D}$, la velocidad de la corriente libre, $U_{\infty}$, y la densidad relativa, $\rho$, quedando en la forma: $\overline{F_{a}}=F_{a} / 1 / 2 \rho U_{\infty}^{2} L_{D}^{2}$.

De esta manera, en la Figuras 5.15, 5.16 y 5.17 están representados los valores de las fuerzas aerodinámicas adimensionales por unidad de longitud proyectadas sobre ejes cuerpo, $\overline{\mathrm{d} F_{a}}$, a lo largo de la envergadura adimensional de ambas alas, para las configuraciones $\mathrm{JWC}_{15}\left(\varphi_{R}=-30^{\circ}\right.$ y $\left.\delta_{R}=-25^{\circ}\right)$ y $\mathrm{JWC}_{51}$ $\left(\varphi_{R}=-10^{\circ}\right.$ y $\left.\delta_{R}=-5^{\circ}\right)$ y para ángulos de ataque $\alpha=5^{\circ}, \alpha=10^{\circ}$ y $\alpha=15^{\circ}$, respectivamente.

Como puede observarse en las gráficas, la componente de las fuerzas aerodinámicas en dirección del eje $z$ en el ala delantera no experimenta cambios significativos independientemente de la configuración de ensayo, sin embargo, esta misma componente de la fuerza en el ala trasera si presenta cambios haciendo que aumente para el caso del modelo de ensayo $\operatorname{JWC}_{51}\left(\varphi_{R}=-10^{\circ}\right.$ y $\left.\delta_{R}=-5^{\circ}\right)$, es decir, según disminuye el ángulo de flecha y el ángulo diedro del ala trasera en valor absoluto. De forma similar este suceso se repite para la componente de la fuerza según la dirección del eje $x$.

Calculadas las fuerzas aerodinámicas es posible determinar los coeficientes de sustentación, $\widehat{C_{L}}$, de resistencia inducida, $\widehat{C_{D i}}$, y de resistencia, $\widehat{C_{D}}$, de cada configuración de ensayo pero adimensionalizados con respecto a la longitud no proyectada del ala delantera. A tal efecto sólo es necesario integrar las fuerzas a lo largo de la envergadura del ala delantera y del ala trasera, descomponerlas en las direcciones de la corriente incidente y perpendicular a ella en cada ala y proyectarlas en ejes cuerpo. De esta forma las fuerzas producidas por el ala delantera derecha son:

$$
\begin{gathered}
\widehat{C_{L F R}}=\overline{F a_{z F R}}(l) \cos \alpha-\overline{F a_{x F R}}(l) \sin \alpha-\overline{F a_{x F R}}(d) \sin \alpha+\overline{F a_{z F R}}(d) \cos \alpha \\
\widehat{C_{D i F R}}=\overline{F a_{z F R}}(l) \sin \alpha-\overline{F a_{x F R}}(l) \cos \alpha \\
\widehat{C_{D F R}}=\overline{F a_{x F R}}(d) \cos \alpha+\overline{F a_{z F R}}(d) \sin \alpha
\end{gathered}
$$




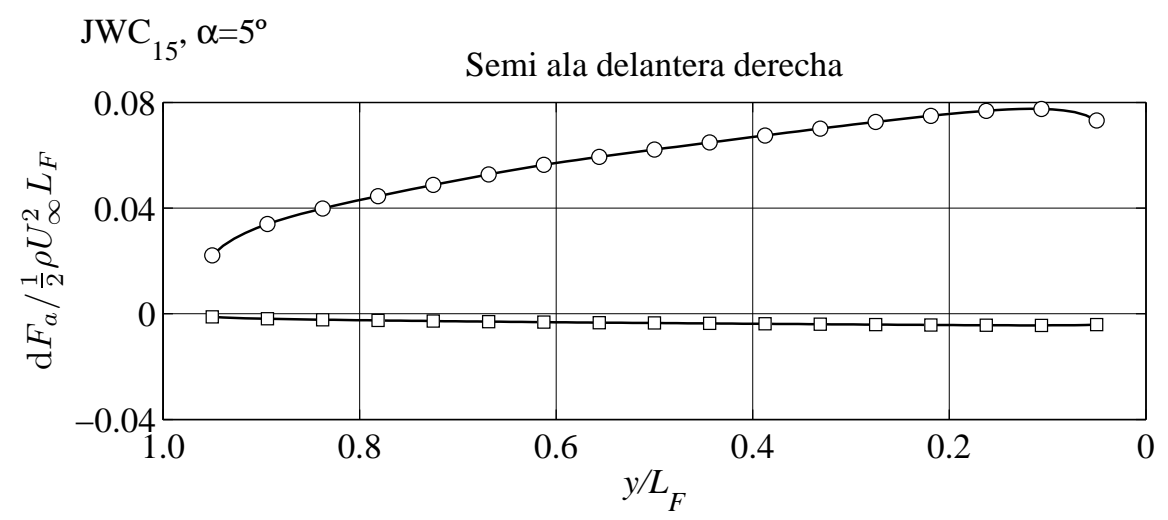

Semi ala trasera derecha
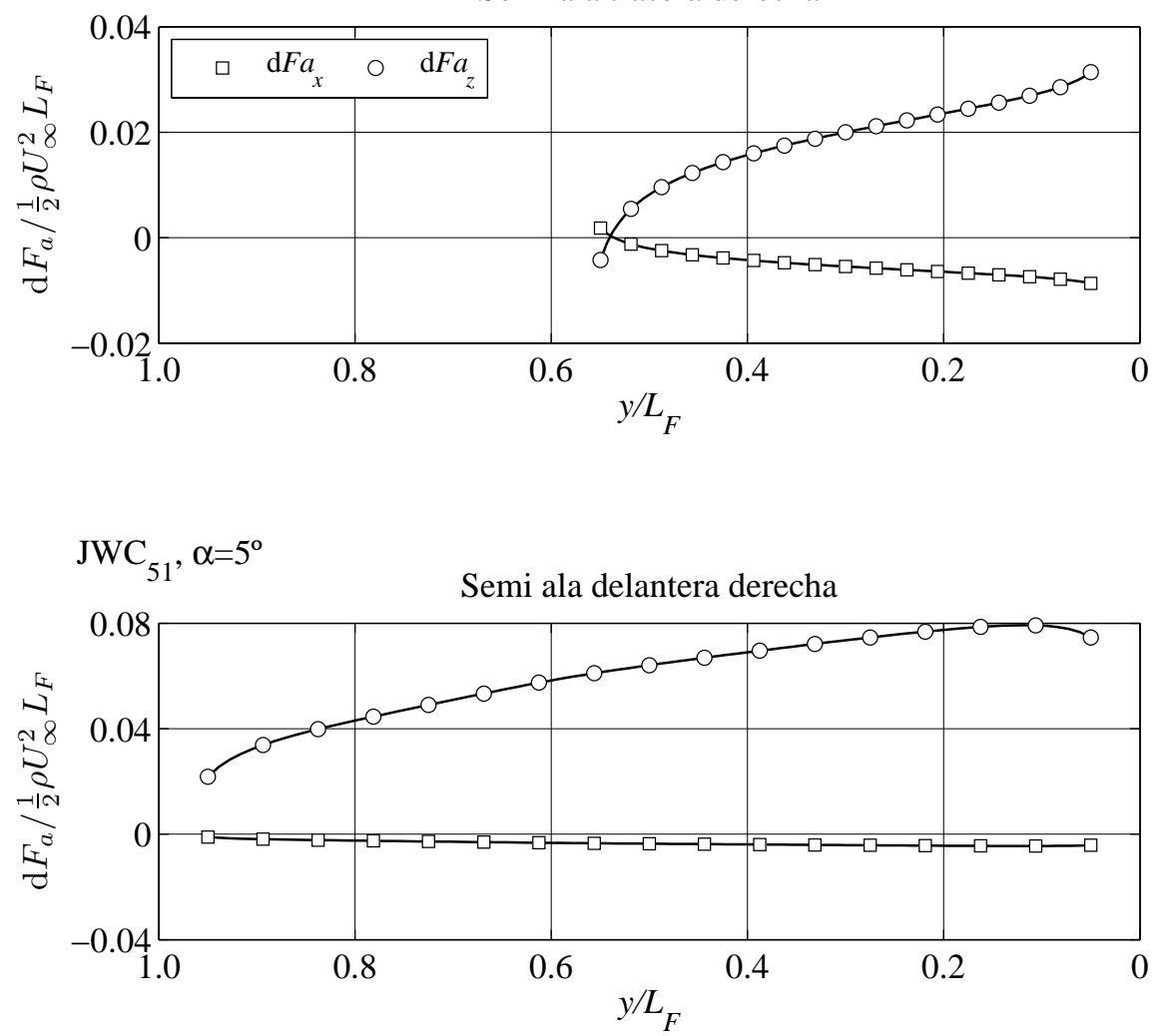

Semi ala trasera derecha

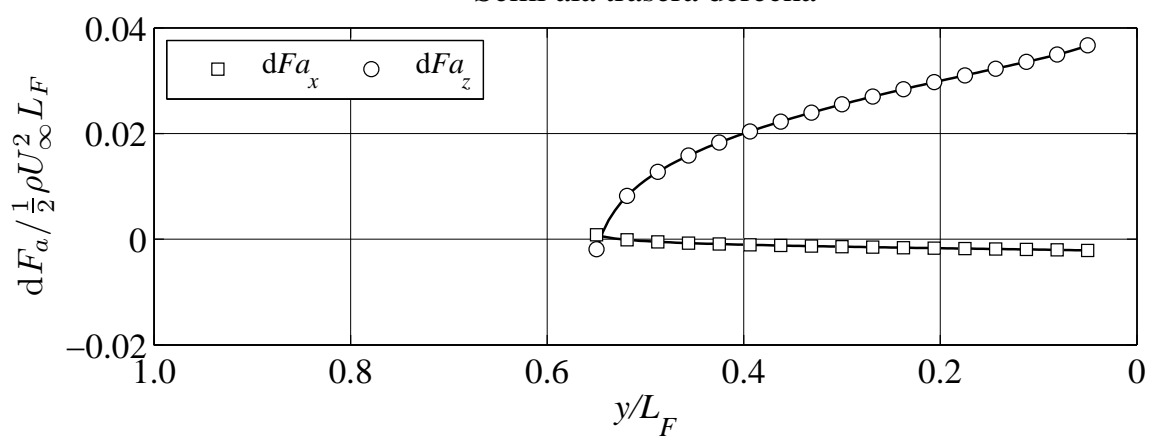

Figura 5.15: Valores de las componentes en dirección del eje $x$ y $z$ de las fuerzas aerodinámicas adimensionales proyectadas sobre ejes cuerpo, $\overline{F_{a}}$, para las configuraciones de ensayo $\operatorname{JWC}_{15}\left(\varphi_{R}=-30^{\circ} \mathrm{y}\right.$, $\left.\delta_{R}=-25^{\circ}\right)$ y $\mathrm{JWC}_{51}\left(\varphi_{R}=-10^{\circ}\right.$ y $\left.\delta_{R}=-5^{\circ}\right)$, para un ángulo de ataque $\alpha=5^{\circ}$. 


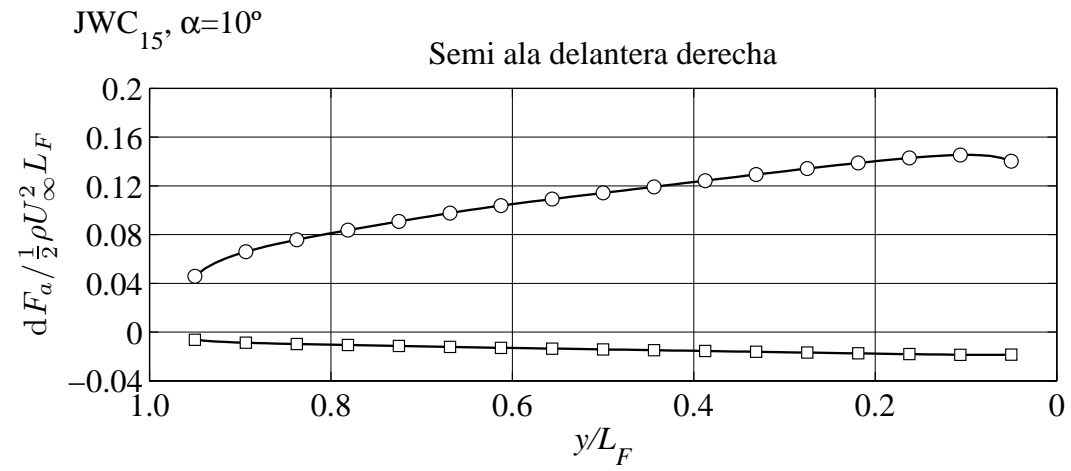

Semi ala trasera derecha
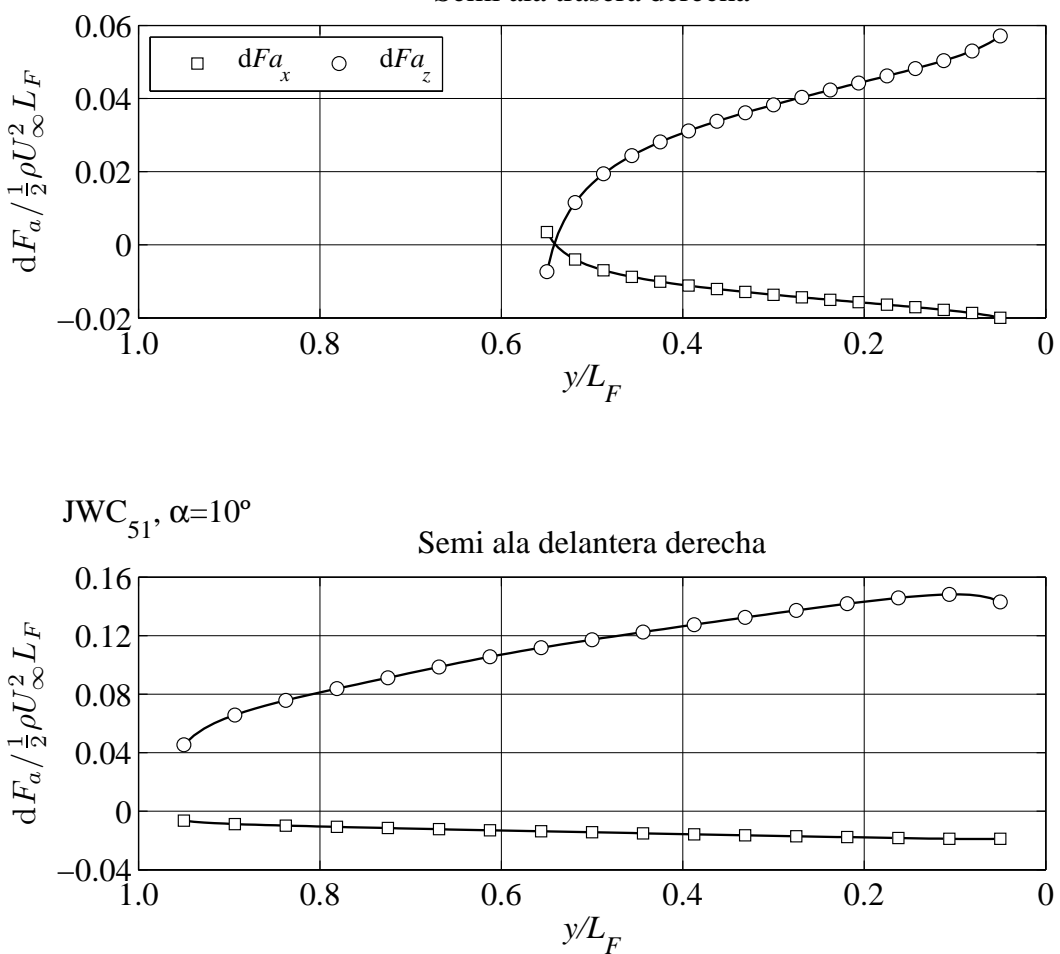

Semi ala trasera derecha

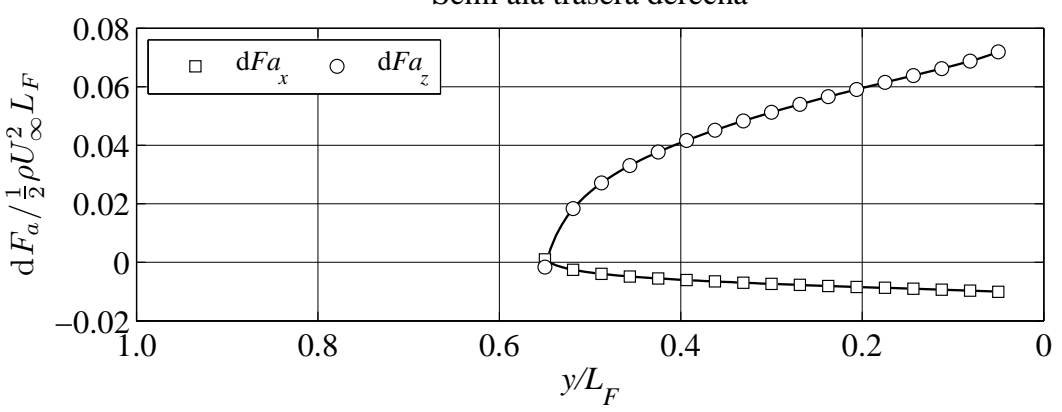

Figura 5.16: Valores de las componentes en dirección del eje $x$ y $z$ de las fuerzas aerodinámicas adimensionales proyectadas sobre ejes cuerpo, $\overline{F_{a}}$, para las configuraciones de ensayo $\operatorname{JWC}_{15}\left(\varphi_{R}=-30^{\circ} \mathrm{y}\right.$, $\left.\delta_{R}=-25^{\circ}\right)$ y $\mathrm{JWC}_{51}\left(\varphi_{R}=-10^{\circ}\right.$ y $\left.\delta_{R}=-5^{\circ}\right)$, para un ángulo de ataque $\alpha=10^{\circ}$. 


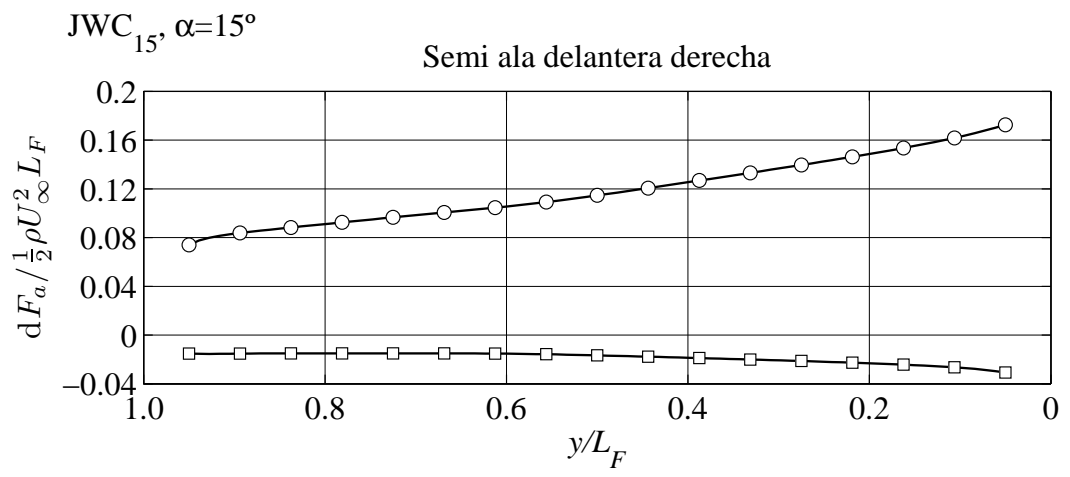

Semi ala trasera derecha
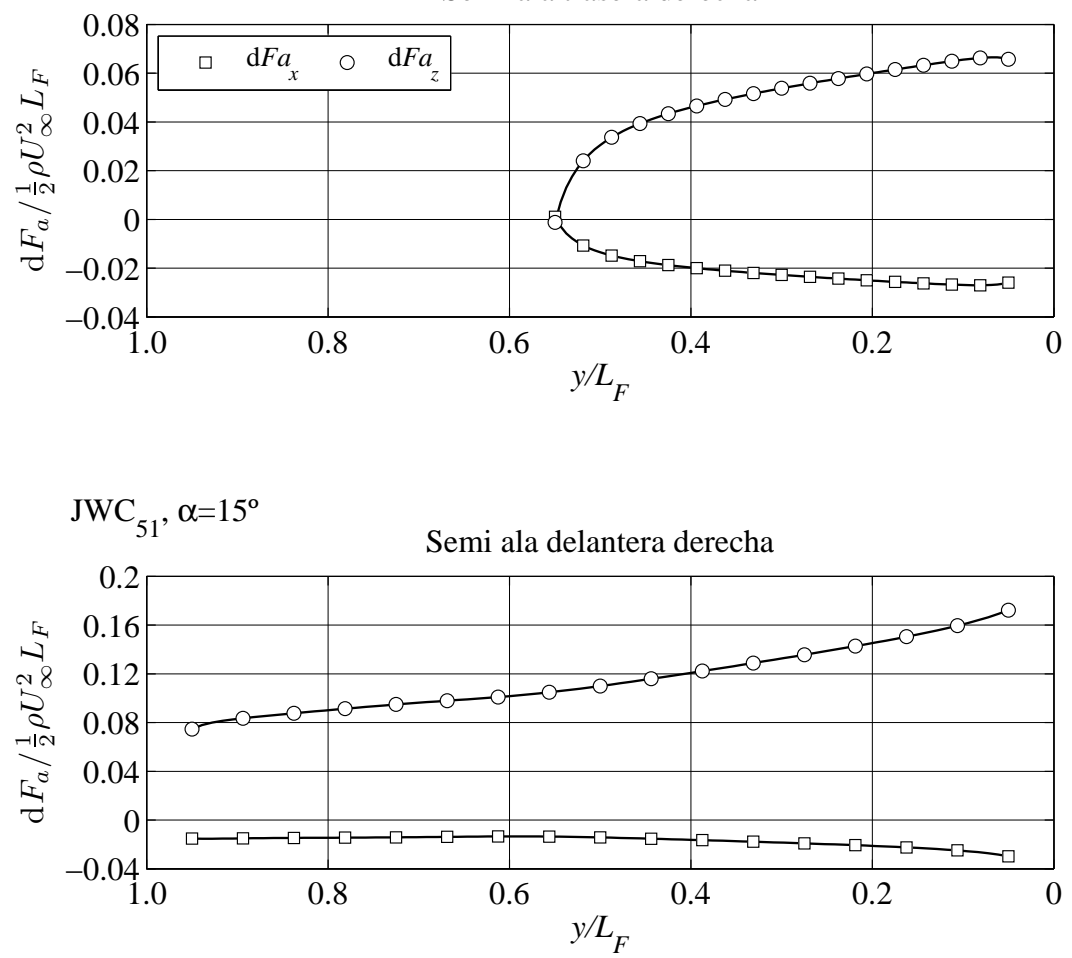

Semi ala trasera derecha

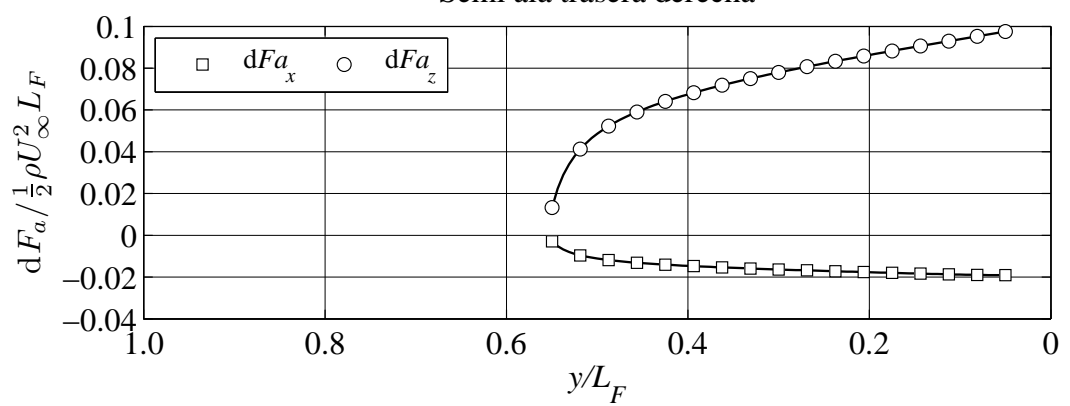

Figura 5.17: Valores de las componentes en dirección del eje $x$ y $z$ de las fuerzas aerodinámicas adimensionales proyectadas sobre ejes cuerpo, $\overline{F_{a}}$, para las configuraciones de ensayo $\operatorname{JWC}_{15}\left(\varphi_{R}=-30^{\circ} \mathrm{y}\right.$, $\left.\delta_{R}=-25^{\circ}\right)$ y $\operatorname{JWC}_{51}\left(\varphi_{R}=-10^{\circ}\right.$ y $\left.\delta_{R}=-5^{\circ}\right)$, para un ángulo de ataque $\alpha=15^{\circ}$. 
De forma similar se calculan los coeficientes del resto de alas. Una vez determinados es posible cálcular los coeficientes totales del sistema como la suma de los coeficientes de cada ala, respectivamente.

Una vez determinados estos valores es posible calcular los coeficientes aerodinámicos, en su forma conocida, teniendo en cuenta que los valores adimensionales han de ser acordes con los característicos en en este tipo de parámetros aerodinámicos, como:

$$
\begin{gathered}
C_{L}=\left(\widehat{C_{L F R}}+\widehat{C_{L F L}}+\widehat{C_{L R R}}+\widehat{C_{L R L}}\right) \frac{L_{F}^{2}}{b \bar{c}} \\
C_{D i}=\left(\widehat{C_{D i F R}}+\widehat{C_{D i F L}}+\widehat{C_{D i R R}}+\widehat{C_{D i R L}}\right) \frac{L_{F}^{2}}{b \bar{c}} \\
C_{D}=\left(\widehat{C_{D F R}}+\widehat{C_{D F L}}+\widehat{C_{D R R}}+\widehat{C_{D R L}}\right) \frac{L_{F}^{2}}{b \bar{c}},
\end{gathered}
$$

donde, $b$, es la envergadura efectiva [Wolkovitch 1986] y, $\bar{c}$, es la cuerda media aerodinámica efectiva del conjunto ala delantera y ala trasera, definidas en (4.8) y en (4.10) respectivamente.

Así, en la Figura 5.18 están representados los valores calculados con el modelo numérico de los coeficientes de sustentación, $C_{L}$, resistencia inducida, $C_{D i}$, resistencia, $C_{D}$, y eficiencia aerodinámica, $E$, en función del ángulo de ataque, $\alpha$, para la configuración de referencia de ensayo $\mathrm{JWC}_{15}\left(\varphi_{R}=-30^{\circ} \mathrm{y}, \delta_{R}=-25^{\circ}\right)$.

\subsubsection{Comparación de resultados numéricos y experimentales}

Calculados los coeficientes aerodinámicos característicos es posible comparar estos resultados con los obtenidos en el capítulo 4, de esta manera en la Figura 5.19 están representados la comparación entre el modelo numérico y el experimental de los valores de los coeficientes de sustentación, $C_{L}$, resistencia inducida, $C_{D i}$, resistencia, $C_{D}$, y eficiencia aerodinámica, $E$, en función del ángulo de ataque, $\alpha$, para valores del ángulo diedro, $\delta_{R}=-25^{\circ}$, del ángulo de flecha, $\varphi_{R}=-30^{\circ}$, del ala trasera.

Como puede observarse en lo datos mostrados en la gráfica los resultados numéricos se ajustan satisfactoriamente a los valores de los resultados experimentales y lo que es más importante, reproducen la misma tendencia que presentan los resultados experimentales al variar la configuración de ensayo (véase apéndice E).

A la vista de los resultados, se puede afirmar, que este modelo sencillo en su definición (y rápido de ejecución) puede ser una herramienta útil en las fases iniciales de un proyecto de aeronave de estas características. 

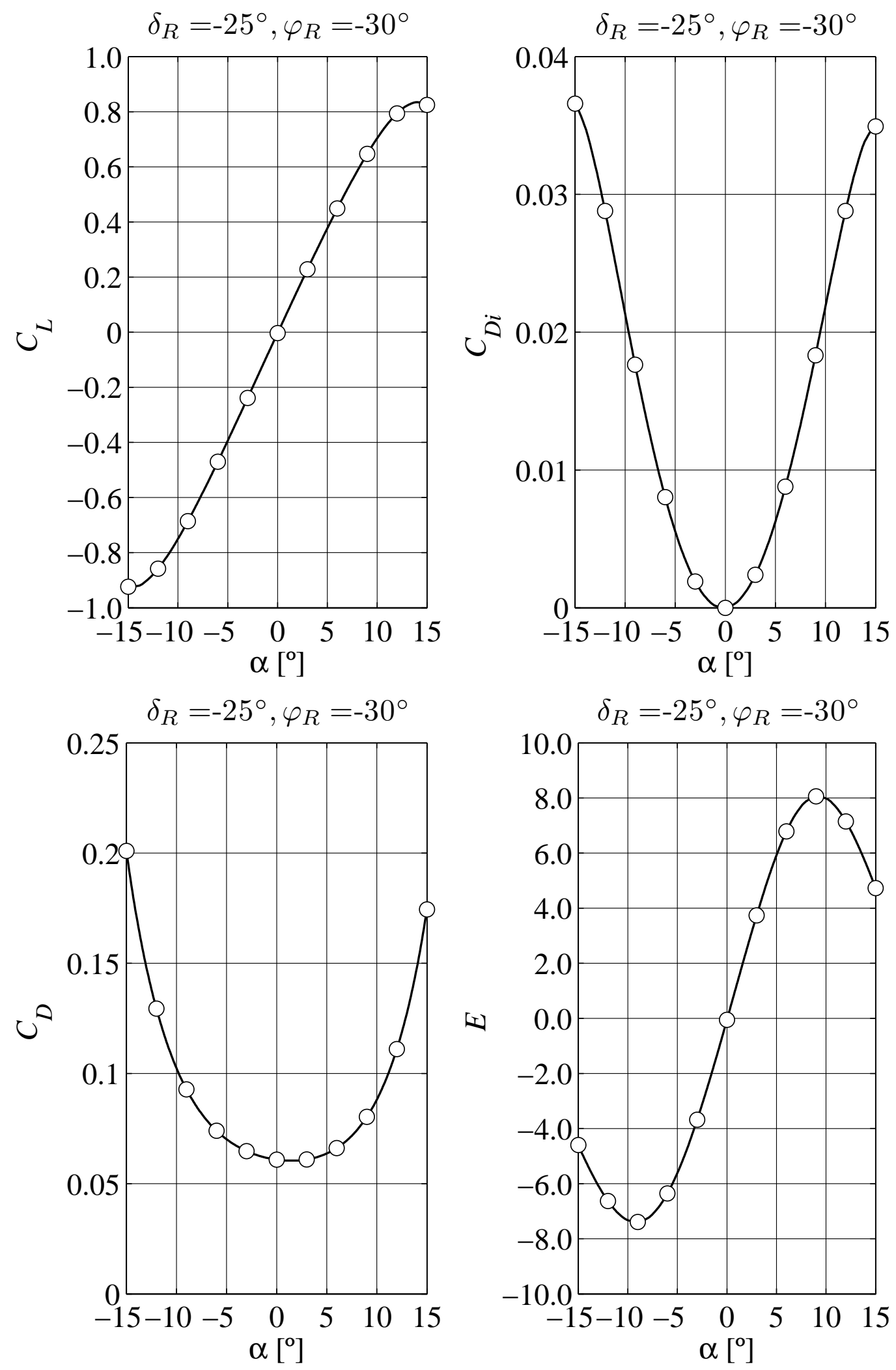

Figura 5.18: Variación de los valores calculados por el modelo numérico de los coeficientes de sustentación, $C_{L}$, de resistencia inducida, $C_{D i}$, de resistencia, $C_{D}$ y de eficiencia aerodinámica, $E$, en función del ángulo de ataque, $\alpha$, para la configuración de referencia de ensayo $\mathrm{JWC}_{15}\left(\varphi_{R}=-30^{\circ}\right.$ y $\left.\delta_{R}=-25^{\circ}\right)$. 

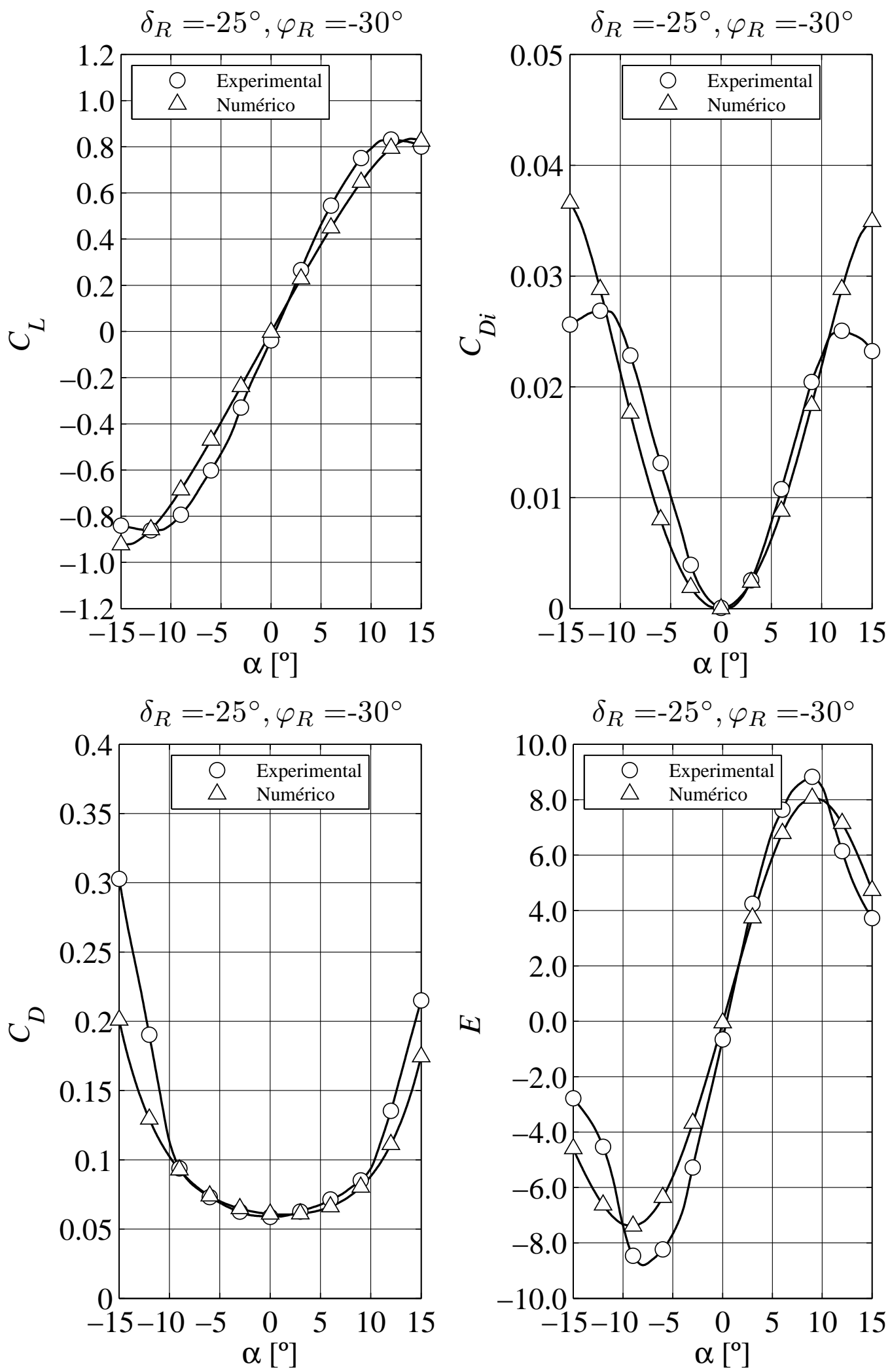

Figura 5.19: Variación de los valores de los coeficientes de sustentación, $C_{L}$, de resistencia inducida, $C_{D i}$, de resistencia, $C_{D}$ y de eficiencia aerodinámica, $E$, en función del ángulo de ataque, $\alpha$, para la configuración de referencia de ensayo $\operatorname{JWC}_{15}\left(\varphi_{R}=-30^{\circ}\right.$ y $\delta_{R}=$ $\left.-25^{\circ}\right)$. Comparación entre el modelo numérico y los resultados experimentales. 



\section{Capítulo 6}

\section{Conclusiones y líneas de investigación futuras}

En este trabajo se ha presentado la investigación realizada sobre el modelo de aeronave de alas convergentes, cuantificando, en primer lugar y por medio de ensayos en túnel aerodinámico, la influencia en los parámetros aerodinámicos de los parámetros geométricos que intervienen en la definición de sus elementos sustentadores y, en segundo, la definición de un modelo número sencillo que permita, por un lado, obtener soluciones de cualquier configuración geométrica del ala delantera y trasera y, por otro, realizar análisis de sensibilidad de parámetros geométricos característicos y todo ello de forma rápida, económica y con la precisión suficiente en la fase de diseño conceptual de una aeronave.

\section{Determinación de los coeficientes aerodinámicos}

Para tal propósito se ha realizado un estudio para comprobar cómo influye esta configuración en los coeficientes de sustentación y resistencia inducida comparada con una configuración tradicional. Para ello se han tomado de referencia las teorías de biplanos y de sistemas de alas múltiples de diferentes autores, adaptándolas, en la medida de lo posible, a la configuración de alas convergentes.

Desde el punto de vista del valor de la fuerza de sustentación, es evidente que el hecho de tener mayor superficie sustentadora, debido a la morfología de la configuración, aumenta el valor de la misma, aunque en el caso de estudio, donde el ala trasera se encuentra en una posición retrasada con respecto a la delantera, este efecto no es tan acusado y el valor proporcional de la sustentación que aporta el ala delantera y el ala trasera a la sustentación total se reduce según aumenta la separación y la altura entre ambas alas.

La velocidad inducida por el ala delantera en el ala trasera y viceversa, provoca un efecto en el valor del ángulo de ataque efectivo, reduciendo su valor en el ala delantera, en la zona donde el ala trasera está presente y por consiguiente la resultante de la fuerza de sustentación es menor, de igual manera, en el ala trasera 
el efecto es aún mayor y el ángulo de ataque efectivo disminuye más, tal como se muestra en el esquema de la Figura 6.1.

Este cambio en el valor del ángulo de ataque es mayor según el ala trasera se aleja, haciendo que la velocidad inducida en el ala delantera aumente tanto en dirección del eje $x$ como en dirección del eje $z$, reduciendo así el ángulo de ataque efectivo. Sin embargo, en el ala trasera el efecto es al contrario haciendo que el ángulo de ataque efectivo aumente, pero debido a la menor envergadura y superficie alar del ala trasera, la fuerza de sustentación del ala delantera es dominante y la combinación de ambos efectos hace que la sustentación total sea menor según aumenta la separación entre ambas alas. Este cambio en las velocidades inducidas puede observarse en la Figura 5.9, 5.10 y 5.11.

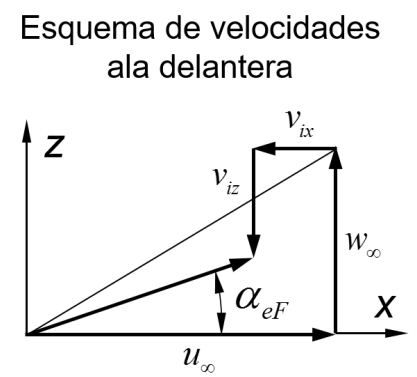

\section{Esquema de velocidades ala trasera}

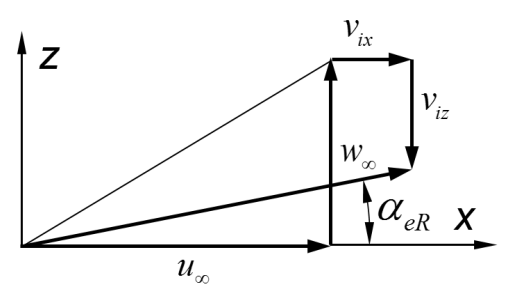

Figura 6.1: Esquema de velocidades inducidas y de ángulo de ataque efectivo para una sección transversal genérica en ala delantera y en ala trasera.

En la Figura 6.2 puede observarse como la presencia del ala trasera varía el valor del ángulo de ataque efectivo a lo largo de la envergadura del ala delantera en su zona de influencia. En el tramo de envergadura adimensional donde el ala trasera está presente, la tendencia de la curva del ángulo efectivo en el ala delantera cambia reduciendo su pendiente.
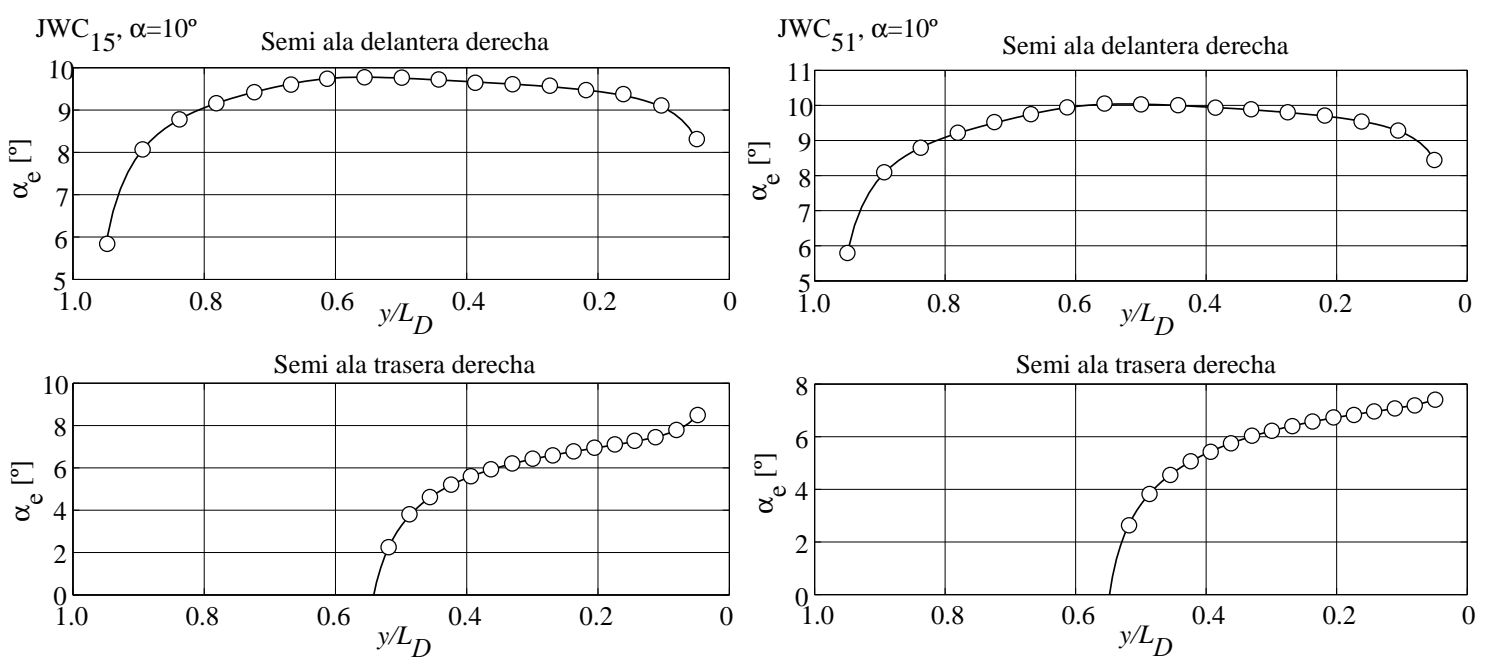

Figura 6.2: Valores del ángulo de ataque efectivo, $\alpha_{e}$, para las configuraciones de ensayo $\operatorname{JWC}_{15}\left(\varphi_{R}=-30^{\circ} \mathrm{y}, \delta_{R}=-25^{\circ}\right)$ y $\operatorname{JWC}_{51}\left(\varphi_{R}=-10^{\circ}\right.$ $\left.\mathrm{y}, \delta_{R}=-5^{\circ}\right)$, para un ángulo de ataque $\alpha=10^{\circ}$.

Desde el punto de vista del valor de la resistencia inducida se ha comprobado cómo las características particulares de esta configuración afectan significativamente al valor de la misma. 
Por otro lado y para el cálculo de la resistencia inducida, la naturaleza de un sistema de alas múltiples no permite utilizar el parámetro de Oswald en la manera que se haría en una aeronave de geometría clásica por lo que para poder comparar las diferentes configuraciones en función del ángulo de flecha y del ángulo diedro del ala trasera ha sido necesario definir un modelo para el cálculo del parámetro de Oswald. Para tal propósito en el anexo A se han estudiado y comparado varios modelos, adaptándolos a la aeronave de alas convergentes y cuyos resultados han sido muy satisfactorios, en primer lugar porque estos han sido acordes a los estudiados en la literatura y por otro porque los resultados obtenidos en los cálculos del modelo numérico realizado en el capítulo 5 son coherentes con los experimentales. En todos los casos el valor del parámetro de Oswald aumenta según aumenta la altura entre el ala delantera y el ala trasera, tal como se muestra en la Figura 6.3.

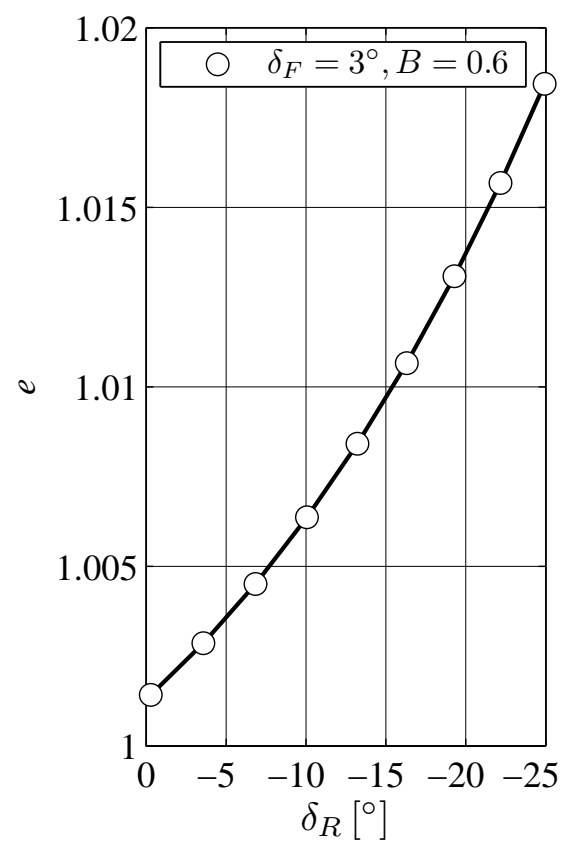

Figura 6.3: Factor de eficiencia $e$ para el modelo de alas convergentes en función del ángulo diedro del ala trasera, $\delta_{R}$.

Este valor ha sido corregido para tener en cuenta el efecto del ángulo de flecha del ala trasera y cuyos resultados se muestran en la Tabla 4.7, y en la Figura 6.4 donde se muestra la variación del valor $1 / \pi \Lambda e$ en función del ángulo de flecha del ala trasera, $\varphi_{R}$, y del ángulo diedro del ala trasera, $\delta_{R}$.

Para la determinación de los coeficientes aerodinámicos se ha realizado el ensayo de 25 configuraciones en túnel aerodinámico (ver Tabla 4.3) cuyas características tanto de ellas como del ensayo se describen en el capítulo 4.

A la vista de los resultados queda de manifiesto que la variación de los coeficientes aerodinámicos es mayor cuando se modifica el ángulo de flecha del ala trasera que con la modificación del ángulo diedro del ala trasera. Mientras que incrementos del ángulo de flecha del ala trasera producen variaciones en el coeficiente 


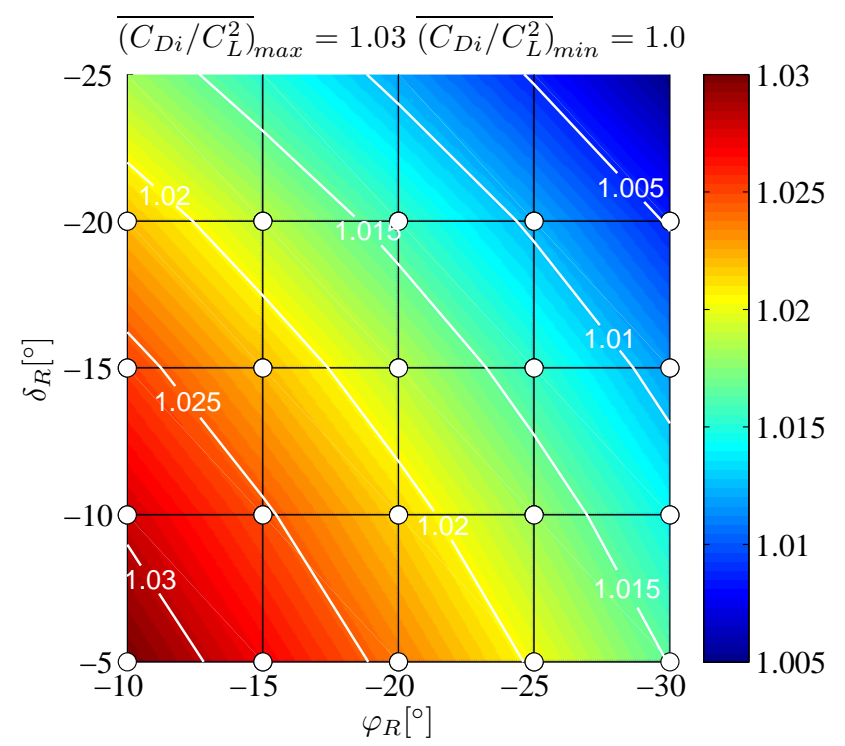

Figura 6.4: Variación de la relación de coeficientes, $\overline{C_{D i} / C_{L}^{2}}$, en función del ángulo de flecha del ala trasera, $\varphi_{R}$, y del ángulo diedro del ala trasera,$\delta_{R}$. Número de Reynolds de la corriente libre $R e=250000$.

de sustentación de hasta un $20 \%$ y en el coeficiente de resistencia de hasta un $40 \%$, incrementos en el ángulo diedro del ala trasera sólo producen variaciones de un $2 \%$ en el coeficiente de sustentación y de un $10 \%$ en el coeficiente de resistencia. Parece claro que, variaciones en el parámetro de distancia relativa, $l_{t}$, y altura relativa, $h_{t}$, del ala trasera tiene una influencia evidente en los coeficientes aerodinámicos. Por otro lado, dependiendo del coeficiente aerodinámico en estudio, la variación en su valor presenta diferentes características, como se muestra a continuación.

El coeficiente de sustentación, $C_{L}$, tiende a aumentar si los ángulos de flecha y diedro del ala trasera se reducen (en valor absoluto), tal y como se muestra en la Figura 4.25. En general, la configuración $\operatorname{JWC}_{51}\left(\delta_{R}=-5^{\circ}, \varphi_{R}=-10^{\circ}\right)$ presenta valores de hasta un $20 \%$ mayores que los que presenta la configuración $\mathrm{JWC}_{15}\left(\delta_{R}=-25^{\circ}, \varphi_{R}=-30^{\circ}\right)$. De forma similar ocurre con el valor máximo del coeficiente de sustentación, $C_{\text {Lmax }}$, tal como se muestra en la Figura 4.31 (a). En conclusión, el coeficiente de sustentación decrece según aumenta la distancia y la altura del ala trasera.

El coeficiente de resistencia, $C_{D}$, tiende a no sufrir variación con el ángulo diedro pero, sin embargo, decrece significativamente si el ángulo de flecha crece (en valor absoluto), tal y como se muestra en la Figura 4.26. En general, la configuración $\mathrm{JWC}_{55}\left(\delta_{R}=-5^{\circ}, \varphi_{R}=-30^{\circ}\right)$ presenta valores de hasta un $40 \%$ menores que la configuración $\mathrm{JWC}_{11}\left(\delta_{R}=-25^{\circ}, \varphi_{R}=-10^{\circ}\right)$. De forma similar ocurre con el valor mínimo del coeficiente de resistencia, $C_{D m i n}$, tal como se muestra en la Figura 4.31 (b). En conclusión, el coeficiente de resistencia decrece según aumenta la distancia y disminuye la altura del ala trasera.

El coeficiente de resistencia inducida, $C_{D i}$, es menor si los ángulos de flecha y diedro del ala trasera aumentan (ambos en valor absoluto), tal y como se muestra en la Figura 4.27. En general, la configuración $\operatorname{JWC}_{51}\left(\delta_{R}=-5^{\circ}, \varphi_{R}=-10^{\circ}\right)$ presenta valore de hasta un $45 \%$ menores que los que presenta la configuración $\mathrm{JWC}_{15}\left(\delta_{R}=-25^{\circ}, \varphi_{R}=-30^{\circ}\right)$. En conclusión, el coeficiente de resistencia 
inducida decrece según aumenta la distancia y la altura del ala trasera.

En general, desde el punto de vista de los valores de la resistencia aerodinámica, tanto total como inducida, se puede afirmar que las configuraciones con mayor valor del ángulo de flecha en valor absoluto $\left(\varphi_{R}=-30^{\circ}\right)$, son las que presentan valores mínimos.

Por otro lado y tal y como se muestra en la Figura 4.32, la configuración $\mathrm{JWC}_{55}\left(\delta_{R}=-5^{\circ}, \varphi_{R}=-30^{\circ}\right)$ es la que presenta mejores actuaciones encontrándose el máximo valor de la eficiencia aerodinámica, $E_{\max }$, y el máximo valor del factor para potencia mínima requerida, $\left(C_{L}^{3 / 2} / C_{D}\right)_{\min }$, el cual, como es sabido, implica mejores valores de alcance y autonomía.

Por último, y a la vista de los resultados expuestos en el anexo $\mathrm{C}$ y el anexo D, se puede comprobar que los valores del coeficiente de sustentación máximo y del coeficiente de resistencia mínimo se dan para ángulos de ataque de $12^{\mathrm{O}} \mathrm{y}$ $0^{\underline{0}}$ respectivamente y para todas las configuraciones, y de forma similar, los valores máximos de la eficiencia aerodinámica y del parámetro de mínima potencia requerida se dan para valores de ángulo de ataque de $9^{\underline{O}}$, e igual que antes para todas las configuraciones de estudio.

Un resumen de la influencia de los parámetros geométricos en la variación de los parámetros aerodinámicos se presenta en la Tabla 6.1 y en la Figura 6.5, donde se muestra como aumentan o disminuyen los coeficientes aerodinámicos del sistema.

Tabla 6.1: Resumen de la variación de los coeficientes aerodinámicos con la altura y la separación del ala trasera.

\begin{tabular}{c|ccccccccc}
\cline { 2 - 8 } \multicolumn{1}{c}{} & $C_{L}$ & $C_{D}$ & $C_{D i}$ & $E$ & $C_{L}^{3 / 2} / C_{D}$ & $C_{\text {Lmax }}$ & $C_{\text {Dmin }}$ & $E_{\max }$ & $\left(C_{L}^{3 / 2} / C_{D}\right)_{\min }$ \\
\hline Si $h_{t} \Uparrow$ & $\Downarrow$ & $\mathbb{1}$ & $\Downarrow$ & $\Downarrow$ & $\Downarrow$ & $\Downarrow$ & $\Uparrow$ & $\Downarrow$ & $\Downarrow$ \\
Si $l_{t} \Uparrow$ & $\Downarrow$ & $\Downarrow$ & $\Downarrow$ & $\Uparrow$ & $\mathbb{1}$ & $\Downarrow$ & $\Downarrow$ & $\Uparrow$ & $\mathbb{1}$ \\
\hline$\Uparrow$ & Aumenta & & & & & & \\
$\Downarrow$ & Disminuye & & & & & & \\
$\mathbb{1}$ & Estable & & & & & & \\
\hline
\end{tabular}

De igual manera, en la Tabla 6.2 se presenta un resumen de las configuraciones que muestran los valores máximo y mínimo de esos parámetros .

Tabla 6.2: Tabla resumen donde se muestra en que configuraciones se presentan los valores máximo y mínimo de los coeficientes aerodinámicos

\begin{tabular}{|c|c|c|c|c|c|}
\hline & $C_{L}$ & $C_{D}$ & $C_{D i}$ & $E$ & $C_{L}^{3 / 2} / C_{D}$ \\
\hline Valor máximo & $\mathrm{JWC}_{51}$ & $\mathrm{JWC}_{11}$ & $\mathrm{JWC}_{51}$ & $\mathrm{JWC}_{55}$ & $\mathrm{JWC}_{55}$ \\
\hline Valor mínimo & $\mathrm{JWC}_{15}$ & $\mathrm{JWC}_{55}$ & $\mathrm{JWC}_{15}$ & $\mathrm{JWC}_{11}$ & $\mathrm{JWC}_{15}$ \\
\hline
\end{tabular}

En una línea futura de investigación, teniendo en cuenta lo anterior, desde el punto de vista del cálculo de coeficientes aerodinámicos y con la firme intención de construir un prototipo de aeronave no tripulada, sería necesario diseñar una campaña de ensayos en túnel aerodinámico de modelos de aeronave completo de aquellas configuraciones más prometedoras, que permita, en primer lugar el estudio 


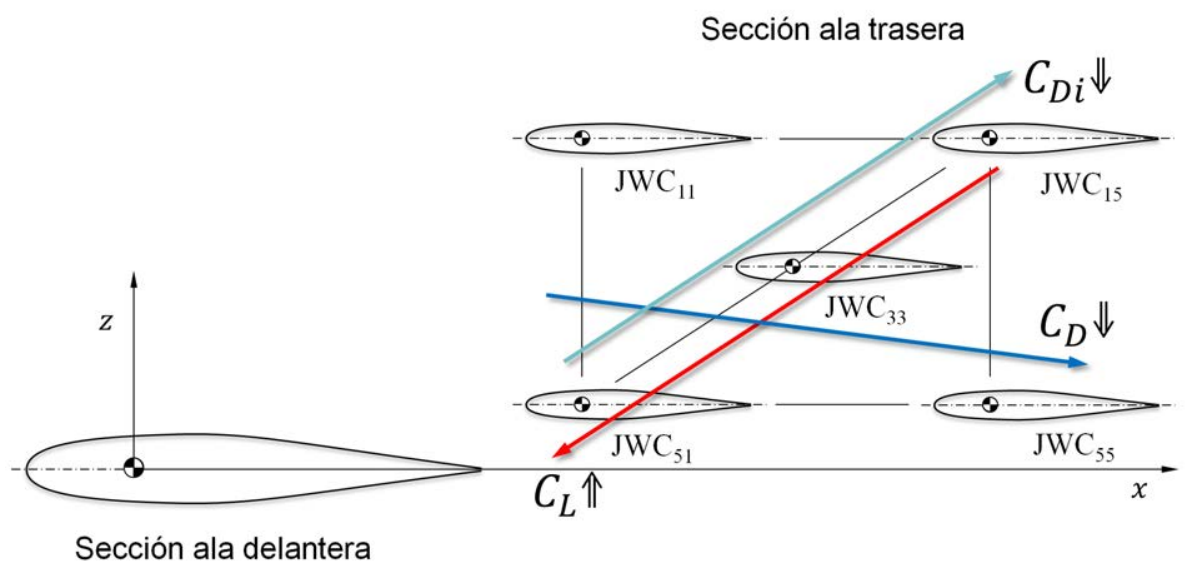

Figura 6.5: Esquema de la tendencia de variación de los coeficientes aerodinámicos de sustentación, $C_{L}$, resistencia, $C_{D}$, y resistencia inducida, $C_{D i}$, al modificar la altura y la separación del ala trasera.

de la interferencia producida por el fuselaje en los coeficientes y, en segundo lugar el estudio de las características de estabilidad y maniobrabilidad del modelo.

Por otro lado, el gran número de resultados experimentales obtenidos permite la creación de una base de datos para su uso en la correlación de resultados de modelos de mallas calculados en aplicaciones CFD para este tipo de configuraciones.

Finalmente en la Figura 6.6 se muestra una imagen con las configuraciones que presentan los mejores valores en los coeficientes aerodinámicos: configuración $\mathrm{JWC}_{15}\left(C_{\text {Dimin }}\right)$, configuración $\mathrm{JWC}_{55}\left(C_{D \min }\right)$ y configuración $\mathrm{JWC}_{15}\left(C_{L \max }\right)$.

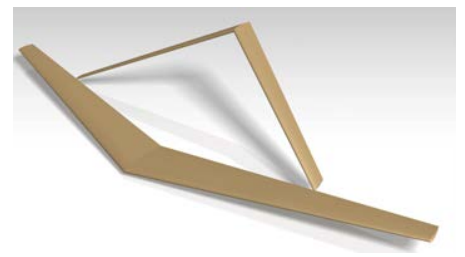

Configuración $\mathrm{JWC}_{15}$

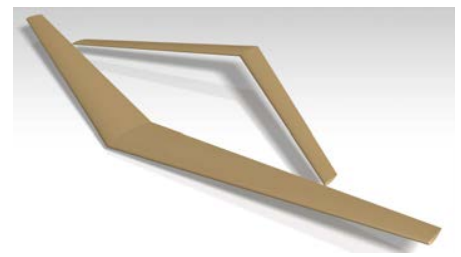

Configuración $\mathrm{JWC}_{55}$

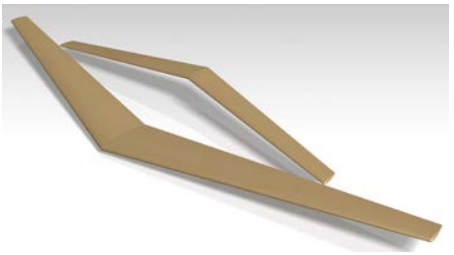

Configuración $\mathrm{JWC}_{51}$

Figura 6.6: Imagen que muestra las configuraciones que presentan los mejores valores en los coeficientes aerodinámicos.

\section{Modelo numérico}

Como es sabido, el alto coste, tanto económico como en tiempo, que exige la realización de ensayos en túnel aerodinámico obliga a la utilización de modelos numéricos que permitan obtener resultados cualquiera que sea la geometría de estudio.

De igual manera el uso de aplicaciones numéricas de simulación complejas reduce en parte el problema económico pero mantiene el problema del tiempo que exige este tipo de herramientas en la definición del modelo teórico. 
En las fases de diseño conceptual, en un proyecto de aeronave de estas características, es necesario disponer de herramientas que proporcionen soluciones rápidas, incluso cuando sean estudiados y modificados un número elevado de parámetros, y que permitan realizar análisis de sensibilidad de los mismos, poniendo mayor énfasis en aquellos que son relevantes en el diseño y dejar aquellos que presenten poca influencia en el mismo.

Para tal propósito en el capítulo 5 se ha desarrollado un modelo numérico sencillo y simplificado que consiste básicamente en aproximar las características aerodinámicas del modelo de alas convergentes por medio de dos lineas o herraduras de torbellinos que se adapten a su geometría.

Este modelo permite la posibilidad de obtener múltiples mapas de resultados de coeficientes en función de múltiples variables geométricas del ala delantera y del ala trasera (envergadura, ángulo de flecha, ángulo diedro, alargamiento, estrechamiento, torsión, valor de la cuerda en el encastre, etc.), así como elementos aerodinámicos como es el tipo de perfil aerodinámico en la definición de sus superficies sustentadoras.

Con el elevado número de datos obtenidos por medio de los ensayos en túnel ha sido posible comparar los resultados experimentales con los teóricos y comprobar que, a pesar de la extremada sencillez del modelo numérico, este proporciona resultados próximos y coherentes con los experimentales.

Como puede comprobarse en el anexo E los valores de los coeficientes obtenidos con el modelo numérico no sufren una desviación significativa con respecto a los valores de los resultados experimentales, especialmente en rango de valores del ángulo de ataque entre $-8^{\mathrm{O}}$ a $12^{\mathrm{O}}$. Además, se puede comprobar que el modelo reproduce fielmente la tendencia de variación de los coeficientes aerodinámicos con respecto a la variación del ángulo diedro y del ángulo de flecha del ala trasera que presentan los resultados experimentales.

A la vista de los resultados, es significativo comprobar que el modelo reproduce, no sólo efectos en los resultados debidos al propio cambio de geometría en cada configuración, sino que también los cambios en los coeficientes producidos por la propia asimetría del modelo de ala convergentes. Por ejemplo, en el caso del coeficiente de resistencia aerodinámica donde los resultados experimentales presentan valores mayores para ángulos de ataque negativos que para ángulos de ataque positivos, los resultados del modelo numérico presentan esa misma tendencia, aunque no con tanta diferencia como el modelo experimental. En la Tabla 6.3 están indicados los valores de los coeficientes aerodinámicos de sustentación, resistencia, resistencia inducida y eficiencia aerodinámica obtenidos numéricamente comparados con los obtenidos experimentalmente para la configuración de referencia $\mathrm{JWC}_{15}$ $\left(\delta_{R}=-25^{\circ}, \varphi_{R}=-30^{\circ}\right)$. Como puede observarse, para valores de ángulo de ataque entre $-8^{\underline{O}}$ y $12^{\underline{O}}$ excepto $0^{\underline{O}}$, la precisión es del orden de entre el $70 \%$ y el $100 \%$ dependiendo del coeficiente.

En conclusión se pude afirmar que el modelo numérico generado puede ser una herramienta de gran utilidad y versátil con el que es posible estudiar y comparar cualquier tipo de configuración de alas convergentes, desde configuración de alas en forma de diamante, configuración de alas convergentes como las estudiadas e incluso, y porque no, configuraciones tradicionales. 
Tabla 6.3: Valores de los coeficientes aerodinámicos obtenidos numéricamente comparados con respecto a los obtenidos experimentalmente de la configuración de referencia $\mathrm{JWC}_{15}\left(\delta_{R}=-25^{\circ}, \varphi_{R}=-30^{\circ}\right)$.

\begin{tabular}{c|cccc}
\hline$\alpha\left[^{\circ}\right]$ & $\frac{C_{L n}}{C_{L e}}$ & $\frac{C_{D n}}{C_{D e}}$ & $\frac{C_{D i n}}{C_{D i e}}$ & $\frac{E_{n}}{E_{e}}$ \\
\hline \hline-14 & 1.08 & 0.65 & 1.33 & 1.66 \\
\hline-12 & 1.00 & 0.68 & 1.07 & 1.46 \\
\hline-10 & 0.90 & 0.90 & 0.84 & 0.99 \\
\hline-8 & 0.83 & 1.01 & 0.71 & 0.82 \\
\hline-6 & 0.78 & 1.01 & 0.61 & 0.77 \\
\hline-4 & 0.71 & 1.03 & 0.49 & 0.69 \\
\hline-2 & 0.71 & 1.05 & 0.43 & 0.68 \\
\hline 0 & 0.09 & 1.04 & 0.08 & 0.08 \\
\hline 2 & 0.96 & 1.00 & 1.24 & 0.96 \\
\hline 4 & 0.82 & 0.95 & 0.84 & 0.87 \\
\hline 6 & 0.82 & 0.93 & 0.82 & 0.89 \\
\hline 8 & 0.85 & 0.93 & 0.86 & 0.91 \\
\hline 10 & 0.89 & 0.94 & 0.96 & 0.94 \\
\hline 12 & 0.95 & 0.82 & 1.15 & 1.16 \\
\hline 14 & 1.02 & 0.78 & 1.40 & 1.30 \\
\hline
\end{tabular}

Por otro lado y desde el punto de vista de líneas futuras de investigación, determinado y comprobado el modelo numérico presentado, es necesario plantear modificaciones sobre el mismo que permitan alcanzar mayor precisión en los resultados obtenidos.

En primer lugar se deben estudiar distribuciones de la circulación más exactas para remplazar, la actual definición del modelo de un único torbellino constante por cada herradura, por una más real.

Un primer paso sería buscar aproximaciones a distribuciones más complejas y realizar una correlación con los resultados ya existentes.

De manera más ambiciosa se propone estudiar, por medio de ensayos en túnel aerodinámico y con otras técnicas de ensayo (Particle image velocimetry, hilo caliente de 3 componentes, tomas de presión, etc.), cómo es la distribución de velocidades a lo largo del borde de salida de ambas alas, en diferentes planos a lo largo del eje $x$, obteniéndose así una aproximación más precisa de la distribución de circulación para cada configuración.

Una vez determinada la distribución de circulación, de forma experimental, es posible crear modelos numéricos de la misma que incluidos en el proceso de cálculo del modelo numérico, proporcione mejores resultados.

Existe varios inconvenientes para alcanzar este objetivo, primero que es complicado obtener la distribución de circulación, y segundo que el coste en tiempo y en infraestructuras que han de emplearse en la realización de estos ensayos es elevado. La realización de los mismos es posible por medio del uso de diferentes técnicas como es el caso de; anemometría de hilo caliente de tres componentes midiendo en una matriz de puntos suficientemente grande para abarcar ambas alas y en un número de planos necesarios, realizándose en un número mínimo de configuraciones y en ángulos de ataque que posibiliten conseguir distribuciones 
espaciales de velocidades que sean determinantes; o por medio del uso tecnología PIV (Particle image velocimetry) que permite la medida de campos de velocidades. 



\section{Bibliografía}

European Commission. Annual Analyses of the EU Air Transport Market 2015. 2016.

T. Aarons, R. Canfield, C. Woolsey, J. Richards, A. Suleman, N. Lindsley and M. Blair. Design for flight test of a scaled joined wing sensorcraft. In Structures, Structural Dynamics, and Materials and Co-located Conferences. American Institute of Aeronautics and Astronautics, 2011.

R. B. Addoms and F. W. Spaid. Aerodynamic design of high-performance biplane wings. Journal of Aircraft, 12(8):629-630, 1975.

T. Bagwill and B. Selberg. Aerodynamic investigation of joined wing configurations for transport aircraft. In Fluid Dynamics and Co-located Conferences. American Institute of Aeronautics and Astronautics, 1996a.

T. Bagwill and B. Selberg. Aerodynamic investigation of joined wing configurations for transport aircraft. In 14th Applied Aerodynamics Conference, 30-41. American Institute of Aeronautics and Astronautics, New Orleans, LA, U.S.A., 1996b.

T. Bagwill and B. Selberg. Aerodynamic investigation of twist and cant angles for joined wing transport aircraft. In Aerospace Sciences Meetings. American Institute of Aeronautics and Astronautics, 1997.

J. Barlow, W. H. J. Rae and A. Pope. Low-speed wind tunnel testing. New York: Wiley-Interscience, 1999.

M. Belardo, N. Paletta, L. di palma and M. Pecora. Structural and aeroelastic design of a joined-wing UAV. Proceedings of the Institution of Mechanical Engineers.Part G, Journal of Aerospace Engineering, 93-111, 2014.

F. Bertagnolio. NACA0015 measurements in LM wind tunnel and turbulence generated noise. Ris $\varnothing-\mathrm{R} 1657$, Ris $\varnothing$ National Laboratory for Sustainable Energy, Technical University of Denmark Roskilde, Denmark, 2008.

J. J. Bertin and M. L. Smith. Aerodynamics for engineers. Prentice-Hall International Editions, 2nd edition, 1989. ISBN: 0-13-018227-3. 
M. Blair and R. A. Canfield. A joined-wing structural weight modeling study. In Structures, Structural Dynamics, and Materials and Co-located Conferences. American Institute of Aeronautics and Astronautics, 2002.

M. Blair, R. A. Canfield and R. W. Roberts. Joined-wing aeroelastic design with geometric nonlinearity. Journal of Aircraft, 42(4):832-848, 2005.

H. J. Bos. Induced drag for non-planar wings. Report LR 521, TUDelft Faculty of Aerospace Engineering, 1987.

A. J. Botero Arango, J. E. Bravo Bolívar and M. Botero Arbeláez. El método de Newton-Raphson - La alternativa del ingeniero para resolver sistemas de ecuaciones no lineales. Scientia et Technica, XI(27):221-224, 2005.

G. G. Brebner and L. A. Wyatt. The velocities induced by distributions of infinite kinked source and vortex lines representing wings with sweep and dihedral in incompressible flow. Reports and Memoranda 3667, Ministry Of Defence: Aeronautical Research Council Reports And Memoranda Aerodynamics Dept., R.A.E., Farnborough, 1970.

J. R. Chambers. Innovation in flight: Research of the NASA Langley Research Center on revolutionary advanced concepts for aeronautics. Technical Report No. 4539, NASA SP, Langley, 2005.

R. V. Churchill and J. W. Brown. Variable compleja y aplicaciones. McGRAW-HILL/ITERAMERICA DE ESPAÑA, S. A., 5ª edition, 1992.

C. D. Cone. The theory of induced lift and minimun induced drag of nonplanar lifting systems. NASA TR R-139, NASA, 1962.

C. Cuerno-Rejado, L. Alonso-Albir and P. Gehse. Conceptual design of a medium-sized joined-wing aircraft. Proceedings of the Institution of Mechanical Engineers.Part G, Journal of Aerospace Engineering, 224(G6):681-696, 2010.

A. Cuerva, A. Sanz-Andres, J. Meseguer and J. Espino. An engineering modification of the blade element momentum equation for vertical descent: An autorotation case study. Journal of the American Helicopter Society, 51(4):349-354, 2006.

L. Demasi. Investigation on the conditions of minimum induced drag of closed wing systems and c-wings. Journal of Aircraft, 44(1):81-99, 2007.

L. Demasi, R. Cavallaro and A. M. Razón. Postcritical analysis of prandtlplane joined-wing configurations. AIAA Journal, 51(1):161-177, 2013.

L. Demasi, A. Dipace, G. Monegato and R. Cavallaro. An invariant formulation for the minimum induced drag conditions of non-planar wing systems. In AIAA SciTech. American Institute of Aeronautics and Astronautics, 2014.

L. Demasi, A. Dipace, G. Monegato and R. Cavallaro. Minimum induced drag theorems for joined wings, closed systems, and generic biwings: Theory. In 56th AIAA Structures, Structural Dynamics, and Materials Conference, 0697-0730. American Institute of Aeronautics and Astronautics, Kissimmee, Florida, U.S.A, 2015a. 
L. Demasi, G. Monegato, E. Rizzo, R. Cavallaro and A. Dipace. Minimum induced drag theorems for joined wings, closed systems, and generic biwings: Results. In AIAA SciTech. American Institute of Aeronautics and Astronautics, 2015b.

J. DeYoung. Minimization theory of induced drag subject to constraint conditions. NASA CR 3140, NASA, 1979.

J. DeYoung. Induced drag ideal efficiency factor of arbritrary lateral-vertical wing forms. NASA CR 3357, NASA, 1980.

W. S. Diehl. Relative loading on biplane wings. NACA TR 458, National Advisory Committee for Aeronautics, 1934.

W. S. Diehl. Engineering Aerodynamics. Research And Development Center, 1986.

W. F. Durand, ed. Aerodynamic Theory, volume II, División E. Dover Publications, Inc., New York, U.S.A., 1963.

A. Frediani. The prandtl wing. In Lecture series on Innovative Configurations and Advanced Concepts for Future Civil Transport Aircraft. Von Karman Institute, 2005.

J. W. Gallman and I. M. Kroo. Structural optimization for joined-wing synthesis. Journal of Aircraft, 33(1):214-223, 1996.

J. W. Gallman, I. M. Kroo and S. C. Smith. Design synthesis and optimization of joined-wing transports. In Aircraft Design and Operations Meetings. American Institute of Aeronautics and Astronautics, 1990.

J. W. Gallman, S. C. Smith and I. M. Kroo. Optimization of joined-wing aircraft. Journal of Aircraft, 30(6):897-905, 1993.

R. E. S. Gómez. Sistemas aéreos no tripulados y espacio aéreo en europa. una combinación estratégica. Documento marco, Instituto Español de Estudios Estratégicos, 2001.

P. Hajela and J. L. Chen. Preliminary weight estimation of conventional and joined wings using equivalent beam models. Journal of Aircraft, 25(6):574-576, 1988.

N. Hirose, T. Ohnuki, H. Masakata and M. Ishikawa. A computational and experimental analysis of joined-wing aerodynamics. NAL TR 1088T, National Aerospace Laboratory, 1990.

R. T. Jones and T. A. Lasinski. Effect of winglets on the induced drag of ideal wing shapes. NASA TM 81230, NASA, 1980.

J. A. Jupp. The design of future passenger aircraft. The environmental and fuel price challenges. The Aeronautical Journal, 120(1223):37-60, 2016.

H. Kang, N. Genco and A. Altman. Gap and stagger effects on biplanes with end plates: Part I. In Aerospace Sciences Meetings. American Institute of Aeronautics and Astronautics, 2009.

J. Katz and A. Plotkin. Low-speed aerodynamics. Cambridge, United Kingdom: Cambridge University Press, 2001. 
D. Küchemann. Investigation of the lift distribution over the separate wings of a biplane. NACA TM 889, National Advisory Committee for Aeronautics, 1938.

M. Knight and R. W. Noyes. Wind tunnel pressure distribution tests on a series of biplanes wing models part I, effects of changes in stagger and gap. NACA TN 310, National Advisory Committee for Aeronautics, 1929.

I. Kroo, J. Gallman and S. Smith. Aerodynamic and structural studies of joined-wing aircraft. Journal of Aircraft, 28(1):74-81, 1991.

I. Kroo, J. McMasters and S. C. Smith. Highly nonplanar lifting systems. In Transportation Beyond 2000: Technologies Needed for Engineering Design. 1995.

E. V. Laitone. Comment on 'Drag reduction factor due to ground effect'. Journal of Aircraft, 27(1):96-96, 1990.

R. H. Latiff, P. M. Banks, A. S. Bicos and E. R. C. amd Ravi B. Deo. 3d printing in space. Technical report, Committee on Space-Based Additive Manufacturing; Aeronautics and Space Engineering Board; National Materials and Manufacturing Board; Division on Engineering and Physical Sciences; National Research Council, 2014.

J. S. Letcher. V-wings and diamond ring-wings of minimum induced drag. Journal of Aircraft, 9(8):605-607, 1972.

P. Mamla and C. Galinski. Basic induced drag study of the joined-wing aircraft. Journal of Aircraft, 46(4):1438-1440, 2009.

R. Mannings. The future, by AIRBUS. Technical report, AIRBUS Industrie, 2011.

J. Meseguer, A. Sanz-Andrés, S. Franchini and S. Pindado. Instalaciones de aerodinámica experimental para ingeniería civil de la Universidad Politécnica de Madrid. In $8^{o}$ Congreso Iberoamericano de Ingeniería Mecánica. Cuzco, 2007.

J. Meseguer Ruiz and A. Sanz Andrés. Aerodinámica básica. Ibergarceta Publicaciones, S.L., $2^{\mathrm{a}}$ edition, 2011.

H. Miura, A. T. Shyuf and J. Wolkovitcht. Parametric weight evaluation of joined wings by structural optimization. Journal of Aircraft, 25(12):1142-1149, 1988.

R. M. Mock. The distribution of loads between the wings of a biplane having decalage. NACA TR 269, National Advisory Committee for Aeronautics, 1927.

M.O.D. The UK Approach to Unmanned Aircraft Systems. Jdn 2/11, Ministry of Defence, Swindon, Wiltshire, SN6 8RF, United Kindong, 2011.

J. Morrison, P. Bonnefoy, R. J. Hansman and S. Sgouridis. Investigation of the impacts of effective fuel cost increase on the U.S. air transportation network and fleet. In Aviation Technology, Integration, and Operations (ATIO) Conferences. American Institute of Aeronautics and Astronautics, 2010.

K. Mortara, D. M. Straussfogel and M. D. Maughmer. Analysis and design of planar and non-planar wings for induced drag minimization. NASA CR 189509, NASA, Langley, 1991. 
M. M. Munk. The minimum induced drag of aerofoils. NACA TR 121, National Advisory Committee for Aeronautics, 1921.

M. M. Munk. General byplane theory. NACA TR 151, National Advisory Committee for Aeronautics, 1923.

R. Nangia, M. Palmer and C. Tilmann. Unconventional high aspect ratio joined-wing aircraft with aft- and forward-swept wing-tips. In Aerospace Sciences Meetings. American Institute of Aeronautics and Astronautics, 2003.

R. K. Nangia and M. E. Palmer. Joined wing configuration for high speeds - a first stage aerodynamic study. 41st AIAA Aerospace Science Meeting 86 Exhibit, $1-21,2006$.

F. H. Norton. The effect of staggering a biplane. NACA TN 70, National Advisory Committee for Aeronautics, 1921.

J. O'Banion, J. Jhou, R. Stearman and S. Smith. A study of joint fixativity in a joined-wing aircraft. TM 112388, NASA, 1982.

B. Oligney, M. Frash and T. Yechout. Aerodynamic evaluation and optimization of the Houck joined wing aircraft. In Aerospace Sciences Meetings. American Institute of Aeronautics and Astronautics, 2008.

PAE. Visión estratégica española sistemas de vehículos aéreos no tripulados (UAS). Doc. No: PAE/UAS-sistemas. rev.06, Plataforma Aeroespacial Española, 2014.

M. J. Patil. Nonlinear aeroelastic analysis of joined-wing aircraft. In Structures, Structural Dynamics, and Materials and Co-located Conferences. American Institute of Aeronautics and Astronautics, 2003.

W. F. Phillips and D. O. Snyder. Modern adaptation of prandtl's classic lifting-line theory. Journal of Aircraft, 37(4):662-670, 2000.

R. Platz. A novel sailplane. Sailplane \& Glider, 21(4):17-18, 1924.

M. Powell. A fortran subroutine for solving systems of non-linear algebraic equations. numerical methods for nonlinear algebraic equations. AERE-R 5947, Atomic Energy Research Establishment, 1968.

L. Prandtl. Theory of lifting ssurface Part I. NACA TN 009, National Advisory Committee for Aeronautics, 1920a.

L. Prandtl. Theory of lifting ssurface Part II. NACA TN 010, National Advisory Committee for Aeronautics, 1920b.

L. Prandtl. Applications of modern hydrodynamics to aeronautics. NACA TR 116, National Advisory Committee for Aeronautics, 1923.

L. Prandtl. Induced drag of multiplanes. NACA TN 182, National Advisory Committee for Aeronautics, 1924.

J. Pérez-Álvarez. Optimización de Avión No Convencional: Joined-Wing. Master's thesis, E.T.S.I. Aeronáuticos, Universidad Politécnica de Madrid, 2010. 
C. Rasmussen, R. Canfield and M. Blair. Joined-wing sensor-craft configuration design. In 45 th Structures, Structural Dynamics, and Materials and Co-located Conferences. American Institute of Aeronautics and Astronautics, Palm Springs, California USA, 2004.

C. C. Rasmussen, R. A. Canfield and M. Blair. Joined-wing sensor-craft configuration design. Journal of Aircraft, 43(5):1470-1478, 2006.

C. C. Rasmussen, R. A. Canfield and M. Blair. Optimization process for configuration of flexible joined-wing. Structural and Multidisciplinary Optimization, 37(3):265-277, 2009.

R. Roberts, R. Canfield and M. Blair. Sensorcraft structural optimization and analytical certification. In Structures, Structural Dynamics, and Materials and Co-located Conferences. American Institute of Aeronautics and Astronautics, 2003.

P. M. Rodríguez, E. M. Rodríguez, A. M. Loredo-Souza and E. B. C. Schettini. Utilización de anemómetro de hilo caliente a temperatura constante para mediciones de velocidad de aire en túnel de viento. RIELAC, XXXV(1):78-92, 2014 .

R. Roedts. Design of a biplane wing for small-scale aircraft. In Aerospace Sciences Meetings. American Institute of Aeronautics and Astronautics, 2009.

M. F. Samuels. Structural weight comparison of a joined wing and a conventional wing. Journal of Aircraft, 19(6):485-491, 1982.

B. P. Selberg and D. L. Cronin. Aerodynamic - structural optimization of positive/negative stagger joined wing configurations. In Aircraft Design and Technology Meeting. American Institute of Aeronautics and Astronautics, 1986.

S. C. Smith. A computational and experimental study of nonlinear aspects of induced drag. NASA TP 3598, NASA, 1996.

S. C. Smith and R. K. Stonum. Experimental aerodynamic characteristics of a joined-wing research aircraft configuration. NASA TM 101083, NASA, 1989.

R. Stearman, L. Hung-Hsi and J. Jhou. Influence of joint fixity on the aeroelastic characteristics of a joined wing structure. In Structures, Structural Dynamics, and Materials and Co-located Conferences, 1442-1454. American Institute of Aeronautics and Astronautics, 1990.

D. Stinton. The design of the aeroplane. Blackweel Science, $2^{\mathrm{a}}$ edition, 1983.

G. A. Tokaty. A History and Philosophy of Fluid Mechanics. Dover Publications, Inc., New York, U.S.A., 1971.

T. Tyler. Annual review 2015. 71st annual general meeting, International Air Transport Association, 2015.

T. Von Karman and J. M. Burgers. General aerodynamic theory-perfect fluids. In W. F. Durand, ed., Aerodynamic Theory, volume II, 201-222. Dover

Publications, New York., 1963. 
J. C. Wai, W. W. Herling and E. L. Roetman. Joined wing configuration aerodynamics. Reston, VA: American Institute of Aeronautics and Astronautics, Inc., 1996.

J. Wolkovitch. Subsonic VSTOL aircraft configurations with tandem wings. Journal of Aircraft, 16(9):605-611, 1979.

J. Wolkovitch. Joined wing aircraft. 1982. US Patent 4,365,773.

J. Wolkovitch. Joined-wing beginnings. Aerospace America, 23:6-6, 1985.

J. Wolkovitch. The joined wing - an overview. Journal of Aircraft, 23(3):161-178, 1986.

K. V. Wong and A. Hernandez. A review of additive manufacturing. International Scholarly Research Network ISRN Mechanical Engineering, 2012(208760), 2012.

D. Zafirov. Joined wing uav maneuverability. International Journal of Research in Engineering and Technology, 02:377-380, 2013. 



\section{Apéndice A}

\section{Modelo analítico y geométrico. Aplicación al modelo de alas convergentes}

\section{A.1. Introducción}

Para la determinación de la resistencia inducida, $C_{D i}$, a partir de datos experimentales de ensayos en túnel, es necesario calcular el valor del parámetro de eficiencia de Oswald, $e$.

Son varios los estudios realizados con la idea de desarrollar modelos para la estimación del parámetro de eficiencia aerodinámica de Oswald para sistemas de alas no planas y sistemas de alas múltiples [Cone 1962; Letcher 1972; DeYoung 1980; Demasi et al. 2014; 2015a;b], aunque, en general, ninguno de ellos aborda directamente la solución para modelo de alas convergentes donde la envergadura del ala delantera, $b_{F}$, es mayor que la envergadura del ala trasera, $b_{R}$.

A continuación se muestra el desarrollo de dos de los métodos, uno estrictamente analítico y otro geométrico e interpolado [Letcher 1972; DeYoung 1980], la comparación de resultados para el modelo de alas en forma de diamante $\left(b_{F}=b_{R}\right)$ cuya solución es bien conocida y la adaptación al modelo de alas convergentes $\left(b_{F}>b_{R}\right)$.

En este estudio sólo se pretende realizar los desarrollos necesarios para la estimación del coeficiente de eficiencia de Oswald, por lo que todos los desarrollos previos necesarios para el cálculo de la solución del problema aerodinámico han sido simplificados o presentados directamente.

\section{A.2. El modelo analítico de Letcher}

John S. Letcher, en 1972 [Letcher 1972], estudió y desarrolló un modelo para el caso de sistemas alas múltiples que establece una solución analítica para el 
cálculo de la masa inducida, $M$, y como consecuencia una estimación del parámetro de eficiencia, e. Para el cálculo se tiene en cuenta que el movimiento del fluido inducido por los torbellinos del ala se considera bidimensional en el plano de Trefftz, y que el movimiento del fluido se supone idéntico en todos los planos paralelos al plano de Trefftz pudiendo considerarse la estela plana aguas abajo en dicho plano.

Como es sabido, para la solución de problemas potenciales bidimensionales de un flujo incompresible se pueden utilizar transformaciones conformes que están representadas por funciones regulares de transformaciones complejas [Meseguer Ruiz and Sanz Andrés 2011] y cuyo desarrollo en serie de Laurent es:

$$
g(z)=z+\sum_{n=1}^{\infty} A_{n} z^{-n}
$$

Por lo que, para el caso de estudio, es posible establecer el potencial complejo que satisface la condición de contorno y presenta circulación nula alrededor del cuerpo, por medio de una función analítica [Bos 1987]. Este potencial complejo a determinar es el correspondiente al perfil bidimensional, $P$, con una velocidad incidente de módulo, 2w, (ver Figura A.1) que, como es conocido, experimenta un velocidad en la estela lejana de valor el doble de la velocidad inducida en el ala, acorde con el segundo teorema de Munk y también conocido como teorema de Froude-Finsterwalder [Durand 1963; Tokaty 1971]. De esta forma el potencial complejo puede expresarse como:

$$
f(z)=2 w\left(z+\sum_{n=1}^{\infty} A_{n} z^{-n}\right),
$$

donde, $w$, es la velocidad inducida, $z$, es la variable compleja, $z=x+\mathrm{i} y, \mathrm{y}$ los términos, $A_{n}$, son constantes de valor real.

Por otro lado es posible establecer la transformación conforme que convierte la forma del perfil bidimensional $P$ (proyección del contorno aparente de la sección transversal del sistema de alas sobre el plano de Trefftz) en una placa plana. La transformada presenta un número finito de singularidades (en los vértices del polígono) sobre el plano, $\varsigma$ :

$$
\zeta=g(z)=z+\sum_{n=1}^{\infty} A_{n} z^{-n}
$$

El valor de la función en el plano $\varsigma$ puede obtenerse analíticamente haciendo uso del teorema global de la función inversa en la forma:

$$
\phi=g^{-1}(\zeta)=\zeta-A_{1} \zeta^{-1}+\sum_{n=2}^{\infty} B_{n} \zeta^{-n}
$$

donde la velocidad conjugada en dicho plano se calcula por medio de su derivada:

$$
\frac{d \phi}{d \zeta}=1+A_{1} \zeta^{-2}-\sum_{n=2}^{\infty} n B_{n} \zeta^{-(n+1)}
$$




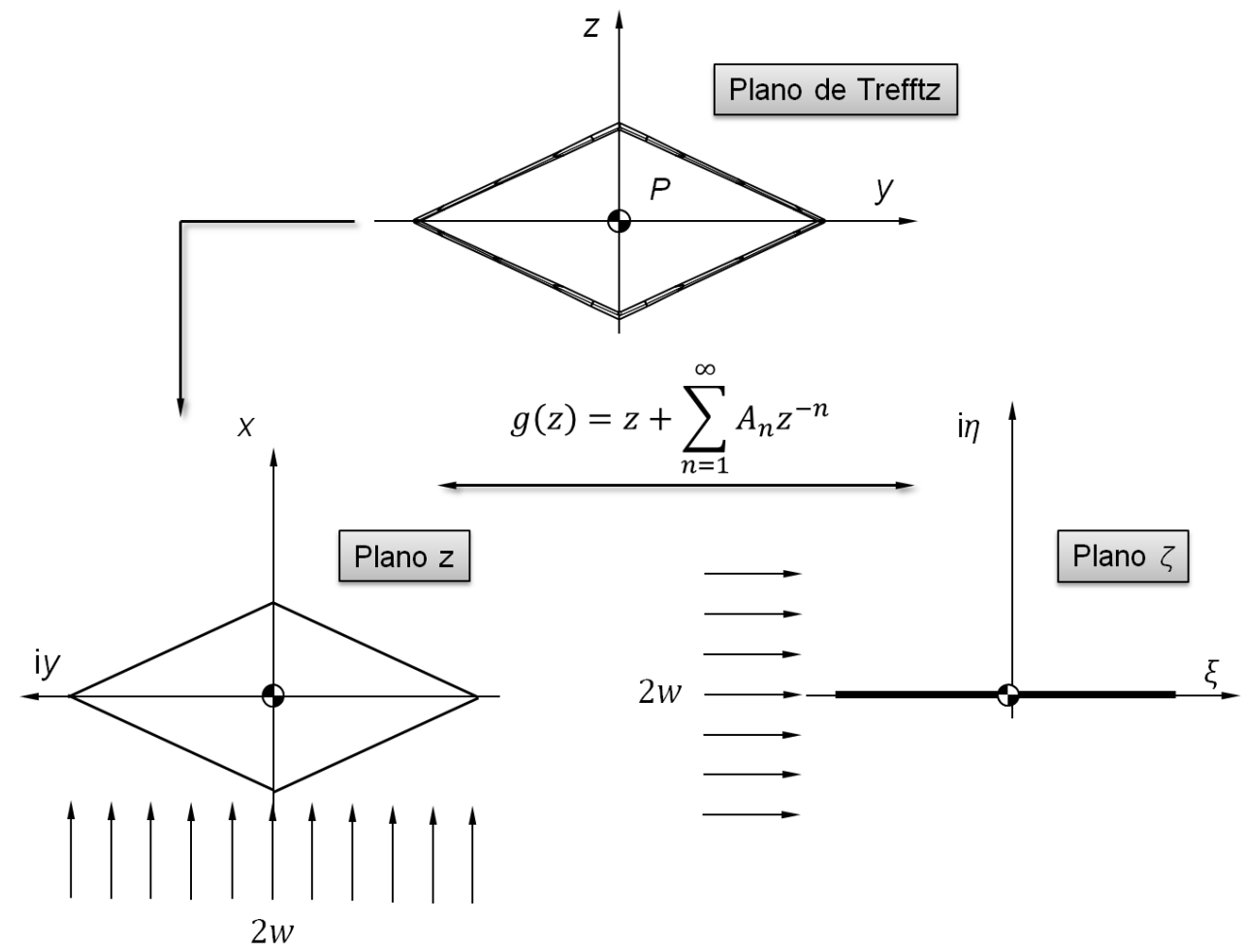

Figura A.1: Esquema de transformación para el modelo de alas en forma de diamante.

Resulta factible ahora, convertir la forma del perfil, $P$, en una línea. Haciendo uso de la transformación Schwarz-Christorffel [Churchill and Brown 1992] es posible aplicar un polígono cerrado, $P$, sobre el eje real, $\xi(\operatorname{Im}\{\zeta\}=0)$ como:

$$
\frac{\mathrm{d} \phi}{\mathrm{d} \zeta}=\prod_{j=1}^{n}\left(\zeta-\xi_{j}\right)^{-k_{j}}
$$

siendo, $\xi_{j}$, son las, $n$ coordenadas en el eje real, $\xi$, de cada vértice transformado del polígono, $P, \mathrm{y}, k_{j}$, el ángulo entre cada lado, y cuyo valor puede relacionarse con el ángulo interior, $\gamma_{j}$, como:

$$
k_{j}=1-\frac{1}{\pi} \theta_{j}
$$

teniendo en cuenta que al tratase de polígonos cerrados debe cumplirse que $\sum_{j=1}^{n} k_{j}=0$, y que $\theta_{j}=\pi / 2-\gamma_{j}$ corresponde al ángulo exterior de los lados del semi polígono.

De esta forma, para el modelo de alas en forma de diamante (ver Figura A.2) la transformada de Schwarz-Christoffel queda:

$$
\frac{\mathrm{d} \phi}{\mathrm{d} \zeta}=(\zeta-c)^{-\gamma} \zeta^{2 \gamma}(\zeta+c)^{-\gamma}
$$

Agrupando términos, la expresión (A.8) puede escribirse como: 


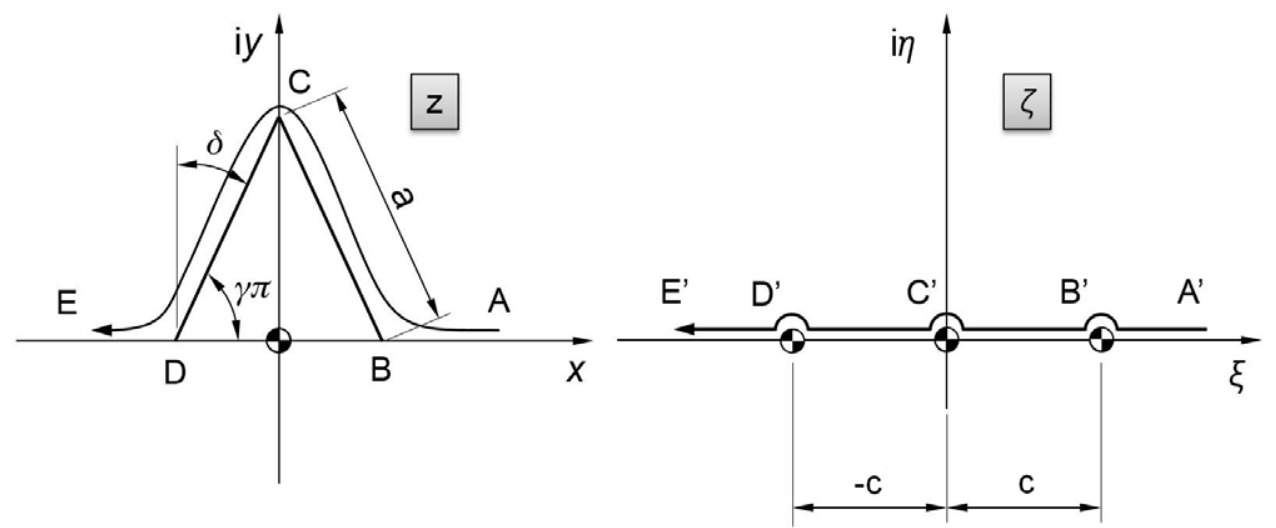

Figura A.2: Esquema de transformación Schwarz-Christoffel para el modelo de alas en forma de diamante. El parámetro $a$ representa el lado del polígono.

$$
\frac{\mathrm{d} \phi}{\mathrm{d} \zeta}=\left(c^{2}-\zeta^{-2}\right)^{-\gamma}
$$

y cuyo desarrollo en serie de Poincaré (serie asintótica) es:

$$
\frac{\mathrm{d} \phi}{\mathrm{d} \zeta}=\sum_{n=0}^{\infty}(-c)^{n}\left(\begin{array}{c}
-\gamma \\
n
\end{array}\right) \zeta^{-2 n}=1+c^{2} \gamma \zeta^{-2}+\frac{1}{2} c^{4}(\gamma+1) \gamma \zeta^{-4}+\mathcal{O}\left(\zeta^{-6}\right)
$$

Comparando este resultado con el obtenido en (A.5) es fácil identificar el término, $A_{1}$, que para este caso corresponde con el valor del ángulo interior, es decir $A_{1}=c^{2} \gamma$, lo que implica que la masa añadida por unidad de longitud es $M=2 \pi \rho c^{2} \gamma$

El ajuste de la dimensión del polígono, $P$, puede realizarse calculando el valor de $a$ en función del ángulo interior, $\gamma$, mediante la integral definida:

$$
a(\gamma)=\int_{0}^{c} \frac{\xi^{2 \gamma}}{\left(c^{2}-\xi^{2}\right)^{\gamma}} \mathrm{d} \xi=c \int_{0}^{1}\left(\frac{t}{1-t}\right)^{\gamma} \mathrm{d} t=c \frac{\Gamma(1-\gamma) \Gamma\left(\frac{1}{2}+\gamma\right)}{\sqrt{\pi}}
$$

donde $\Gamma$ hace referencia a la función matemática gamma y siendo el valor de de coordenada $c$ :

$$
c=a \frac{\sqrt{\pi}}{\Gamma(1-\gamma) \Gamma\left(\frac{1}{2}+\gamma\right)}
$$

Teniendo en cuenta este resultado, la masa añadida por unidad de longitud adimensional, $\bar{M}$, puede escribirse como: $M / \rho \pi a^{2}$ y cuyo valor queda:

$$
\bar{M}=\frac{2 \pi \gamma}{\left[\Gamma(1-\gamma) \Gamma\left(\frac{1}{2}+\gamma\right)\right]^{2}}
$$




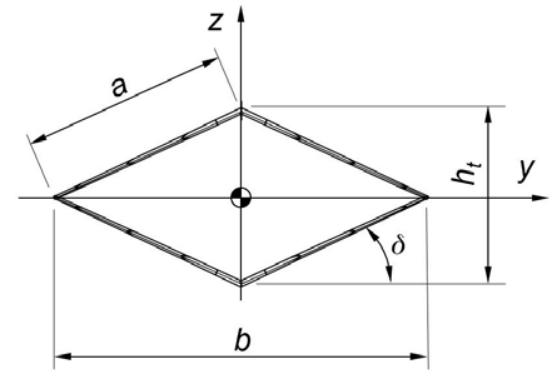

(a)

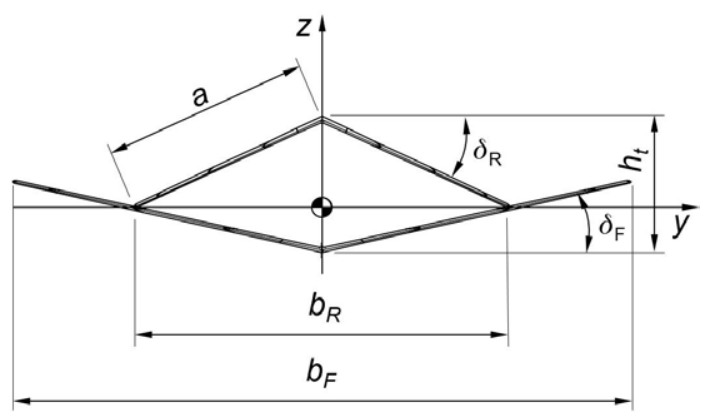

(b)

Figura A.3: Esquema de parámetros geométricos: (a) Modelo de alas en forma de diamante; (b) Modelo de alas convergentes

Por otro lado Letcher establece, aplicando los teoremas de conservación de la cantidad de movimiento y de energía cinética [Von Karman and Burgers 1963], la relación entre la resistencia inducida, $D_{i}$, la sustentación, $L$, y la masa añadida, $M$, como:

$$
D_{i}=\frac{L^{2}}{2 M U^{2}}=\frac{L^{2}}{4 \pi \frac{1}{2} \rho U^{2} b^{2} \frac{a^{2}}{b^{2}}\left(\frac{M}{\rho \pi a^{2}}\right)}
$$

Por lo tanto el valor del coeficiente de resistencia inducida $C_{D i}$ puede expresarse como:

$$
C_{D i}=\frac{C_{L}^{2}}{\pi \Lambda 4 \frac{a^{2}}{b^{2}} \bar{M}}
$$

siendo entonces el factor de eficiencia de Oswald, $e$ :

$$
e=4 \frac{a^{2}}{b^{2}} \bar{M}
$$

Teniendo en cuenta que el valor de la longitud del lado, $a$, está relacionado con la envergadura, $b$, y la separación vertical entre el ala delantera y el ala trasera, $h_{t}$, en la forma (ver Figura A.3):

$$
a^{2}=\frac{b^{2}+h_{t}^{2}}{4}
$$

y considerando el parámetro $H$ como el valor adimensional de la separación vertical, que para el caso de alas en forma de diamante puede ser calculado directamente en función del valor del ángulo diedro común, $\delta$, como:

$$
H=\frac{h_{t}}{b}=\tan \delta
$$

el factor de eficiencia puede expresarse como: 


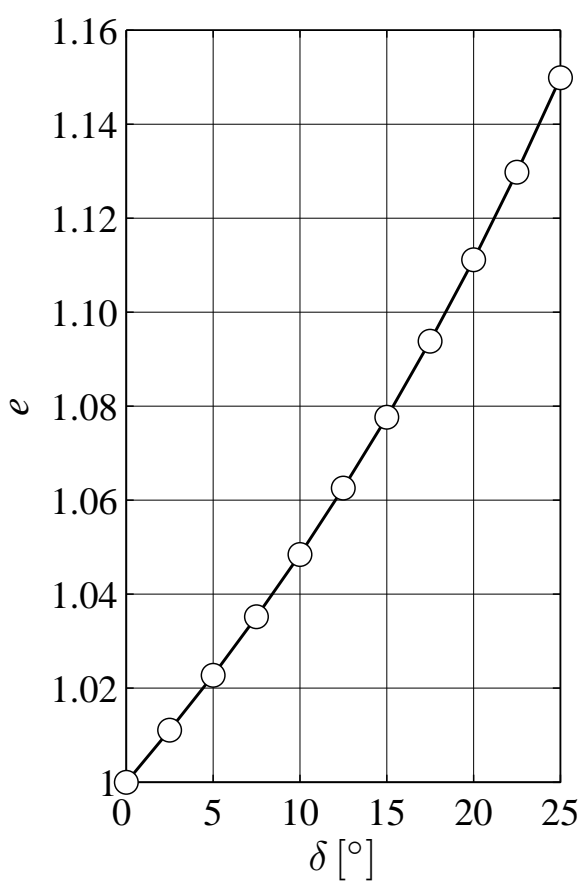

Figura A.4: Modelo de Letcher del factor de eficiencia aerodinámica $e$ en función del ángulo diedro $\delta$ para alas en forma de diamante [Letcher 1972].

$$
e=\frac{\pi\left(1+H^{2}\right)\left(1-\frac{2}{\pi} \tan ^{-1} H\right)}{\left[\Gamma\left(\frac{1}{2}+\frac{1}{\pi} \tan ^{-1} H\right) \Gamma\left(1-\frac{1}{\pi} \tan ^{-1} H\right)\right]^{2}}
$$

Este valor está representado en la Figura A.4 y muestra su variación en función del ángulo diedro, $\delta$.

\section{A.3. El modelo geométrico de DeYoung.}

John DeYoung, en 1980 [DeYoung 1980], desarrolló una ecuación a partir del cálculo de la solución exacta del factor de eficiencia de Oswald, e. Esta ecuación es aplicable, de forma general, para cualquier configuración de alas y para cualquier forma en su sección transversal. El cálculo se basa en la solución de 8 configuraciones básicas: alas en forma de caja rectangular (rectangular boxwing) [Von Karman and Burgers 1963], en forma elíptica (eliptical boxwing) [Cone 1962], en forma de diamante (diamond wing) [Letcher 1972], alas en forma de v ( $v$-wing) [Letcher 1972], alas con planos en punta de ala (end-plate wing [Von Karman and Burgers 1963], biplanos cruciformes a $90^{\circ}$ [DeYoung 1979], alas circulares y biplanos en general.

La función que determina el factor de eficiencia está basada en parámetros de forma y tiene en cuenta criterios geométricos (ver Figura A.3) como el perímetro y el área encerrada en la sección transversal, $P$, la unión relativa entre ala delantera y ala trasera, $B$, así como parámetros relacionados con el número de elementos sustentadores. El valor de esta función es: 


$$
e=\left[1+\frac{4}{\pi} H_{a}\left[1-\left(1-\left(\frac{4}{\pi}\right)^{2} \frac{H_{a}}{H} B_{a}\right)\left(1-\frac{H_{a}}{H} B_{a}\right) G_{0}\right]\right](1+f)+f_{1}
$$

donde el parámetro, $H_{a}=S_{e} / b^{2}$, representa el valor adimensional del área encerrada, $S_{e}$, en la sección transversal del perfil, $P, H$, es el valor adimensional de la separación vertical entre el ala delantera y el ala trasera y, $B_{a}$, es el valor medio de la coordenada lateral adimensional, $2 \frac{y}{b}$, del sistema de alas en la sección transversal.

Por otro lado la función $G_{0}$ está relacionada con el valor adimensional de la separación vertical entre alas, $H$, y la relación entre envergaduras, $B$. La función $f$ relaciona el valor adimensional del perímetro, $p$, de la sección transversal, $P$, por medio de la integral elíptica completa de segunda especie de módulo $K$. La función $f_{1}$ establece correcciones para los casos donde el sistema incorpora planos verticales finales en punta de alas con diedro, siendo el valor de esta función nulo para el caso de estudio.

Teniendo en cuenta todo lo anterior para el caso de alas en forma de diamante estos valores quedan:

$$
\begin{aligned}
& H_{a}=\frac{\frac{1}{2} b h_{t}}{b^{2}}=\frac{h_{t}}{2 b}=\frac{H}{2} \\
& B_{a}=\int_{0}^{1} \frac{2 y}{b} \mathrm{~d}\left(\frac{h}{h_{t}}\right)=\frac{1}{2} \\
& B=1 \\
& p=2 \sqrt{h_{t}^{2}+b^{2}} \\
& k= \begin{cases}\sqrt{1-H^{2}} & \text { si } H \leq 1 \\
\sqrt{1-H^{-2}} & \text { si } H \geq 1\end{cases}
\end{aligned}
$$

Con los datos de partida es fácil calcular el parámetro de Oswald y cuyo valor está representado en la Figura A.5 en función del ángulo diedro, $\delta$.

En términos generales se puede afirmar que, independientemente de la configuración del sistema de alas, el método de DeYoung es más sencillo de aplicar y cuyos resultados son prácticamente idénticos a los calculados por el método de Letcher, tal y como se puede observarse en la Tabla A.1.

\section{A.4. Aplicación del método para el modelo de alas convergentes.}

El modelo de alas convergentes difiere del modelo de alas en forma de diamante en tres parámetros: la relación de envergaduras entre el ala trasera y el ala delantera, $B$, el ángulo diedro del ala delantera, $\delta_{F}$ y el ángulo diedro del ala trasera, $\delta_{R}$ (ver Figura A.3)

Haciendo uso del planteamiento propuesto en el modelo de Letcher y con los parámetros de referencia que se muestran en el esquema de la transformación (ver Figura A.6), cuyos valores están relacionados con los parámetros geométricos del modelo como: 


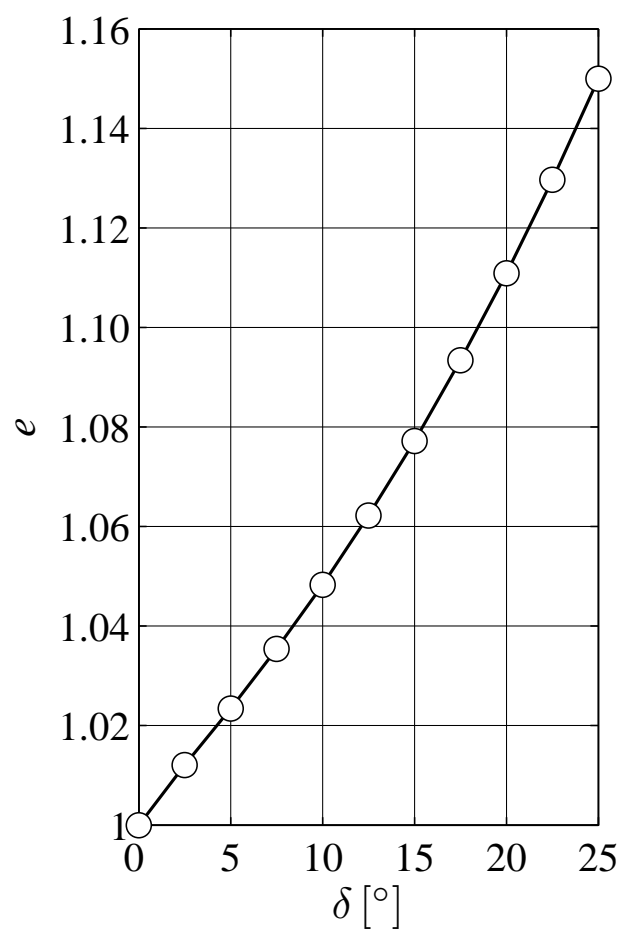

Figura A.5: Modelo de DeYoung del factor de eficiencia aerodinámica $e$ en función del ángulo diedro $\delta$ para alas en forma de diamante

Tabla A.1: Valores del factor de eficiencia según el modelo de Letcher y el modelo de DeYoung

\begin{tabular}{ccc}
\hline Ángulo diedro $\delta\left[^{\circ}\right]$ & $e$ Letcher & $e$ DeYoung \\
\hline 0 & 1 & 1 \\
2.5 & 1.011 & 1.012 \\
5.0 & 1.022 & 1.023 \\
7.5 & 1.035 & 1.035 \\
10.0 & 1.048 & 1.048 \\
12.5 & 1.062 & 1.062 \\
15.0 & 1.077 & 1.077 \\
17.5 & 1.094 & 1.093 \\
20.0 & 1.111 & 1.110 \\
22.5 & 1.130 & 1.129 \\
25.0 & 1.149 & 1.150 \\
\hline
\end{tabular}

$$
\begin{gathered}
L_{F_{t}}=\frac{b_{F}}{2 \cos \delta_{F}} \\
L_{R_{t}}=\frac{b_{R}}{2 \cos \delta_{R}}
\end{gathered}
$$



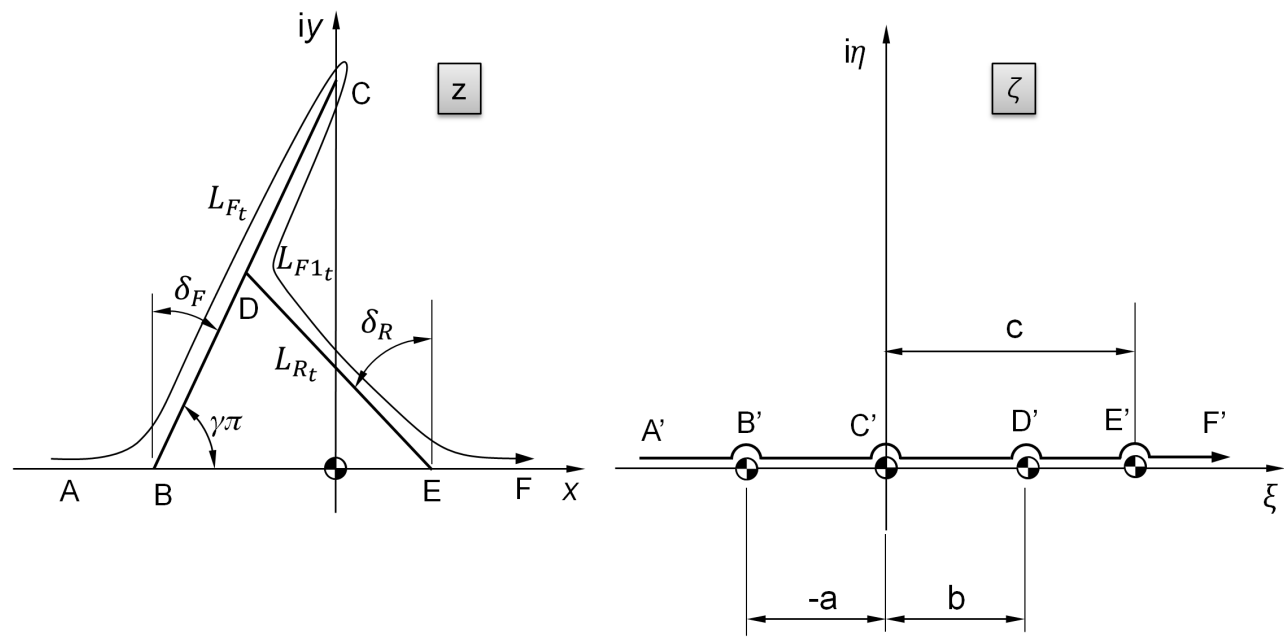

Figura A.6: Esquema de transformación Schwarz-Christoffel para el modelo de alas convergentes. El parámetro $L_{F_{t}}$ representa la longitud proyectada en el plano de Trefftz de la semiala delantera, $L_{R_{t}}$ es la longitud proyectada de la semiala trasera y $L_{F 1_{t}}$ corresponde a la longitud proyectada de la parte de la semiala delantera después del punto de unión de ambas alas.

$$
L_{F 1_{t}}=\frac{b_{F}(1-B)}{2 \cos \delta_{F}}
$$

donde el parámetro $L_{F_{t}}$ representa la longitud proyectada en el plano de Trefftz de la semiala delantera, $L_{R_{t}}$ es la longitud proyectada plano de Trefftz de la semiala trasera y $L_{F 1_{t}}$ corresponde a la longitud proyectada plano de Trefftz de la parte de la semiala delantera después del punto de unión de ambas alas, es posible adaptar la solución al modelo de alas convergentes

De esta forma, para el modelo de alas convergentes la transformada de Schwarz-Christoffel queda:

$$
\frac{\mathrm{d} \phi}{\mathrm{d} \zeta}=(\zeta+a)^{-\left(\frac{1}{2}-\delta_{F}\right)} \zeta(\zeta-b)^{-\left(\delta_{F}+\delta_{R}\right)}(\zeta-c)^{-\left(\frac{1}{2}-\delta_{R}\right)}
$$

como el valor del ángulo diedro del ala delantera, $\delta_{F}=3^{\circ}$, es pequeño, este puede despreciarse simplificando (A.25) en la forma:

$$
\frac{\mathrm{d} \phi}{\mathrm{d} \zeta}=\frac{\zeta(\zeta-b)^{-\delta_{R}}(\zeta-c)^{-\left(\frac{1}{2}-\delta_{R}\right)}}{\sqrt{\zeta+a}}
$$

y cuyo desarrollo en serie es: 


$$
\begin{aligned}
\frac{\mathrm{d} \phi}{\mathrm{d} \zeta}= & 1+\left(\frac{c}{2}+\delta_{R}(b-c)-\frac{a}{2}\right) \zeta^{-1}+ \\
& \left(\frac{3}{4} c^{2}-c^{2} \delta_{R}+\frac{1}{2} c^{2} \delta_{R}^{2}+\frac{1}{2} c b \delta_{R}-\frac{1}{4} c a-c b \delta_{R}^{2}+\right. \\
& \left.\frac{1}{2} c a \delta_{R}+\frac{1}{2} b^{2} \delta_{R}-\frac{1}{2} b a \delta_{R}+\frac{3}{4} a^{2}\right) \zeta^{-2} \\
& \mathcal{O}\left(\zeta^{-3}\right)
\end{aligned}
$$

Comparando este resultado con el obtenido en (A.5) es fácil identificar el término $A_{1}$, que corresponde con el término en $\zeta^{-2}$, es decir:

$$
\begin{aligned}
A_{1}= & \left(\frac{3}{4} c^{2}-c^{2} \delta_{R}+\frac{1}{2} c^{2} \delta_{R}^{2}+\frac{1}{2} c b \delta_{R}-\frac{1}{4} c a-c b \delta_{R}^{2}+\right. \\
& \left.\frac{1}{2} c a \delta_{R}+\frac{1}{2} b^{2} \delta_{R}-\frac{1}{2} b a \delta_{R}+\frac{3}{4} a^{2}\right)
\end{aligned},
$$

lo que implica que la masa añadida por unidad de longitud es $M=2 \pi \rho A_{1}$.

Teniendo en cuenta este resultado, la masa añadida por unidad de longitud adimensional, $\bar{M}$, puede escribirse como: $M / \rho \pi b_{F}^{2}$

El valor de $A_{1}$, se determina por medio del cálculo de de las constantes $a$, $b$ y $c$, en función de la envergadura del ala delantera, $b_{F}, \mathrm{y}$ de los ángulos diedros, $\delta_{F}$, y $\delta_{R}$, de ambas alas lo que implica que el valor de la masa añadida queda como:

$$
M=2 \pi \rho f\left(b_{F}, \delta_{F}, \delta_{R}\right)
$$

El cálculo de la constante $a$, es sencillo y pasa por identificar el primer término de (A.27), quedando:

$$
a=c+2(b-c) \delta_{R}
$$

El cálculo de las constantes $b$ y $c$, se realiza mediante el ajuste de las dimensiones del polígono $P$, (ver Figura A.6), resolviendo numéricamente las integrales definidas:

$$
\begin{gathered}
L_{F_{t}}=\frac{b_{F}}{2 \cos \delta_{F}}=\int_{0}^{a} \frac{\zeta(\zeta-b)^{-\delta_{R}}(\zeta-c)^{-\left(\frac{1}{2}-\delta_{R}\right)}}{\sqrt{\zeta+a}} \mathrm{~d} \xi \\
L_{R_{t}}+L_{F 1_{t}}=\frac{b_{R}}{2 \cos \delta_{R}}+\frac{b_{F}(1-B)}{2 \cos \delta_{F}}=\int_{0}^{c} \frac{\zeta(\zeta-b)^{-\delta_{R}}(\zeta-c)^{-\left(\frac{1}{2}-\delta_{R}\right)}}{\sqrt{\zeta+a}} \mathrm{~d} \xi
\end{gathered}
$$

Finalmente, operando de forma similar que en la sección A.2 el factor de eficiencia de Oswald, $e$, del modelo de alas convergentes queda: 


$$
e=4 \frac{M\left(b_{F}, \delta_{F}, \delta_{R}\right)}{\rho \pi b_{F}^{2}}
$$

En la Figura A.7 se muestra la variación del parámetro e, para el modelo de alas convergentes en función del ángulo diedro del ala trasera, $\delta_{R}$, para un valor de relación de envergaduras, $B=0,3$, y un ángulo diedro del ala delantera $\delta_{F}=3^{\circ}$ calculado por este método.

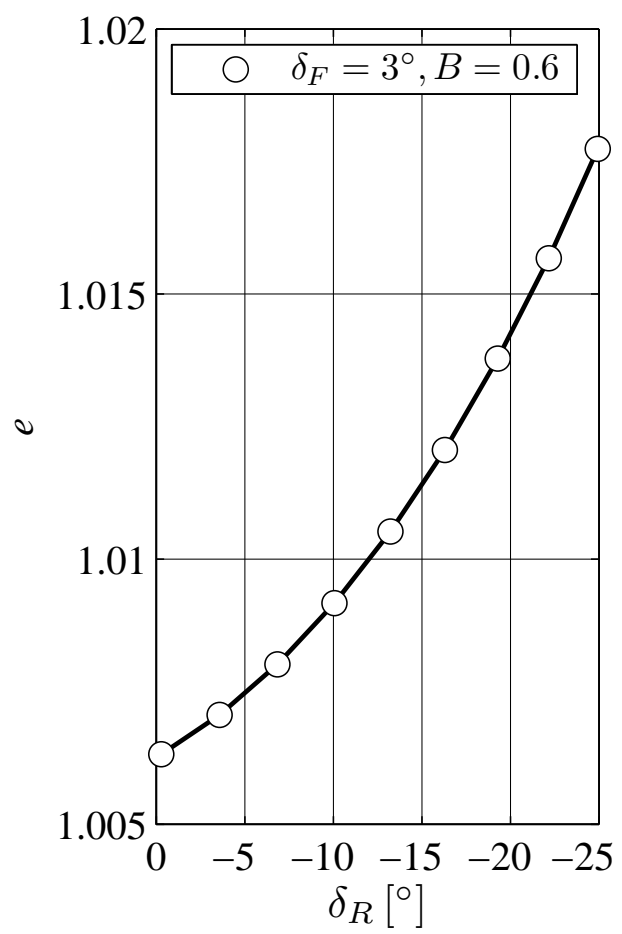

Figura A.7: Modelo de Letcher del factor de eficiencia de Oswald, e, para el modelo de alas convergentes en función del ángulo diedro del ala trasera $\delta_{R}$.

Este resultado también puede obtenerse por medio del cálculo propuesto en el modelo de DeYoung, donde es factible encontrar una solución del parámetro de Oswald adaptando el cálculo a las condiciones geométricas que se muestran en la Figura A.3, donde los valores de los parámetros de la función definida en (A.20) quedan ahora:

$$
\begin{gathered}
H_{a}=\frac{\frac{1}{2} b h_{t}}{b^{2}}=\frac{h_{t}}{2 b}=\frac{H}{2} B \\
B_{a}=\int_{0}^{1} \frac{2 y}{b} \mathrm{~d}\left(\frac{h}{h_{t}}\right)=\frac{1}{2} \\
B=\frac{b_{R}}{b_{F}} \\
h_{t}=h_{t 1}+h_{t 2}
\end{gathered}
$$




$$
\begin{gathered}
p=2\left[\sqrt{h_{t 1}^{2}+\frac{b_{F}^{2}}{4} B^{2}}+\sqrt{h_{t 2}^{2}+\frac{b_{F}^{2}}{4} B^{2}}\right] \\
h_{t 1}=B \frac{b_{F}}{2} \tan \delta_{R}, h_{t 2}=B \frac{b_{F}}{2} \tan \delta_{F} \\
k= \begin{cases}\sqrt{1-H^{2}} & \text { si } H \leq 1 \\
\sqrt{1-H^{-2}} & \text { si } H \geq 1,\end{cases}
\end{gathered}
$$

por lo que la función definida en (A.20), para el modelo de alas convergentes queda en la forma:

$$
e=\left[\left(1+\frac{2}{\pi} B H\right)\left(\frac{1}{2}+\left(\frac{2}{\pi}\right)^{2} B\right) G_{0}\right](1+f)
$$

siendo la función $G_{0}$ :

$$
G_{0}=\frac{(1-0,2 B H)\left(1+0,5(B H)^{2}\right)}{(1+1,81 B H)}
$$

Como ya se ha comentado, existen ahora tres parámetros posibles con influencia directa en el cálculo del factor de Oswald, e: la relación de envergaduras, $B$, el ángulo diedro del ala delantera, $\delta_{F}$ y el ángulo diedro del ala trasera, $\delta_{R}$. En la Figura A.8 se muestra la variación del parámetro $e$, para el modelo de alas convergentes en función del ángulo diedro del ala trasera, $\delta_{R}$, para un valor de relación de envergaduras, $B=0,3$, y un ángulo diedro del ala delantera $\delta_{F}=3^{\circ}$.

De la misma forma en la Figura A.9 se representa el valor del parámetro, $\bar{e}=e / e_{12525}$, en función del ángulo diedro del ala delantera, $\delta_{F}$, y del ala trasera, $\delta_{R}$, para varios valores de la relación de envergadura, $B=[0,5-1]$, para el modelo de alas convergentes, donde el valor $e_{12525}$ corresponde al valor del parámetro $e$ para, $B=1, \delta_{F}=25^{\circ}$ y $\delta_{R}=25^{\circ}$. 


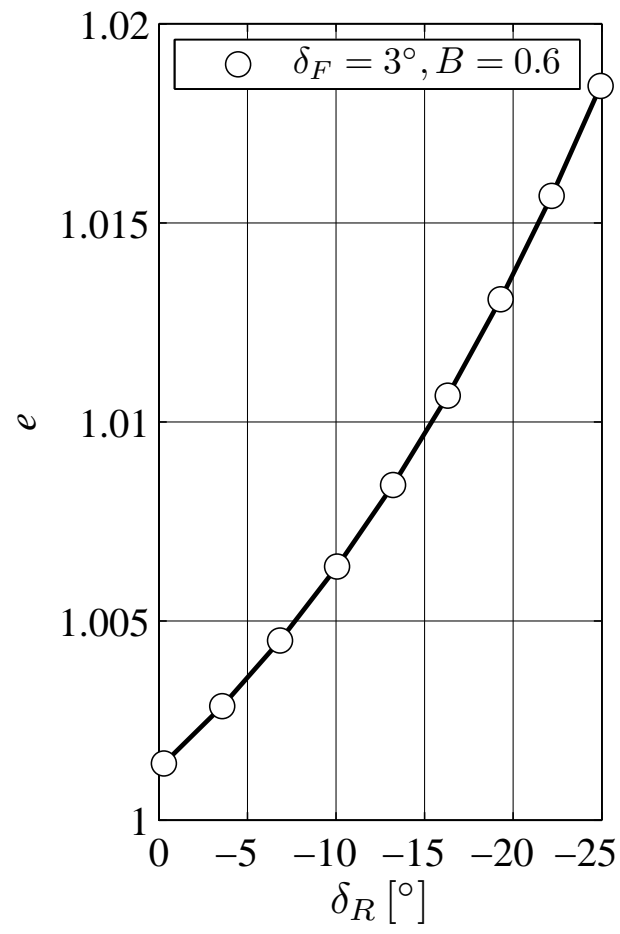

(a)

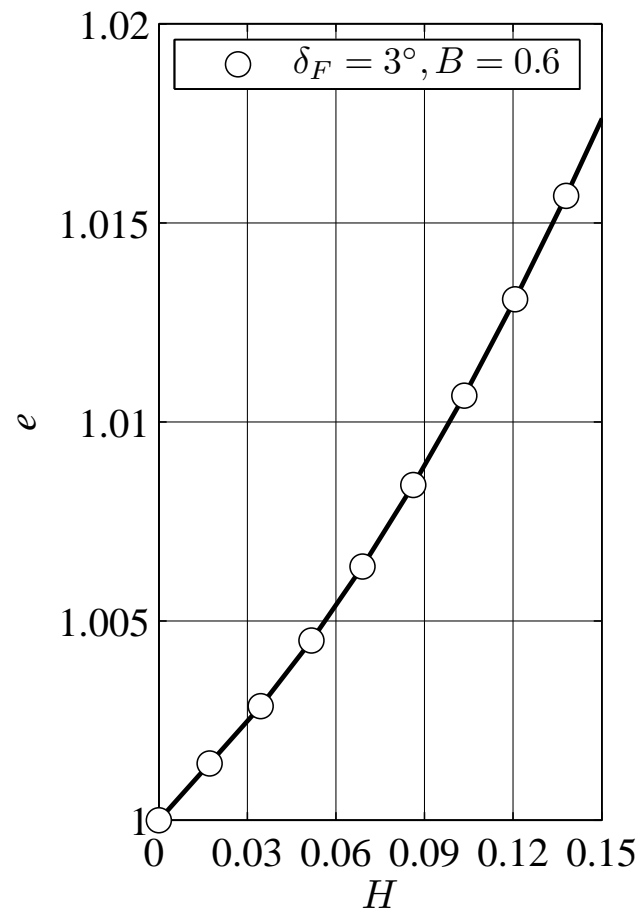

(b)

Figura A.8: Modelo de DeYoung del factor de eficiencia e para el modelo de alas convergentes: (a) en función del ángulo diedro del ala trasera $\delta_{R}$, y (b) en función de la separación vertical entre las dos alas $H$. 

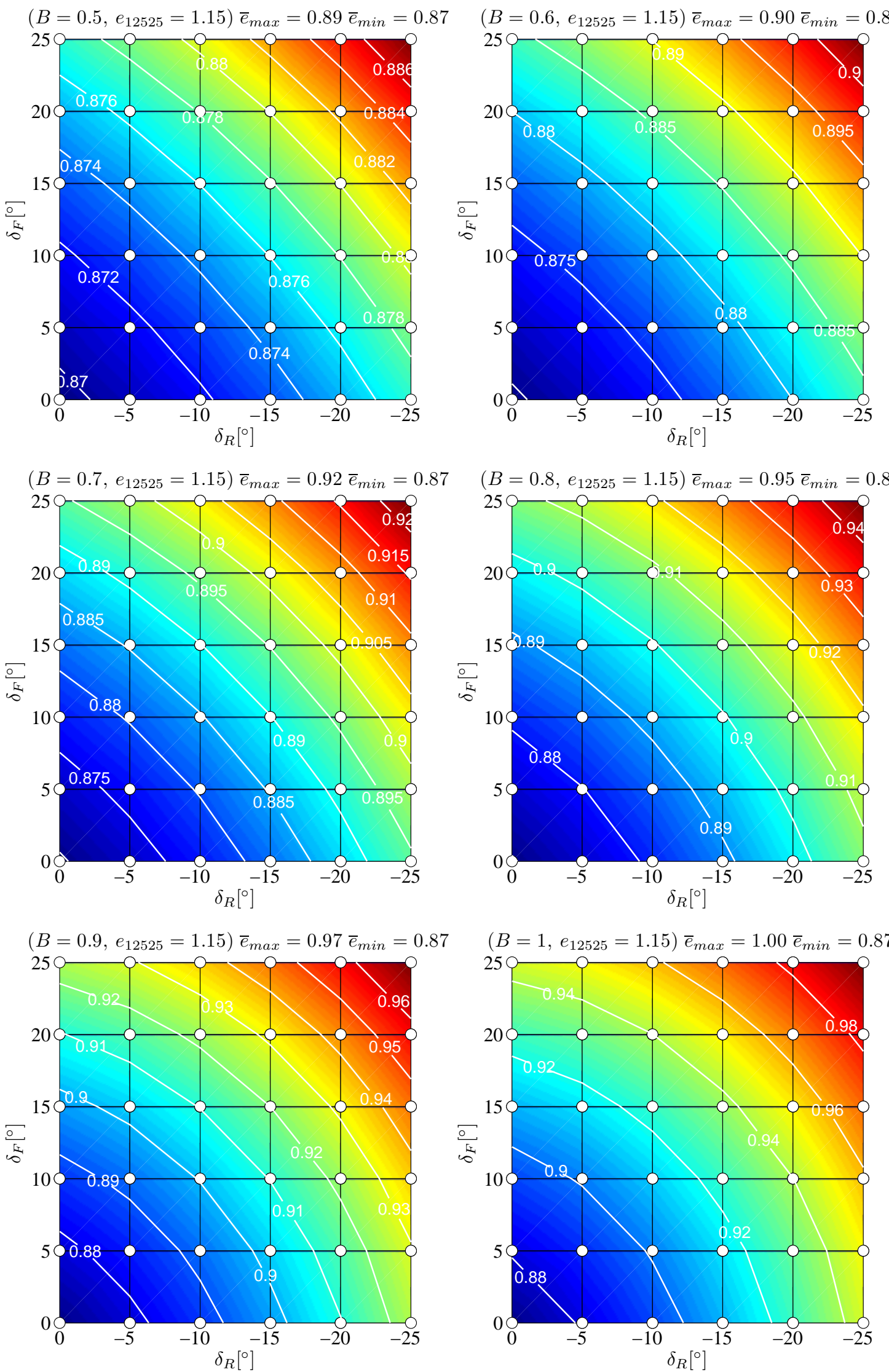

Figura A.9: Modelo de DeYoung. Factor de eficiencia aerodinámica $\bar{e}=$ $e / e_{12525}$, en función del ángulo diedro del ala delantera $\delta_{F} \mathrm{y}$ del ala trasera $\delta_{R}$, para varios valores de $B$ en el modelo de alas convergentes. 


\section{Apéndice B}

\section{Calibración túnel A9}

\section{B.1. Introducción.}

Previo a la realización de las medidas en el túnel A9 y debido a la naturaleza de los ensayos, es necesario estudiar el comportamiento del flujo en la cámara de ensayo con el fin de determinar la distribución de intensidad de turbulencia, $I_{T}$, de la velocidad, $U_{x}$, en dirección del eje $x_{T}$ del túnel.

Para la determinación de la intensidad de turbulencia se mide de la velocidad del viento incidente en la cámara de ensayo del túnel A9 en vacío, es decir sin modelo alguno, usando un equipo de anemometría de hilo caliente DANTEC con sonda de una componente y sistema de guías orientables programables para el posicionamiento del sensor de manera automática.

\section{B.2. Procedimiento de calibración.}

Para obtener una amplia resolución espacial se mide la velocidad en 132 puntos contenidos en el plano transversal a la cámara de ensayo (plano $y_{T} z_{T}$ con $x_{T}=0$, Figura 4.9), repartidos en una matriz de 11 filas por 12 columnas, con un tiempo de medida de $t_{T}=120 \mathrm{~s}$ por punto, una frecuencia de $f_{T}=100 \mathrm{~Hz} \mathrm{y}$ con una velocidad del túnel medida con el tubo Pitot en la cámara de ensayo de $U_{x}=28 \mathrm{~m} / \mathrm{s}$.

La posición y las dimensiones de la matriz de coordenadas de los puntos de medida con respecto al centro de la cámara están representadas en la Figura B.1.

Obtenidas las series temporales de velocidad en cada punto es posible determinar el valor de la intensidad de turbulencia calculada mediante la expresión:

$$
I_{T}=\frac{\sigma_{U_{X}}}{\overline{U_{X}}}
$$

donde $\overline{U_{x}}$ es la velocidad media de cada serie temporal de velocidades y $\sigma_{U_{x}}$ es la desviación estándar. 

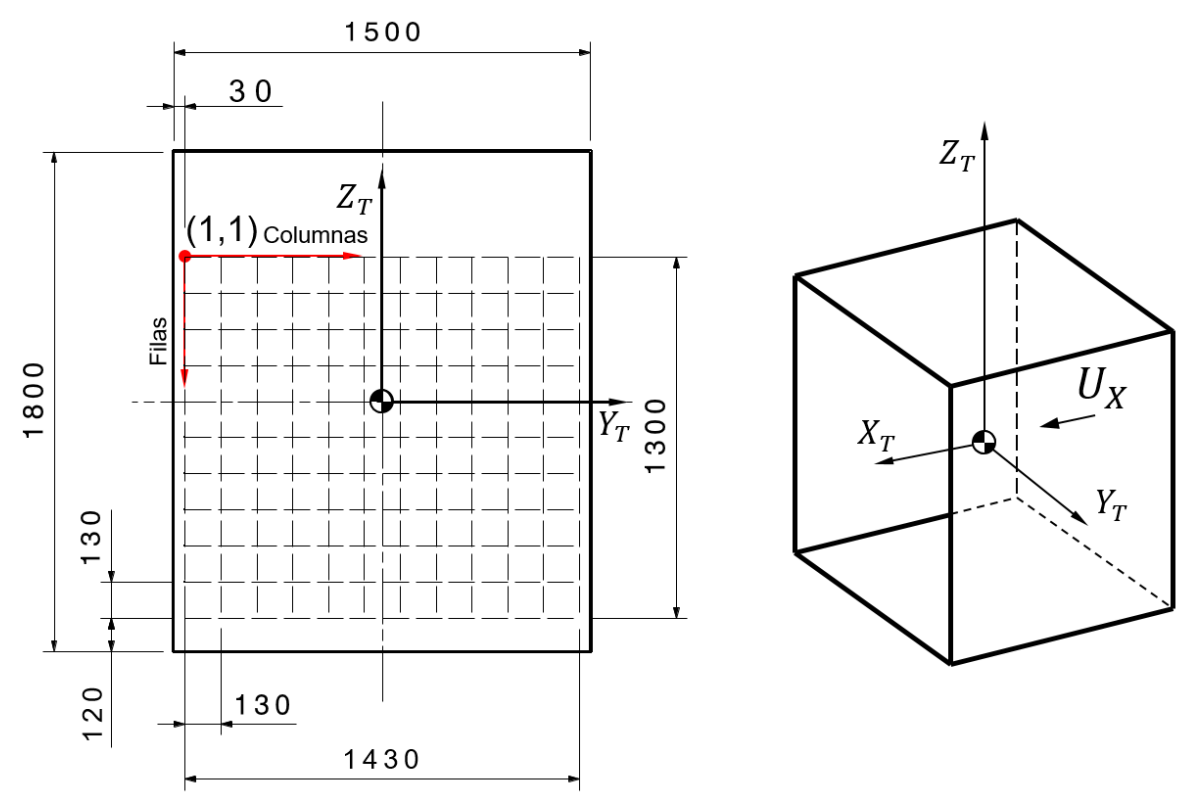

Figura B.1: Coordenadas de la matriz de puntos de medida y sistema de referencia para la determinación de la intensidad de turbulencia, $I_{T}$. Dimensiones en $\mathrm{mm}$.

En primer lugar es necesario verificar que los parámetros elegidos de frecuencia y tiempo de muestreo proporcionan resultados válidos de cara al cálculo de la intensidad de turbulencia. Para ello se analizan las medidas realizadas en un punto arbitrario de la matriz de medidas (fila 5, columna 6) y se estudia la convergencia del valor de la media de la velocidad, $\overline{U_{x}}$, y de su desviación estándar, $\sigma_{U_{x}}$, comparando el valor de la medida para cada instante $t(\triangle t=0,5 \mathrm{~s})$, con el valor de la medida para $t_{T}$, es decir para $t=120 \mathrm{~s}$. Como se observa en la Figura B.2 el valor de la desviación de la media de la velocidad es menor de $0.1 \%$ para tiempos mayores de $t=40 \mathrm{~s}$ (Figura B.2 a) y el valor de la desviación de la desviación estándar es menor de un $0.25 \%$ para tiempos mayores de $t=40 \mathrm{~s}$ (Figura B.2 b).

A la vista de los resultados se puede concluir que los valores elegidos para la realización de las medidas son válidos de cara a los ensayos que incluyen el modelo de ensayo.

Con estos datos es fácil representar la distribución de velocidad en la cámara de ensayo medida con sonda de hilo caliente, $\overline{U_{x h w}}$, (Figura B.3), la distribución de velocidad medida con hilo caliente con respecto a la medida con tubo Pitot, $\overline{U_{x p t}}$, (Figura B.4) y la distribución de intensidad de turbulencia media medida en la cámara de ensayo (Figura B.5).

Como puede comprobarse existen tres zonas claramente diferenciadas: una zona entre las filas 3 y 6 donde la intensidad de turbulencia es del $2.2 \%$, otra zona entre las filas 7 y 11 donde la intensidad de turbulencia es del $3 \%$ y una tercera zona entre las columnas 1 y 2 y 11 y 12, próximas a las paredes laterales de la cámara de ensayo, donde la intensidad de turbulencia es mayor de $3 \%$. 


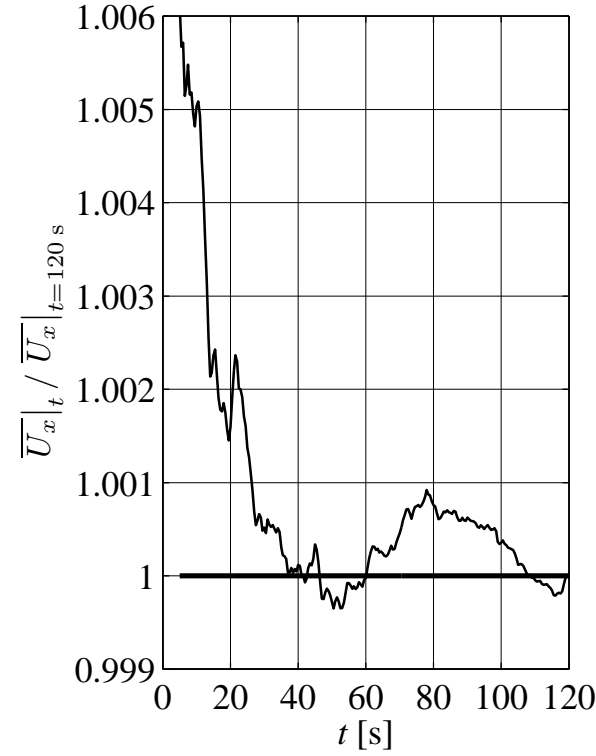

(a)

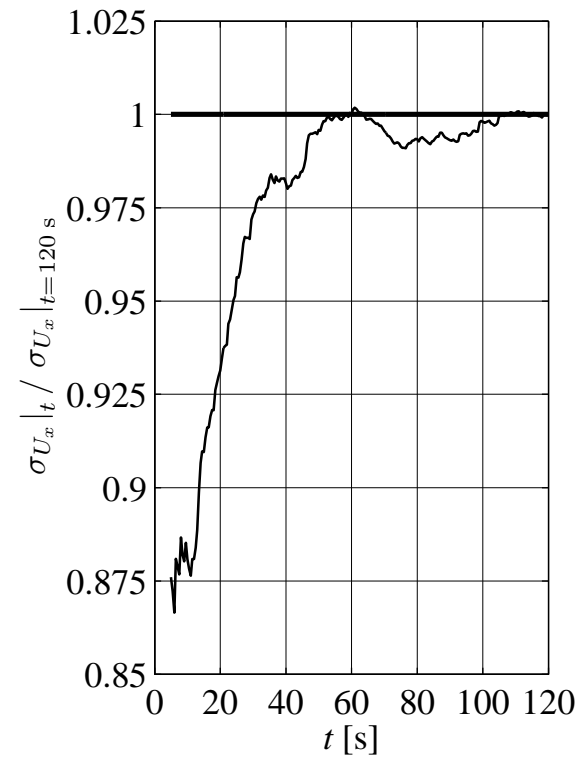

(b)

Figura B.2: Valores de la convergencia del valor de la media de la velocidad, $\overline{U_{x}}$ (a) y de la desviación estándar de la velocidad, $\sigma_{U_{x}}$ (b) en función del tiempo de medida, $t$, para un punto situado en la fila 5 y columna 6 .

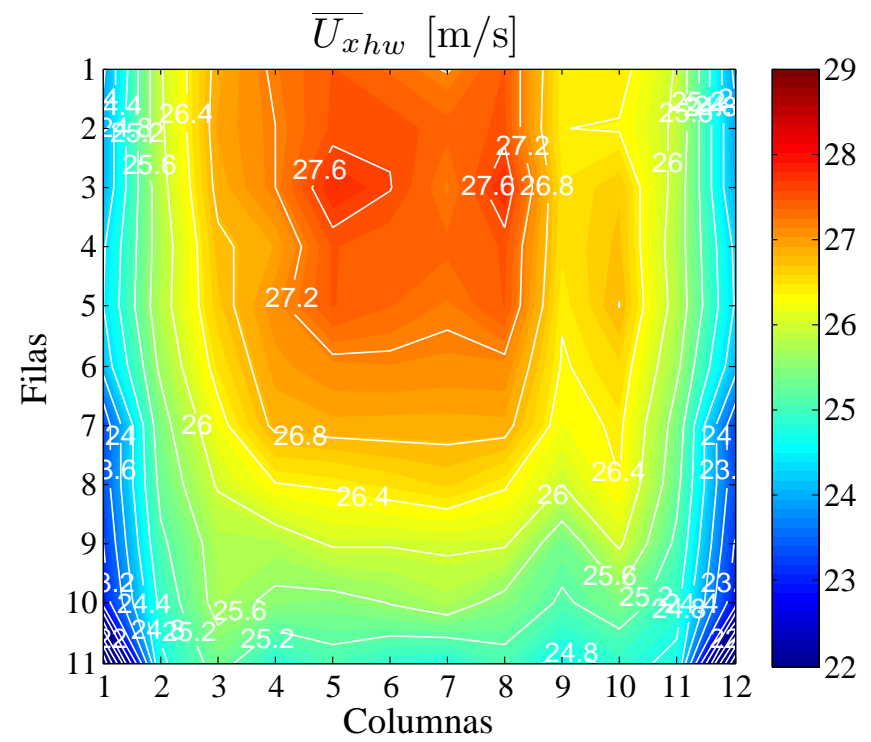

Figura B.3: Distribución de velocidad en la cámara de ensayo medida con sonda de hilo caliente, $\overline{U_{x}}$, en la cámara de ensayo del túnel A9. 


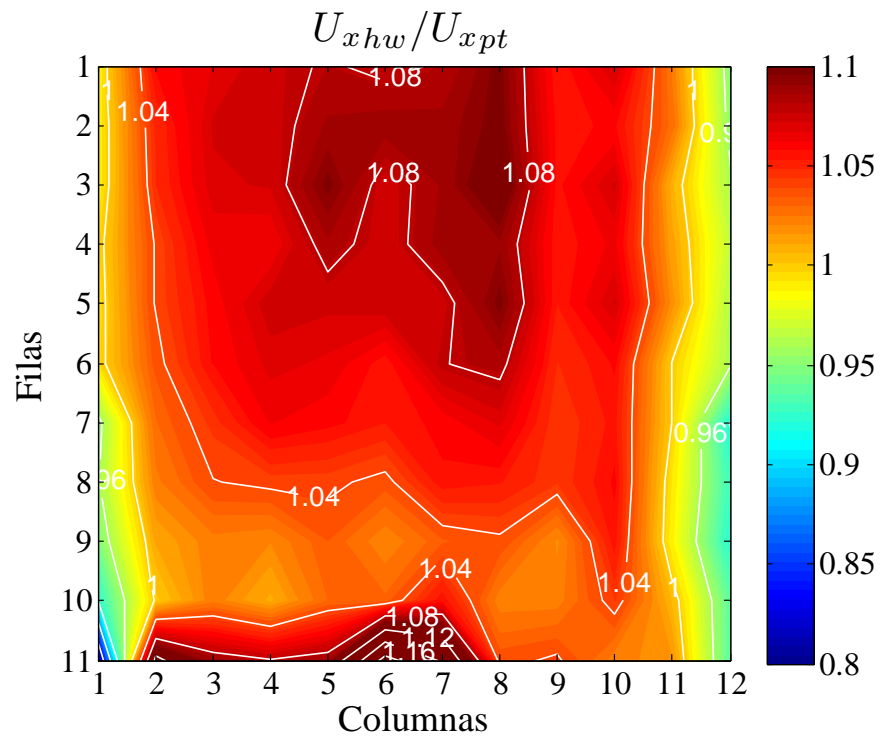

Figura B.4: Distribución de velocidad en la cámara de ensayo medida con sonda de hilo caliente, $\bar{U}_{x}$ ww , comparada con la velocidad medida por tubo Pitot, $\overline{U_{x p t}}$, en la cámara de ensayo del túnel A9.

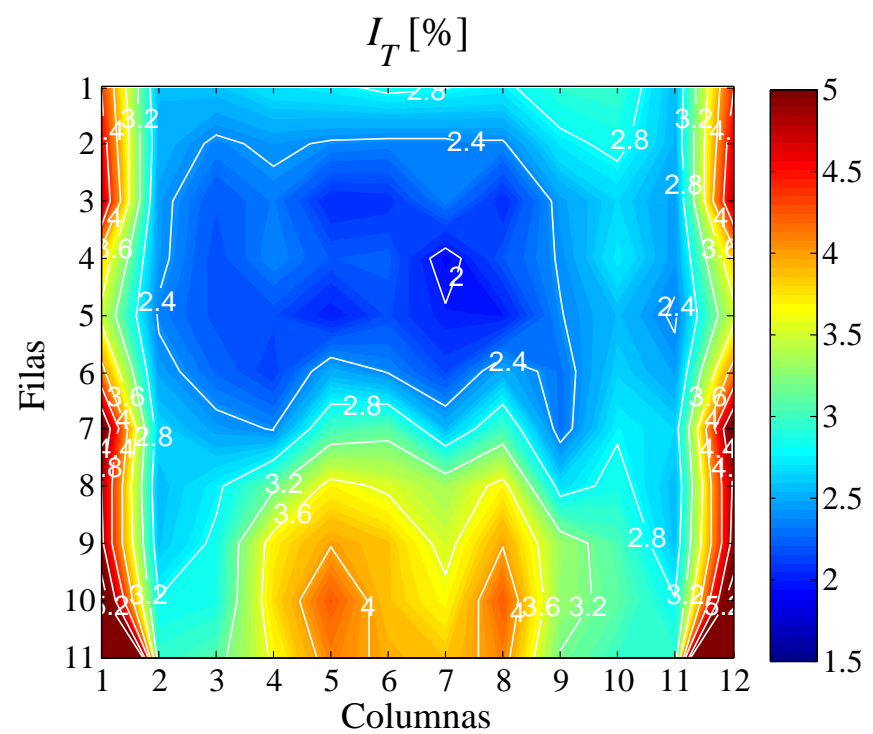

Figura B.5: Distribución de la intensidad de turbulencia en el plano transversal $y_{T} z_{T}$ de la cámara de ensayo del túnel A9. 


\section{Apéndice C}

\section{Resultados de ensayo 1}

\section{C.1. Introducción.}

En este apéndice se presenta los resultados completos de los valores de los coeficientes aerodinámicos del modelo de alas convergentes en función del ángulo diedro $\delta_{R}=\left[-5^{\circ},-25^{\circ}\right]$, del ángulo de flecha del ala trasera $\varphi_{R}=\left[-10^{\circ},-30^{\circ}\right]$, para ángulos de ataque $\alpha=\left[-14^{\circ}, 15^{\circ}\right]$.

En la sección C.2 están representados los valores de los coeficientes aerodinámicos en función del ángulo de ataque, $\alpha$, agrupados en función del ángulo diedro y del ángulo de flecha del ala trasera. Los coeficientes representados son:

- En la Figura C.1 están representados los valores del coeficiente de sustentación, $C_{L}$, para ángulos de ataque $\alpha=\left[-15^{\circ}, 15^{\circ}\right]$.

- En la Figura C.2 están representados los valores del coeficiente de resistencia, $C_{D}$, para ángulos de ataque $\alpha=\left[-15^{\circ}, 15^{\circ}\right]$.

- En la Figura C.3 están representados los valores del coeficiente de resistencia inducida, $C_{D i}$, para ángulos de ataque $\alpha=\left[-15^{\circ}, 15^{\circ}\right]$.

- En la Figura C.4 están representados los valores de la eficiencia aerodinámica, $E=C_{L} / C_{D}$, para ángulos de ataque $\alpha=\left[-15^{\circ}, 15^{\circ}\right]$.

- En la Figura C.5 están representados los valores de coeficiente de momento de cabeceo, $C_{M y}$, para ángulos de ataque $\alpha=\left[-15^{\circ}, 15^{\circ}\right]$.

- En la Figura C.6 están representados los valores de la relación de coeficientes, $C_{L}^{3 / 2} / C_{D}$, para ángulos de ataque $\alpha=\left[-15^{\circ}, 15^{\circ}\right]$.

- En la Figura C.7 están representados los valores del coeficiente de resistencia, $C_{D}$, pero en este caso sólo para ángulos de ataque $\alpha=\left[-9^{\circ}, 9^{\circ}\right]$.

- En la Figura C.8 están representados los valores del coeficiente de resistencia inducida, $C_{D i}$, pero en este caso sólo para ángulos de ataque $\alpha=\left[-9^{\circ}, 9^{\circ}\right]$. 
- En la Figura C.9 están representados los valores de la eficiencia aerodinámica, $E=C_{L} / C_{D}$, para ángulos de ataque $\alpha=\left[3^{\circ}, 12^{\circ}\right]$.

- En la Figura C.10 están representados los valores de la relación de coeficientes, $C_{L}^{3 / 2} / C_{D}$, para ángulos de ataque $\alpha=\left[6,12^{\circ}\right]$.

En la sección C.3 están representados los valores de los coeficientes de los coeficientes aerodinámicos en función del coeficiente de resistencia, $C_{D}$, agrupados en función del ángulo diedro y del ángulo de flecha del ala trasera. Los coeficientes representados son:

- En la Figura C.11 están representados los valores del coeficiente de sustentación, $C_{L}$, para valores de $C_{D}=[0,0,35]$.

- En la Figura C.12 están representados los valores del cuadrado del coeficiente de sustentación, $C_{L}^{2}$, para valores de $C_{D}=[0,0,35]$.

- En la Figura C.13 están representados los valores del coeficiente de sustentación, $C_{L}$, para valores de $C_{D}=[0,04,0,16]$.

En la sección C.4 están representados los valores de los coeficientes de los coeficientes aerodinámicos en función del coeficiente de resistencia, $C_{L}$, agrupados en función del ángulo diedro y del ángulo de flecha del ala trasera. Los coeficientes representados son:

- En la Figura C.14 están representados los valores de la eficiencia aerodinámica, $E=C_{L} / C_{D}$.

- En la Figura C.15 están representados los valores de coeficiente de momento de cabeceo, $C_{M y}$. 


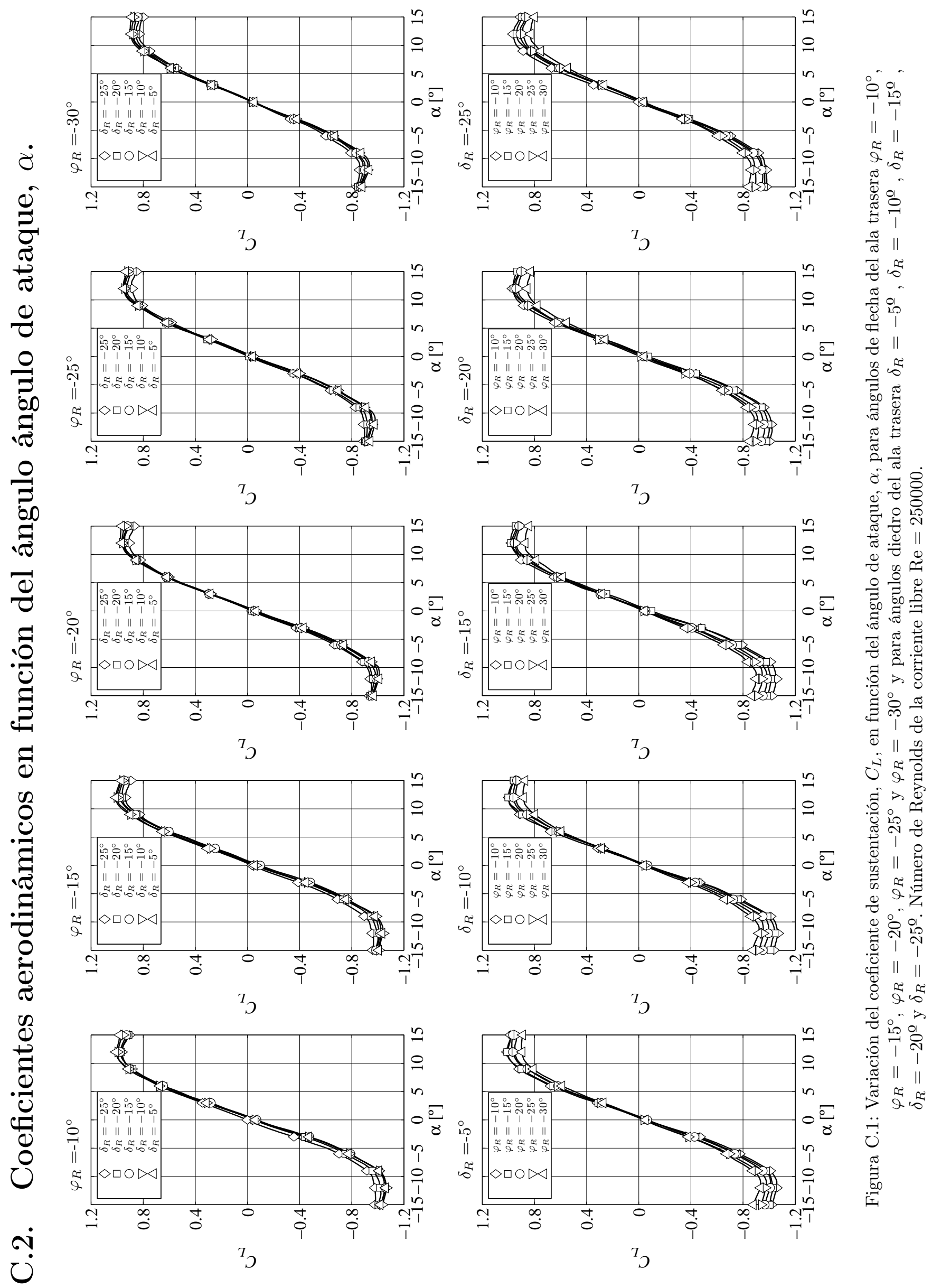



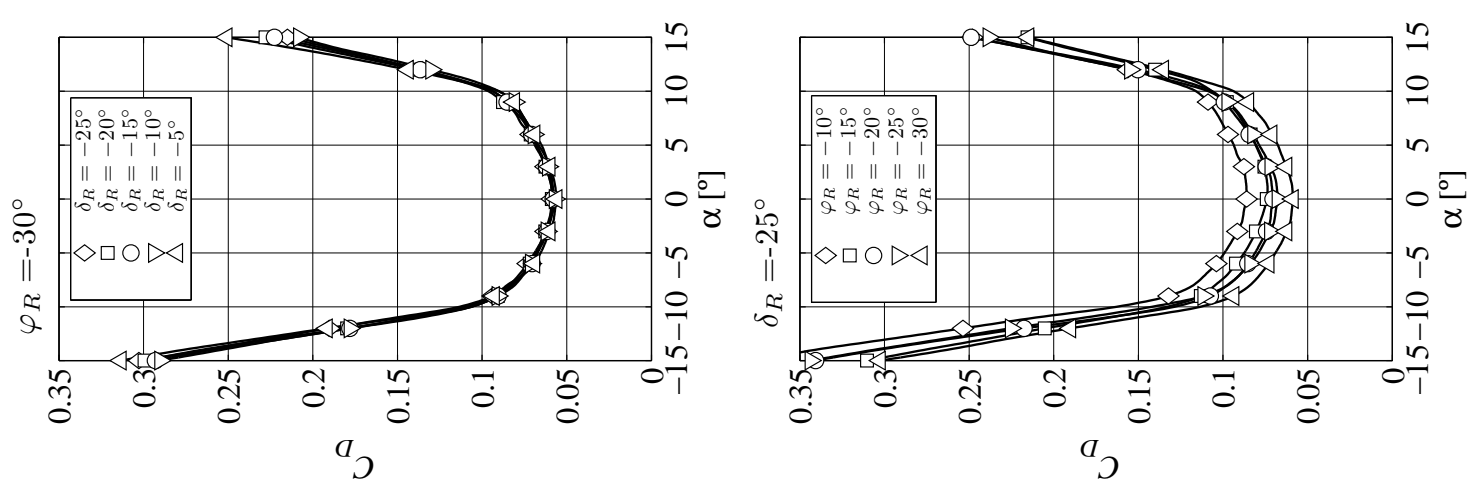

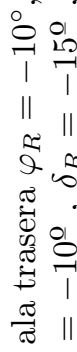
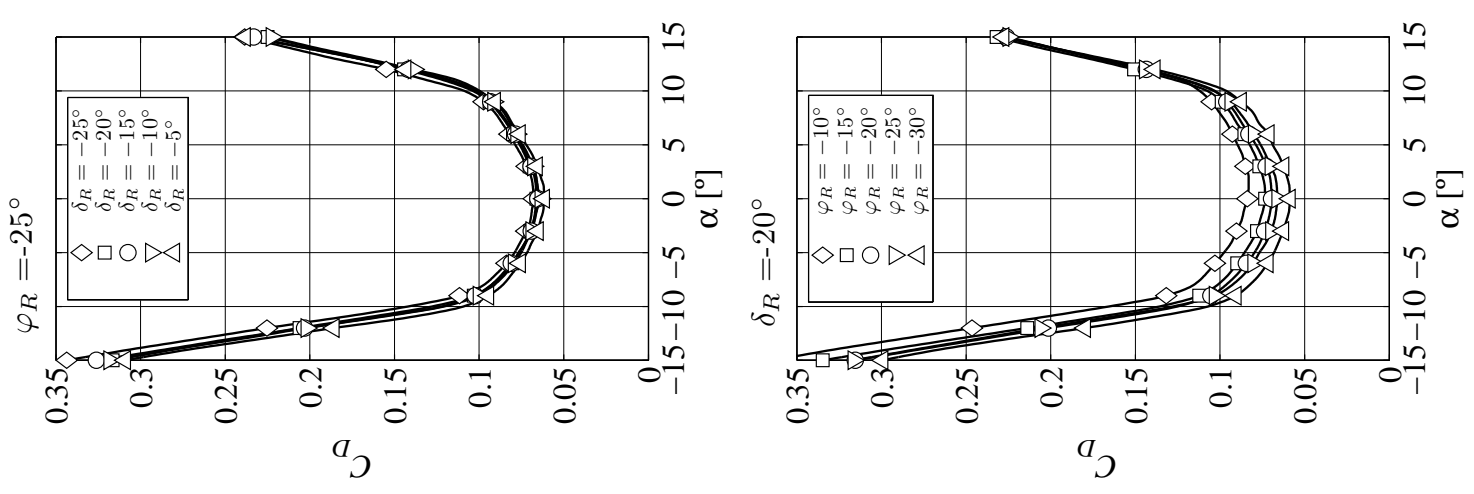

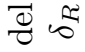

承 i

ช $\|$

告

ప్రు

范

๘ $\frac{\pi}{\pi}$

ठ응

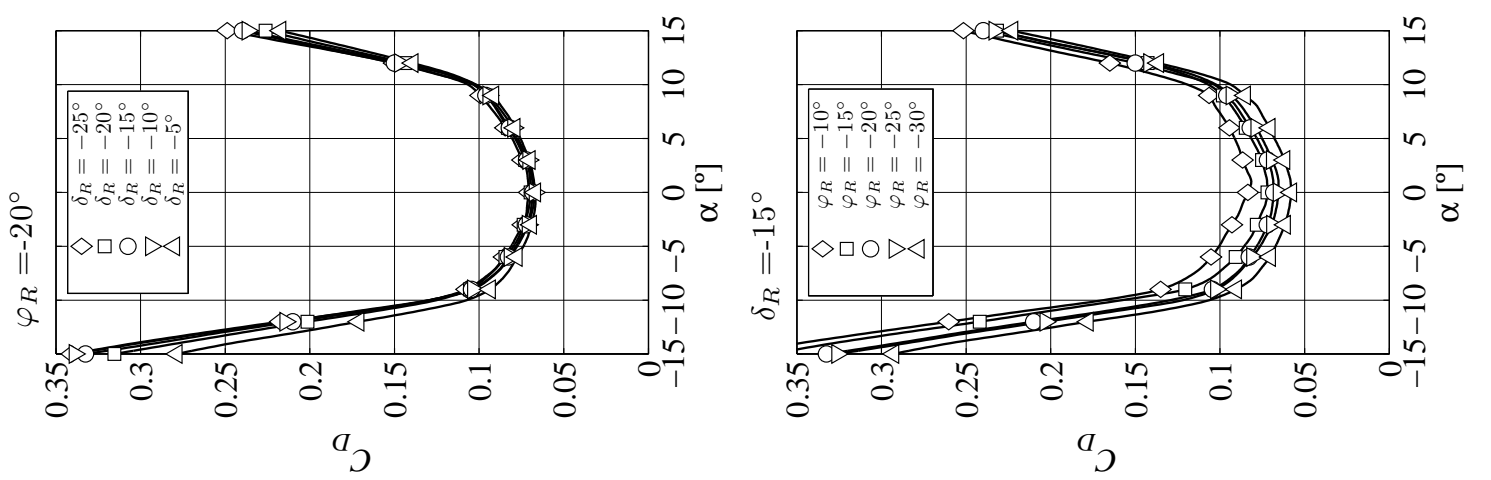

앙 옹

๘

ช 0 م

을 을 을

雨

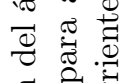

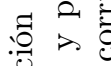

异 융

궁

- 11 告

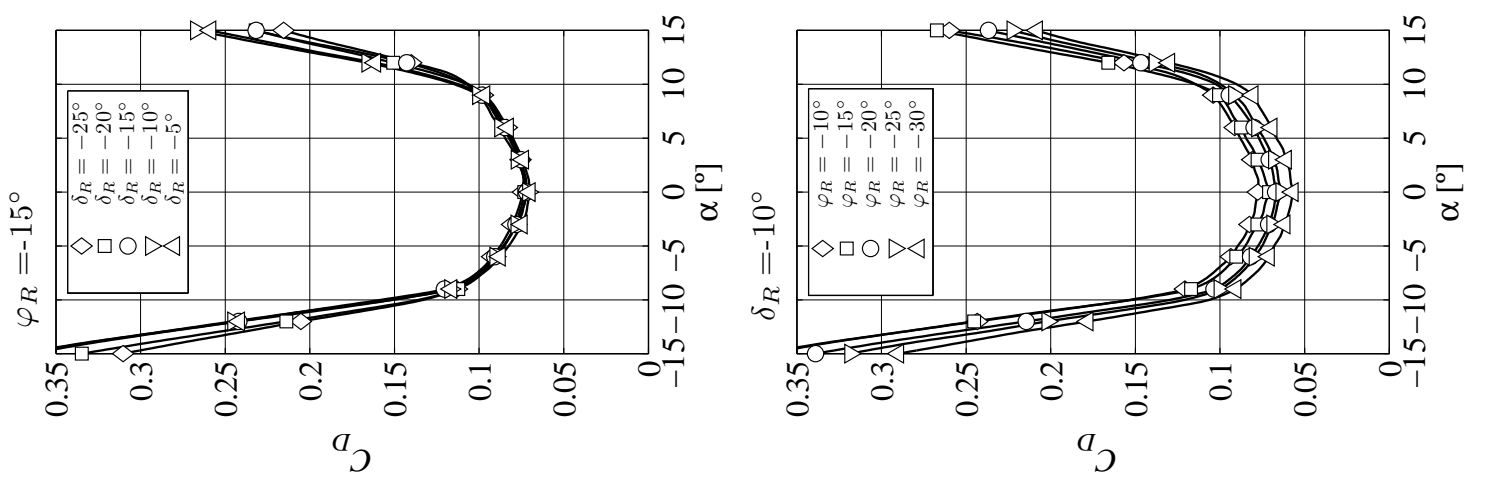

نे

वृं > है

얼

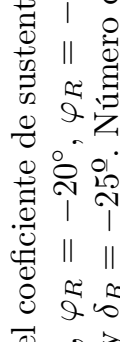
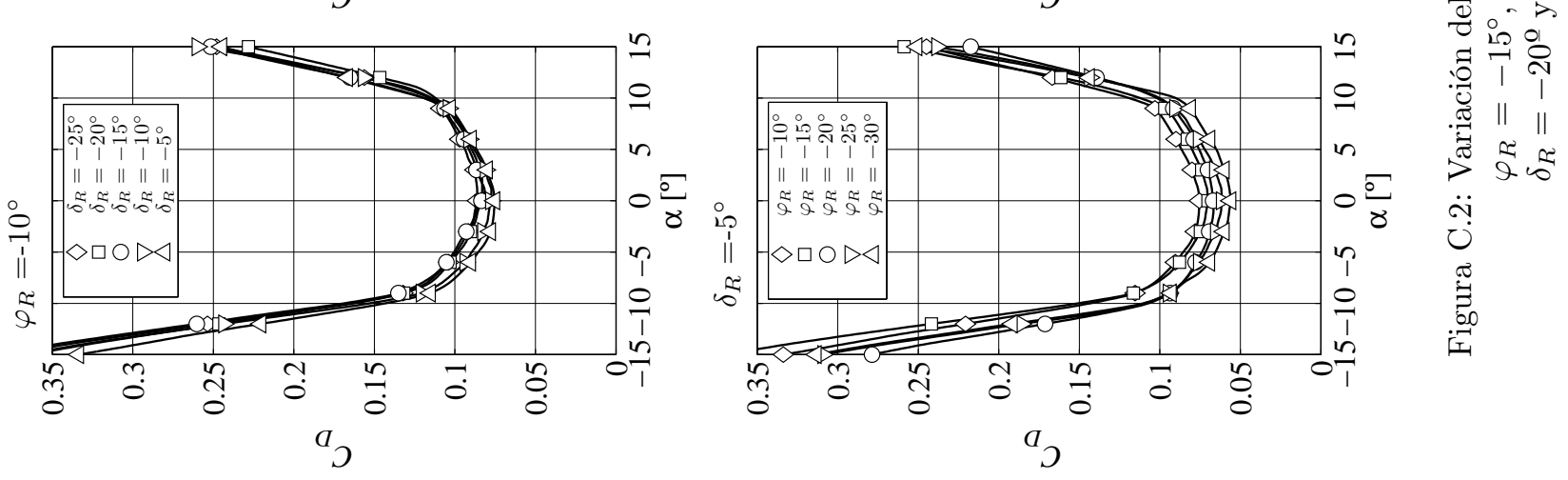

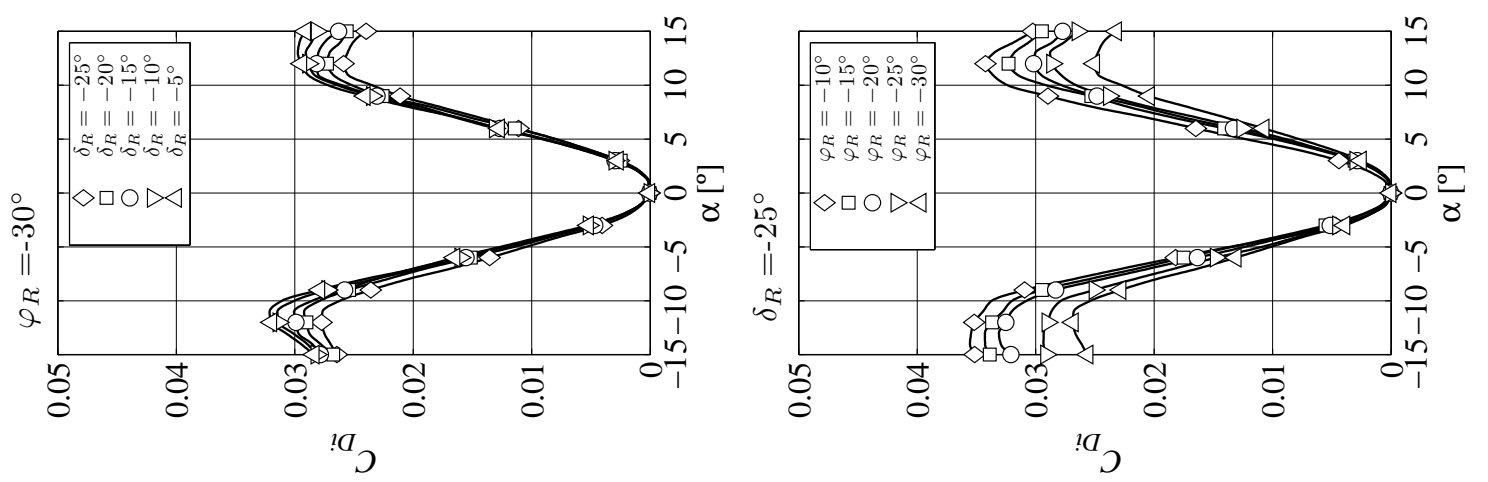

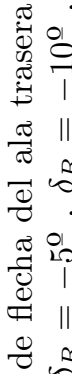
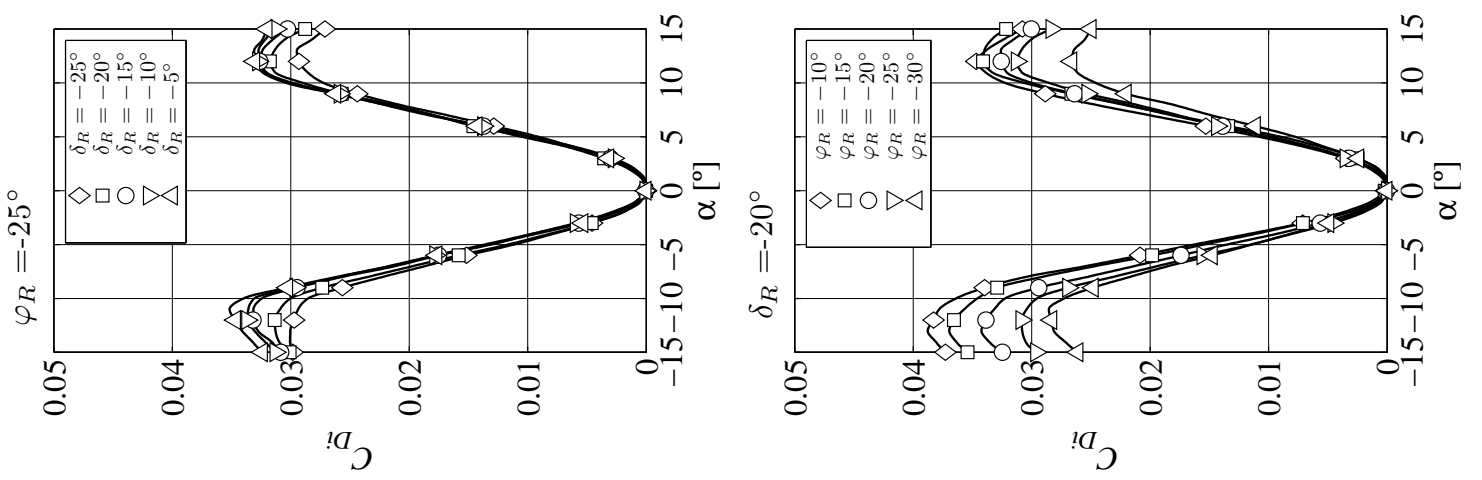

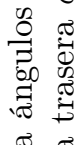

๘ี $\frac{\sigma}{\sigma}$

ช 항

๑융

奈:

o ?

유

윽
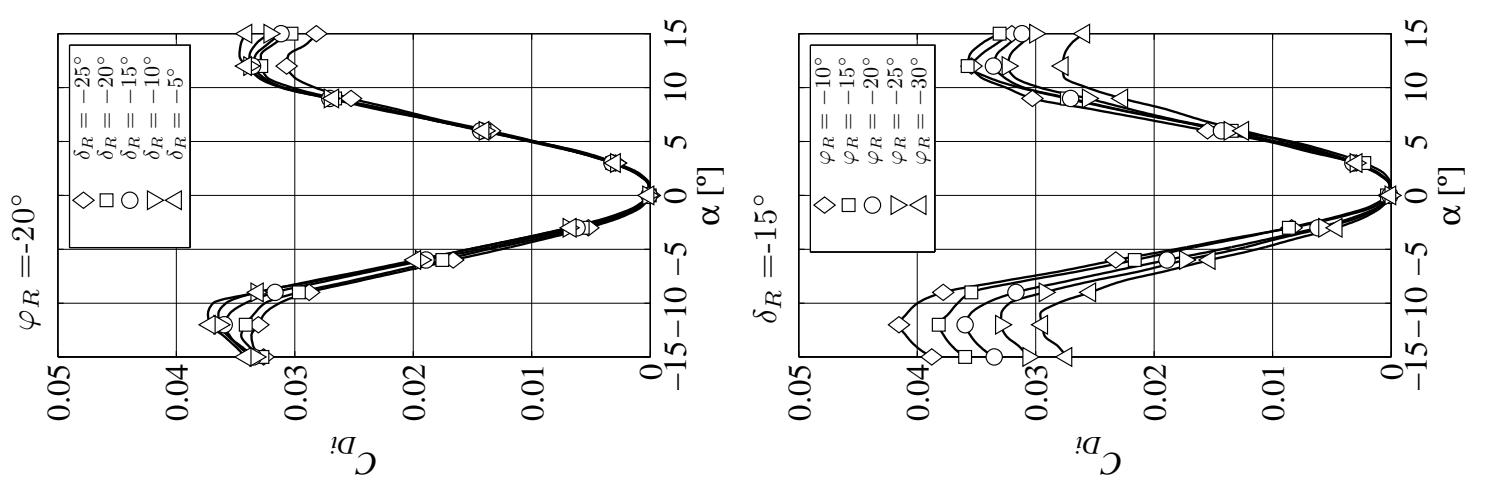

灾芯苛

윰

두요

氖

단정

$\dot{\tilde{a}}>5$

in

|
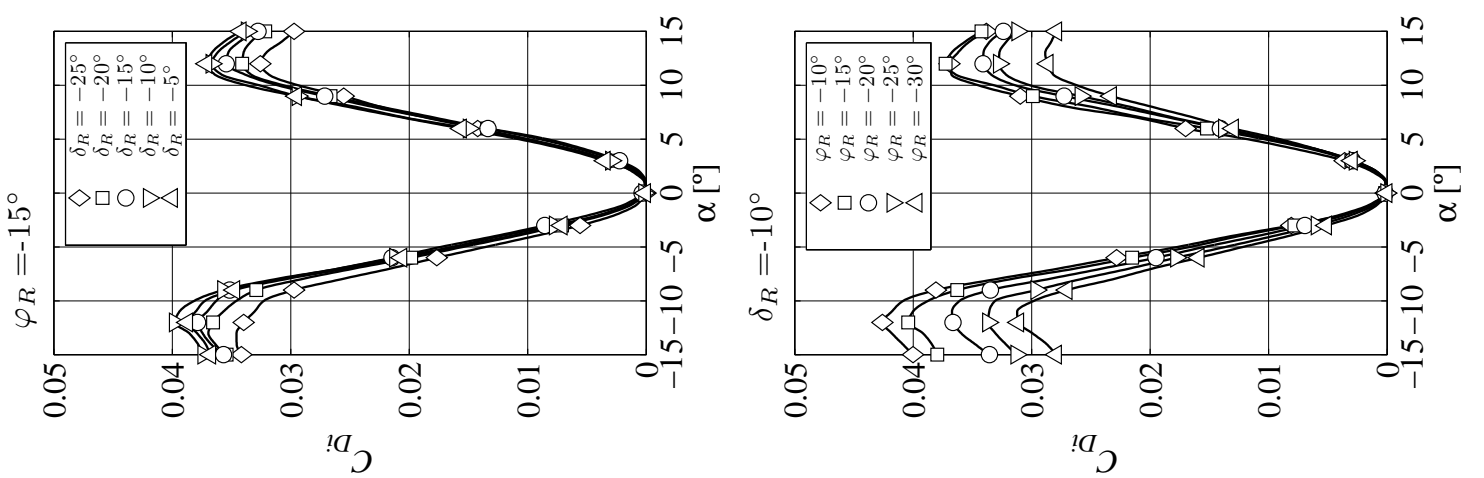

量要

월 $\stackrel{0}{\circ} \stackrel{0}{\circ}$

W

$-20$

- $>$

늘 옹 용

¿ ।

25
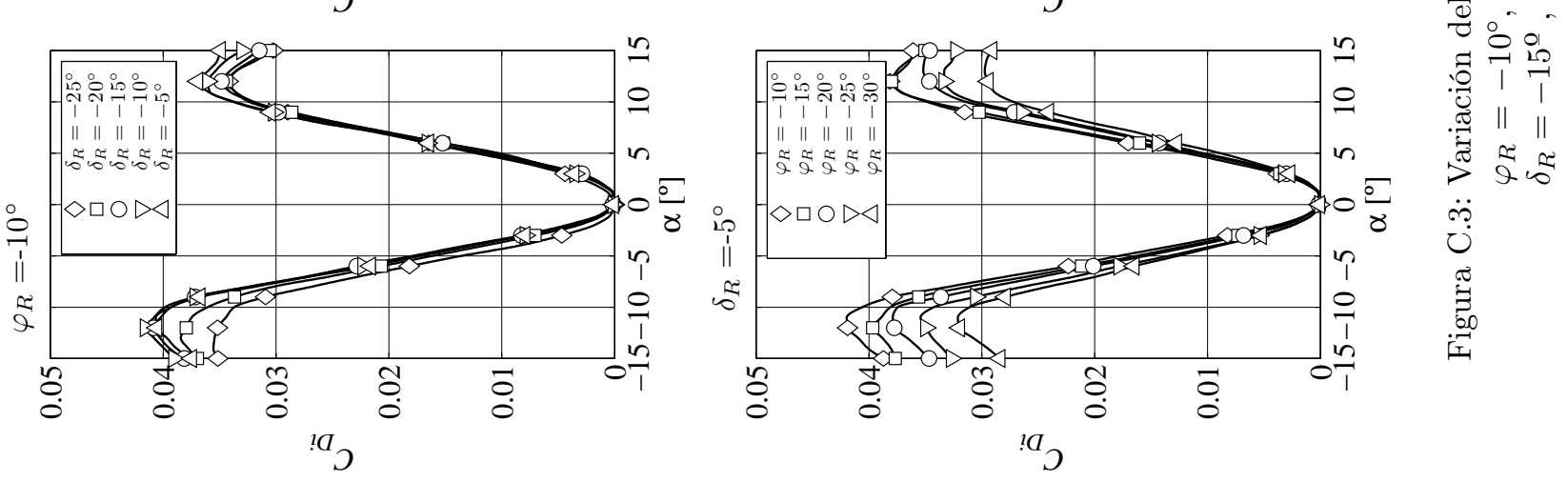

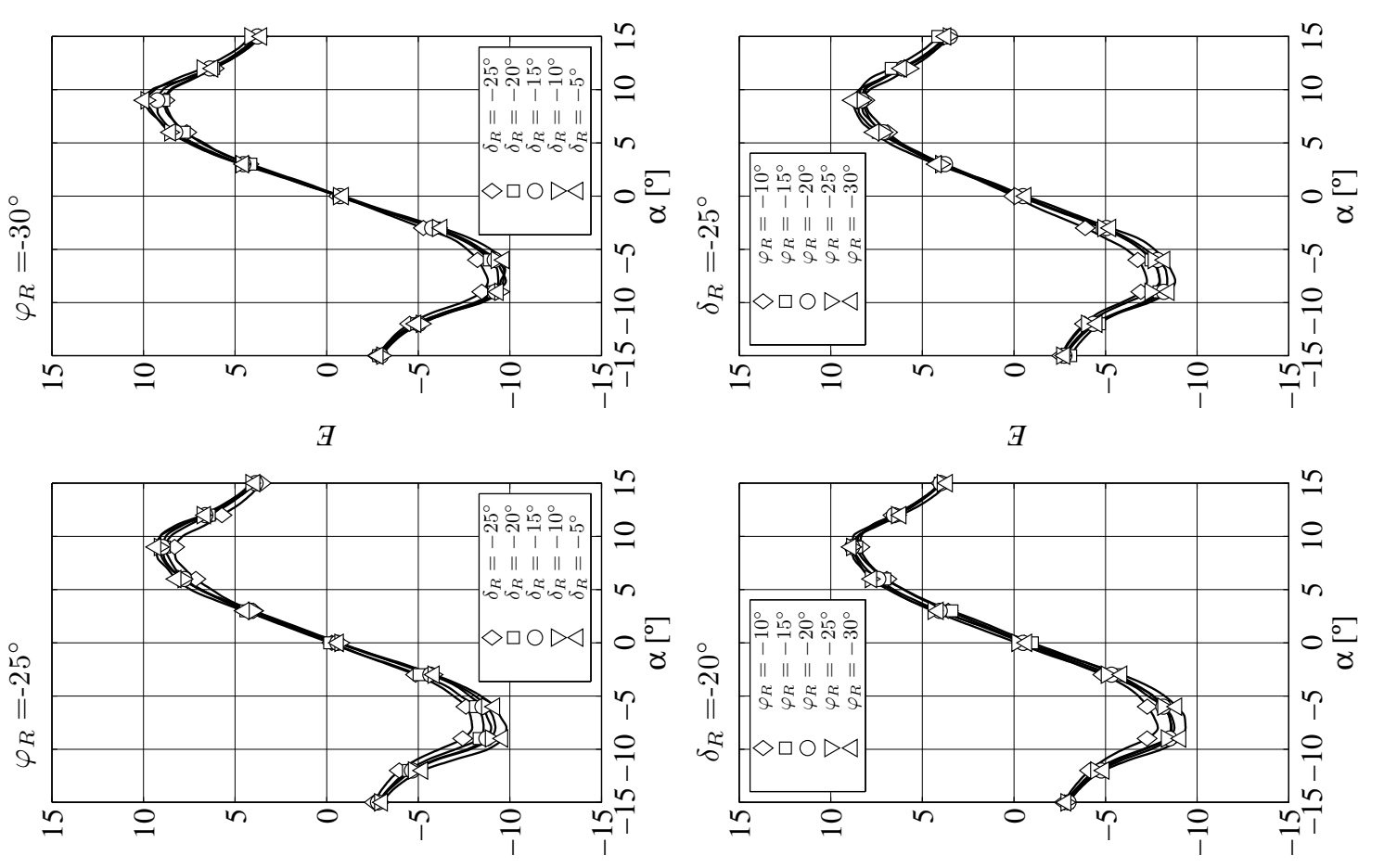

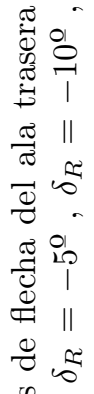

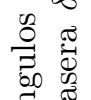
范 สี ช 옥용 蛋. त क ज ช $\frac{0}{3}$
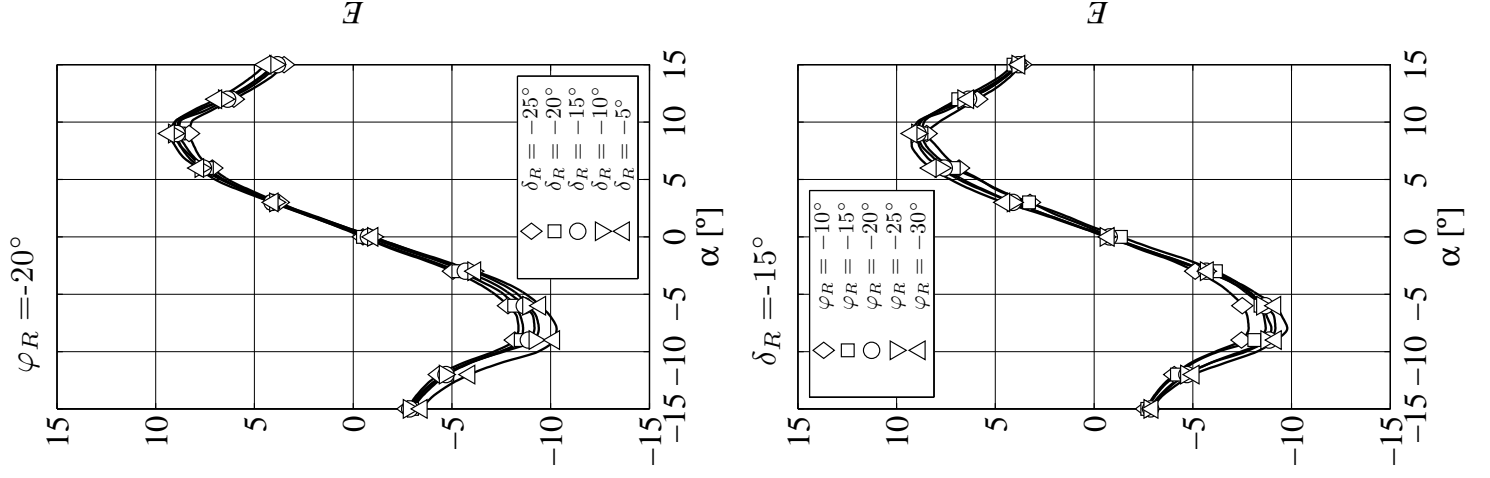
욜

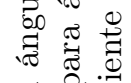
诺 : 苛 च

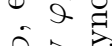
0 ชึथ
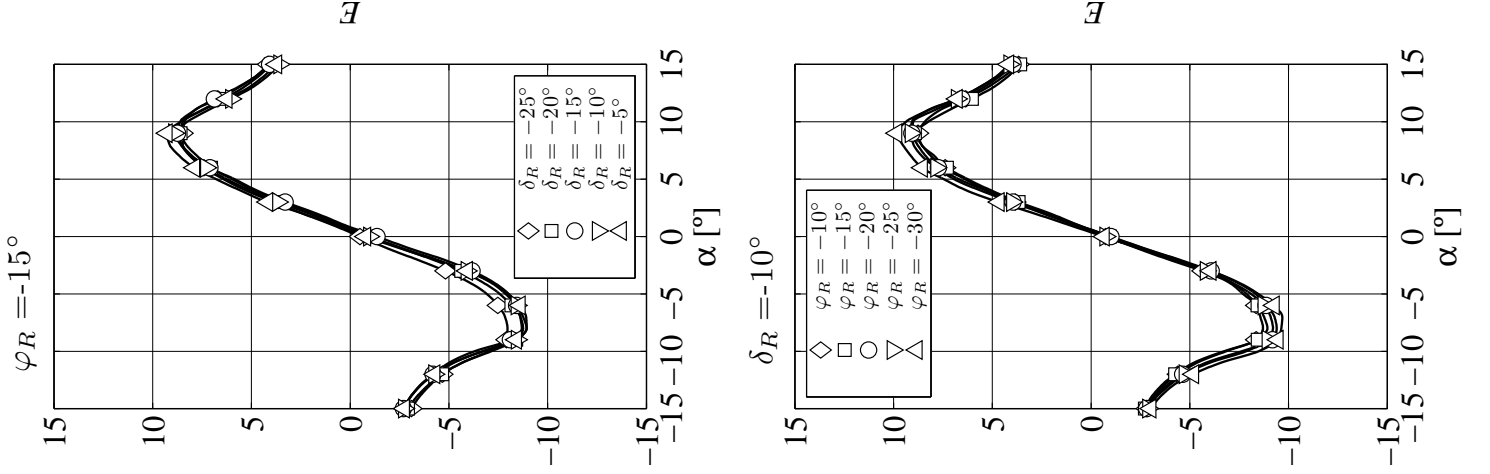
\|\|$\stackrel{0}{0}$ 되 2 宩

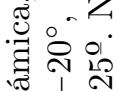

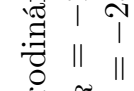
橧

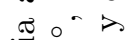

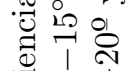
䔅 || $\approx 2,5$
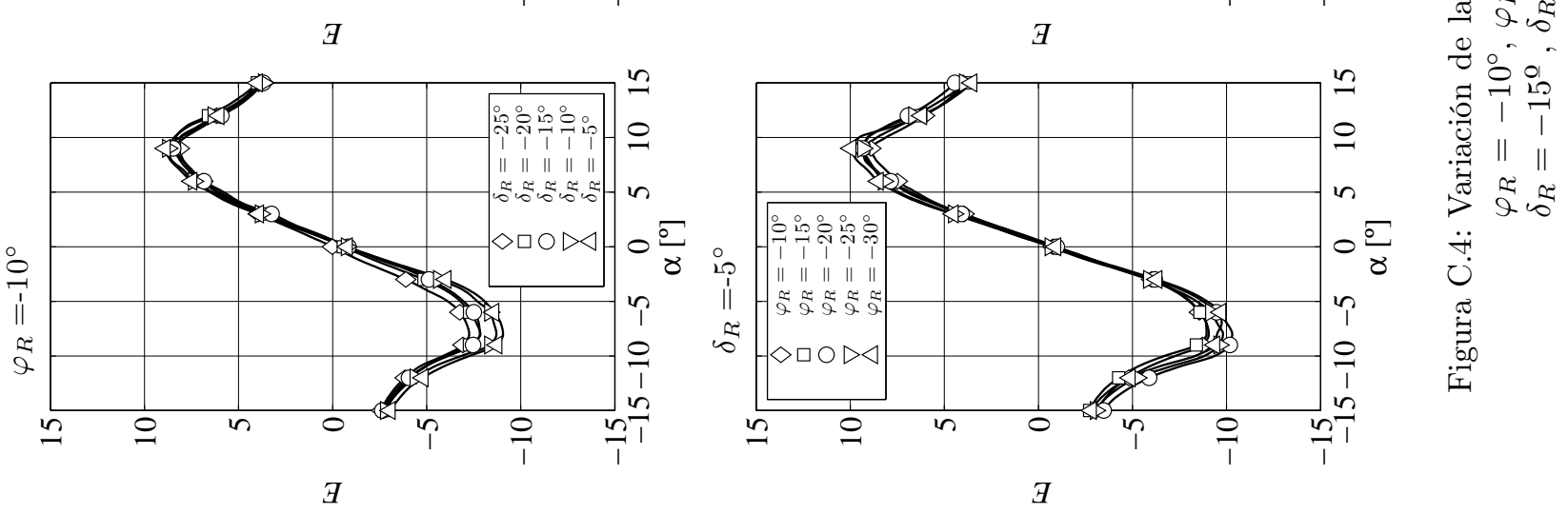

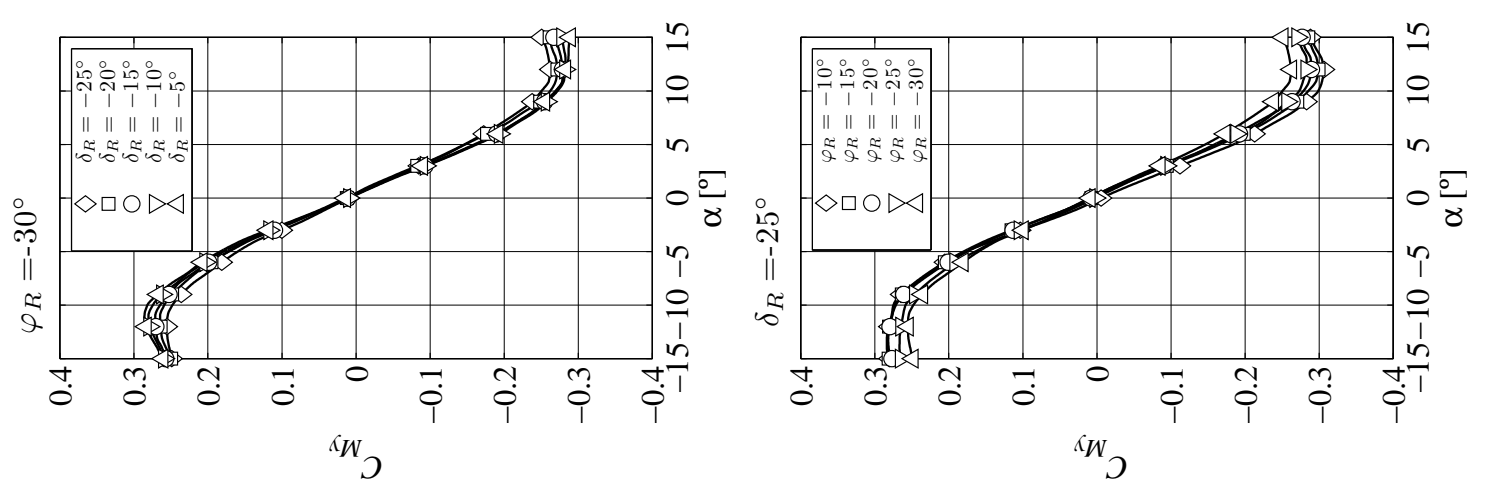

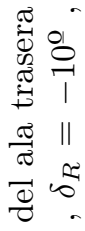

궁 옹

난
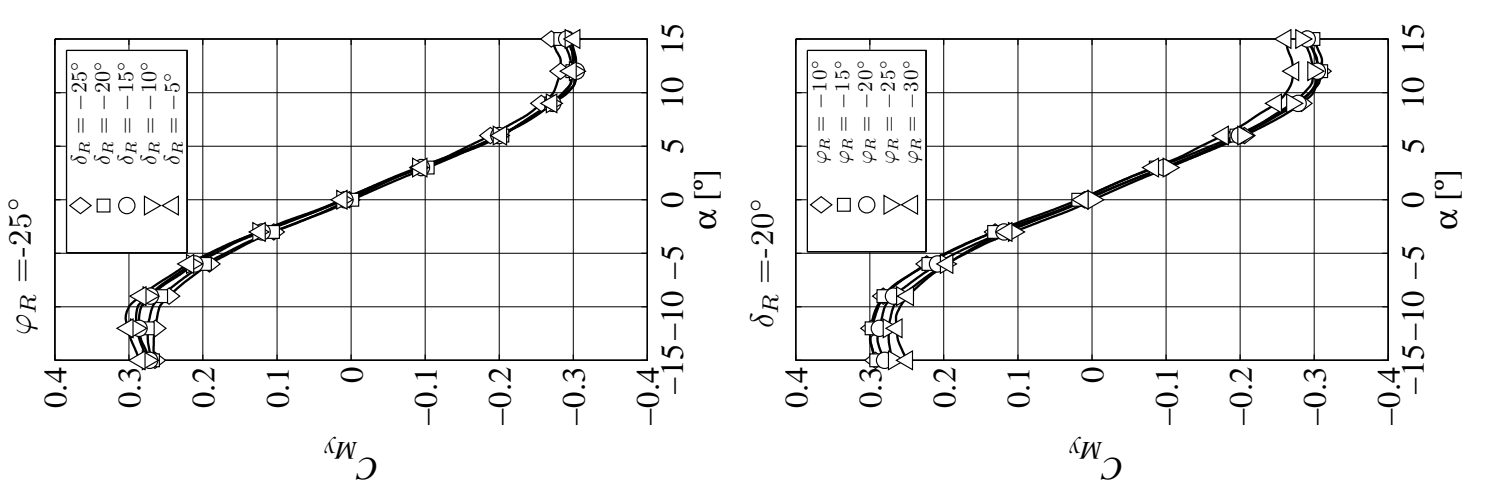

요

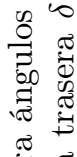

䒕

ช용

원융

灾

o : 0

을
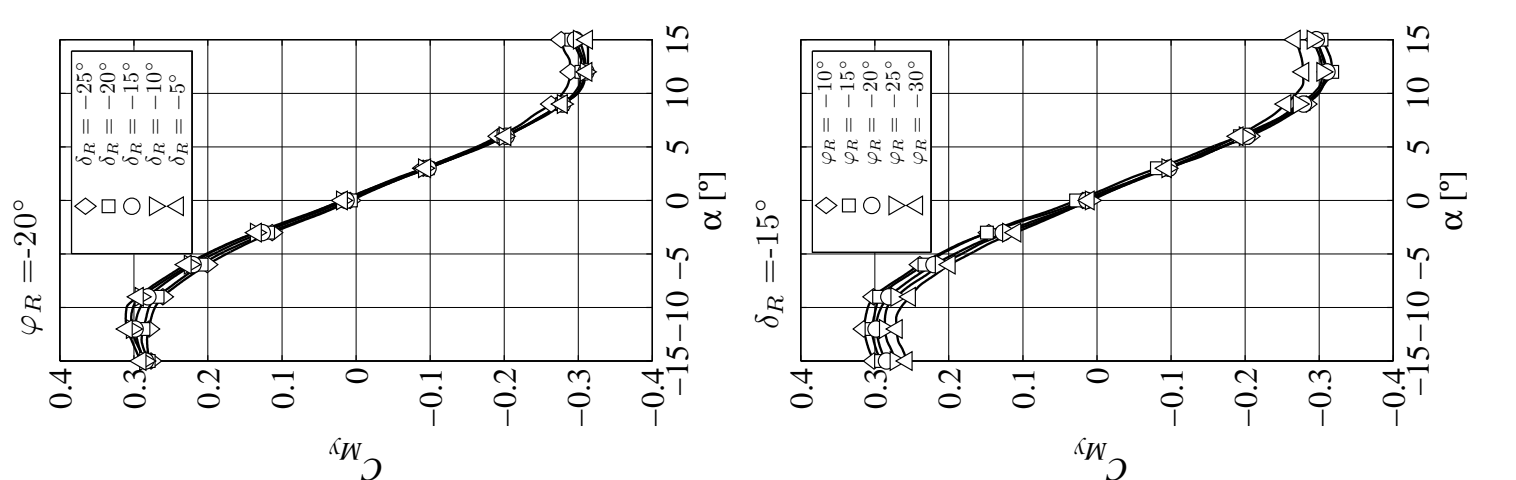

ส

๙

> $>$

ํㅠㅇำ

:

क्ष 닝

क 9

दे ब্

ำ ำ

仓
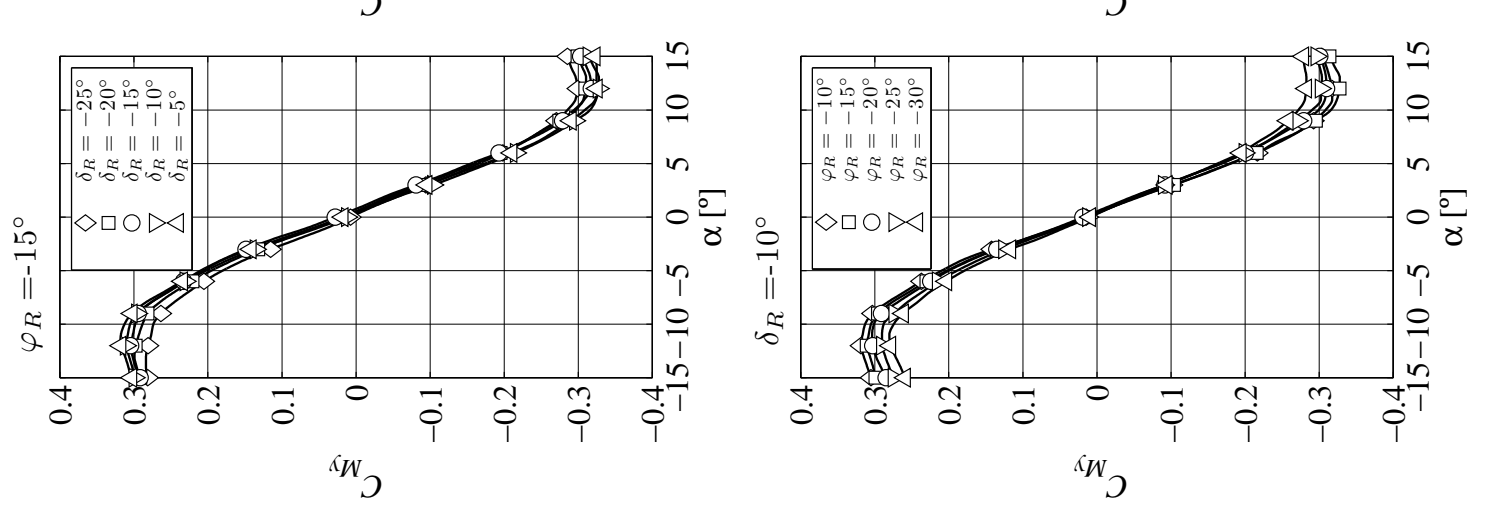

ن 2 只罗

$\circ \stackrel{\circ}{\circ}$.

울ㅇํㅇำ

ह્व ||

吾 25

o in $\rightarrow$

쿵 ते

ए।

ㄴ.5

요

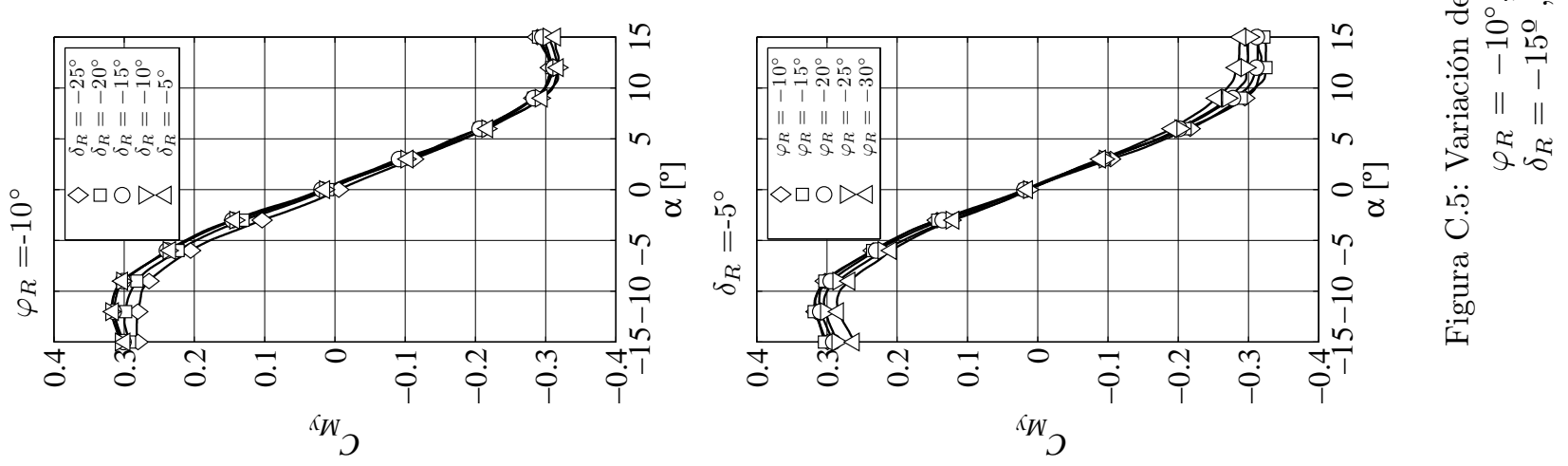



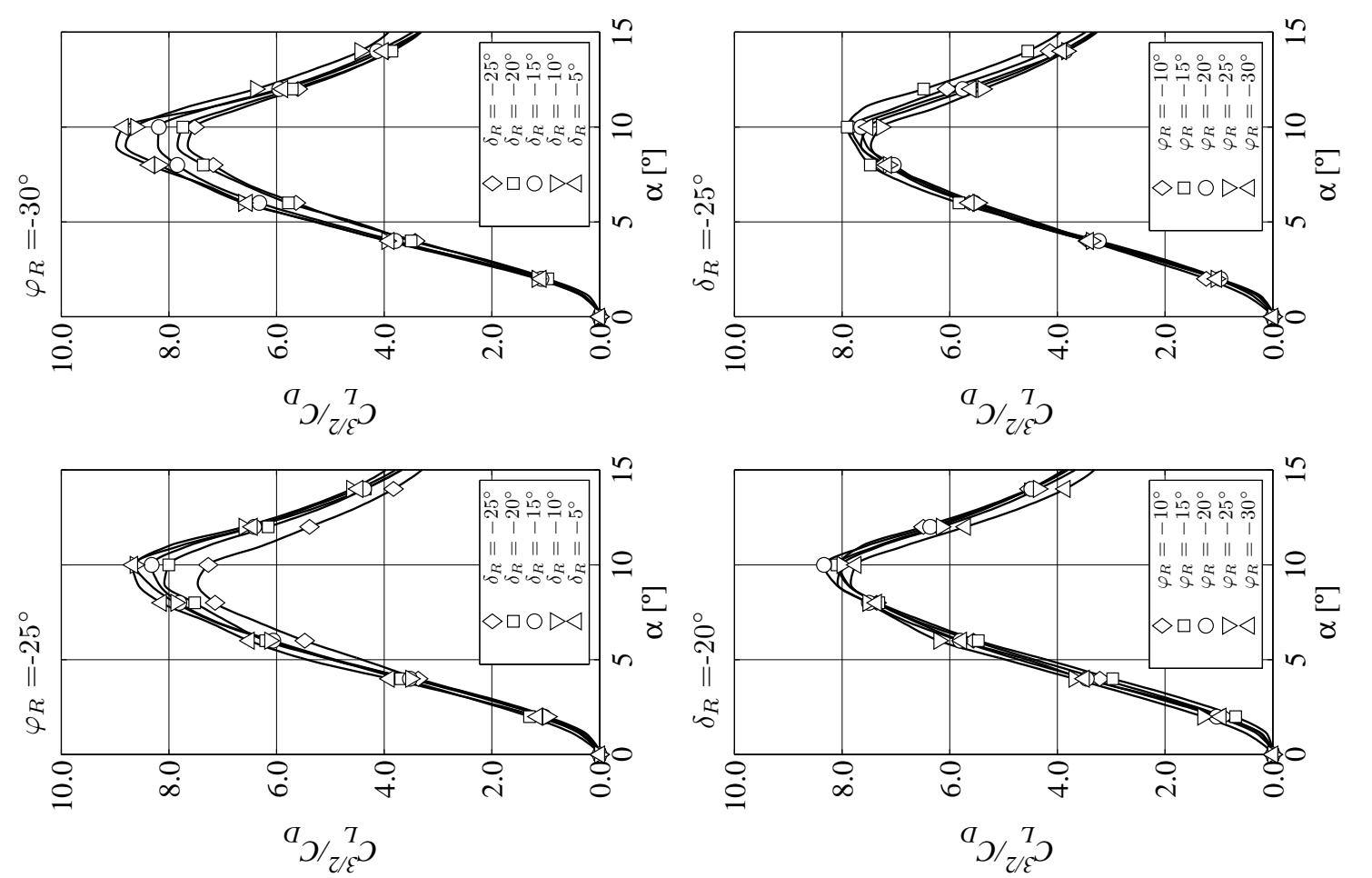

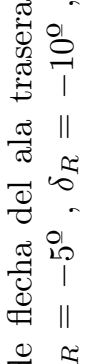

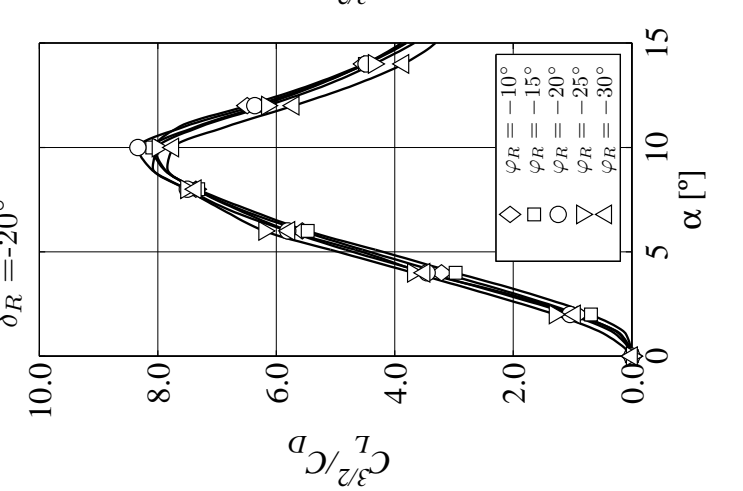

串

ర్

कृ $\frac{\pi}{\sigma}$

ڤ

- 0

E.

.

ช
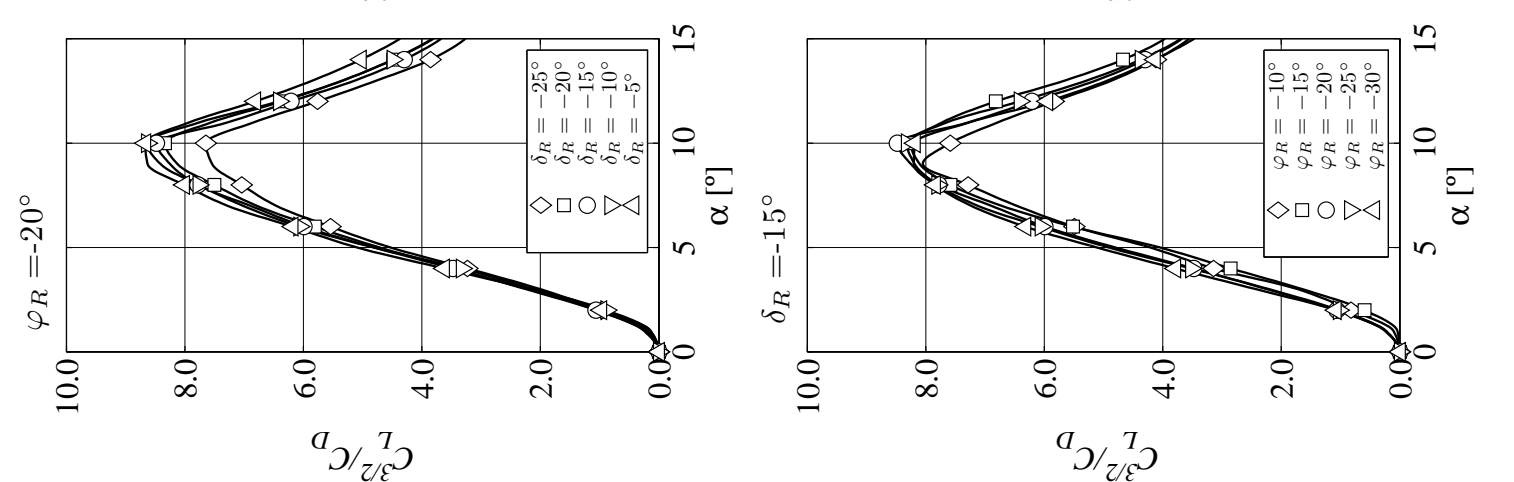

잉

ర0

$\rightarrow>0$

ช

:0ี

灵先

가용

Ain

$\checkmark \stackrel{0}{1}$
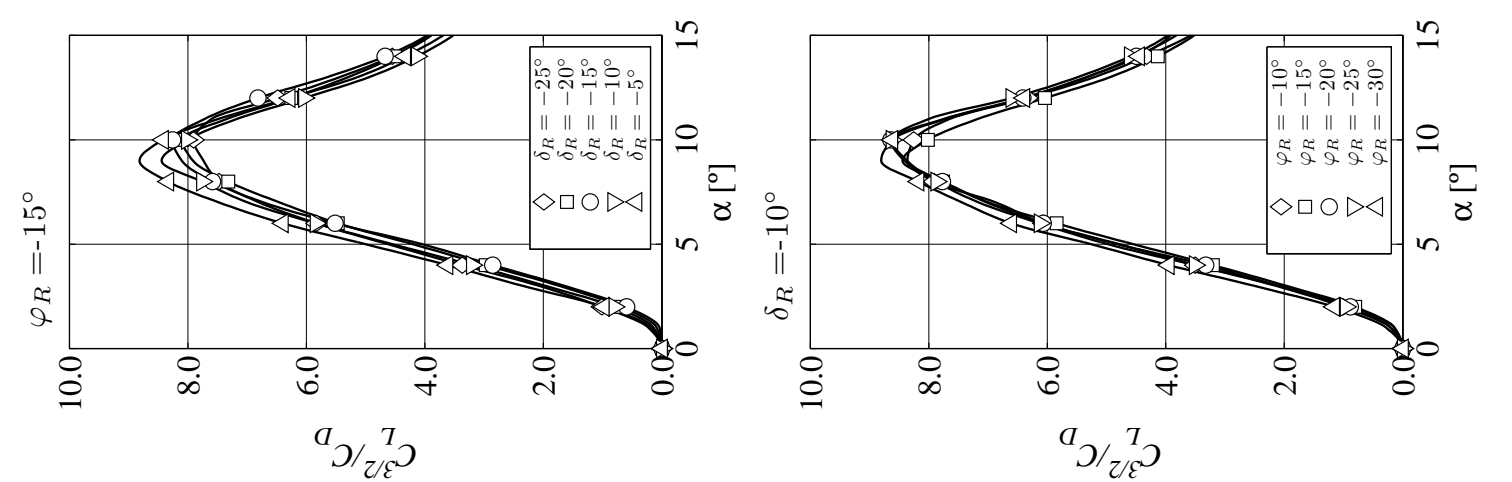

ชิ

क 学

잉 ㅇํㅇ

ن । - 0 $\rightarrow \infty$

요용ㅇㅇ

胥 |

$\approx 2$

웅

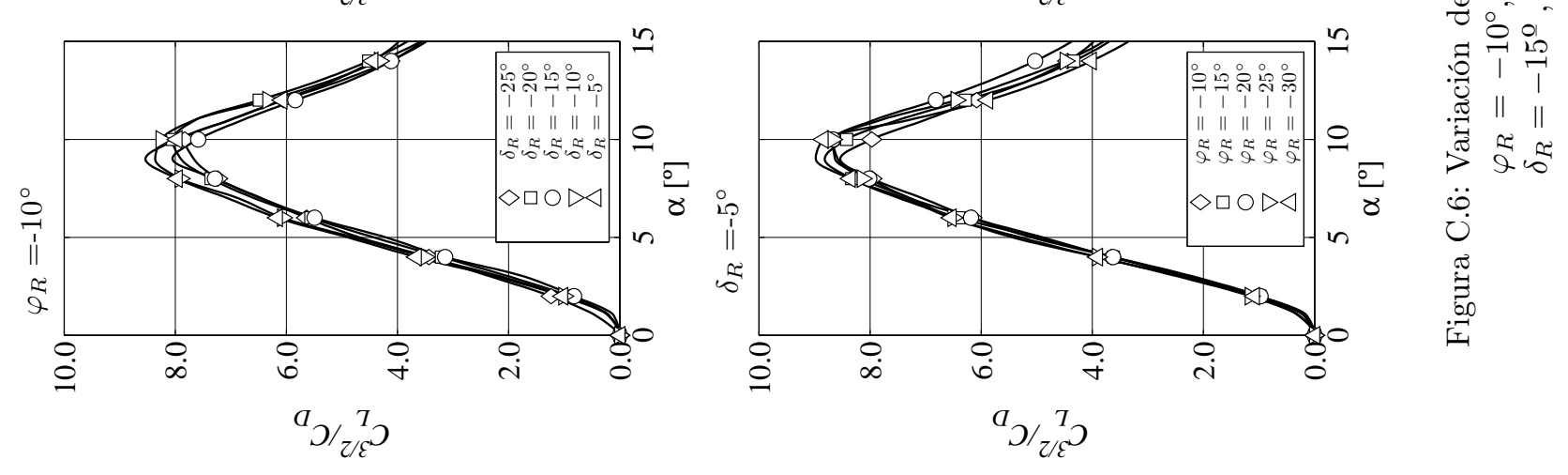



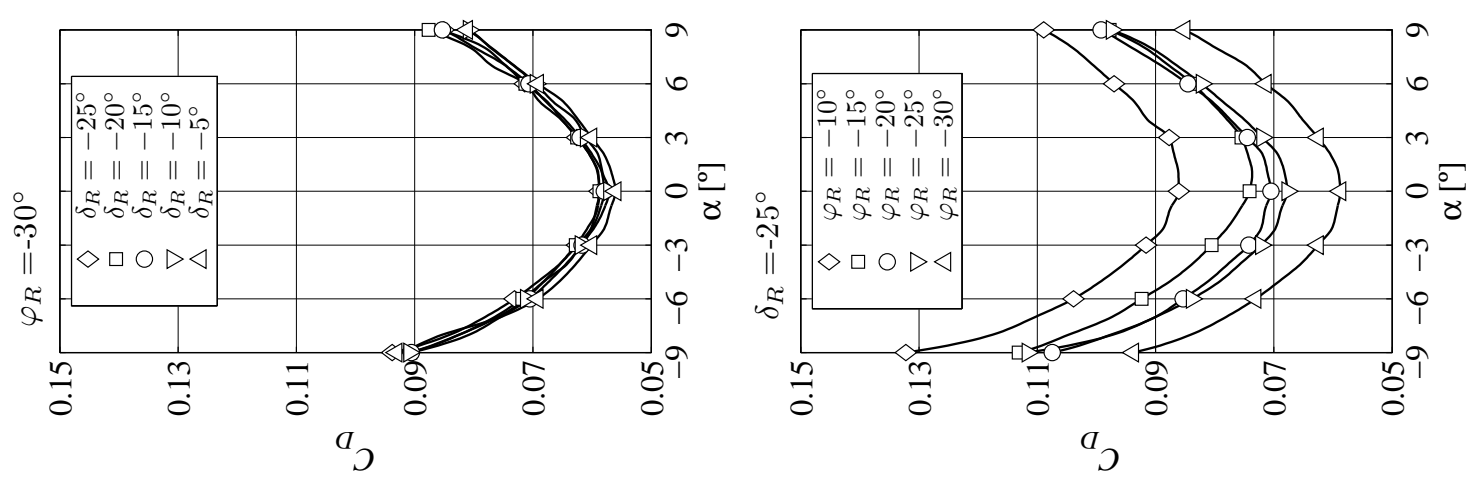

$\stackrel{\circ}{\circ}$ 인

a

2,5

苟

$\frac{\sigma}{\sigma} \|$
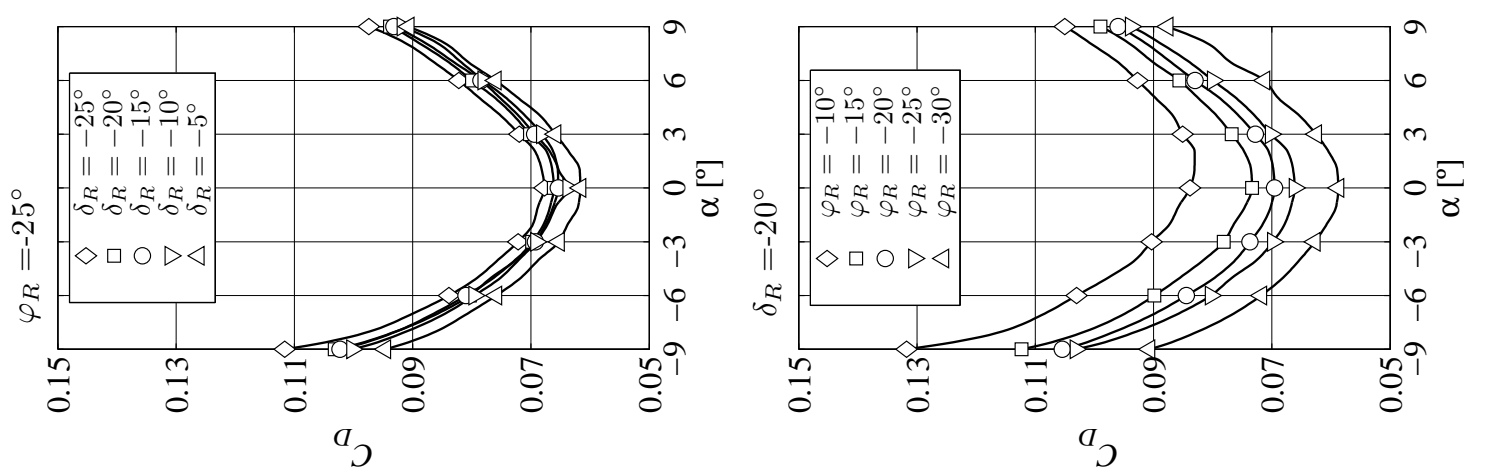

항

궁

\& 1

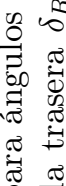

完 $\frac{\pi}{\pi}$

ช
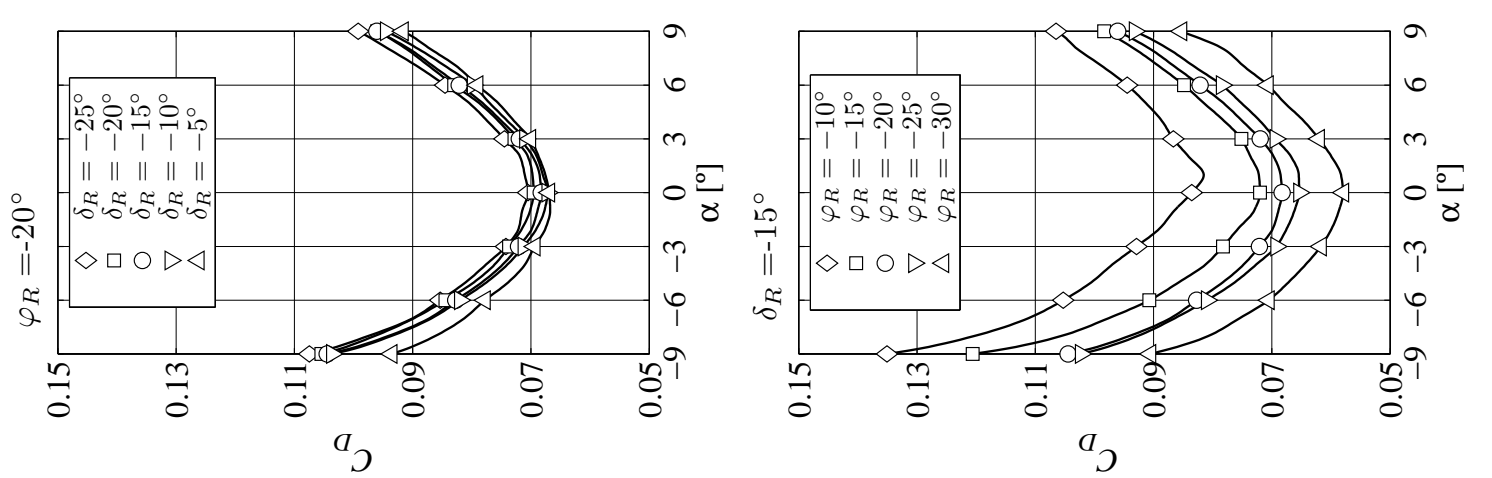

은 는

蛋

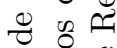

을 200

ชี

ป

궁 $\rightarrow$ 잉

얼 용

1 ช

하 $\| \frac{\text { क }}{0}$
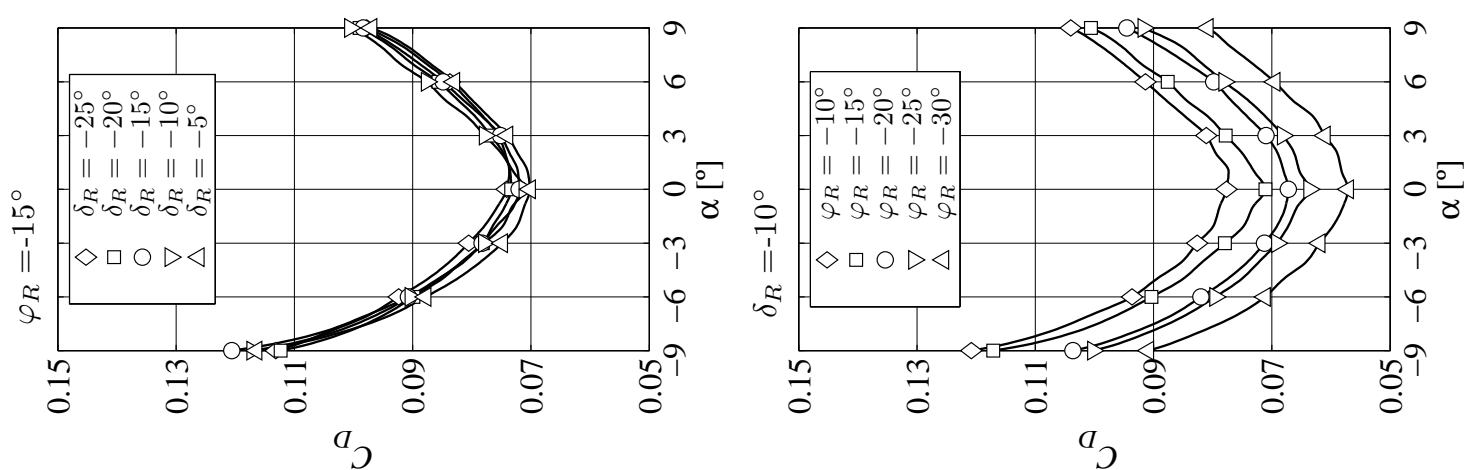

is

乙2

त

نั

.

○点

궝 ㅇํㅇ 옹

ن |

$80^{\circ}$

\& $>$
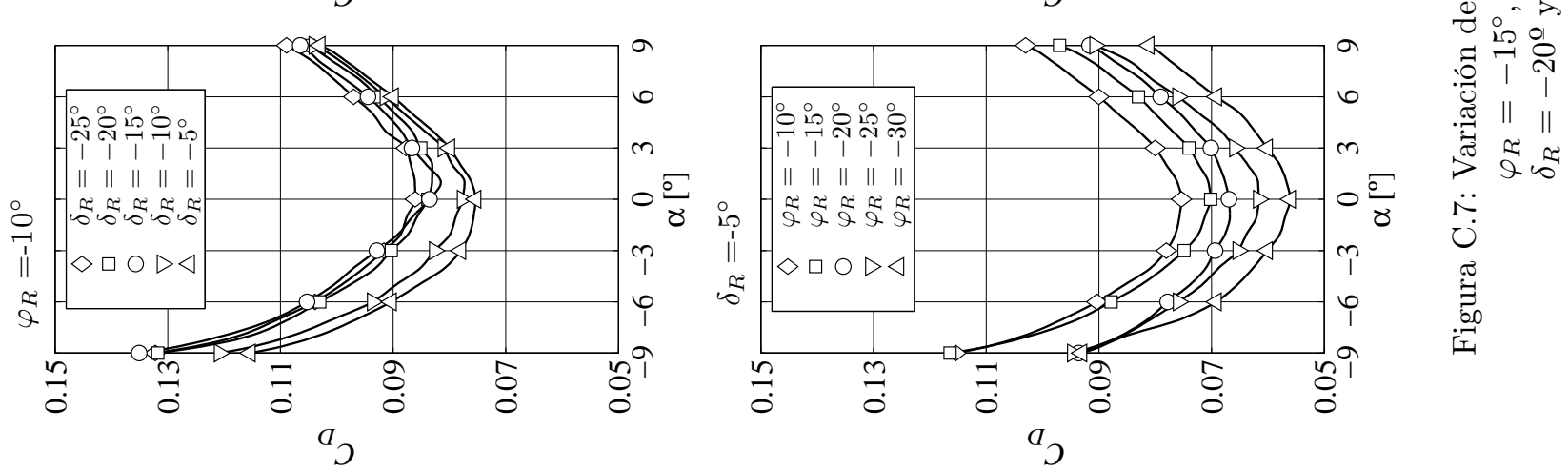

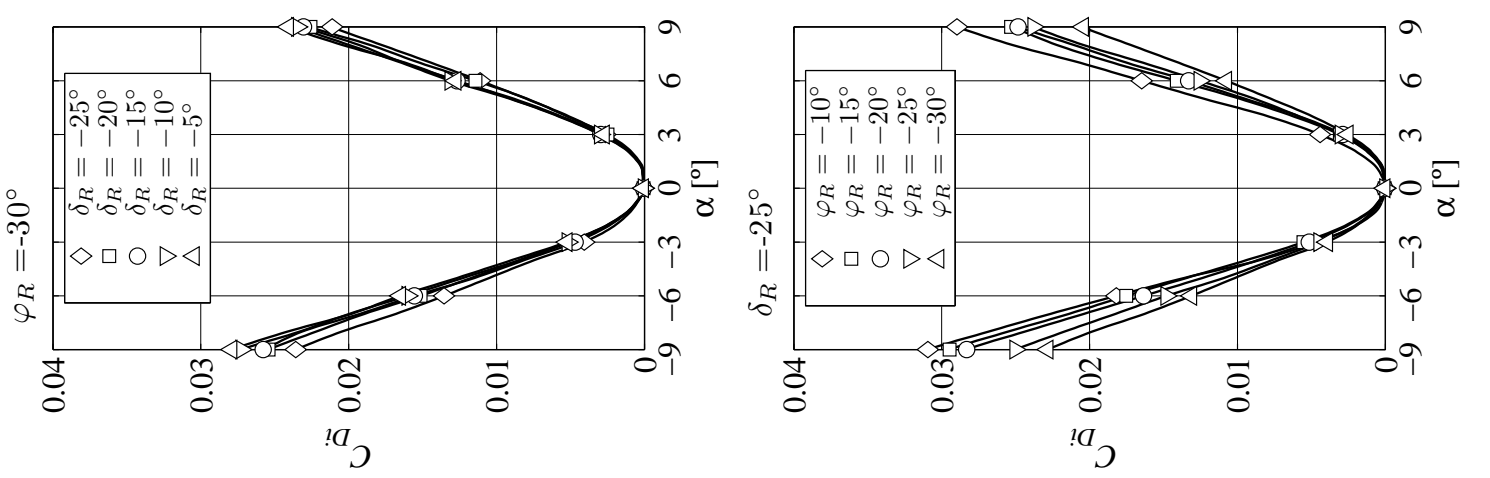

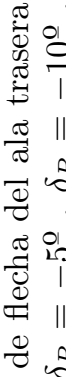
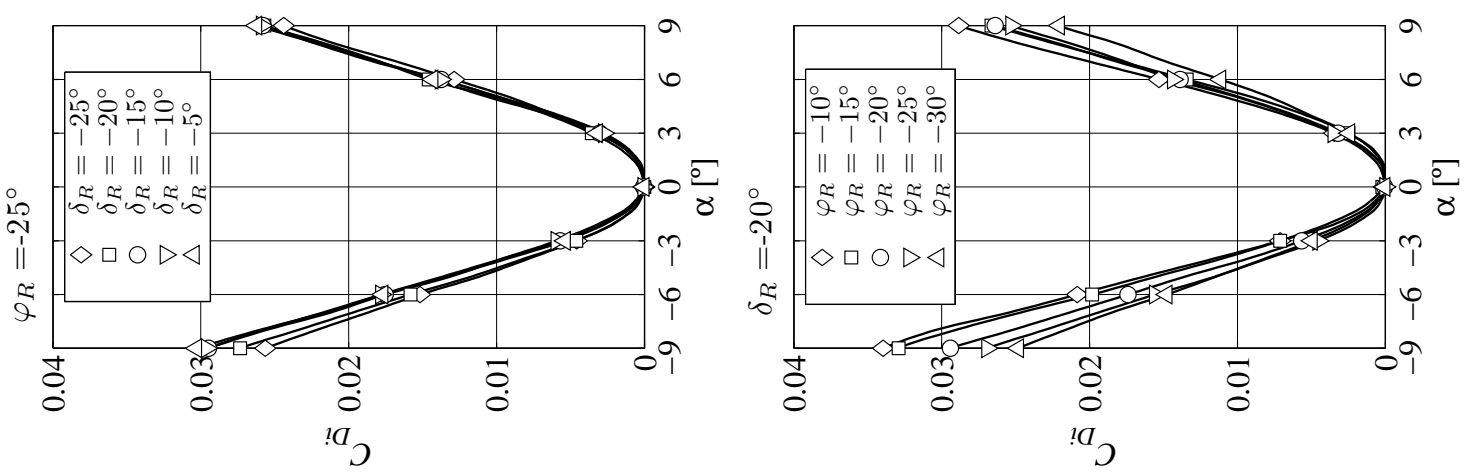

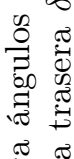

敢 $\frac{\pi}{\sigma}$

웡

仓융

蛋:

0. 오

을
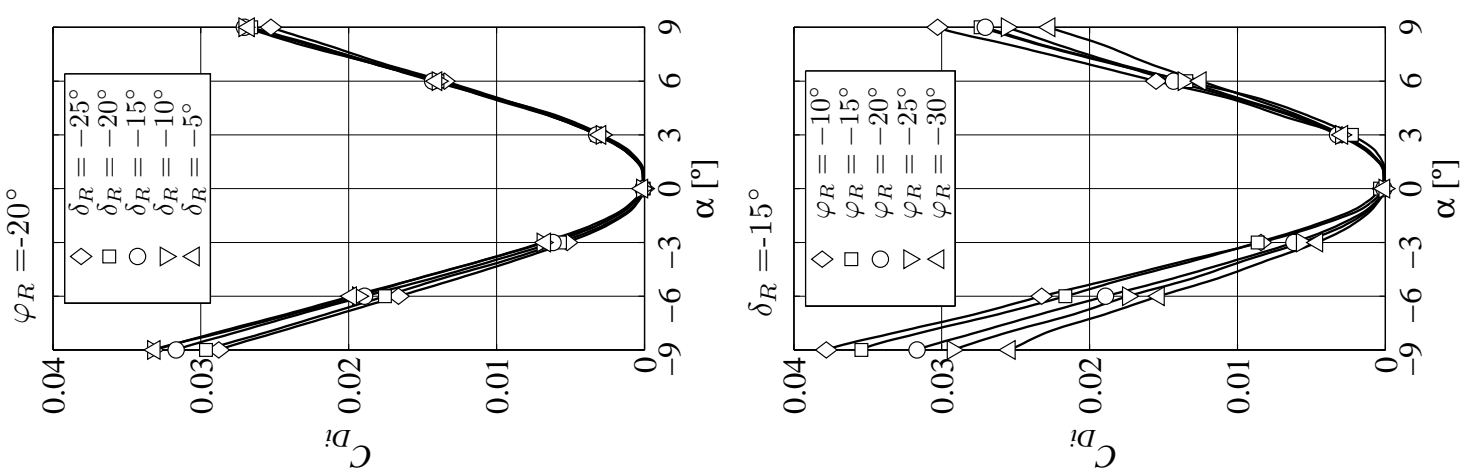

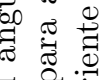

西 $\rightarrow$

:융 용

包

$=\frac{n}{0}$

구웡

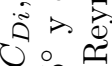

ชิ ขึ
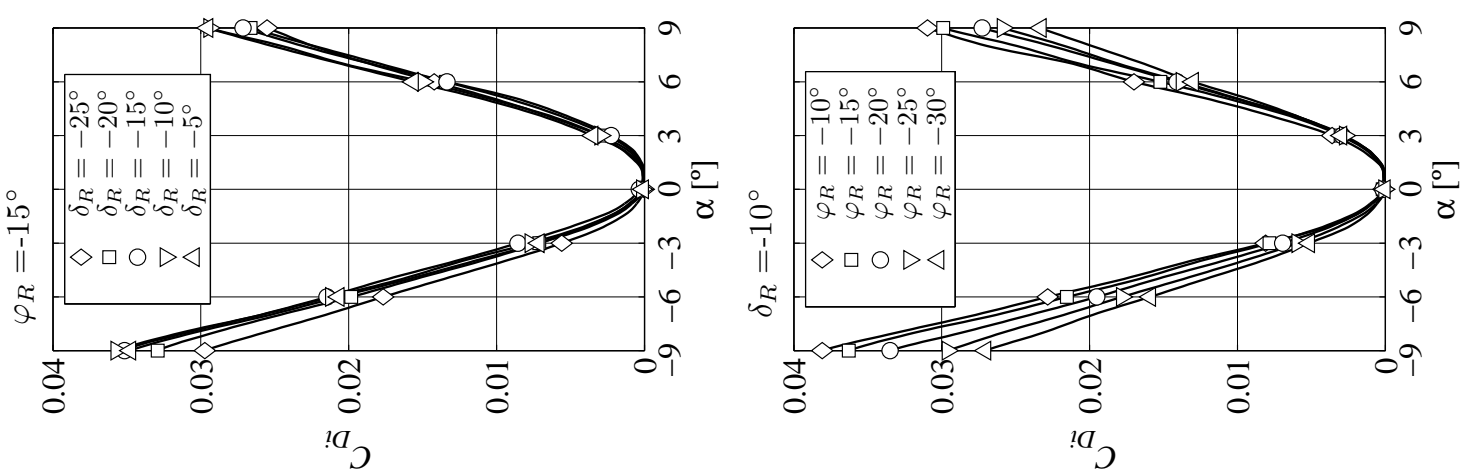

总造

胥。

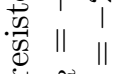

$-2$

ช 2 .

o in $>$

늘 잉

II

० 25
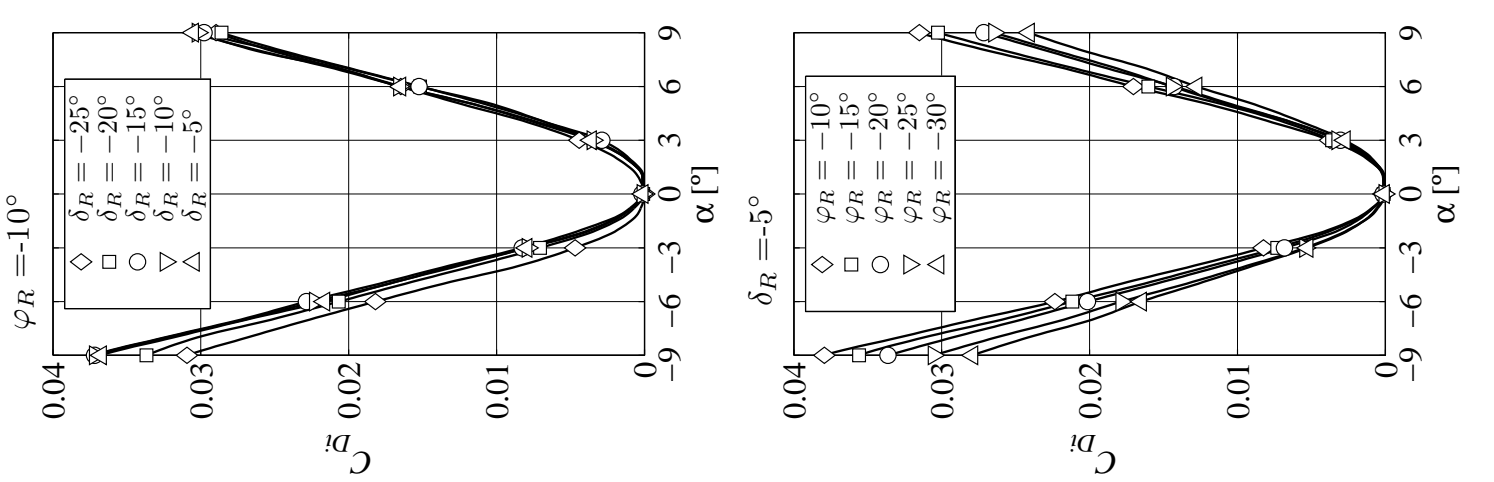

:

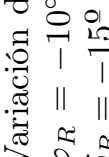

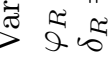



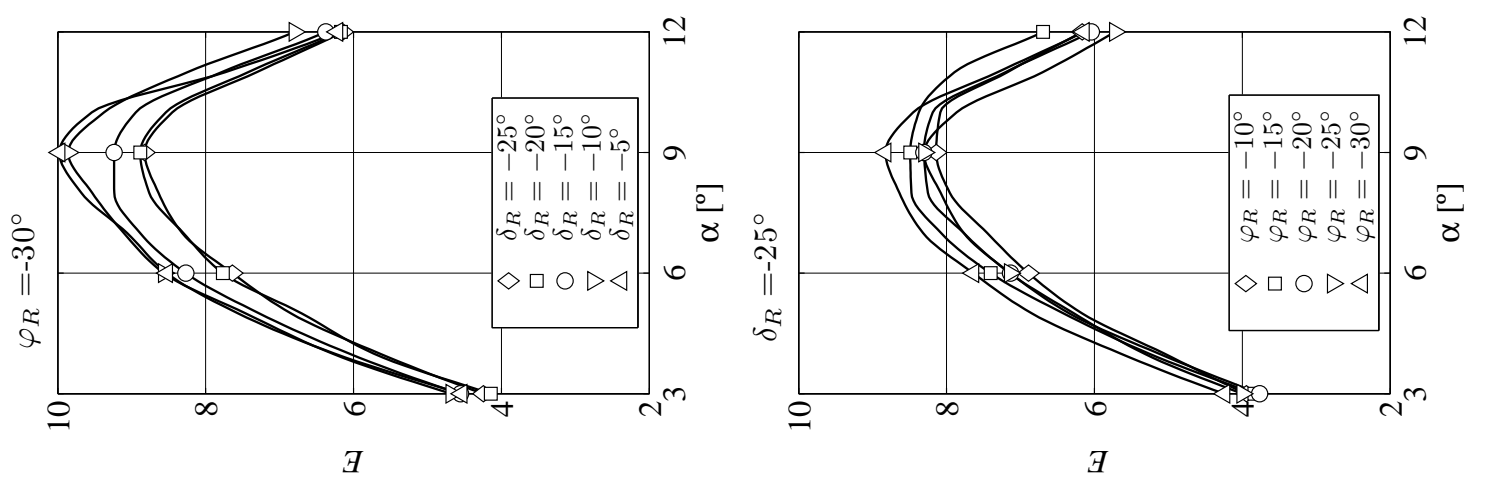

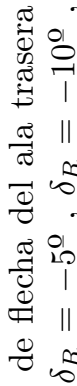
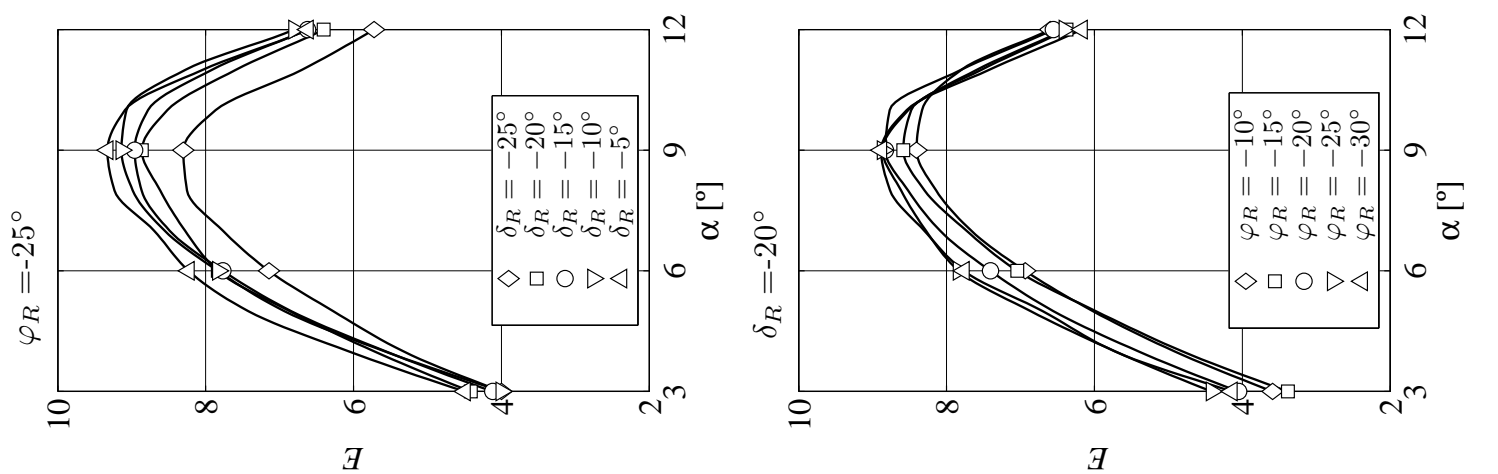

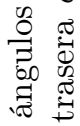

तె $\frac{\sigma}{\sigma}$

o

욤

F.

का को

을

올

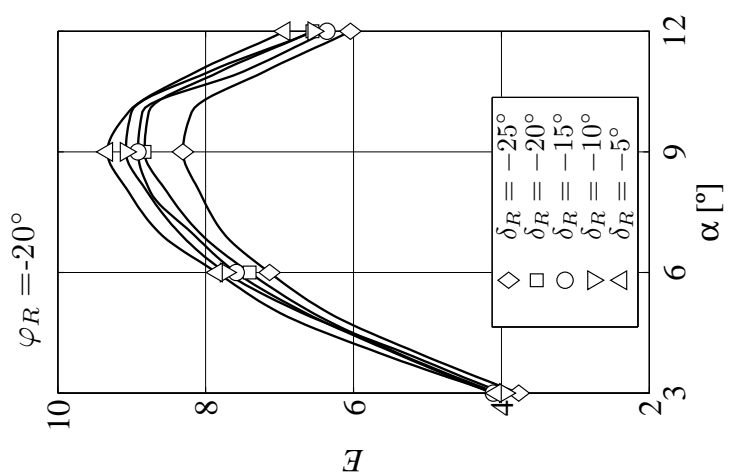

马

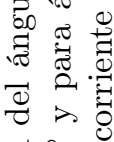

둥요

过

$\Xi \|$ n

동

is $\rightarrow$

0 is

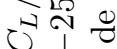

|| ||
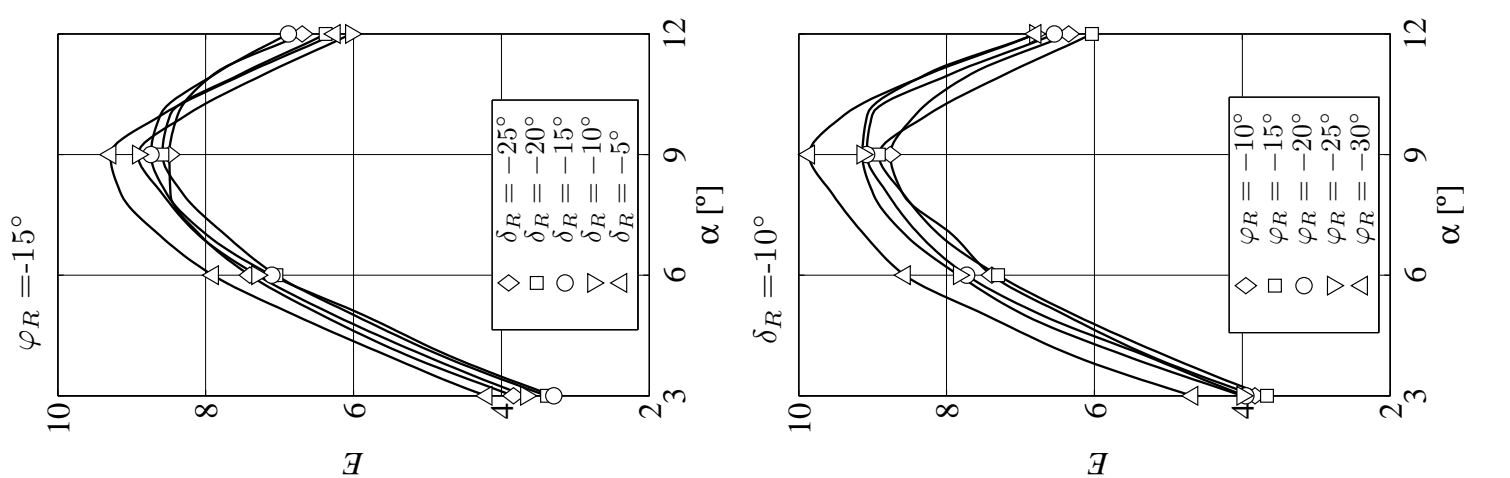

四 吐

क 9

을 ㅇํㅇ

西

छ ॥

苟

.

웡

岂 ||

$\approx \int^{2} \infty$

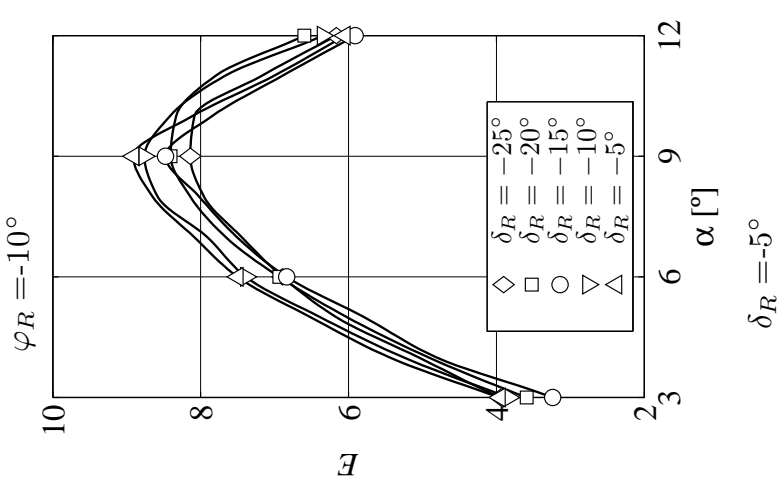

¿ 80

둥으용

\& $\|$

సี

$\ddot{\circ}$ 

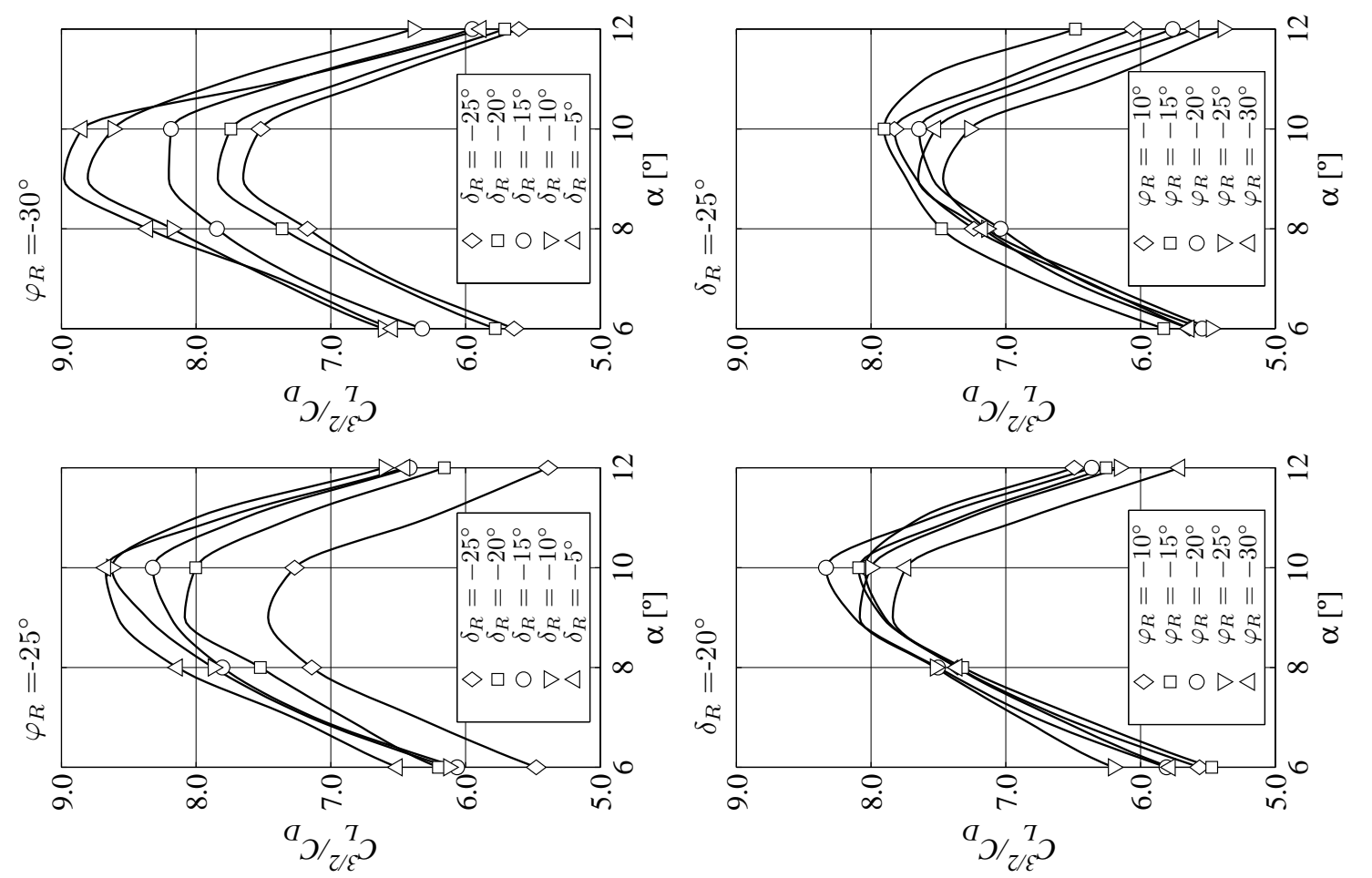

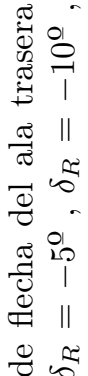

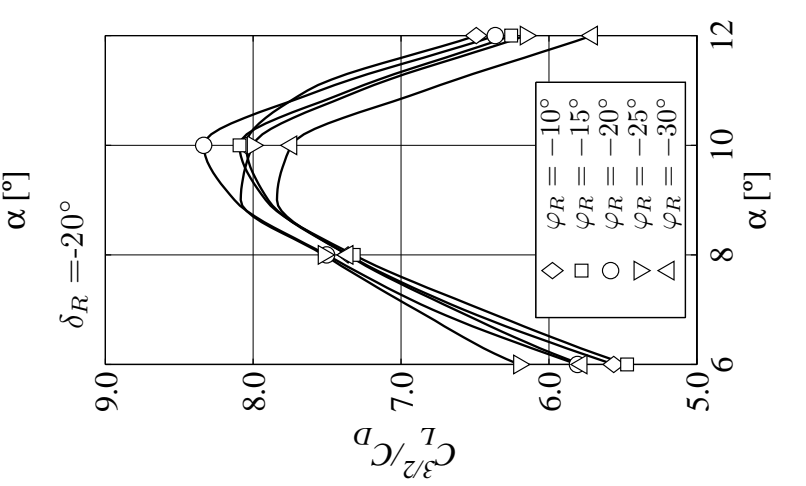

先

20

สำ $\frac{\pi}{\sigma}$

용

8 용

点 矛

규 의

๑
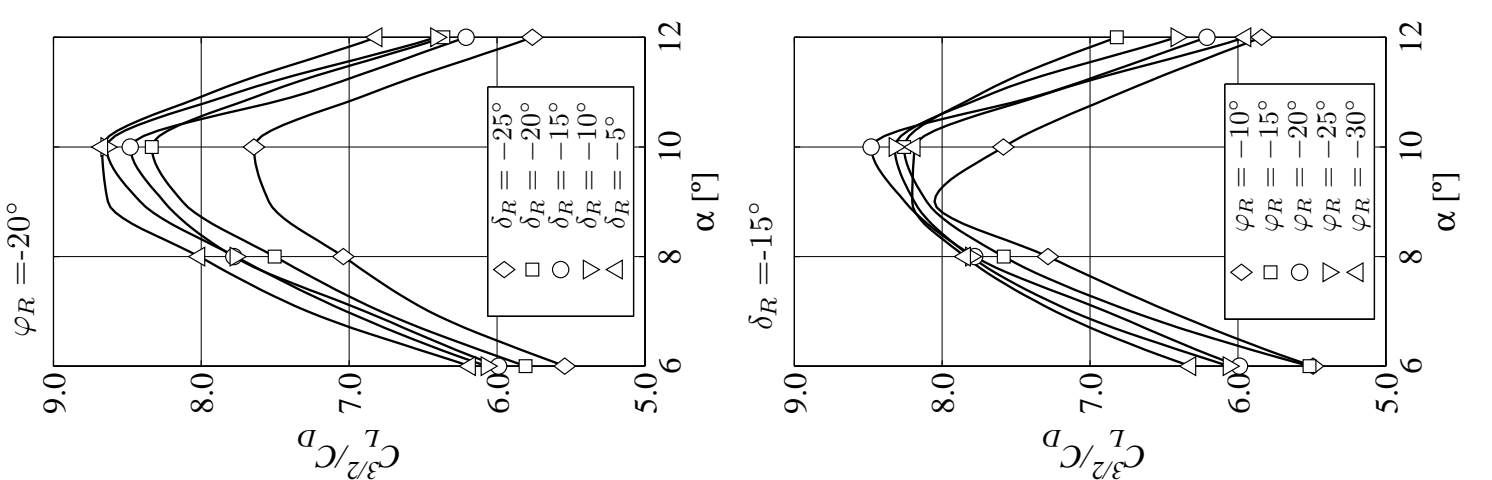

일

承

웡

象

잉

当 $\|$

궁

$\begin{array}{cc}0 \\ 0 & 0\end{array}$

ํํำ ำ

ขึ
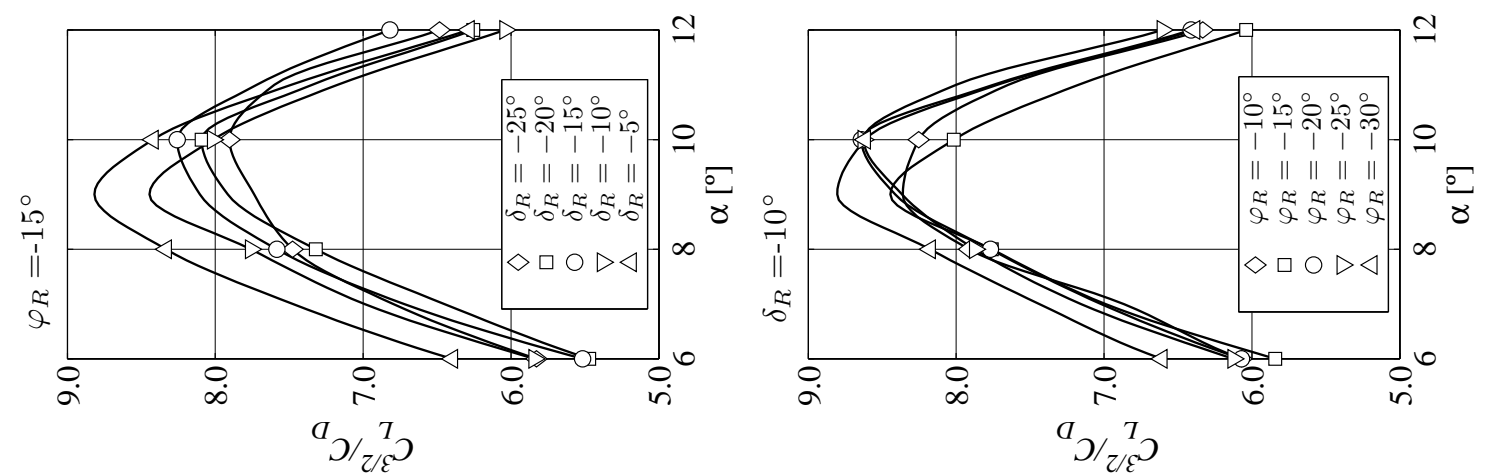

क 2 步

눙 ㅇำ

歎 $\|$

0 皮

क $2+\infty$

등 in

† 尺

\|\|

$\stackrel{5}{2}$

웅
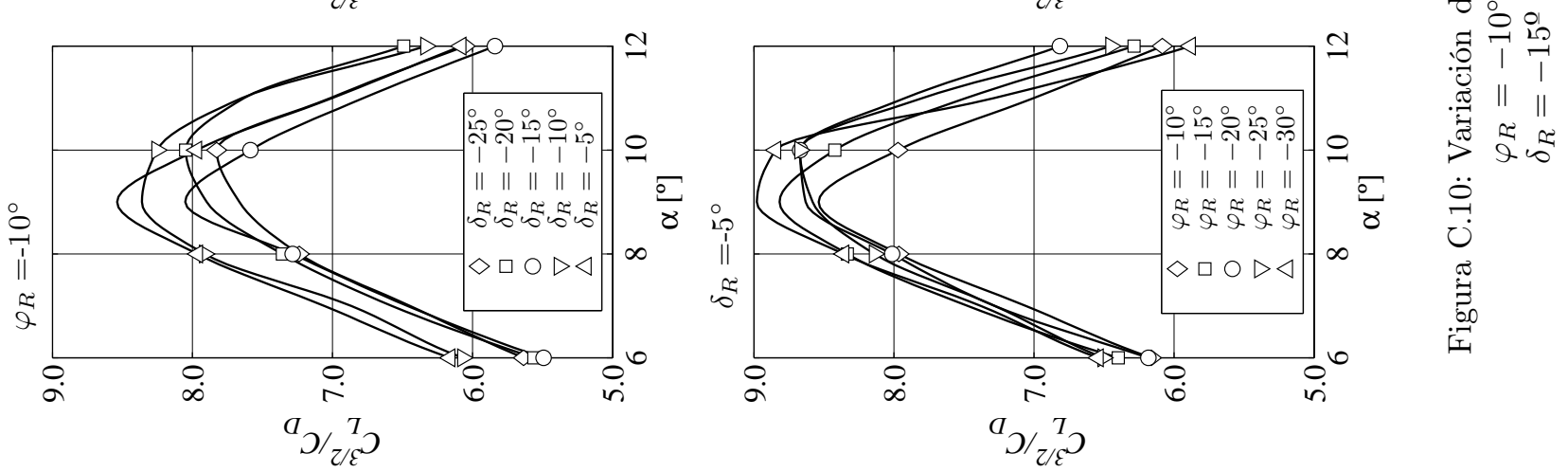


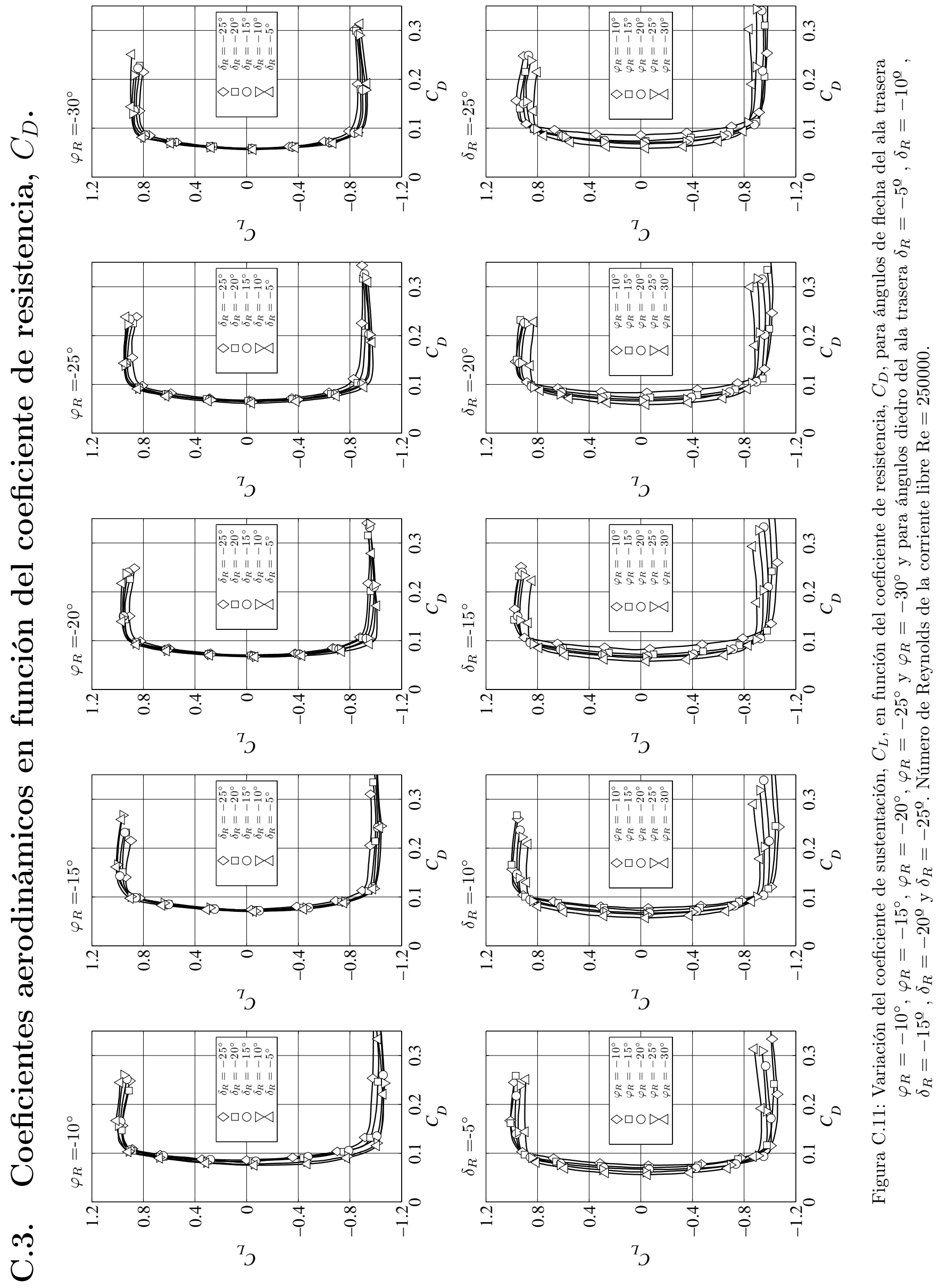



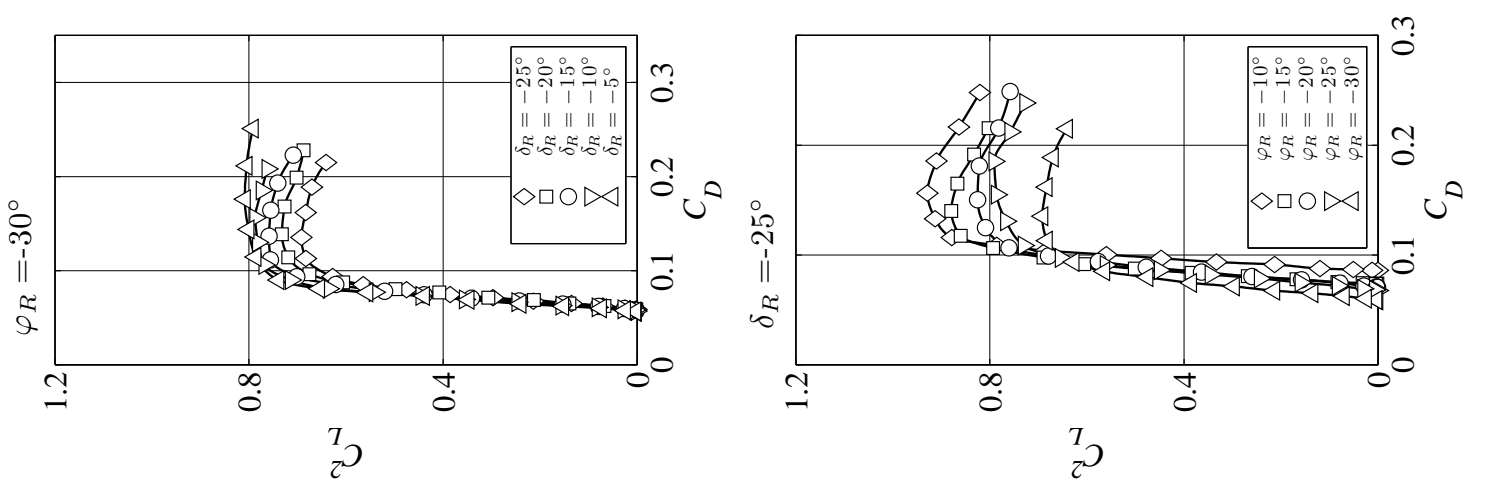

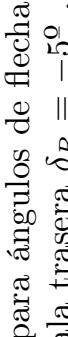
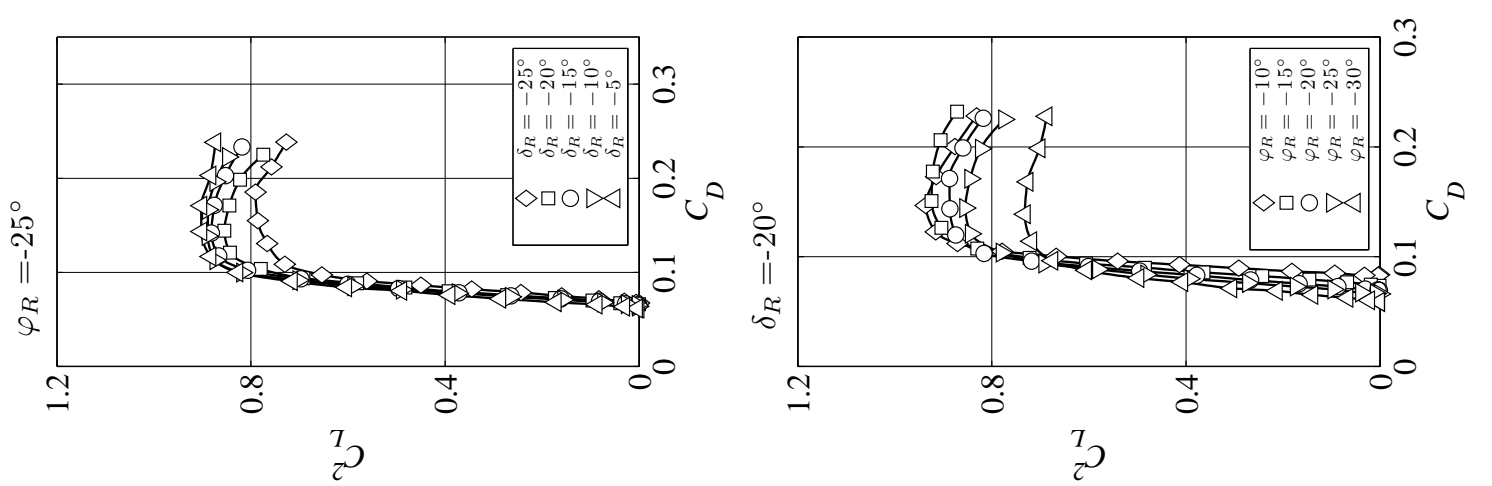

i。

ชे

ชీ

卷

th

$\sum_{0 \rightarrow 0}$

范 :

苛芯.

车

ठำ
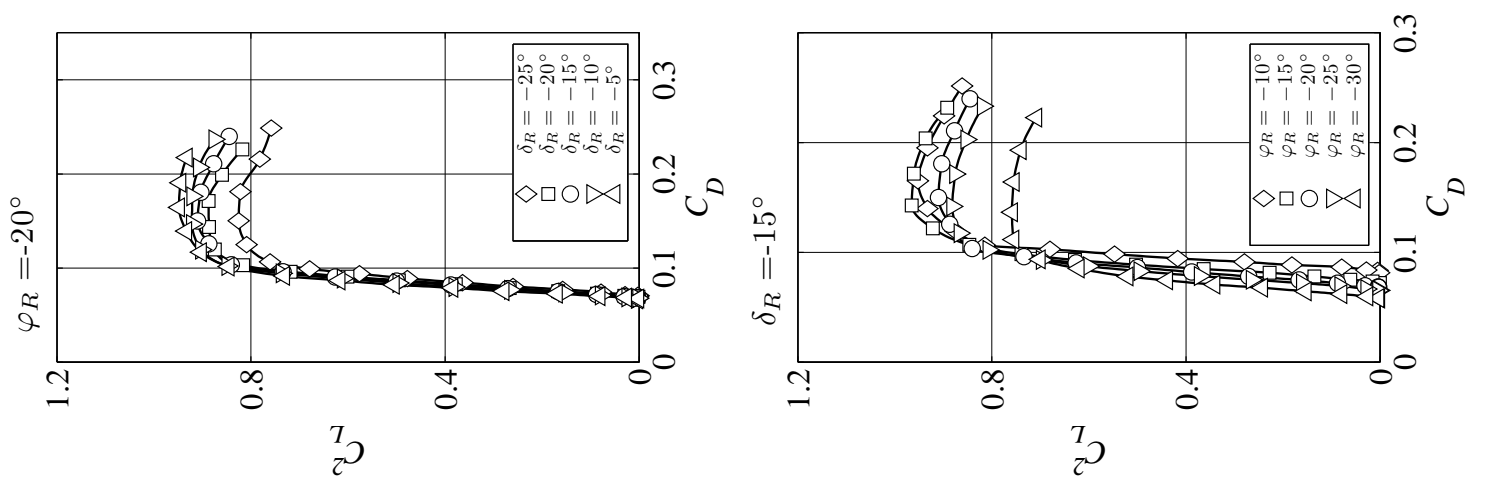

ष्ष $\|$

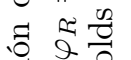

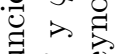

$\exists$ is

₹ $\uparrow$

बิ่ 11 .

ध 2 ह

:

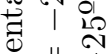

荡 11

के
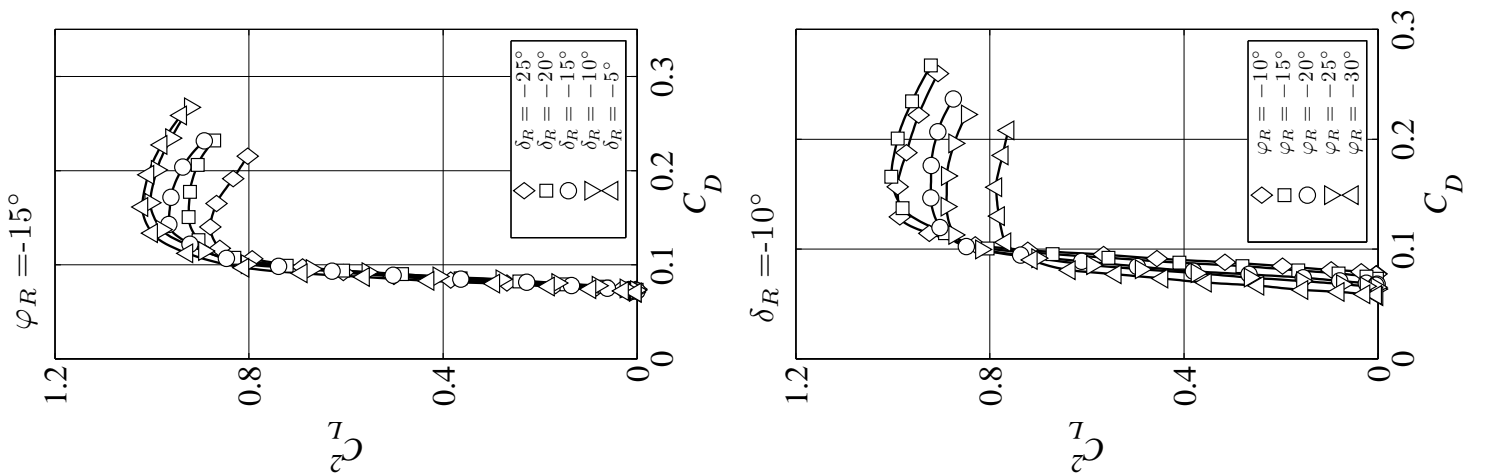

- 0.0

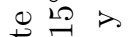

.

c

\& \&

용

유일

䒕

త
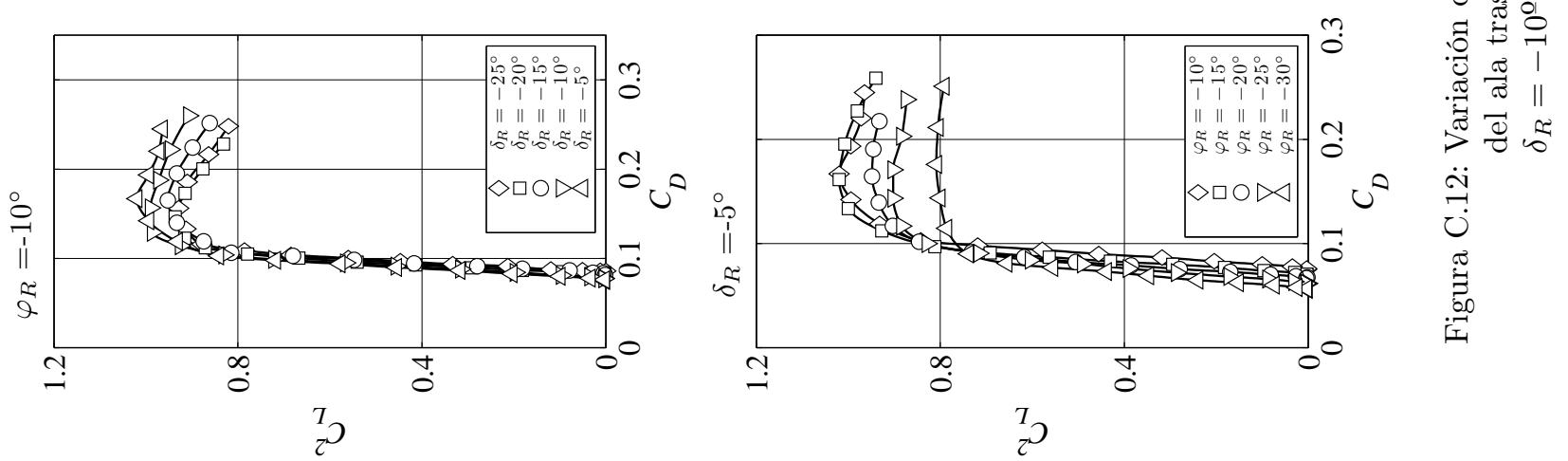

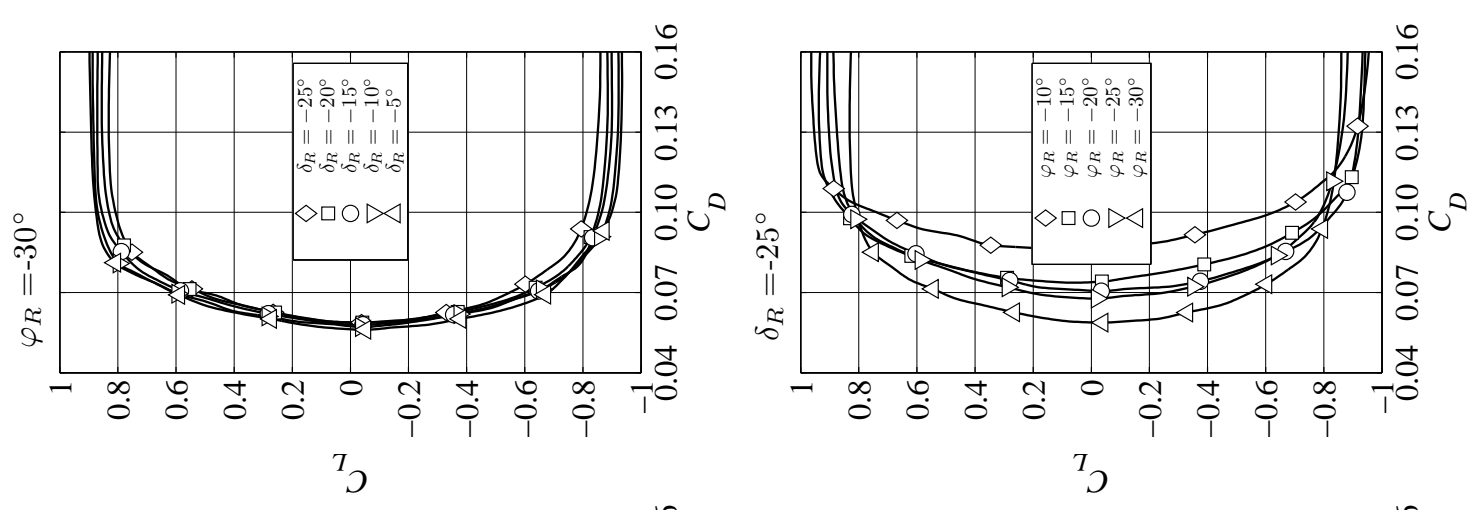

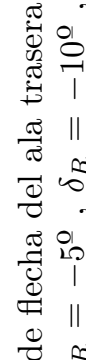
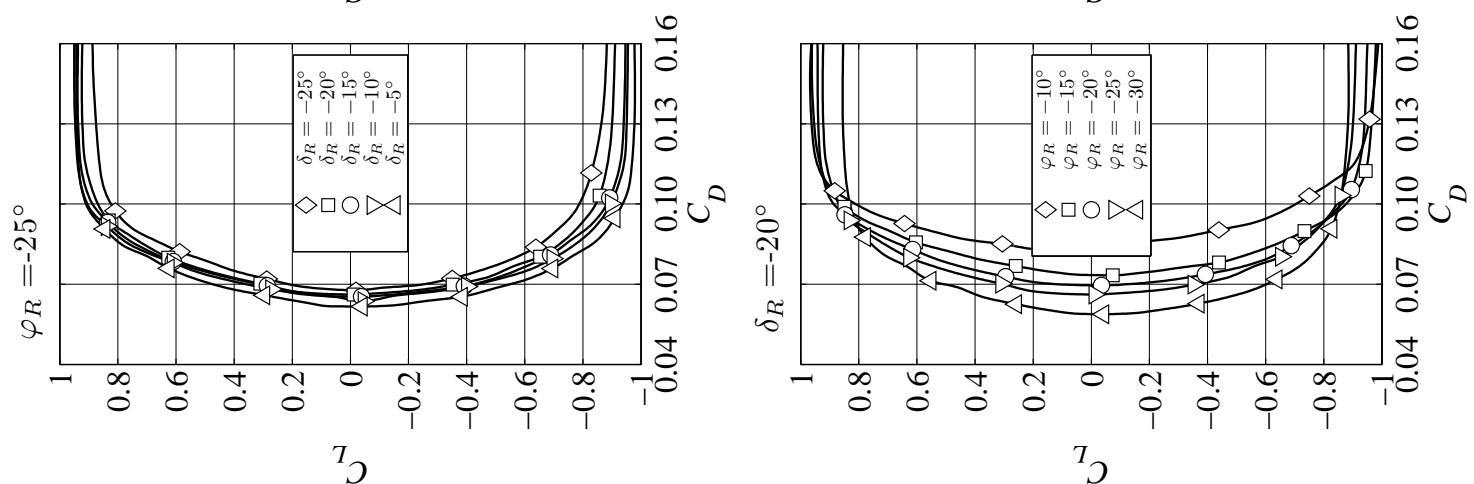

茴

䒕

A

O ㅇํㅇ

总

预

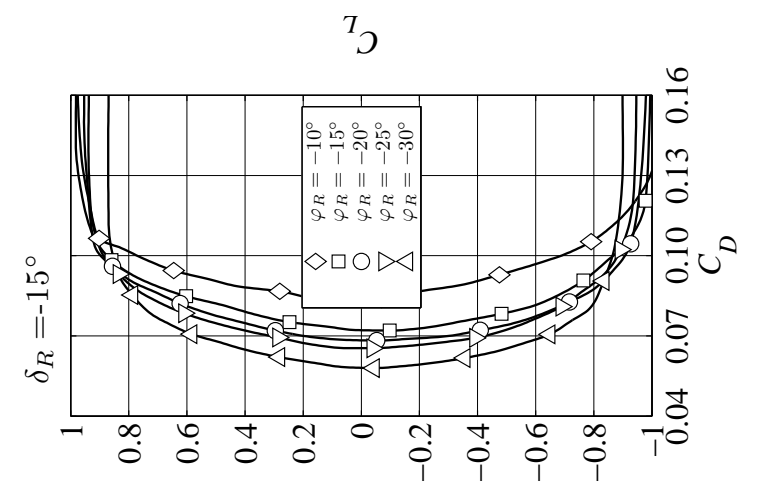

过

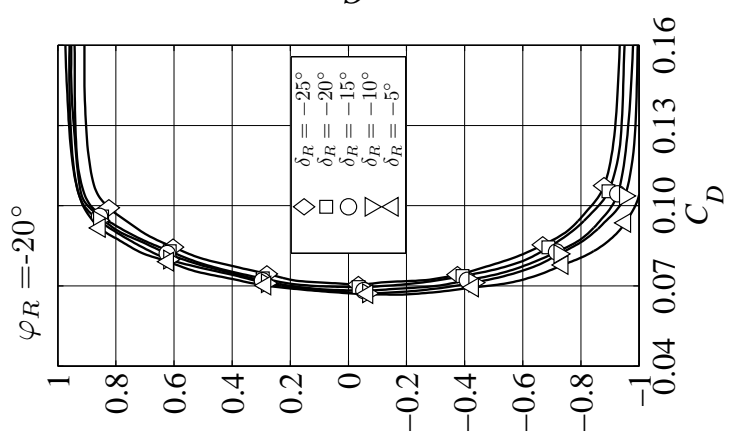

${ }^{7} \mathrm{~J}$
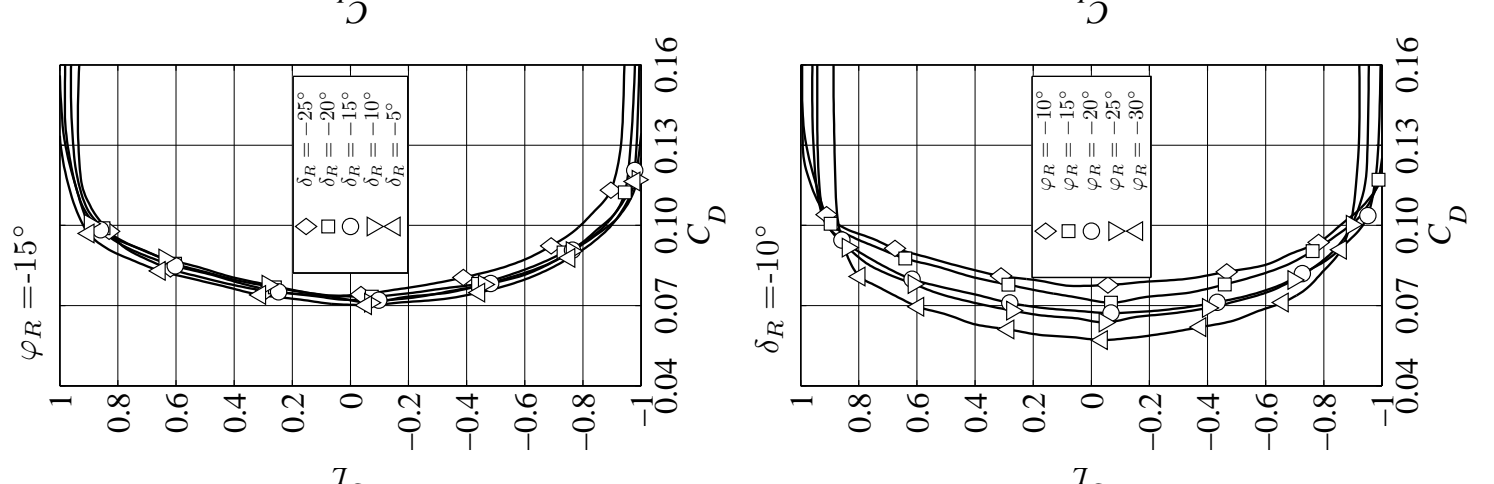

ข

窇

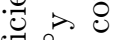

要 -

811 㐘

:유 9

苛 $\rightarrow$

농

जा 11

0

ริ 9

웡 ㅇำ

द्ष

类 ||

क

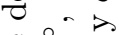

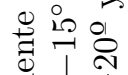

或 ||

8 造

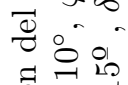
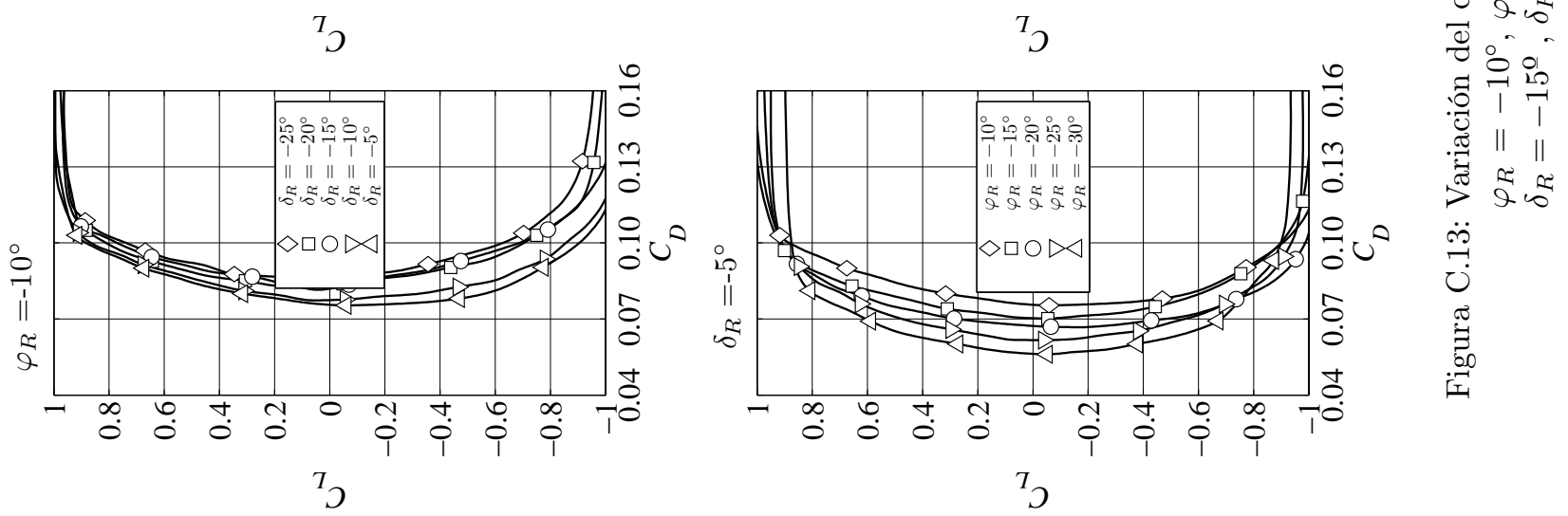

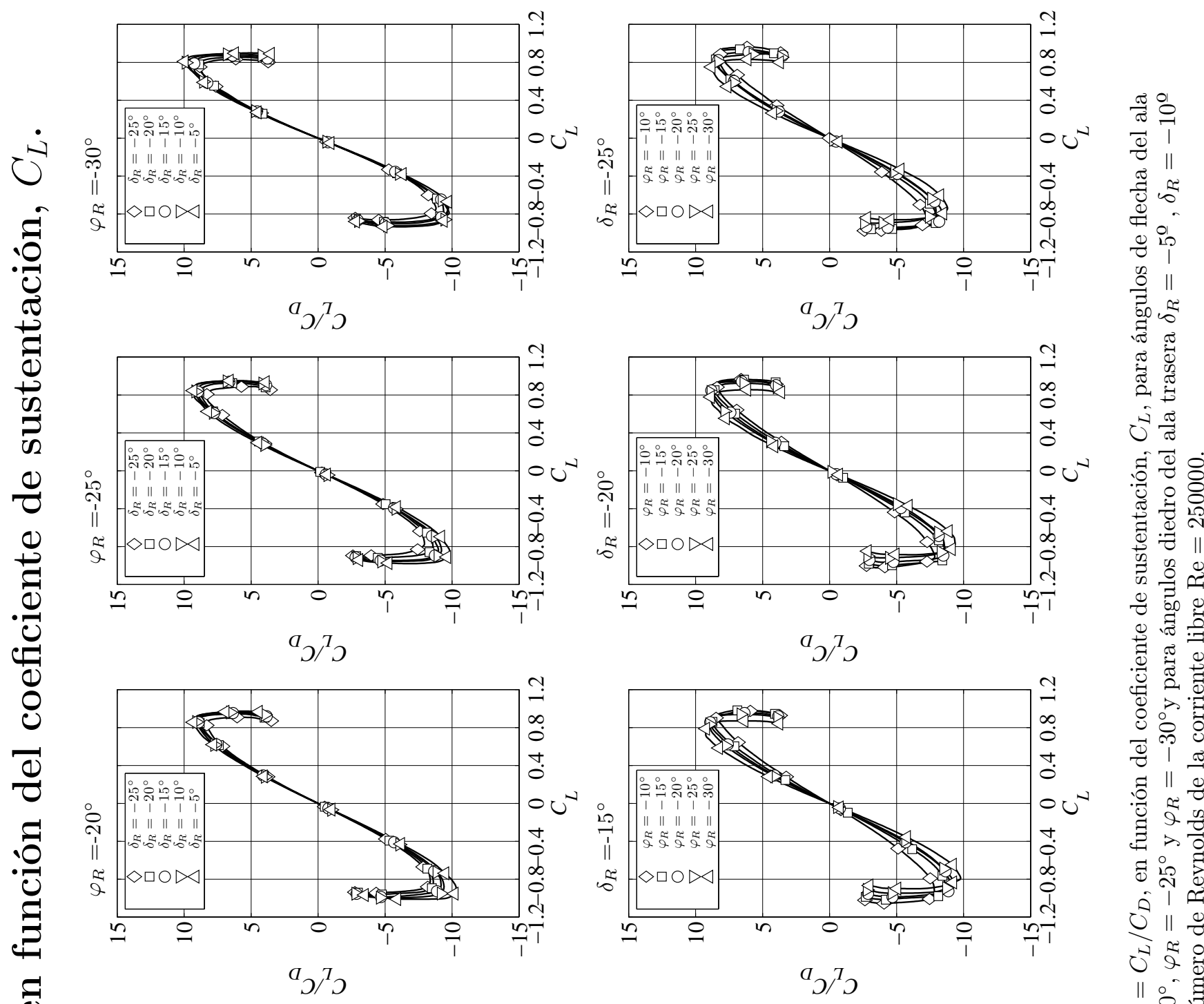

苞

0

$\bar{\theta}$ i

유 응

承造

4

ปิ

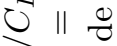

$0^{2} x^{2}$

$\| \circ \circ^{\circ}$ 多
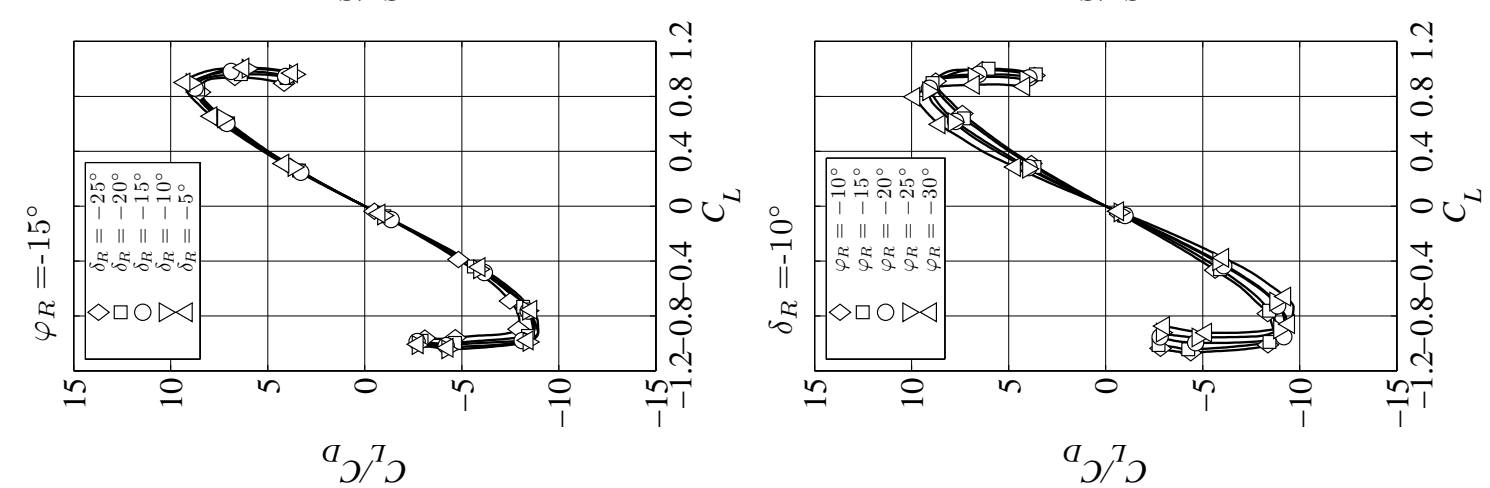

종ำ

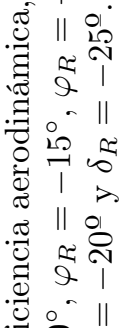

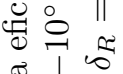

\& 110

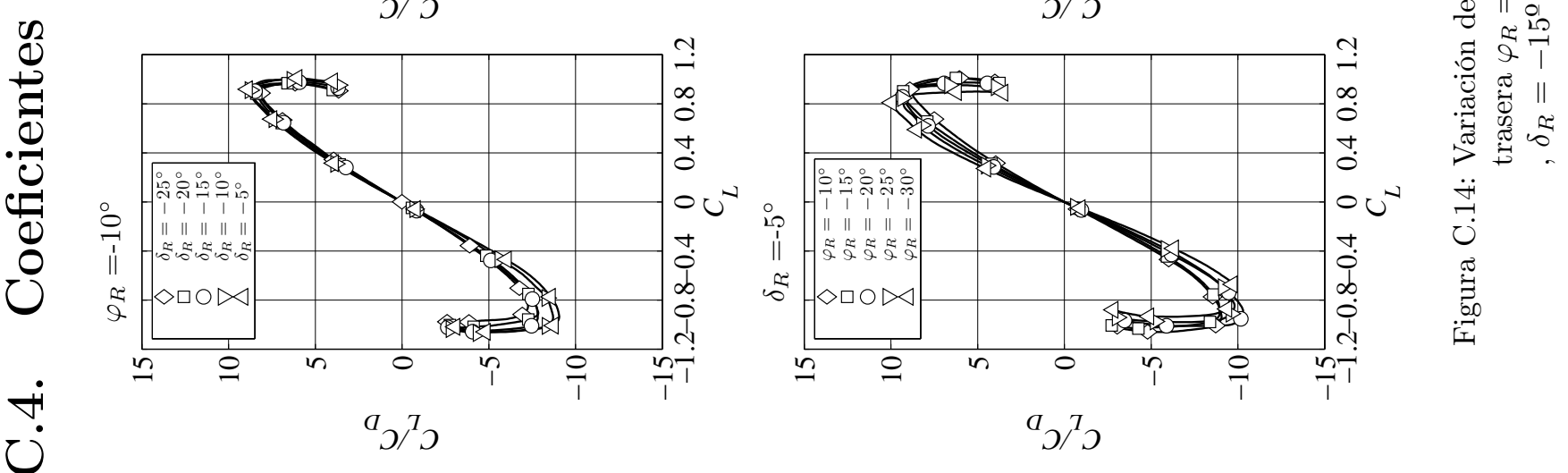



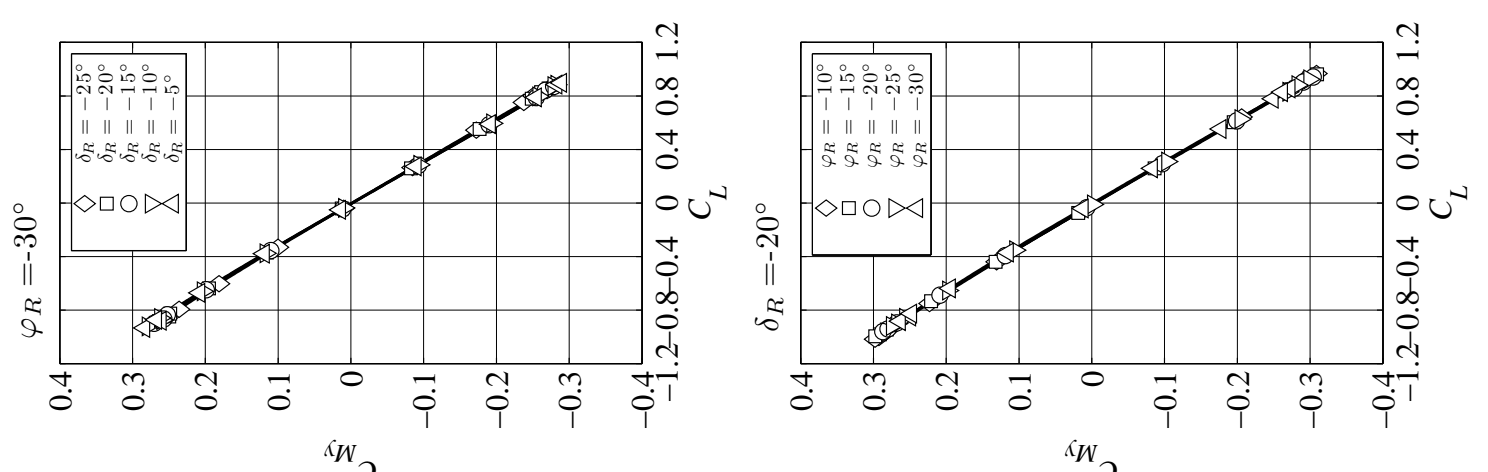

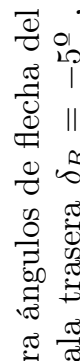
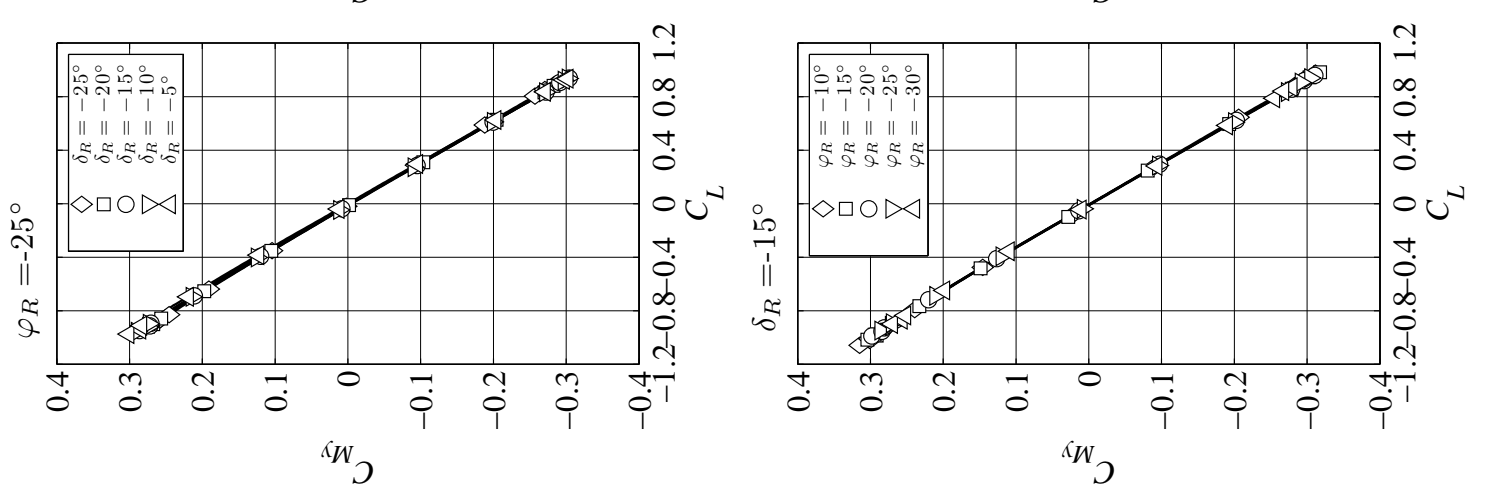

ใี

U

0

:0ี :

हैं

菏

का

ช

事落

.
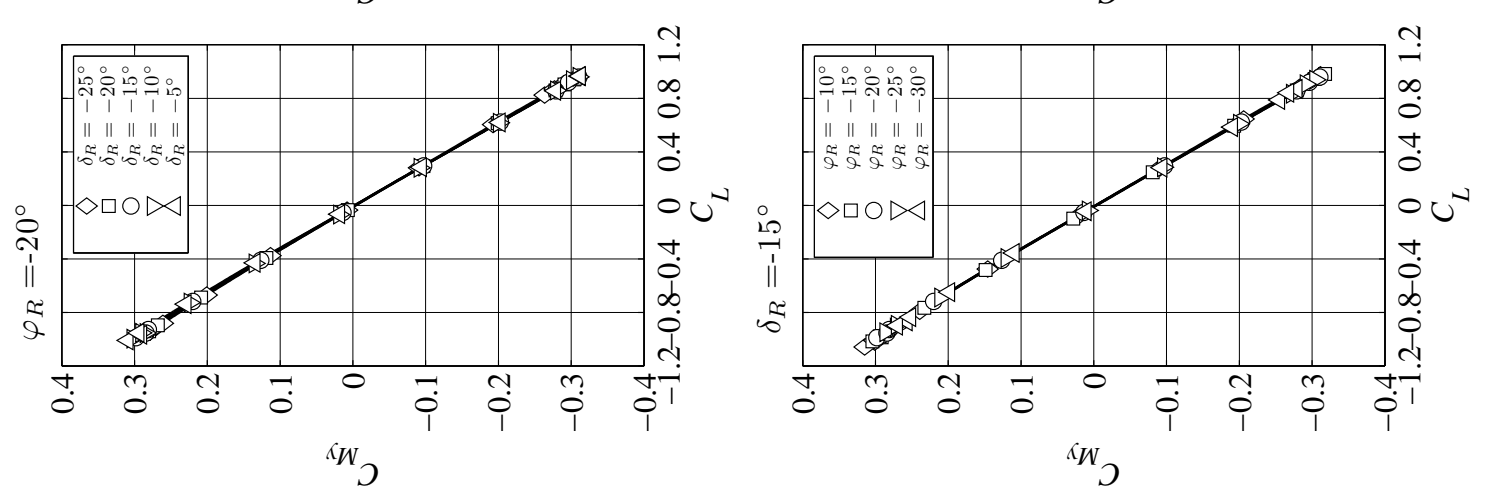

10

\& || in

용

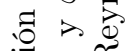

웝 웡

$\rightleftarrows 10$

ฮ ||

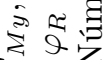

ᄋำ

(1)
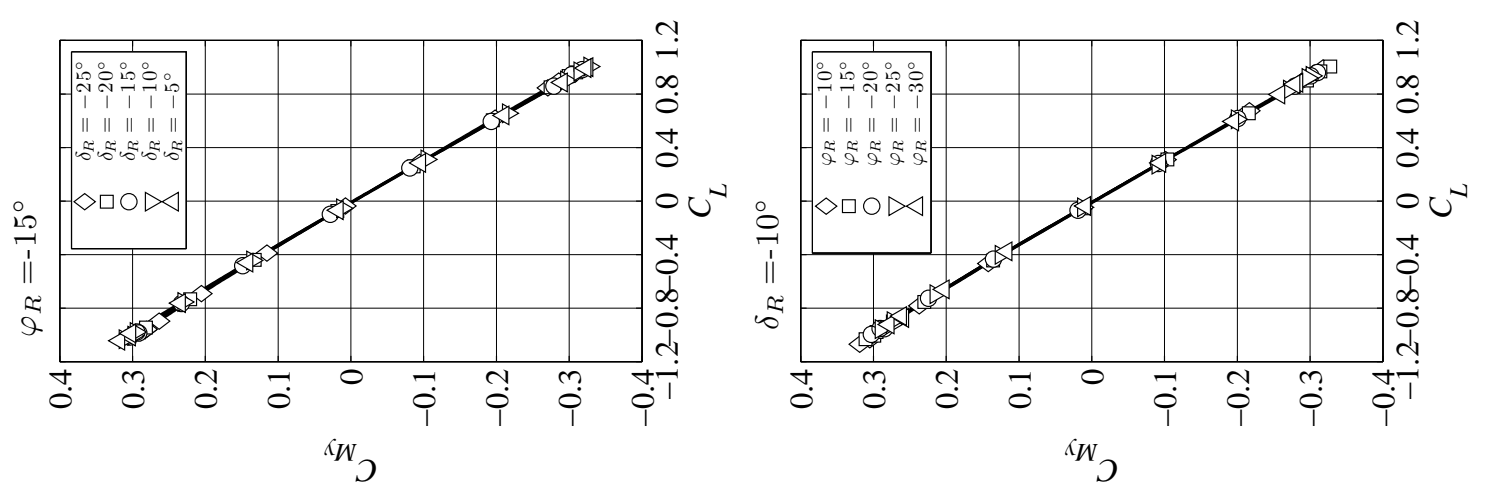

o एक

ช $2 \infty$

율 옥 ᄋ

I ।

\& $\|$

¿ $2 x^{2}$

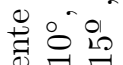

। ।

\& $\|$

동.
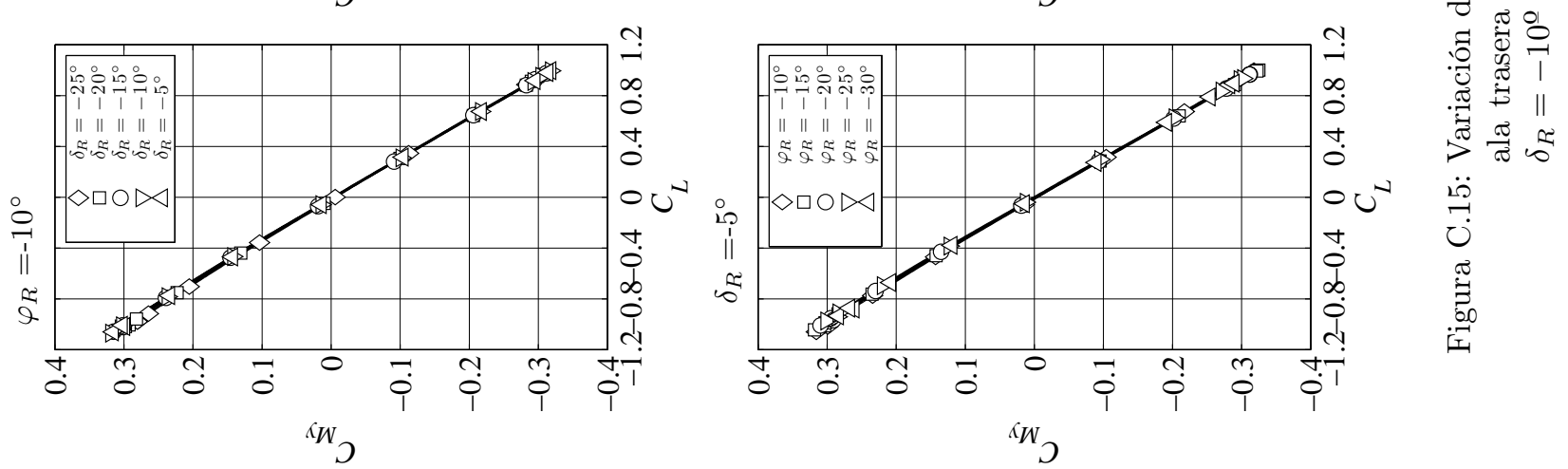



\section{Apéndice D}

\section{Resultados de ensayo 2}

\section{D.1. Introducción.}

En este apéndice se presenta los resultados completos de los valores de los coeficientes aerodinámicos del modelo de alas convergentes en formato de mapa de color con líneas de isovalores en función del ángulo diedro, $\delta_{R}=\left[-5^{\circ},-25^{\circ}\right]$, del ángulo de flecha del ala trasera, $\varphi_{R}=\left[-10^{\circ},-30^{\circ}\right]$, para ángulos de ataque $\alpha=\left[-14^{\circ}, 15^{\circ}\right]$.

Los valores representados en los mapas de color corresponden a los normalizados con respecto al valor del coeficiente correspondiente determinados en la configuración $\mathrm{JWC}_{15}\left(\delta_{R}=-25^{\circ}, \varphi_{R}=-30^{\circ}\right)$ para cada ángulo de ataque, $\alpha$, mostrándose además y de forma explícita los valores máximo y mínimo del coeficiente normalizado para cada ángulo de ataque.

Los coeficientes representados son:

- Figura D.1 a Figura D.5 está representado el valor del coeficiente de sustentación normalizado, $\overline{C_{L}}$.

- Figura D.6 a Figura D.10 está representado el valor del coeficiente de resistencia normalizado, $\overline{C_{D}}$.

- Figura D.11 a Figura D.15 está representado el valor del coeficiente de resistencia inducida normalizado, $\overline{C_{D i}}$.

- Figura D.16 a Figura D.20 está representado el valor del coeficiente de eficiencia aerodinámica normalizado, $\bar{E}$.

- Figura D.21 a Figura D.23 está representado el valor del coeficiente para potencia mínima requerida normalizado, $\bar{P}=\overline{C_{L}^{3 / 2} / C_{D}}$. Para este parámetro la representación sólo se hace para valores de ángulo de ataque $\alpha=\left[0^{\circ}, 15^{\circ}\right.$. 

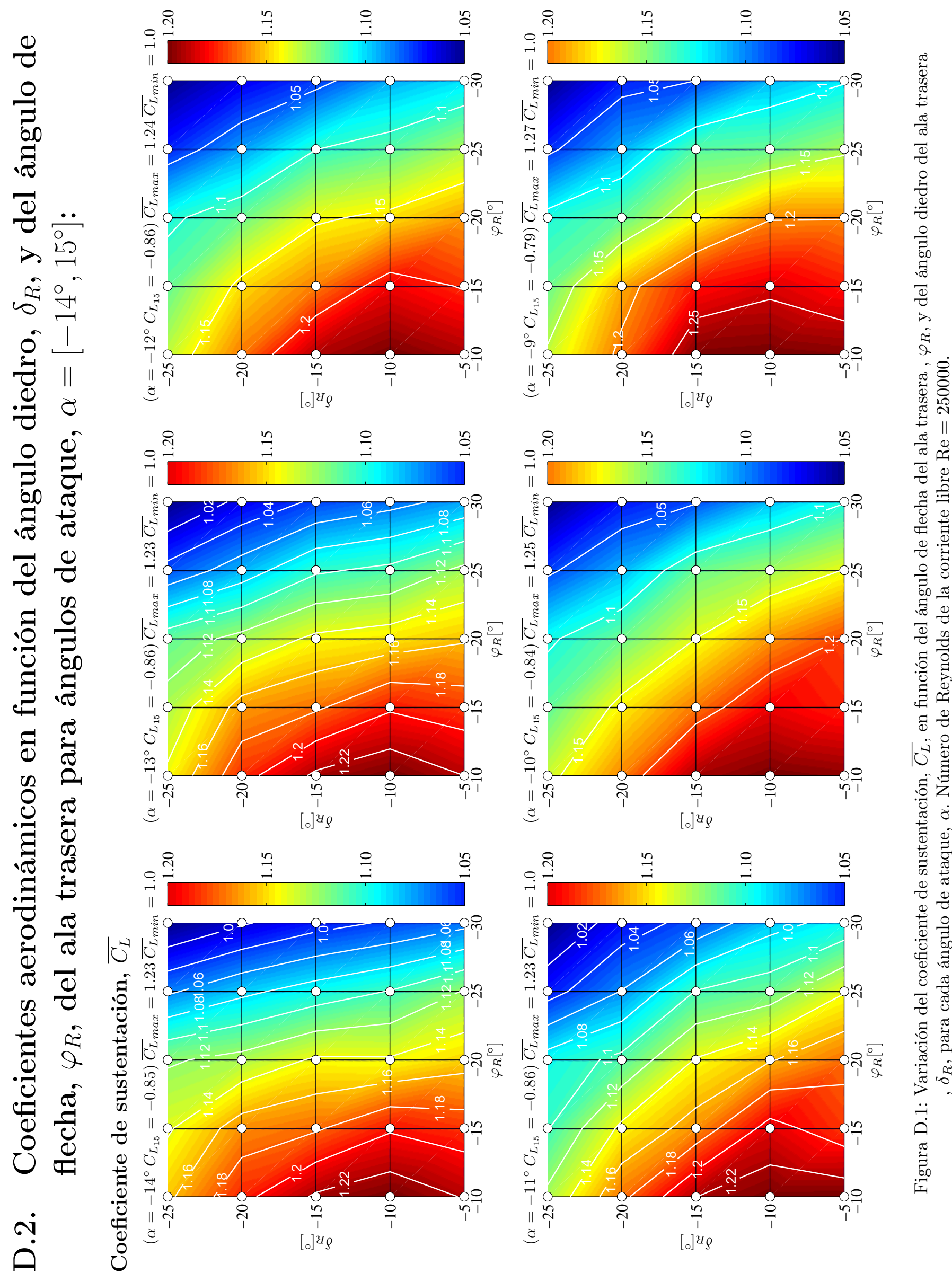

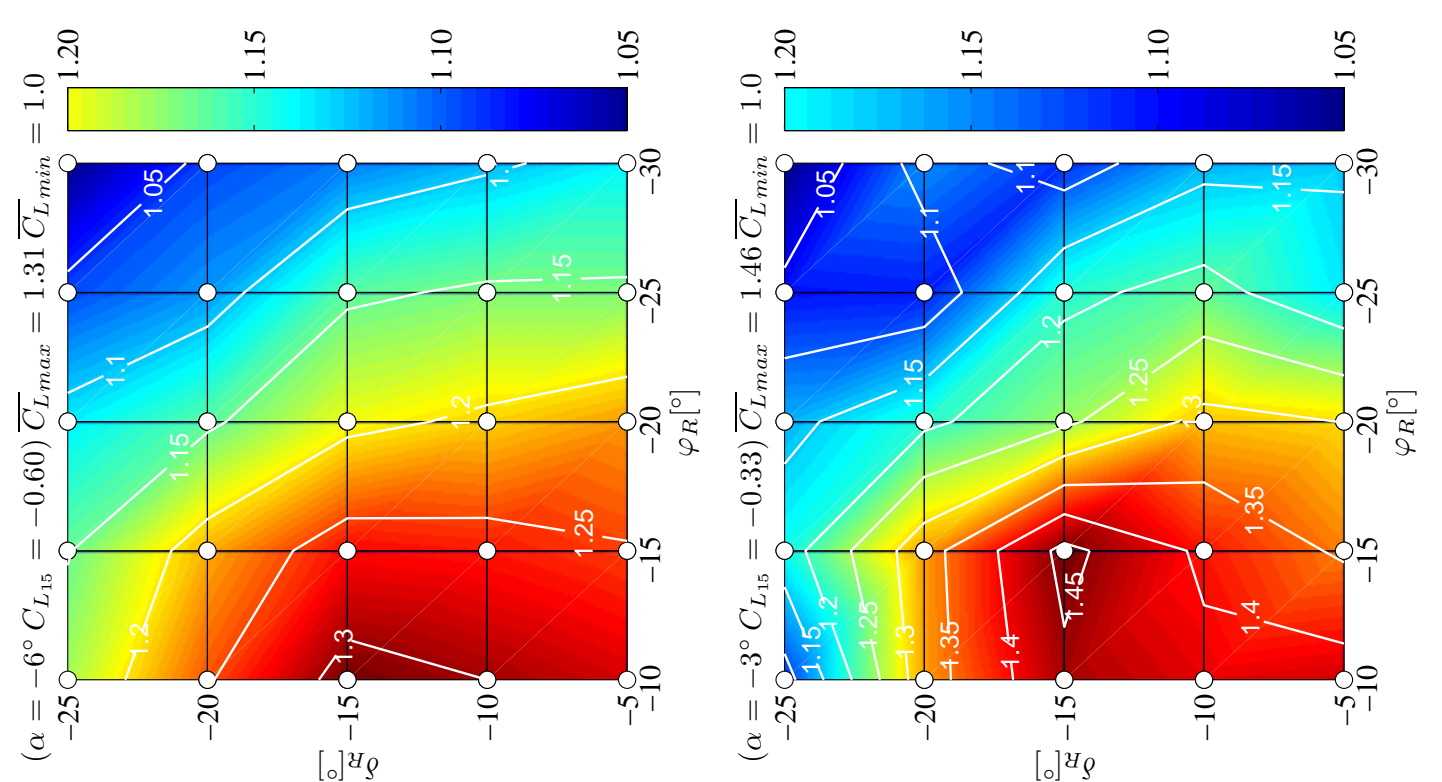

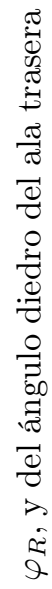
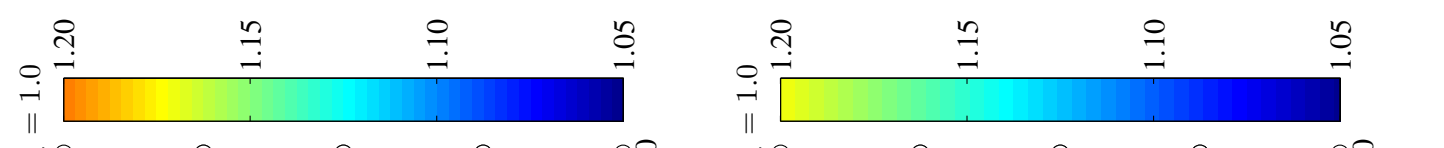

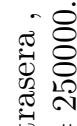
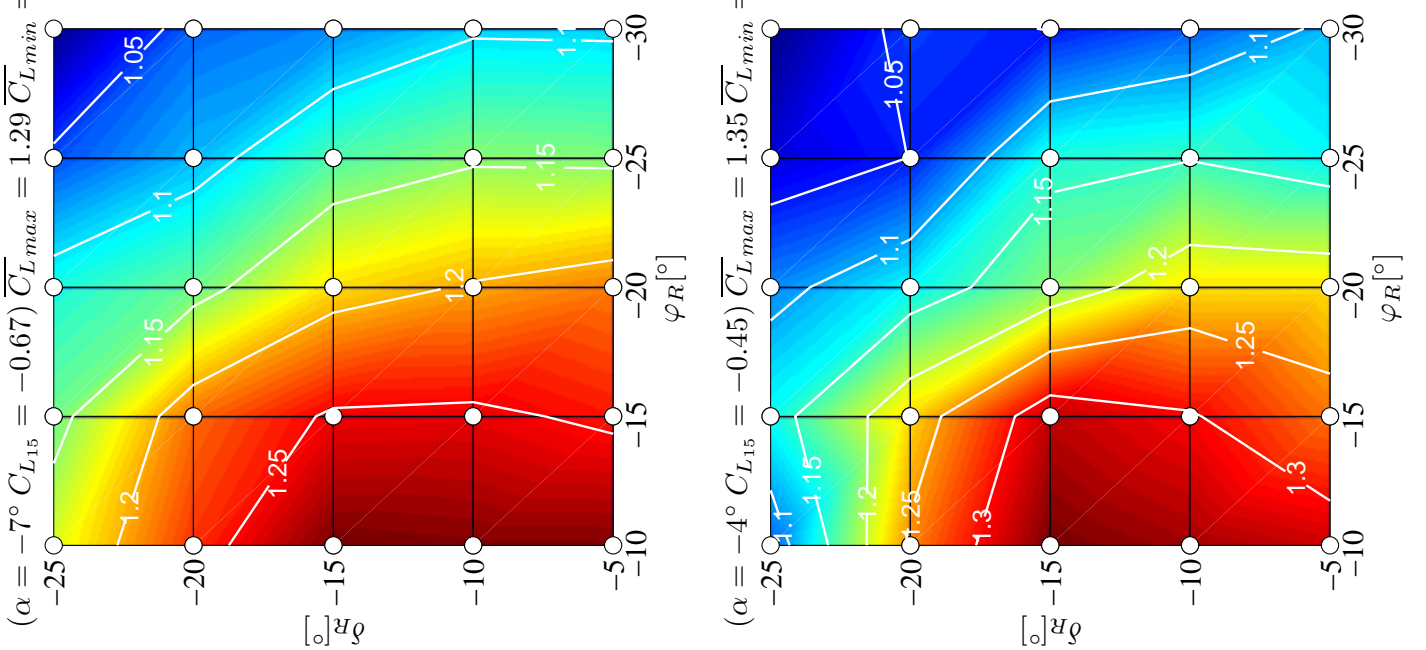

蛋

웝

ช

을

跑

웜

:월

灵

하용

过兽

音

苟
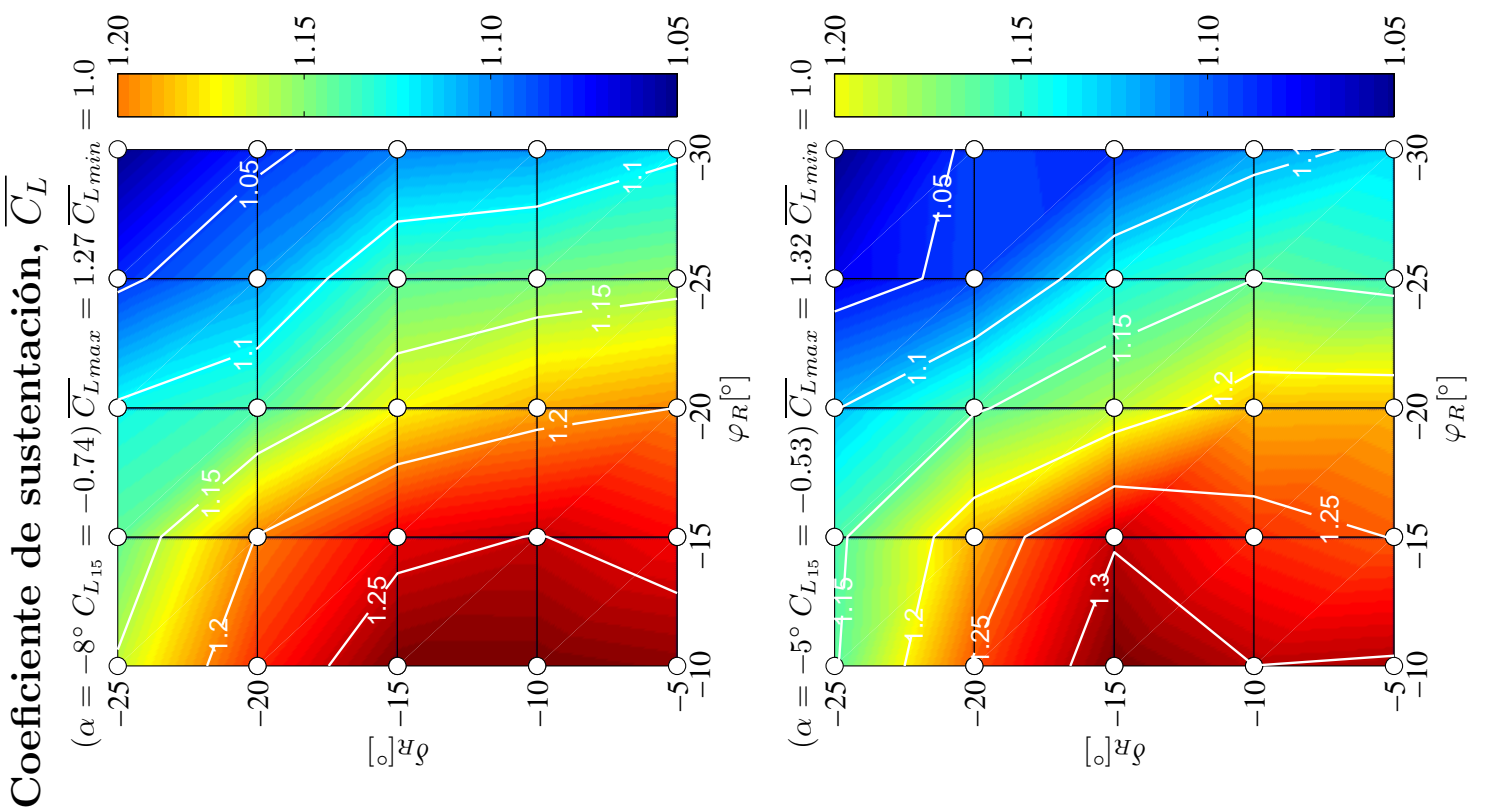

范 

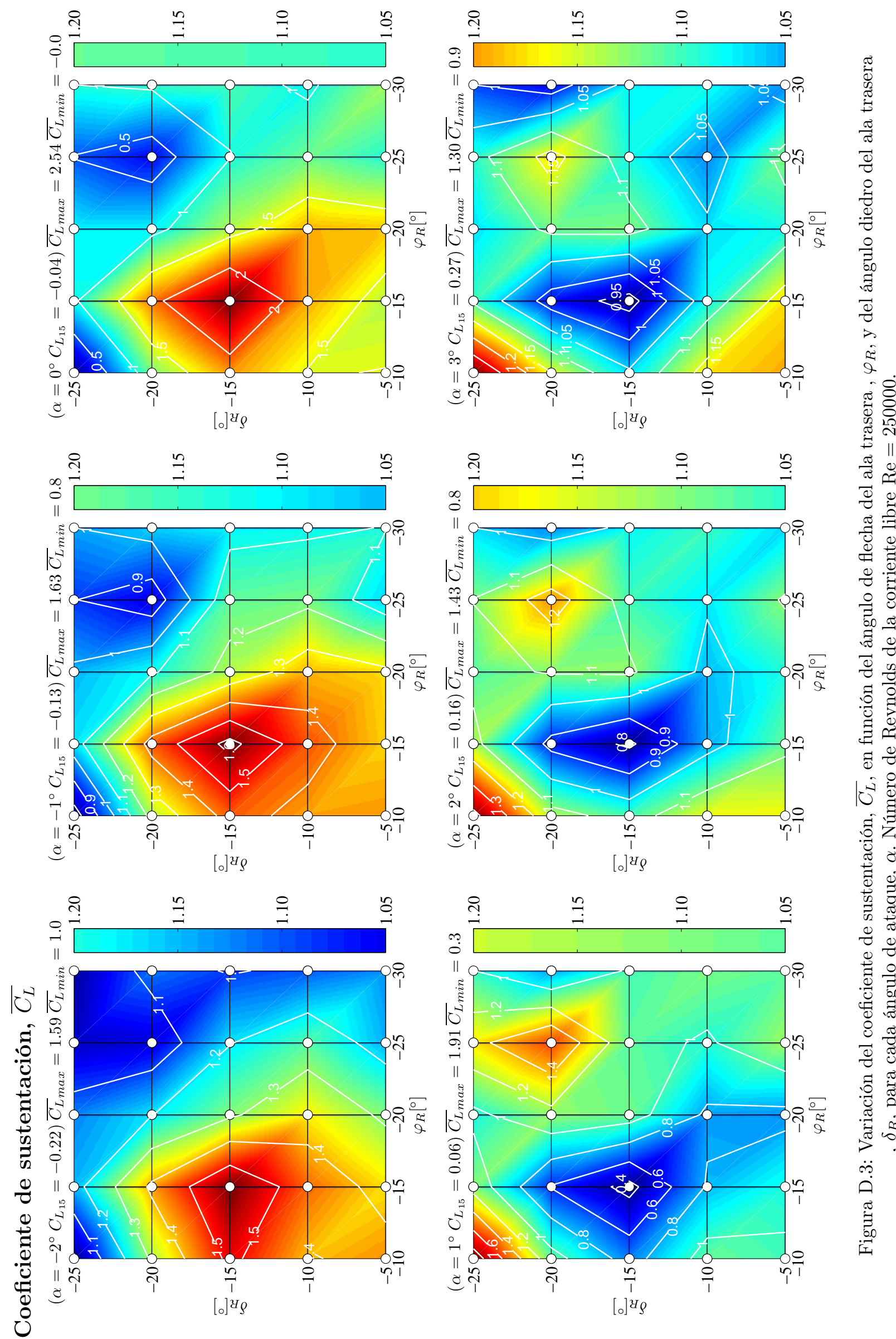

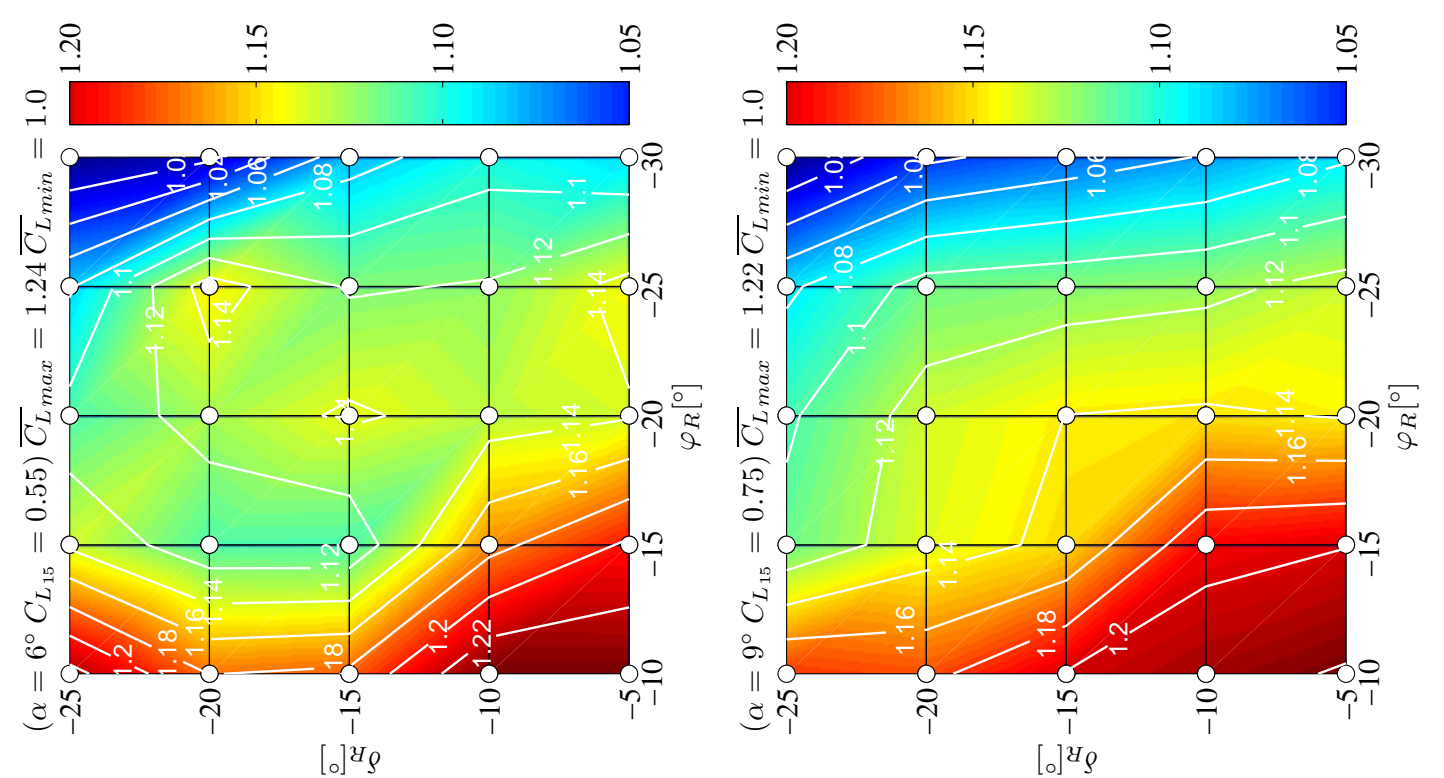

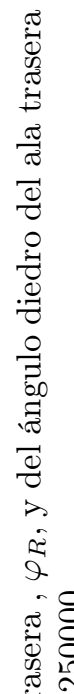
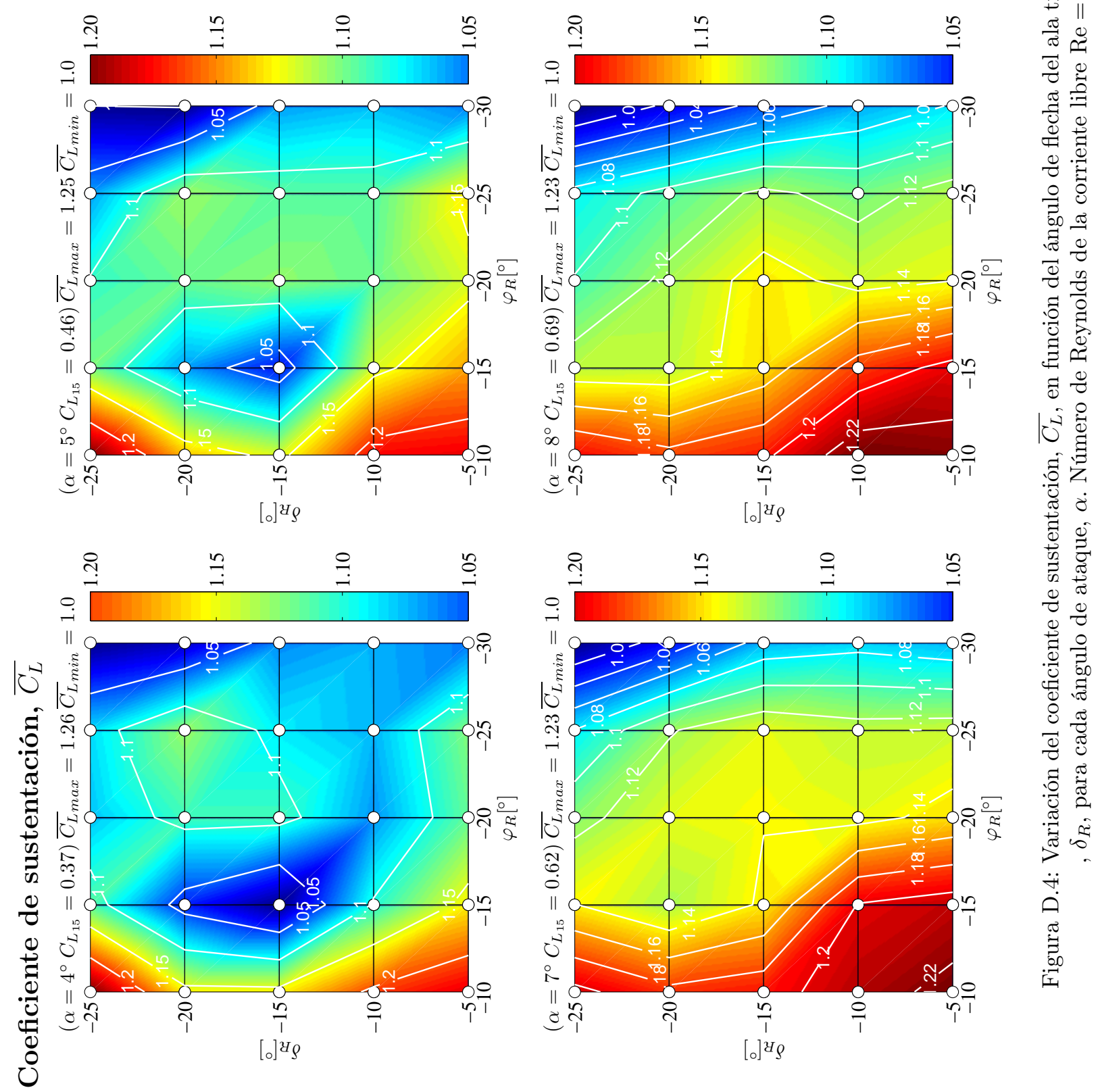

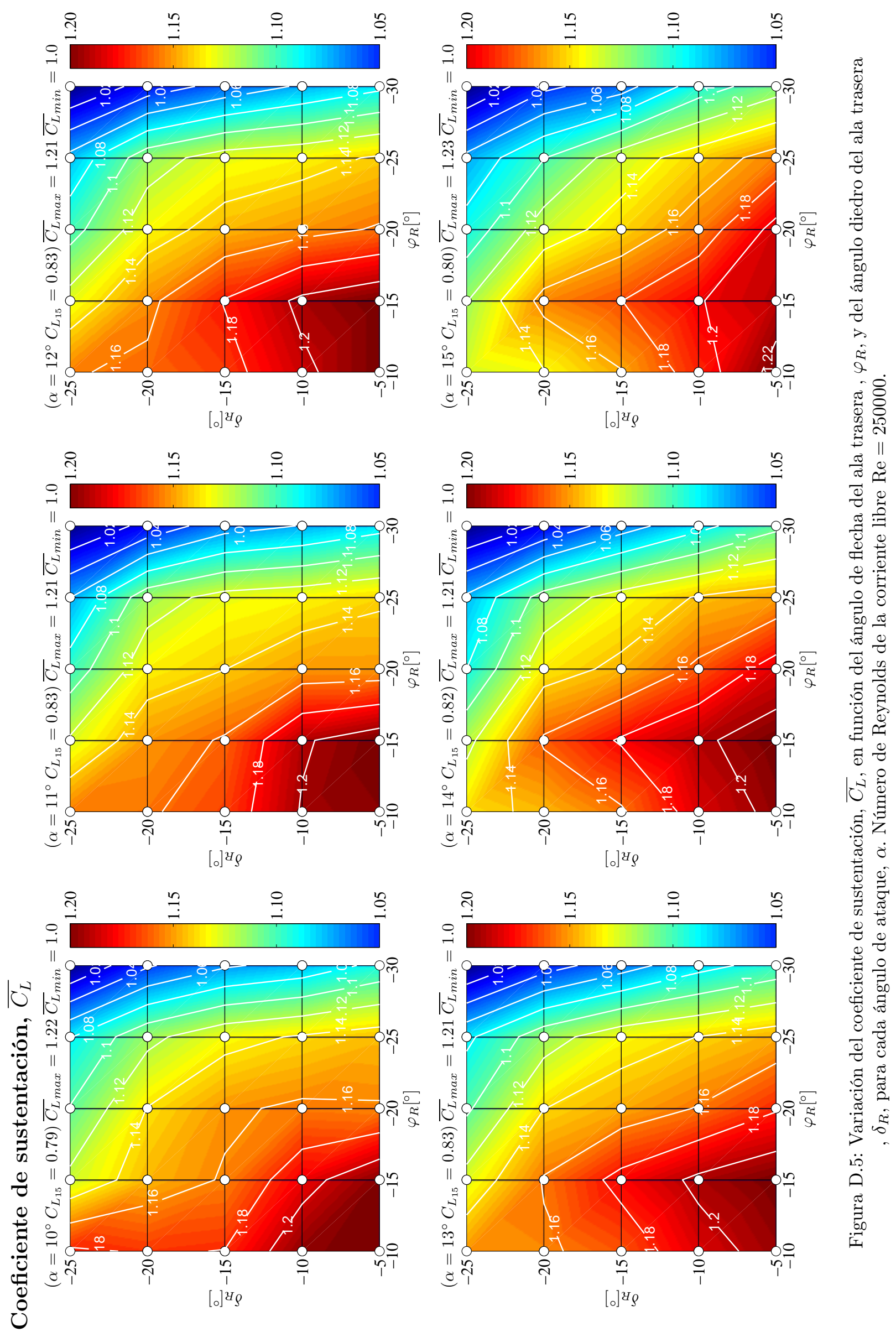

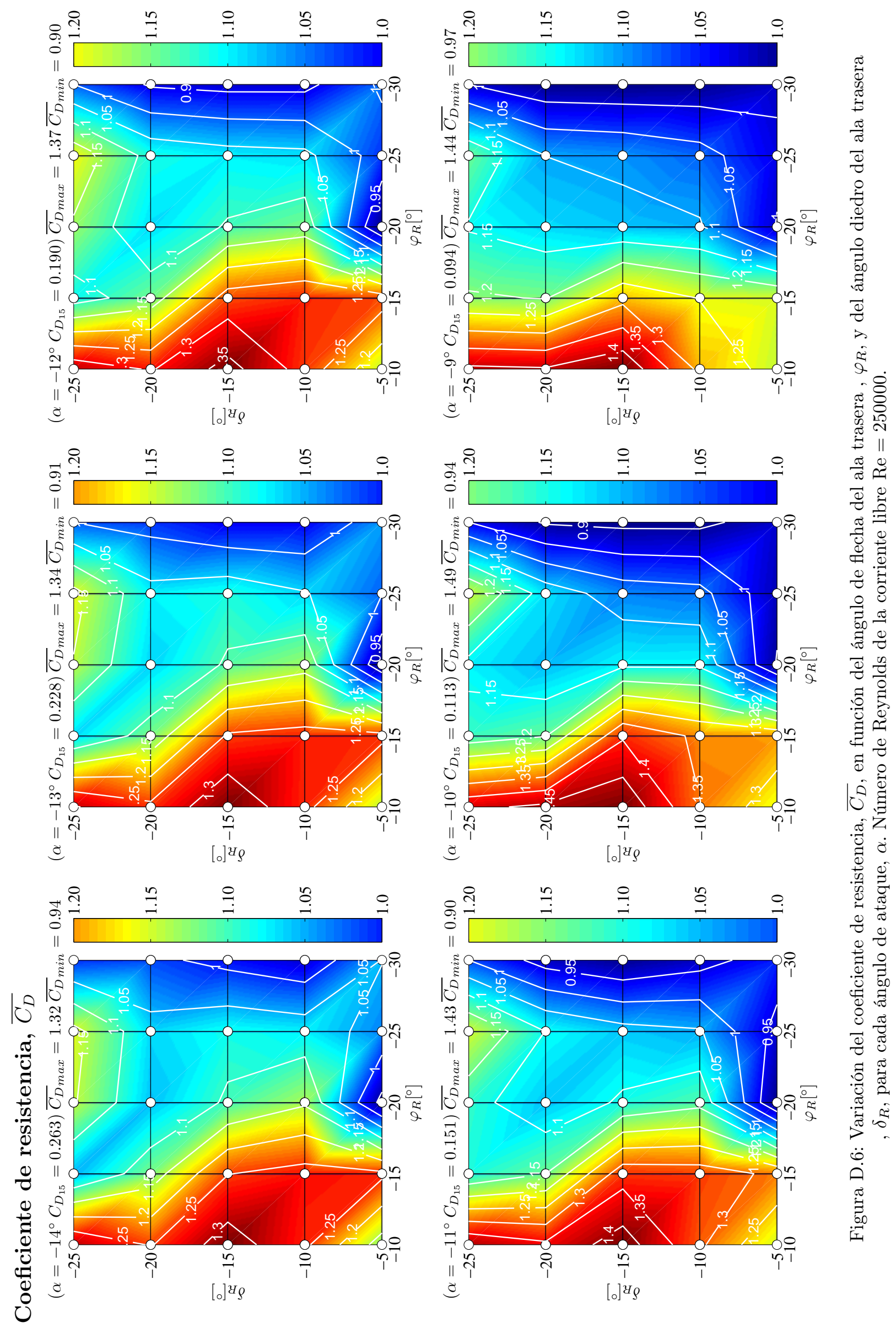

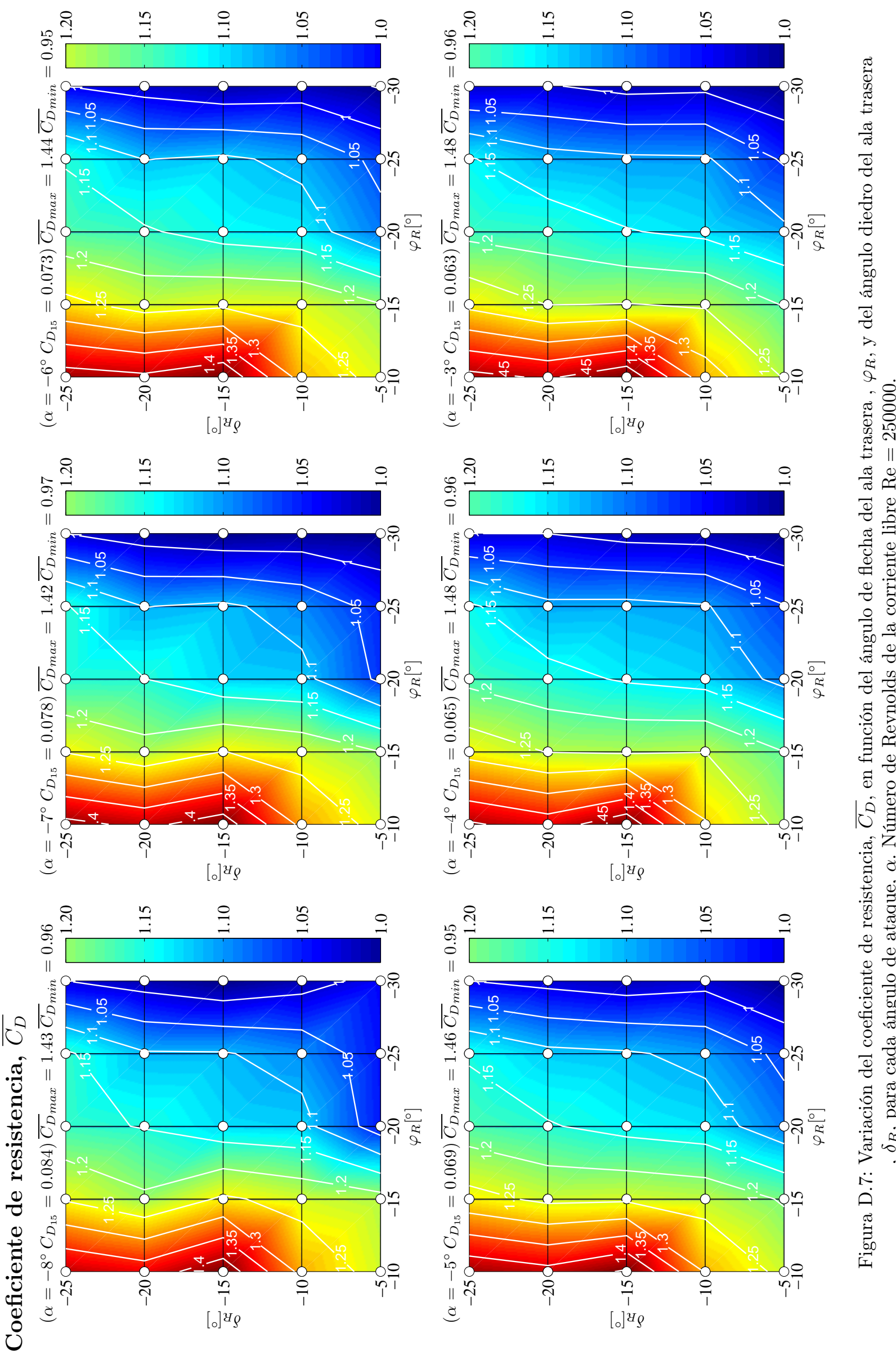

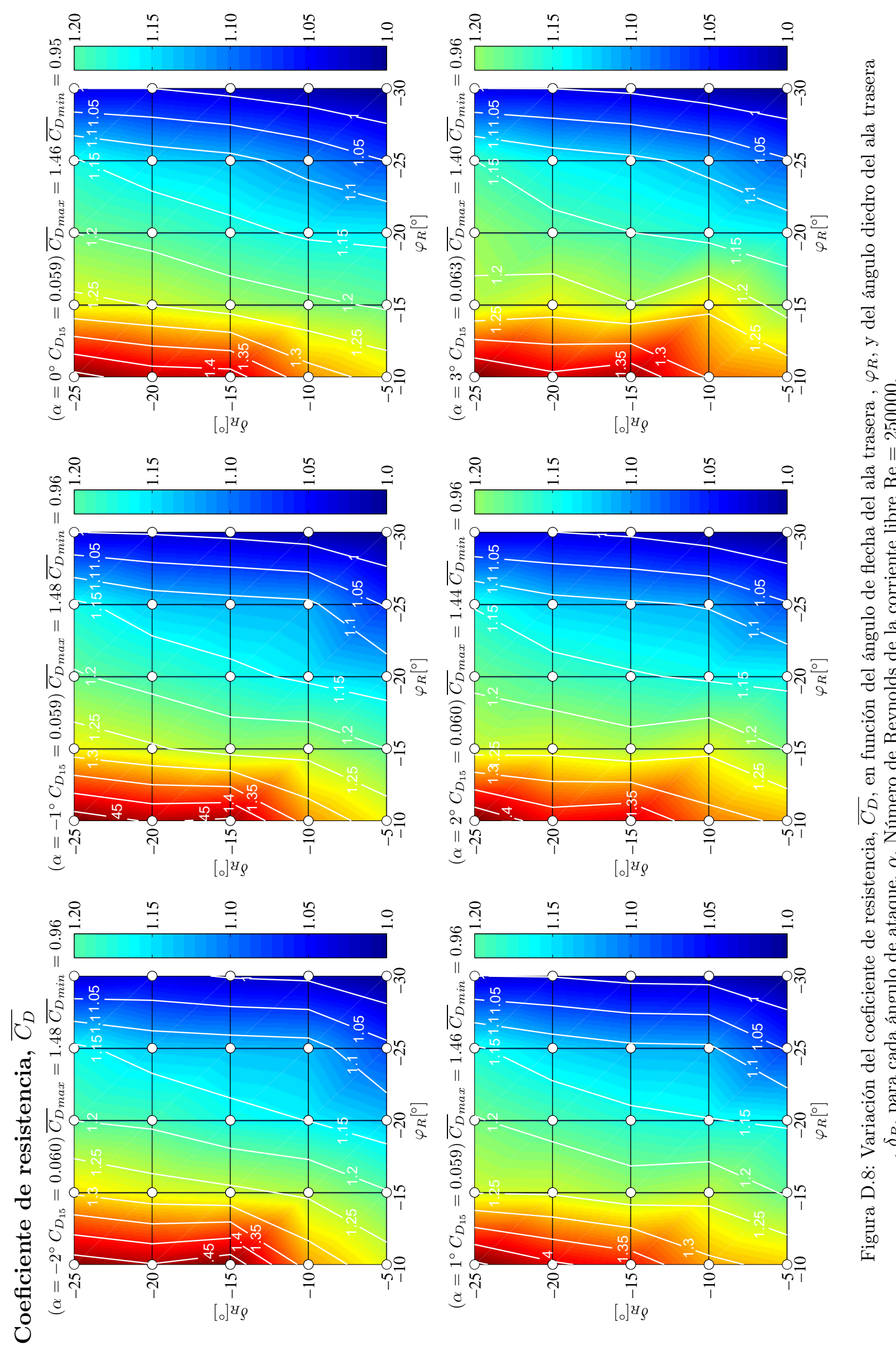

○

\%

홍

궝

응

응

สี

षृ

:웅

드

하

过

D

0

$\stackrel{0}{0}$

可 

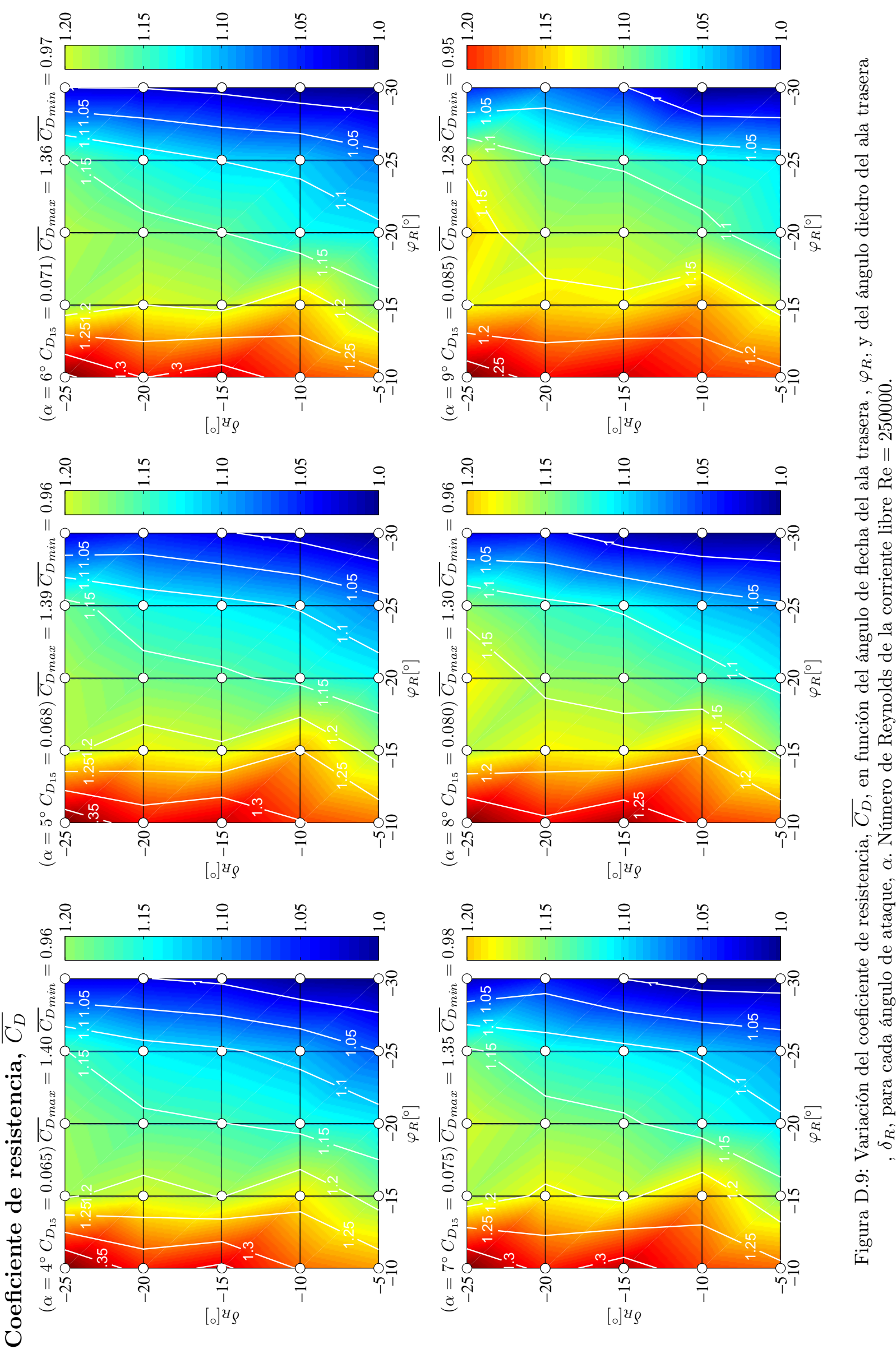

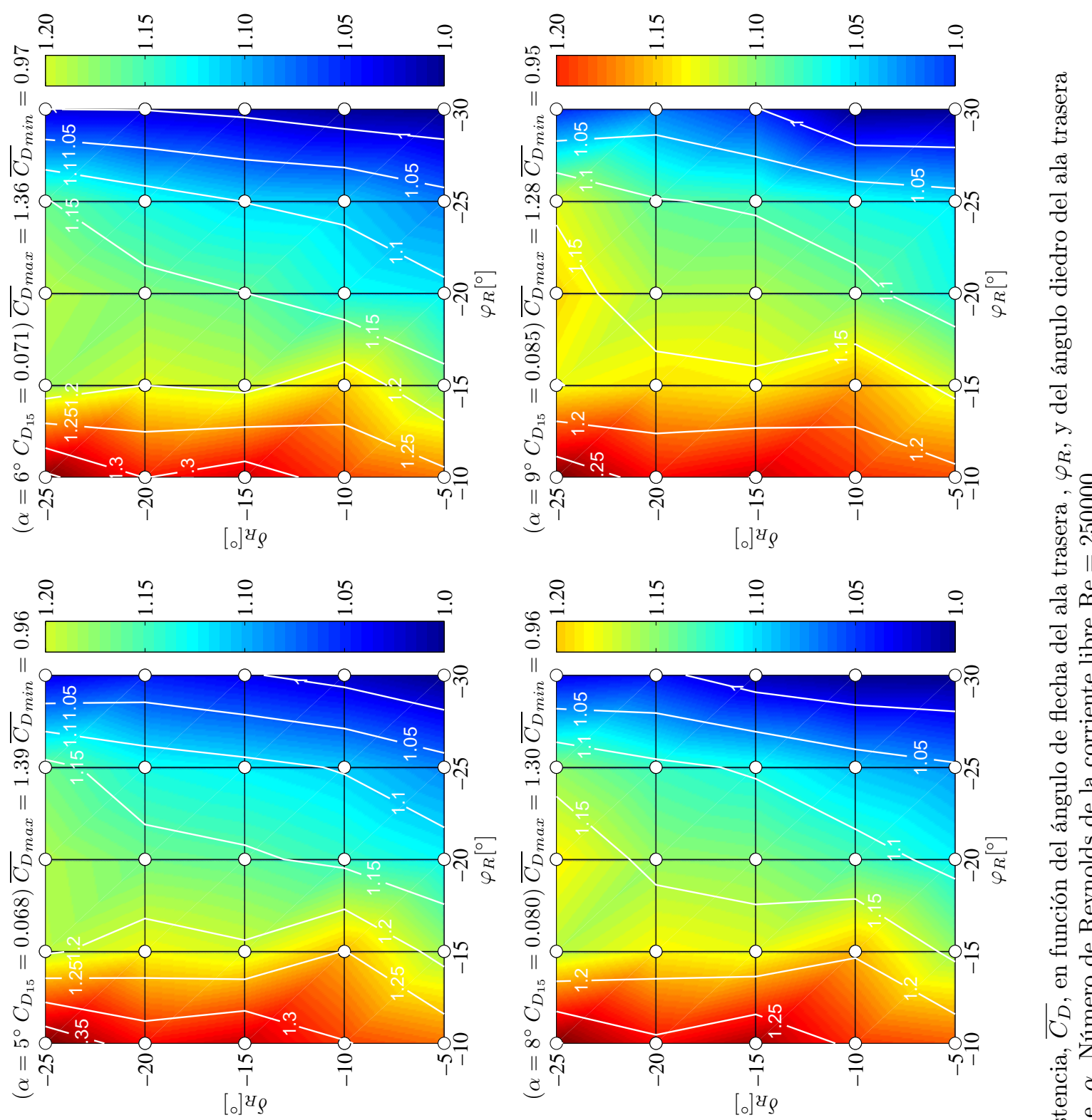

\&
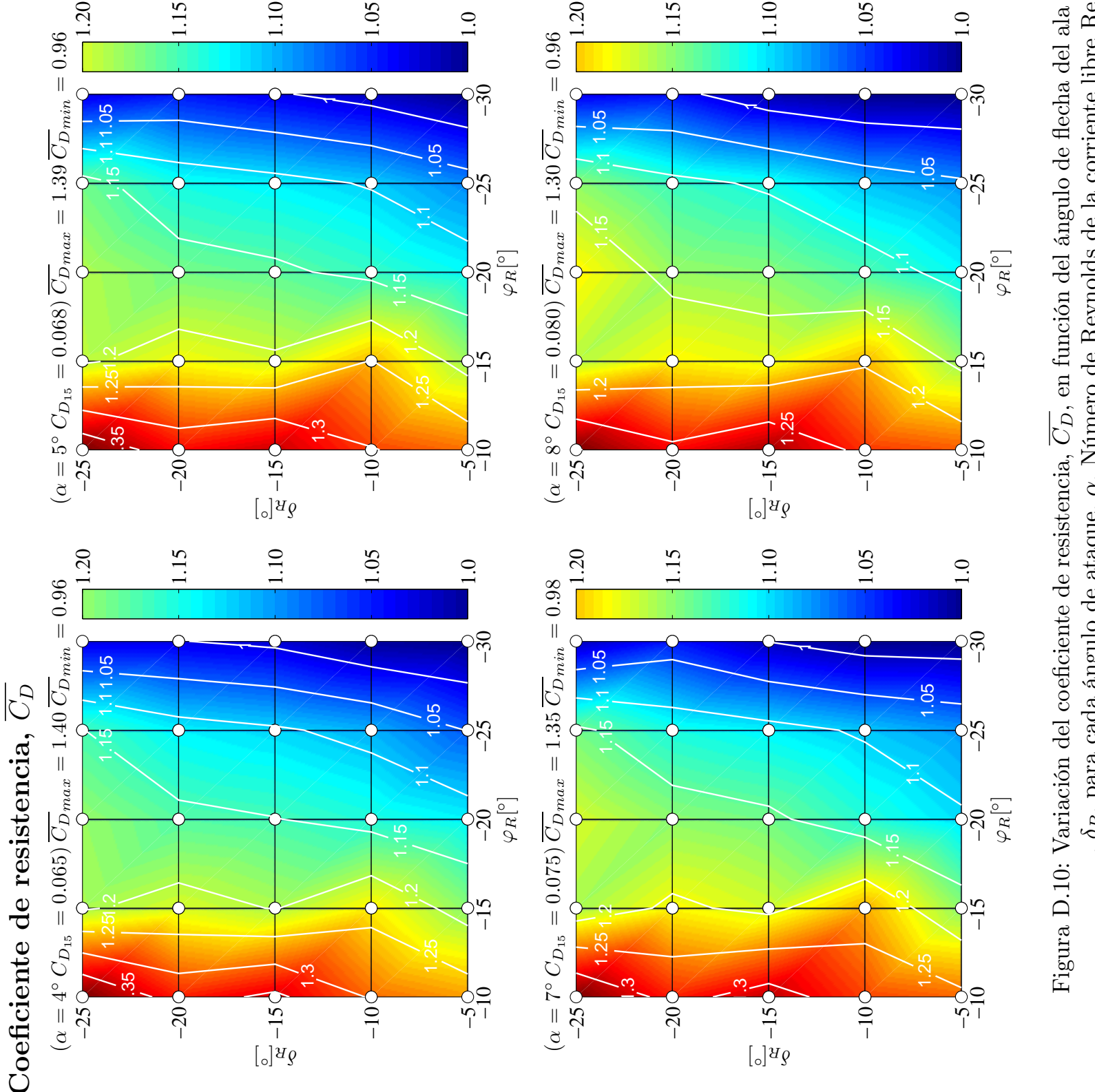

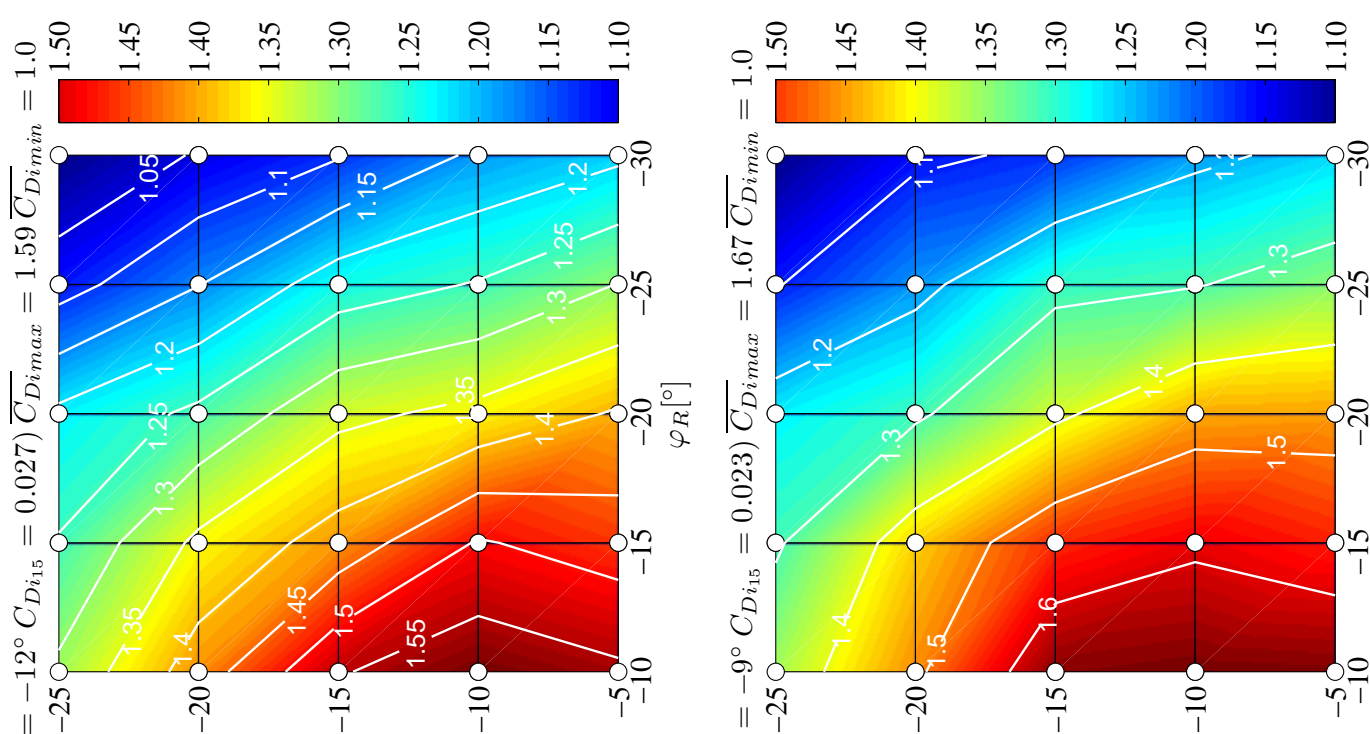

$$
\text { ఠ }
$$

[o] ${ }^{4} \rho$
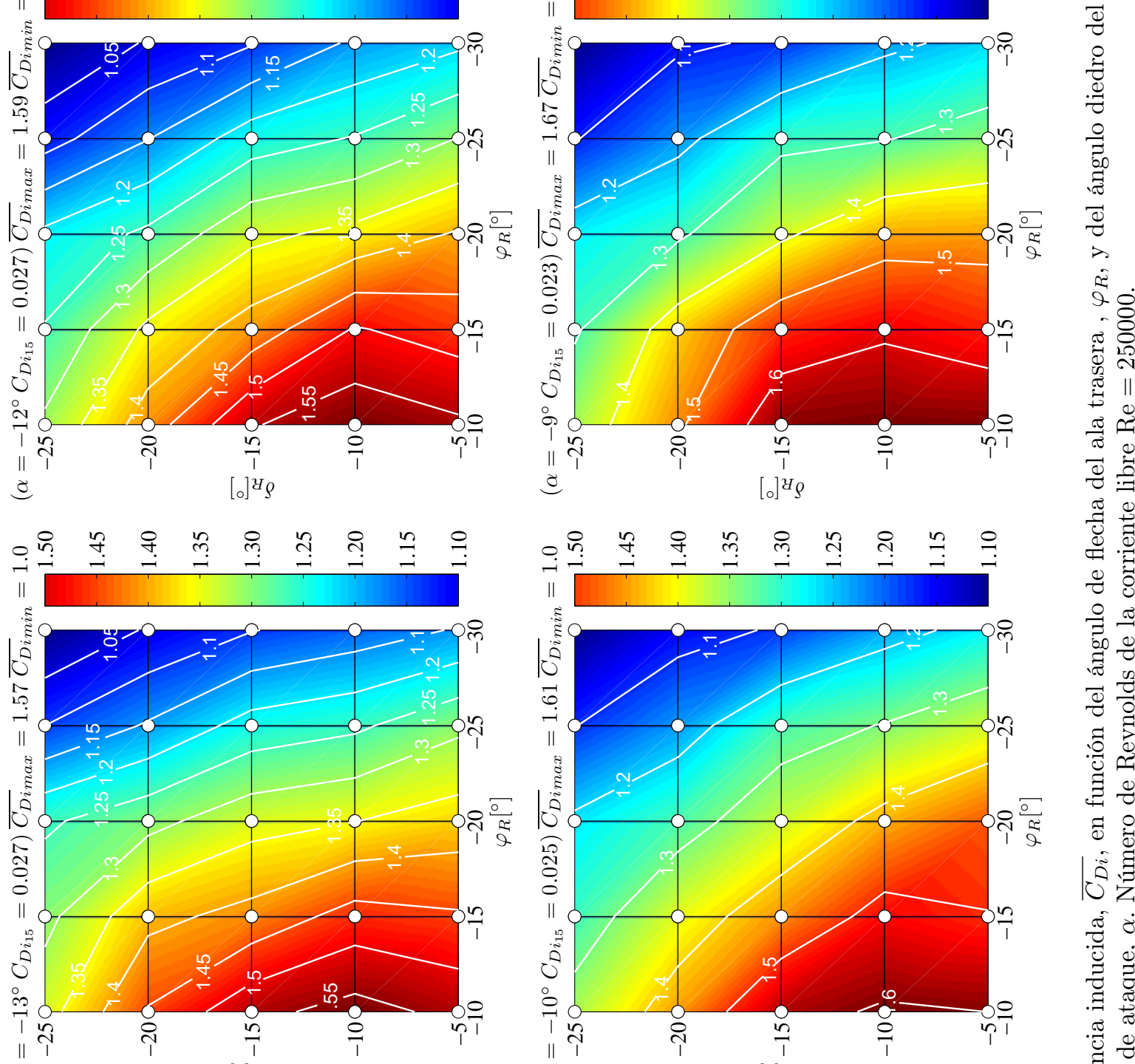

○

$\left[{ }_{0}\right]^{4} \rho$

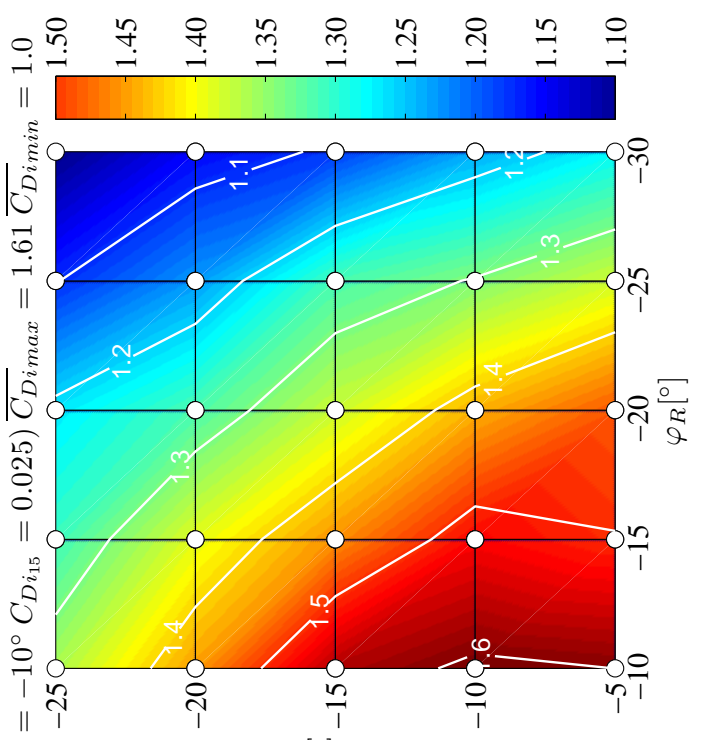

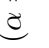

[o] ${ }^{4} \rho$
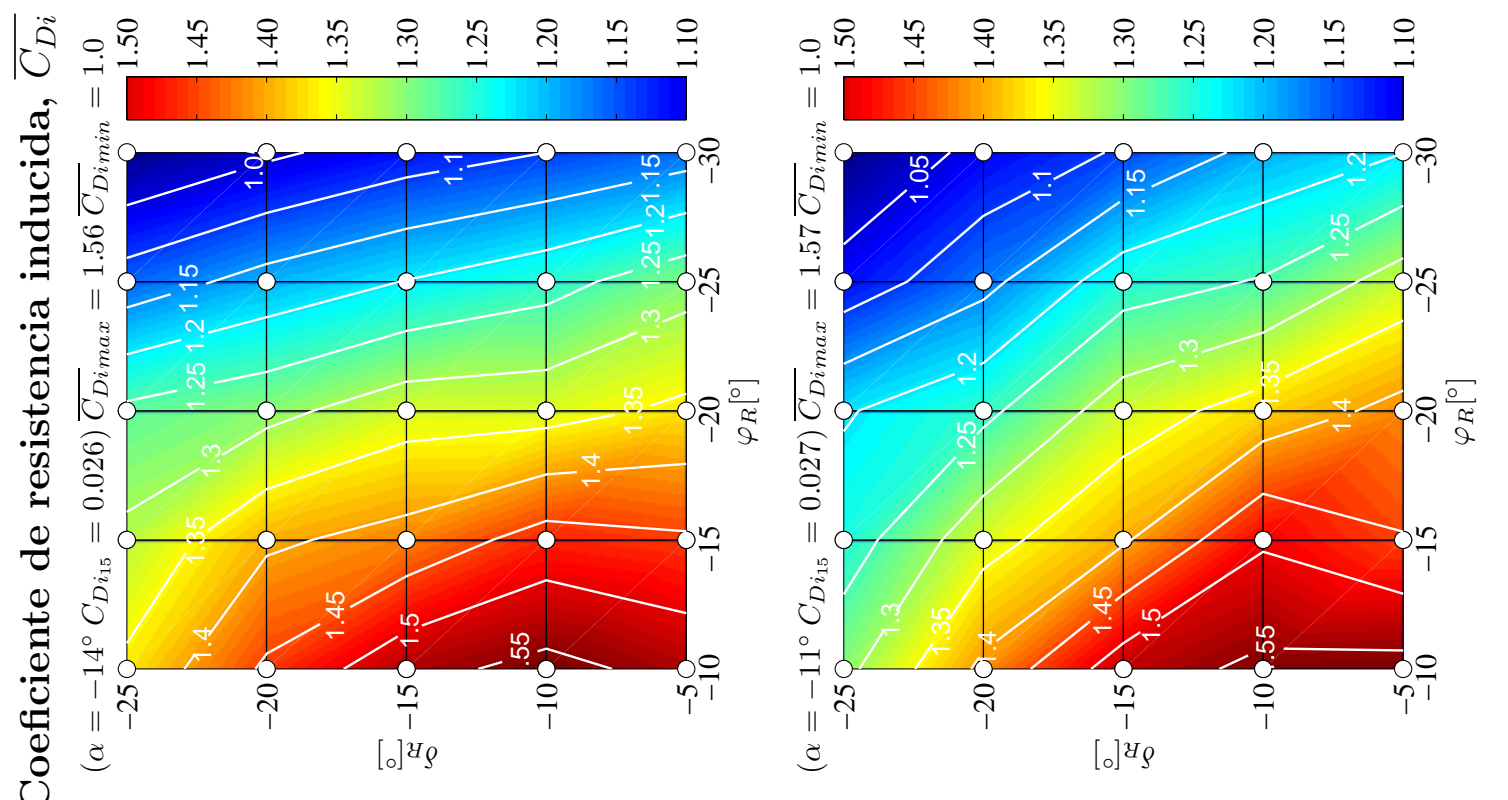

总苛

윽 증

20

훙

:

夏

하 엉

运学

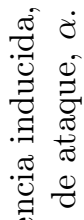

.

ช

0

.

०

임

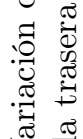

官 $\frac{\sigma}{\sigma}$

금

$\ddot{\theta}$

范 

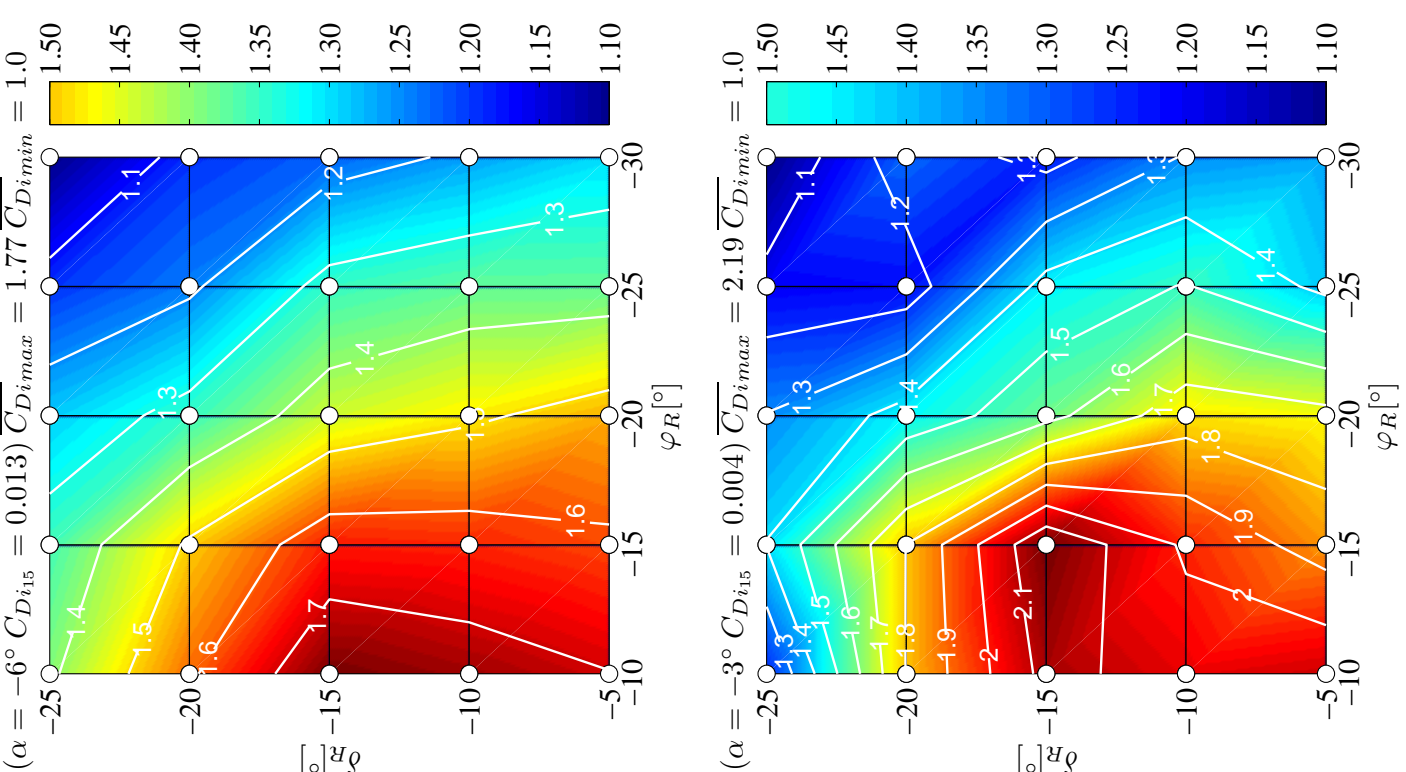

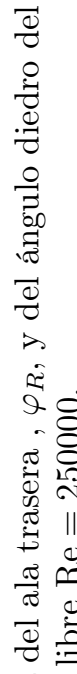

$[0]^{2} \rho$

ठ

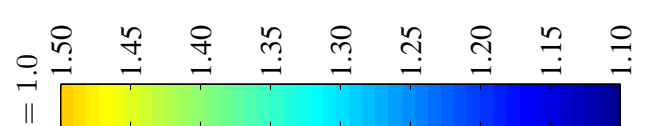

in

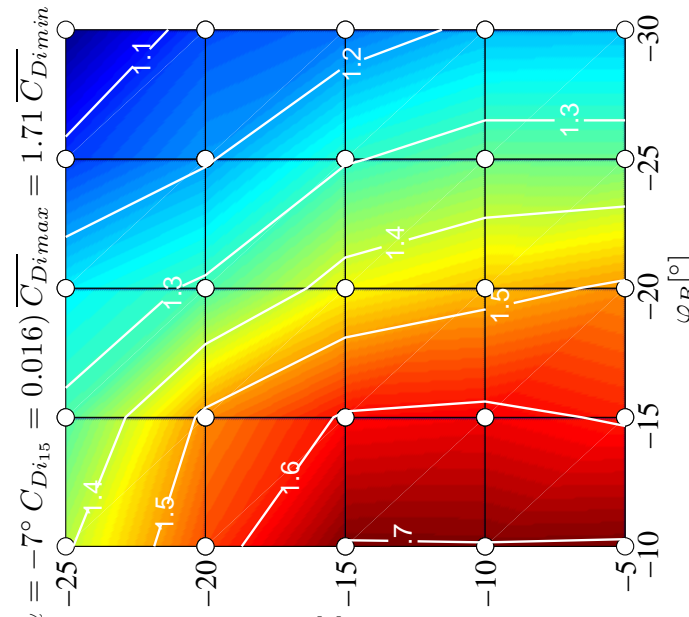

ฮ

$[0]^{4} \mathrm{~g}$

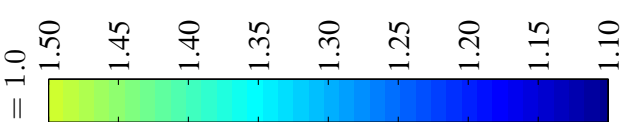

焉

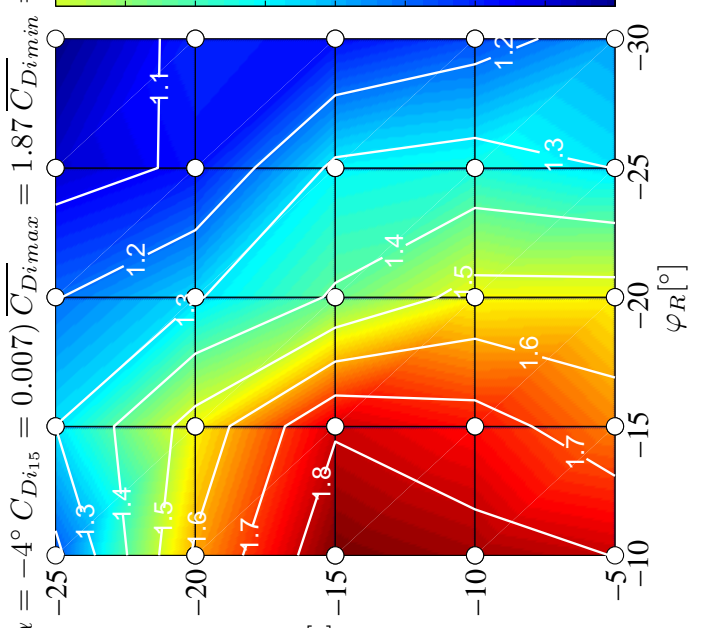

을

कृ

莗

㐘

कृ 웡

运范

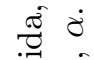

窇

.

Uี่

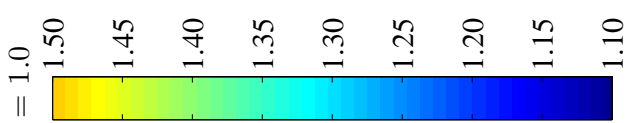

焉要
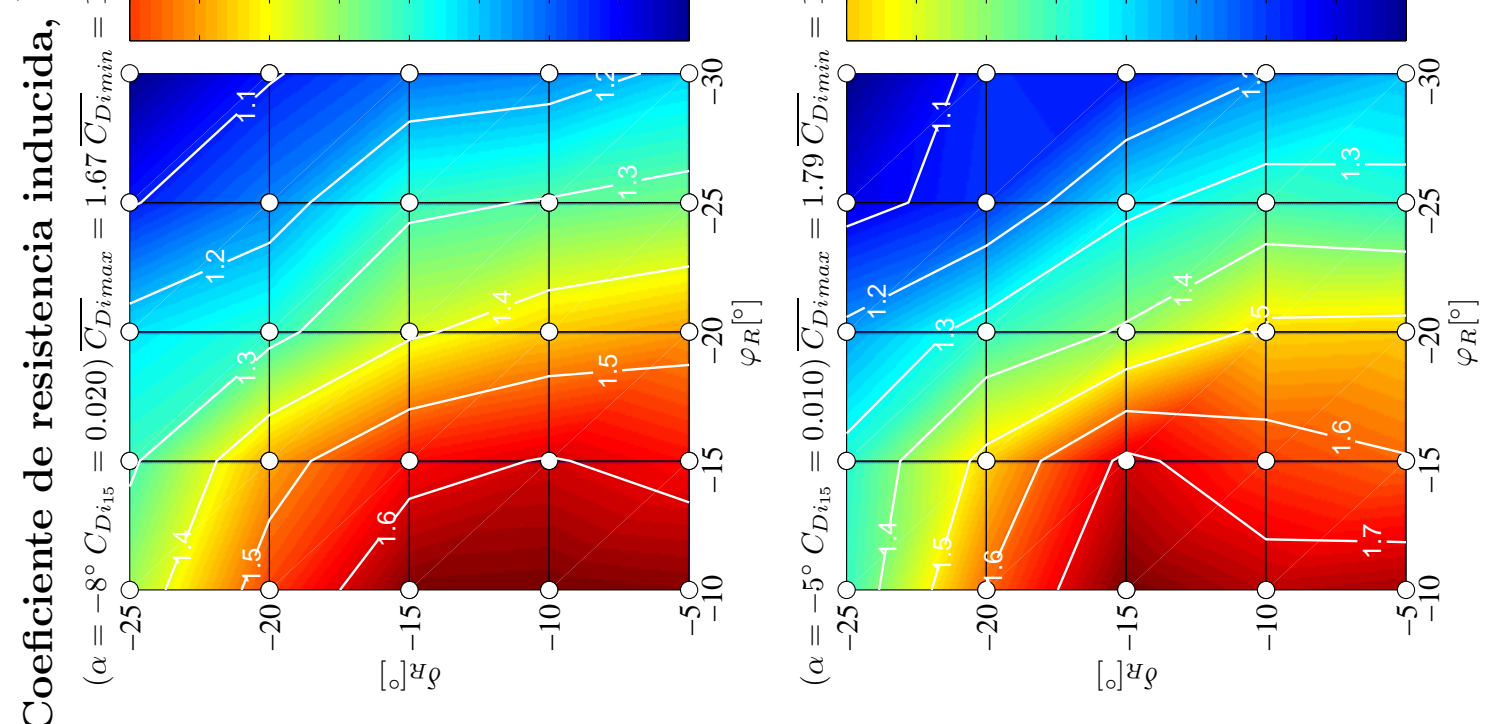

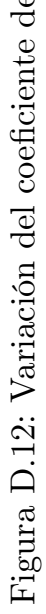



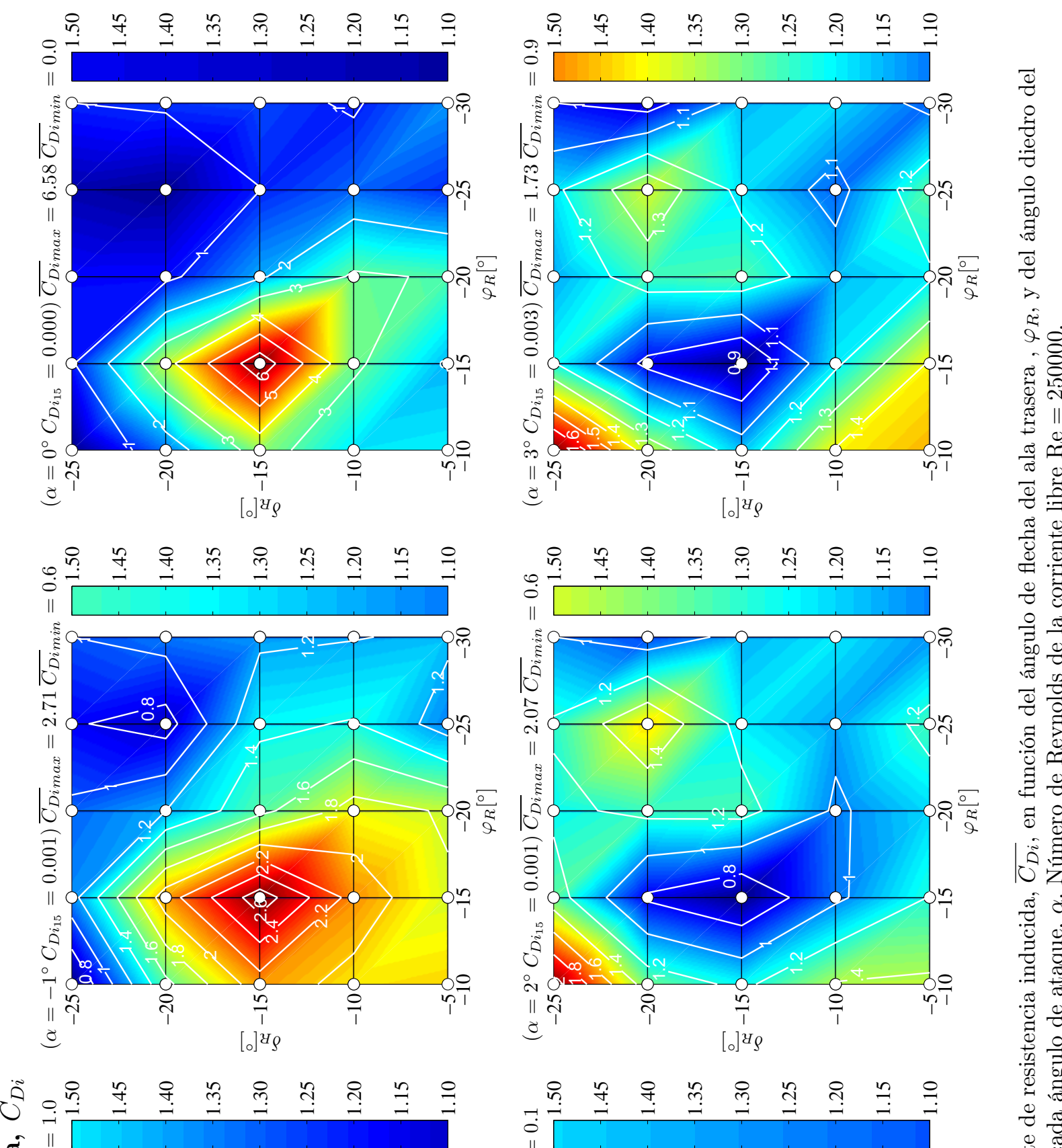

营过

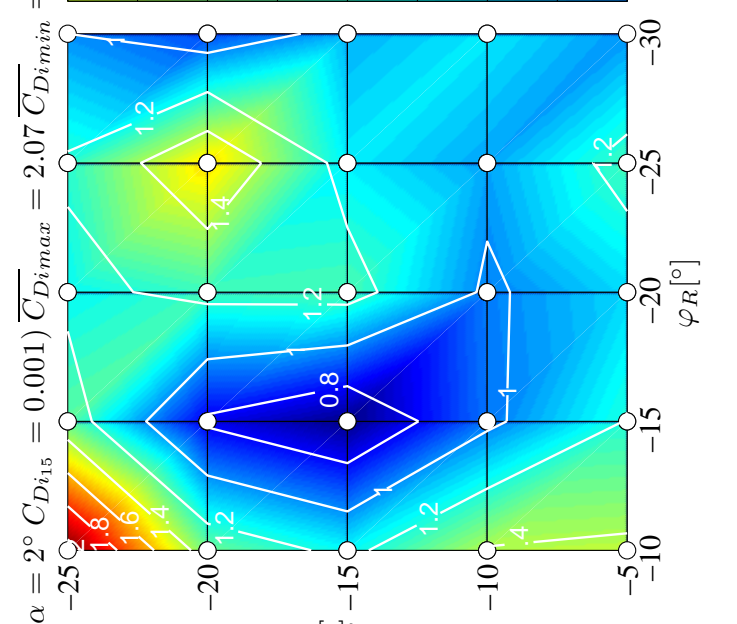

$\frac{0}{3} \frac{\pi}{0}$

50

के

:

当。

뭉워

记窎

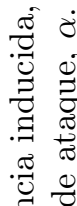

¿

-
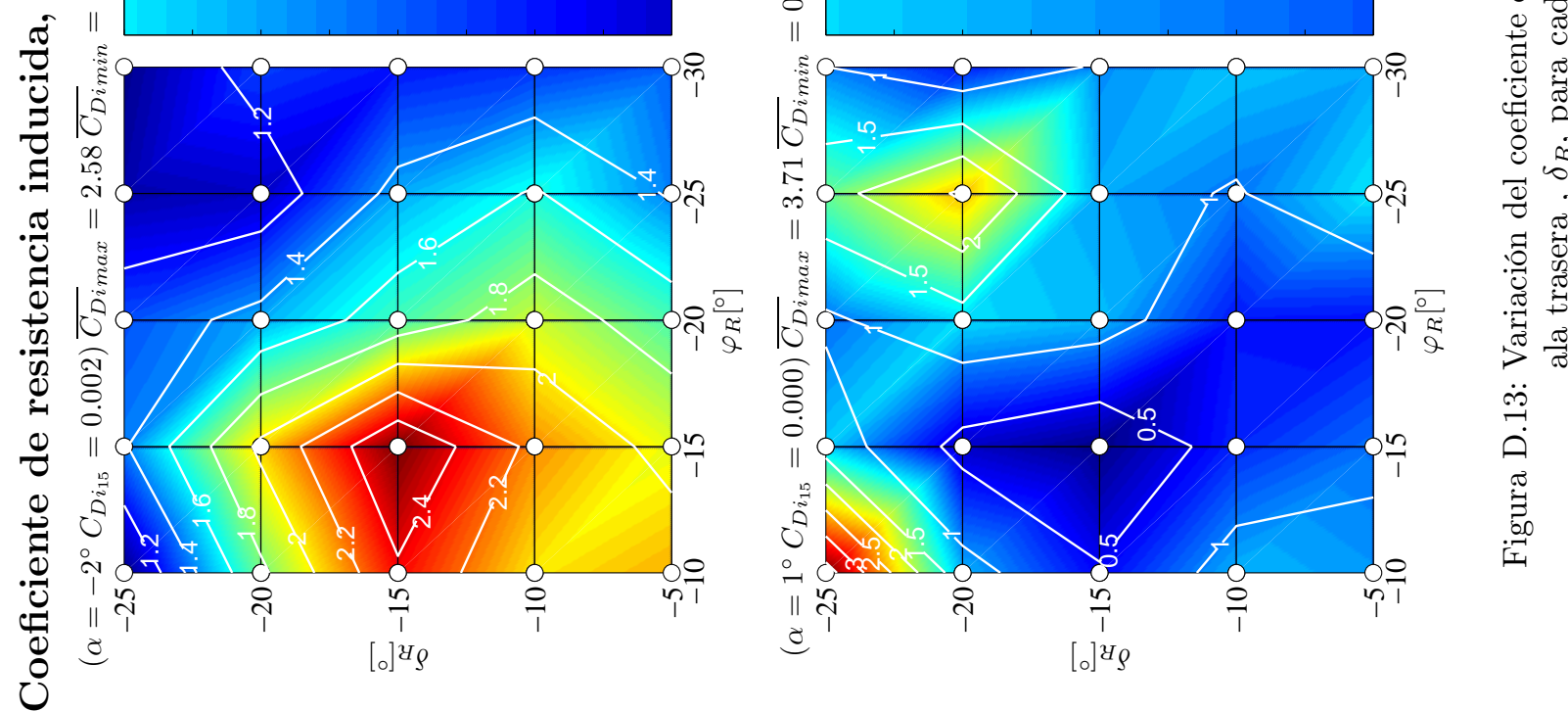

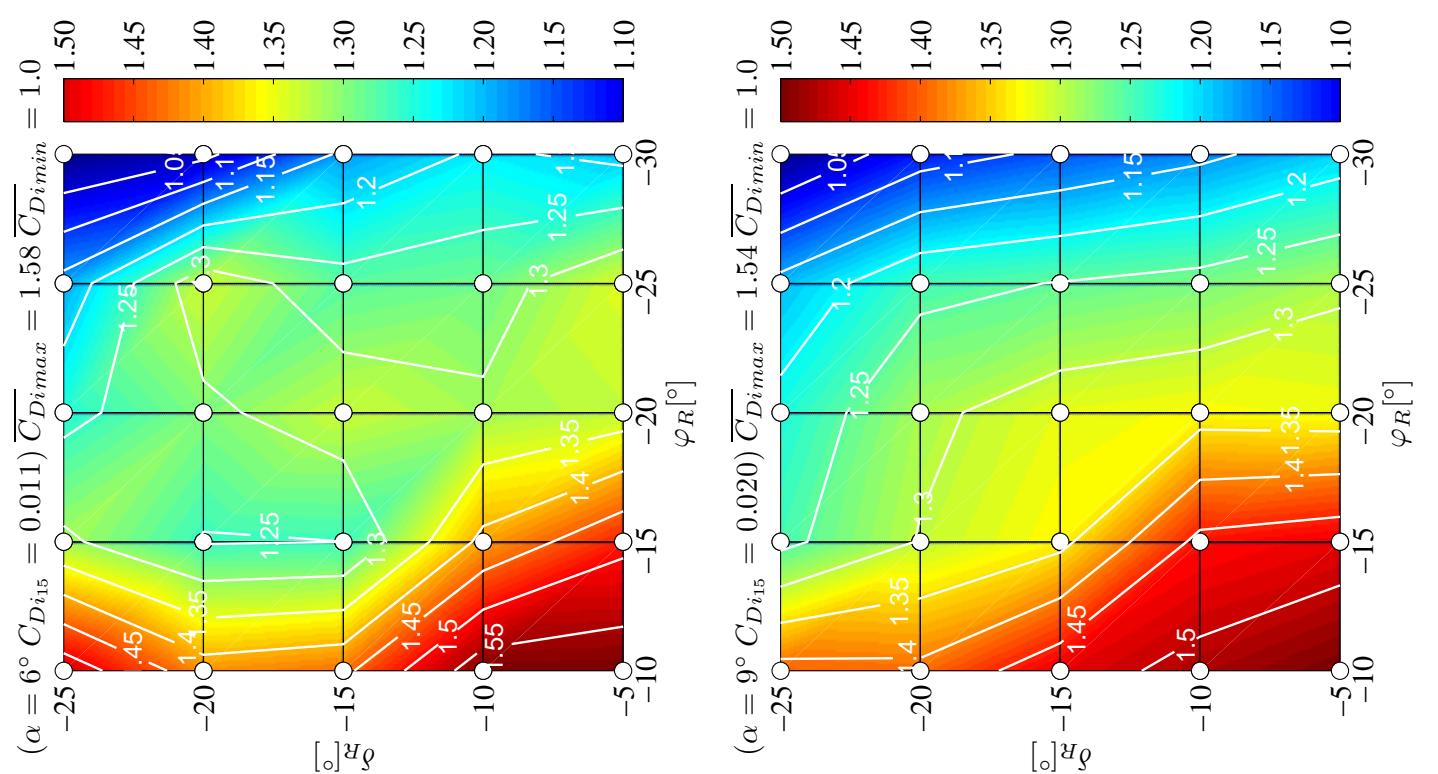

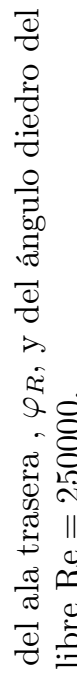
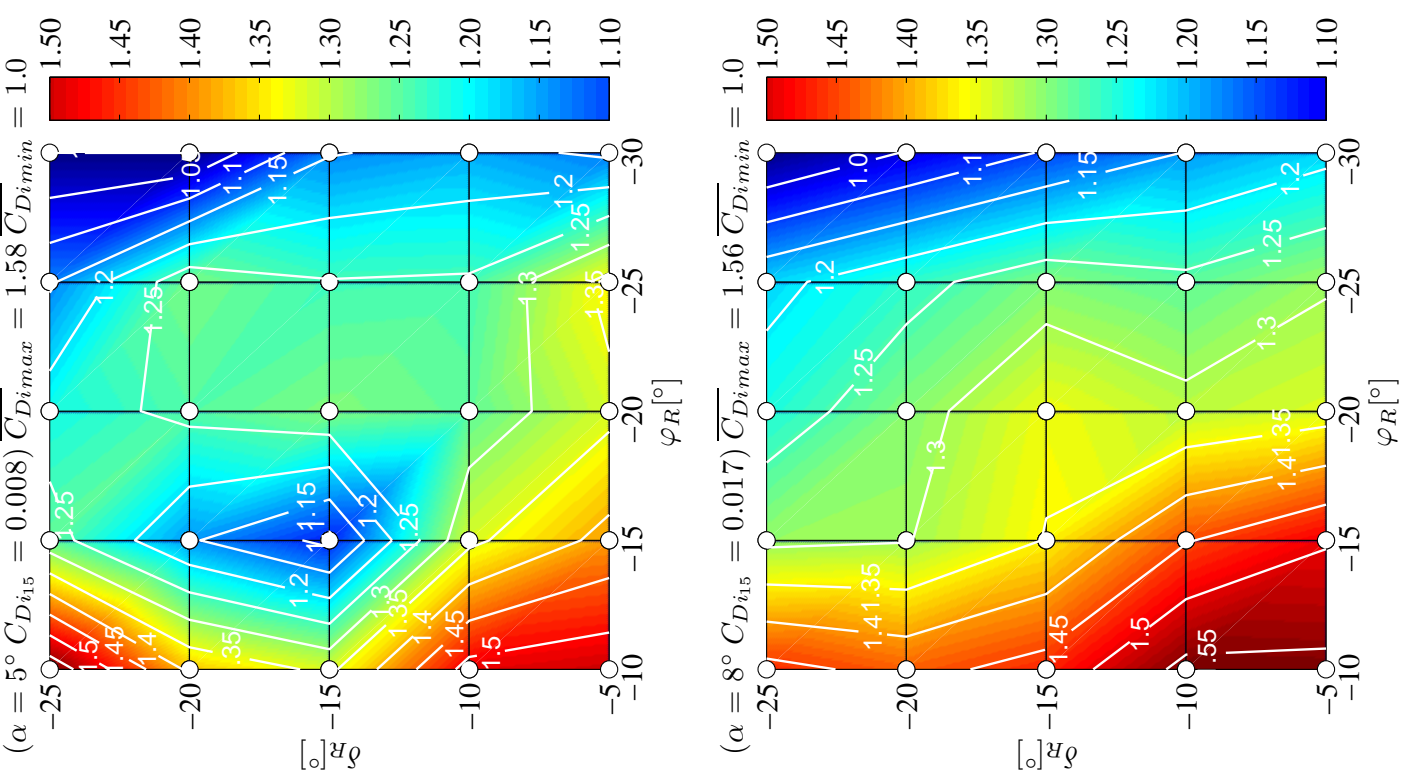

兽苞
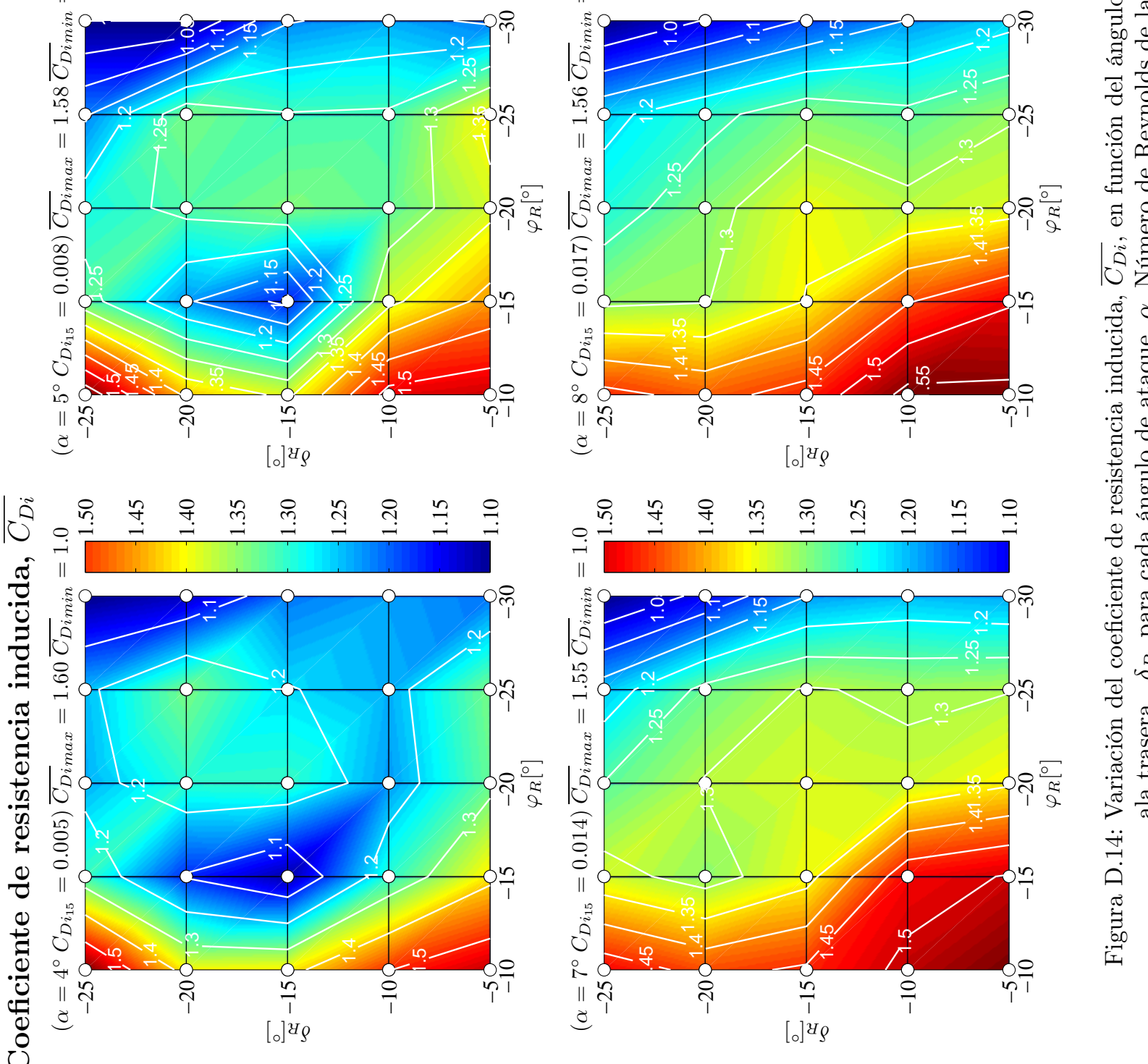

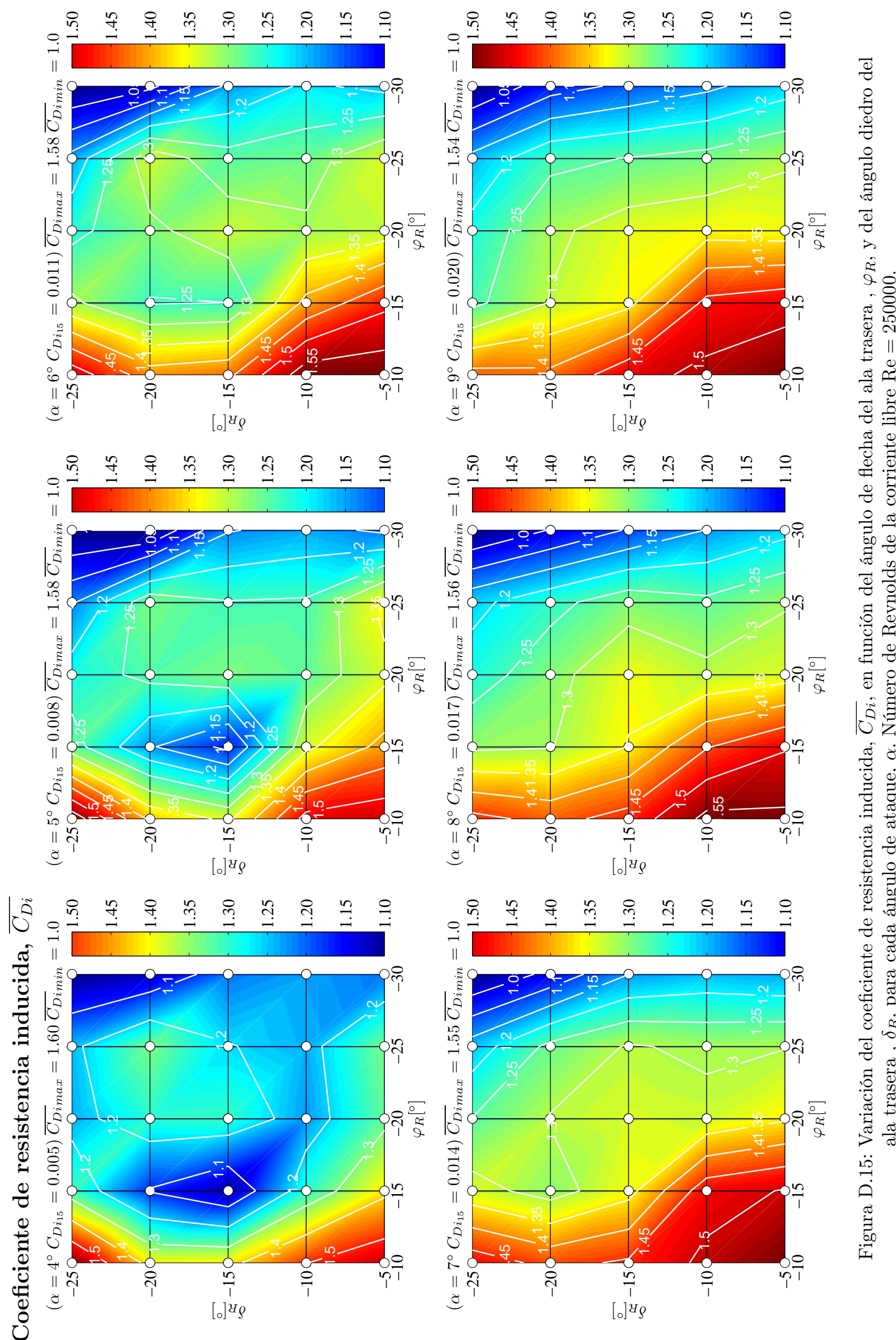

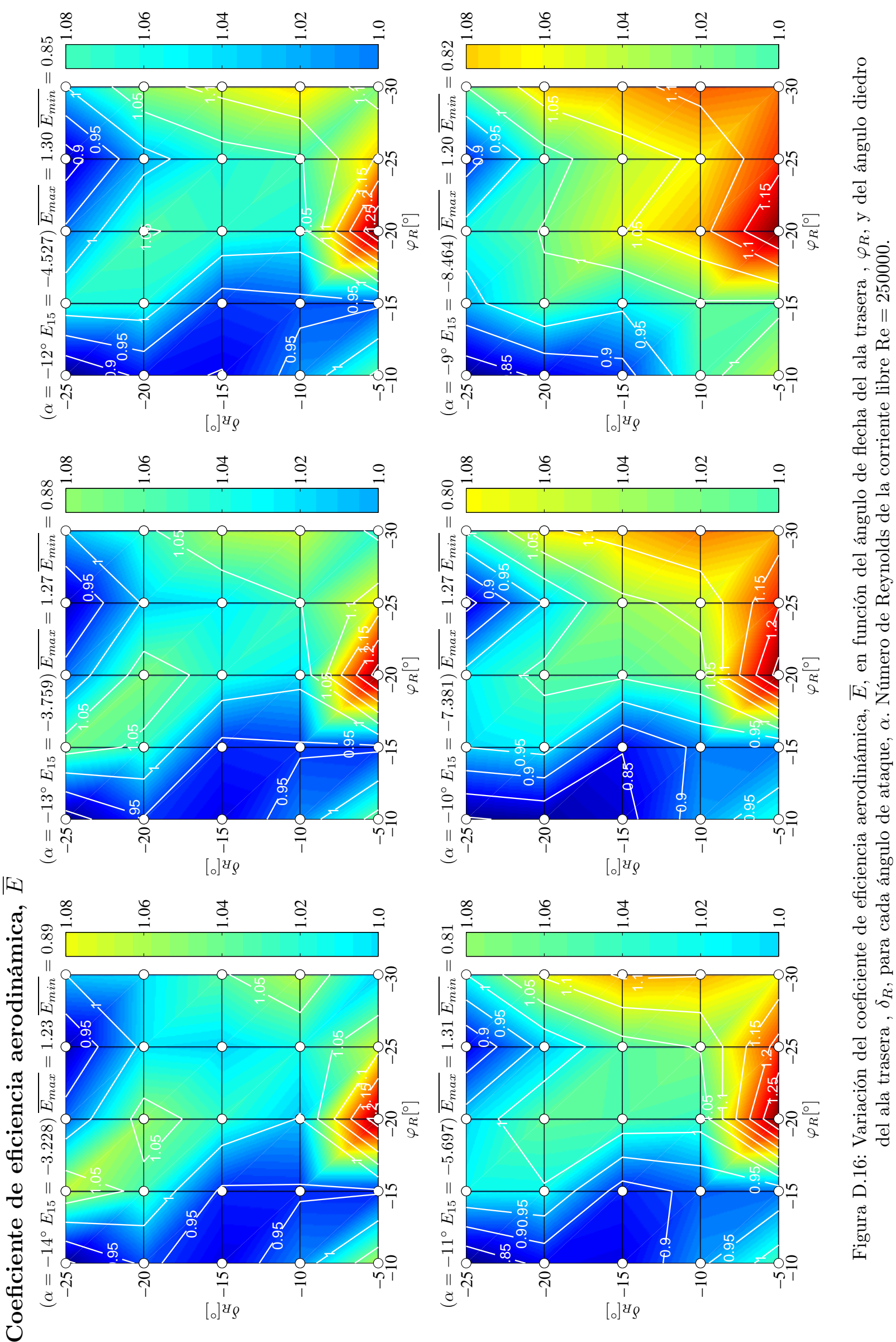

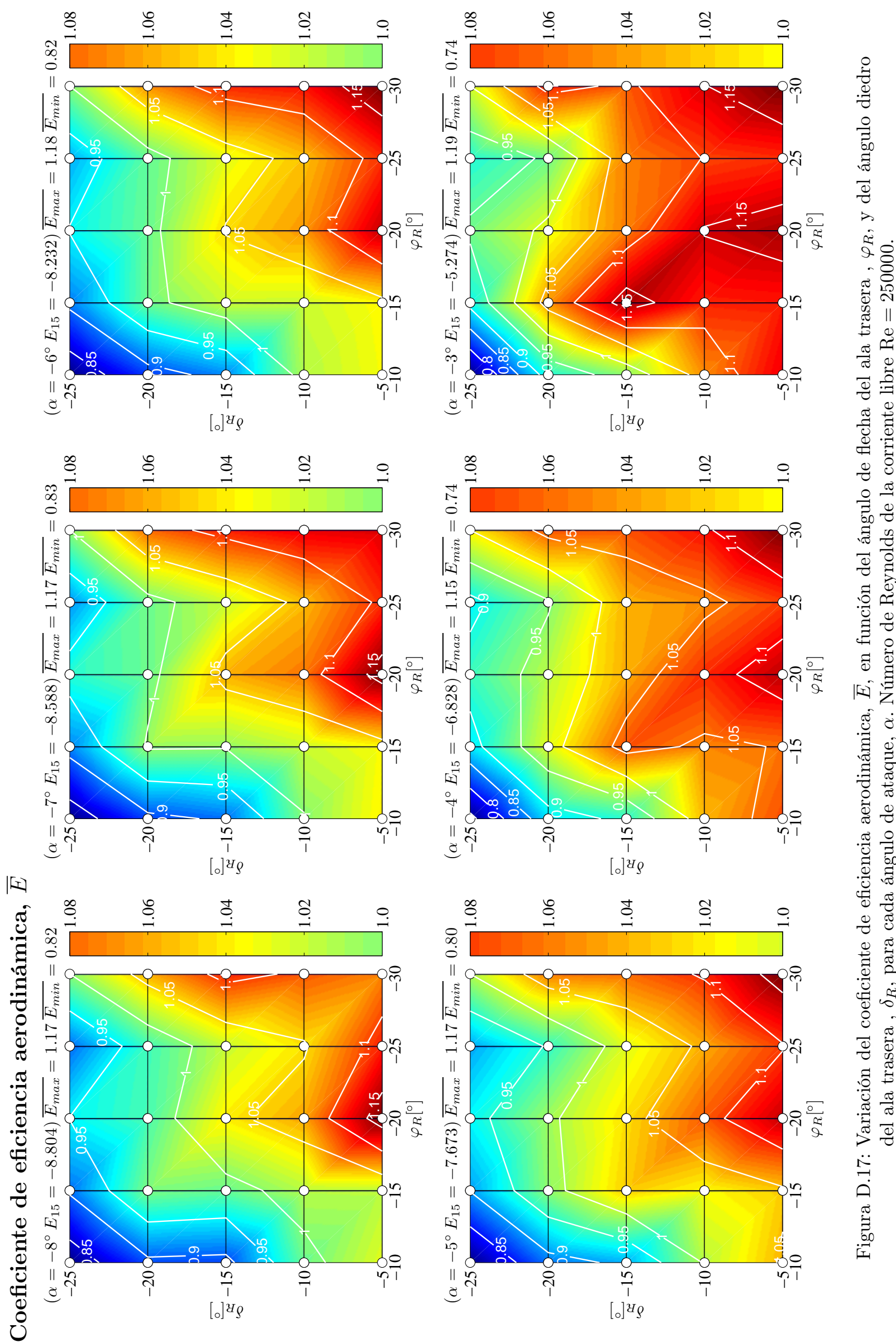

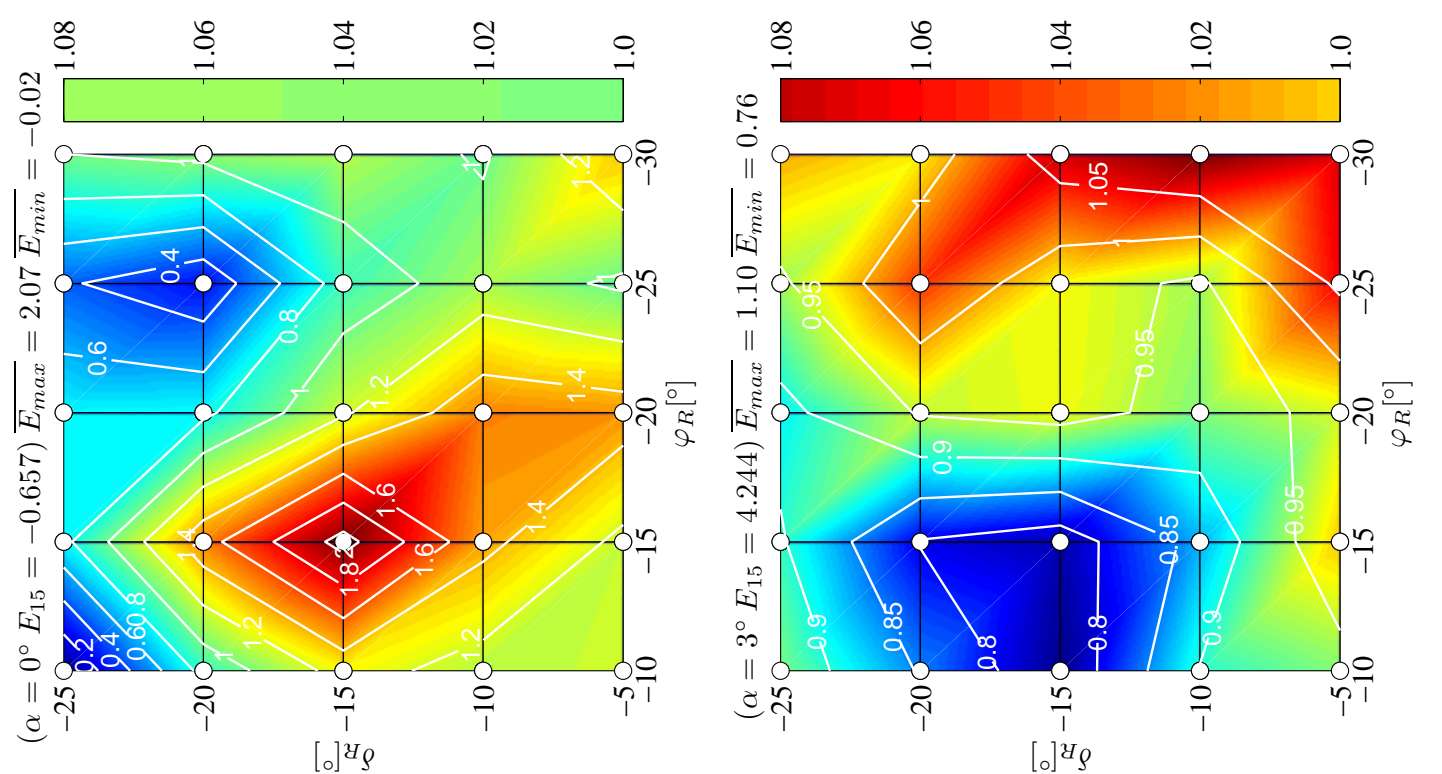

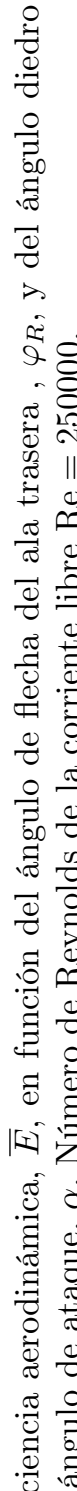
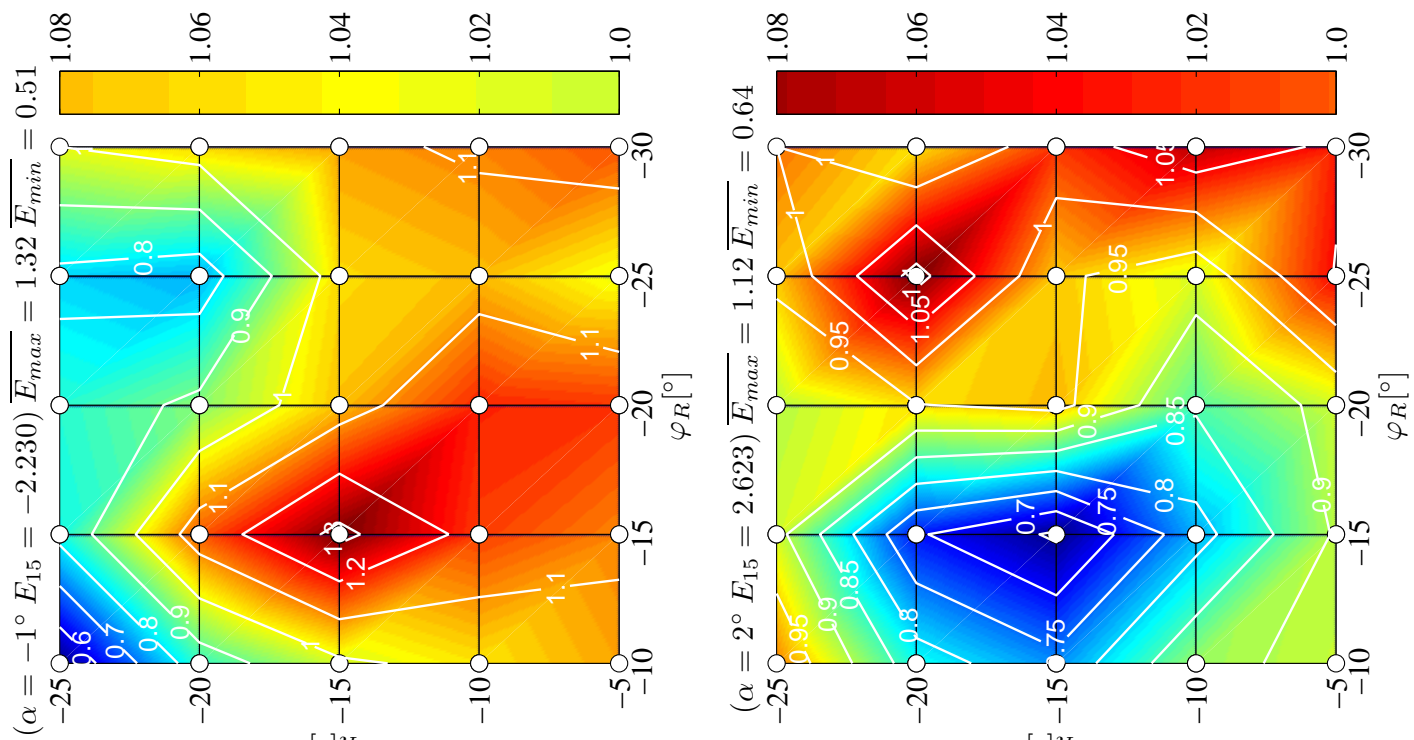

|[氏工正
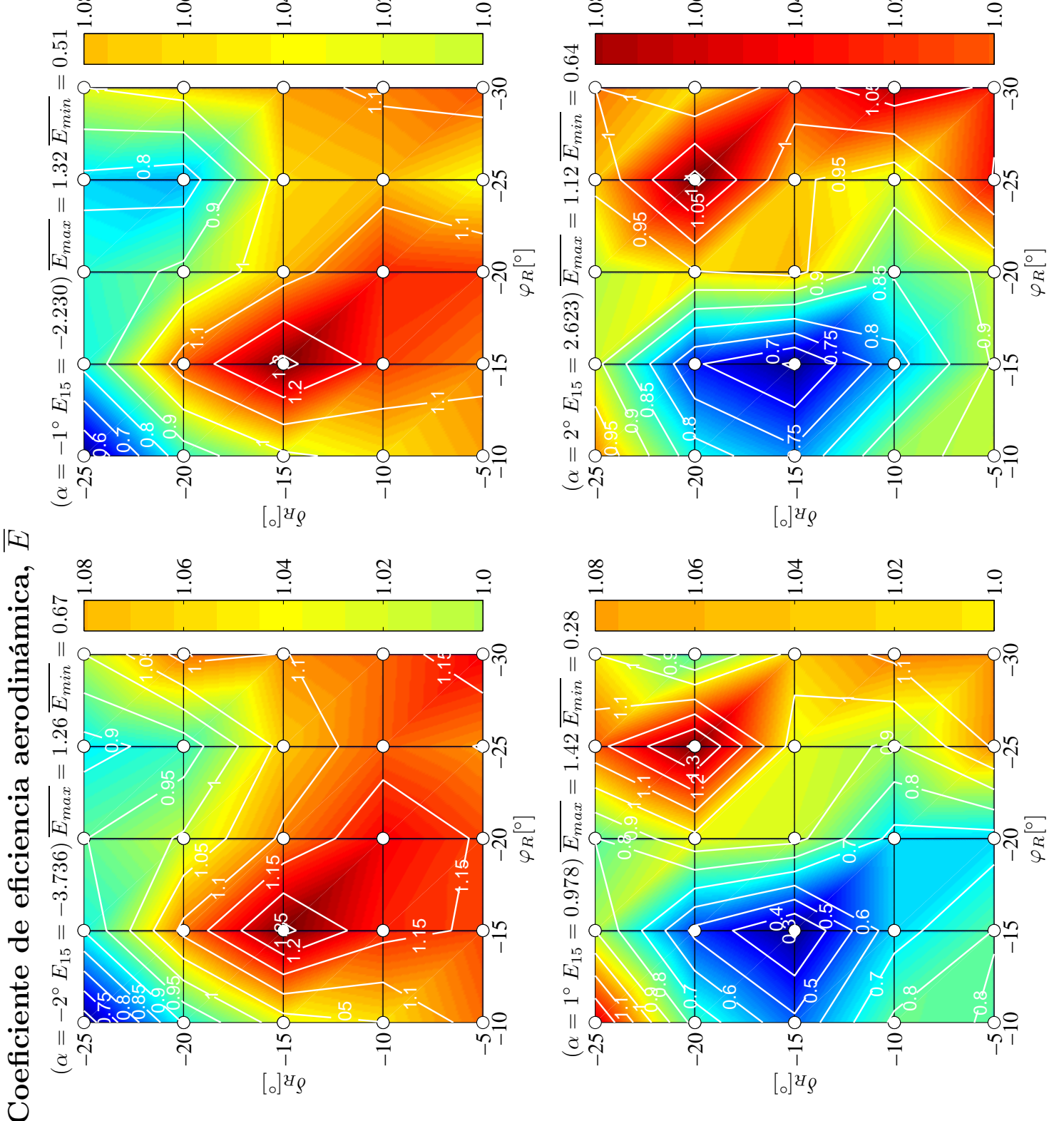

0
0
0
0
0
0
0
0
0
0
0
0
0
0
0
0
0
0
0
0
0
0
0
0
0
0
0
0
0
0
0
0
0
0 

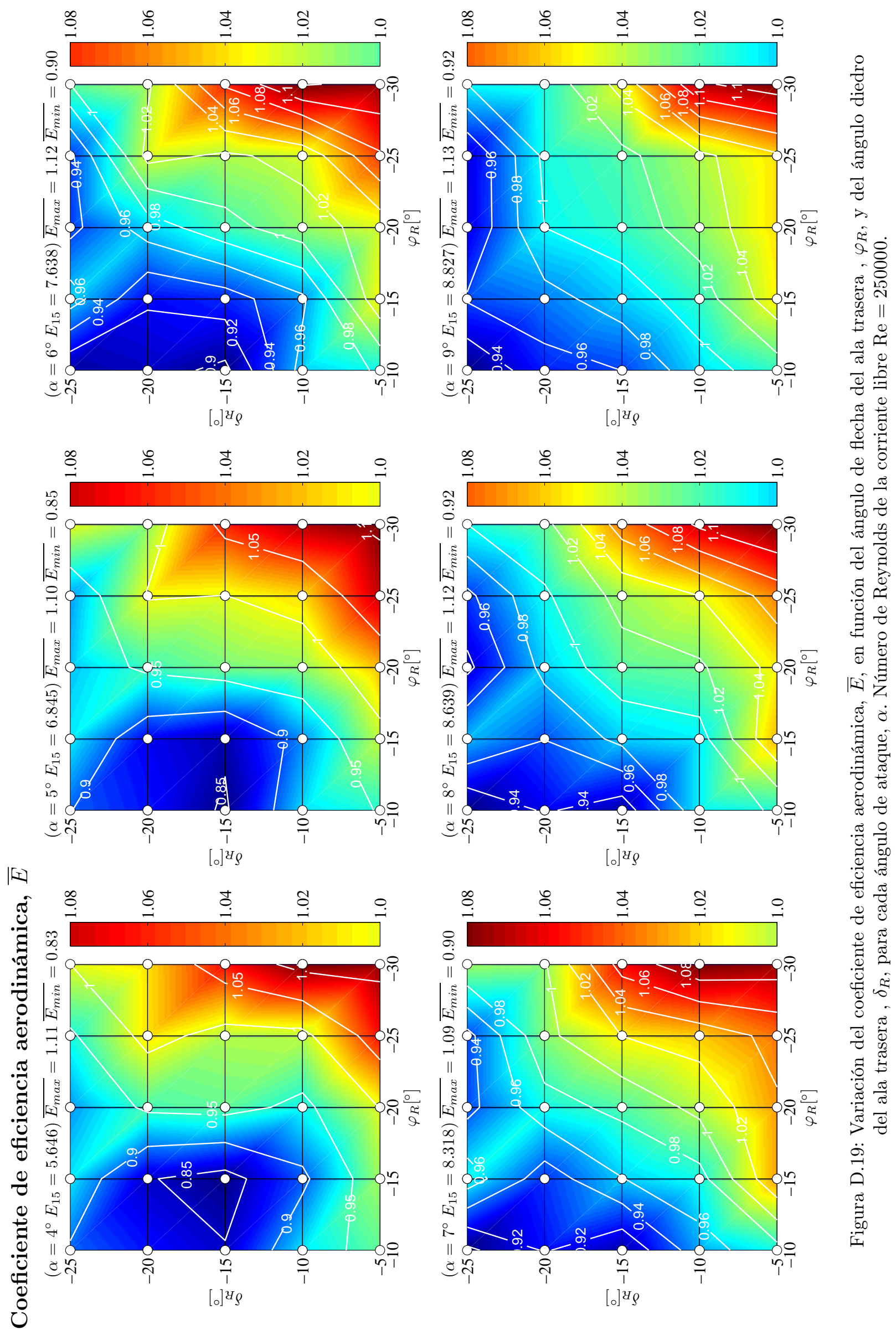

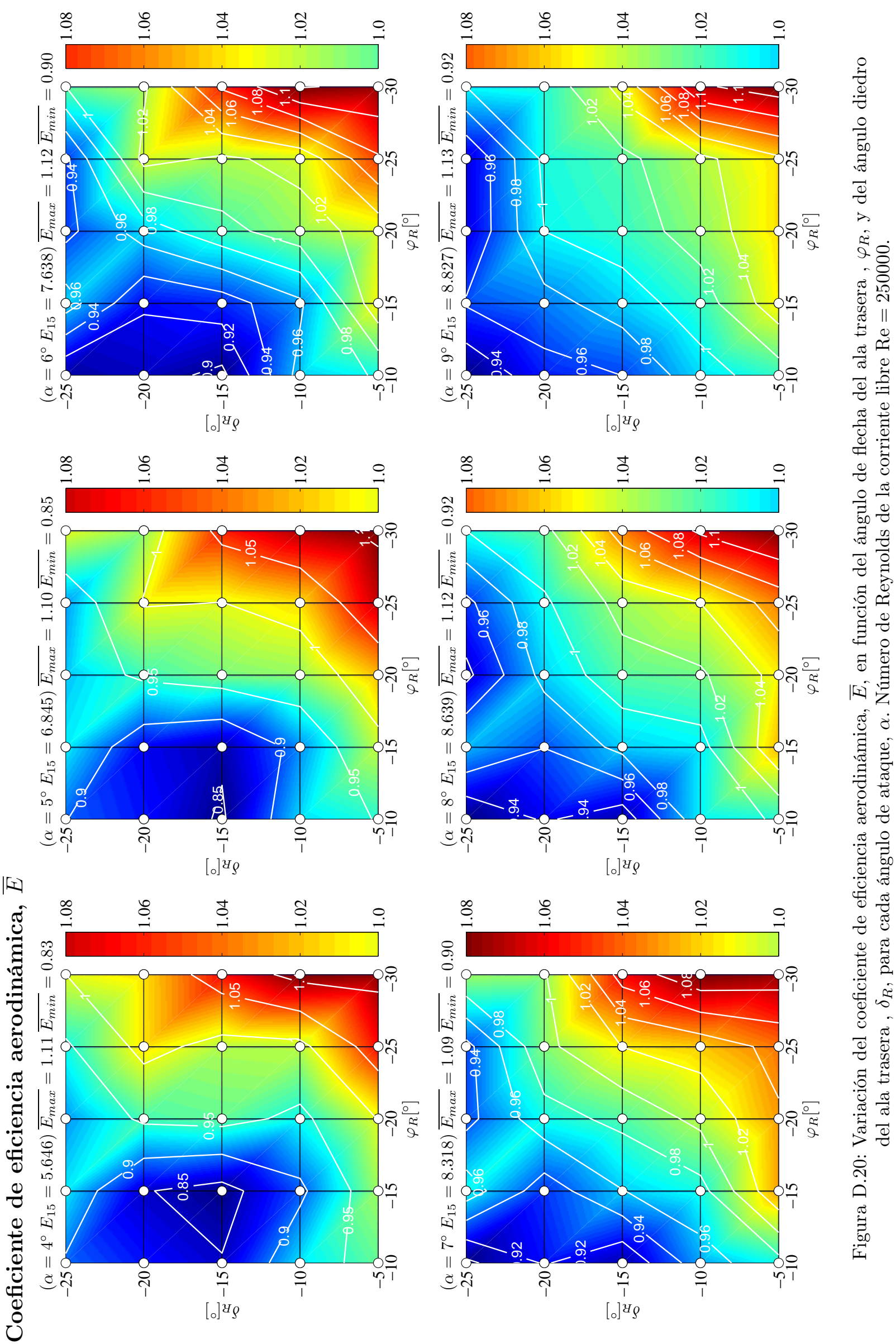

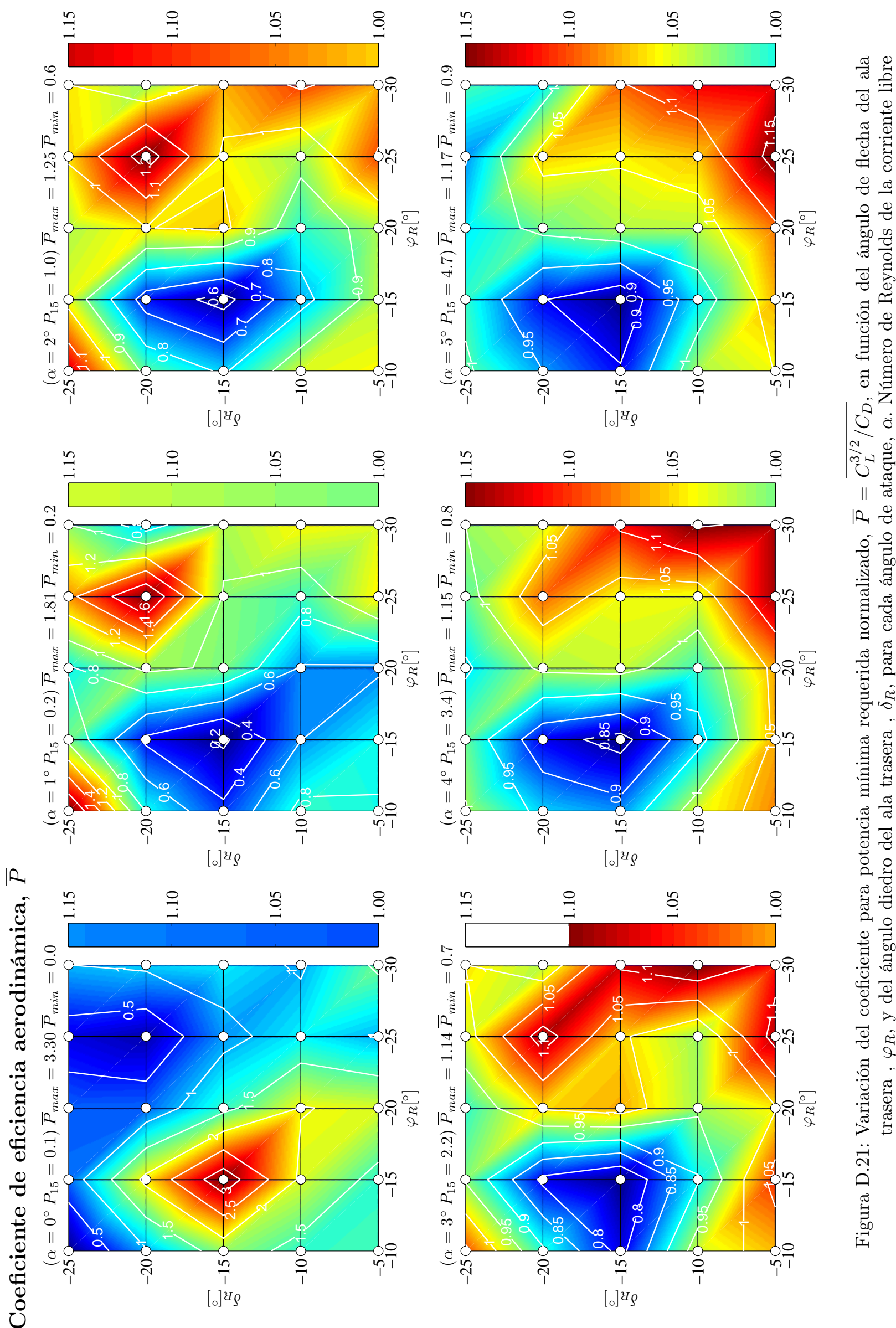
نें ฟ

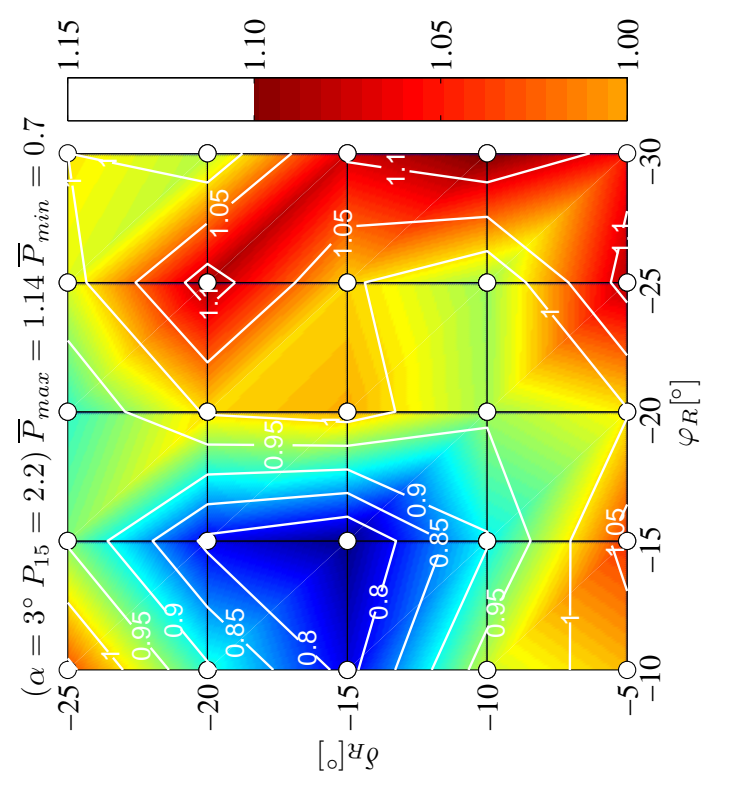



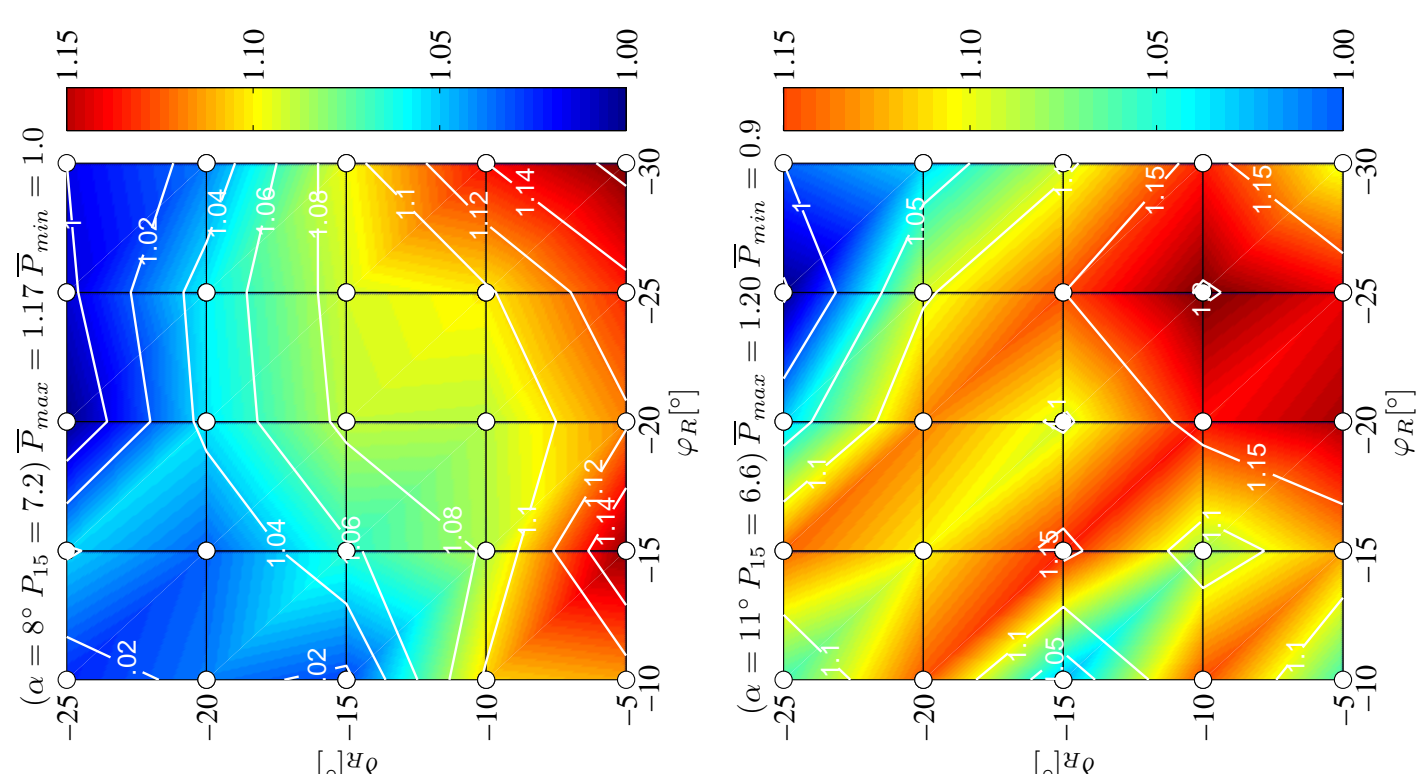

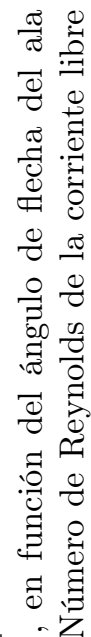
ขैं

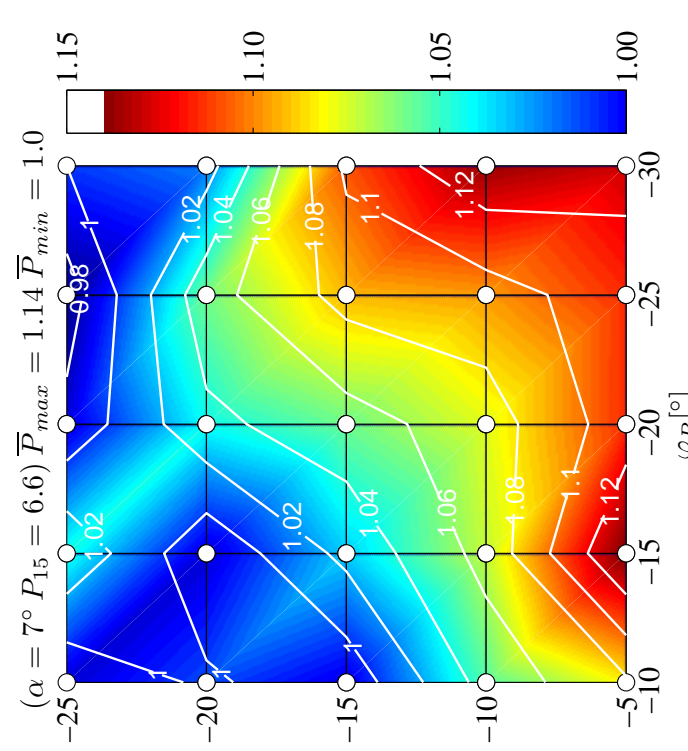
[o] $]^{2} \rho$
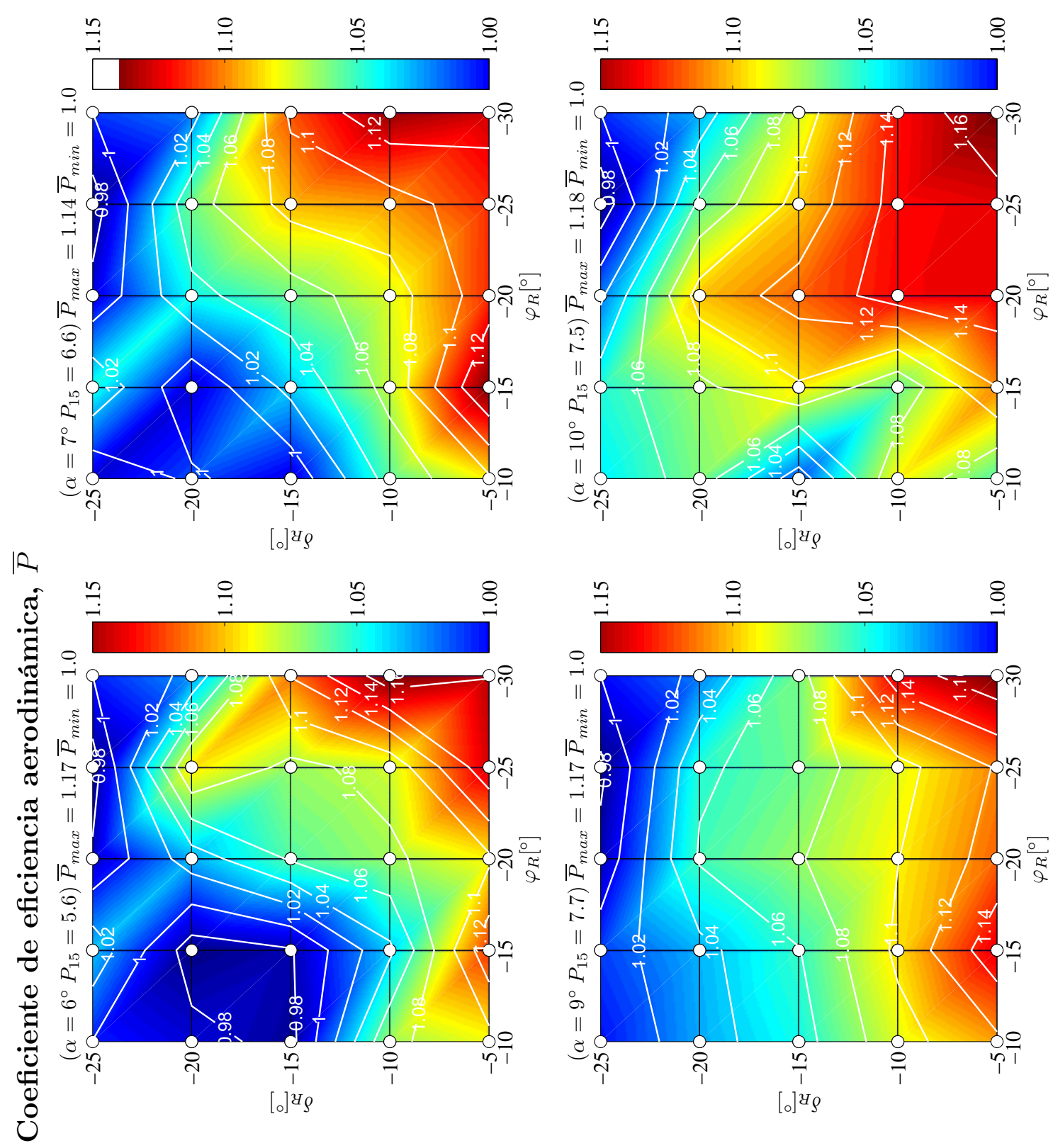

$[0]^{3} \rho$

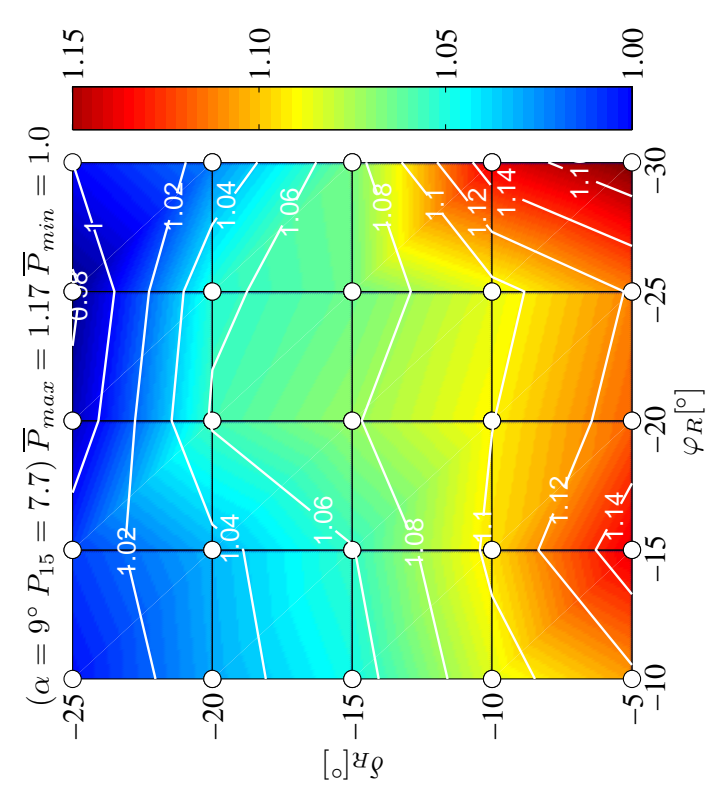

กิ้

$\|$

R. 응

융요

⿶ี สี

ฮี

궁

苂

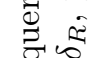

边

苟

当

.

엉

용

๘.

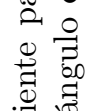

矛芯

:

च

:

赵

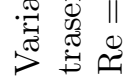

๙ิ่

ก)

苟 

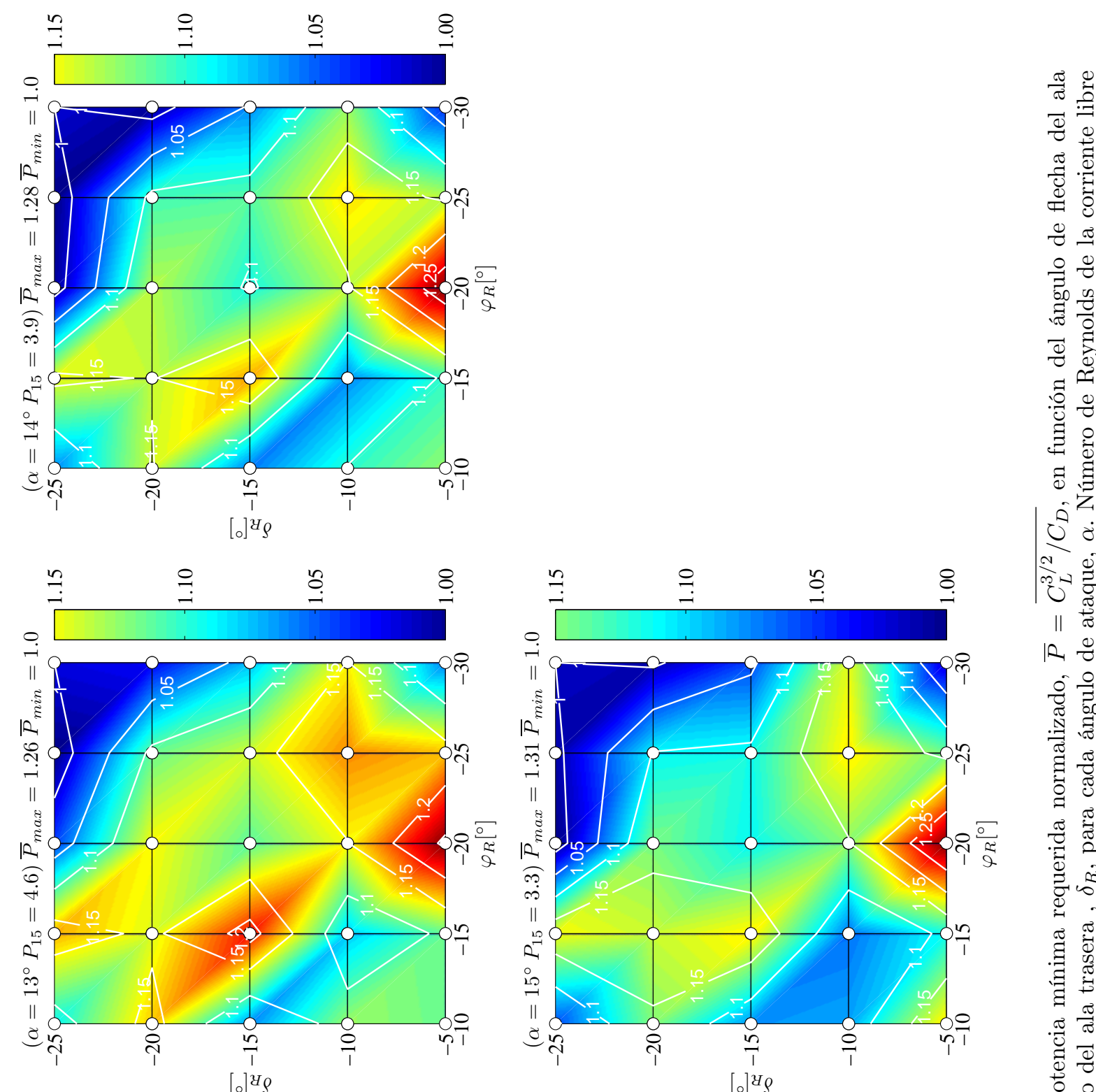

ขैं

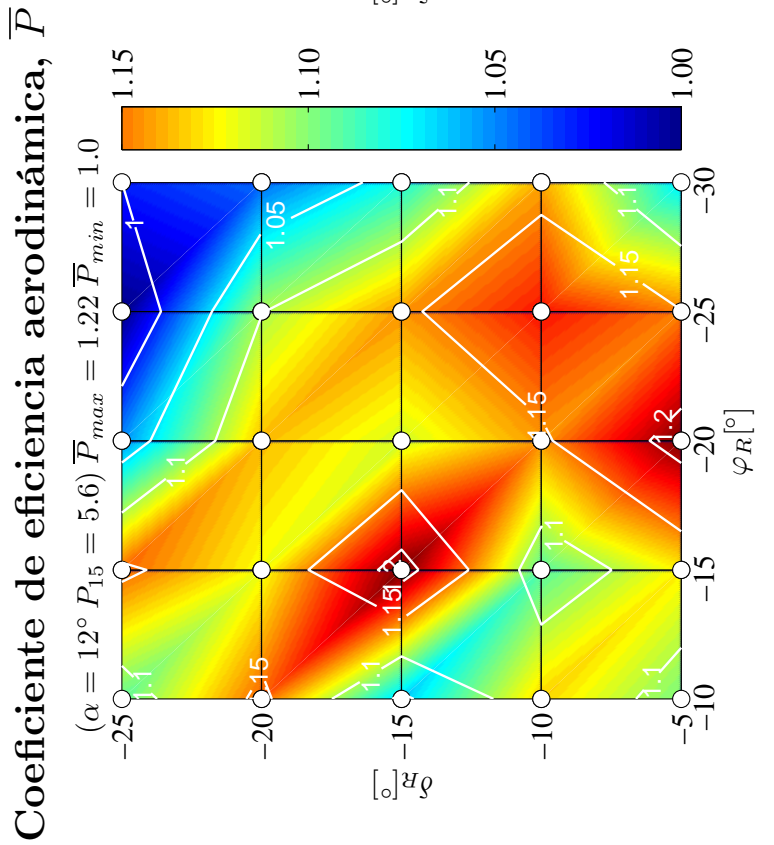

$[0]^{3} \rho$

要

R. 8

융용

สี สิ

:

हี

엉

节

当

. $\frac{\pi}{\sigma}$

웡

응

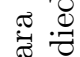

2 .

总

过

क्ष

:

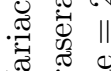

$\ddot{\sim}$

$\dot{0}$

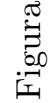




\section{Apéndice E}

\section{Resultados modelo numérico}

\section{E.1. Introducción.}

En este apéndice se presenta los resultados completos derivados de la comparación de los valores de los coeficientes aerodinámicos entre los resultados del modelo numérico y los resultados del ensayo.

\section{E.2. Coeficientes aerodinámicos en función del ángulo ángulo de ataque, $\alpha$.}

Los datos representados corresponden a los valores de los coeficientes de sustentación, $C_{L}$, resistencia inducida, $C_{D i}$, resistencia, $C_{D}$, y eficiencia aerodinámica, $E$, en función del ángulo de ataque, $\alpha$, para valores del ángulo diedro, $\delta_{R}=\left[-5^{\circ},-25^{\circ}\right]$, del ángulo de flecha del ala trasera, $\varphi_{R}=\left[-10^{\circ},-30^{\circ}\right]$.

Los coeficientes representados son:

- En Figura E.1 a Figura E.5 están representados los valores de los coeficientes para ángulo diedro del ala trasera, $\delta_{R}=-5^{\circ}$, para ángulos de flecha del ala trasera $\varphi_{R}=\left[-10^{\circ},-15^{\circ},-20^{\circ},-25^{\circ},-30^{\circ}\right]$.

- En Figura E.6 a Figura E.10 están representados los valores de los coeficientes para ángulo diedro del ala trasera, $\delta_{R}=-10^{\circ}$, para ángulos de flecha del ala trasera $\varphi_{R}=\left[-10^{\circ},-15^{\circ},-20^{\circ},-25^{\circ},-30^{\circ}\right]$.

- En Figura E.11 a Figura E.15 están representados los valores de los coeficientes para ángulo diedro del ala trasera, $\delta_{R}=-15^{\circ}$, para ángulos de flecha del ala trasera $\varphi_{R}=\left[-10^{\circ},-15^{\circ},-20^{\circ},-25^{\circ},-30^{\circ}\right]$.

- En Figura E.16 a Figura E.20 están representados los valores de los coeficientes para ángulo diedro del ala trasera, $\delta_{R}=-20^{\circ}$, para ángulos de flecha del ala trasera $\varphi_{R}=\left[-10^{\circ},-15^{\circ},-20^{\circ},-25^{\circ},-30^{\circ}\right.$. 
- En Figura E.21 a Figura E.25 están representados los valores de los coeficientes para ángulo diedro del ala trasera, $\delta_{R}=-25^{\circ}$, para ángulos de flecha del ala trasera $\varphi_{R}=\left[-10^{\circ},-15^{\circ},-20^{\circ},-25^{\circ},-30^{\circ}\right]$. 
E.2. COEFICIENTES AERODINÁMICOS EN FUNCIÓN DEL ÁNGULO ÁNGULO DE ATAQUE, $\alpha$.
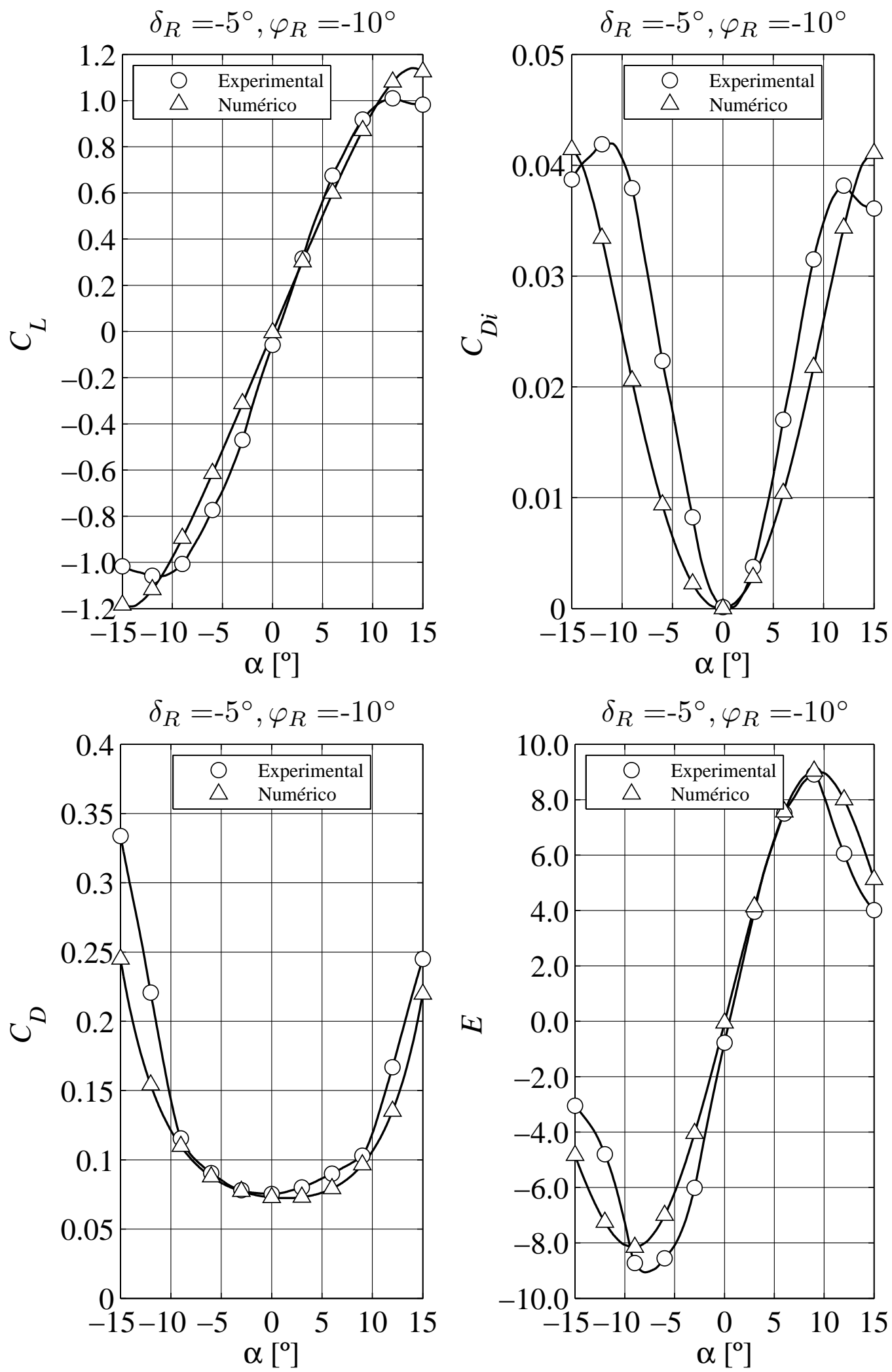

Figura E.1: Variación de los valores de los coeficientes de sustentación, $C_{L}$, de resistencia inducida, $C_{D i}$, de resistencia, $C_{D}$, y de eficiencia aerodinámica, $E$, en función del ángulo de ataque, $\alpha$, para la configuración de ensayo $\operatorname{JWC}_{11}\left(\varphi_{R}=-10^{\circ}\right.$ y $\delta_{R}=$ $\left.-5^{\circ}\right)$. Comparación entre el modelo numérico y los resultados experimentales. 

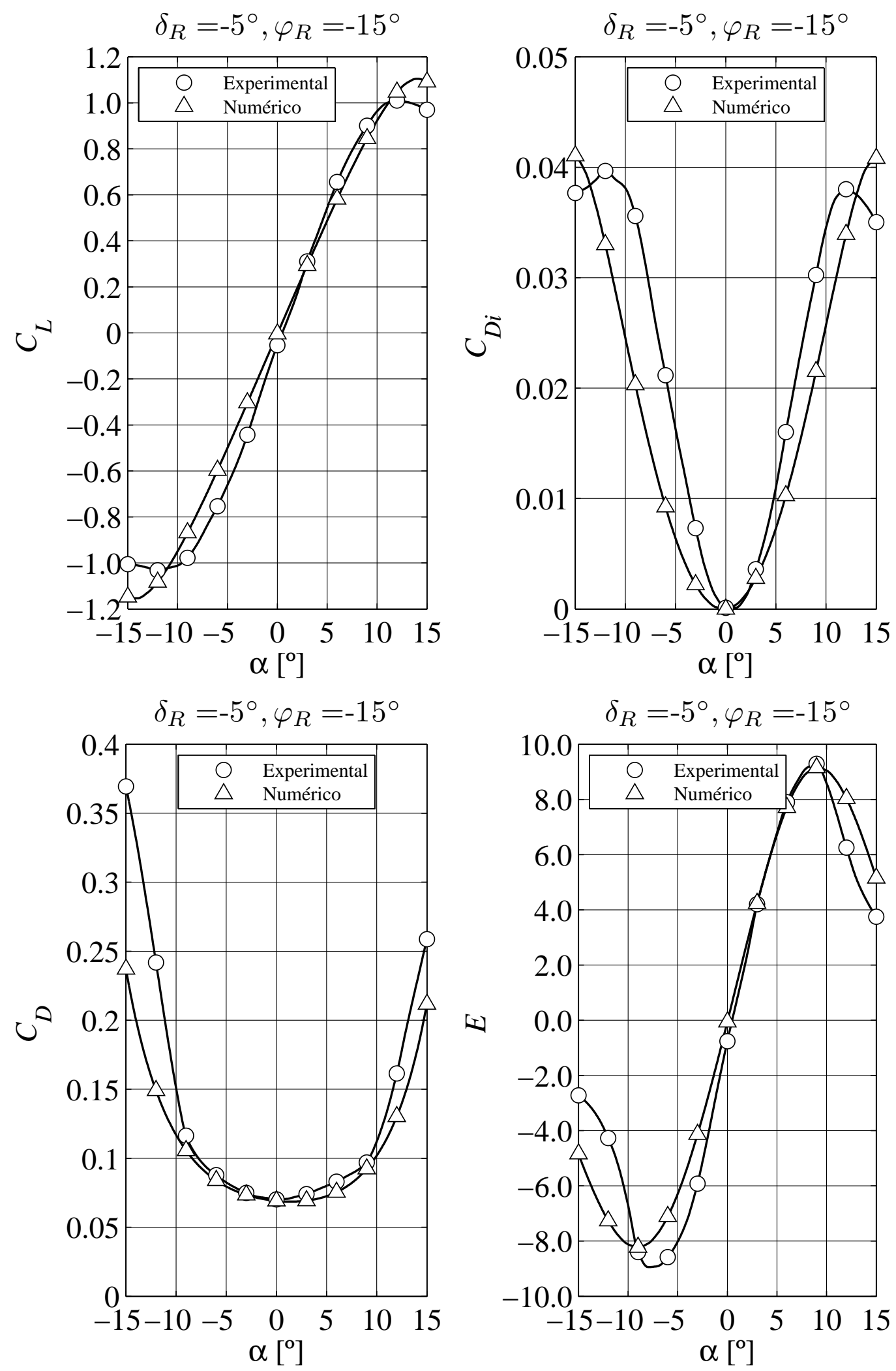

Figura E.2: Variación de los valores de los coeficientes de sustentación, $C_{L}$, de resistencia inducida, $C_{D i}$, de resistencia, $C_{D}$, y de eficiencia aerodinámica, $E$, en función del ángulo de ataque, $\alpha$, para la configuración de ensayo $\operatorname{JWC}_{21}\left(\varphi_{R}=-15^{\circ}\right.$ y $\delta_{R}=$ $\left.-5^{\circ}\right)$. Comparación entre el modelo numérico y los resultados experimentales. 
E.2. COEFICIENTES AERODINÁMICOS EN FUNCIÓN DEL ÁNGULO ÁNGULO DE ATAQUE, $\alpha$.


Figura E.3: Variación de los valores de los coeficientes de sustentación, $C_{L}$, de resistencia inducida, $C_{D i}$, de resistencia, $C_{D}$ y de eficiencia aerodinámica, $E$, en función del ángulo de ataque, $\alpha$, para la configuración de ensayo $\operatorname{JWC}_{31}\left(\varphi_{R}=-20^{\circ}\right.$ y $\delta_{R}=$ $\left.-5^{\circ}\right)$. Comparación entre el modelo numérico y los resultados experimentales. 

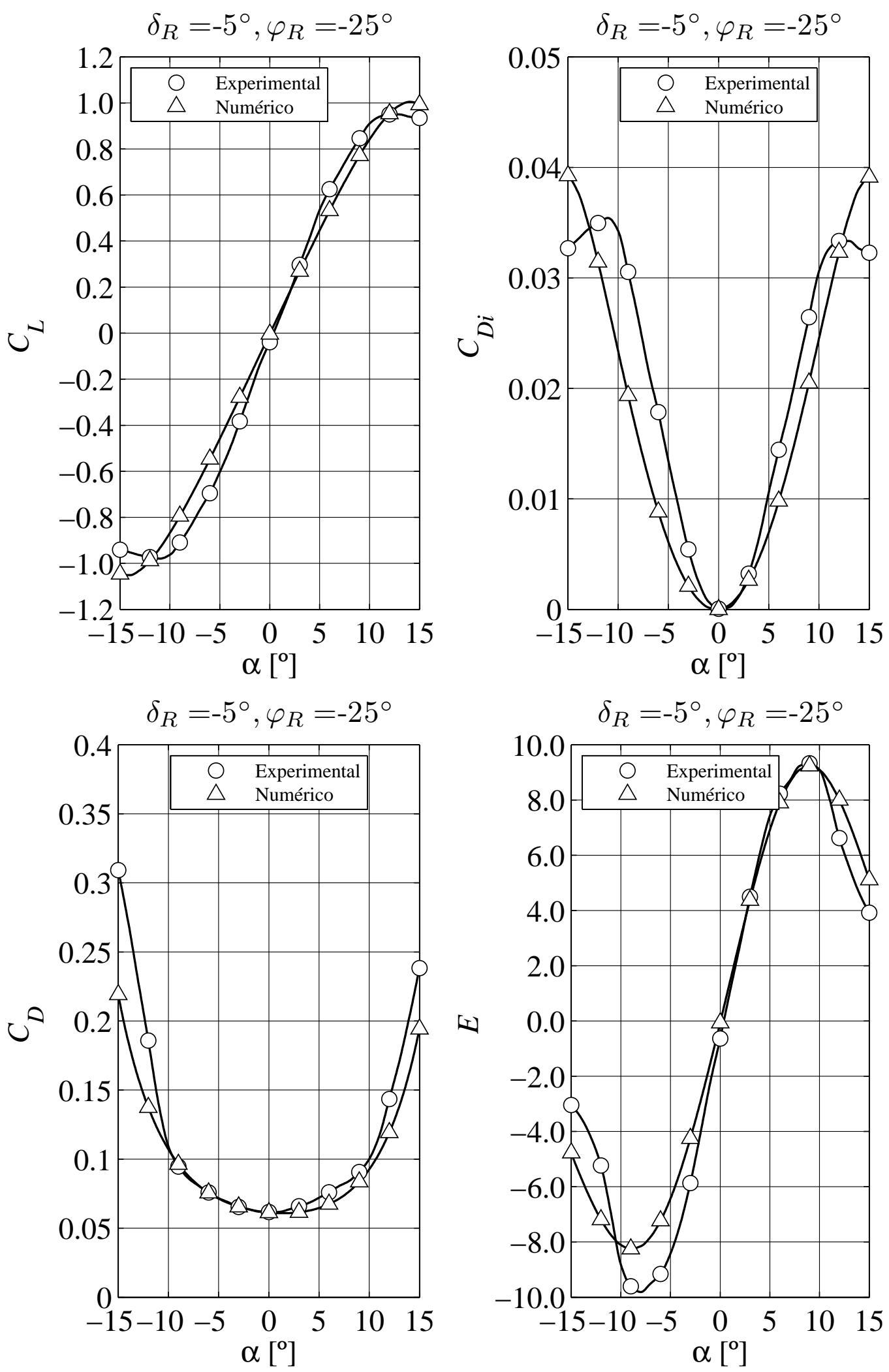

Figura E.4: Variación de los valores de los coeficientes de sustentación, $C_{L}$, de resistencia inducida, $C_{D i}$, de resistencia, $C_{D}$ y de eficiencia aerodinámica, $E$, en función del ángulo de ataque, $\alpha$, para la configuración de ensayo $\operatorname{JWC}_{41}\left(\varphi_{R}=-25^{\circ}\right.$ y $\delta_{R}=$ $\left.-5^{\circ}\right)$. Comparación entre el modelo numérico y los resultados experimentales. 
E.2. COEFICIENTES AERODINÁMICOS EN FUNCIÓN DEL ÁNGULO ÁNGULO DE ATAQUE, $\alpha$.
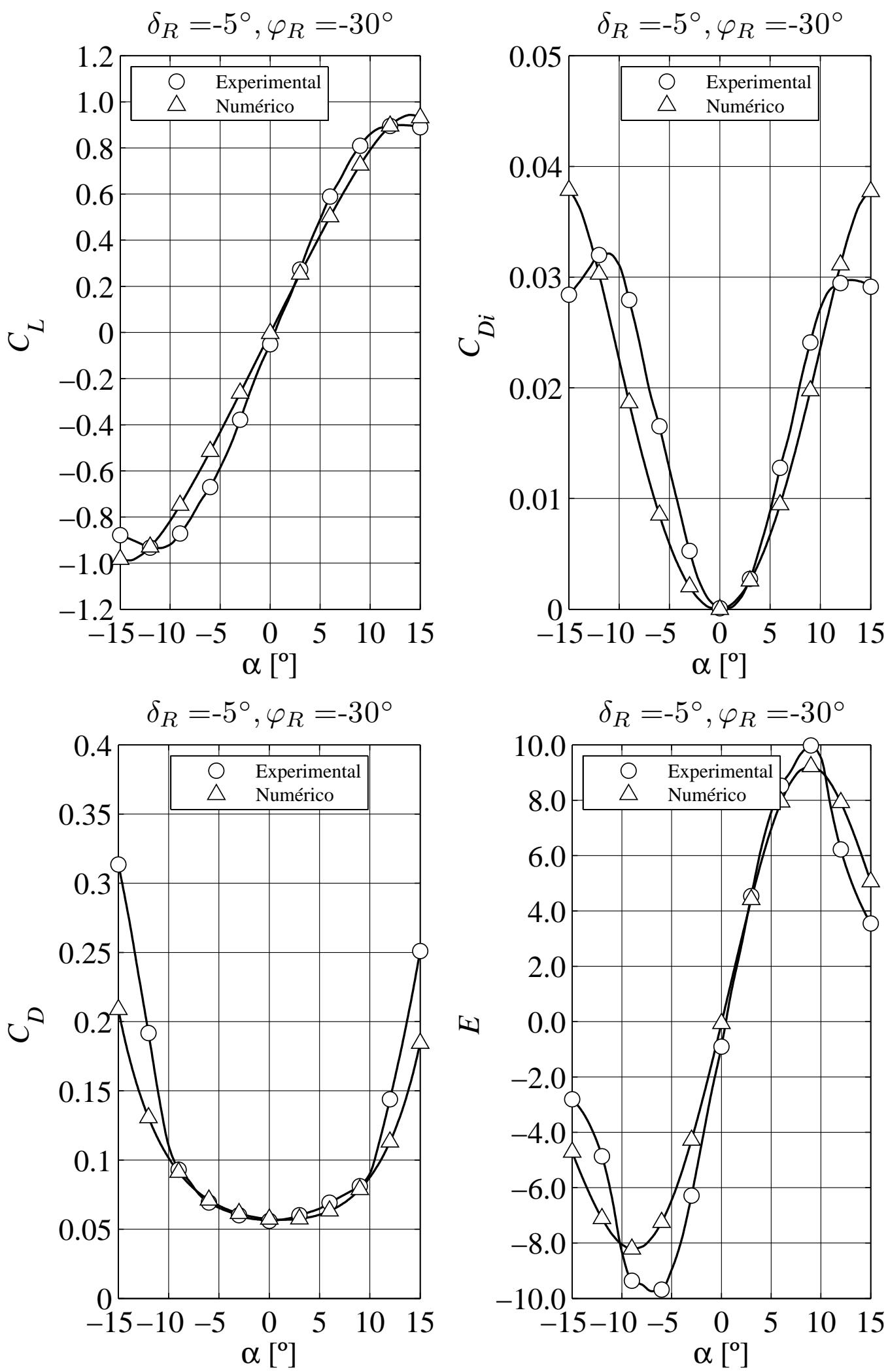

Figura E.5: Variación de los valores de los coeficientes de sustentación, $C_{L}$, de resistencia inducida, $C_{D i}$, de resistencia, $C_{D}$, y de eficiencia aerodinámica, $E$, en función del ángulo de ataque, $\alpha$, para la configuración de ensayo $\operatorname{JWC}_{51}\left(\varphi_{R}=-30^{\circ}\right.$ y $\delta_{R}=$ $\left.-5^{\circ}\right)$. Comparación entre el modelo numérico y los resultados experimentales. 

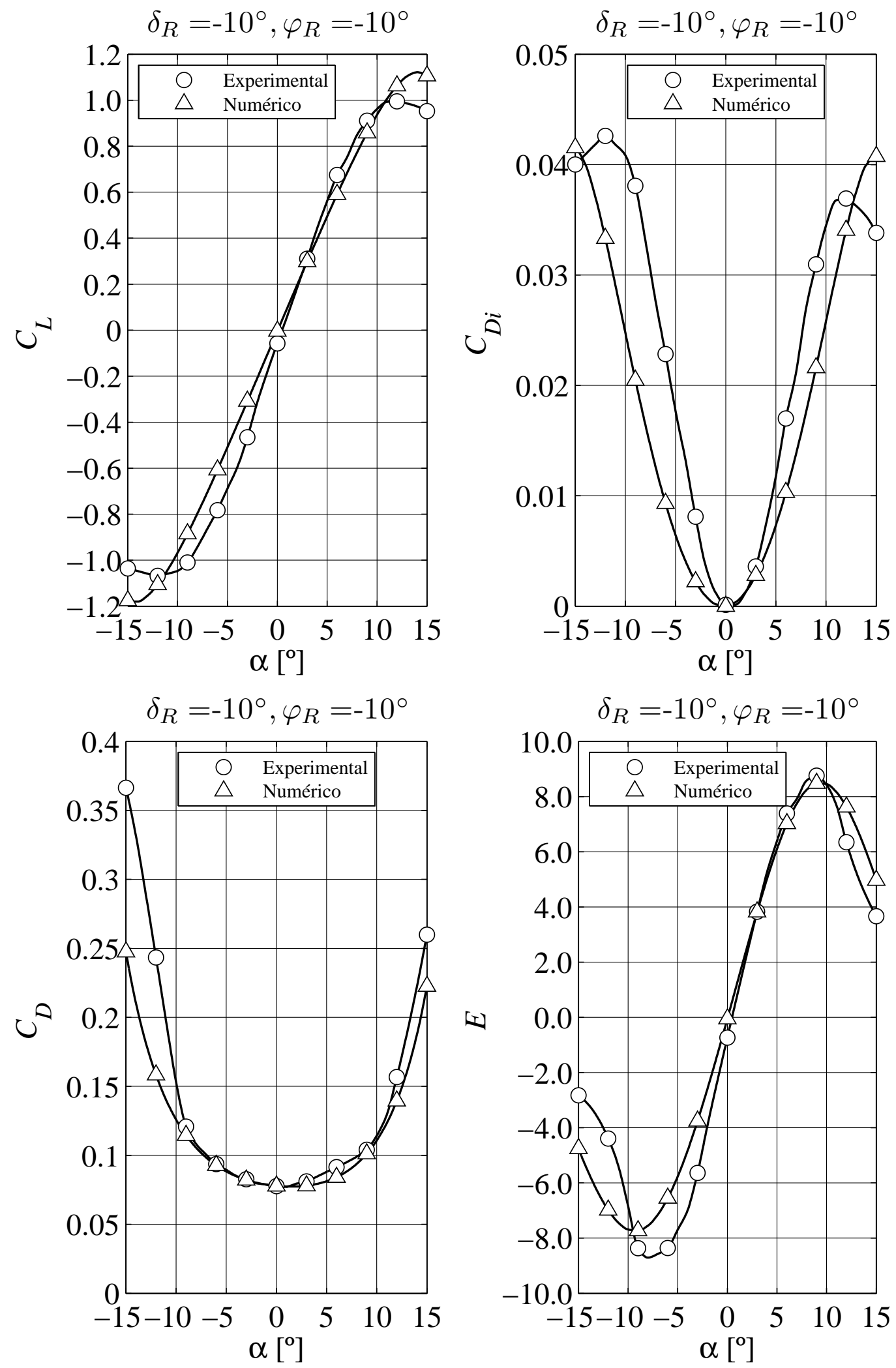

Figura E.6: Variación de los valores de los coeficientes de sustentación, $C_{L}$, de resistencia inducida, $C_{D i}$, de resistencia, $C_{D}$, y de eficiencia aerodinámica, $E$, en función del ángulo de ataque, $\alpha$, para la configuración de ensayo $\operatorname{JWC}_{12}\left(\varphi_{R}=-10^{\circ}\right.$ y $\delta_{R}=$ $-10^{\circ}$ ). Comparación entre el modelo numérico y los resultados experimentales. 
E.2. COEFICIENTES AERODINÁMICOS EN FUNCIÓN DEL ÁNGULO ÁNGULO DE ATAQUE, $\alpha$.
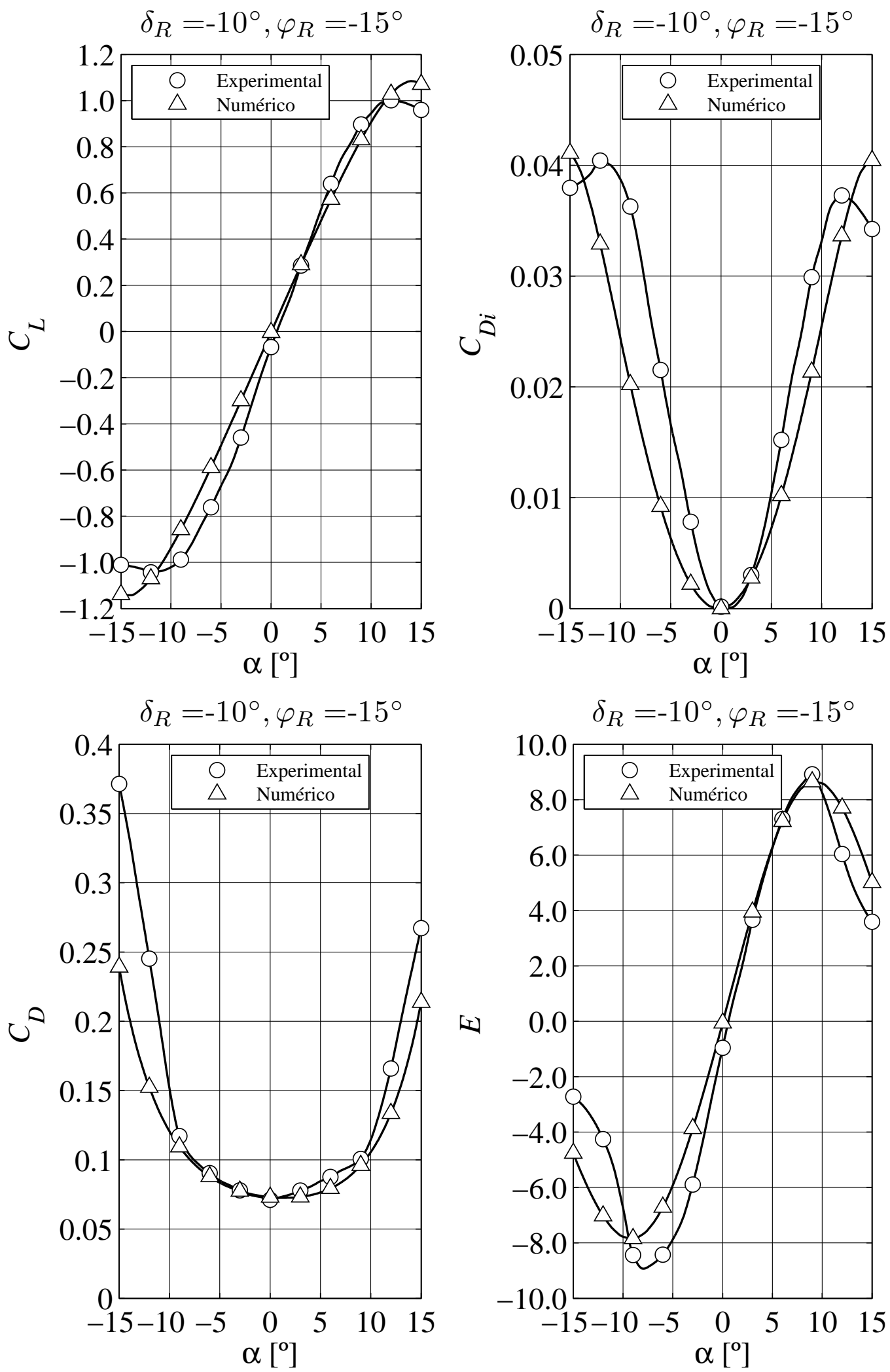

Figura E.7: Variación de los valores de los coeficientes de sustentación, $C_{L}$, de resistencia inducida, $C_{D i}$, de resistencia, $C_{D}$, y de eficiencia aerodinámica, $E$, en función del ángulo de ataque, $\alpha$, para la configuración de ensayo $\operatorname{JWC}_{22}\left(\varphi_{R}=-15^{\circ}\right.$ y $\delta_{R}=$ $\left.-10^{\circ}\right)$. Comparación entre el modelo numérico y los resultados experimentales. 

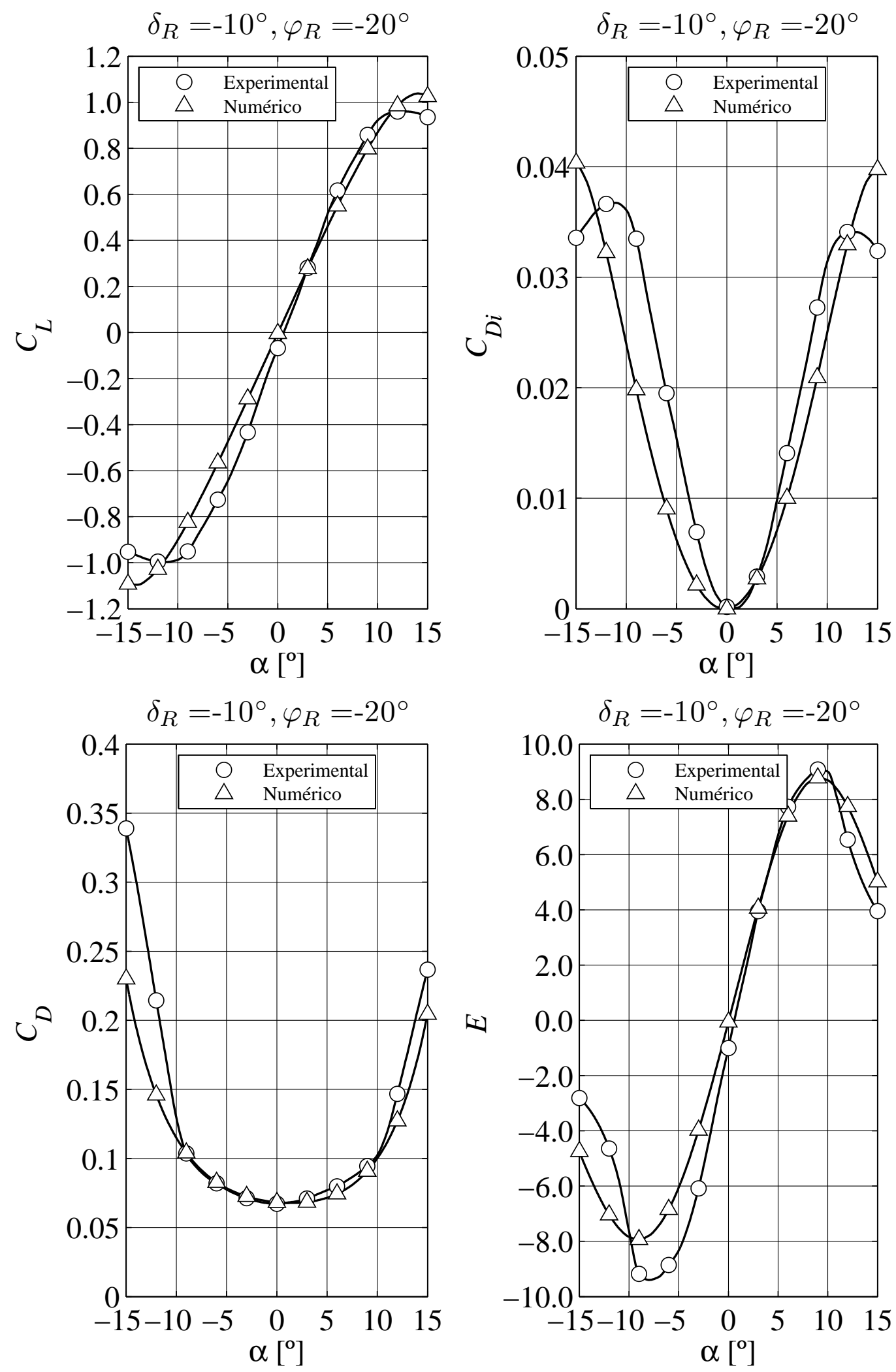

Figura E.8: Variación de los valores de los coeficientes de sustentación, $C_{L}$, de resistencia inducida, $C_{D i}$, de resistencia, $C_{D}$, y de eficiencia aerodinámica, $E$, en función del ángulo de ataque, $\alpha$, para la configuración de ensayo $\operatorname{JWC}_{32}\left(\varphi_{R}=-20^{\circ}\right.$ y $\delta_{R}=$ $-10^{\circ}$ ). Comparación entre el modelo numérico y los resultados experimentales. 
E.2. COEFICIENTES AERODINÁMICOS EN FUNCIÓN DEL ÁNGULO ÁNGULO DE ATAQUE, $\alpha$.
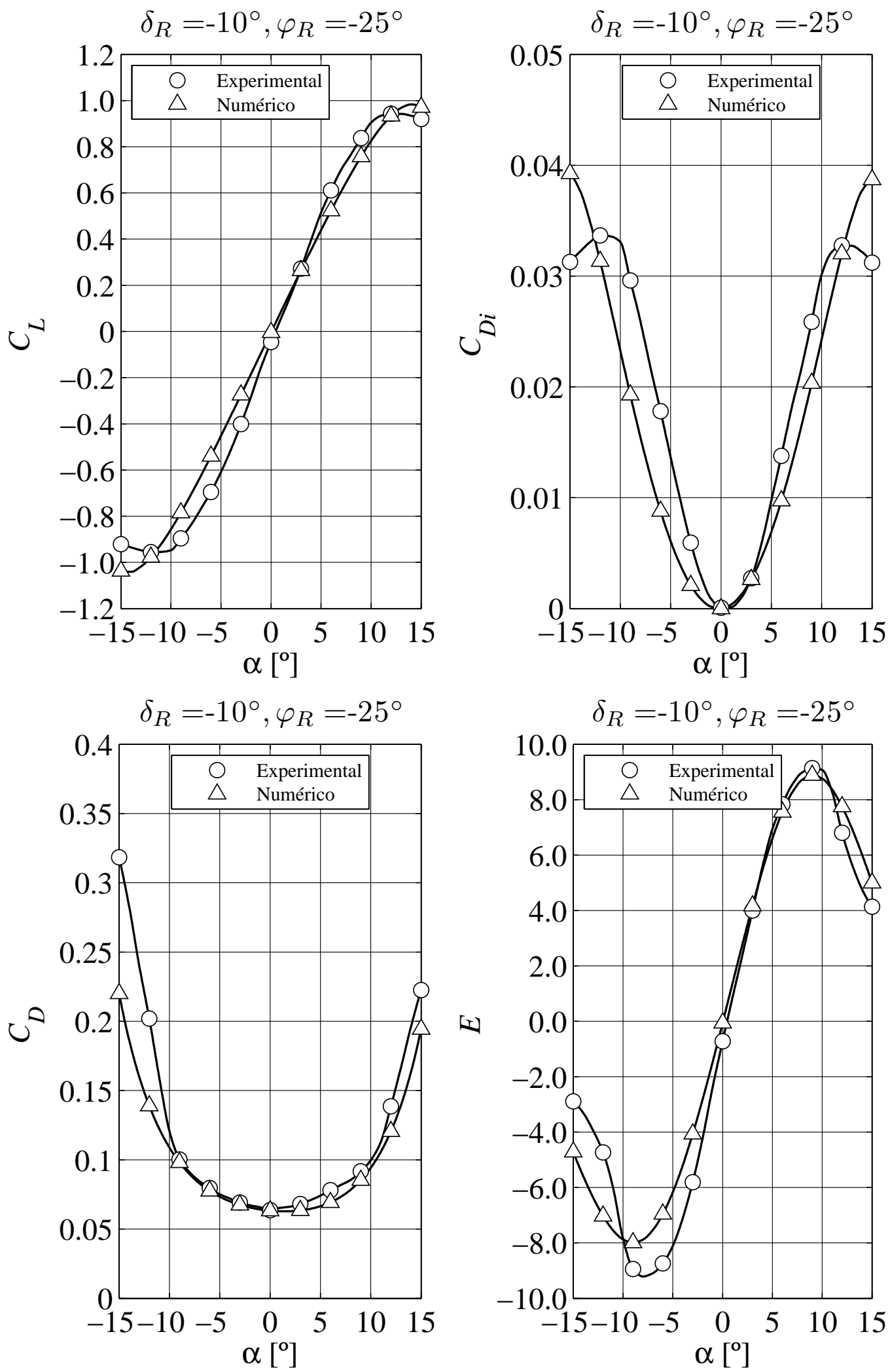

Figura E.9: Variación de los valores de los coeficientes de sustentación, $C_{L}$, de resistencia inducida, $C_{D i}$, de resistencia, $C_{D}$, y de eficiencia aerodinámica, $E$, en función del ángulo de ataque, $\alpha$, para la configuración de ensayo $\mathrm{JWC}_{42}\left(\varphi_{R}=-25^{\circ}\right.$ y $\delta_{R}=$ $\left.-10^{\circ}\right)$. Comparación entre el modelo numérico y los resultados experimentales. 

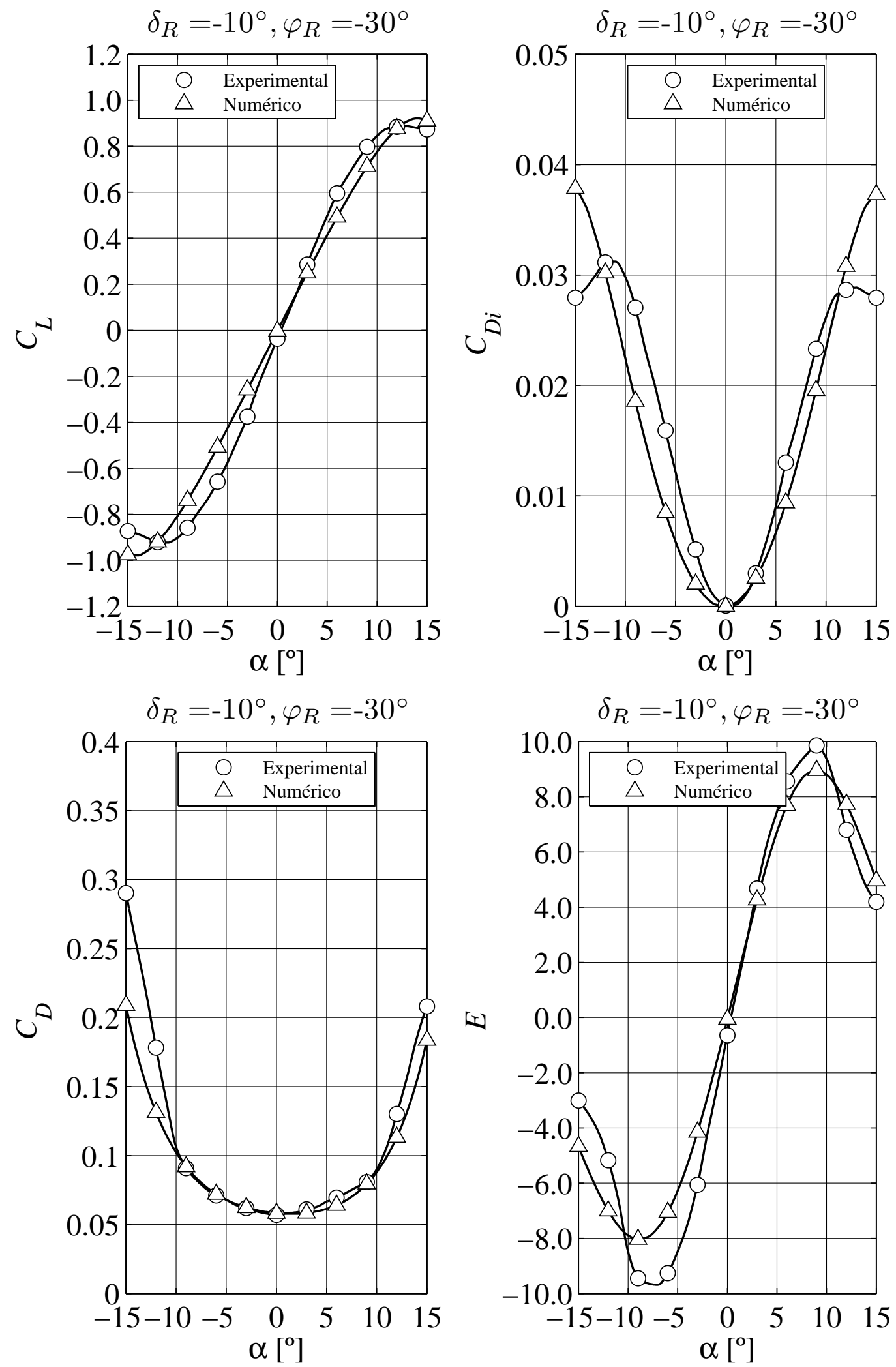

Figura E.10: Variación de los valores de los coeficientes de sustentación, $C_{L}$, de resistencia inducida, $C_{D i}$, de resistencia, $C_{D}$, y de eficiencia aerodinámica, $E$, en función del ángulo de ataque, $\alpha$, para la configuración de ensayo $\operatorname{JWC}_{52}\left(\varphi_{R}=-30^{\circ}\right.$ y $\delta_{R}=$ $-10^{\circ}$ ). Comparación entre el modelo numérico y los resultados experimentales. 
E.2. COEFICIENTES AERODINÁMICOS EN FUNCIÓN DEL ÁNGULO ÁNGULO DE ATAQUE, $\alpha$.
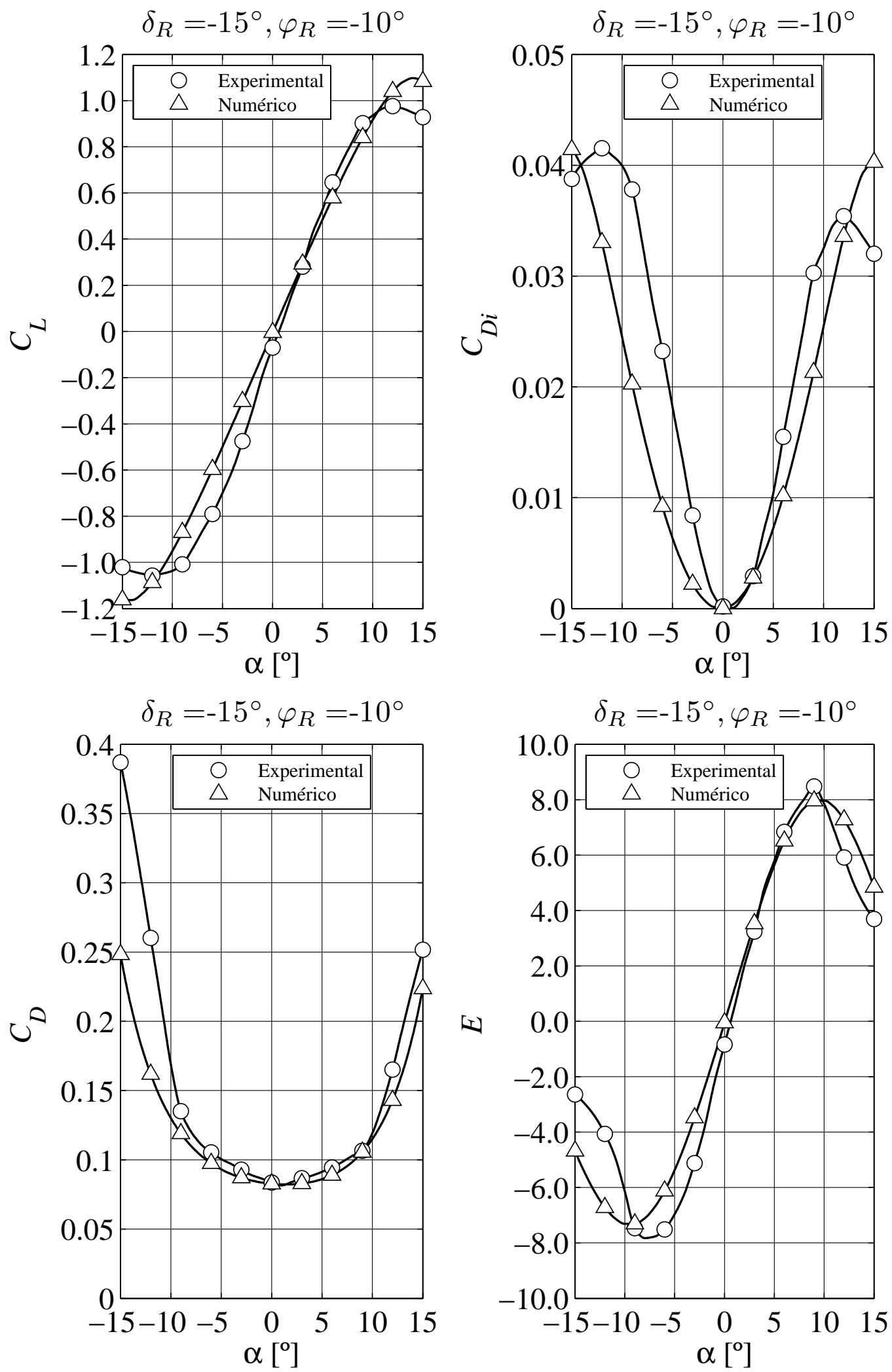

Figura E.11: Variación de los valores de los coeficientes de sustentación, $C_{L}$, de resistencia inducida, $C_{D i}$, de resistencia, $C_{D}$, y de eficiencia aerodinámica, $E$, en función del ángulo de ataque, $\alpha$, para la configuración de ensayo $\operatorname{JWC}_{13}\left(\varphi_{R}=-10^{\circ}\right.$ y $\delta_{R}=$ $-15^{\circ}$ ). Comparación entre el modelo numérico y los resultados experimentales. 

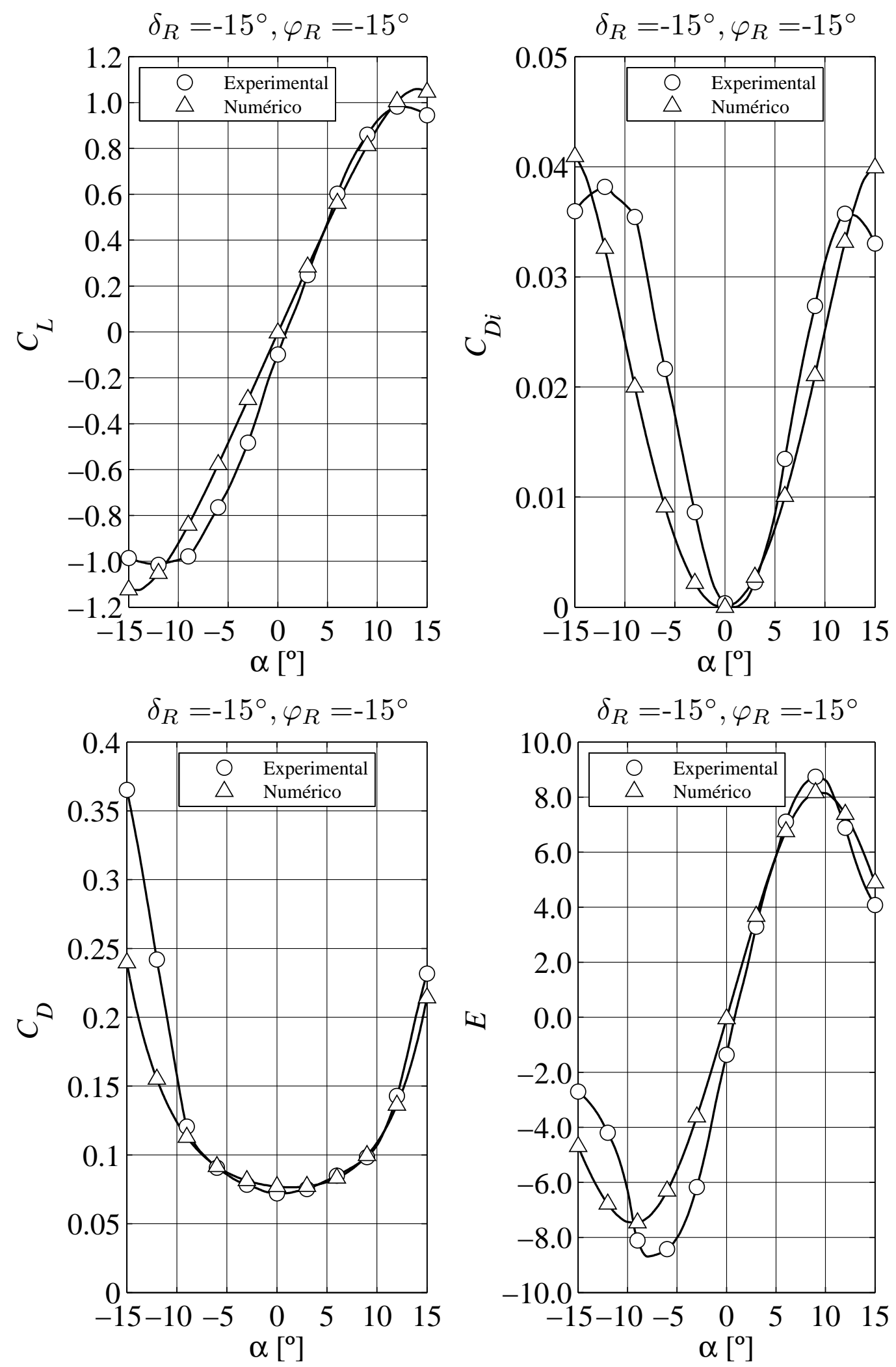

Figura E.12: Variación de los valores de los coeficientes de sustentación, $C_{L}$, de resistencia inducida, $C_{D i}$, de resistencia, $C_{D}$, y de eficiencia aerodinámica, $E$, en función del ángulo de ataque, $\alpha$, para la configuración de ensayo $\operatorname{JWC}_{23}\left(\varphi_{R}=-15^{\circ}\right.$ y $\delta_{R}=$ $\left.-15^{\circ}\right)$. Comparación entre el modelo numérico y los resultados experimentales. 
E.2. COEFICIENTES AERODINÁMICOS EN FUNCIÓN DEL ÁNGULO ÁNGULO DE ATAQUE, $\alpha$.
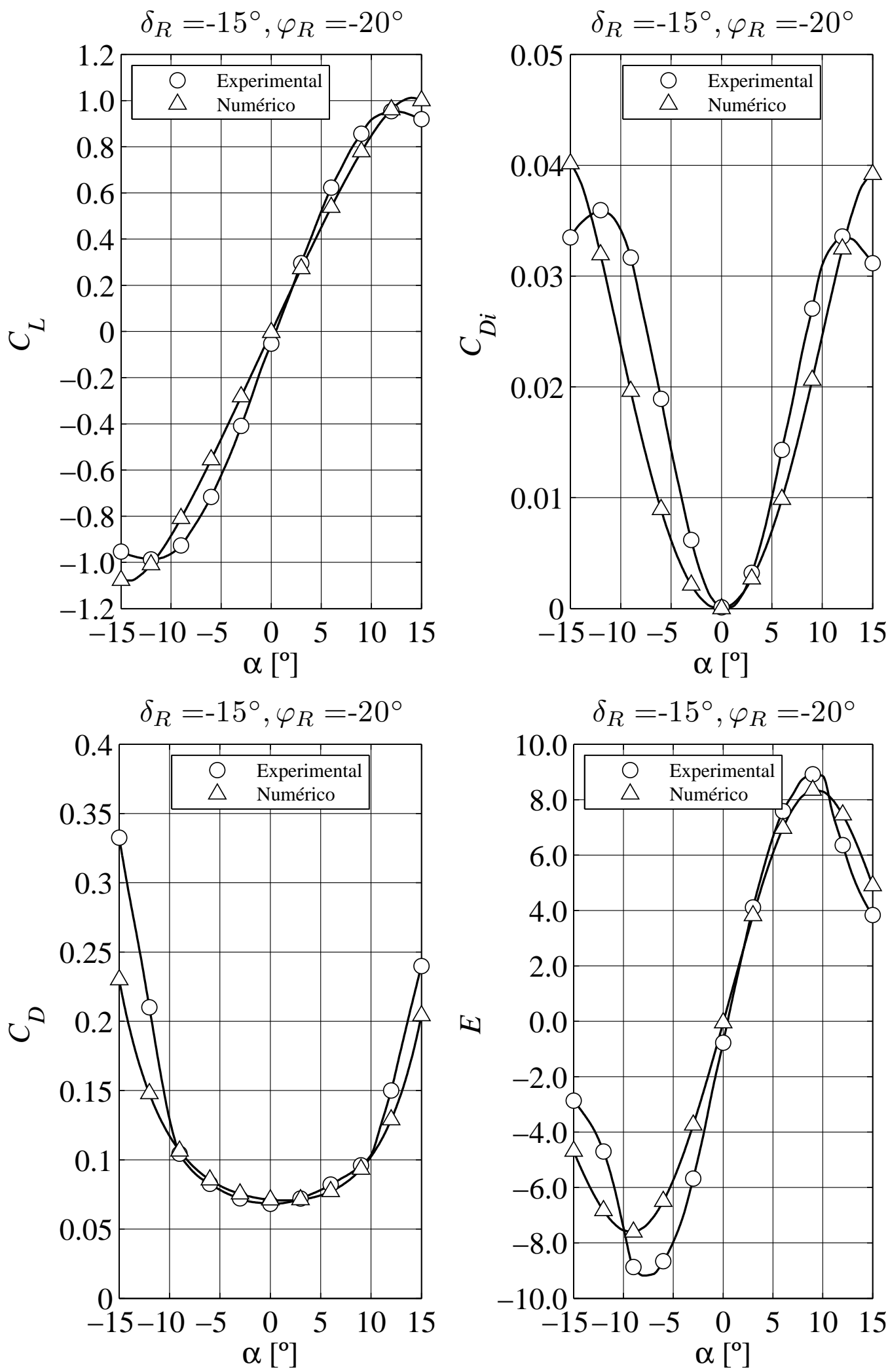

Figura E.13: Variación de los valores de los coeficientes de sustentación, $C_{L}$, de resistencia inducida, $C_{D i}$, de resistencia, $C_{D}$, y de eficiencia aerodinámica, $E$, en función del ángulo de ataque, $\alpha$, para la configuración de ensayo $\mathrm{JWC}_{33}\left(\varphi_{R}=-20^{\circ}\right.$ y $\delta_{R}=$ $-15^{\circ}$ ). Comparación entre el modelo numérico y los resultados experimentales. 

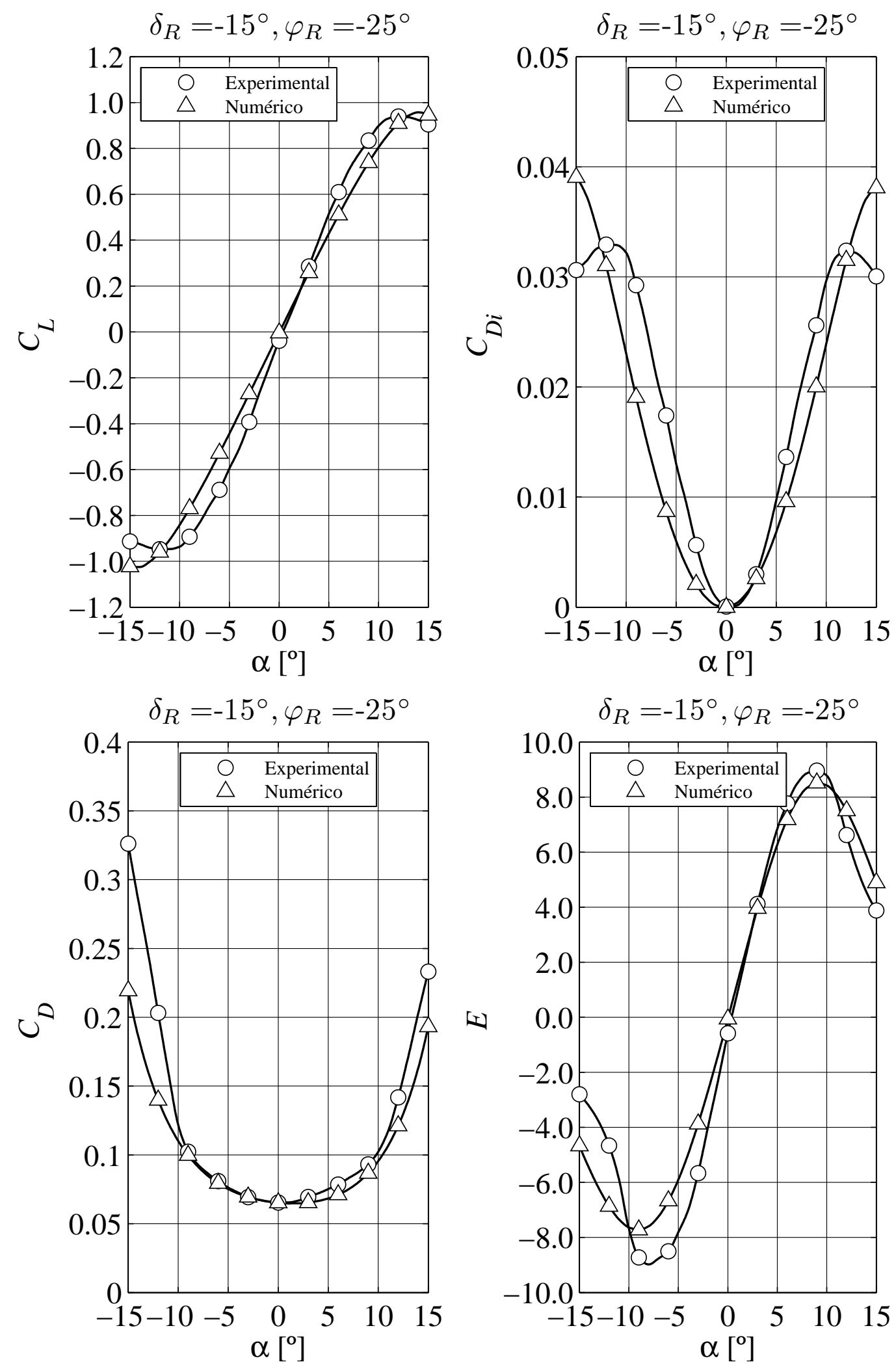

Figura E.14: Variación de los valores de los coeficientes de sustentación, $C_{L}$, de resistencia inducida, $C_{D i}$, de resistencia, $C_{D}$, y de eficiencia aerodinámica, $E$, en función del ángulo de ataque, $\alpha$, para la configuración de ensayo $\mathrm{JWC}_{43}\left(\varphi_{R}=-25^{\circ}\right.$ y $\delta_{R}=$ $-15^{\circ}$ ). Comparación entre el modelo numérico y los resultados experimentales. 
E.2. COEFICIENTES AERODINÁMICOS EN FUNCIÓN DEL ÁNGULO ÁNGULO DE ATAQUE, $\alpha$.
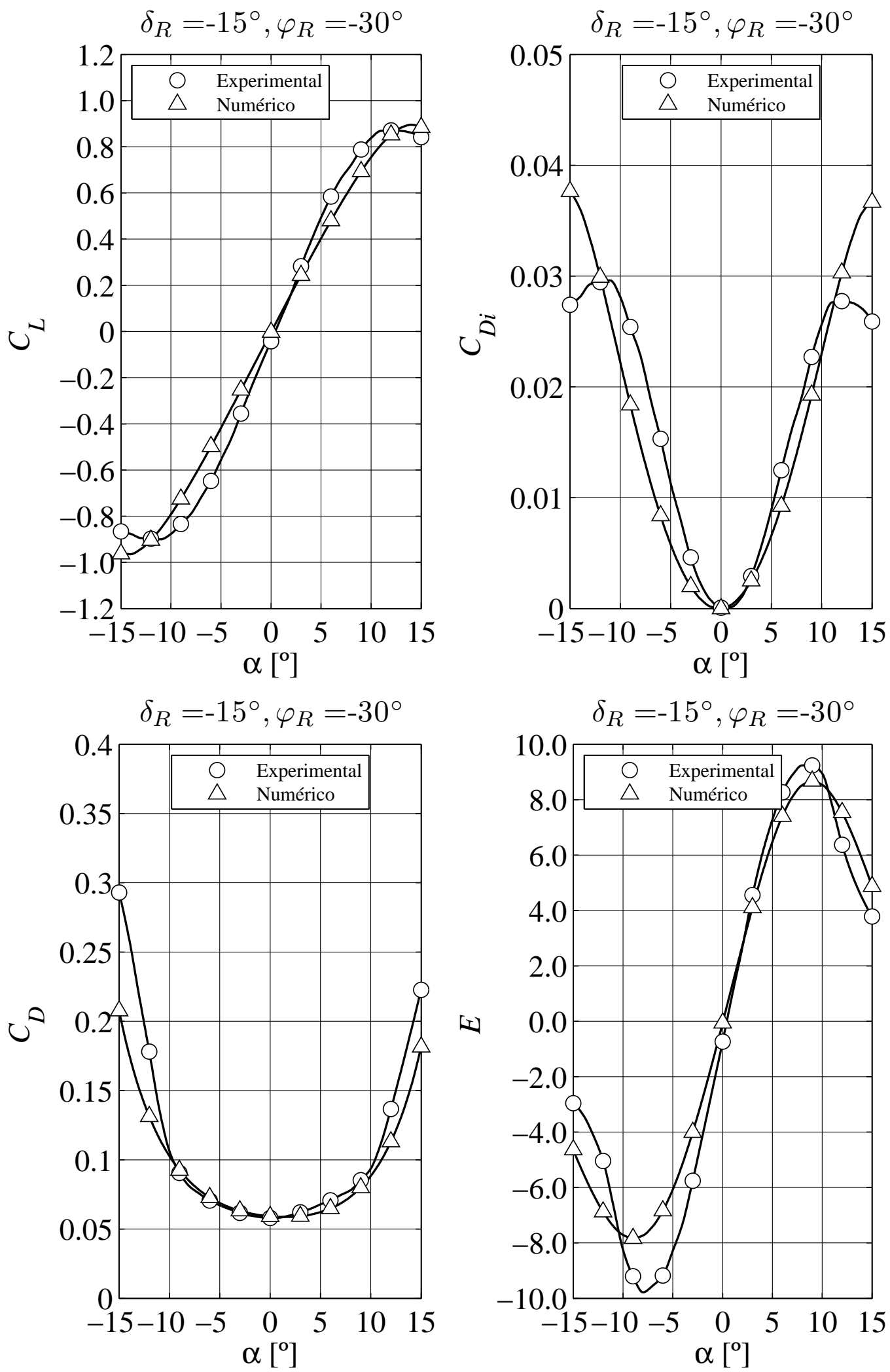

Figura E.15: Variación de los valores de los coeficientes de sustentación, $C_{L}$, de resistencia inducida, $C_{D i}$, de resistencia, $C_{D}$, y de eficiencia aerodinámica, $E$, en función del ángulo de ataque, $\alpha$, para la configuración de ensayo $\operatorname{JWC}_{53}\left(\varphi_{R}=-30^{\circ}\right.$ y $\delta_{R}=$ $\left.-15^{\circ}\right)$. Comparación entre el modelo numérico y los resultados experimentales. 

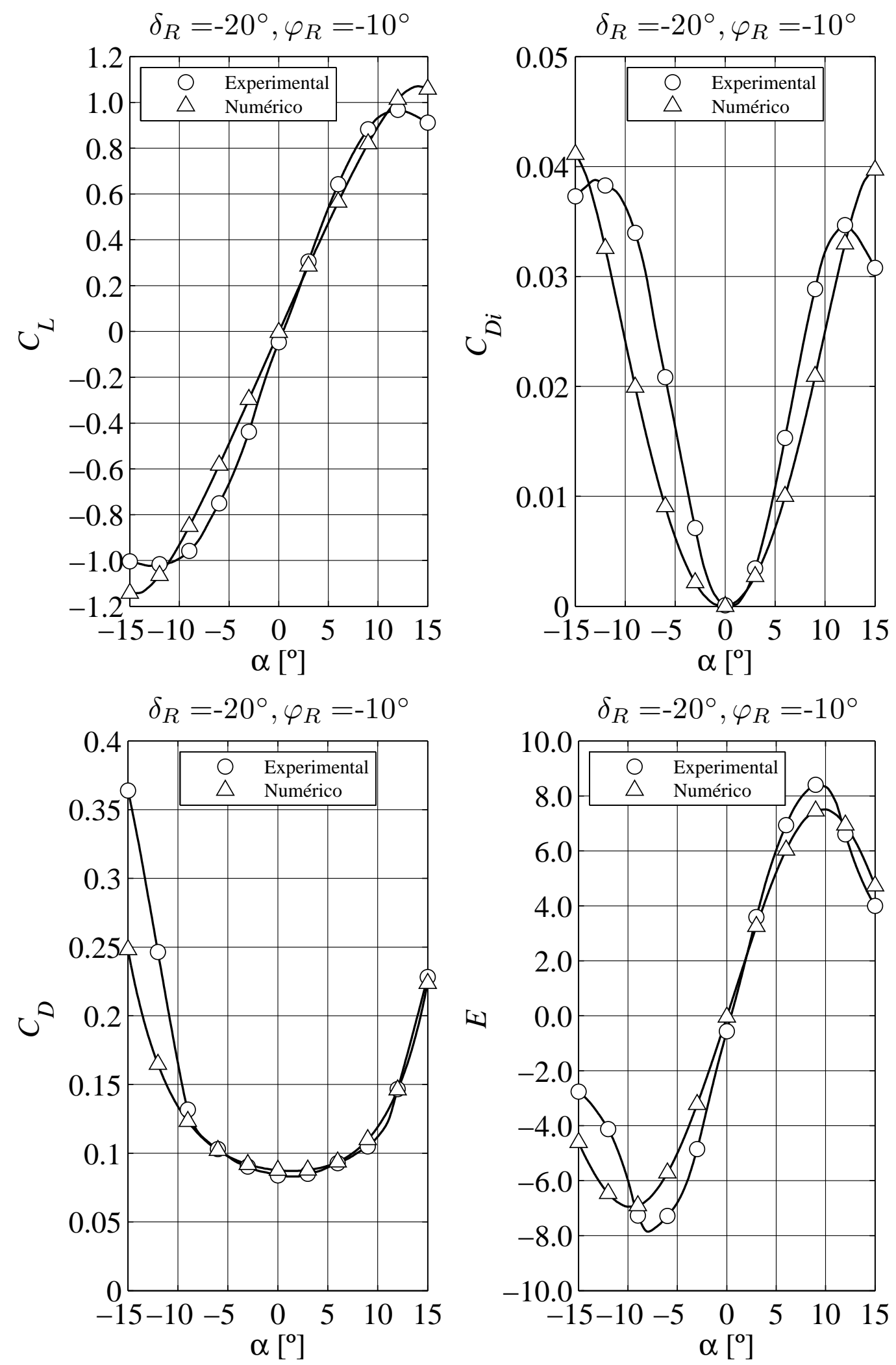

Figura E.16: Variación de los valores de los coeficientes de sustentación, $C_{L}$, de resistencia inducida, $C_{D i}$, de resistencia, $C_{D}$, y de eficiencia aerodinámica, $E$, en función del ángulo de ataque, $\alpha$, para la configuración de ensayo $\operatorname{JWC}_{14}\left(\varphi_{R}=-10^{\circ}\right.$ y $\delta_{R}=$ $-20^{\circ}$ ). Comparación entre el modelo numérico y los resultados experimentales. 
E.2. COEFICIENTES AERODINÁMICOS EN FUNCIÓN DEL ÁNGULO ÁNGULO DE ATAQUE, $\alpha$.
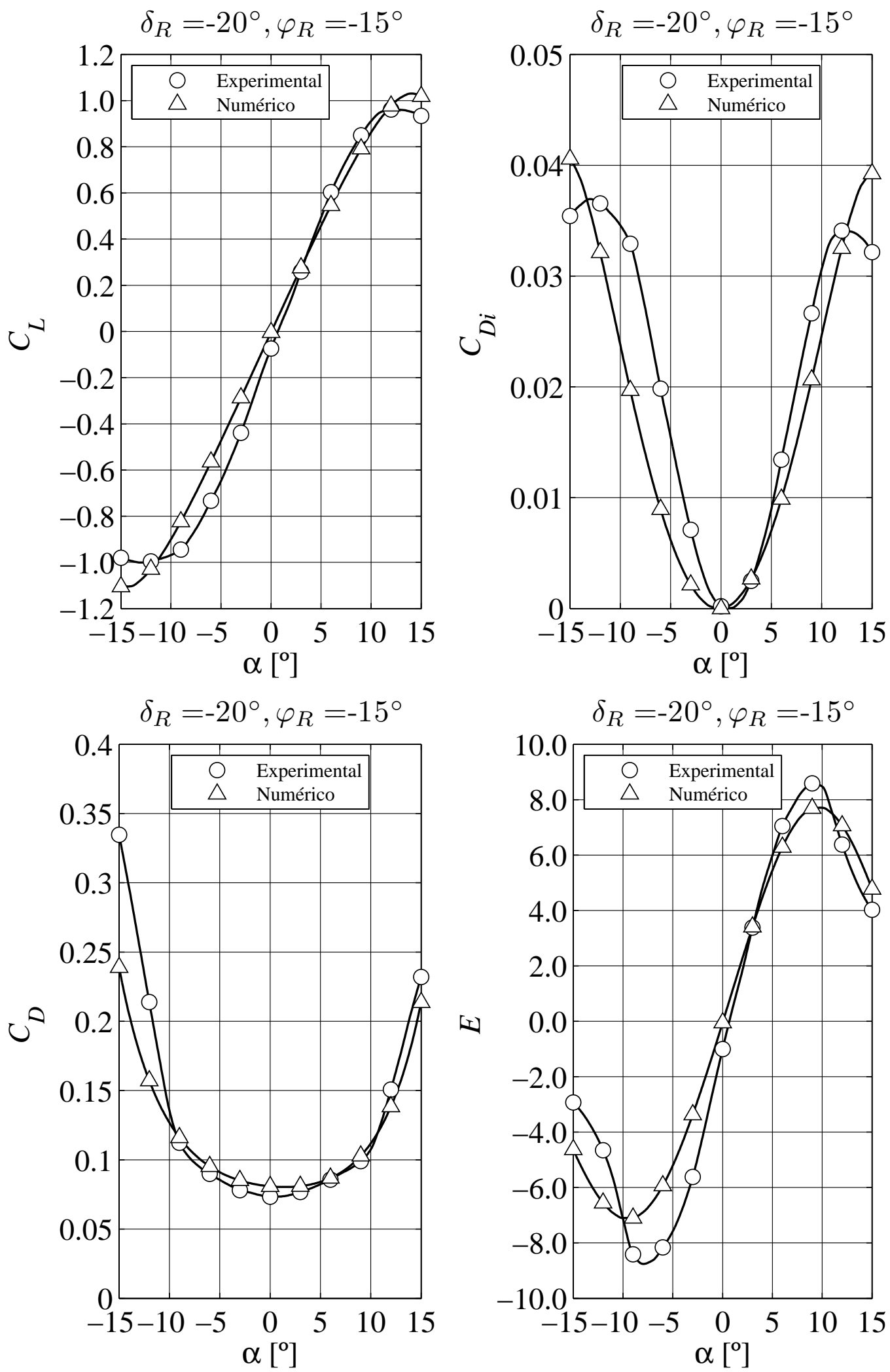

Figura E.17: Variación de los valores de los coeficientes de sustentación, $C_{L}$, de resistencia inducida, $C_{D i}$, de resistencia, $C_{D}$, y de eficiencia aerodinámica, $E$, en función del ángulo de ataque, $\alpha$, para la configuración de ensayo $\mathrm{JWC}_{24}\left(\varphi_{R}=-15^{\circ}\right.$ y $\delta_{R}=$ $\left.-20^{\circ}\right)$. Comparación entre el modelo numérico y los resultados experimentales. 

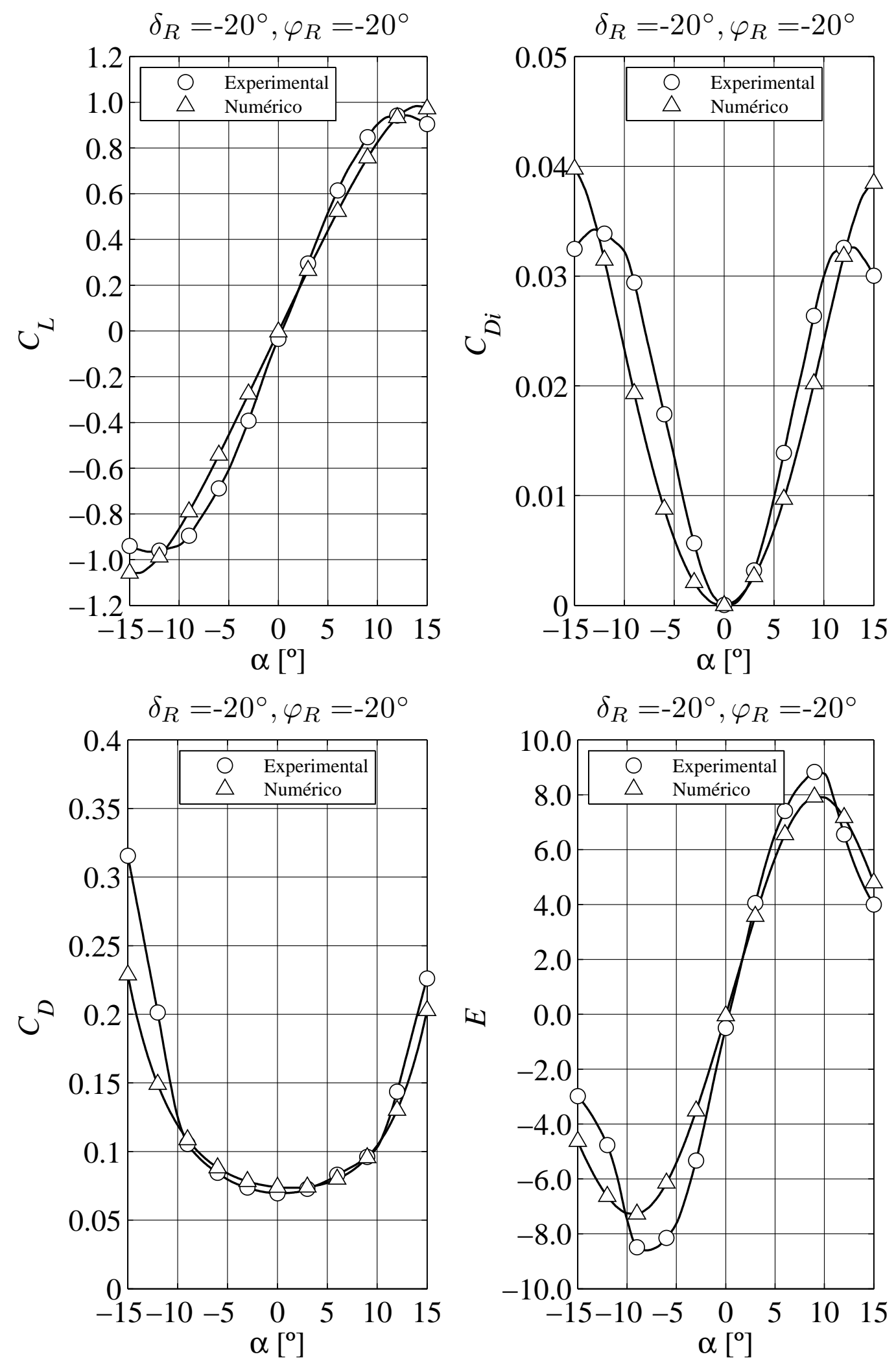

Figura E.18: Variación de los valores de los coeficientes de sustentación, $C_{L}$, de resistencia inducida, $C_{D i}$, de resistencia, $C_{D}$, y de eficiencia aerodinámica, $E$, en función del ángulo de ataque, $\alpha$, para la configuración de ensayo $\operatorname{JWC}_{34}\left(\varphi_{R}=-20^{\circ}\right.$ y $\delta_{R}=$ $\left.-20^{\circ}\right)$. Comparación entre el modelo numérico y los resultados experimentales. 
E.2. COEFICIENTES AERODINÁMICOS EN FUNCIÓN DEL ÁNGULO ÁNGULO DE ATAQUE, $\alpha$.
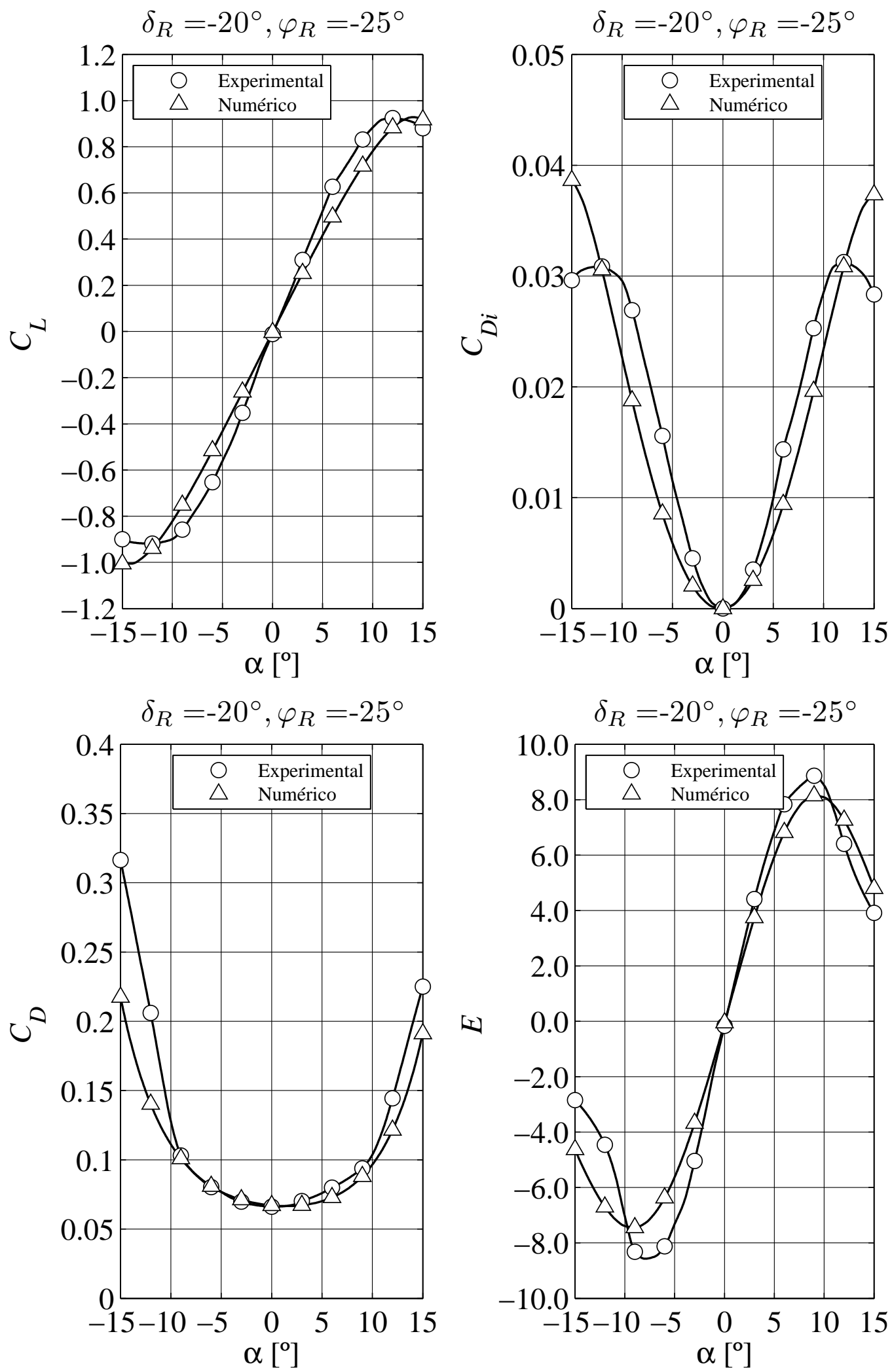

Figura E.19: Variación de los valores de los coeficientes de sustentación, $C_{L}$, de resistencia inducida, $C_{D i}$, de resistencia, $C_{D}$, y de eficiencia aerodinámica, $E$, en función del ángulo de ataque, $\alpha$, para la configuración de ensayo $\operatorname{JWC}_{44}\left(\varphi_{R}=-25^{\circ}\right.$ y $\delta_{R}=$ $\left.-20^{\circ}\right)$. Comparación entre el modelo numérico y los resultados experimentales. 

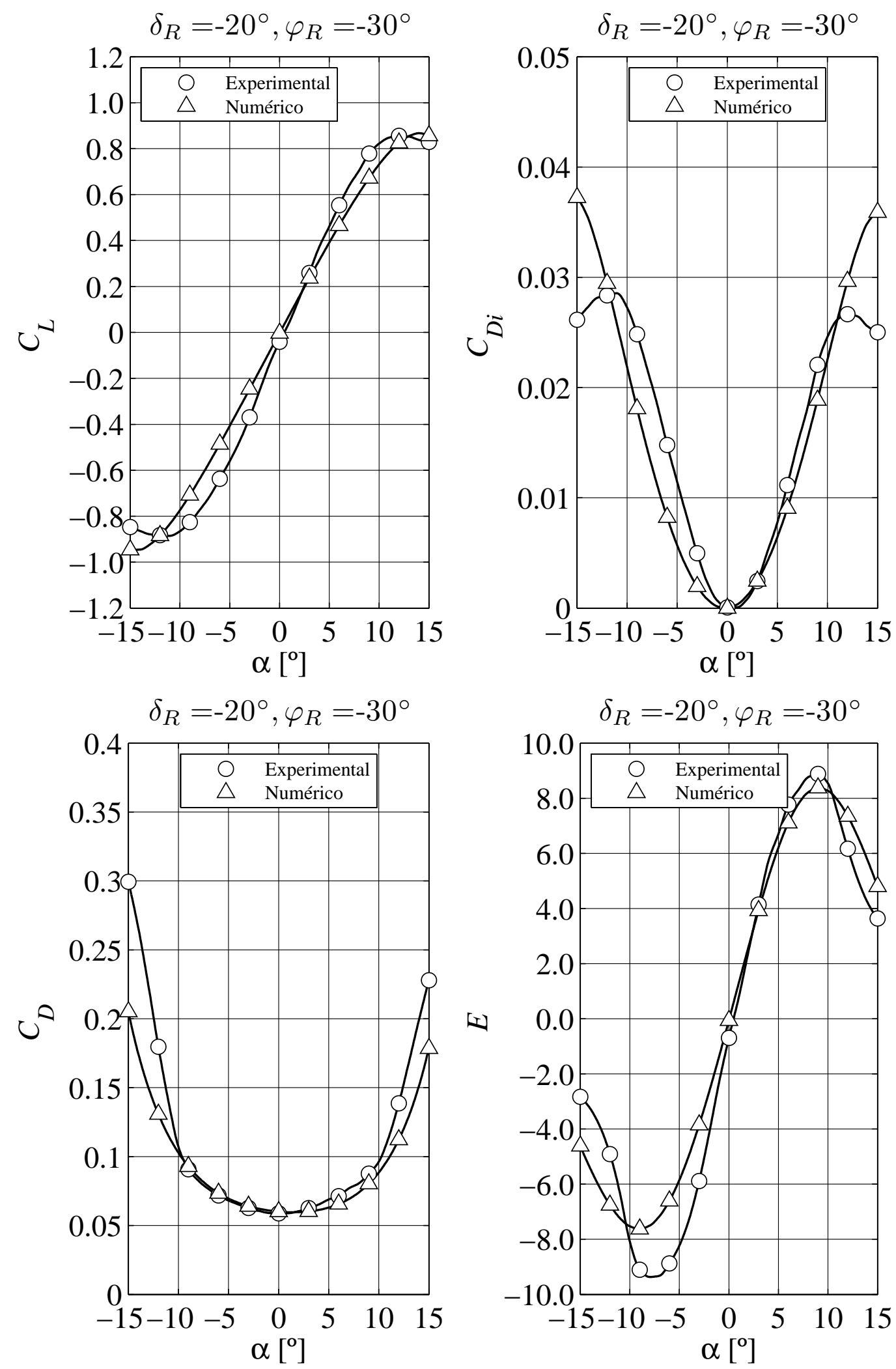

Figura E.20: Variación de los valores de los coeficientes de sustentación, $C_{L}$, de resistencia inducida, $C_{D i}$, de resistencia, $C_{D}$, y de eficiencia aerodinámica, $E$, en función del ángulo de ataque, $\alpha$, para la configuración de ensayo $\operatorname{JWC}_{54}\left(\varphi_{R}=-30^{\circ}\right.$ y $\delta_{R}=$ $\left.-20^{\circ}\right)$. Comparación entre el modelo numérico y los resultados experimentales. 
E.2. COEFICIENTES AERODINÁMICOS EN FUNCIÓN DEL ÁNGULO ÁNGULO DE ATAQUE, $\alpha$.
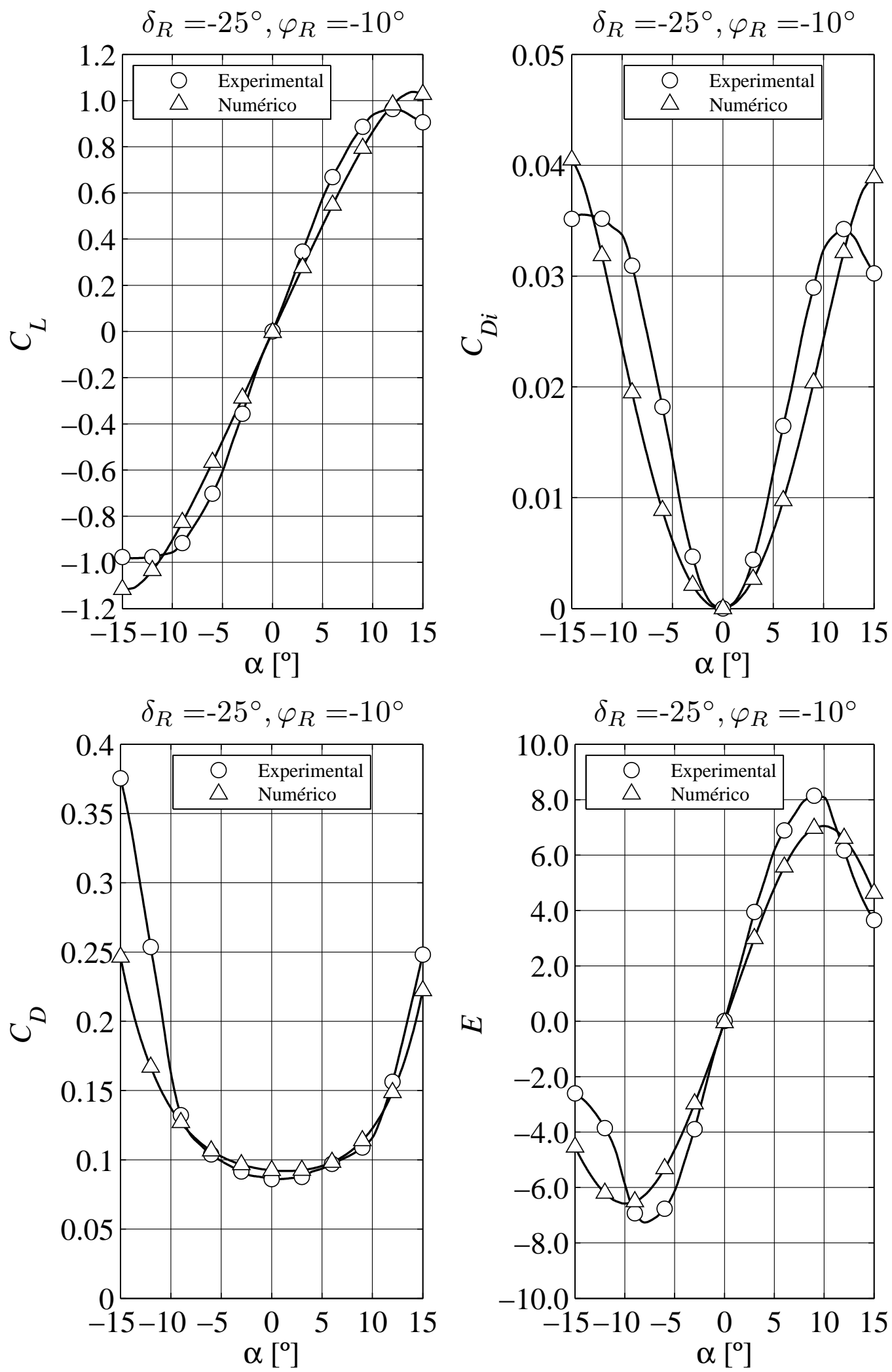

Figura E.21: Variación de los valores de los coeficientes de sustentación, $C_{L}$, de resistencia inducida, $C_{D i}$, de resistencia, $C_{D}$, y de eficiencia aerodinámica, $E$, en función del ángulo de ataque, $\alpha$, para la configuración de ensayo $\operatorname{JWC}_{15}\left(\varphi_{R}=-10^{\circ}\right.$ y $\delta_{R}=$ $\left.-25^{\circ}\right)$. Comparación entre el modelo numérico y los resultados experimentales. 

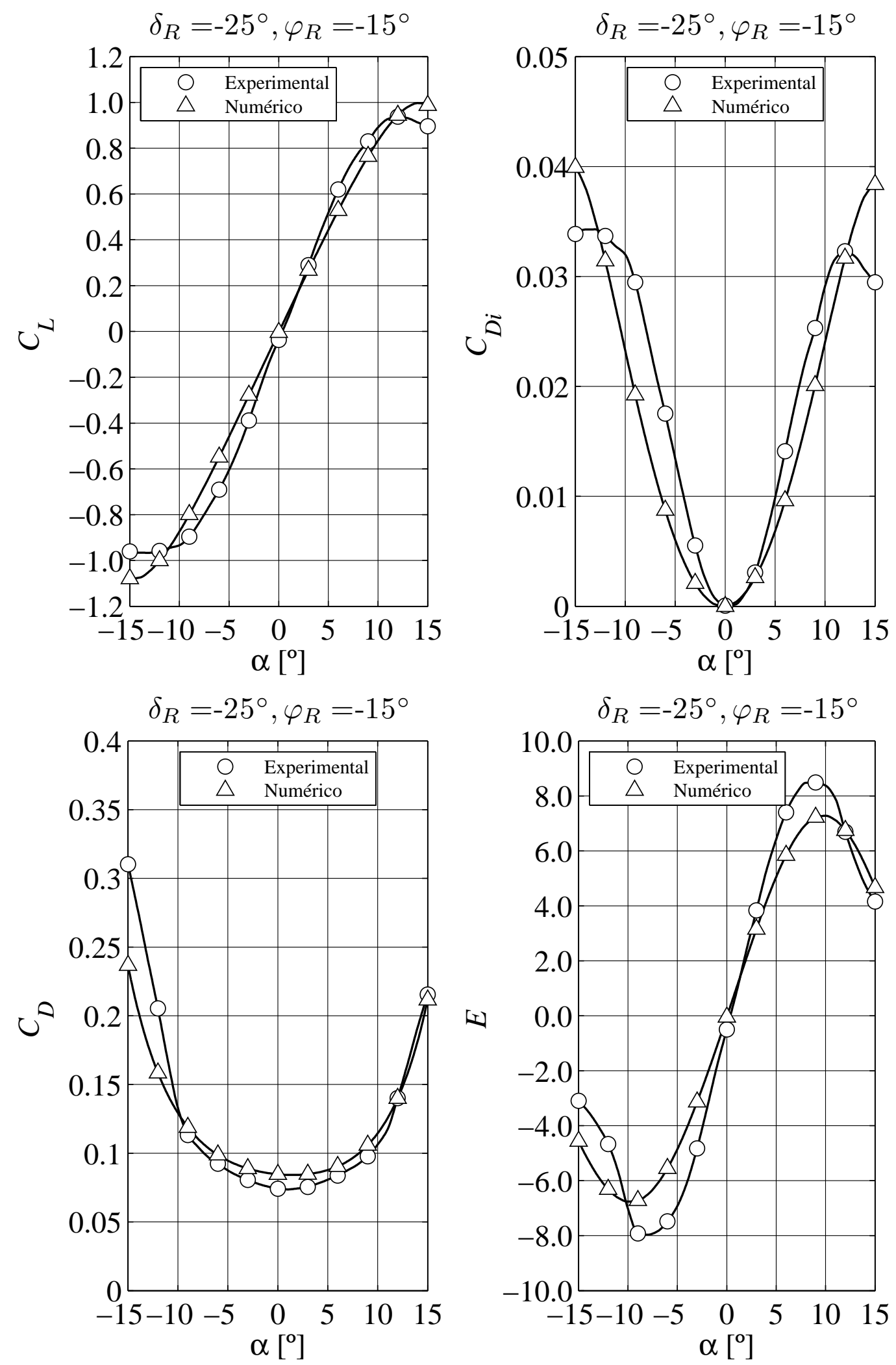

Figura E.22: Variación de los valores de los coeficientes de sustentación, $C_{L}$, de resistencia inducida, $C_{D i}$, de resistencia, $C_{D}$, y de eficiencia aerodinámica, $E$, en función del ángulo de ataque, $\alpha$, para la configuración de ensayo $\operatorname{JWC}_{25}\left(\varphi_{R}=-15^{\circ}\right.$ y $\delta_{R}=$ $-25^{\circ}$ ). Comparación entre el modelo numérico y los resultados experimentales. 
E.2. COEFICIENTES AERODINÁMICOS EN FUNCIÓN DEL ÁNGULO ÁNGULO DE ATAQUE, $\alpha$.
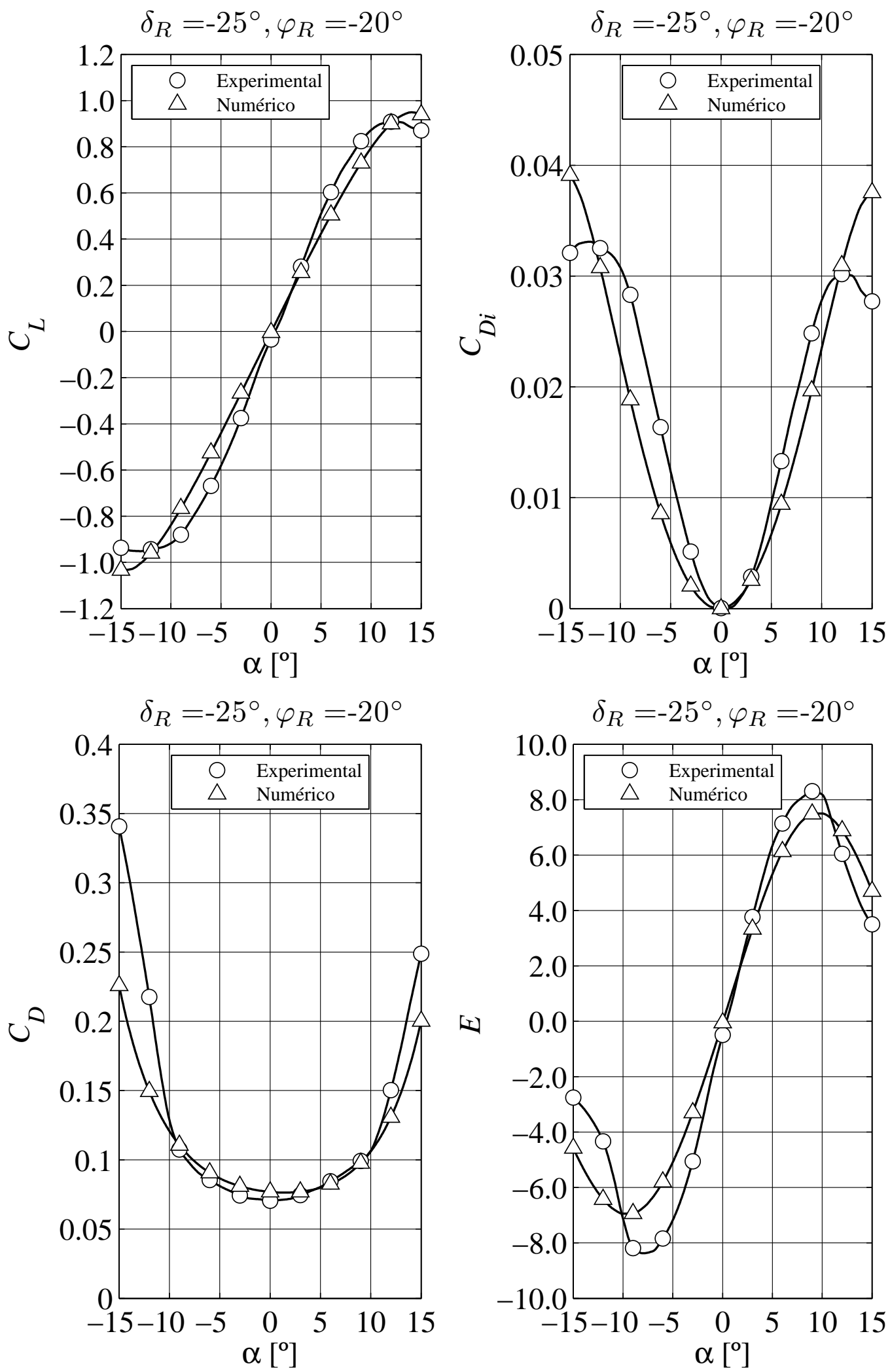

Figura E.23: Variación de los valores de los coeficientes de sustentación, $C_{L}$, de resistencia inducida, $C_{D i}$, de resistencia, $C_{D}$, y de eficiencia aerodinámica, $E$, en función del ángulo de ataque, $\alpha$, para la configuración de ensayo $\operatorname{JWC}_{35}\left(\varphi_{R}=-20^{\circ}\right.$ y $\delta_{R}=$ $\left.-25^{\circ}\right)$. Comparación entre el modelo numérico y los resultados experimentales. 

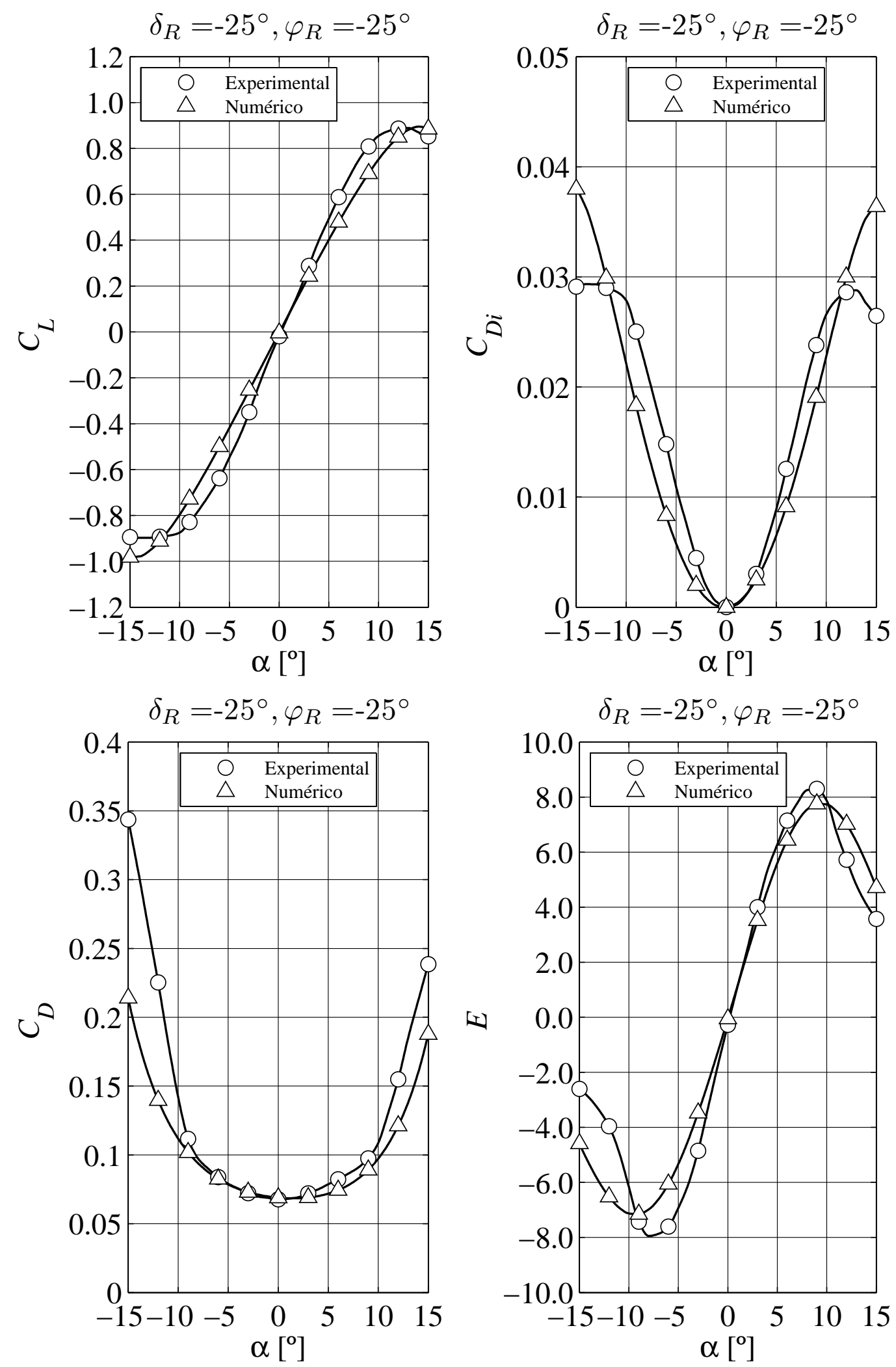

Figura E.24: Variación de los valores de los coeficientes de sustentación, $C_{L}$, de resistencia inducida, $C_{D i}$, de resistencia, $C_{D}$, y de eficiencia aerodinámica, $E$, en función del ángulo de ataque, $\alpha$, para la configuración de ensayo $\operatorname{JWC}_{45}\left(\varphi_{R}=-25^{\circ}\right.$ y $\delta_{R}=$ $-25^{\circ}$ ). Comparación entre el modelo numérico y los resultados experimentales. 
E.2. COEFICIENTES AERODINÁMICOS EN FUNCIÓN DEL ÁNGULO ÁNGULO DE ATAQUE, $\alpha$.
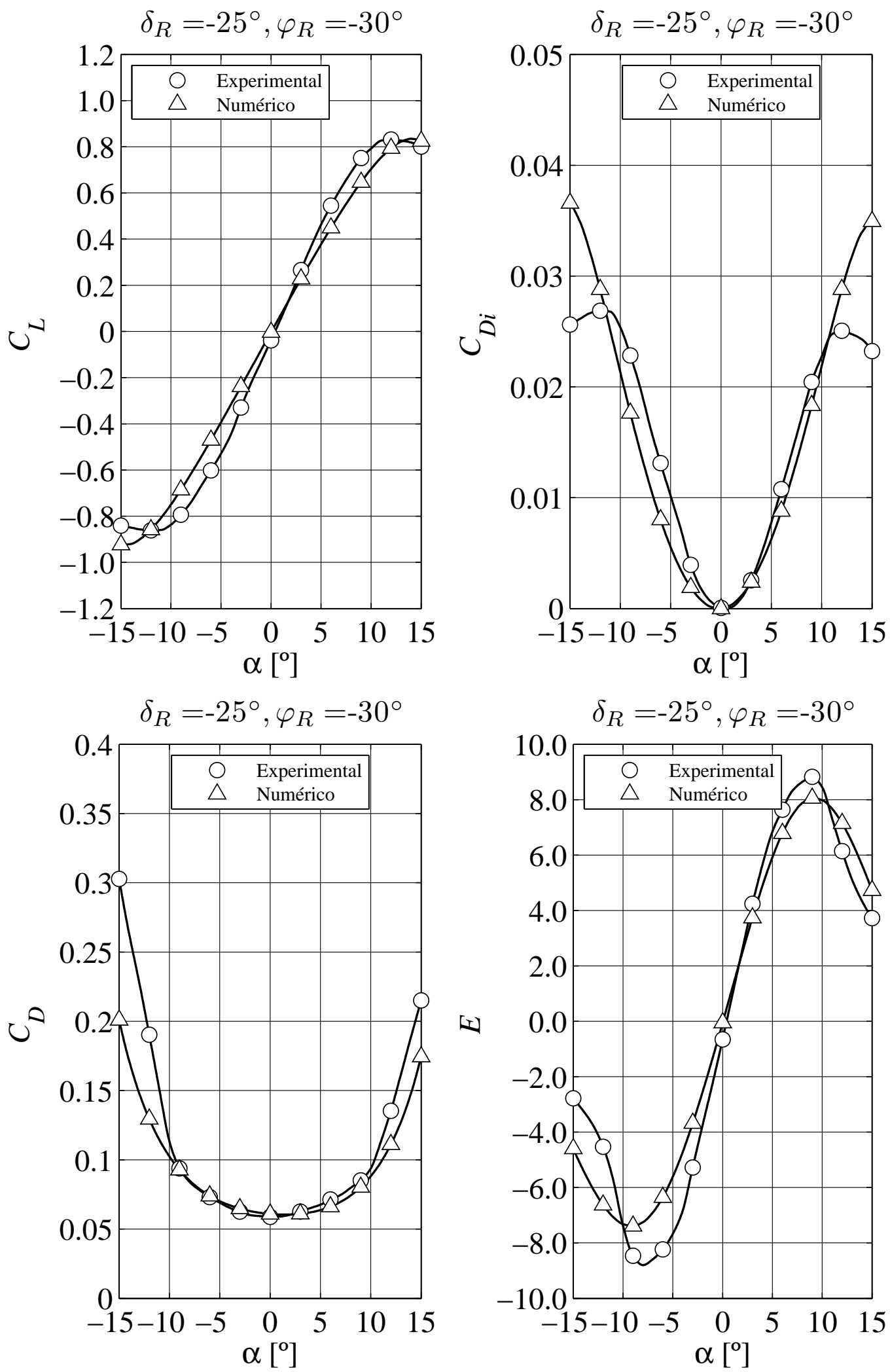

Figura E.25: Variación de los valores de los coeficientes de sustentación, $C_{L}$, de resistencia inducida, $C_{D i}$, de resistencia, $C_{D}$, y de eficiencia aerodinámica, $E$, en función del ángulo de ataque, $\alpha$, para la configuración de ensayo $\operatorname{JWC}_{55}\left(\varphi_{R}=-30^{\circ}\right.$ y $\delta_{R}=$ $-25^{\circ}$ ). Comparación entre el modelo numérico y los resultados experimentales. 
